\title{
AN INTEGRATED MODELING FRAMEWORK FOR CARBON MANAGEMENT TECHNOLOGIES
}

Volume 1 - Technical Documentation: Amine-Based $\mathrm{CO}_{2}$ Capture and Storage Systems

for Fossil Fuel Power Plant

Final Report of

Work Performed Under Contract No.: DE-FC26-00NT40935

Reporting Period Start, October 2000

Reporting Period End, December 2003

Report Submitted, March 2004

to

U.S. Department of Energy

National Energy Technology Laboratory

626 Cochrans Mill Road, P.O. Box 10940

Pittsburgh, Pennsylvania 15236-0940

by

Anand B. Rao

Edward S. Rubin

Michael B. Berkenpas

Carnegie Mellon University

Center for Energy and Environmental Studies

Department of Engineering and Public Policy

Pittsburgh, PA 15213-3890 


\section{DISCLAIMER}

This report was prepared as an account of work sponsored by an agency of the United States Government. Neither the United States Government nor any agency thereof, nor any of their employees, makes any warranty, express or implied, or assumes any legal liability or responsibility for the accuracy, completeness, or usefulness of any information, apparatus, product, or process disclosed, or represents that its use would not infringe privately owned rights. Reference herein to any specific commercial product, process, or service by trade name, trademark, manufacturer, or otherwise does not necessarily constitute or imply its endorsement, recommendation, or favoring by the United States Government or any agency thereof. The views and opinions of authors expressed herein do not necessarily state or reflect those of the United States Government or any agency thereof. 


\section{ACKNOWLEDGEMENTS}

This report is an account of research sponsored by the U.S. Department of Energy's National Energy Technology Center (DOE/NETL) under Contract No. DE-FC2600NT40935. Additional support for this research was provided by the Carnegie Mellon University Center for Integrated Study of the Human Dimensions of Global Change under a Cooperative Agreement with the National Science Foundation (SBR-9521914), and by The Heinz Foundation Teresa Heinz Scholarship for Environmental Research.

Special thanks are due to members of the Project Advisory Team: Manoj Guha (AEP), Howard Herzog (MIT), Richard Rhudy (EPRI) and Dale Simbeck (SFA Pacific), and also to Jack Dingman (Optimized Gas Treating, Inc.) and Carl Mariz (Fluor Daniel) for their assistance and insights in the modeling of amine-based systems. Valuable support and assistance also were provided by Adisorn (Andy) Aroonwilas (U. Regina), Paul Feron (TNO), Bill Gunter (Alberta Research Council), J.C. Gupta (ABB), Neeraj Gupta (Battelle), Chris Hendriks (Ecofys), Masaki Iijima (MHI), Nobuo Imai (MHI), Mohamed Kanniche (EDF), Gelein de Koeijer (Statoil), Gary Rochelle (U. Texas-Austin), Bob Stobbs (Sask Power), Brian Strazisar (DOE/NETL), Hallvard Svendsen (NTNU), John Topper (IEA GHG), Frank van Bergen (TNO), Amomvadee Veawab (U. Regina), David Vogel (ABB), Malcom Wilson (U. Regina), and Sam Wong (Alberta Research Council). Finally, we would like to express sincere appreciation for the support and guidance of the

DOE/NETL project managers Sean Plasynski and Karen Cohen. Responsibility for the content of this report, however, remains solely with the authors of this report. 


\begin{abstract}
$\mathrm{CO}_{2}$ capture and storage (CCS) is gaining widespread interest as a potential method to control greenhouse gas emissions from fossil fuel sources, especially electric power plants. Commercial applications of $\mathrm{CO}_{2}$ separation and capture technologies are found in a number of industrial process operations worldwide. Many of these capture technologies also are applicable to fossil fuel power plants, although applications to large-scale power generation remain to be demonstrated. This report describes the development of a generalized modeling framework to assess alternative $\mathrm{CO}_{2}$ capture and storage options in the context of multi-pollutant control requirements for fossil fuel power plants. The focus of the report is on post-combustion $\mathrm{CO}_{2}$ capture using amine-based absorption systems at pulverized coal-fired plants, which are the most prevalent technology used for power generation today. The modeling framework builds on the previously developed Integrated Environmental Control Model (IECM). The expanded version with carbon sequestration is designated as IECM-cs. The expanded modeling capability also includes natural gas combined cycle (NGCC) power plants and integrated coal gasification combined cycle (IGCC) systems as well as pulverized coal (PC) plants.
\end{abstract}

This report presents details of the performance and cost models developed for an aminebased $\mathrm{CO}_{2}$ capture system, representing the baseline of current commercial technology. The key uncertainties and variability in process design, performance and cost parameters which influence the overall cost of carbon mitigation also are characterized.

The new performance and cost models for $\mathrm{CO}_{2}$ capture systems have been integrated into the IECM-cs, along with models to estimate $\mathrm{CO}_{2}$ transport and storage costs. The $\mathrm{CO}_{2}$ control system also interacts with other emission control technologies such as flue gas desulfurization (FGD) systems for $\mathrm{SO}_{2}$ control. The integrated model is applied to study the feasibility and cost of carbon capture and sequestration at both new and existing PC plants as well as new NGCC plants. The cost of $\mathrm{CO}_{2}$ avoidance using amine-based $\mathrm{CO}_{2}$ capture technology is found to be sensitive to assumptions about the reference plant design and operation, as well as assumptions about the $\mathrm{CO}_{2}$ capture system design. The 
case studies also reveal multi-pollutant interactions and potential tradeoffs in the capture of $\mathrm{CO}_{2}, \mathrm{SO}_{2}, \mathrm{NO}_{2}$ and $\mathrm{NH}_{3}$.

The potential for targeted $\mathrm{R} \& \mathrm{D}$ to reduce the cost of $\mathrm{CO}_{2}$ capture also is explored using the IECM-cs in conjunction with expert elicitations regarding potential improvements in key performance and cost parameters of amine-based systems. The results indicate that the performance of amine-based $\mathrm{CO}_{2}$ capture systems can be improved significantly, and the cost of $\mathrm{CO}_{2}$ capture reduced substantially over the next decade or two, via innovations such as new or improved sorbents with lower regeneration heat requirements, and improvements in power plant heat integration to reduce the (currently large) energy penalty of $\mathrm{CO}_{2}$ capture. Future work will explore in more detail a broader set of advanced technology options to lower the costs of $\mathrm{CO}_{2}$ capture and storage.

Volume 2 of this report presents a detailed User's Manual for the IECM-cs computer model as a companion to the technical documentation in Volume 1. 


\section{Table of Contents}

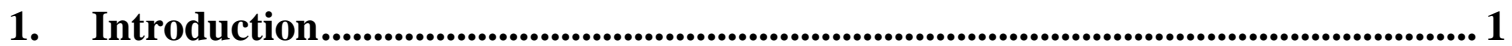

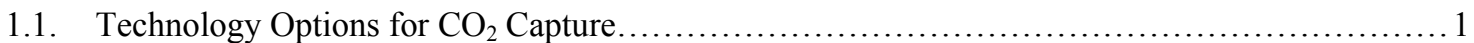

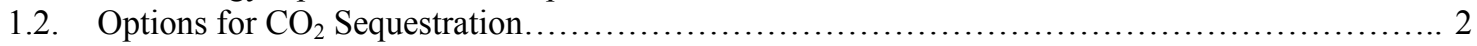

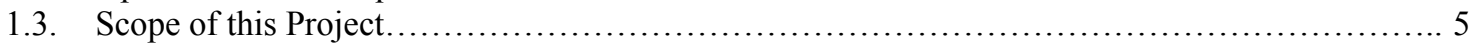

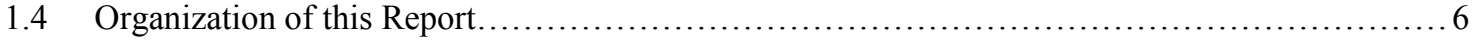

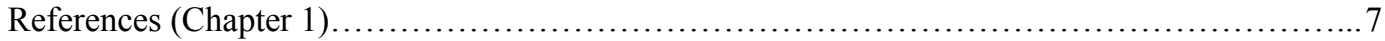

2. Amine-based $\mathrm{CO}_{2}$ Capture Systems: An overview ............................................ 9

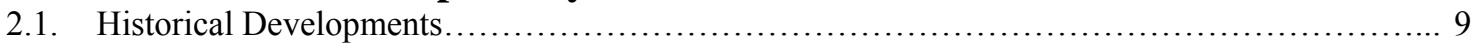

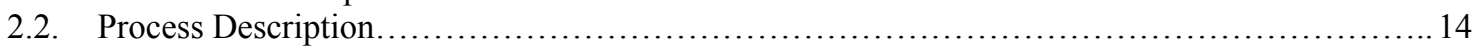

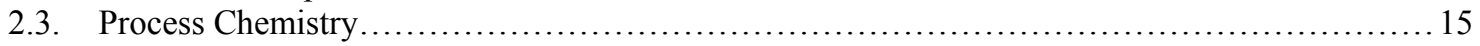

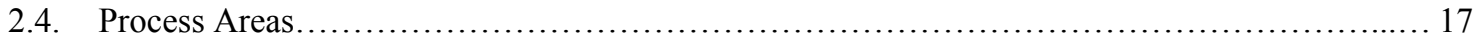

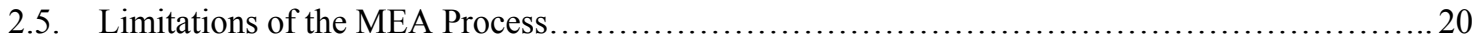

References (Chapter 2) 23

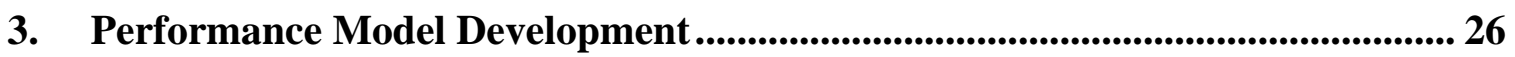

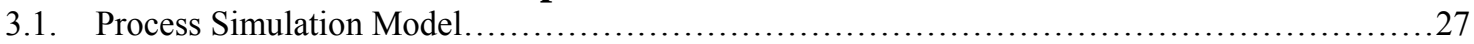

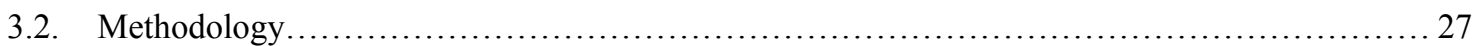

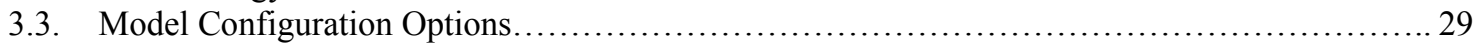

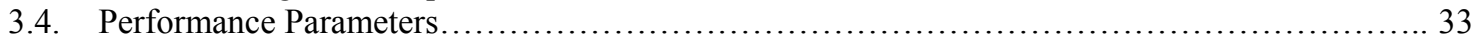

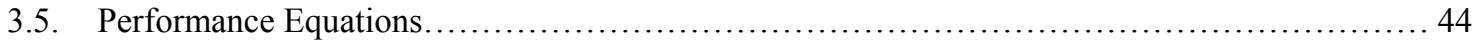

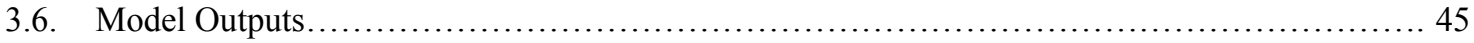

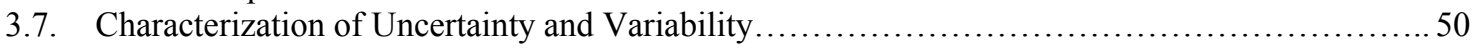

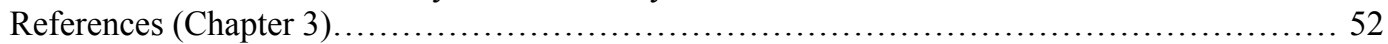

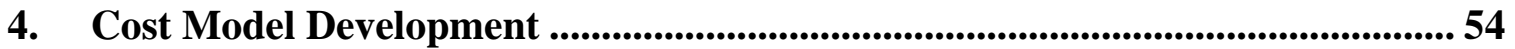

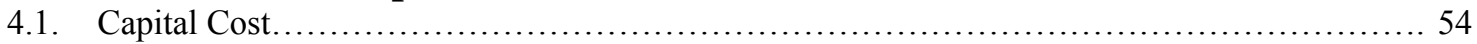

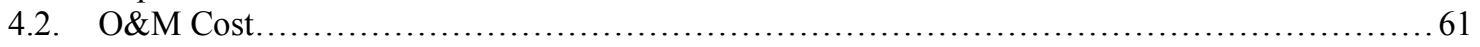

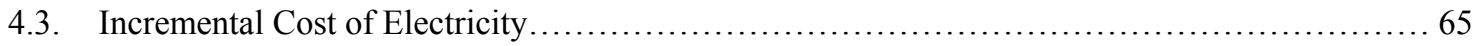

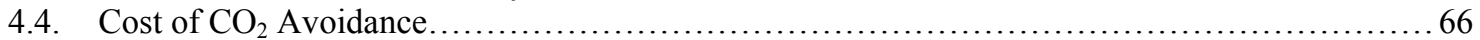

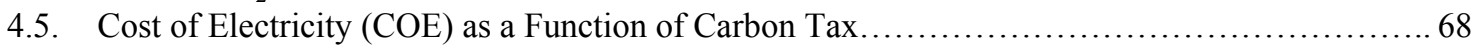

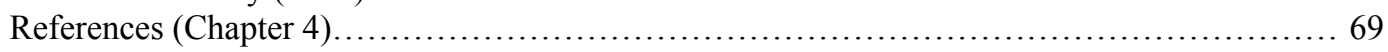

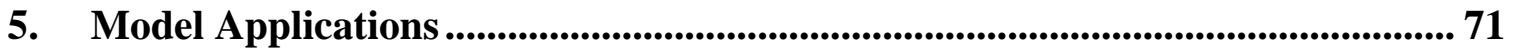

5.1. Case Study of a New Coal-fired Power Plant............................................... 72

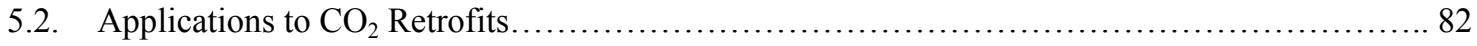

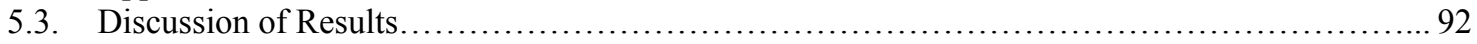

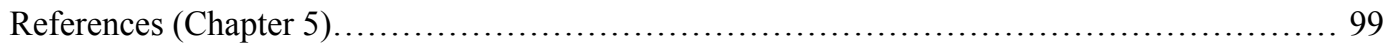

6. R\&D Management: Potential Improvements and Cost Reduction.................. 100

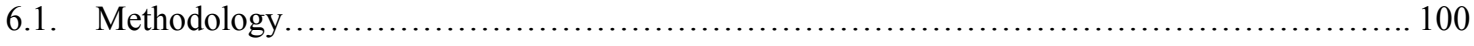

6.2. Expert Judgments on Current Amine-based $\mathrm{CO}_{2}$ Capture Systems......................... 103

6.3. Expert judgments on Future Amine-based $\mathrm{CO}_{2}$ Capture Systems.......................... 105

6.4. Estimation of Future Cost Reductions..................................................... 113

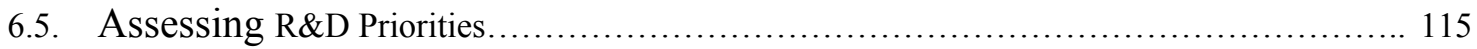

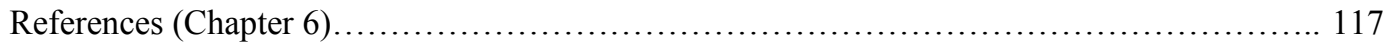




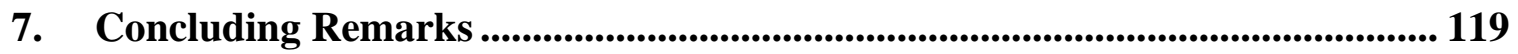

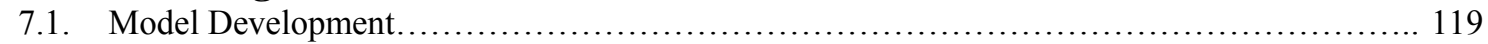

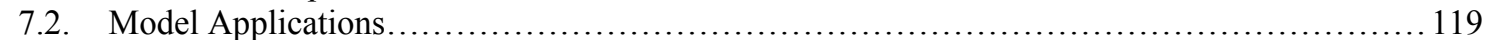

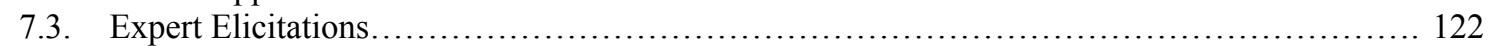

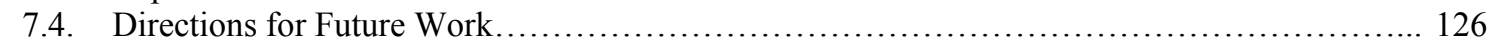

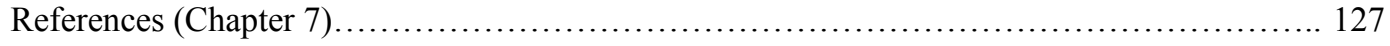

Appendix A. Potential Environmental Impacts of Amine Systems............................... 128

Appendix B. Characterization of Uncertainty and Variability ...................................... 134

Appendix C. Probability Distributions for Amine System Model Parameters.............. 139

Appendix D. Case Study Results for New Coal Plants …………………………….... 151

Appendix E. Questionnaire to Experts on Amine-Based $\mathrm{CO}_{2}$ Capture Technology ..... 154 


\section{List of Figures}

Figure 1.1. Technology options for $\mathrm{CO}_{2}$ separation and capture …....................................... 2

Figure 1.2. Technology options for fossil-fuel based power generation ..................................... 4

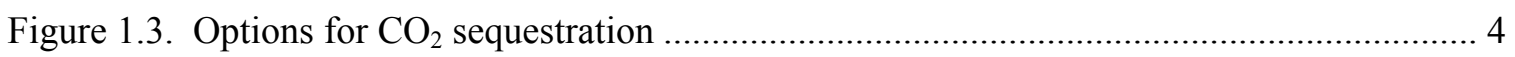

Figure 2.1. Major industrial applications of amine-based $\mathrm{CO}_{2}$ capture systems ......................... 11

Figure 2.2. Flowsheet for $\mathrm{CO}_{2}$ capture from flue gases using amine-based system................... 15

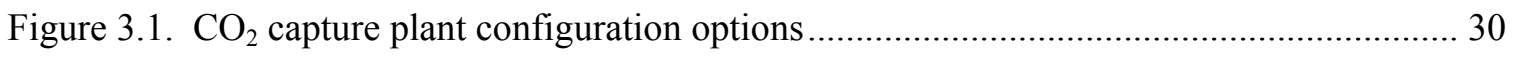

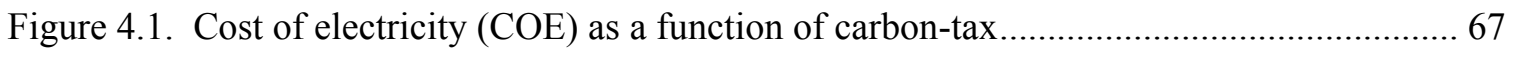

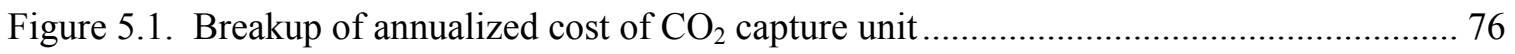

Figure 5.2. Estimation of $\mathrm{CO}_{2}$ avoidance cost for a new low-S coal plant ............................... 77

Figure 5.3. Probability distribution for the $\mathrm{CO}_{2}$ avoidance cost, with only the uncertainties in performance parameters of amine system considered .......................................... 80

Figure 5.4. Probability distribution for the $\mathrm{CO}_{2}$ avoidance cost, with the uncertainties in performance and cost parameters of amine system considered

Figure 5.5. Effect of considering three different options for $\mathrm{CO}_{2}$ storage/disposal, assuming either no cost $(\mathrm{A}(0))$, storage cost of $\$ 5 /$ tonne $\mathrm{CO}_{2}(\mathrm{~B}(5))$, or revenue of $\$ 18 /$ tonne $\mathrm{CO}_{2}$ from EOR $(\mathrm{C}(-18))$, on the distribution of the $\mathrm{CO}_{2}$ avoidance cost (with uncertainties in amine system parameters)

Figure 5.6. Effect of considering uncertainty in base plant parameters (viz. base plant gross cycle heat rate, plant capacity factor, fixed charge factor and coal price) on the distribution of $\mathrm{CO}_{2}$ avoidance cost derived in Figure 5.5 81

Figure 5.7. Comparison of $\mathrm{CO}_{2}$ retrofitting options: cost of electricity ................................... 93

Figure 5.8. Comparison of $\mathrm{CO}_{2}$ retrofitting options: cost of $\mathrm{CO}_{2}$ avoidance …........................ 93

Figure 5.9. Estimation of $\mathrm{CO}_{2}$ avoidance cost for pulverized coal plants: greenfield vs. retrofit

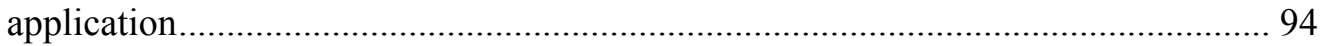

Figure 5.10. Effect of natural gas price on $\mathrm{COE}$ in $\mathrm{CO}_{2}$ retrofit applications .......................... 95

Figure 5.11. Effect of natural gas price on $\mathrm{CO}_{2}$ avoidance cost in $\mathrm{CO}_{2}$ retrofit applications....... 96

Figure 5.12. Comparison of the cost of $\mathrm{CO}_{2}$ control from a new NGCC and a new PC plant (the numbers in the circles represent the cost of $\mathrm{CO}_{2}$ avoidance) 
Figure 6.1. Experts' affiliations $(\mathrm{N}=12)$

Figure 6.2. Expert judgements on current commercial amine systems for $\mathrm{CO}_{2}$ capture from flue gases: absorber inlet flue gas temperature $(\operatorname{deg} \mathrm{C})$

Figure 6.3. Expert judgements on current commercial amine systems for $\mathrm{CO}_{2}$ capture from flue gases: absorber inlet flue gas pressure $(\mathrm{kPa})$

Figure 6.4. Expert on current commercial amine systems for $\mathrm{CO}_{2}$ capture from flue gases: lean sorbent $\mathrm{CO}_{2}$ loading (mole $\mathrm{CO}_{2} /$ mole MEA).....

Figure 6.5. Expert on current commercial amine systems for $\mathrm{CO}_{2}$ capture from flue gases: energy required for $\mathrm{CO}_{2}$ compression to $2000 \mathrm{psig}\left(\mathrm{kWh} /\right.$ tonne $\left.\mathrm{CO}_{2}\right)$......

Figure 6.6. Expert judgments on future commercial amine systems: Parameter 1: sorbent regeneration heat $\left(\mathrm{kJ} / \mathrm{kgCO}_{2}\right)$

Figure 6.7. Expert judgments on future commercial amine systems: Parameter 2: sorbent concentration $(\% \mathrm{w} / \mathrm{w})$

Figure 6.8. Expert judgments on future commercial amine systems: Parameter 3: sorbent loss (kg $\mathrm{MEA} /$ tonne $\mathrm{CO}_{2}$ )

Figure 6.9. Expert judgments on future commercial amine systems: Parameter 4: sorbent loss (US\$/ tonne $\mathrm{CO}_{2}$ )

Figure 6.10. Sorbent regeneration heat requirement $\left(\mathrm{kj} / \mathrm{kgco}_{2}\right)$ : current baseline vs. future projections.

Figure 7.1. Probabilistic estimate of future cost reduction 


\section{List of Tables}

Table 1.1. Comparison of technology options for $\mathrm{CO}_{2}$ separation and capture ............................ 3

Table 3.1. ProTreat parameter ranges (total number of simulation runs $=1983) \ldots \ldots \ldots \ldots \ldots \ldots \ldots . . . . .28$

Table 3.2. Removal efficiency of acid gases due to MEA sorbent $\left(90 \% \mathrm{CO}_{2}\right.$ removal $) \ldots \ldots \ldots \ldots . . . . .37$

Table 3.3. Amine system performance model parameters and uncertainties .............................. 51

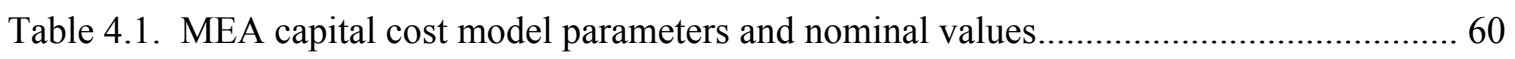

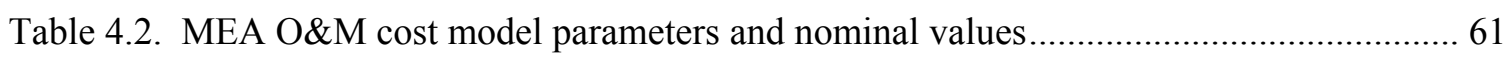

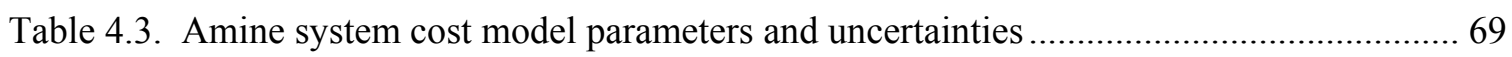

Table 5.1. Design parameters for case study of new pulverized coal plant................................ 72

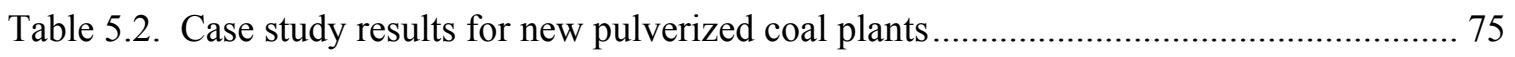

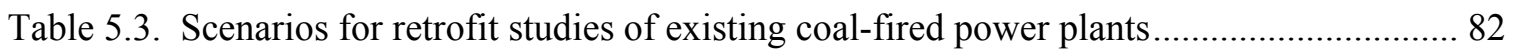

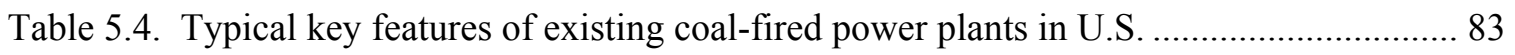

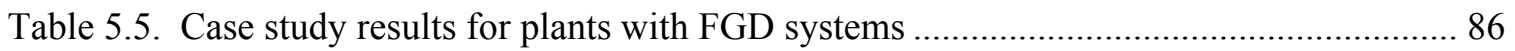

Table 5.6. Case study results for plants without FGD systems: sensitivity analysis .................... 87

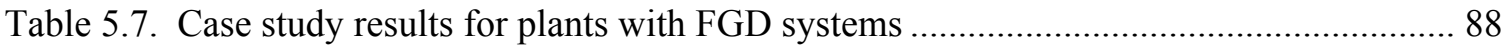

Table 5.8. Case study results for plants with FGD systems: sensitivity analysis ........................ 89

Table 5.9. Comparison of amine-based $\mathrm{CO}_{2}$ capture and IGCC re-powering options for an existing pulverized coal plant .................................................................................. 91

Table 5.10. Comparison of $\mathrm{CO}_{2}$ control from a new NGCC and a new PC plant...................... 97

Table 6.1. Relative improvement in sorbent regeneration heat requirement............................ 111

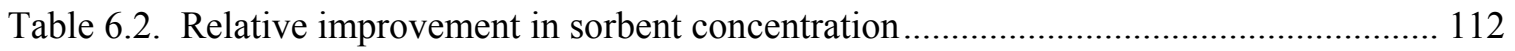

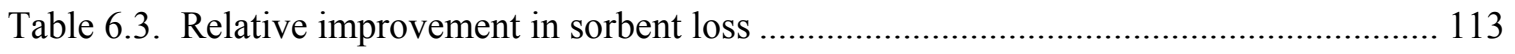

Table 6.4. Relative improvement in sorbent cost ............................................................. 113

Table 6.5. Expected reduction in capital cost from improvements in four process parameters (relative to current baseline) .............................................................................. 114 
Table 6.6. Expected reduction in incremental COE from improvements in four process parameters (relative to current baseline)

Table 6.7. Expected reduction in cost of $\mathrm{CO}_{2}$ avoidance from improvements in four process parameters (relative to current baseline) ............................................................... 114

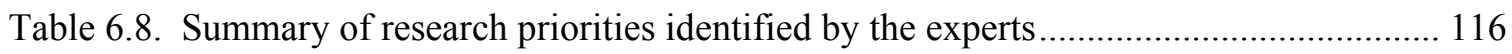

Table 7.1. Relative improvement in amine-system parameters............................................ 123

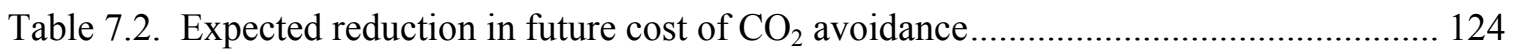




\section{INTRODUCTION}

Today fossil fuels provide a major share of the total electricity generated in the United States The $300 \mathrm{GW}$ of coal-fired power generation capacity in the U.S. provides $51 \%$ of all power generation and accounts for $79 \%$ of carbon emissions coming from electric utilities (EIA 2000). Even with the expected growth in natural gas for new generating capacity, coal's share of the electricity supply is still projected to be about $44 \%$ in 2020 , and higher in absolute amount compared to today, according to recent DOE/EIA projections. Natural gas use is projected to account for $31 \%$ of power generation in 2020 . At the same time, there is significant concern about the potential climate change impacts of increased $\mathrm{CO}_{2}$ emissions to the atmosphere, to which fossil fuel power plants are the largest contributor. Thus, any new policies to significantly reduce $\mathrm{CO}_{2}$ emissions during the next few decades must consider not only the technology options for new power plants, but also the retrofitting or repowering of existing coal and natural gas plants which will continue to operate for several decades to come. In all cases, improved modeling tools are needed to reliably estimate the performance and cost of alternative options, including the potential use of $\mathrm{CO}_{2}$ capture and storage (CCS) as a $\mathrm{CO}_{2}$ reduction strategy.

\subsection{Technology Options for $\mathrm{CO}_{2}$ Capture}

A wide range of technologies currently exist for separation and capture of $\mathrm{CO}_{2}$ from gas streams, although they have not been designed for power plant scale operations (Desideri and Corbelli 1998). Figure 1.1 and Table 1.1 briefly summarize the salient features of these technology options (Riemer, Audus et al. 1993; Hendriks 1994; Mimura, Satsumi et al. 1999; Audus 2000; Jeremy 2000; White, Strazisar et al. 2003). They are based on different physical and chemical processes including absorption, adsorption, membranes and cryogenics. Apart from these four types of processes that generate a concentrated stream of $\mathrm{CO}_{2}$ product (which needs to be sequestered later), there is another set of biological systems (e.g. forestry, microbial/ algal processes etc.) that capture $\mathrm{CO}_{2}$ and bio-chemically transform it into some other compounds (and hence also get sequestered simultaneously). 
The choice of a suitable technology depends upon the characteristics of the gas stream from which $\mathrm{CO}_{2}$ needs to be separated, which mainly depends on the power plant technology. At present, the majority of the plants in the U.S. are conventional combustion-based plants that generate a flue gas that is a dilute stream of $\mathrm{CO}_{2}$ due to large amount of nitrogen in the combustion air. Future power plants may be designed so as to separate out $\mathrm{CO}_{2}$ from coal before combustion (e.g. coal gasification systems), or they may employ pure oxygen combustion instead of air so as to obtain a concentrated $\mathrm{CO}_{2}$ stream for treatment. Error! Reference source not found. shows the variety of power plant fuels and technologies that affect the choice of $\mathrm{CO}_{2}$ capture systems.

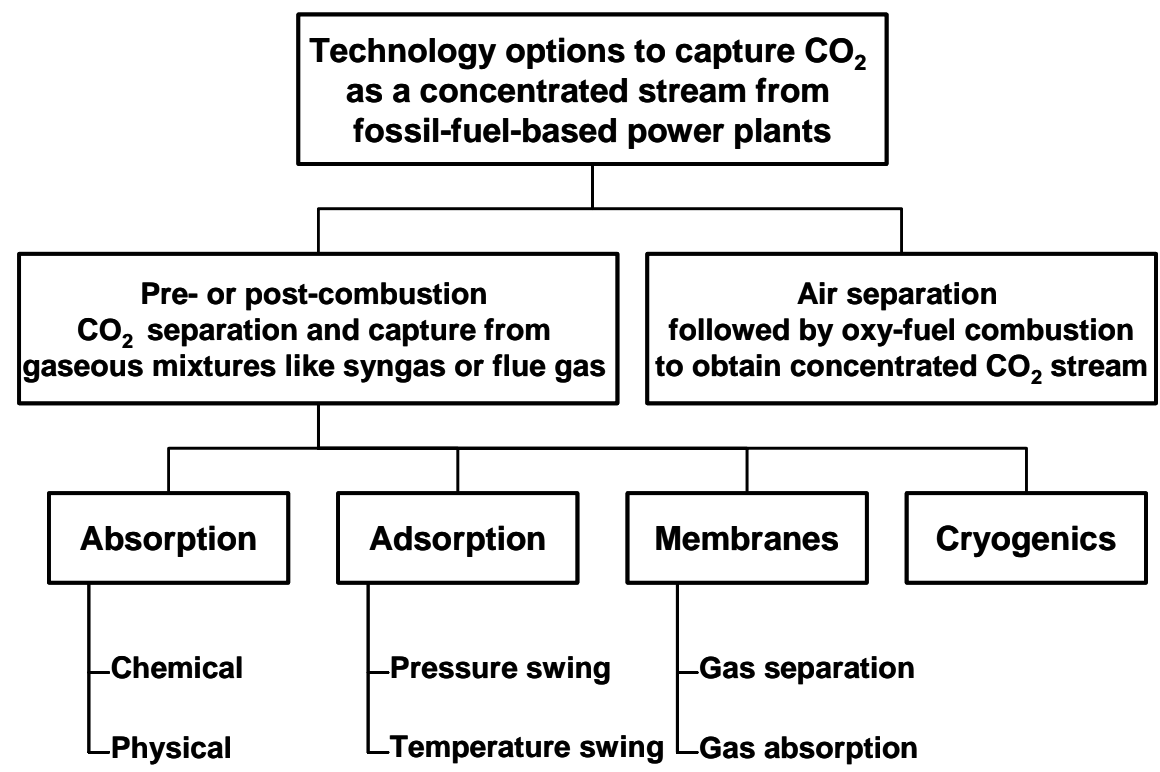

Figure 1.1. Technology options for $\mathrm{CO}_{2}$ separation and capture

\subsection{Options for $\mathrm{CO}_{2}$ Sequestration}

Once the $\mathrm{CO}_{2}$ is captured, it needs to be securely stored (sequestered). Again, there is a range of options potentially available, as shown in Error! Reference source not found.. Geologic formations including deep saline reservoirs, depleted oil and gas wells, and unmineable coal seams are some of the potentially attractive disposal sites (Adams, Ormerod et al. 1993; Byrer and Guthrie 1998; Lindeberg and Holloway 1998). Some of these options, such as enhanced oil recovery (EOR) and enhanced coal bed methane 
(ECBM), can be revenue generating as well, as they lead to production of valuable products like oil and methane,.

Table 1.1. Comparison of technology options for $\mathrm{CO}_{2}$ separation and capture

\begin{tabular}{|c|c|c|c|}
\hline $\begin{array}{l}\text { Technology } \\
\text { Option }\end{array}$ & $\begin{array}{c}\text { System } \\
\text { Requirements }\end{array}$ & Advantages & Problems/ Drawbacks \\
\hline $\begin{array}{l}\text { Absorption } \\
\text { (Chemical) }\end{array}$ & $\begin{array}{l}\text { Absorber and } \\
\text { stripper sections } \\
\text { Chemical sorbent } \\
\text { (e.g. MEA, HPC) }\end{array}$ & $\begin{array}{l}\text { Suitable even for dilute } \\
\mathrm{CO}_{2} \text { streams (typical flue } \\
\text { gas from power plants) } \\
\text { Operates at ordinary } \\
\text { temperature \& pressure } \\
\text { Commercially available, } \\
\text { proven technology }\end{array}$ & $\begin{array}{l}\text { The heat of sorbent } \\
\text { regeneration is very } \\
\text { high } \\
\text { Significant sorbent } \\
\text { losses; pre-processing } \\
\text { (e.g. S removal) may } \\
\text { be required }\end{array}$ \\
\hline $\begin{array}{l}\text { Absorption } \\
\text { (Physical) }\end{array}$ & $\begin{array}{l}\text { Absorber and } \\
\text { stripper sections } \\
\text { Physical sorbent } \\
\text { (e.g. Selexol) }\end{array}$ & $\begin{array}{l}\text { Suitable only for gas } \\
\text { streams with high partial } \\
\text { pressure of } \mathrm{CO}_{2} \text { (typical } \\
\text { syngas from gasification } \\
\text { systems) } \\
\text { Less energy required, } \\
\text { assuming that the gas } \\
\text { stream to be processed is } \\
\text { already at high pressure } \\
\text { Sorbents are less } \\
\text { susceptible to the } \\
\text { impurities in the gas } \\
\text { stream }\end{array}$ & $\begin{array}{l}\text { Requires high } \\
\text { operating pressure } \\
\text { Works better only with } \\
\text { gas streams having } \\
\text { high } \mathrm{CO}_{2} \text { content; so it } \\
\text { is not suitable for flue } \\
\text { gas processing }\end{array}$ \\
\hline Adsorption & Adsorber bed(s) & $\begin{array}{l}\text { Commercially available } \\
\text { gas separation process }\end{array}$ & $\begin{array}{l}\text { Low capacity and } \mathrm{CO}_{2} \\
\text { selectivity of available } \\
\text { adsorbents }\end{array}$ \\
\hline Membranes & $\begin{array}{l}\text { Membrane } \\
\text { filter(s) }\end{array}$ & $\begin{array}{l}\text { Upcoming, promising } \\
\text { technology with diverse } \\
\text { applications } \\
\text { Space efficient }\end{array}$ & $\begin{array}{l}\text { Requires high } \\
\text { operating pressures } \\
\text { Lower product purity; } \\
\text { need for multiple } \\
\text { stages/ recycle }\end{array}$ \\
\hline Cryogenics & $\begin{array}{l}\text { Refrigeration and } \\
\text { distillation units }\end{array}$ & $\begin{array}{l}\text { Direct production of } \\
\text { liquid } \mathrm{CO}_{2}\end{array}$ & $\begin{array}{l}\text { Requires very large } \\
\text { amount of energy for } \\
\text { refrigeration (not } \\
\text { suitable for dilute } \\
\text { streams) }\end{array}$ \\
\hline
\end{tabular}




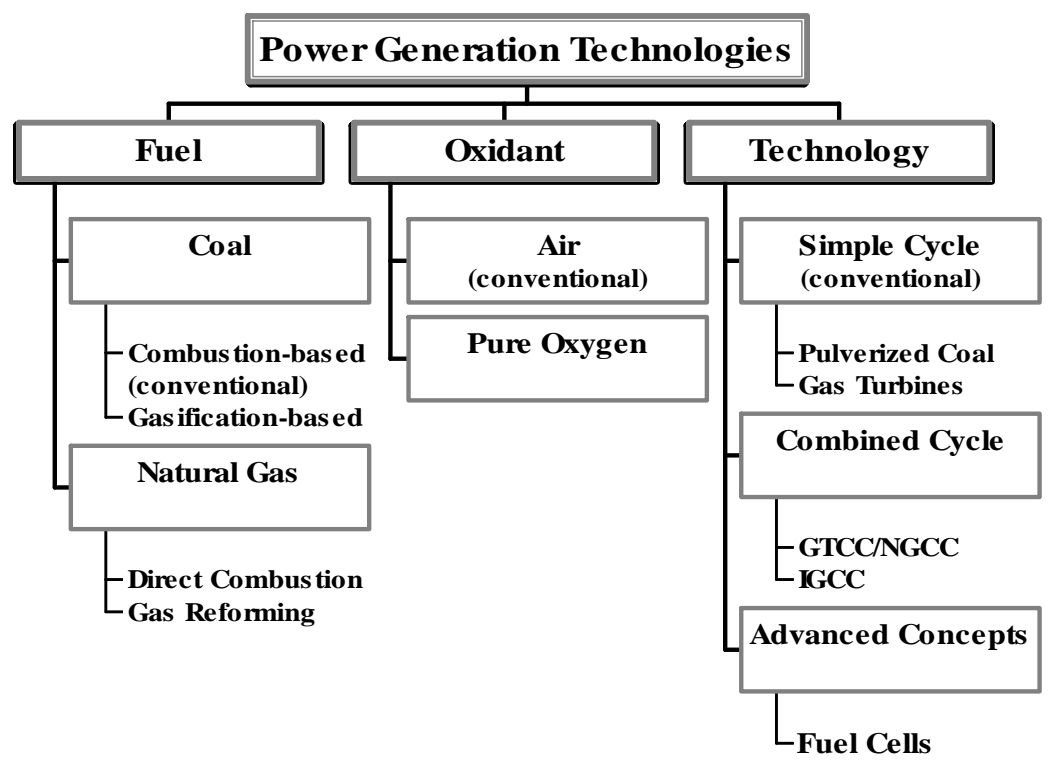

Figure 1.2. Technology options for fossil-fuel based power generation

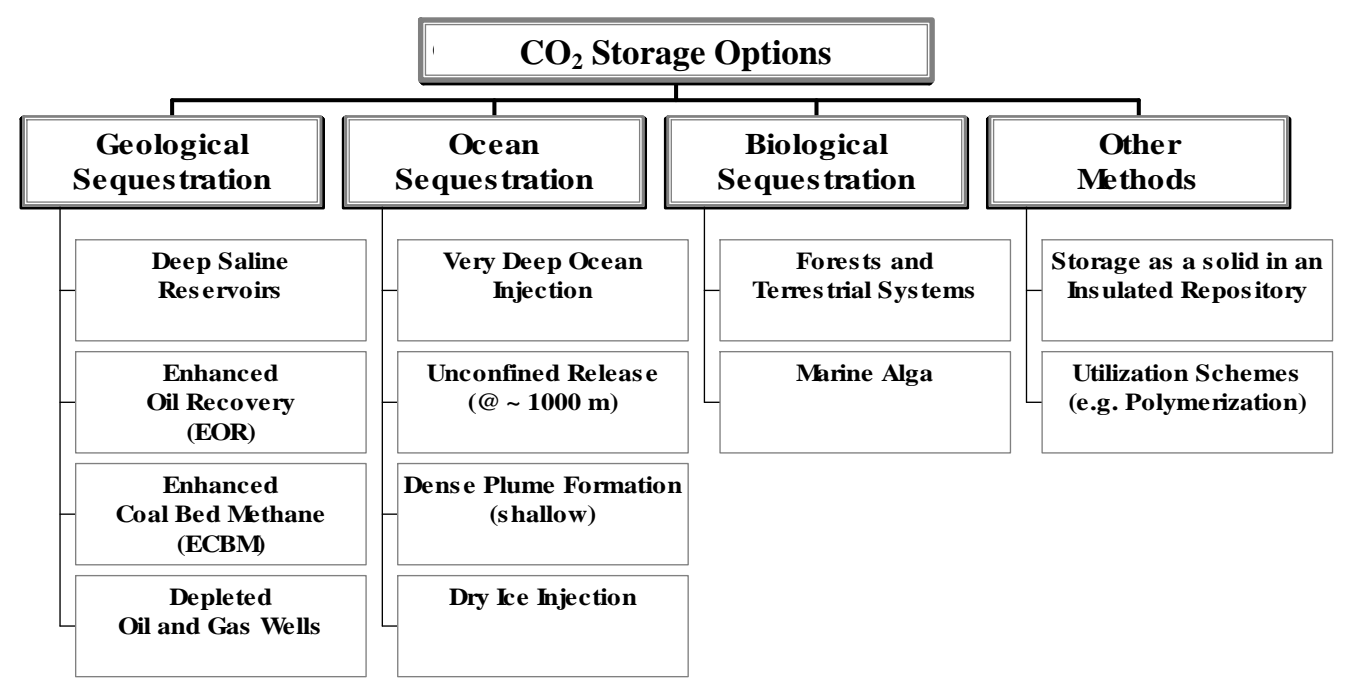

Figure 1.3. Options for $\mathrm{CO}_{2}$ sequestration

respectively. Ocean storage is another option being studied (Herzog 1998; Spencer 1998)

The distance to a secure storage site, the availability and cost of transportation infrastructure, and the regulatory framework all affect the choice of disposal option. In general, studies indicate that geologic formations are the most plentiful and attractive 
option for U.S. power plants (Bergman and Winter 1995). While the economic costs of $\mathrm{CO}_{2}$ storage appear to be low, its social and political acceptability are not yet clear, especially with regard to ocean sequestration (CO2-Experiment 2001; USDOE/NETL 2001). Issues related to geologic sequestration of $\mathrm{CO}_{2}$, including. potential sites, storage capacities, environmental impacts, monitoring requirements and leakage are well summarized elsewhere (White, Strazisar et al. 2003).

\subsection{Scope of this Project}

Efforts by DOE/NETL and others to develop new or improved technologies for $\mathrm{CO}_{2}$ capture and storage (CCS) can be assisted substantially by improved analytical tools to assess the overall effectiveness, costs, and carbon sequestration potential of alternative CCS approaches. Especially important for R\&D planning and management is an ability to systematically compare advanced systems and concepts to current commercial technologies that also are evolving. To help provide this capability, this project described in this report builds upon the Integrated Environmental Control Model (IECM) developed under a prior DOE/NETL research contract (DE-AC22-92PC91346). The IECM was designed to provide preliminary performance, emissions and cost estimates for alternative environmental control options for coal-fired power plants, with a focus on $\mathrm{SO}_{2}, \mathrm{NO}_{\mathrm{x}}$, particulates and (most recently) mercury emissions. The model simulates a single userspecified facility, and is built in a modular fashion that allows models of individual technologies to be incorporated into the overall framework. A user can then select different options to configure and evaluate a particular plant design involving current commercial technology or advanced emission control systems.

The current project extends the IECM capabilities to include technology options for carbon management. This version of the model is referred to as the IECM-cs. The CCS options include a set of "baseline" technologies representing currently available fossilfuel power plants, plus a selected set of advanced process designs that promise improved performance and/or lower cost. In addition to coal combustion plants, the IECM-cs framework has been expanded to include natural gas combined cycle (NGCC) plants plus integrated coal gasification combined cycle (IGCC) systems. Pipeline transport and alternative $\mathrm{CO}_{2}$ storage options also have been added. The IECM-cs thus provides a 
comprehensive yet easy-to-use modeling tool for evaluating technological options in a total systems context.

The focus of this report is on the modeling of CCS systems for a pulverized coal-fired (PC) power plant, which is the most widely used technology for electric power generation. Models of $\mathrm{NGCC}$ and IGCC plants with $\mathrm{CO}_{2}$ capture systems are undergoing further refinement as part of a continuing project which will issue reports on these technologies later in 2004. As detailed in the section below, the scope of the present report covers the development and illustrative applications of the baseline coal-fired power plant model employing an amine $\mathrm{CO}_{2}$ capture system together with pipeline transport to a geological storage site. An advanced post-combustion $\mathrm{CO}_{2}$ capture system design also is developed and evaluated to assess the potential benefits of R\&D. Further details on the design and use of the IECM-cs software package can be found in Volume 2 of this report, which provides a complete User's Manual for the coal-fired plant model.

Some of the key research questions that are addressed in this report include:

- What technologies and design configurations are most suitable for capture and storage of $\mathrm{CO}_{2}$ from coal or gas-fired power plants?

- What are the key parameters that affect the performance, cost and environmental acceptability of different options?

- How do alternative options compare in terms of these considerations?

- What are the technical and economic uncertainties associated with different options?

- What are the potential benefits of continued R\&D on CCS technology?

\subsection{Organization of this Report}

The organization of this report is as follows. Chapter 2 gives a brief overview of aminebased $\mathrm{CO}_{2}$ capture systems, including historical developments and process description. Chapter 3 documents the performance model developed in this research, including the model configurations, methodology, parameters and performance equations. Chapter 4 next describes the associated cost model, which is directly linked to the performance model. Then, Chapter 5 illustrates the applications of the new performance and cost models. Several case studies applying current amine-based $\mathrm{CO}_{2}$ capture technology to coal and natural gas power plants (new and retrofits) are presented. Following this, 
together with an expert elicitation study, Chapter 6 , uses the model to explore potential future cost reductions and $R \& D$ management. Finally, Chapter 7 presents some concluding remarks and directions to future work. Additional details concerning amine systems, characterization of uncertainties and variability, probability distributions for model parameters, case study results, and expert elicitation study are included as Appendices A-E.

\section{References (Chapter 1)}

Adams, D., W. Ormerod, et al. (1993). Carbon Dioxide Disposal from Power Stations. UK, a report published by IEA Greenhouse Gas R\&D Programme.

Audus, H. (2000). Leading options for the capture of CO2 at power stations. Presented at the Fifth International Conference on Greenhouse Gas Control Technologies, 13-16 August, Cairns, Australia.

Bergman, P. D. and E. M. Winter (1995). "Disposal of carbon dioxide in aquifers in the U.S." Energy Conversion and Management 36(6-9): 523-526.

Byrer, C. W. and H. D. Guthrie (1998). Coal deposits: potential geological sink for sequestering carbon dioxide emissions from power plants. in the proceedings of the AWMA's Second International Specialty Conference, Oct 13-15, 1998, Washington, DC.

CO2-Experiment (2001). Ocean sequestration of CO2: Field experiment (www.co2experiment.org).

Desideri, U. and R. Corbelli (1998). "CO2 capture in small size cogeneration plants: Technical and economical considerations." Energy Conversion and Management 39(9): 857-867.

EIA (2000). Annual Energy Outlook 2001 (with Projections to 2020), A report published by Energy Information Administration, U.S. Department of Energy, Washington, DC.

Hendriks, C. (1994). Carbon Dioxide Removal from Coal-fired Power Plants. The Netherlands, Kluwer Academic Publishers.

Herzog, H. J. (1998). Ocean sequestration of CO2 - an overview. in the proceedings of the AWMA's Second International Specialty Conference, Oct 13-15, 1998, Washington, DC.

Jeremy, D. (2000). Economic Evaluation of Leading Technology Options for Sequestration of Carbon Dioxide, M.S. Thesis, Massachusetts Institute of Technology, Cambridge, MA .

Lindeberg, E. and S. Holloway (1998). The next steps in geo-storage of carbon dioxide. Fourth International Conference on Greenhouse Gas Control Technologies, 30 August - 2 September, Interlaken, Switzerland, Elsevier Science Ltd. 
Mimura, T., S. Satsumi, et al. (1999). Developments on energy saving technology for flue gas carbon dioxide recovery by the chemical absorption method and steam system in power plant. Proceedings of the 4th International Conference on Greenhouse Gas Control Technologies, 30 August - 2 September 1998, Interlaken, Switzerland, Elsevier Science Ltd.

Riemer, P., H. Audus, et al. (1993). Carbon Dioxide Capture from Power Stations. UK, a report published by IEA Greenhouse Gas R\&D Programme.

Spencer, D. F. (1998). Integration of an advanced CO2 separation process with methods for disposing of $\mathrm{CO} 2$ in oceans and terrestrial deep aquifers. Fourth International Conference on Greenhouse Gas Control Technologies, 30 August - 2 September, Interlaken, Switzerland, Elsevier Science Ltd.

USDOE/NETL (2001). Environmental Assessment: Ocean sequestration of $\mathrm{CO}_{2}$ field experiment (report \# DOE/EA-1336), U.S. Department of Energy, National Energy Technology Laboratory (NETL), Pittsburgh, PA 15236.

White, C. M., B. R. Strazisar, et al. (2003). "Separation and capture of CO2 from large stationary sources and sequestration in geological formations - coalbeds and deep saline aquifers." Journal of the Air \& Waste Management Association 53: 645-715. 


\section{AMINE-BASED CO2 CAPTURE SYSTEMS: AN OVERVIEW}

In conventional coal plants, which use air for combustion, $\mathrm{CO}_{2}$ can be separated from the post-combustion flue gas stream. Past studies have shown that amine-based $\mathrm{CO}_{2}$ absorption systems are the most suitable for combustion-based power plants for the following reasons:

- These systems are effective for dilute $\mathrm{CO}_{2}$ streams, such as coal combustion flue gas which typically contains about $10 \%-15 \% \mathrm{CO}_{2}$ by volume.

- Amine-based $\mathrm{CO}_{2}$ capture system is a proven technology that is commercially available today.

- Amine-based systems are similar to other post-combustion environmental control units used at power plants. These units are operated at ordinary temperature and pressure.

- A major effort is being made worldwide to improve this process in the light of its potential role in $\mathrm{CO}_{2}$ abatement. Thus, one can anticipate future benefits from technology advances.

\subsection{Historical Developments}

Although the amine-based $\mathrm{CO}_{2}$ capture technology has gained increasing attention in the recent times, this technology has been around for several decades now. Amine-based absorption/stripping has been a commercial technology for $\mathrm{CO}_{2}$ removal from natural gas and hydrogen (Yeh 2003). The basic amine process was first patented by R. R. Bottom in 1930 as absorbents for acidic gas treating (Bottoms 1930). Alkanolamines (referred to as "amines" in this document) are a family of organic compounds that are derivatives of alkanols (compounds with functional group "OH" group, commonly called as alcoholic group), that also have an "amino" $\left(\mathrm{NH}_{2}\right)$ group attached to one of the carbon atoms. The amine-based gas treating basic process flow scheme has remained relatively unchanged over the years. Members of alkanolamine family were subsequently introduced into gas purification market and still remain the technology of choice for the removal of $\mathrm{H}_{2} \mathrm{~S}$ and $\mathrm{CO}_{2}$ for gas purification (Kohl and Blohm 1950; Kohl and Nielsen 1997). 
The idea of separating $\mathrm{CO}_{2}$ from flue gas streams started back in 1970's, not with concern about the greenhouse effect, but as a possible economic source of $\mathrm{CO}_{2}$, mainly for enhanced oil recovery (EOR) operations. Flue gas, a gaseous product stream resulting from the combustion of fossil fuels in air, mainly contains nitrogen, carbon dioxide, and water vapor along with small quantities of many other gases. Depending upon the carbon content of the fuel and the quantity of air used for combustion of the fuel, the flue gas stream may contain as high as $15 \% \mathrm{CO}_{2}$ and is a potential source of $\mathrm{CO}_{2}$ which is available at no cost. Even today, about $80 \%$ of $\mathrm{CO}_{2}$ production is used for EOR (Chapel et al., 1999), most of which is obtained from natural $\mathrm{CO}_{2}$ domes. $\mathrm{CO}_{2}$ is also produced for other industrial applications such as carbonation of brine and production of products like dry ice, urea and beverages.

Several commercial $\mathrm{CO}_{2}$ capture plants were constructed in the US in the late 1970's and early 1980's (Kaplan 1982; Pauley, Simiskey et al. 1984). Some of these plants are still in operation today. But all these plants are much smaller than a typical power plant in terms of tonnage of $\mathrm{CO}_{2}$ handled. Figure 2.1 gives a rough idea about the various industrial applications of $\mathrm{CO}_{2}$ capture technologies and their relative magnitude of operations.

Unless there is an application that can make use of the captured $\mathrm{CO}_{2}$, it has to be securely stored (sequestered) to prevent it from entering the atmosphere. However, there is little experience with $\mathrm{CO}_{2}$ sequestration technologies other than EOR. The first commercial $\mathrm{CO}_{2}$ sequestration facility started in Norway in September 1996 in response to a Norwegian carbon tax. Since then, Statoil has been storing about 1 million tonnes of $\mathrm{CO}_{2}$ per year from the Sleipner West gas field into a sandstone aquifer $1000 \mathrm{~m}$ beneath the North Sea (DOE 1999; Statoil 2001). The international research community is closely monitoring this facility to enhance the understanding of this geological experiment.

All these plants (Figure 2.1) capture $\mathrm{CO}_{2}$ with processes based on chemical absorption using a monoethanolamine (MEA) based sorbent. MEA is a primary amine with high $\mathrm{pH}$ 


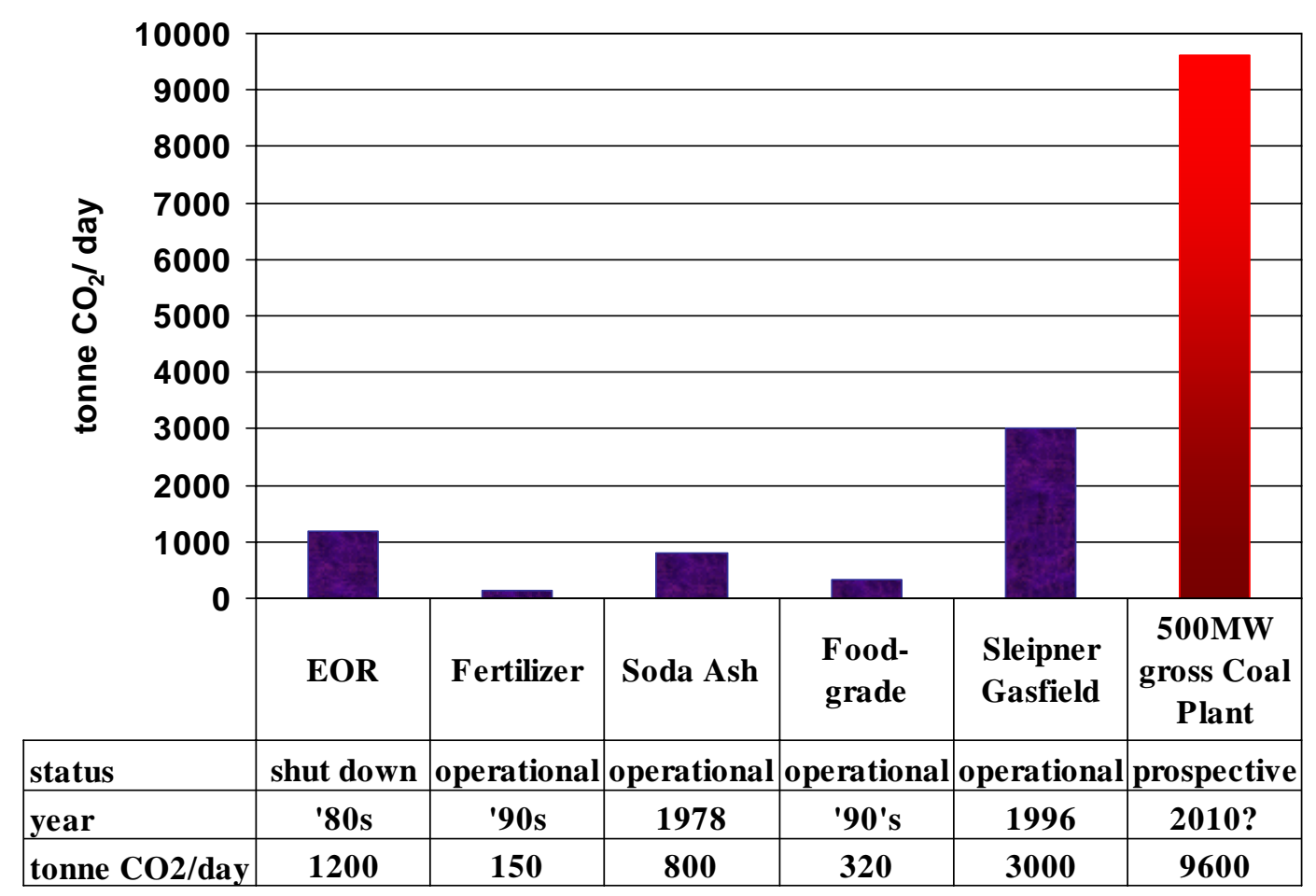

Figure 2.1. Major industrial applications of amine-based $\mathrm{CO}_{2}$ capture systems

and has the lowest molecular weight of the available amines. It is completely soluble in water and is readily biodegradable (Dow 2000). It is a fairly reactive compound and is classified as a corrosive material. It was developed over 60 years ago as a general, nonselective sorbent to remove acidic gas impurities (e.g. $\mathrm{H}_{2} \mathrm{~S}, \mathrm{CO}_{2}$ ) from natural gas streams (Herzog 1998). The process was then adapted to treat flue gas streams for $\mathrm{CO}_{2}$ capture. Dow Chemical Co. (and later Fluor Daniel Inc.), Kerr-McGee Chemical Corp. and ABB Lummus Crest Inc., are some of the key players in the development of MEAbased technology for $\mathrm{CO}_{2}$ capture. About $75 \%-96 \% \mathrm{CO}_{2}$ may be captured using this technology to yield a fairly pure ( $>99 \%) \mathrm{CO}_{2}$ product stream.

Today there are two main MEA-based processes available for commercial $\mathrm{CO}_{2}$ recovery plants: the Fluor Daniel Econamine FG process and the ABB Lummus Crest MEA process (Wong, Gunter et al. 2000). The brief commercial history of these processes is as follows. 


\subsubsection{Fluor Daniel's Econamine FG process}

Dow Chemical and Union Carbide developed inhibited 30 wt.\% MEA processes. These absorption processes use a sorbent containing MEA diluted in water to $30 \% \mathrm{w} / \mathrm{w}$ (by weight) i.e., each $100 \mathrm{~kg}$ of total sorbent contains $30 \mathrm{~kg}$ of MEA, for the recovery of $\mathrm{CO}_{2}$ from flue gases in the 1970's and 1980's primarily for the EOR market. Dow Chemical's

process was known as the GAS/SPEC FT-1 ${ }^{\mathrm{TM}}$ process till 1989. Several plants were built in West Texas to recover $\mathrm{CO}_{2}$ from boiler flue gas for EOR purposes between 1982 and 1986. These plants were technical and commercial successes and were shut down only because of the crude oil price collapse in 1986. As the price of crude oil dropped dramatically in 1986, the EOR market disappeared and these licensors (Dow Chemical and Union Carbide) became less active. Dow Chemical sold its GAS/SPEC FT-1тм process to Fluor Daniel, Inc. in 1989. Fluor Daniel renamed the technology as Econamine $\mathrm{FG}^{\mathrm{sm}}$. Sixteen commercial plants have been built, including nine large plants (60 tonne/d and larger) and seven skid-mounted plants (Chapel, Ernst et al. 1999). Seven of the large plants are still operating. Fluor Daniel licensed three of these commercial plants: a 320 tonne/d $\mathrm{CO}_{2}$ plant in Bellingham, Massachusetts for Northeast Energy Associates, a 150 tonne/d plant for Sumitomo Chemical in Chiba, Japan, and a 90 tonne/d plant for Prosint Produtos Sintéticos in Rio de Janeiro, Brazil. Fluor Daniel also constructed the Bellingham plant. Prior to this, Dow had licensed six commercial plants ranging from 6 to 1000 tonne/d. The seven facilities employing skid-mounted Econamine FG process units have capacities ranging from 6 to 40 tonne/d and were manufactured by the Wittemann Company of Palm Coast, Florida.

It may be noted that all of the large commercial plants use flue gas resulting from the combustion of natural gas, except the Sumitomo plant that fires a variety of fuels including heavy fuel oil. The Econamine FG process has also been demonstrated with coal-derived flue gas in three pilot plants: a 4.5 tonne/d plant in Yokosuka, Japan for Tokyo Electric Power Co, a 2 tonne/d unit at the Sundance Generating Plant in Alberta, and a 4 tonne/d unit at the Boundary Dam Power Plant in Saskatchewan (IEA-GHGRDP 2002). Fluor Daniel has also licensed a natural gas-fired 2 tonne/d pilot plant in Osaka, Japan for KEPCO. Commercial operations at the Warrior Run Power Plant in Maryland 
started in February 2000. The Econamine FG scrubber system is used to capture $\mathrm{CO}_{2}$ from a portion of the flue gases from this 180 MWe AES cogeneration plant that burns only Western Maryland coal with a clean coal technology using a circulating fluidized bed boiler. The extracted $\mathrm{CO}_{2}$ from this commercial-scale production facility is used for food processing and related processes. Recently, Fluor has announced an improved version of this process named as "Fluor Econamine FG Plus" that addresses some of the problems in the Econamine FG process (Reddy and Roberts 2003), although no commercial plants are yet in existence.

The performance and cost model developed in this thesis for current commercial systems is based primarily on the Econamine FG process, which uses 30\% w/w MEA solution with an oxygen inhibitor. The inhibitor helps in two ways - reduced sorbent degradation and reduced equipment corrosion (Chapel, Ernst et al. 1999). It may be noted that this process is not applicable to reducing gas streams that contain large amounts of $\mathrm{CO}$ and $\mathrm{H}_{2}$, or contain more than $1 \mathrm{ppm}$ of $\mathrm{H}_{2} \mathrm{~S}$, or contain less than $1 \% \mathrm{O}_{2} \mathrm{v} / \mathrm{v}$.

\subsubsection{The Kerr-McGee/ ABB Lummus amine process}

Kerr-McGee started up their 800 tonne/d $\mathrm{CO}_{2}$ recovery unit in 1978. The Trona unit has been fed flue gases from boilers fired with natural gas, coal and coke. During the first several years of operation, the installation was improved in terms of reliability and cost effectiveness. In late 1990, Kerr-McGee and Lummus Global concluded a joint licensing agreement whereby Lummus gained worldwide exclusive marketing rights to KerrMcGee's $\mathrm{CO}_{2}$ recovery technology and became responsible for marketing and basic engineering. Kerr-McGee maintains the continuing role in technology transfer, process improvement, quality control of new designs, operator training, and licensing(ALSTOM, AEP et al. 2001).

Two units have been licensed using this technology. They are:

1) Applied Energy System, Poteau, Oklahoma. This $300 \mathrm{MW}$ coal-fired cogeneration plant incorporates a 200 TPD food-grade liquid $\mathrm{CO}_{2}$ unit as the steam host. Startup was completed in January 1991. Lummus Crest was responsible for engineering, procurement and construction of the entire facility, including the 
power plant. Lummus Crest made several design improvements to the AES facility.

2) Soda Ash Botswana, Pty. Ltd., Sue pan, Botswana. This soda ash facility, incorporating a 300 TPD $\mathrm{CO}_{2}$ unit, started up in March 1991.

The Kerr-McGee/ ABB Lummus amine process uses a 15\%-20\% w/w MEA solution without any inhibitor (Marion, Nsakala et al. 2001). This technology can capture more than $96 \%$ of the $\mathrm{CO}_{2}$ from flue gases, but the lower sorbent concentration leads to economic disadvantages in terms of greater capital requirements due to larger equipment size and higher energy requirements due to higher amount of dilution water per unit of sorbent. At the same time, it may be noted that this process has already been proven for coal-fired flue gas applications at commercial scale.

\subsection{Process Description}

Amine-based $\mathrm{CO}_{2}$ capture process is based on the principle of chemical absorption. Chemical absorption systems tend to be more efficient than the other systems shown in Figure 1.1, as the process is accompanied by a chemical reaction that enhances the overall mass transfer from gas phase to liquid phase. Here, a continuous scrubbing system is used to separate $\mathrm{CO}_{2}$ from a gaseous stream. The system consists of two main elements, an absorber, where $\mathrm{CO}_{2}$ is absorbed into a sorbent and a regenerator (or stripper), where $\mathrm{CO}_{2}$ is released (in concentrated form) and the original sorbent is recovered.

In a power plant application (Figure 2.2) cooled flue gases flow vertically upwards through the absorber countercurrent to the sorbent (MEA in a water solution, with some additives). The MEA reacts chemically with the $\mathrm{CO}_{2}$ in the flue gases to form a weakly bonded compound (carbamate). The scrubbed gases are then washed and vented to the atmosphere. The $\mathrm{CO}_{2}$-rich sorbent stream leaves the absorber and passes through a heat exchanger, then further heated in a reboiler using low-pressure steam. The weakly bonded compound formed during absorption is broken down by the application of heat, regenerating the sorbent, and producing a concentrated $\mathrm{CO}_{2}$ stream. The hot $\mathrm{CO}_{2}$-lean sorbent is then returned to the heat exchanger, where it is cooled, and then sent back to 
the absorber. Some fresh MEA is added to make up for the losses incurred in the process (see section 2.5.2 for details).

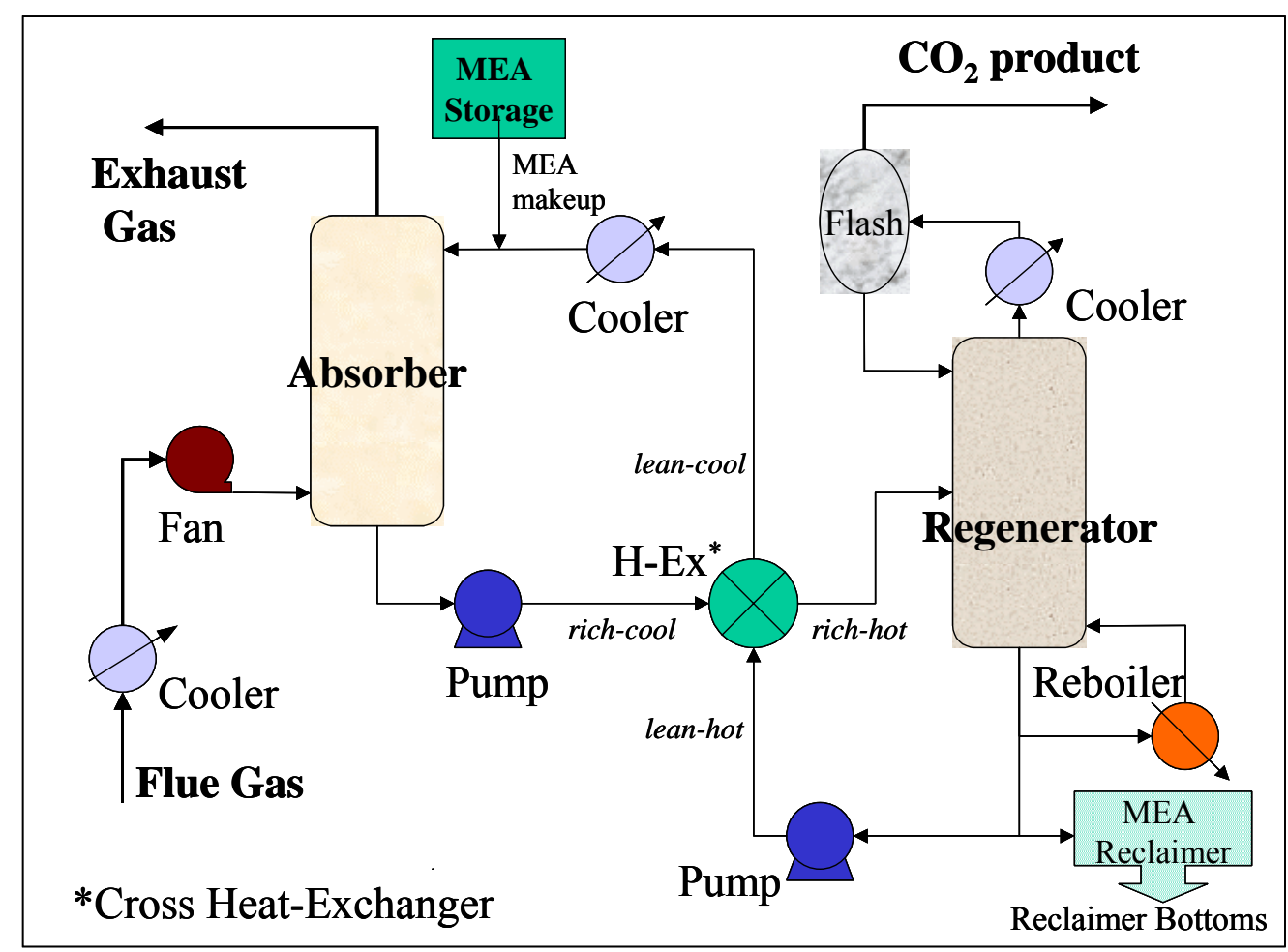

Figure 2.2. Flowsheet for $\mathrm{CO}_{2}$ capture from flue gases using amine-based system

The $\mathrm{CO}_{2}$ product is separated from the sorbent in a flash separator, and then taken to the drying and compression unit. It is compressed to very high pressures (about 2000 psig) so that it is liquefied and easily transported over long distances to the designated storage or disposal facility.

\subsection{Process Chemistry}

There are mainly three compounds, viz. carbon dioxide $\left(\mathrm{CO}_{2}\right)$, amine (MEA) and water $\left(\mathrm{H}_{2} \mathrm{O}\right)$ that are active in this system. The following equilibrium reactions occur in the bulk of the liquid (Al-Baghil, Pruess et al. 2001):

Water hydrolysis: $2 \mathrm{H}_{2} \mathrm{O} \leftrightarrow \mathrm{H}_{3} \mathrm{O}^{+}+\mathrm{OH}^{-}$

Bicarbonate formation: $2 \mathrm{H}_{2} \mathrm{O}+\mathrm{CO}_{2} \leftrightarrow \mathrm{H}_{3} \mathrm{O}^{+}+\mathrm{HCO}_{3}^{-}$ 


$$
\begin{aligned}
& \text { Carbonate formation: } \mathrm{H}_{2} \mathrm{O}+\mathrm{HCO}_{3}^{-} \leftrightarrow \mathrm{H}_{3} \mathrm{O}^{+}+\mathrm{CO}_{3}^{-2} \\
& \text { Amine protonation: } \mathrm{H}_{3} \mathrm{O}^{+}+\mathrm{R}-\mathrm{NH}_{2} \leftrightarrow \mathrm{H}_{2} \mathrm{O}+\mathrm{R}_{-}-\mathrm{NH}_{3}^{+} \\
& \text {Carbamate formation: } \mathrm{R}-\mathrm{NH}_{2}+\mathrm{HCO}_{3}^{-} \leftrightarrow \mathrm{H}_{2} \mathrm{O}+\mathrm{R}-\mathrm{NH}-\mathrm{COO}^{-}
\end{aligned}
$$

Here, MEA has been represented as $\mathrm{R}-\mathrm{NH}_{2}$, where " $\mathrm{R}$ " stands for $\mathrm{HO}-\mathrm{CH}_{2} \mathrm{CH}_{2}$.

The process chemistry is complex, but the main reactions taking place are (Desideri and Paolucci 1999):

\section{$\mathrm{CO}_{2}$ Absorption: $2 \mathrm{R}-\mathrm{NH}_{2}+\mathrm{CO}_{2} \rightarrow{\mathrm{R}-\mathrm{NH}_{3}}^{+}+\mathrm{R}-\mathrm{NH}-\mathrm{COO}^{-}$ \\ MEA Regeneration: $\mathrm{R}-\mathrm{NH}-\mathrm{COO}^{-}+\mathrm{R}_{-} \mathrm{NH}_{3}{ }^{+}+($Heat $) \rightarrow \mathrm{CO}_{2}+2 \mathrm{R}-\mathrm{NH}_{2}$}

Pure MEA (with $\mathrm{R}=\mathrm{HO}-\mathrm{CH}_{2} \mathrm{CH}_{2}$ ) is an "unhindered" amine that forms a weakly bonded intermediate called "carbamate" that is fairly stable. Only half a mole of $\mathrm{CO}_{2}$ is absorbed per mole of amine, as shown in the $\mathrm{CO}_{2}$ absorption equation above. On application of heat, this carbamate dissociates to give back $\mathrm{CO}_{2}$ and amine sorbent, as shown in the second equation above. Since the carbamate formed during absorption is quite stable, it takes a large amount of heat energy to break the bonds and to regenerate the sorbent. When MEA is used as the sorbent, the theoretical minimum heat requirement to reverse this reaction is about $1900 \mathrm{~kJ} / \mathrm{kgCO}_{2}$.

MEA is a reactive compound. The reversible reactions allow us to make use of the same sorbent mass repeatedly, by capturing $\mathrm{CO}_{2}$ in the absorber column and then regenerating the sorbent in the stripper or the regenerator column. In spite of dilution with water and use of inhibitors, a small quantity of MEA is lost through various unwanted reactions, mainly the polymerization reaction (to form long-chained compounds) and the oxidation reaction forming organic acids and liberating ammonia. Appropriate measures must be taken in order to avoid accumulation of these unwanted chemical species in the circulating sorbent.

Acid gas impurities that might be present in the flue gas are another potential source of sorbent loss. This is especially true for the flue gas from coal-fired combustion. Acid 
gases like $\mathrm{SO}_{2}, \mathrm{HCl}$, and $\mathrm{NO}_{2}$ react with MEA to form heat-stable salts that reduce the $\mathrm{CO}_{2}$ absorption capacity of the sorbent. Thus, very low concentrations of these gases (on the order of $10 \mathrm{ppm}$ ) are desirable to avoid excessive loss of expensive sorbent. The problem is especially acute for $\mathrm{SO}_{2}$ because its concentration in flue gases is typically 700 to 2500 ppm at coal-fired plants. $\mathrm{NO}_{\mathrm{x}}$ is less of a problem because most of the $\mathrm{NO}_{\mathrm{x}}$ is nitric oxide (NO), whereas only $\mathrm{NO}_{2}$ (typically about $5 \%$ of total $\mathrm{NO}_{\mathrm{x}}$ ) is reactive with MEA. Hence, from a multi-pollutant perspective, there are important interactions between the $\mathrm{CO}_{2}$ capture system and the control of other air pollutants, especially $\mathrm{SO}_{2}$ and $\mathrm{NO}_{\mathrm{x}}$ emissions.

For other "hindered" amines (e.g., where $\mathrm{R}$ is a bulky group), the carbamate formed is not stable, and an alternate reaction leads to formation of bicarbonate ions and hence a higher theoretical capacity of one mole of $\mathrm{CO}_{2}$ per mole of amine, as shown in the $\mathrm{CO}_{2}$ absorption equation below (Sartori, Ho et al. 1994; Herzog, Drake et al. 1997).

CO 2 Absorption: $\mathrm{R}-\mathrm{NH}_{2}+\mathrm{CO}_{2}+\mathrm{H}_{2} \mathrm{O} \rightarrow \mathrm{R}_{-} \mathrm{NH}_{3}{ }^{+}+\mathrm{HCO}_{3}{ }^{-}$

MEA Regeneration: $\mathrm{HCO}_{3}{ }^{-}+\mathrm{R}_{-}-\mathrm{NH}_{3}{ }^{+}+$(less Heat) $\rightarrow \mathrm{CO}_{2}+\mathrm{H}_{2} \mathrm{O}+\mathrm{R}-\mathrm{NH}_{2}$

The regeneration of these amines requires lesser amount of heat energy as compared to the unhindered amines. But the $\mathrm{CO}_{2}$ uptake rate of hindered amines is very low. Efforts are underway to formulate better sorbents by combining favorable properties of these two groups of amines.

\subsection{Process Areas}

The $\mathrm{CO}_{2}$ capture and separation system consists of the following process equipment:

Direct contact cooler: The flue gases coming out of a power plant are quite hot. The temperature of flue gas may be ranging from as low as $60 \mathrm{deg}$. C (in case of coal-fired power plants with wet $\mathrm{SO}_{2}$ scrubbers) to more than 550 deg. $\mathrm{C}$ (in case of natural gas fired simple cycle power plants). It is desirable to cool down the flue gases to about 4550 deg. $\mathrm{C}$, in order to improve absorption of $\mathrm{CO}_{2}$ into the amine sorbent, to minimize sorbent losses, and to avoid excessive loss of moisture with the exhaust gases. Absorption being an exothermic process is favored by low temperatures, and higher 
temperature may also lead to sorbent losses due to evaporation and degradation. In case of gas-fired power plants or majority of coal-fired power plants without wet scrubbers for $\mathrm{SO}_{2}$ removal, a direct contact cooler needs to be installed to reduce the temperature of the flue gas stream to acceptable levels. In case of coal-fired power plant applications that have a wet FGD (flue gas desulfurization) unit upstream of the amine system, the wet scrubber helps in substantial cooling of the flue gases, and additional cooler may not be required.

Flue gas blower: The flue gas enters at the bottom of the absorber column and flows upwards, countercurrent to the sorbent flow. Thus, it needs to overcome a substantial pressure drop as it passes through a very tall absorber column. Hence the cooled flue gas must be pressurized using a blower before it enters the absorber.

Absorber: This is the vessel where the flue gas is made to contact with the MEA-based sorbent, and where the $\mathrm{CO}_{2}$ from the flue gas is dissolved into the sorbent. The column may be plate-type or packed. Most of the $\mathrm{CO}_{2}$ absorbers are packed columns using some kind of polymer-based packing to provide large interfacial area.

Rich/lean cross heat exchanger: The $\mathrm{CO}_{2}$-loaded sorbent must be heated in order to strip off $\mathrm{CO}_{2}$ and regenerate the sorbent. On the other hand, the regenerated (lean) sorbent coming out of the regenerator must be cooled down before it can be circulated back to the absorber column. Hence, these two sorbent streams are passed through a cross heat exchanger, where the rich $\left(\mathrm{CO}_{2}\right.$-loaded) sorbent gets heated and the lean (regenerated) sorbent gets cooled.

Regenerator: This is the column where the weak intermediate compound (carbamate) formed between the MEA-based sorbent and dissolved $\mathrm{CO}_{2}$ is broken down by the application of heat. The $\mathrm{CO}_{2}$ is separated from the sorbent to leave reusable sorbent behind. In case of unhindered amines like MEA, the carbamate formed is stable and requires large amounts of energy to dissociate. It also consists of a flash separator, where $\mathrm{CO}_{2}$ is separated from most of the moisture and evaporated sorbent to produce a fairly concentrated $\mathrm{CO}_{2}$ stream. 
Reboiler: The regenerator is connected with a reboiler that is basically a heat exchanger where low-pressure steam extracted from the power plant is used to heat the loaded sorbent.

Steam extractor: In case of coal-fired power plants that generate electricity in a steam turbine, a part of the LP (low pressure)/IP (intermediate pressure) steam has to be diverted to the reboiler for sorbent regeneration. Steam extractors are installed to take out steam from the steam turbines.

MEA reclaimer: The presence of acid gas impurities $\left(\mathrm{SO}_{2}, \mathrm{SO}_{3}, \mathrm{NO}_{2}\right.$ and $\left.\mathrm{HCl}\right)$ in the flue gas leads to formation of heat stable salts in the sorbent stream, which can not be dissociated even on application of heat. In order to avoid accumulation of these salts in the sorbent stream and to recover some of this lost MEA sorbent, a part of the sorbent stream is periodically distilled in this vessel. Addition of a strong alkali such as caustic helps in release of some of the MEA. The recovered MEA is recycled to the sorbent stream while the bottom sludge (reclaimer waste) is sent for proper disposal.

Sorbent processing area: The regenerated sorbent needs to be further cooled even after passing through the rich/lean cross heat exchanger using a cooler. This is so that the sorbent temperature can be brought back to an acceptable level (about 40 deg C). In order to make up for the sorbent losses, a small quantity of fresh MEA sorbent must be added to the sorbent stream. So, the sorbent processing area primarily consists of a sorbent cooler, MEA storage tank, and a mixer. It also consists of an activated carbon bed filter that adsorbs impurities (degradation products of MEA) from the sorbent stream.

$\mathrm{CO}_{2}$ drying and compression unit: The $\mathrm{CO}_{2}$ product may have to be carried over very long distances via pipelines. Hence it is desirable that it does not contain any moisture in order to avoid corrosion in the pipelines. Of course, the $\mathrm{CO}_{2}$ product specifications may vary depending upon the end use (or storage/ disposal method) and the material of construction of the pipeline. Also, it has to be compressed to very high pressures so that it gets liquefied and can overcome the pressure losses during the pipeline transport. The multi-stage compression unit with inter-stage cooling and drying yields a final $\mathrm{CO}_{2}$ 
product at the specified pressure (about 2000 psig) that contains acceptable levels of moisture and other impurities (e.g. $\mathrm{N}_{2}$ ).

$\mathbf{C O}_{2}$ transport facility: The $\mathrm{CO}_{2}$ captured at the power plant site has to be carried to the appropriate storage/ disposal site. Considering the scale of the operation (thousands of tonnes of $\mathrm{CO}_{2}$ per day), pipelines are the suitable mode of transportation. There is fair amount of industrial experience and expertise in the field of the construction (and operation) of pipelines for $\mathrm{CO}_{2}$ transport. Recently, a $325-\mathrm{km}$ pipeline carrying $\mathrm{CO}_{2}$ from the Great Plains Synfuels Plant in Beulah, North Dakota (owned by Dakota Gasification Company of Bismarck, North Dakota) to the Weyburn oil fields in Saskatchewan, Canada went operational (EnCana 2003). In case of retrofit applications, where construction of new pipelines might be prohibitively expensive (and questionable in terms of public acceptance, especially in densely populated regions), transport via tankers may have to be considered.

$\mathrm{CO}_{2}$ disposal facility: Once the $\mathrm{CO}_{2}$ is captured, it needs to be securely stored (sequestered). Again, there are a wide range of options potentially available (see Figure 1.3). Geologic formations such as underground deep saline reservoirs, depleted oil and gas wells, and abandoned coal seams are some of the potentially attractive disposal sites (Adams, Ormerod et al. 1993; Byrer and Guthrie 1998; Lindeberg and Holloway 1998). Ocean disposal and terrestrial sinks are additional options being studied (Herzog 1998; Spencer 1998). The distance to a secure storage site and the availability and cost of transportation infrastructure also affect the choice of disposal option. In general, studies indicate that geologic formations are the most plentiful and attractive option for U.S. power plants (Bergman and Winter 1995). Transport of $\mathrm{CO}_{2}$ to a storage site is typically assumed to be via pipeline. While the economic costs of $\mathrm{CO}_{2}$ storage appear to be low, the social and political acceptability of different options are not yet clear.

\subsection{Limitations of the MEA Process}

Although MEA-based absorption process is the most suitable technology available today for post-combustion capture of $\mathrm{CO}_{2}$ from power plant flue gases, it has several important drawbacks. The main problems may be listed as follows: 


\subsubsection{Energy Penalty}

The stable carbamate ion requires substantial energy to break the bonds. Because MEA is diluted with lot of water, a large amount of heat is required to regenerate the sorbent. Substantial energy also is needed to compress the captured $\mathrm{CO}_{2}$ for pipeline transport to a storage site. This heat and electricity requirement reduces the net efficiency of the power plant if it is extracted internally by de-rating the power plant. Alternatively, a much bigger power plant must be built in order to achieve the same "net" power generation capacity as it would have been without $\mathrm{CO}_{2}$ capture. In case of retrofit applications, an auxiliary gas-fired boiler and steam turbine might be added to maintain the power output.

\subsubsection{Loss of Sorbent}

Sorbent is lost during the process because of a variety of reasons including degradation, entrainment, vaporization and mechanical losses (Stewart and Lanning 1994; Stewart and Lanning 1994). All the sorbent entering the stripper does not get regenerated. Flue gas impurities, especially oxygen, sulfur oxides and nitrogen dioxide react with MEA to form heat-stable salts, thus reducing the $\mathrm{CO}_{2}$-absorption capacity of the sorbent. Proprietary inhibitors are available that make the sorbent tolerant to oxygen. Flue gas $\mathrm{NO}_{\mathrm{x}}$ is not a major problem since nitric oxide (NO) is the predominant form $(\sim 90-95 \%)$ of total $\mathrm{NO}_{\mathrm{x}}$ in the flue gas, and does not react with inhibited amines (Suda, Fujii et al. 1992; Leci 1996). But, $\mathrm{SO}_{2}$ does degenerate MEA sorbent, so very low inlet concentrations (10 ppm) are desirable to avoid excessive loss of sorbent. However, untreated flue gases of coal-fired power plants contain about 700 to 2500 ppm $\mathrm{SO}_{2}$ (plus roughly 10-40 ppm $\mathrm{NO}_{2}$ ). The interaction of $\mathrm{SO}_{2}$ with $\mathrm{CO}_{2}$ control system is thus particularly important. The heat-stable salts that are formed may be treated in a side stream MEA-reclaimer, which can regenerate some of the MEA. Technologies such as electro-dialysis are also being proposed for this purpose (Yagi, Shibuya et al. 1992).

Most of the previous studies on reactions of MEA had focused on natural gas purification processes and were conducted with pure gases under laboratory-controlled conditions. However, the flue gas generated by a fossil-fuel-fired boiler is a complex mixture of various chemical species. It has been shown that there are chemical degradation reactions that occur under plant conditions which do not occur in laboratory experiments 
with pure gases (Strazisar, Anderson et al. 2003). Better understanding of the mechanisms and chemical pathways associated with MEA degradation in actual power plant applications may help reduce or eliminate its negative impacts.

\subsubsection{Corrosion}

MEA is a reactive compound and the MEA solution in water in the presence of oxygen and carbon dioxide is a highly corrosive system. Irreversible side reactions with $\mathrm{CO}_{2}$ and other flue gas components lead to the formation of various degradation products. These byproducts are associated with increased corrosion in this system. Hence, corrosion control is very important in amine systems processing oxygen-containing flue gases. In

order to reduce corrosion rates, corrosion inhibitors, lower concentrations of MEA, appropriate materials of construction, and mild operating conditions are recommended (Barchas and Davis 1992).

\subsubsection{Environmental Emissions}

In case of MEA-based $\mathrm{CO}_{2}$ capture systems, environmental problems may arise from the spent sorbent slurry discharged from the MEA reclaimer (also called "reclaimer bottoms") and the emissions of MEA and ammonia $\left(\mathrm{NH}_{3}\right)$ carried by the treated flue gas. Details about the potential environmental impacts of these emissions are discussed in Appendix A. The spent sorbent is treated as a hazardous waste. Entrainment of MEA with the treated flue gas is at most a few ppm and is likely to go down with the improvements in the absorber design (wash section at the top). The emission rate of $\mathrm{NH}_{3}$ from a $\mathrm{CO}_{2}$ capture plant is substantially higher than that from a power plant without amine-based $\mathrm{CO}_{2}$ capture system. However, these $\mathrm{NH}_{3}$ emissions are much smaller than those coming from other sources (animals, farms). At this time, it is not very clear if these emissions are likely to have significant environmental impacts. Hence, these aspects must be carefully looked into before a widespread application of this technology. 


\section{References (Chapter 2)}

Adams, D., W. Ormerod, et al. (1993). Carbon Dioxide Disposal from Power Stations. UK, a report published by IEA Greenhouse Gas R\&D Programme.

Al-Baghil, N. A., S. A. Pruess, et al. (2001). "A rate-based model for the design of gas absorbers for the removal of $\mathrm{CO} 2$ and $\mathrm{H} 2 \mathrm{~S}$ using aqueous solutions of MEA and DEA." Fluid Phase Equilibria 185(2001): 31-43.

ALSTOM, AEP, et al. (2001). Engineering feasibility and economics of CO2 capture on an existing coal-fired power plant, Final report prepared by ALSTOM Power Inc., ABB Lummus Global Inc., ALSTOM Power Environmental Systems and American Electric Power (AEP). report no. (PPL-01-CT-09) submitted to Ohio Department of Development, Columbus, OH and US Department of Energy/ NETL, Pittsburgh, PA: 163.

Barchas, R. and R. Davis (1992). "The Kerr-McGee/ ABB Lummus Crest technology for the recovery of CO2 from stack gases." Energy Conversion and Management 33(5-8): 333340 .

Bergman, P. D. and E. M. Winter (1995). "Disposal of carbon dioxide in aquifers in the U.S." Energy Conversion and Management 36(6-9): 523-526.

Bottoms, R. R. (1930). Process for Separating Acid Gases.

Byrer, C. W. and H. D. Guthrie (1998). Coal deposits: potential geological sink for sequestering carbon dioxide emissions from power plants. in the proceedings of the AWMA's Second International Specialty Conference, Oct 13-15, 1998, Washington, DC.

Chapel, D., J. Ernst, et al. (1999). Recovery of CO2 from flue gases: commercial trends (paper no. 340). presented at the Canadian Society of Chemical Engineers Annual Meeting, 4-6 October, Saskatoon, Saskatchewan, Canada.

Desideri, U. and A. Paolucci (1999). "Performance modelling of a carbon dioxide removal system for power plants." Energy Conversion and Management 40: 1899-1915.

DOE (1999). Carbon sequestration: research and development, A U.S. Department of Energy Report, Office of Science, Office of Fossil Energy, U.S. Department of Energy, Washington, DC.

Dow (2000). "Specialty Alkanolamines": Product information available at the website of The Dow Chemical Company (www.dow.com/alkanolamines/).

EnCana (2003). Weyburn carbon dioxide miscible flood project, report available online at: http://www.encana.com/operations_and_projects/weyburn.shtml.

Herzog, H. J. (1998). The economics of CO2 capture. Fourth International Conference on Greenhouse Gas Control Technologies, 30 August - 2 September, Interlaken, Switzerland, Elsevier Science Ltd.

Herzog, H. J. (1998). Ocean sequestration of CO2 - an overview. in the proceedings of the AWMA's Second International Specialty Conference, Oct 13-15, 1998, Washington, DC. 
Herzog, H. J., E. Drake, et al. (1997). CO2 capture, reuse and storage technologies for mitigating global climate change. A White Paper (Final Report), January 1997.

IEA-GHGRDP (2002). International test network for CO2 capture: report no. PH4/11 on 3rd workshop (May 16-17 2002, Apeldoorn, The Netherlands), IEA Greenhouse Gas R\&D Programme, UK.

Kaplan, L. J. (1982). "Cost-saving process recovers CO2 from power-plant flue gas." Chemical Engineering 89(24): 30-31.

Kohl, A. L. and C. L. Blohm (1950). "Technical aspects of glycol-amine gas treating." Petroleum Engineer 22(June): C-37.

Kohl, A. L. and R. B. Nielsen (1997). Gas Purification. Houston, TX, Gulf Publishing Company.

Leci, C. L. (1996). "Financial implications on power generation costs resulting from the parasitic effect of $\mathrm{CO} 2$ capture using liquid scrubbing technology from power station flue gases." Energy Conversion and Management 37(6-8): 915-921.

Lindeberg, E. and S. Holloway (1998). The next steps in geo-storage of carbon dioxide. Fourth International Conference on Greenhouse Gas Control Technologies, 30 August - 2 September, Interlaken, Switzerland, Elsevier Science Ltd.

Marion, J., N. Nsakala, et al. (2001). Engineering feasibility of CO2 capture on an existing US coal-fired power plant. in the proceedings of the Twenty-sixth International Conference on Coal Utilization and Fuel Systems, 5-8 March, Clearwater, FL, USA.

Pauley, C. R., P. L. Simiskey, et al. (1984). "N-Ren recovers CO2 from flue gas economically." Oil and Gas Journal 82(20): 87-92.

Reddy, S. and C. A. Roberts (2003). ECONOMINE FGSM Plus: An Enhanced Amine Based $\mathrm{CO}_{2}$ Capture Process. 2nd Annual Conference on Carbon Sequestration, Alexandria, VA, USA.

Sartori, G., W. A. Ho, et al. (1994). Sterically hindered amines for acid gas absorption. Carbon Dioxide Chemistry: Environmental Issues. J. Paul and C. Pradier. Cambridge, UK, The Royal Society of Chemistry.

Spencer, D. F. (1998). Integration of an advanced CO2 separation process with methods for disposing of $\mathrm{CO} 2$ in oceans and terrestrial deep aquifers. Fourth International Conference on Greenhouse Gas Control Technologies, 30 August - 2 September, Interlaken, Switzerland, Elsevier Science Ltd.

Statoil (2001). CO2 storage $1000 \mathrm{~m}$ down, report available online at: http://www.statoil.com/STATOILCOM/SVG00990.nsf/web/sleipneren?opendocument.

Stewart, E. J. and R. A. Lanning (1994). "Reduce amine plant solvent losses (Part 1)." Hydrocarbon Processing May: 67-81.

Stewart, E. J. and R. A. Lanning (1994). "Reduce amine plant solvent losses (Part 2)." Hydrocarbon Processing June: 51-54. 
Strazisar, B. R., R. R. Anderson, et al. (2003). "Degradation pathways for monoethanolamine in a $\mathrm{CO}_{2}$ capture facility." Energy \& Fuels 17(4): 1034-1039.

Suda, T., M. Fujii, et al. (1992). "Development of flue gas carbon dioxide recovery technology." Energy Conversion and Management 33(5-8): 317-324.

Wong, S., W. D. Gunter, et al. (2000). Economics of CO2 sequestration in coal bed methane reservoirs. presented at the 2000 SPE/CERI Gas Technology Symposium, 3-5 April, Calgary, Alberta, Canada.

Yagi, T., H. Shibuya, et al. (1992). "Application of chemical absorption process to CO2 recovery from flue gas generated in power plants." Energy Conversion and Management 33(5-8): 349-355.

Yeh, S. (2003). The historical developments in amine-based CO2 capture technology - personal communication. 


\section{PERFORMANCE MODEL DEVELOPMENT}

A performance model of a chemical technology essentially consists of the simulation of mass and energy balances around the system. In case of an environmental control technology such as amine-based $\mathrm{CO}_{2}$ capture, it keeps track of various material flows (e.g. flow of flue gas and its individual components, flow of the sorbent and its degradation products, flow of other chemical reagents) and energy flows (e.g. thermal energy requirement for sorbent regeneration, electrical energy required for mechanical devices such as pumps, fans, compressors).

The rate of removal of $\mathrm{CO}_{2}$ from flue gas using an amine scrubber depends on the gasliquid mass transfer process. The chemical reactions that permit diffusion of $\mathrm{CO}_{2}$ in the liquid film at the gas-liquid interface enhance the overall rate of mass transfer. So, the $\mathrm{CO}_{2}$ removal efficiency in the absorber is a function of various parameters that affect the gas-liquid equilibrium (e.g., flow rates, temperature, pressure, flue gas composition, MEA concentration, equipment design, etc.). Absorption of $\mathrm{CO}_{2}$ in an alkaline medium may be considered as a first order reaction. Higher $\mathrm{CO}_{2}$ concentration thus improves the efficiency of the absorption system. Even at low concentration of $\mathrm{CO}_{2}, \mathrm{MEA}$ has great affinity for $\mathrm{CO}_{2}$. The solubility of $\mathrm{CO}_{2}$ in MEA is much higher as compared to many other conventional sorbents. Similarly, the design of and conditions in the regenerator affect the energy requirement and the overall performance of the system.

A number of previous studies have reported some cost and/or performance data for specific amine-based systems, including hypothetical applications to coal-fired power plants (Smelser, Stock et al. 1991; Hendriks 1994; Kohl and Nielsen 1997; Chakma and Tontiwachwuthikul 1998; Herzog 1998; Simbeck 1998; Chapel, Ernst et al. 1999; Marion, Nsakala et al. 2001). However, there are no generally available process models that can be used or modified for detailed studies of $\mathrm{CO}_{2}$ removal options. Cost data also are relatively limited and often incomplete.

The performance model for amine-based $\mathrm{CO}_{2}$ capture system was developed for use in the IECM modeling framework, which simulates the performance and cost of a coal-fired power plant equipped with multiple emission control systems (Rubin, Kalagnanam et al. 
1997). The performance model of the $\mathrm{CO}_{2}$ capture system is a response-surface model with about two dozen parameters; developed from numerous runs of a detailed process simulation model. The performance model estimates all of the key flow rates and energy requirements. The output from the performance model is then used by the cost model to estimate various costs of the system.

In this chapter, the process simulation packages used are described in section 3.1, the overall model development methodology is laid out in section 3.2 and the various performance parameters are discussed in section 3.3. The actual performance equations employed in the model are listed in section 3.4 and the model outputs are explained in section 3.5. Finally, section 3.6 discusses the characterization of uncertainty in the performance parameters.

\subsection{Process Simulation Model}

Two process simulators, viz. ASPEN-Plus and ProTreat have been used to derive the performance equations. The $\mathrm{CO}_{2}$ capture and separation model is based on the ProTreat simulations while the $\mathrm{CO}_{2}$ compression model is based on the ASPEN-Plus simulations.

- ProTreat is a software package for simulating processes for the removal of $\mathrm{H}_{2} \mathrm{~S}$, $\mathrm{CO}_{2}$, and mercaptans from a variety of high and low pressure gas streams by absorption into thermally regenerable aqueous solutions containing one or more amines (ProTreat ${ }^{\mathrm{TM}}$ 2002). The ProTreat package makes exclusive use of a column model that treats the separation as a mass transfer rate process.

- ASPEN-Plus is a widely used process engineering tool for the design and steadystate simulation and optimization of process plants (Aspen-Plus ${ }^{(\mathrm{R})} 2002$ ).

\subsection{Methodology}

A large number of process simulation runs have been conducted to cover a reasonable range of values for the key parameters. The details are presented in the following sections. 


\subsubsection{ProTreat Simulation Runs for $\mathrm{CO}_{2}$ capture and separation from flue gas}

The $\mathrm{CO}_{2}$ capture and separation system consists of a flue gas cooler, compressor, absorber, heat exchangers, regenerator, MEA reclaimer, sorbent circulation pumps and other accessories. Based on the literature review, parameters relevant to the overall performance of the $\mathrm{CO}_{2}$-capture process were identified and were varied within reasonable ranges in the ProTreat model runs. Table 3.1 summarizes the parameters that were varied and the ranges for each parameter.

Table 3.1. ProTreat parameter ranges (total number of simulation runs $=1983$ )

\begin{tabular}{|c|c|c|c|c|}
\hline No. & Parameter & Type & Units & Range \\
\hline 1 & $\mathrm{CO}_{2}$ content in flue gas $\left(\mathrm{y}_{\mathrm{CO} 2}\right)$ & Input & $\mathrm{mole} \%$ & $3.5-13.5$ \\
\hline 2 & Flue gas flow rate $(\mathrm{G})$ & Input & $\mathrm{kmole} / \mathrm{hr}$ & $9000-24000$ \\
\hline 3 & Inlet flue gas temperature $\left(\mathrm{T}_{\mathrm{fg}}\right)$ & Input & $\mathrm{deg} \mathrm{C}$ & $40-65$ \\
\hline 4 & MEA concentration $(\mathrm{C})$ & Input & $\mathrm{wt} \%$ & $15-40$ \\
\hline 5 & Sorbent flow rate $(\mathrm{L})$ & Input & $\mathrm{kmole} / \mathrm{hr}$ & $16000-70000$ \\
\hline 6 & L/G (calc.) & Input & - & $0.7-5.6$ \\
\hline 7 & Reboiler heat duty $(\mathrm{Q})$ & Input & $\mathrm{GJ} / \mathrm{hr}$ & $95-664$ \\
\hline 8 & Q/L (calc.) & Input & $\mathrm{MJ} / \mathrm{kmole}$ & $2.4-22.5$ \\
\hline 9 & $\mathrm{CO}_{2}$ capture efficiency & Output & $\%$ & $40-99$ \\
\hline 10 & $\mathrm{CO}_{2}$ product flow rate & Output & $\mathrm{kmole} / \mathrm{hr}$ & $333-2840$ \\
\hline 11 & Lean sorbent $\mathrm{CO}_{2}$ loading & Output & $\mathrm{moleCO}_{2} / \mathrm{mole} \mathrm{MEA}$ & $0.05-0.34$ \\
\hline 12 & Rich sorbent $\mathrm{CO}_{2}$ loading & Output & $\mathrm{moleCO}_{2} / \mathrm{mole} \mathrm{MEA}$ & $0.27-0.55$ \\
\hline 13 & Absorber diameter & Output & $\mathrm{ft}$ & $26-42$ \\
\hline 14 & Regenerator diameter & Output & $\mathrm{ft}$ & $12-42$ \\
\hline 15 & Exhaust flue gas temperature & Output & $\mathrm{deg} \mathrm{C}$ & $40-72$ \\
\hline
\end{tabular}

The following set of parameters related to the design/configuration of the $\mathrm{CO}_{2}$ capture system were held constant. These values were set for those of a typical commercial application based on experts' suggestions.

- Absorber height: $40 \mathrm{ft}$

- Absorber packing: Rasching rings, metallic, 1-inch packing size

- Inlet flue gas pressure: $3 \mathrm{psi}$

- Sorbent pumping pressure: $30 \mathrm{psi}$ 
- Number of trays in regenerator: 24 (tray spacing $=2 \mathrm{ft}$, weir height $=3$ inches)

\subsubsection{ASPEN-Plus Simulation Runs for $\mathrm{CO}_{2}$ Compression}

The concentrated $\mathrm{CO}_{2}$ product stream obtained from sorbent regeneration is compressed and dried using a multi-stage compressor with inter-stage cooling. The ASPEN-Plus module used for this simulation consists of 4 stages of compression with inter-stage cooling that deliver the compressed product at $35^{\circ} \mathrm{C}$. The compressor efficiency, $\mathrm{CO}_{2}$ product pressure and purity were used as the main control variables. These parameters were varied over the following ranges

- Compressor efficiency: $60-100 \%$

- $\mathrm{CO}_{2}$ product pressure: $500-2500 \mathrm{psi}$

- $\mathrm{CO}_{2}$ stream purity: $99-100 \%$

\subsubsection{Regressions to derive performance equations}

A set of algebraic equations were derived from the process simulation runs to provide a performance model for use in the IECM (Rubin, Kalagnanam et al. 1997; IECM 2001). The key performance output variables were regressed against all the input variables to obtain a set of relationships among them. The data collected from the process simulation runs was used to carry out these multivariate regressions using a statistical package called SAS (SAS 1999-2001). Only those variables with significance value greater than 0.9995 were retained in the performance equations. Details are presented later in section 3.5.

\subsection{Model Configuration Options}

For post-combustion $\mathrm{CO}_{2}$ capture from flue gas, the amine-based $\mathrm{CO}_{2}$ capture system requires substantial thermal energy in the form of heat or steam for sorbent regeneration. Depending upon how this energy is supplied, there are three configuration options available in the model. These are shown graphically in Figure 3.1 and described below. 


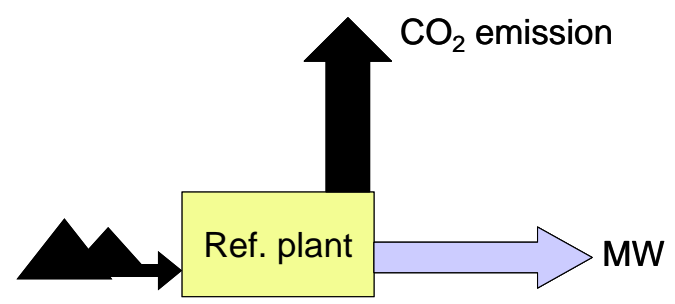

a) Reference base plant

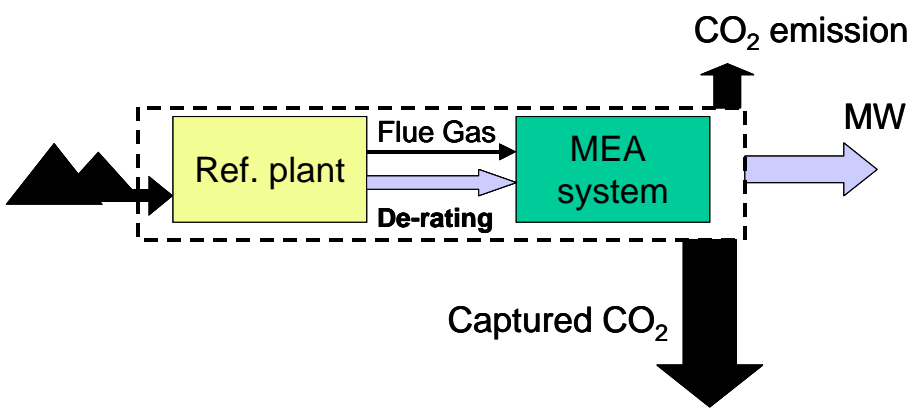

b) Capture plant with internal derating

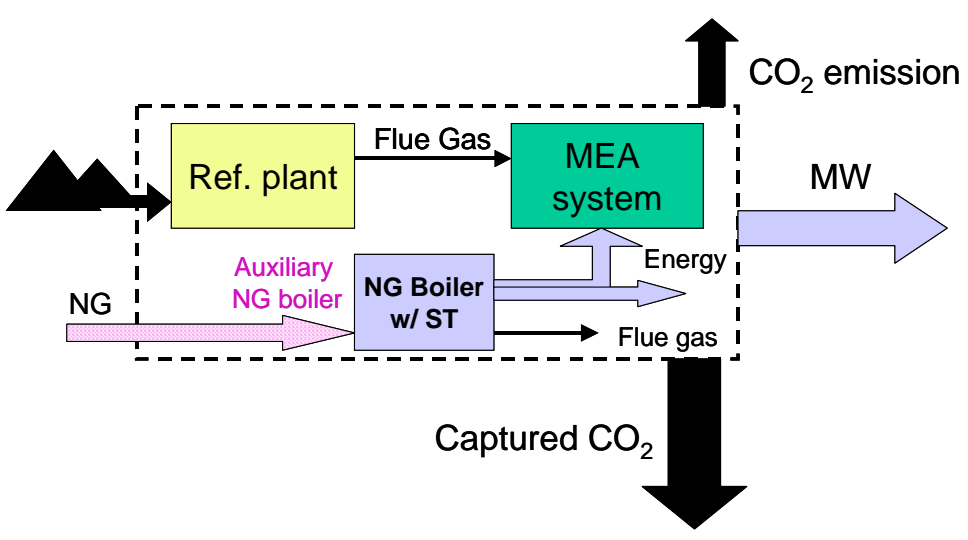

c) Capture plant with an auxiliary boiler and a secondary steam turbine

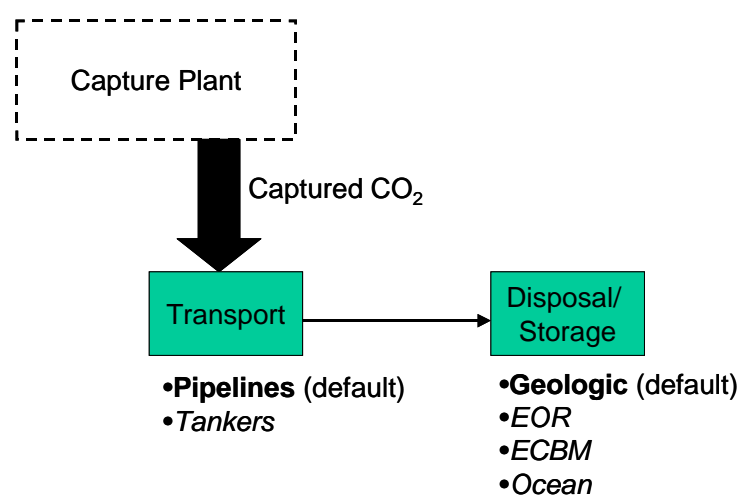

d) Post-capture $\mathrm{CO}_{2}$ transport and disposal options

Figure 3.1. $\mathrm{CO}_{2}$ capture plant configuration options 
Base plant de-rating: Heat extracted from the base plant can provide energy for the amine system. Here, the low-pressure (LP) steam is extracted from the steam cycle of the power plant and supplied to the reboiler for sorbent regeneration. Extraction of steam leads to loss of power generation capacity, and the net plant output decreases substantially. In case of a new plant to be designed with a $\mathrm{CO}_{2}$ capture system, it is possible to optimally design the steam cycle to take care of the steam requirement of the amine system, and proper heat integration may help in reducing the energy penalty. In case of an existing coal plant to be retrofitted with an amine system, optimal heat integration may not be achievable, and is likely to lead to a much higher energy penalty due to steam extraction.

Auxiliary Natural Gas Boiler (w/ Steam Turbine): Another potential option to provide the energy for the amine system is an auxiliary natural gas (NG)-fired boiler. Often it would be combined with a steam turbine to generate some additional power (e.g. to supply electrical energy demand of $\mathrm{CO}_{2}$ capture unit), where the LP steam would be then used for sorbent regeneration. Thus, the original steam cycle of the power plant remains unperturbed and the net power generation capacity of the power plant does not get adversely affected. Again, it comes at an additional cost of capital requirement for the boiler (and turbine) and the cost of supplemental natural gas fuel. Also, the combustion of natural gas leads to additional $\mathrm{CO}_{2}$ emissions (and $\mathrm{NO}_{\mathrm{x}}$ emissions). So, there are at least two possible sub-options available:

1. The flue gas from the auxiliary boiler is cooled down to acceptable exhaust temperature and then directly vented to the atmosphere. Here, the net $\mathrm{CO}_{2}$ capture efficiency of the system is substantially lowered because of the additional $\mathrm{CO}_{2}$ emissions from natural gas boiler. Also, the total $\mathrm{NO}_{\mathrm{x}}$ emissions may exceed the allowable levels of emission.

2. The $\mathrm{CO}_{2}$ capture system may be designed so as to capture $\mathrm{CO}_{2}$ from the auxiliary boiler as well. In this case, the secondary flue gas stream (after cooling and $\mathrm{NO}_{\mathrm{x}}$ removal, if required) may be merged with the main flue gas stream before it enters the $\mathrm{CO}_{2}$ capture system. With higher amount of flue gas to be treated (and 
more $\mathrm{CO}_{2}$ to be captured), the amine-system would require more steam and thus a still bigger auxiliary NG boiler would be required (which means more secondary flue gas!). The auxiliary NG boiler size may be determined by an iterative calculation procedure, so that it matches the sorbent regeneration steam requirement of the $\mathrm{CO}_{2}$ capture system treating the total flue gas. Thus, the $\mathrm{CO}_{2}$ capture level is maintained at the originally desired level, but it requires a large auxiliary NG boiler facility. Our preliminary analysis as well as other studies suggest that this option is quite costly (Simbeck and McDonald 2000), hence in the present version of IECM, this configuration option is not included.

In terms of the configuration of the $\mathrm{CO}_{2}$ capture system shown in Figure 3.1, the user can make the following choices as well.

Direct contact cooler: The default design of an amine system includes a direct contact cooler (DCC) to cool the flue gas before it enters the amine system. The temperature of the flue gas affects the absorption reaction because absorption of $\mathrm{CO}_{2}$ in $\mathrm{MEA}$ sorbent is an exothermic process favored by lower temperatures. Also, the volumetric flow rate of the flue gas stream, which is a key determinant of the sizes of various equipments (e.g., direct contact cooler, flue gas blower, absorber), is directly related to the flue gas temperature. Hence lower flue gas temperature is desired. The typically acceptable range of flue gas temperature is less than $50-60{ }^{\circ} \mathrm{C}$. If the flue gas is coming from wet sulfur scrubber, additional DCC may not be required. But in case of flue gas from NGfired boiler, which often does not pass through a sulfur scrubber, DCC is essential. Exclusion of the DCC is an option available in the model.

Choice of sorbent: At this time, MEA is the only sorbent used in the model. The nominal values of various parameters are based on a process simulation model that uses only MEA. However, the users can overwrite the nominal values of these parameters if they wish to use a different sorbent and have the relevant data. The model could include other sorbents in the future by providing the appropriate values for the key parameters.

$\mathrm{CO}_{2}$ transport: As shown in Figure 3.1(d), the default mode of $\mathrm{CO}_{2}$ transport is via pipelines. This is the suggested mode of transporting large quantities of $\mathrm{CO}_{2}$ that would 
be captured in a typical power plant application. The user can specify the distance over which $\mathrm{CO}_{2}$ needs to be carried to, and the unit cost of $\mathrm{CO}_{2}$ transportation. This module may be expanded in the future to include more detailed parameters regarding pipeline transport and other transport options.

$\mathrm{CO}_{2}$ storage/ disposal: The default option for $\mathrm{CO}_{2}$ disposal is underground geological storage (see Figure 3.1(d)). Studies indicate that geologic formations are the most plentiful and attractive option for U.S. power plants (Bergman and Winter 1995). A nominal cost of $\$ 5 /$ tonne $\mathrm{CO}_{2}$ has been suggested, which can be changed by the user to match the specific details about the location. If $\mathrm{CO}_{2}$ is being used as a byproduct for EOR or ECBM activity, it may generate some revenue. This module, which is represented by a single cost parameter, may be expanded in future to include details about the various storage/ disposal options.

\subsection{Performance Parameters}

There are three types of input parameters to the $\mathrm{CO}_{2}$ performance model:

- Parameters from the "reference plant": These include the flow rate, temperature, pressure and composition of the flue gas inlet to the $\mathrm{CO}_{2}$ absorber, and the gross power generation capacity of the power plant.

- Parameters to configure the $\mathrm{CO}_{2}$ system: The $\mathrm{CO}_{2}$ module provides a menu of options from which the user may select a $\mathrm{CO}_{2}$ capture technology, $\mathrm{CO}_{2}$ product pressure, mode and distance of $\mathrm{CO}_{2}$ product transport, and $\mathrm{CO}_{2}$ storage/ disposal method. At this stage, a model of the MEA-based absorption system with pipeline transport and geologic sequestration has been developed; other options are still under construction.

- Parameters controlling the performance of the $\mathbf{C O}_{2}$ system: The main parameters include the $\mathrm{CO}_{2}$ capture efficiency, MEA concentration, lean sorbent $\mathrm{CO}_{2}$ loading, regeneration heat requirement, pressure drop across the system, MEA make-up requirement, pump efficiency, compressor efficiency and several others. 
These parameters are used to calculate the sorbent flow rate, MEA requirement, and energy penalty of the $\mathrm{CO}_{2}$ system.

Functional relationships and default values for all model parameters were developed based on engineering fundamentals, a detailed review of the literature, and contacts with experts in the field. All of these performance parameters directly affect the cost of the system. Here is a brief description of the various input parameters to the $\mathrm{CO}_{2}$ system.

\subsubsection{Parameters obtained from the "reference base plant"}

The amine-based $\mathrm{CO}_{2}$ capture system gets the following inputs from the (reference) base plant:

- $\quad$ Gross plant size $=\mathrm{MW}_{\mathrm{g}}$

- Net plant size ( prior to environmental controls) $=\mathrm{MW}_{\text {noctl }}$

- Flue gas composition and flow rate (as entering into the amine system): This is an array of molar flow rates of different gas components that include $\mathrm{N}_{2}$, $\mathrm{O}_{2}, \mathrm{H}_{2} \mathrm{O}, \mathrm{CO}_{2}, \mathrm{CO}, \mathrm{HCl}, \mathrm{SO}_{2}, \mathrm{SO}_{3}, \mathrm{NO}, \mathrm{NO}_{2}$ and mass flow rate of particulates.

- The total molar flow rate of the flue gas $=\mathrm{G}$,

- The molar fraction of $\mathrm{CO}_{2}$ in the flue gas $=\mathrm{y}_{\mathrm{CO} 2}$.

- $\quad$ Temperature of flue gas $=\mathrm{T}_{\mathrm{fg}}$

- $\quad$ Plant capacity factor $=$ PCF $(\%)$

- Annual hours of operation $=\mathrm{HPY}=(\mathrm{PCF} / 100) * 365.25 * 24 \mathrm{hrs} / \mathrm{yr}$

\subsubsection{Parameters to configure the $\mathrm{CO}_{2}$ system}

These are the choices the user can make in order to configure the $\mathrm{CO}_{2}$ capture system. These configuration choices have been described in section 3.3, and could be summarized as follows:

- Flue gas cooler: Whether to include DCC (default) or exclude it 
- Sorbent regeneration steam source: Steam extraction from the base plant (default, internal derating) or steam generated from an auxiliary natural gas boiler with (or without) a secondary steam turbine

- Mode of $\mathrm{CO}_{2}$ product transportation: Via pipelines (default) or any other means.

- Mode of $\mathrm{CO}_{2}$ storage/ disposal: Underground geologic reservoir (default), EOR, ECBM, Depleted oil/gas wells, or Ocean

\subsubsection{Parameters controlling the performance of the $\mathrm{CO}_{2}$ system}

Input parameters are given to allow the model user to control the performance of the $\mathrm{CO} 2$ capture system. These parameters then determine the intermediate and final output results. It may be noted that the user can override any of these values, but may want to change values of all the relevant parameters to avoid inconsistencies. These are the key input parameters below:

\section{i) $\mathrm{CO}_{2}$ capture efficiency ( $\eta_{\mathrm{CO}_{2}}$ )}

The overall $\mathrm{CO}_{2}$ capture efficiency of the system is the fraction of $\mathrm{CO}_{2}$ present in the incoming flue gas stream captured in this system.

$$
\eta_{\mathrm{CO}_{2}}=100 \times\left(\text { Moles } \mathrm{CO}_{2} \text { in }- \text { Moles } \mathrm{CO}_{2} \text { out }\right) /\left(\text { Moles } \mathrm{CO}_{2} \text { in }\right)
$$

Most of the studies report the $\mathrm{CO}_{2}$ capture efficiency of the amine-based systems to be $90 \%$, with few others reporting as high as 96\% capture efficiency. Here, it has been assumed to be $90 \%$ as the nominal value, but the user can specify the desired level of $\mathrm{CO}_{2}$ capture efficiency.

\section{ii) $\mathrm{MEA}$ concentration $\left(\mathrm{C}_{M E A}\right)$}

The sorbent used for $\mathrm{CO}_{2}$ absorption is a mixture of mono-ethanol-amine (MEA) with water. The presence of water enhances the chemical absorption reaction of MEA with $\mathrm{CO}_{2}$. MEA is a highly corrosive liquid, especially in the presence of oxygen and carbon dioxide, and hence needs to be diluted. Today the commercially available MEA-based technology supplied by Fluor Daniel uses 30\% w/w MEA sorbent with the help of some corrosion inhibitors. Other suppliers, who do not use this inhibitor, prefer to use lower 
MEA concentrations in the range of $15 \%-20 \% \mathrm{w} / \mathrm{w}$. Here we use $30 \%$ as the nominal value for the sorbent concentration with an acceptable range between $15-40 \%$.

\section{iii) Lean sorbent $\mathrm{CO}_{2}$ loading ( $\left.\phi_{\text {min }}\right)$}

Ideally, the sorbent will be completely regenerated on application of heat in the regenerator section. Actually, even on applying heat, not all the MEA molecules are freed from $\mathrm{CO}_{2}$. So, the regenerated (or lean) sorbent contains some "left-over" $\mathrm{CO}_{2}$. The level of lean sorbent $\mathrm{CO}_{2}$ loading mainly depends upon the initial $\mathrm{CO}_{2}$ loading in the sorbent and the amount of regeneration heat supplied, or alternatively, the regeneration heat requirement depends on the allowable level of lean sorbent loading. Here we use a nominal value of 0.2 based on the values reported in the literature, and the user may specify any desired value in the range (0.1-0.25).

\section{iv) Liquid to gas ratio (L/G)}

The liquid to gas ratio is the ratio of total molar flow rate of the liquid (MEA sorbent plus water) to the total molar flow rate of flue gas being treated in the absorber. This is a calculated parameter and is derived by the process simulation model.

\section{v) Liquid flow rate $(L)$}

The liquid flow rate is the total molar flow rate of sorbent plus dilution water being circulated in the $\mathrm{CO}_{2}$ capture system. It is obtained by multiplying (L/G), which is derived from the process simulation model, by the total flue gas flow rate $(\mathrm{G})$ entering the $\mathrm{CO}_{2}$ capture system.

$$
\mathrm{L}=(\mathrm{L} / \mathrm{G}) \times(\mathrm{G})
$$

vi) Acid gases removal efficiency ( $\eta_{\text {acid gas }}$ ) and stoichiometric MEA loss ( $\boldsymbol{n}_{\text {MEA,acidgas }}$ ) As discussed before, MEA is an alkaline sorbent that has strong affinity for various acid gases. In fact, gases such as hydrogen chloride and oxides of sulfur are much more reactive towards MEA than carbon dioxide itself. These gases form heat stable salts (HSS) with MEA that can not be broken down even after application of heat. So, they cause a permanent loss of MEA sorbent that may be estimated according the stoichiometry of their reactions with MEA. The typical removal efficiencies of these gases in the absorber using MEA sorbent designed for $90 \%$ removal of $\mathrm{CO}_{2}$ are given in Table 3.2. 
Table 3.2. Removal efficiency of acid gases due to MEA sorbent ( $90 \% \mathrm{CO}_{2}$ removal)

\section{Acid gas Removal efficiency (\%) MEA loss (mole MEA/mole acid gas)}

\begin{tabular}{ccc}
\hline $\mathrm{SO}_{2}$ & $\eta_{\mathrm{SO}_{2}}=99.5 \%$ & $\mathrm{n}_{\mathrm{MEA}, \mathrm{SO}_{2}}=2$ \\
$\mathrm{SO}_{3}$ & $\eta_{\mathrm{SO}_{3}}=99.5 \%$ & $\mathrm{n}_{\mathrm{MEA}, \mathrm{SO}_{3}}=2$ \\
$\mathrm{NO}_{2}$ & $\eta_{\mathrm{NO}_{2}}=25 \%$ & $\mathrm{n}_{\mathrm{MEA}, \mathrm{NO}_{2}}=2$ \\
$\mathrm{NO}$ & $\eta_{\mathrm{NO}}=0$ & $\mathrm{n}_{\mathrm{MEA}, \mathrm{NO}}=0$ \\
$\mathrm{HCl}$ & $\eta_{\mathrm{HCl}}=95 \%$ & $\mathrm{n}_{\mathrm{MEA}, \mathrm{HCl}}=1$ \\
\hline
\end{tabular}

\section{v) Temperature of the flue gas entering the $\mathrm{CO}_{2}$ capture system $\left(\mathrm{T}_{f g, \text { in }}\right)$}

The desirable temperature of the flue gas entering the $\mathrm{CO}_{2}$ capture system is about 45-50 deg $\mathrm{C}$. If a direct contact cooler is installed upstream of $\mathrm{CO}_{2}$ capture system, then this temperature level may be achieved.

The temperature of the flue gas affects the absorption reaction as absorption of $\mathrm{CO}_{2}$ in MEA sorbent is an exothermic process favored by lower temperatures. Also, the flue gas temperature directly affects the volumetric flow rate of the flue gas stream, which is a key determinant of the sizes of various equipments (e.g., direct contact cooler, flue gas blower, absorber).

\section{vi) Nominal MEA loss ( $\left.\dot{\mathrm{m}}_{M E A, \text { nom }}\right)$}

MEA is a reactive sorbent. In spite of dilution with water and use of inhibitors, a small quantity of MEA is lost through various unwanted reactions, mainly the polymerization reaction to form long-chained compounds and the oxidation reaction forming organic acids and liberating ammonia. In general, this nominal loss of MEA is estimated as about $1.5 \mathrm{~kg} \mathrm{MEA} /$ tonne $\mathrm{CO}_{2}$.

It is assumed that $50 \%$ of this MEA loss is due to polymerization:

$$
\dot{\mathrm{m}}_{\text {MEA, polym }}==50 \% \text { of }\left(\dot{\mathrm{m}}_{\text {MEA, nom }}\right)
$$

The remaining $50 \%$ of the MEA loss is due to oxidation to acids: 


$$
\dot{\mathrm{m}}_{\text {MEA, oxid }}=50 \% \text { of }\left(\dot{\mathrm{m}}_{\text {MEA, nom }}\right)
$$

\section{vii) $\mathrm{NH}_{3}$ Generation $\left(\mathrm{n}_{\mathrm{NH}_{3}}\right)$}

The oxidation of MEA to organic acids (oxalic, formic, etc.) also leads to formation of $\mathrm{NH}_{3}$. Each mole of MEA lost in oxidation liberates a mole of ammonia $\left(\mathrm{NH}_{3}\right)$.

$$
\text { Rate of ammonia generation, } \mathrm{n}_{\mathrm{NH}_{3}}=\frac{1 \text { mole } \mathrm{NH}_{3}}{\text { mole MEA oxidized }}
$$

\section{viii) Heat-Stable Salts (HSS)}

The organic acids (product of MEA oxidation) combine with MEA to form some other heat stable salts (HSS). The exact nature of these salts is not known. The most conservative estimate, assuming that the organic acids are mono-basic, is that each mole of organic acid takes up one mole of fresh MEA. So, each mole of MEA lost in oxidation takes up additional mole of MEA in HSS formation.

$$
\mathrm{n}_{\text {MEA, organics }}=\frac{1 \text { mole } M E A}{\text { mole organic acid }}
$$

\section{ix) Caustic Consumption in Reclaimer $\left(\dot{\mathrm{m}}_{\mathrm{NaOH}}\right)$}

Caustic in the form of $\mathrm{NaOH}$ is added in the reclaimer so that some of the MEA could be regenerated from HSS. $\dot{\mathrm{m}}_{\mathrm{NaOH}}$ is the quantity (mass) of caustic (as $\mathrm{NaOH}$ ) consumed in MEA reclaimer per tonne of $\mathrm{CO}_{2}$ captured. A typical value is $0.13 \mathrm{~kg} \mathrm{NaOH} /$ tonne $\mathrm{CO}_{2}$.

\section{x) Reclaimed MEA}

Caustic regenerates stoichiometric amount of MEA from the HSS in the reclaimer. Each mole of $\mathrm{NaOH}$ regenerates 1 mole of MEA, and adds the corresponding sodium salt of organic acid to the reclaimer bottoms.

$$
\begin{aligned}
& \dot{\mathrm{n}}_{\text {MEA, reclaimed }}=\text { no. of moles of MEA reclaimed using caustic } \\
& =\text { no. of moles of caustic added } \\
& =\dot{\mathrm{n}}_{\mathrm{NaOH}}
\end{aligned}
$$




$$
\begin{aligned}
& =\dot{\mathrm{m}}_{\mathrm{NaOH}} /(\text { Molecular Weight of } \mathrm{NaOH}) \\
& =\dot{\mathrm{m}}_{\mathrm{NaOH}} / 40
\end{aligned}
$$

\section{xi) Removal efficiency for particulates ( $\left.\eta_{\text {partic }}\right)$}

Amine-based absorption system for $\mathrm{CO}_{2}$ removal is a wet scrubbing operation. So, it also leads to removal of particulate matter from the flue gas to certain extent. Based on the experience of other scrubbing systems, the removal efficiency for particulates has been assumed to be $50 \%$.

\section{xii) Density of sorbent $\left(\rho_{\text {sorbent }}\right)$}

MEA has a density of $1.022 \mathrm{~g} / \mathrm{cc}$ that is similar to that of water. So, the overall density of the MEA based sorbent (with almost $70 \%$ water) is assumed to be same as that of water $\sim 1$ tonne/ $\mathrm{m}^{3}$.

\section{xiii) Activated Carbon Requirement ( $\dot{\mathrm{m}}_{\text {act-C }}$ )}

An activated carbon bed in the sorbent circuit removes the long chained/ cyclic polymeric compounds formed from the degenerated MEA. Over a period of time ( $\sim-6$ months) the carbon-bed needs to be replaced and the used bed is sent back to the suppliers. $\dot{\mathrm{m}}_{\text {act-C }}$ is the average amount of activated carbon consumed per tonne of $\mathrm{CO}_{2}$ captured. This consumption is estimated to be about $0.075 \mathrm{~kg} \mathrm{C} /$ tonne $\mathrm{CO}_{2}$.

\section{xiv) Total moles of $\mathrm{CO}_{2}$ captured ( $\left.\mathrm{n}_{\mathrm{CO} 2}\right)$}

This is the molar flow rate of $\mathrm{CO}_{2}$ captured from the flue gas. It is obtained by multiplying the total $\mathrm{CO}_{2}$ content in the inlet flue gas $\left(\mathrm{kmole} \mathrm{CO}_{2} / \mathrm{hr}\right)$ by the $\mathrm{CO}_{2}$ capture efficiency of the system.

$$
\mathrm{n}_{\mathrm{CO} 2}=\left(\eta_{\mathrm{CO}_{2}} / 100\right) *\left(\text { Moles } \mathrm{CO}_{2} \text { in }\right)=\left(\eta_{\mathrm{CO}_{2}} / 100\right) *\left(\mathrm{G}^{*} \mathrm{y}_{\mathrm{CO} 2}\right)
$$

Since the molecular weight of $\mathrm{CO}_{2}$ is 44 , the total amount of $\mathrm{CO}_{2}$ captured $\left(\mathrm{m}_{\mathrm{CO} 2}\right.$, tonne/ $\mathrm{hr}$ ) is

$$
\mathrm{m}_{\mathrm{CO} 2}=\mathrm{n}_{\mathrm{CO} 2} *(44 / 1000)
$$




\section{xv) $\mathrm{CO}_{2}$ product purity $(\alpha)$}

The final $\mathrm{CO}_{2}$ product has to meet certain specifications depending upon the mode of transport and final destination. Impurities such as nitrogen are undesirable as they may pose problems during compression and liquefaction of $\mathrm{CO}_{2}$. In order to avoid corrosion in the pipelines during transport, the moisture levels must be controlled. The acceptable level of purity of $\mathrm{CO}_{2}$ product for most of the applications is about $99.8 \%$.

\section{xvi) Reboiler duty per mole of liquid $(Q / L)$}

This is the total amount of heat energy input required for the regeneration of the sorbent per unit of liquid circulated. This is mainly dependent on lean sorbent loading, $\mathrm{CO}_{2}$ capture efficiency, MEA concentration and $\mathrm{CO}_{2}$ content of the flue gas and is a calculated value derived from the process simulation model.

\section{xvii) Total heat requirement for sorbent regeneration (Q)}

This is the total amount of heat energy required in the reboiler for sorbent regeneration. It is obtained by multiplying $(\mathrm{Q} / \mathrm{L})$ above, by the total sorbent circulation molar flow rate (MEA sorbent plus dilution water) in the $\mathrm{CO}_{2}$ capture system.

$$
\mathrm{Q}=(\mathrm{Q} / \mathrm{L}) \mathrm{x}(\mathrm{L})
$$

\section{$x v i i i)$ Unit heat of sorbent regeneration $\left(q_{\text {regen }}\right)$}

This is the amount of heat required for the regeneration of the MEA sorbent (loaded with $\mathrm{CO}_{2}$ ) in the stripper/ regenerator section. It is expressed as the amount of heat (in $\mathrm{kJ}$ or Btu) per unit mass ( $\mathrm{kg}$ or $\mathrm{lb}$ ) of $\mathrm{CO}_{2}$ captured. Theoretically, the heat of reaction that needs to be supplied in order to reverse the absorption reaction between $\mathrm{CO}_{2}$ and MEA is about $1900 \mathrm{~kJ} / \mathrm{kg} \mathrm{CO}$ (i.e. about $825 \mathrm{Btu} / \mathrm{lb} \mathrm{CO}_{2}$ ). The actual amount of heat required for regeneration of the sorbent is much higher, about 2-3 times higher than this theoretical minimum. This is because of the large amount of latent heat taken up by the dilution water in the sorbent. A wide range of numbers have been reported for the regeneration heat requirement of MEA system. The majority of the sources report a heat requirement of about $4000 \mathrm{~kJ} / \mathrm{kg} \mathrm{CO}$. Here it is obtained by dividing the total heat requirement for sorbent regeneration $(\mathrm{Q})$ by the total amount of $\mathrm{CO}_{2}$ captured $\left(\mathrm{m}_{\mathrm{CO} 2}\right)$.

$$
\mathrm{q}_{\text {regen }}=\mathrm{Q} / \mathrm{m}_{\mathrm{CO} 2}
$$




\section{xix) Enthalpy of regenerating steam ( $\left.h_{\text {steam }}\right)$}

The regeneration heat is provided in the form of LP (low pressure) steam extracted from the steam turbine in case of coal-fired power plants and combined-cycle gas plants, through the reboiler (a heat exchanger). In case of simple cycle natural gas fired power plants, a heat recovery unit maybe required. $\left(\mathrm{h}_{\text {steam }}\right)$ is the enthalpy or heat content of the steam used for sorbent regeneration. Typically, the LP steam is around $300^{\circ} \mathrm{C}$ and $60-80$ psi. From the steam-tables, the enthalpy (heat content) of such steam is found to be about $2000 \mathrm{~kJ} / \mathrm{kg}$ steam.

\section{$x x)$ Heat-to-electricity equivalence factor $\left(F_{H E}\right)$}

The extraction of LP (low pressure) steam for sorbent regeneration results in some loss of power generation capacity of the plant. The factor $\left(\mathrm{F}_{\mathrm{HE}}\right)$ gives the equivalent loss of power generation capacity due to the heat requirement for sorbent regeneration.

Let us try to understand what this factor stands for and how it is derived. According to the Second Law of Thermodynamics, thermal energy cannot be fully converted to work. Electrical energy is a form of work. The efficiency of converting heat into electricity depends upon the temperature and pressure conditions of the working fluid, i.e. steam in case of a conventional coal plant. Even the advanced steam plants (using super-critical or ultra-super-critical boilers) can achieve net plant efficiencies of about 42-45 percent by using high quality steam (steam at high pressure and temperature, e.g. $31 \mathrm{MPa}$ and 590 $\left.{ }^{\circ} \mathrm{C}\right)$ (Rubin 2001). In general, steam at lower pressure and temperature will have still lower efficiency to convert heat into electric energy. The steam used for regeneration of sorbent is low quality steam (low pressure, $\sim 0.5 \mathrm{MPa}$ and low temperature, $\sim 120{ }^{\circ} \mathrm{C}$ ), and hence the equivalent loss of power generation is much lower than the power plant efficiency. Again, the electric energy penalty due to steam extraction also depends on the overall heat integration within the plant. Factor $\left(\mathrm{F}_{\mathrm{HE}}\right)$ may be looked upon as the power generation efficiency of the LP steam extracted from the steam cycle of the power plant for sorbent regeneration. So, the equivalent loss in power generation capacity of the base plant may be estimated by multiplying the total sorbent regeneration heat requirement by this factor. 
From the data obtained from the available studies (Smelser, Stock et al. 1991; Hendriks 1994; Mimura, Simoyoshi et al. 1997; Bolland and Undrum 1998; Marion, Nsakala et al. $2001)$, this factor has been found to lie in the range $(9,19)$ for a new plant and $(20,25)$ for retrofit cases. So, the nominal value (for this new plant application) has been taken as $14 \%$.

For example, if $10,000 \mathrm{~kJ} / \mathrm{hr}$ is the regeneration heat requirement for the $\mathrm{CO}_{2}$ capture operation, then the corresponding loss in power generation capacity of the power plant is estimated as $14 \%$ of $10,000 \mathrm{~kJ} / \mathrm{hr}$ i.e. $1400 \mathrm{~kJ} / \mathrm{hr}$, or $(1400 / 3600=) 0.39 \mathrm{~kW}$. It may be noted that, in case of retrofit applications, the energy penalty might be significantly higher due to poor heat integration, and $\mathrm{F}_{\mathrm{HE}}$ may be approximately $22 \%$.

\section{xxi) Blower pressure head $\left(\Delta P_{f g}\right)$}

Prior to entering into the absorber column, the flue gas has to be compressed in a flue gas blower so that it can overcome the pressure drop in the absorber tower. $\left(\Delta \mathrm{P}_{\mathrm{fg}}\right)$ is the pressure head that needs to be provided to the flue gas in the blower, and is about $14 \mathrm{kPa}$ $(\sim 2 \mathrm{psi})$.

\section{xxii) Blower (fan) efficiency ( $\eta_{\text {blower }}$ )}

This is the efficiency of the fan/blower to convert electrical energy input into mechanical work output. Typically, the value of blower efficiency ( $\left.\eta_{\text {blower }}\right)$ is about $75 \%$.

\section{xxiii) Sorbent pumping head $\left(\Delta P_{\text {sorbent }}\right)$}

The sorbent has to flow through the absorber column through packed media, countercurrent to the flue gas flowing upwards. So, some pressure loss is encountered in the absorber column and sufficient sorbent head has to be provided to overcome these pressure losses. $\left(\Delta \mathrm{P}_{\text {sorbent }}\right)$ is the pressure head to be provided to the sorbent using sorbent circulation pumps. A typical value is about $200 \mathrm{kPa}(\sim 30 \mathrm{psi})$.

\section{xxiv) Pump efficiency ( $\eta_{\text {pump }}$ )}

This is the efficiency of the sorbent circulation pumps to convert electrical energy input into mechanical energy output. Typically, the value of ( $\left.\eta_{\text {pump }}\right)$ is assumed to be $75 \%$. 


\section{xxv) $\mathrm{CO}_{2}$ product pressure $\left(\mathrm{P}_{\mathrm{CO}_{2}}\right)$}

The $\mathrm{CO}_{2}$ product may have to be carried over long distances. Hence it is necessary to compress and liquefy it to very high pressures $\left(\mathrm{P}_{\mathrm{CO}_{2}}\right)$, so that it may be delivered to the required destination in liquid form and with minimal recompression facilities en route. The critical pressure is the minimum pressure required to liquefy a gas at its critical temperature, which is the temperature above which it is not possible to liquefy a gas. For $\mathrm{CO}_{2}$ this is about $1070 \mathrm{psig}$. The typically reported value of final pressure to which the product $\mathrm{CO}_{2}$ stream is pressurized using compressors before it is transported is about 2000 psig (about $137 \mathrm{~atm}$ ).

xxvi) Energy required for $\mathrm{CO}_{2}$ compression ( $\left.e_{\text {comp }}\right)$

This is the electrical energy required $\left(\mathrm{kWh}\right.$ per tonne $\left.\mathrm{CO}_{2}\right)$ to compress a unit mass of $\mathrm{CO}_{2}$ product stream to the designated pressure $\left(\mathrm{P}_{\mathrm{CO}_{2}}\right)$ expressed in psig. Compression of $\mathrm{CO}_{2}$ to high pressures requires substantial energy, and is a principle contributor to the overall energy penalty of a $\mathrm{CO}_{2}$ capture unit in a power plant.

\section{xxvii) $\mathrm{CO}_{2}$ compression efficiency ( $\eta_{\text {comp }}$ )}

This is the effective efficiency of the compressors used to compress $\mathrm{CO}_{2}$ to the desirable pressure. Typically, the value of compressor efficiency ( $\left.\eta_{\text {comp }}\right)$ is about $80 \%$. It may be noted that the energy requirement calculated from the performance equation $\left(\mathrm{e}_{\mathrm{comp}}\right)$ has to be corrected by this efficiency factor in order to get the total energy required for $\mathrm{CO}_{2}$ compression.

The following set of parameters are relevant only if the $\mathrm{CO}_{2}$ capture system has been configured to include an auxiliary NG boiler to supply sorbent regeneration heat.

\section{xxviii) Heating value of natural gas (NGHV)}

This is the high heating value (HHV, MJ/ kmole NG) of the natural gas used as fuel for the auxiliary boiler.

$x x i x)$ Density of natural gas $\left(\rho_{N G}\right)$

This is the density $\left(\mathrm{lb} / \mathrm{ft}^{3}\right)$ of the natural gas used as fuel for the auxiliary boiler. 
$x x x)$ Average molecular weight of natural gas ( $m w_{N G}$ )

This is the average molecular weight $(\mathrm{kg} / \mathrm{kmole} \mathrm{NG})$ of the natural gas used as fuel for the auxiliary boiler. This is a function of the molar composition of the natural gas.

xxxi) Flow rate of natural gas $\left(m_{N G}\right)$

This is the total molar flow rate (kmole NG/ hr) of the natural gas used as fuel for the auxiliary boiler. It is a function of the total heat requirement for sorbent regeneration in the amine system.

xxxii) Auxiliary NG boiler efficiency ( $\eta_{N G B}$ )

This is the efficiency of the auxiliary boiler that uses natural gas as fuel input. It is defined as the ratio of total thermal energy (in the from of steam) delivered by the boiler divided by the total heat energy input (in the form of heating value of the natural gas input).

xxxiii) Secondary steam turbine power generation efficiency $\left(\eta_{S T 2}\right)$

This is the efficiency of the secondary steam turbine added with the auxiliary NG boiler to generate electrical power. It may be defined as the ratio of electrical energy generated $\left(\mathrm{MW}_{\mathrm{ST2}}\right)$ by the steam turbine divided by the total thermal energy (in the form of steam) input from the auxiliary NG boiler. It is assumed that the rest of the thermal energy is contained in the LP exhaust steam from the turbine, which is sent to the reboiler for sorbent regeneration.

\subsection{Performance Equations}

The performance equations define the functional relationships among various key performance parameters. They have been derived as multivariate linear or log-linear regression equations from the data obtained from the process simulation model runs.

$$
\begin{gathered}
(\mathrm{L} / \mathrm{G})=\exp \left(-1.4352+0.1239 * \mathrm{y}_{\mathrm{CO} 2}+3.4863 * \phi_{\text {lean }}+0.0174 * \eta_{\mathrm{CO} 2}-0.0397 * \mathrm{C}+\right. \\
{\left[\operatorname{adj} . \mathrm{R}^{2}=0.92\right]} \\
(\mathrm{Q} / \mathrm{L})=\exp \left(-2.4452-0.0037 * \mathrm{y}_{\mathrm{CO} 2}-6.2743 * \phi_{\text {lean }}+0.0254 * \mathrm{C}\right) \\
{\left[\operatorname{adj} . \mathrm{R}^{2}=0.96\right]} \\
\left(\mathrm{T}_{\mathrm{fg}, \text { out }}\right)=41.15+0.062 * \mathrm{~T}_{\mathrm{fg}, \text { in }}+1.307 * \mathrm{y}_{\mathrm{CO} 2}-18.872 * \phi_{\text {lean }}+0.270 * \mathrm{C} \\
\text { [adj. } \left.\mathrm{R}^{2}=0.92\right]
\end{gathered}
$$




$$
\left(\mathrm{mw}_{\text {lean }}\right)=16.907+2.333 * \phi_{\text {lean }}+0.204 * \mathrm{C}
$$

$\left(\mathrm{e}_{\mathrm{comp}}\right)=-51.632+19.207 * \ln \left(\mathrm{P}_{\mathrm{CO} 2}+14.7\right)$

where,

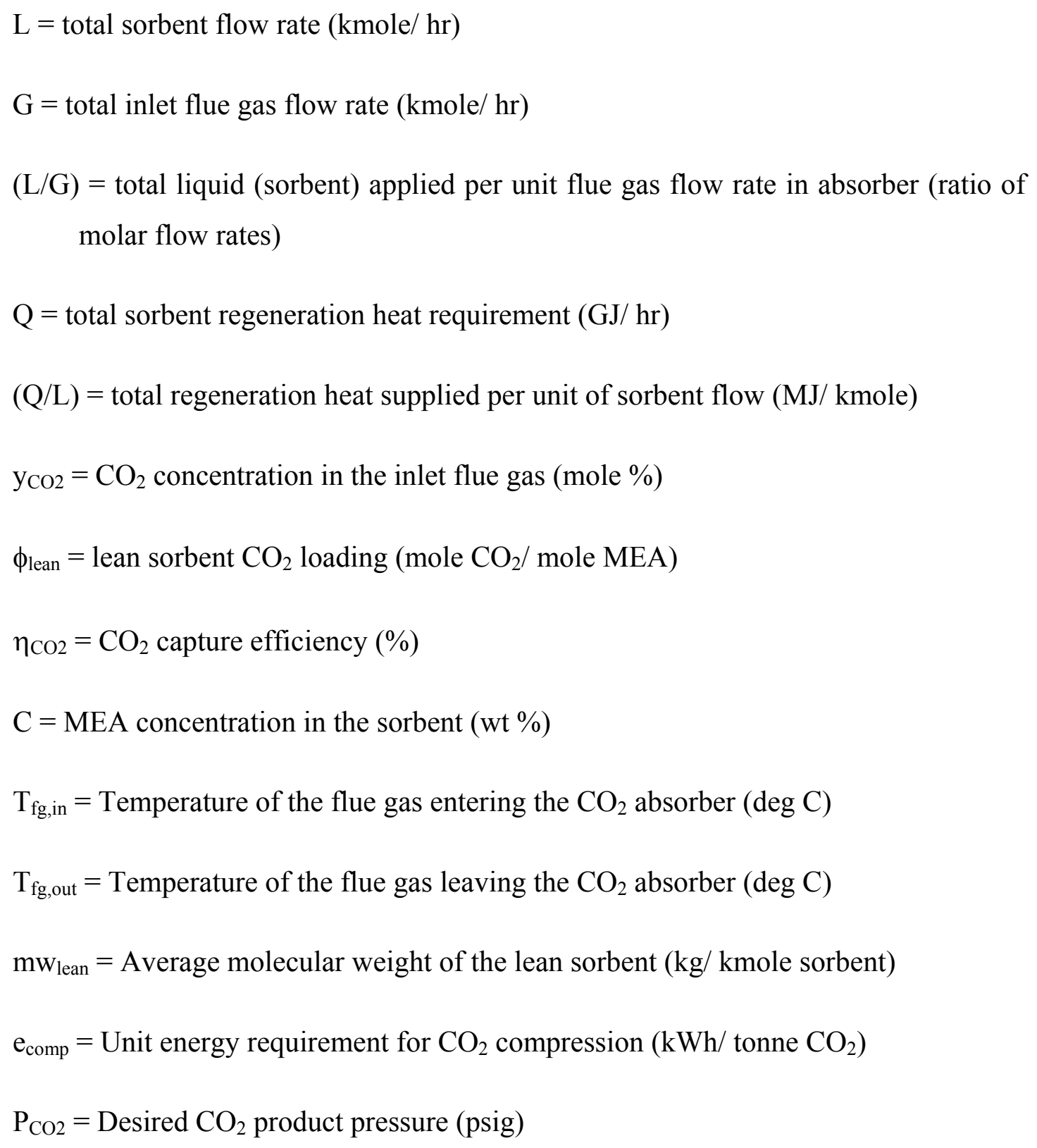

\subsection{Model Outputs}

The key outputs of the amine system performance model include: 
- MEA makeup requirement. This depends mainly on the mass flow rate of $\mathrm{CO}_{2}$ in the flue gas, the desired $\mathrm{CO}_{2}$ capture efficiency, MEA concentration, and $\mathrm{CO}_{2}$ loadings in the sorbent. Depending on the level of impurities in the flue gas, there is some loss of sorbent. If the power plant does not have emission controls for $\mathrm{SO}_{\mathrm{x}}$ and $\mathrm{NO}_{\mathrm{x}}$, the cost imposed due to amine loss may be significant.

- Energy requirement. Heat for sorbent regeneration is derived from low-pressure steam available in the power plant, which decreases power generation efficiency. Additional electrical energy is required for $\mathrm{CO}_{2}$ product compression, sorbent circulation, and other system requirements. The energy requirement is one of the most important results, as it dictates the net size of the power plant, and hence the net cost of power generation and $\mathrm{CO}_{2}$ avoidance.

The following material and energy flows are estimated using the above stated inputs

\subsubsection{Total quantity of $\mathrm{CO}_{2}$ captured:}

$$
\begin{aligned}
& \mathrm{m}_{\mathrm{CO} 2}(\text { tonne } / \mathrm{hr})=\mathrm{n}_{\mathrm{CO} 2} \times(\mathrm{MolWt})_{\mathrm{CO} 2} \\
& =\eta_{\mathrm{CO} 2} \times \mathrm{n}_{\mathrm{CO} 2 \text {,inlet }} \times(\mathrm{MolWt})_{\mathrm{CO} 2}
\end{aligned}
$$

where,

$$
\mathrm{n}_{\mathrm{CO} 2}=\text { Total moles of } \mathrm{CO}_{2} \text { captured }\left(\mathrm{kmole} \mathrm{CO}_{2} / \mathrm{hr}\right)
$$

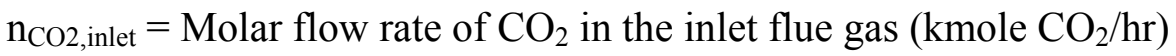

$(\mathrm{MolWt})_{\mathrm{CO} 2}=$ Molecular weight of $\mathrm{CO}_{2}=0.044$ tonne $/ \mathrm{kmole} \mathrm{CO}_{2}$

\subsubsection{MEA makeup requirement:}

3.2.1.

$$
\dot{\mathrm{m}}_{\text {MEA,makeup }}
$$

$=$ Net loss of MEA

$$
\begin{aligned}
= & \left(\begin{array}{c}
\text { loss due to acid gas } \\
\text { impurities }
\end{array}\right)+\left(\begin{array}{c}
\text { loss due to } \\
\text { polymerization }
\end{array}\right) \\
& +\left(\begin{array}{cc}
\text { loss due to } & \text { Gain in } \\
\text { HSS formation }- \text { Reclaimer }
\end{array}\right)+\left(\begin{array}{c}
\text { loss with } \\
\text { fluegas exhaust }
\end{array}\right)
\end{aligned}
$$




\subsubsection{Total sorbent circulation flow rate:}

From the performance equations, we find

$$
\mathrm{L} / \mathrm{G}=\mathrm{f}\left(\mathrm{y}_{\mathrm{CO} 2}, \phi_{\text {lean }}, \eta_{\mathrm{CO} 2}, \mathrm{C}, \mathrm{T}_{\mathrm{fg}, \mathrm{in}}\right), \text { and } \mathrm{L}=\mathrm{G}^{*}(\mathrm{~L} / \mathrm{G})
$$

Including the MEA makeup requirement gives the total sorbent flow rate $\left(\mathrm{m}^{3} / \mathrm{hr}\right)$

$$
\mathrm{L}_{\text {tot, } \mathrm{v}}=\left\{\mathrm{G} *(\mathrm{~L} / \mathrm{G}) * \mathrm{mw}_{\text {lean }}+\dot{\mathrm{m}}_{\mathrm{MEA}, \text { makeup }} *(100 / \mathrm{C})\right\}^{*} \rho_{\text {sorbent }}
$$

\subsubsection{Waste generated from reclaimer:}

$$
\begin{aligned}
\mathrm{m}_{\text {waste }}= & \left(\begin{array}{c}
\text { MEAlost due to } \\
\text { acid gases }
\end{array}+\begin{array}{c}
\text { Total qty of } \\
\text { acid gases removed }
\end{array}\right)+\left(\begin{array}{c}
\text { MEAloss due } \\
\text { to oxidation }
\end{array}\right) \\
& +\left(\begin{array}{c}
\text { MEA loss due to } \\
\text { HSS format } \\
- \text { Gain in reclaimer }
\end{array}\right)+\left(\begin{array}{c}
\text { Caustic added } \\
\text { to } \\
\text { Reclaimer }
\end{array}\right)
\end{aligned}
$$

Considering $\left(\mathrm{f}_{\mathrm{w}, \text { waste }}\right)$ as the water content $(\% \mathrm{w} / \mathrm{w})$ in the waste, the actual mass flow rate of waste is obtained as:

$\mathrm{M}_{\text {waste,total }}=\mathrm{m}_{\text {waste }} /\left(1-\mathrm{f}_{\mathrm{w}, \text { waste }}\right) \mathrm{kg} / \mathrm{hr}$

Typically, the reclaimer waste contains about $40 \%$ water.

\subsubsection{Activated carbon consumption:}

$$
\mathrm{m}_{\text {act-C }}=\dot{\mathrm{m}}_{\text {act-C }} \times \mathrm{m}_{\mathrm{CO}_{2}} \mathrm{~kg} \text { act-C/hr }
$$

\subsubsection{Caustic consumption in reclaimer:}

$$
\mathrm{m}_{\text {Caustic }}=\dot{\mathrm{m}}_{\mathrm{NaOH}} \times \mathrm{m}_{\mathrm{CO}_{2}} \mathrm{~kg} \mathrm{NaOH} / \mathrm{hr}
$$

\subsubsection{Process water requirement:}

Unit process water makeup $=\dot{\mathrm{m}}_{\mathrm{pw}}$ (tonne/ hr)/MW(net) 
Typically, the value of $\dot{\mathrm{m}}_{\mathrm{pw}}$ is about 0.114 tonne/hr per $\mathrm{MW}$ (net)

(Smelser, Stock et al. 1991). Therefore, the process water requirement is:

$\left(\mathrm{M}_{\mathrm{pw}}\right)=\dot{\mathrm{m}}_{\mathrm{pw}} \times \mathrm{MW}_{\text {net }}$ tonne $/ \mathrm{hr}$

\subsubsection{Cooling water requirement:}

If there is a direct contact cooler installed, the required flow rate of cooling water is estimated based on the following assumptions

Specific heat of water, $\mathrm{SH}_{\mathrm{w}}=4.2 \mathrm{~kJ} / \mathrm{kg}{ }^{\circ} \mathrm{C}$

Specific heat of flue gas $=\mathrm{SH}_{\mathrm{fg}}$ (Generally, this is around $1.2 \mathrm{~kJ} / \mathrm{kg}{ }^{\circ} \mathrm{C}$ )

Temperature rise in the cooling water (once through system) $=\Delta \mathrm{T}_{\mathrm{w}}$

Drop in flue gas temperature $=\Delta \mathrm{T}_{\mathrm{fg}}=\left(\mathrm{T}_{\mathrm{fg}, \mathrm{i}}-\mathrm{T}_{\mathrm{fg}}\right){ }^{\circ} \mathrm{F}$

where,

$\mathrm{T}_{\mathrm{fg}, \mathrm{i}}=$ Temperature of flue gas entering the direct contact cooler

$\mathrm{T}_{\mathrm{fg}}=$ Temperature of flue gas exiting the direct contact cooler

Mass flow rate of flue gas $=\mathrm{m}_{\mathrm{fg}}$ tonne/ $\mathrm{hr}$

So, the required cooling water flow rate,

$\mathrm{M}_{\mathrm{cw}}=\mathrm{m}_{\mathrm{fg}} *\left(\Delta \mathrm{T}_{\mathrm{fg}} / \Delta \mathrm{T}_{\mathrm{w}}\right) *\left(\mathrm{SH}_{\mathrm{fg}} / \mathrm{SH}_{\mathrm{w}}\right) \quad$ tonne $/ \mathrm{hr}$

\subsubsection{Steam requirement:}

LP steam is extracted from the power plant steam turbine or secondary steam turbine in order to provide the sorbent regeneration heat in the reboiler. Based on the regeneration heat requirement and enthalpy of regeneration steam, the flow rate of steam may be estimated as follows:

From the performance equations,

$(\mathrm{Q} / \mathrm{L})=\mathrm{f}\left(\mathrm{y}_{\mathrm{CO} 2}, \phi_{\text {lean }}, \mathrm{C}\right)$ 
Total regeneration heat requirement,

$\mathrm{Q}(\mathrm{MJ} / \mathrm{hr})=(\mathrm{Q} / \mathrm{L}) *(\mathrm{~L})$

Mass flow rate of steam,

$\mathrm{m}_{\text {steam }}($ tonne $/ \mathrm{hr})=\mathrm{Q} / \mathrm{q}_{\text {steam }}$

The equivalent energy penalty due to regeneration steam requirement is ( $\mathrm{E}_{\text {regen }}$ ). Depending upon the $\mathrm{CO}_{2}$ capture system configuration (source of regeneration steam supply), $E_{\text {regen }}$ has to be estimated in two different ways.

1. In case of steam extraction from the base plant steam cycle (derating)

$$
\mathrm{E}_{\text {regen }}=\mathrm{Q}^{*} \mathrm{~F}_{\mathrm{HE}}
$$

2. In case of steam supplied from an auxiliary NG boiler,

$$
\mathrm{E}_{\text {regen }}=-\mathrm{E}_{\mathrm{ST} 2}=-\left(\mathrm{m}_{\mathrm{NG}} * \mathrm{NG}_{\mathrm{HV}} * \eta_{\mathrm{NGB}} * \eta_{\mathrm{ST}}\right)
$$

It maybe noted that in the case of auxiliary NG boiler, the energy penalty term is negative, implying that there is an increase in the net power generation of the plant.

\subsubsection{Electrical energy requirement:}

Total electricity requirement of $\mathrm{CO}_{2}$ capture system is:

$$
\mathrm{E}_{\mathrm{CO}_{2} \text {, tot }}=\mathrm{E}_{\text {regen }}+\mathrm{E}_{\text {pumping }}+\mathrm{E}_{\text {compr }}
$$

where,

$$
\begin{aligned}
& \mathrm{E}_{\text {regen }}=\text { as explained above } \\
& \mathrm{E}_{\text {pumping }}=\mathrm{E}_{\text {blower }}+\mathrm{E}_{\text {pump }} \\
& E_{\text {blower }}(\mathrm{hp})=\frac{144 \mathrm{Q}_{\mathrm{fg}} \Delta \mathrm{P}_{\mathrm{fg}}}{33000 \cdot \eta_{\text {blower }}}
\end{aligned}
$$

where $\mathrm{Q}_{\mathrm{fg}}$ and $\Delta \mathrm{P}_{\mathrm{fg}}$ are expressed in $\mathrm{ft}^{3} / \mathrm{min}$ and psi respectively, 


$$
E_{\text {pump }}(\mathrm{hp})=\frac{\mathrm{Q}_{\text {solvent }} \Delta P_{\text {solvent }}}{1714 \cdot \eta_{\text {pump }}}
$$

where $\mathrm{Q}_{\text {sorbent }}$ and $\Delta \mathrm{P}_{\text {sorbent }}$ are expressed in gal/min and psi respectively, and

$$
\mathrm{E}_{\mathrm{compr}}=\mathrm{e}_{\mathrm{comp}} * \mathrm{~m}_{\mathrm{CO} 2} / \eta_{\mathrm{comp}}
$$

where $\mathrm{e}_{\text {comp }}$ is given by equation (3-5).

\subsection{Characterization of Uncertainty and Variability}

Any techno-economic analysis, and especially that of new energy and environmental control technologies that are still in the research phase, involves uncertainties regarding the performance and costs. These uncertainties come from incomplete information available and numerous assumptions and approximations built into simulations. Some parameters, especially the cost parameters, are influenced by a larger set of factors outside the scope of the particular study and fluctuations in these quantities may be seen as "inherent randomness" when viewed within this limited focus area. In addition, there may be significant variability in plant or process design assumptions across different studies or organizations.

One of the distinguishing features of this modeling effort is a probabilistic capability that allows model inputs to be represented by probability distributions rather than single deterministic values. Probability distributions for these parameters reflect the ranges of values reported in the literature, the evolving nature of the technology, and practical considerations in running such plants. Also, it is possible to use probability distributions for more than one parameter (or all the parameters together) simultaneously.

Table 3.3 lists the uncertainty distributions developed for performance model parameters based on the current literature on amine-based (MEA) systems. These distributions reflect both uncertainty and variability in system designs. Details are presented in Appendix B and Appendix C. The data sources from which the parameter values were 
Table 3.3. Amine system performance model parameters and uncertainties

\begin{tabular}{|c|c|c|c|c|}
\hline $\begin{array}{c}\text { Performance } \\
\text { Parameter }\end{array}$ & Units & $\begin{array}{c}\text { Data } \\
\text { (Range) }\end{array}$ & $\begin{array}{c}\text { Nominal } \\
\text { Value }\end{array}$ & $\begin{array}{c}\text { Unc. Representation } \\
\text { (Distribution Function) }\end{array}$ \\
\hline $\mathrm{CO}_{2}$ removal efficiency & $\%$ & Mostly 90 & 90 & - \\
\hline $\mathrm{SO}_{2}$ removal efficiency & $\%$ & Almost 100 & 99.5 & Uniform $(99,100)$ \\
\hline $\mathrm{NO}_{2}$ removal efficiency & $\%$ & $20-30$ & 25 & Uniform $(20,30)$ \\
\hline $\mathrm{HCl}$ removal efficiency & $\%$ & $90-95$ & 95 & Uniform $(90,95)$ \\
\hline Particulate removal eff. & $\%$ & 50 & 50 & Uniform $(40,60)$ \\
\hline MEA concentration & $\mathrm{wt} \%$ & $15-50$ & 30 & Triangular $(20,30,40)$ \\
\hline $\begin{array}{l}\text { Lean sorbent } \mathrm{CO}_{2} \\
\text { loading }\end{array}$ & $\mathrm{mol} \mathrm{CO} 2 / \mathrm{mol} \mathrm{MEA}$ & $0.15-0.30$ & 0.2 & Triangular( $(0.1,0.2,0.25)$ \\
\hline Nominal MEA make-up & kg MEA/tonne $\mathrm{CO}_{2}$ & $0.5-3.1$ & 1.5 & Triangular $(0.5,1.5,3.1)$ \\
\hline MEA loss $\left(\mathrm{SO}_{2}\right)$ & $\mathrm{mol} \mathrm{MEA} / \mathrm{mol} \mathrm{SO}_{2}$ & 2 & 2 & - \\
\hline MEA loss $\left(\mathrm{NO}_{2}\right)$ & $\mathrm{mol} \mathrm{MEA} / \mathrm{mol} \mathrm{NO}_{2}$ & 2 & 2 & - \\
\hline MEA loss $(\mathrm{HCl})$ & $\mathrm{mol} \mathrm{MEA} / \mathrm{mol} \mathrm{HCl}$ & 1 & 1 & - \\
\hline $\mathrm{NH}_{3}$ generation & $\begin{array}{c}\mathrm{mol} \mathrm{NH}_{3} / \mathrm{mol} \mathrm{MEA} \\
\text { oxidized }\end{array}$ & 1 & 1 & - \\
\hline $\begin{array}{l}\text { Caustic consumption in } \\
\text { MEA reclaimer }\end{array}$ & $\mathrm{kg} \mathrm{NaOH} /$ tonneCO${ }_{2}$ & 0.13 & 0.13 & - \\
\hline Activated carbon use & $\mathrm{kg} \mathrm{C} /$ tonne $\mathrm{CO}_{2}$ & 0.075 & 0.075 & - \\
\hline Gas-phase pressure drop & $\mathrm{kPa}$ & $4-37$ & 14 & Triangular(4,14,37) \\
\hline Fan efficiency & $\%$ & $70-80$ & 75 & Uniform $(70,80)$ \\
\hline Sorbent pumping head & $\mathrm{kPa}$ & 200 & 200 & Triangular(150,200,250) \\
\hline Pump efficiency & $\%$ & $70-80$ & 75 & Uniform $(70,80)$ \\
\hline Equiv. elec. requirement & $\%$ regeneration heat & $9-19$ & $14^{\mathrm{a}}$ & Uniform $(9,19)$ \\
\hline $\mathrm{CO}_{2}$ product pressure & $\mathrm{MPa}$ & $7.58-15.16$ & 13.79 & Triangular( $(7.58,13.79,15.16)$ \\
\hline Compressor efficiency & $\%$ & $75-88$ & 80 & Uniform $(75,88)$ \\
\hline
\end{tabular}

${ }^{\mathrm{a}}$ For retrofit applications, nominal value is 22 . 
obtained also included peer-reviewed journal articles, conference papers, books, technical reports, and technical judgments given by experts.

Almost all the sources reported a $\mathrm{CO}_{2}$ capture efficiency of $90 \%$. Hence this value has been used as the default nominal value without any default probability distribution. Nonetheless, the user is allowed to use a probability distribution for this parameter as well.

\section{References (Chapter 3)}

Aspen-Plus $^{(\mathrm{R})}$ (2002). Aspen Technology, Inc. Cambridge, MA.

Bergman, P. D. and E. M. Winter (1995). "Disposal of carbon dioxide in aquifers in the U.S." Energy Conversion and Management 36(6-9): 523-526.

Bolland, O. and H. Undrum (1998). Removal of CO2 from gas turbine power plants: Evaluation of pre- and post-combustion methods. Fourth International Conference on Greenhouse Gas Control Technologies, 30 August - 2 September, Interlaken, Switzerland, Elsevier Science Ltd.

Chakma, A. and P. T. Tontiwachwuthikul (1998). Designer solvents for energy efficient CO2 separation from flue gas streams. Fourth International Conference on Greenhouse Gas Control Technologies, 30 August - 2 September, Interlaken, Switzerland, Elsevier Science Ltd.

Chapel, D., J. Ernst, et al. (1999). Recovery of CO2 from flue gases: commercial trends (paper no. 340). presented at the Canadian Society of Chemical Engineers Annual Meeting, 4-6 October, Saskatoon, Saskatchewan, Canada.

Hendriks, C. (1994). Carbon Dioxide Removal from Coal-fired Power Plants. The Netherlands, Kluwer Academic Publishers.

Herzog, H. J. (1998). The economics of CO2 capture. Fourth International Conference on Greenhouse Gas Control Technologies, 30 August - 2 September, Interlaken, Switzerland, Elsevier Science Ltd. 
IECM (2001). Integrated Environmental Control Model and User Documentation (available at www.iecm-online.com), Center for Energy and Environmental Studies, Carnegie Mellon University, Pittsburgh, PA.

Kohl, A. L. and R. B. Nielsen (1997). Gas Purification. Houston, TX, Gulf Publishing Company.

Marion, J., N. Nsakala, et al. (2001). Engineering feasibility of CO2 capture on an existing US coal-fired power plant. in the proceedings of the Twenty-sixth International Conference on Coal Utilization and Fuel Systems, 5-8 March, Clearwater, FL, USA.

Mimura, T., H. Simoyoshi, et al. (1997). "Development of energy saving technology for flue gas carbon dioxide recovery in power plants by chemical absorption method and steam system." Energy Conversion and Management 38(Suppl): S57-S62.

ProTreat $^{\mathrm{TM}}$ (2002). Optimized Gas Treating, Inc.,. Houston, TX.

Rubin, E. S. (2001). Introduction to engineering and the environment. New York, NY, The McGraw-Hill Companies, Inc.

Rubin, E. S., J. R. Kalagnanam, et al. (1997). "Integrated environmental control modeling of coalfired power systems." Journal of Air and\& Water Management Association 47: 11801188 .

SAS (1999-2001). SAS Institute Inc. Cary, NC, USA.

Simbeck, D. (1998). A portfolio selection approach for power plant CO2 capture, separation and $\mathrm{R} \& \mathrm{D}$ options. Fourth International Conference on Greenhouse Gas Control Technologies, 30 August - 2 September, Interlaken, Switzerland, Elsevier Science Ltd.

Simbeck, D. and M. McDonald (2000). Existing coal power plant retrofit $\mathrm{CO}_{2}$ control options analysis. presented at the Fifth International Conference on Greenhouse Gas Control Technologies, 13-16 August, Cairns, Australia.

Smelser, S. C., R. M. Stock, et al. (1991). Engineering and economic evaluation of CO2 removal from fossil-fuel-fired power plants, EPRI IE-7365, Volume 1, Project 2999-10, a research project final report prepared by Fluor Daniel Inc., for EPRI and IEA. 


\section{COST MODEL DEVELOPMENT}

The $\mathrm{CO}_{2}$ capture and sequestration system cost model is directly linked to the performance model. The cost model follows the framework used in the IECM (Rubin, Kalagnanam et al. 1997) to ensure consistency in economic calculations. There are four types of cost calculated by this model based on the available data (Smelser, Stock et al. 1991; Hendriks 1994; Leci 1996; Mariz 1998; Simbeck 1998; Chapel, Ernst et al. 1999; Desideri and Paolucci 1999; Jeremy and Herzog 2000). They are capital cost, operation and maintenance $(\mathrm{O} \& \mathrm{M})$ cost, incremental cost of electricity $(\mathrm{COE})$ and cost of $\mathrm{CO}_{2}$ avoidance.

\subsection{Capital Cost}

The total capital requirement (TCR) of a system is calculated as the sum of direct equipment costs (which depend on one or more performance variables that determine the size or capacity of the component), plus various indirect costs that are estimated as fractions of the total direct cost following the EPRI cost estimating guidelines (TAG 1993; TAG 1999).

The capital cost model is primarily based on detailed information obtained from Fluor Daniel Inc. (Fluor 1998). To develop a generalized cost model applicable to different plant sizes and material flow rates, the Fluor Daniel data are used as a reference point for the application of engineering "scaling laws". For example, where multiple trains are required to perform the $\mathrm{CO}_{2}$ capture operation, the maximum train size is taken to be 5000 tonnes per day of $\mathrm{CO}_{2}$, based on current design. Based on the actual $\mathrm{CO}_{2}$ capture rate $\left(\mathrm{m}_{\mathrm{CO} 2}\right)$ the number of trains required to be installed $\left(\mathrm{N}_{\min }\right)$ is determined.

Different components have different maximum capacity limits. $E_{n, i}$ defines the number of components required per train. Each train consists of the following pieces of equipment $\left(\mathrm{E}_{\mathrm{n}, \mathrm{i}}\right)$ :

- Direct contact cooler (DCC), flue gas blower, absorber, heat exchanger, regenerator, steam extractor, MEA reclaimer - 1 per each train 
- Pumps - 2 per train

- Reboilers - 4 per train

Special cases:

1. Only one installation is required for the sorbent processing area, auxiliary natural gas boiler, secondary steam turbine, $\mathrm{CO}_{2}$ transport facility and $\mathrm{CO}_{2}$ disposal facility.

2. In case of $\mathrm{CO}_{2}$ compressors, which have higher capacity $(\sim 7200$ tonnes per day of $\mathrm{CO}_{2}$ ), the number of compressors required is calculated accordingly.

The unit cost of each component of this system (absorber, regenerator, flue gas blower etc.) is scaled based on the flow rate of the material being handled by that particular device, using the 0.6 power law commonly used in chemical engineering costing (Peters and Timmerhaus 1991). For example, the cost of an absorber is scaled on the basis of the flue gas flow rate entering the $\mathrm{CO}_{2}$ system, with the data obtained from Fluor Daniel serving as the reference basis for this scaling. Thus, in general, the capital cost of a component of arbitrary size (less than the maximum size) may be estimated as:

$$
C_{i}=\mathrm{C}_{\mathrm{i}, \text { ref }} \cdot\left(\frac{\mathrm{X}_{\mathrm{i}}}{\mathrm{X}_{\mathrm{i}, \text { ref }}}\right)^{0.6}
$$

where,

$$
\begin{aligned}
& \mathrm{C}_{\mathrm{i}}=\text { Component cost } \\
& \mathrm{C}_{\mathrm{i}, \text { ref }}=\text { Cost of reference size } \\
& \mathrm{X}=\text { actual flow rate (for scaling) } \\
& \mathrm{X}_{\mathrm{i}, \text { ref }}=\text { Flow rate of reference plant }
\end{aligned}
$$

Once the cost of a particular equipment $\left(\mathrm{C}_{\mathrm{i}}\right)$ is calculated, it needs to be multiplied by the total number of equipment installed $\left(Z_{i}\right)$ in order to get the total cost of installation for that process area (i). 
The overall amine system is divided into a number of process areas for which some physical quantity is identified as the basis for scaling of the capital cost. For example, flue gas flow rate, sorbent flow rate, $\mathrm{CO}_{2}$ product flow rate, $\mathrm{CO}_{2}$ compression energy requirement, steam flow rate, and makeup MEA flow rate are used for scaling he capital cost of various process areas.

The direct capital cost (process facilities cost) of $\mathrm{CO}_{2}$ capture and separation system consists of the following cost areas:

Direct contact cooler: In case of coal-fired power plant applications that have a wet FGD (flue gas desulfurization) unit upstream of the amine system, the wet scrubber helps in substantial cooling of the flue gases, and additional cooler may not be required. In case of gas-fired power plants or majority of coal-fired power plants that do not have wet scrubbers for $\mathrm{SO}_{2}$ removal, a direct contact cooler has to be installed to bring down the temperature of the flue gas stream to acceptable levels. A direct contact cooler is a large vessel where the incoming hot flue gas is made to contact with the cooling water. The size of this unit is a function of the volumetric flow rate of the flue gas, which in turn depends upon the temperature and pressure conditions of the flue gas stream. The capital cost of the unit is estimated as:

$$
C_{d c c}=\mathrm{C}_{\mathrm{dcc}, \text { ref }} \cdot\left(\frac{\mathrm{V}_{\mathrm{fg}}}{V_{f g, r e f}} \cdot \frac{\mathrm{T}_{\mathrm{fg}}}{T_{f g, r e f}}\right)^{0.6}
$$

Flue gas blower: The cooled flue gas is pressurized using a blower before it enters the absorber. The size (and the cost) of the blower is again a function of the volumetric flow rate of the flue gas as it enters the blower. So, the cost may be estimated as:

$$
C_{\text {blower }}=\mathrm{C}_{\text {blower, ref }} \cdot\left(\frac{\mathrm{V}_{\mathrm{fg}, 1}}{V_{f g, 1, \text { ref }}} \cdot \frac{\mathrm{T}_{\mathrm{fg}, 1}}{T_{f g, 1, \text { ref }}}\right)^{0.6}
$$

Absorber: This is the vessel where the flue gas is made to contact with the MEA-based sorbent, and some of the $\mathrm{CO}_{2}$ from the flue gas gets dissolved in the sorbent. Again, the size of this unit is mainly a function of the volumetric flow rate of the flue gas, which in turn depends upon the temperature and pressure conditions of the flue gas stream, as it 
enters this vessel. The cost of the unit is again estimated on the basis of the cost information available for a particular reference case:

$$
C_{\text {absorber }}=\mathrm{C}_{\mathrm{absorber,} \mathrm{ref}} \cdot\left(\frac{\mathrm{V}_{\mathrm{fg}, \text { in }}}{V_{f g, \text { in }, \text { ref }}} \cdot \frac{\mathrm{T}_{\mathrm{fg}, \mathrm{in}}}{T_{f g, i n, r e f}}\right)^{0.6}
$$

Rich/lean cross heat exchanger: The rich $\left(\mathrm{CO}_{2}\right.$-loaded) and lean (regenerated) sorbent streams are passed through this cross heat exchanger, where the rich sorbent gets heated and the lean sorbent gets cooled. So, the size (and cost) of this unit is a function of the volumetric sorbent flow rate in the absorber. It is assumed that this volumetric flow rate is constant in the range of temperature and pressure conditions found in this system. The capital cost of the unit is estimated as:

$$
C_{\text {crossHEx }}=\mathrm{C}_{\text {crossHEx, ref }} \cdot\left(\frac{\mathrm{V}_{\text {solvent }}}{V_{\text {solvent }, \text { ref }}}\right)^{0.6}
$$

Regenerator: This is the column where the $\mathrm{CO}_{2}$-loaded sorbent is regenerated with the application of heat. Sorbent flow rate is the main physical quantity that decides the size (and cost) of this unit, for a given residence time, which is a function of many parameters including the sorbent concentration, desired $\mathrm{CO}_{2}$ capture efficiency, etc. So, the cost may be estimated as above:

$$
C_{\text {regenerator }}=\mathrm{C}_{\text {regenerator, ref }} \cdot\left(\frac{\mathrm{V}_{\text {solvent }}}{V_{\text {solvent,ref }}}\right)^{0.6}
$$

Reboiler: The regenerator is connected with a reboiler, which is a heat exchanger where low-pressure steam extracted from the power plant is used to heat the loaded sorbent. So, the size (and cost) of this unit is a function of mainly the flow rate of the sorbent as well as the flow rate of steam. The cost of the unit is estimated as:

$$
C_{\text {reboiler }}=\mathrm{C}_{\text {reboiler, ref }} \cdot\left(\frac{\mathrm{V}_{\text {solvent }}}{V_{\text {solvent,ref }}} \cdot \frac{\mathrm{M}_{\text {steam }}}{M_{\text {steam }, \text { ref }}}\right)^{0.6}
$$


It may be noted that the ratio of mass flow rates of LP steam $\left(M_{\text {steam }} / M_{\text {steam,ref }}\right)$ has been used in place of the ratio of volumetric flow rates of LP steam, assuming that the temperature and pressure conditions of the LP steam in both cases (actual and reference) are approximately the same.

Steam extractor: Steam extractors are installed to take LP steam from the steam turbines in the power plant. The size (and the cost) of the steam extractor is assumed to be a function of the steam flow rate.

$$
C_{\text {steam_extractor }}=C_{\text {steam_extractor, ref }} \cdot\left(\frac{\mathrm{M}_{\text {steam }}}{M_{\text {steam,ref }}}\right)^{0.6}
$$

This cost item is included if the $\mathrm{CO}_{2}$ capture system is configured to make use of steam extracted from the steam cycle of the base plant. Alternatively, an auxiliary NG boiler and a secondary steam turbine may be used, and the next two cost items $\left(\mathrm{C}_{\mathrm{NG} \text { _boiler }}\right.$ and $\mathrm{C}_{\mathrm{ST} 2}$ ) are included in its place.

Auxiliary boiler with steam turbine: The cost of the NG boiler is estimated on the basis of the steam flow rate generated by the boiler using a cost estimation formula reported by Simbeck (Simbeck and McDonald 2000):

$$
\mathrm{C}_{\mathrm{NG}_{-} \text {boiler }}=\$ 15 \cdot(\text { steam flow rate expressed in } \mathrm{lb} / \mathrm{hr} \text { ) }
$$

Since the steam flow rate $\left(\mathrm{m}_{\text {steam }}\right)$ in the model is given as tonnes $/ \mathrm{hr}$, the following expression is obtained after accounting for the unit conversions

$$
\mathrm{C}_{\mathrm{NG} \_ \text {boiler }}=\$ 33000 \cdot\left(\mathrm{m}_{\text {steam }}\right)
$$

The cost of the secondary steam turbine is estimated on the basis of the electrical power generated from this new turbine again using a cost estimation formula reported by Simbeck (Simbeck and McDonald 2000):

$$
\mathrm{C}_{\mathrm{ST2}}=\$ 300 \cdot\left(\mathrm{E}_{\mathrm{ST} 2}\right)
$$

Where, 


\section{$\mathrm{E}_{\mathrm{ST2}}=$ Power generation from secondary steam turbine expressed in MWe}

Auxiliary boiler without steam turbine: If a NG boiler is installed to provide only LP steam (and not to generate any electrical power using a secondary steam turbine), then the boiler cost is lower than that of high pressure boiler discussed earlier. According to Simbeck (Simbeck 2002)its cost is estimated as:

$$
\mathrm{C}_{\mathrm{NG}_{\text {_boiler_only }}}=\$ 22000 \cdot\left(\mathrm{m}_{\text {steam }}\right)
$$

MEA reclaimer: In order to avoid accumulation of the heat stable salts in the sorbent stream and to recover some of the lost MEA sorbent, a part of the sorbent stream is periodically distilled in this vessel. Addition of caustic helps in freeing of some of the MEA. The amount of MEA makeup requirement may be taken as an indicative of the amount of heat stable salts formed and the quantity of sorbent to be distilled in the reclaimer. So, the mass flow rate of makeup MEA requirement is used as a scaling parameter to estimate the cost of this unit:

$$
C_{\text {MEA_reclaimer }}=\mathrm{C}_{\text {MEA_reclaimer, ref }} \cdot\left(\frac{\mathrm{M}_{\text {MEA_makeup }}}{M_{\text {MEA_makeup,ref }}}\right)^{0.6}
$$

Sorbent processing area: The sorbent processing area primarily consists of sorbent cooler, MEA storage tank, and a mixer. It also consists of an activated carbon bed filter that adsorbs impurities (degradation products of MEA) from the sorbent stream. So, the size (and cost) of this unit (together) will be a function of the total sorbent flow rate, and may be estimated as follows:

$$
C_{\text {solvent_proc }}=\mathrm{C}_{\text {solvent_proc, ref }} \cdot\left(\frac{\mathrm{V}_{\text {solvent }}}{V_{\text {solvent,ref }}}\right)^{0.6}
$$

$\mathrm{CO}_{2}$ drying and compression unit: The multi-stage compression unit with inter-stage cooling and drying yields the final $\mathrm{CO}_{2}$ product at the specified pressure (about 2000 psig) that contains only acceptable levels of moisture and other impurities (e.g. $\mathrm{N}_{2}$ ). The size (and cost) of this unit will be a function of the $\mathrm{CO}_{2}$ product flow rate, and may be estimated as follows: 


$$
C_{\mathrm{CO} 2 \_c o m p r}=\mathrm{C}_{\mathrm{CO} 2 \_ \text {compr, ref }} \cdot\left(\frac{\mathrm{M}_{\mathrm{CO} 2}}{M_{\mathrm{CO}, \text { ref }}}\right)^{0.6}
$$

The sum of all these individual process area equipment costs is termed as process facilities capital (PFC). The various indirect costs are then estimated as fractions of the PFC following the EPRI cost estimating guidelines (TAG 1993; TAG 1999). Table 4.1 lists the elements of total capital cost. Because of data limitations some of the indirect cost factors for the amine system are estimated based on other similar technologies.

Table 4.1. MEA capital cost model parameters and nominal values

\begin{tabular}{|c|c|c|}
\hline & Capital Cost Elements & Value \\
\hline A & Process Area Equipment Costs & $\mathrm{A}_{1}, \mathrm{~A}_{2}, \mathrm{~A}_{3}, \ldots, \mathrm{A}_{10}$ \\
\hline $\bar{B}$ & Total Process Facilities Capital (PFC) & $\Sigma \mathrm{A}_{\mathrm{i}}$ \\
\hline $\mathrm{C}$ & Engineering and Home Office & $7 \% \mathrm{PFC}$ \\
\hline $\mathrm{D}$ & General Facilities & $10 \% \mathrm{PFC}$ \\
\hline $\mathrm{E}$ & Project Contingency & $15 \% \mathrm{PFC}$ \\
\hline $\bar{F}$ & Process Contingency & $5 \%$ PFC \\
\hline G & Total Plant Cost $(\mathrm{TPC})=$ sum of above & $\mathrm{B}+\mathrm{C}+\mathrm{D}+\mathrm{E}+\mathrm{F}$ \\
\hline $\mathrm{H}$ & AFUDC (interest during construction) & Calculated \\
\hline $\mathrm{I}$ & Royalty Fees & $0.5 \% \mathrm{PFC}$ \\
\hline $\mathrm{J}$ & Pre-production & 1 month's fixed O\&M cost \\
\hline $\bar{K}$ & Pre-production & 1 month's variable O\&M cost \\
\hline$\overline{\mathrm{L}}$ & Inventory (startup) Cost & $0.5 \% \mathrm{TPC}$ \\
\hline $\mathrm{M}$ & Total Capital Requirement (TCR) ${ }^{\mathrm{a}}$ & $\mathrm{G}+\mathrm{H}+\mathrm{I}+\mathrm{J}+\mathrm{K}+\mathrm{L}$ \\
\hline
\end{tabular}

The total plant cost (TPC) is the sum of the process facilities capital (PFC), general facilities capital (GFC, which is the total construction cost of the general facilities, including roads, office buildings, shops, laboratories etc.), engineering and home office (EHO) overhead, and contingencies - project and process. The project contingency is a capital cost contingency factor covering the cost of additional equipment or other costs that would result from a more detailed design at an actual site. On the other hand, the process contingency is a capital cost contingency factor (added cost) applied to a technology to reflect its level of maturity. TPC is developed on the basis of instantaneous 
("overnight") construction - occurring at a single point in time, and is generally expressed in mid-year dollars of a reference year.

The total capital requirement (TCR) includes all the capital necessary to complete the entire project, including interest during construction (AFUDC, allowance for funds during construction) and owner costs, which include royalties, startup costs, inventory capital etc.

\subsection{O\&M Cost}

The major operating and maintenance $(\mathrm{O} \& \mathrm{M})$ cost consists of fixed costs and variable cost elements as listed in Table 4.2

Table 4.2 MEA O\&M cost model parameters and nominal values

\begin{tabular}{||l|l||}
\hline \multicolumn{1}{|c|}{ O\&M Cost Elements } & \multicolumn{1}{|c||}{ Typical Value } \\
\hline \hline \multicolumn{2}{|c||}{ Fixed O\&M Costs } \\
\hline Total Maintenance Cost & $2.5 \%$ TPC \\
\hline Maintenance Cost Allocated to Labor $\left(\mathrm{f}_{\text {maintlab }}\right)$ & $40 \%$ of total maint. cost \\
\hline Admin. \& Support Labor Cost $\left(\mathrm{f}_{\text {admin }}\right)$ & $30 \%$ of total labor cost \\
\hline Operating Labor $\left(\mathrm{N}_{\text {labor }}\right)$ & 2 jobs/shift \\
\hline \hline \multicolumn{2}{|c|}{ Variable O\&M Costs } \\
\hline Reagent $(\mathrm{MEA})$ Cost & $\$ 1200 /$ tonne \\
\hline Water Cost & $\$ 0.8 / 1000$ gallon \\
\hline Solid Waste Disposal Cost & $\$ 175 /$ tonne waste \\
\hline $\mathrm{CO}_{2}$ Transport Cost & $\$ 0.02 /$ tonne $\mathrm{CO}_{2}$ per $\mathrm{km}$ \\
\hline $\mathrm{CO}_{2}$ Storage/Disposal Cost & $\$ 5 /$ tonne $\mathrm{CO}_{2}$ \\
\hline
\end{tabular}

\subsubsection{Fixed O\&M Costs}

The fixed O\&M (FOM) costs in the model include the costs of maintenance (materials and labor) and labor (operating labor, administrative and support labor). They are estimated on annual basis $(\$ \mathrm{M} / \mathrm{yr})$ as follows:

$$
\begin{aligned}
& \mathrm{FOM}=\mathrm{FOM}_{\text {labor }}+\mathrm{FOM}_{\text {maint }}+\mathrm{FOM}_{\mathrm{admin}} \\
& \mathrm{FOM}_{\text {labor }}=\text { labor } \times \mathrm{N}_{\text {labor }} \times 40(\mathrm{hrs} / \text { week }) \times 52(\text { weeks } / \mathrm{yr})
\end{aligned}
$$




$$
\begin{aligned}
& \mathrm{FOM}_{\text {maint }}=\Sigma_{\mathrm{i}}\left(\mathrm{f}_{\text {maint }}\right)_{\mathrm{i}} \times \mathrm{TPC}_{\mathrm{i}} \text { where } \mathrm{i}=\text { process area } \\
& \mathrm{FOM}_{\text {admin }}=\mathrm{f}_{\text {admin }} \times\left(\mathrm{FOM}_{\text {labor }}+\mathrm{f}_{\text {maintlab }} \times \mathrm{FOM}_{\text {maint }}\right)
\end{aligned}
$$

where,

$$
\begin{aligned}
& \text { labor }=\text { the hourly wages to the labor }(\$ / \mathrm{hr}) \\
& \begin{array}{r}
\mathrm{N}_{\text {labor }}=\text { number of operating labor required } \\
\left(\mathrm{f}_{\text {maint }}\right)_{\mathrm{i}}=\text { total annual maintenance cost expressed as the fraction of the } \\
\text { total plant cost (TPC) } \\
\mathrm{f}_{\text {admin }}=\text { the administrative labor cost expressed as the fraction of the total } \\
\text { labor cost }
\end{array}
\end{aligned}
$$

\subsubsection{Variable O\&M Costs}

The variable O\&M (VOM) costs depend on the capacity factor (or load factor) of the plant. They include costs of chemicals consumed (MEA, inhibitor, other reagents such as caustic and activated carbon), utilities (water, steam, power), fuel (natural gas, in case of auxiliary boiler) and services used (waste disposal, $\mathrm{CO}_{2}$ transport and storage). These quantities are determined in the performance model. The unit cost of each item (e.g., dollars per ton of reagent, or dollars per ton of $\mathrm{CO}_{2}$ stored) is a parameter specified as a cost input to the model. The total annual cost of each item is then calculated by multiplying the unit cost by the total annual quantity used or consumed. Total annual quantities depend strongly on the plant capacity factor, which is defined as the ratio of total annual generation ( $\mathrm{kWh}$ per year) to maximum possible generation (which is the product of plant capacity times total hours per year). The plant capacity factor is one of the crucial assumptions that influences the overall economics of the plant, especially in the case of a capital-intensive technology.

The individual components of variable O\&M costs are as follows: 
Cost of MEA reagent $\left(V O M_{M E A}\right)$ : The makeup MEA requirement estimated in the performance model is transformed into dollar amount by using the unit cost of MEA, which is a user-controlled cost input variable.

$$
\mathrm{VOM}_{\text {MEA }}=\mathrm{M}_{\text {MEA,makeup }} \times \mathrm{UC}_{\text {MEA }} \times \mathrm{HPY}
$$

where, $\mathrm{UC}_{\mathrm{MEA}}$ is the unit cost of MEA, and HPY is the equivalent annual hours per year of plant operation at full capacity (e.g., a capacity factor of $75 \%$ is equivalent to about 6575 hours per year, assuming an average of 365.25 days per year).

Cost of inhibitor $\left(\mathrm{VOM}_{\text {inhibitor }}\right)$ : Addition of inhibitor makes it possible to use higher concentrations of MEA sorbent in the system with minimal corrosion problems. Inhibitors are special compounds that come at a cost premium. The cost of inhibitor is estimated as $20 \%$ of the cost of MEA.

$$
\mathrm{VOM}_{\text {inhibitor }}=0.2 \times \mathrm{VOM}_{\mathrm{MEA}}
$$

Cost of other reagents $\left(\mathrm{VOM}_{\text {reagents }}\right)$ : The cost of other reagents, such as, caustic and activated carbon are also calculated from their physical quantities estimated in the performance model and the unit costs of these reagents.

$$
\begin{aligned}
& \text { VOMreagents }=\mathrm{VOM}_{\text {Caustic }}+\mathrm{VOM}_{\text {act-C }} \\
& =\left\{\left(\mathrm{m}_{\text {Caustic }} \times \mathrm{UC}_{\text {Caustic }}\right)+\left(\mathrm{m}_{\text {act-C }} \times \mathrm{UC}_{\text {act-C }}\right)\right\} \times \mathrm{HPY}
\end{aligned}
$$

where $\mathrm{UC}_{\text {Caustic }}$ and $\mathrm{UC}_{\text {act-C }}$ are the unit costs of the reagents caustic and activated carbon, respectively.

Cost of waste disposal $\left(\mathrm{VOM}_{\text {waste }}\right)$ : Another important variable operating cost item is the cost incurred in disposal of the spent sorbent i.e., the reclaimer waste. The quantity estimated in the performance model is:

$$
\mathrm{VOM}_{\text {waste }}=\mathrm{M}_{\text {waste,total }} \times \mathrm{UC}_{\text {waste }} \times \mathrm{HPY}
$$

where, $\mathrm{UC}_{\mathrm{waste}}$ is the unit cost of waste disposal for the reclaimer waste. 
Cost of $\mathrm{CO}_{2}$ transport $\left(\mathrm{VOM}_{\text {transport }}\right)$ : Transportation of $\mathrm{CO}_{2}$ product is assumed to take place via pipelines. The cost of $\mathrm{CO}_{2}$ transport is estimated on the basis of two userspecified parameters, viz., transportation distance (TD, in $\mathrm{km}$ ) and unit cost of transport ( $\mathrm{UC}_{\text {transport }} \$$ / $\mathrm{km}$ per tonne $\mathrm{CO}_{2}$ ), plus the $\mathrm{CO}_{2}$ product flow rate (calculated result from performance model).

$$
\mathrm{VOM}_{\text {transport }}=\mathrm{M}_{\mathrm{CO} 2} \times \mathrm{UC}_{\text {transport }} \times \mathrm{TD} \times \mathrm{HPY}
$$

Cost of $\mathrm{CO}_{2}$ storage $\left(\mathrm{VOM}_{\text {disposal }}\right)$ : Depending upon the method of $\mathrm{CO}_{2}$ disposal or storage, either there may be some revenue generated (as in enhanced oil recovery, or enhanced coal bed methane), or an additional cost (all other disposal methods). The total cost or revenue of $\mathrm{CO}_{2}$ disposal/ storage is estimated from the unit cost and $\mathrm{CO}_{2}$ product flow rate $\left(\mathrm{UC}_{\text {disp }}\right)$.

$$
\mathrm{VOM}_{\text {disposal }}=\mathrm{M}_{\mathrm{CO} 2} \times \mathrm{UC}_{\text {disp }} \times \mathrm{HPY}
$$

Cost of energy $\left(\mathrm{VOM}_{\text {energy }}\right)$ : By default, all energy costs are handled internally in the model by de-rating the overall power plant based on the calculated power requirement. The $\mathrm{CO}_{2}$ capture unit is charged for the total electricity production foregone because of $\mathrm{CO}_{2}$ capture and compression $\left(\mathrm{E}_{\mathrm{CO} 2 \text {, tot }}\right)$.

For power plants with multi-pollutant controls the desire to quantify costs for a single pollutant requires an arbitrary choice of how to charge or allocate certain costs. This is especially relevant for energy-intensive processes like $\mathrm{CO}_{2}$ capture systems.

The unit cost of electricity $\left(\mathrm{COE}_{\text {noctl }}\right)$ is estimated by the base plant module, or may be overridden by a user-specified value if this energy is assumed to be supplied from an external source. Since energy cost is one of the biggest O\&M cost items for the $\mathrm{CO}_{2}$ unit, the way in which it is accounted for is important when calculating the mitigation cost.

$$
\mathrm{VOM}_{\text {energy }}=\mathrm{E}_{\mathrm{CO} 2, \text { tot }} \times \mathrm{HPY} \times \mathrm{COE}_{\text {noctl }}
$$

Alternatively, when regeneration steam and additional electricity is provided by an auxiliary NG boiler, the cost of energy is estimated from the total annualized cost of the 
new boiler and secondary steam turbine, which takes into account their capital cost requirements and cost of natural gas fuel.

Cost of water $\left(\mathrm{VOM}_{\text {water }}\right)$ : Water is mainly required for process cooling and also as process makeup. Generally this is a minor cost item in the overall plant operation, but it is included over here for the sake of completeness, based on the amount of water needed $\left(\mathrm{M}_{\mathrm{w}}\right)$ and the unit cost of water $\left(\mathrm{UC}_{\mathrm{water}}\right)$ :

$$
\mathrm{VOM}_{\text {water }}=\mathrm{M}_{\mathrm{w}} \times \mathrm{UC}_{\mathrm{water}} \times \mathrm{HPY}
$$

The total variable O\&M (VOM, $\$ / \mathrm{yr})$ cost is obtained by adding all these costs:

$$
\begin{aligned}
\mathrm{VOM}= & \mathrm{VOM}_{\mathrm{MEA}}+\mathrm{VOM}_{\text {reagents }}+\mathrm{VOM}_{\text {waste }}+\mathrm{VOM}_{\text {transport }}+\mathrm{VOM}_{\text {disposal }}+ \\
& \mathrm{VOM}_{\text {energy }}+\mathrm{VOM}_{\text {water }}
\end{aligned}
$$

Finally, the total annual O\&M cost (TOM, \$/yr) may be obtained as:

$$
\mathrm{TOM}=\mathrm{FOM}+\mathrm{VOM}
$$

\subsection{Incremental Cost of Electricity}

Once the total capital requirement and the total O\&M costs are known, the total annualized cost of the power plant may be estimated as follows:

Total annual revenue requirement, $\mathrm{TRR}(\$ / \mathrm{yr})=(\mathrm{TCR} \times \mathrm{CRF})+\mathrm{TOM}$ where, $\mathrm{TCR}=$ Total capital requirement of the power plant $(\$)$, and

$$
\mathrm{CRF}=\text { Capital recovery factor (fraction) }
$$

The capital recovery factor, or fixed charge factor (FCF), is the factor that annualizes the total capital requirement of the plant. It depends on the applicable interest rate (or discount rate) and useful lifetime of the plant. 
It can be seen that a higher value of this factor (e.g. from assumptions of shorter plant life and/or higher interest rate) leads to a higher overall annualized cost. Hence the assumption about this factor (a user-defined parameter) is crucial in the overall economics of the plant.

The IECM framework calculates the cost of electricity (COE) for the overall power plant by dividing the total annualized plant cost $(\$ / \mathrm{yr})$ by the net electricity generated $(\mathrm{kWh} / \mathrm{yr})$. Results are expressed in units of $\$ / M W h$ (equivalent to mills $/ \mathrm{kWh}$ ).

$$
\text { Cost of electricity, } \mathrm{COE}(\$ / \mathrm{MWh})=\mathrm{TRR} /\left(\mathrm{MW}_{\mathrm{net}} * \mathrm{HPY}\right)
$$

where, $\mathrm{TRR}=\mathrm{Total}$ annual revenue requirement $(\$ / \mathrm{yr})$

$\mathrm{MW}_{\text {net }}=$ Net power generation capacity (MW)

$\mathrm{HPY}=$ Annual hours of operation (hrs/yr)

Note that the COE includes the cost of all environmental control systems, not just the $\mathrm{CO}_{2}$ control system. Thus, by running two scenarios of the power plant model, one without $\mathrm{CO}_{2}$ capture (reference plant) and one with $\mathrm{CO}_{2}$ capture $\left(\mathrm{CO}_{2}\right.$ capture plant), we obtain the incremental capital costs, O\&M costs, and total annualized costs attributed to $\mathrm{CO}_{2}$ capture. The addition of a $\mathrm{CO}_{2}$ capture and sequestration system increases the $\mathrm{COE}$ for the plant; this incremental cost of electricity is attributed to $\mathrm{CO}_{2}$ control.

\subsection{Cost of $\mathrm{CO}_{2}$ Avoidance}

Analysts often express the cost of an environmental control system in terms of the cost per unit mass of pollutant removed. However, for energy-intensive $\mathrm{CO}_{2}$ controls there is a big difference between the cost per tonne $\mathrm{CO}_{2}$ "removed" and the cost per tonne "avoided" based on net plant capacity. Since the purpose of adding a $\mathrm{CO}_{2}$ unit is to reduce the $\mathrm{CO}_{2}$ emissions per net $\mathrm{kWh}$ delivered, the "cost of $\mathrm{CO}_{2}$ avoidance" is the economic indicator that is widely used in this field. It can be calculated as:

Cost of $\mathrm{CO}_{2}$ Avoided $(\$ /$ tonne $)=\frac{(\$ / k W h)_{\text {after }}-(\$ / k W h)_{\text {before }}}{\left(\text { tonne } \mathrm{CO}_{2} / \mathrm{kWh}\right)_{\text {before }}-\left(\text { tonne } \mathrm{CO}_{2} / \mathrm{kWh}\right)_{\text {after }}}$

In contrast, the cost per unit of $\mathrm{CO}_{2}$ removed or captured is simply the additional expenses incurred in the capture of $\mathrm{CO}_{2}$, divided by the total quantity of $\mathrm{CO}_{2}$ captured. 
This can be calculated as the difference between the total annualized cost of the plant (TRR, M\$/yr) with and without $\mathrm{CO}_{2}$ control, divided by the total quantity of $\mathrm{CO}_{2}$ captured (tonne $\mathrm{CO}_{2} / \mathrm{yr}$ ), with the net power generated by the two plants remaining the same. Hence, the $\mathrm{CO}_{2}$ avoidance cost, as calculated in equation 4-1, is quite different from the cost per unit of $\mathrm{CO}_{2}$ captured. In case of $\mathrm{CO}_{2}$ control using an energy-intensive technology like amine-scrubbing, the cost of $\mathrm{CO}_{2}$ avoidance may be substantially higher than cost of $\mathrm{CO}_{2}$ capture.

The cost of $\mathrm{CO}_{2}$ avoidance has another interpretation in terms of a carbon-tax scenario. Consider a scenario where a power plant must pay a fixed tax (C-tax) that is proportional to its $\mathrm{CO}_{2}$ emissions. Now consider a reference plant (that does not control its $\mathrm{CO}_{2}$ emissions) and the same plant with $\mathrm{CO}_{2}$ capture (e.g., $90 \%$ of its $\mathrm{CO}_{2}$ emissions). The $\mathrm{COE}$ for the reference plant is initially lower, but increases much faster as compared to the COE for the capture plant, in response to increasing levels of the C-tax. Eventually, a C-tax level is reached where the COE for both the plants are the same (see Figure 4.1).

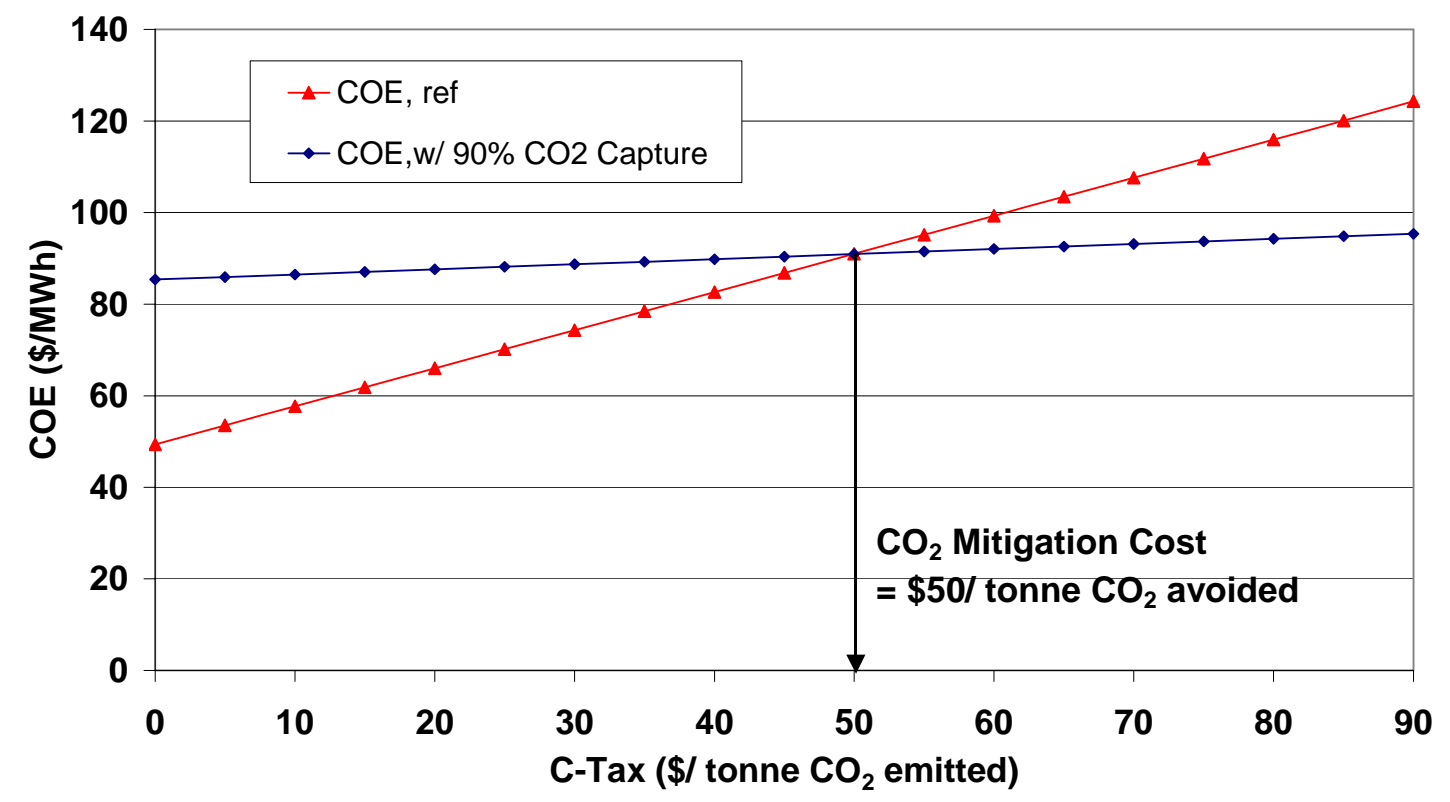

Figure 4.1. Cost of electricity (COE) as a function of carbon-tax

At this C-tax level, the power plant might be indifferent between paying the C-tax or incurring the cost of the $\mathrm{CO}_{2}$ capture unit. The cost of $\mathrm{CO}_{2}$ avoidance is this $\mathrm{C}$-tax level, 
where the $\mathrm{COE}$ for the reference plant and capture plant become equal. Note that this comparison assumes that both the reference plant and capture plant operate at the same annual capacity factor. In practice, load factors may vary for plants with and without emission controls in the context of a specific regional situation (Johnson 2002).

\subsection{Cost of Electricity (COE) as a Function of Carbon Tax}

As mentioned earlier, the IECM modeling framework has probabilistic capabilities that allow model inputs to be represented by probability distributions. In addition to uncertainties or variability in performance model parameters, there is also uncertainty and/ or variability in cost model parameters.

Table 4.3 lists the uncertainty distributions developed for the cost model parameters based on the current literature on amine-based (MEA) systems, the evolving nature of the technology, and views expressed by professionals working in this field. These distributions reflect both uncertainty and variability in the cost of these systems. Additional details are presented in Appendix B and Appendix $C$.

The last three parameters listed in Table 4.3 reflect the cost of $\mathrm{CO}_{2}$ transport and storage. The storage cost or revenue expected from $\mathrm{CO}_{2}$ product stream is the area of largest uncertainty. Various factors including the possibility of government regulation of $\mathrm{CO}_{2}$ emission, the evolution of national and/or international markets for $\mathrm{CO}_{2}$, and site-specific conditions will significantly influence the cost (or credits) for $\mathrm{CO}_{2}$ disposal. The distributions in Table 4.3 reflect the variety of options and costs commonly cited in the current literature.

Other parameters that directly contribute to the overall distribution of cost estimates for this technology include fuel prices, plant capacity factor, and fixed charge factor or capital recovery factor. These are the parameters that essentially belong to the reference plant (base plant) itself, and have nothing to do with amine-based $\mathrm{CO}_{2}$ capture. So, in order to assess the uncertainty associated with the amine-based $\mathrm{CO}_{2}$ capture technology, distributions on these parameters as well for the $\mathrm{CO}_{2}$ transport and disposal cost parameters should not be used. 
Table 4.3. Amine system cost model parameters and uncertainties

\begin{tabular}{|c|c|c|c|}
\hline $\begin{array}{c}\text { Cost } \\
\text { Parameter }\end{array}$ & Units & $\begin{array}{c}\text { Nominal } \\
\text { Value }\end{array}$ & $\begin{array}{c}\text { Unc. Representation } \\
\text { (Distribution Function) }\end{array}$ \\
\hline Process facilities & M\$ & calc & $* \operatorname{Normal}(1.0,0.1)$ \\
\hline Engineering and home office & $\% \mathrm{PFC}$ & 7 & Triangular $(5,7,10)$ \\
\hline General facilities & $\% \mathrm{PFC}$ & 10 & Triangular $(5,10,15)$ \\
\hline Project contingency & $\% \mathrm{PFC}$ & 15 & Triangular $(10,15,20)$ \\
\hline Process contingency & $\% \mathrm{PFC}$ & 5 & Triangular $(2,5,10)$ \\
\hline Royalty fees & $\% \mathrm{PFC}$ & 0.5 & Triangular $(0,0.5,0.5)$ \\
\hline Startup cost & Month of TOM & 1 & Triangular $(0.5,1,1)$ \\
\hline Inventory cost & $\%$ TPC & 0.5 & Triangular $(0.4,0.5,0.6)$ \\
\hline Total maintenance cost & $\% \mathrm{TPC}$ & 2.5 & Triangular $(1,2.5,5)$ \\
\hline Operating labor & Jobs/shift & 2 & Triangular $(1,2,3)$ \\
\hline MEA cost & \$/ tonne MEA & 1200 & Uniform $(1100,1300)$ \\
\hline Waste disposal cost & \$/ tonne waste & 175 & $* \operatorname{Normal}(1.0,0.1)$ \\
\hline $\mathrm{CO}_{2}$ transport cost & $\$ /$ tonne $\mathrm{CO}_{2} / \mathrm{km}$ & 0.02 & Triangular $(0.004,0.02,0.08)$ \\
\hline $\mathrm{CO}_{2}$ storage/disposal cost & $\$ /$ tonne $\mathrm{CO}_{2}$ & 5 & - \\
\hline $\begin{array}{l}\text { Revenue from } \mathrm{CO}_{2} \text { usage in } \\
\text { EOR or } \mathrm{ECBM}^{\mathrm{b}}\end{array}$ & $\$ /$ tonne $\mathrm{CO}_{2}$ & $-18^{a}$ & - \\
\hline
\end{tabular}

*This function is used as a multiplier to the nominal value.

${ }^{a}$ The negative sign indicates that it is a revenue rather than cost to the system.

${ }^{b}$ Individual scenarios may be run with the assumptions about revenue generation from $\mathrm{CO}_{2}$ product usage in EOR/ECBM applications, instead of additional cost for $\mathrm{CO}_{2}$ storage/ disposal. Alternatively, a chance function may be used that will assign either cost or revenue for $\mathrm{CO}_{2}$ product stream during each individual run.

\section{References (Chapter 4)}

Chapel, D., J. Ernst, et al. (1999). Recovery of CO2 from flue gases: commercial trends (paper no. 340). presented at the Canadian Society of Chemical Engineers Annual Meeting, 4-6 October, Saskatoon, Saskatchewan, Canada.

Desideri, U. and A. Paolucci (1999). "Performance modelling of a carbon dioxide removal system for power plants." Energy Conversion and Management 40: 1899-1915. 
Fluor (1998). Evaluation of technologies for the removal of CO2 from coal fired power plant flue gas, a report prepared by Fluor Daniel Canada Inc. for TransAlta, November 1998.

Hendriks, C. (1994). Carbon Dioxide Removal from Coal-fired Power Plants. The Netherlands, Kluwer Academic Publishers.

Jeremy, D. and H. J. Herzog (2000). The cost of carbon capture. Fifth Greenhouse Gas Control Technologies, Cairns, Australia.

Johnson, T. L. (2002). Electricity without carbon dioxide: assessing the role of carbon capture and sequestration in US electric markets. Department of Engineering and Public Policy. Pittsburgh, PA 15213, Carnegie Mellon University: 247.

Leci, C. L. (1996). "Financial implications on power generation costs resulting from the parasitic effect of $\mathrm{CO} 2$ capture using liquid scrubbing technology form power station flue gases." Energy Conversion and Management 37(6-8): 915-921.

Mariz, C. L. (1998). "Carbon dioxide recovery: large scale design trends." The Journal of Canadian Petroleum Technology 37(7).

Peters, M. S. and K. D. Timmerhaus (1991). Plant design and economics. New York, NY, McGraw-Hill.

Rubin, E. S., J. R. Kalagnanam, et al. (1997). "Integrated environmental control modeling of coalfired power systems." Journal of Air and\& Water Management Association 47: 11801188 .

Simbeck, D. (1998). A portfolio selection approach for power plant CO2 capture, separation and $\mathrm{R} \& \mathrm{D}$ options. Fourth International Conference on Greenhouse Gas Control Technologies, 30 August - 2 September, Interlaken, Switzerland, Elsevier Science Ltd.

Simbeck, D. (2002). "Personal communication."

Simbeck, D. and M. McDonald (2000). Existing coal power plant retrofit $\mathrm{CO}_{2}$ control options analysis. Sixth Greenhouse Gas Control Technologies, Cairns, Australia.

Smelser, S. C., R. M. Stock, et al. (1991). Engineering and economic evaluation of CO2 removal from fossil-fuel-fired power plants, EPRI IE-7365, Volume 1, Project 2999-10, a research project final report prepared by Fluor Daniel Inc., for EPRI and IEA.

TAG (1999). Technical Assessment Guide. Palo Alto, CA., EPRI TR 102276, EPRI. 


\section{MODEL APPLICATIONS}

One of the major objectives behind building the IECM-CS model is to provide an analytical tool to compare various technological options for controlling $\mathrm{CO}_{2}$ emissions from fossil-fuel based power plants on a consistent basis. Such assessments of the technical, environmental, and economic impacts of including $\mathrm{CO}_{2}$ control systems in power plant applications are important for greenhouse gas control policy analysis. Policy aspects of controlling $\mathrm{CO}_{2}$ emissions from power plants might include questions such as:

1) Within the spectrum of fossil-fuel based power plants, are there any particular categories that are better candidates for applying this technology?

2) What levels of emission reduction are possible? Are there any secondary environmental benefits associated with this process? Are there any potential environmental concerns? Are there any local pollution control problems or benefits arising from this process?

3) How does $\mathrm{CO}_{2}$ capture affect the overall performance (and cost) of the power plant?

4) How much would it cost in terms of capital requirement, incremental cost of electricity and cost per unit of $\mathrm{CO}_{2}$ avoided? What are the uncertainties associated with these estimates?

5) In case of conventional combustion-based power plants, is it cheaper to control $\mathrm{CO}_{2}$ emissions from a coal plant than from a gas plant?

6) Is it feasible to retrofit the existing power plants with this technology? If yes, are there any crucial issues of system integration in this case? How would the costs for a retrofit application compare to that for a greenfield case?

7) What are the key factors that affect these costs? Is there any scope for improvement in this technology through targeted R\&D efforts? What are the possible cost reductions that could be realized through such improvements? 
The IECM-CS model may be used to generate various scenarios that can provide useful insights into these questions. It may be noted that all these questions address issues related to the techno-economic and environmental feasibility of $\mathrm{CO}_{2}$ capture and separation alone. There is another whole set of questions related to the issues concerning the large-scale transport and storage/disposal of concentrated $\mathrm{CO}_{2}$ stream generated in this process, viz. technical feasibility, long-term environmental impacts, public perceptions, potential risks, uncertainties in the cost estimates etc. However, these are beyond the scope of the present study.

This chapter will address such questions by means of specific case studies of greenfield and retrofit applications of this technology presented in subsequent sections. The last question will be addressed later in the next chapter.

\subsection{Case Study of a New Coal-fired Power Plant}

Let us consider the case of a new conventional coal-fired power plant, and impact of adding a post-combustion amine-based $\mathrm{CO}_{2}$ capture unit to it. The basic assumptions and input parameters are listed in Table 5.1.

Table 5.1 Design parameters for case study of new pulverized coal plant

\begin{tabular}{|c|c|c|c|}
\hline Parameter & Value & Parameter & Value \\
\hline Gross plant size $(\mathrm{MW})$ & 500,670 & Emission standards & 2000 NSPS $^{\mathrm{d}}$ \\
\hline Base plant steam cycle type & $\mathrm{SC}^{\mathrm{a}}$ & $\mathrm{NO}_{\mathrm{x}}$ Controls & $\mathrm{LNB}^{\mathrm{e}}+\mathrm{SCR}^{\mathrm{f}}$ \\
\hline Gross plant heat rate $(\mathrm{kJ} / \mathrm{kWh})$ & $8359^{\mathrm{a}}$ & Particulate Control & $\mathrm{ESP}^{\mathrm{g}}$ \\
\hline Plant capacity factor $(\%)$ & 75 & $\mathrm{SO}_{2}$ Control & $\mathrm{FGD}^{\mathrm{h}}$ \\
\hline \multicolumn{2}{|l|}{ Coal characteristics } & $\mathrm{CO}_{2}$ Control & MEA $^{\mathrm{i}}$ \\
\hline Rank & Sub-bit. & $\mathrm{CO}_{2}$ capture efficiency (\%) & 90 \\
\hline $\mathrm{HHV}(\mathrm{kJ} / \mathrm{kg})$ & 19,346 & $\mathrm{CO}_{2}$ product pressure $(\mathrm{kPa})$ & $13,790^{\mathrm{j}}$ \\
\hline$\% \mathrm{~S}$ & 0.48 & Distance to storage $(\mathrm{km})$ & 165 \\
\hline$\% \mathrm{C}$ & 47.85 & & \\
\hline Mine-mouth cost (\$/tonne) & 13.73 & Cost year basis (constant dollars) & 2000 \\
\hline Delivered cost (\$/tonne) & $23.19^{c}$ & Fixed charge factor & $0.15^{\mathrm{k}}$ \\
\hline
\end{tabular}

${ }^{a}$ Nominal case is a super-critical unit. Unc. $=$ Uniform $(8167,8560) ;{ }^{b}$ Unc $=$ Triangular $(65,75,85) ;{ }^{c}$ Unc $=$ Triangular(15.94,23.19,26.81); ${ }^{\mathrm{d}} \mathrm{NO}_{\mathrm{x}}=65 \mathrm{ng} / \mathrm{J}, \mathrm{PM}=13 \mathrm{ng} / \mathrm{J}, \mathrm{SO}_{2}=70 \%$ removal (upgraded to $99 \%$ with MEA systems); ${ }^{\mathrm{e}} \mathrm{LNB}=$ Low- $\mathrm{NO}_{\mathrm{x}}$ Burner; ${ }^{\mathrm{f}} \mathrm{SCR}=$ Selective Catalytic Reduction; ${ }^{\mathrm{g}} \mathrm{ESP}=$ Electrostatic Precipitator; ${ }^{\mathrm{h}} \mathrm{FGD}=$ Flue Gas Desulfurization; ${ }^{\mathrm{i}} \mathrm{MEA}=$ Monoethanolamine system; ${ }^{\mathrm{j}}$ Unc. $=$ Triangular $(7580,13790,15160) ;{ }^{\mathrm{k}}$ Corresponds to a 30 -year plant lifetime with a $14.8 \%$ real interest rate (or, a 20 -year life with $13.9 \%$ interest); Unc $=\operatorname{Uniform}(0.10,0.20)$ 
The reference plant (without $\mathrm{CO}_{2}$ control unit) is New Source Performance Standard (NSPS) compliant coal-fired power plant and the complete plant with multi-pollutant environmental controls is simulated using IECM. The FGD system is assumed to remove $95 \%$ of the $\mathrm{SO}_{2}$ emissions, reflecting the best available technology, yielding lower emissions than what is required under the present NSPS. This assumption is crucial as the " $\mathrm{CO}_{2}$ avoidance cost" depends on the reference plant design as well. Wyoming Powder River Basin coal has been assumed to be used. The model outputs are presented later in Table 5.2 in comparison with the estimates for the $\mathrm{CO}_{2}$ capture plant.

In case of the $\mathrm{CO}_{2}$ capture plant, the following changes have been assumed as compared to the reference plant:

1) Air leakage has been reduced to $10 \%$ (reference plant uses the default value which is about $19 \%$ ).

2) The FGD system is assumed to be further upgraded to $99 \% \mathrm{SO}_{2}$ removal efficiency.

3) MEA-based $\mathrm{CO}_{2}$ capture system including $\mathrm{CO}_{2}$ product compression, transport and storage has been added.

The values for other parameters are listed in Tables 3.3, 4.1 and 4.2 in the previous chapters.

Since we know that the $\mathrm{CO}_{2}$ capture system is going to consume a lot of energy, we will be comparing two alternative cases of $\mathrm{CO}_{2}$ capture plant:

A) Using the same coal input (same $\mathrm{MW}_{\text {gross }}$ size), so that the $\mathrm{CO}_{2}$ capture plant will end up with lower net power generation, called as Case-A.

B) Starting with a bigger base plant (higher $\mathrm{MW}_{\text {gross }}$ size, larger coal input), so that the $\mathrm{CO}_{2}$ capture plant produces the same net power as generated by the reference plant. The exact size of this plant has to be found by trialand-error method, and this case will be referred as Case-B. From a practical point of view, this is a more useful case to consider, since the 
ultimate objective of a power plant is to generate (a certain amount of) electricity. Hence, this case will be used as a base case in further analysis.

\subsubsection{Deterministic Results}

We examine the model first for the reference power plant (without $\mathrm{CO}_{2}$ capture) and then for the same plant including $\mathrm{CO}_{2}$ capture (case $\mathrm{A}$ ) and for a similar but bigger plant including $\mathrm{CO}_{2}$ capture (case $\mathrm{B}$ ). Table 5.2 quantifies the impact of the $\mathrm{CO}_{2}$ unit on plant performance, plant discharges and plant costs. More detailed results (including those for Case-A) have been provided in Appendix D. Here we refer to the results from the case-B capture plant in comparison to the reference plant.

The $\mathrm{CO}_{2}$ capture system is energy intensive, and puts a large parasitic load on the base plant. Hence, the net heat rate of the capture plant $(11,550 \mathrm{Btu} / \mathrm{kWh})$ is higher than that of the reference plant $(8,657 \mathrm{Btu} / \mathrm{kWh})$. Energy requirements consume about $22 \%$ of gross plant capacity, mostly for sorbent regeneration (54\%) and $\mathrm{CO}_{2}$ product compression (36\%). Sorbent circulation and fan power account for the remaining share $(10 \%)$ of the total energy consumption of $\mathrm{CO}_{2}$ capture unit.

It may be observed that the new $\mathrm{CO}_{2}$ capture plant is significantly bigger $(+34 \%)$ than the reference plant. This implies that the capture plant has greater environmental impacts in terms of higher emissions of solid wastes (bottom ash, fly ash, FGD waste etc.) and ammonia in absolute quantities. This plant uses much more coal, hence it is also responsible for the externalities associated with extraction and transport of this extra coal, although these environmental impacts are beyond the scope of this study. It must be noted that the capture plant has lower $\mathrm{SO}_{\mathrm{x}}, \mathrm{NO}_{\mathrm{x}}$, and particulate emissions, in addition to lower $\mathrm{CO}_{2}$ emissions. However, it has higher emission rate of $\mathrm{NO}_{\mathrm{x}}$ (mass of $\mathrm{NO}_{\mathrm{x}}$ emitted per unit of power generated, $0.78 \mathrm{~g} / \mathrm{kWh})$ as compared to the reference plant $(0.59$ $\mathrm{g} / \mathrm{kWh}$ ). This happens because of the large energy penalty of the $\mathrm{CO}_{2}$ capture system. Finally, the capture plant generates two new discharge streams, viz. spent sorbent (which is treated as a hazardous waste) and a large concentrated $\mathrm{CO}_{2}$ stream. It is assumed that the $\mathrm{CO}_{2}$ product is transported via pipelines to the final storage or disposal site. 
Table 5.2. Case study results for new pulverized coal plants

\begin{tabular}{|c|c|c|c|}
\hline Parameter & $\begin{array}{c}\text { Reference } \\
\text { Plant }\end{array}$ & $\begin{array}{l}\text { Capture } \\
\text { Plant (B) }\end{array}$ & \% change \\
\hline Gross capacity (MW) & 500 & 670 & $+34 \%$ \\
\hline FGD efficiency (\%) & 95 & 99 & \\
\hline $\mathrm{CO}_{2}$ capture efficiency $(\%)$ & - & 90 & \\
\hline Net power generation (MW) & 458 & 458 & NO CHANGE \\
\hline Net cycle heat rate $(\mathrm{Btu} / \mathrm{kWh})$ & 8,657 & 11,550 & $+33 \%$ \\
\hline Coal consumption (tonne/hr) & 215.5 & 287.5 & $+33 \%$ \\
\hline Limestone consumption (tonne/hr) & 2.3 & 3.2 & $+39 \%$ \\
\hline $\mathrm{CO}_{2}$ emission (tonne/ hr) & 381.3 & 50.9 & $-87 \%$ \\
\hline $\mathrm{NO}_{\mathrm{x}}$ emission $(\mathrm{kg} / \mathrm{hr})$ & 269.5 & 355.2 & $+32 \%$ \\
\hline $\mathrm{SO}_{\mathrm{x}}$ emission $(\mathrm{kg} / \mathrm{hr})$ & 76.4 & 0.14 & $-99.8 \%$ \\
\hline $\mathrm{NH}_{3}$ emission $(\mathrm{kg} / \mathrm{hr})$ & 5.35 & 40.0 & $+648 \%$ \\
\hline $\mathrm{CO}_{2}$ emission $\left(\mathrm{gCO}_{2} / \mathrm{kWh}\right)$ & 833.3 & 111.2 & $-87 \%$ \\
\hline $\mathrm{NO}_{\mathrm{x}}$ emission $\left(\mathrm{gNO}_{\mathrm{x}} / \mathrm{kWh}\right)$ & 0.59 & 0.78 & $+32 \%$ \\
\hline $\mathrm{SO}_{\mathrm{x}}$ emission $\left(\mathrm{gNO}_{\mathrm{x}} / \mathrm{kWh}\right)$ & 0.17 & 0.0003 & $-99.8 \%$ \\
\hline FGD waste (tonne/hr) & 4.2 & 5.8 & $+39 \%$ \\
\hline Spent MEA sorbent (tonne/hr) & - & 1.4 & \\
\hline $\mathrm{CO}_{2}$ product (tonne/ hr) & - & 458 & \\
\hline TCR (M\$) & 616 & 963 & $+56 \%$ \\
\hline TCR $(\$ / \mathrm{kW})$ & 1345 & 2104 & $+56 \%$ \\
\hline $\mathrm{COE}(\$ / \mathrm{MWh})$ & 49.4 & 85.7 & $+73 \%$ \\
\hline$\$ /$ tonne $\mathrm{CO}_{2}$ avoided & - & 50.2 & \\
\hline$\$ /$ tonne $\mathrm{CO}_{2}$ delivered & - & 36.2 & \\
\hline
\end{tabular}

Because the $\mathrm{CO}_{2}$ capture plant is a bigger plant (670 MW gross), the total plant capital cost increases from $\$ 616 \mathrm{M}$ (for the reference plant) to $\$ 963 \mathrm{M}$. It maybe noted that this plant benefits from economies of scale. This is evident from the fact that the total capital requirement for case A (500 $\mathrm{MW}_{\text {gross }}$ with $\mathrm{CO}_{2}$ capture) is $\$ 760 \mathrm{M}(\$ 2228 / \mathrm{kW})$. In terms of operational costs, the steam and power cost and $\mathrm{CO}_{2}$ transport and storage/ disposal cost are two most important cost items, and contribute to about $34 \%$ and $23 \%$ to the total 
annualized cost of $\mathrm{CO}_{2}$ capture. MEA makeup requirements contribute to about $8 \%$ of this cost. Figure 5.1 shows the relative contributions from various cost areas in the overall annualized cost of $\mathrm{CO}_{2}$ capture unit.

The presence of $\mathrm{SO}_{2}$ impurities can substantially increase the reagent costs, so the cost of $\mathrm{CO}_{2}$ avoidance is sensitive to the $\mathrm{SO}_{2}$ removal efficiency of the FGD unit located upstream of the MEA system. Tradeoff studies showed that overall plant costs were minimized by reducing the $\mathrm{SO}_{2}$ concentration to about $10 \mathrm{ppm}$ inlet to the MEA absorber. Therefore, in this case study the $\mathrm{SO}_{2}$ removal system was upgraded to $99 \%$ removal with $\mathrm{CO}_{2}$ controls, as against $95 \% \mathrm{SO}_{2}$ removal in the reference case. The costs of this upgrade were charged to the $\mathrm{CO}_{2}$ unit.

Figure 5.2 shows graphically the relationship of electricity cost to $\mathrm{CO}_{2}$ emissions for the two cases; the slope of the line connecting the two points represents the cost of $\mathrm{CO}_{2}$ avoided, which is $\$ 50 /$ tonne for this case. Most of this cost $(77 \%)$ is associated with the $\mathrm{CO}_{2}$ capture process (including product compression); $\mathrm{CO}_{2}$ transport (9\%) and storage (14\%) account for the remainder.

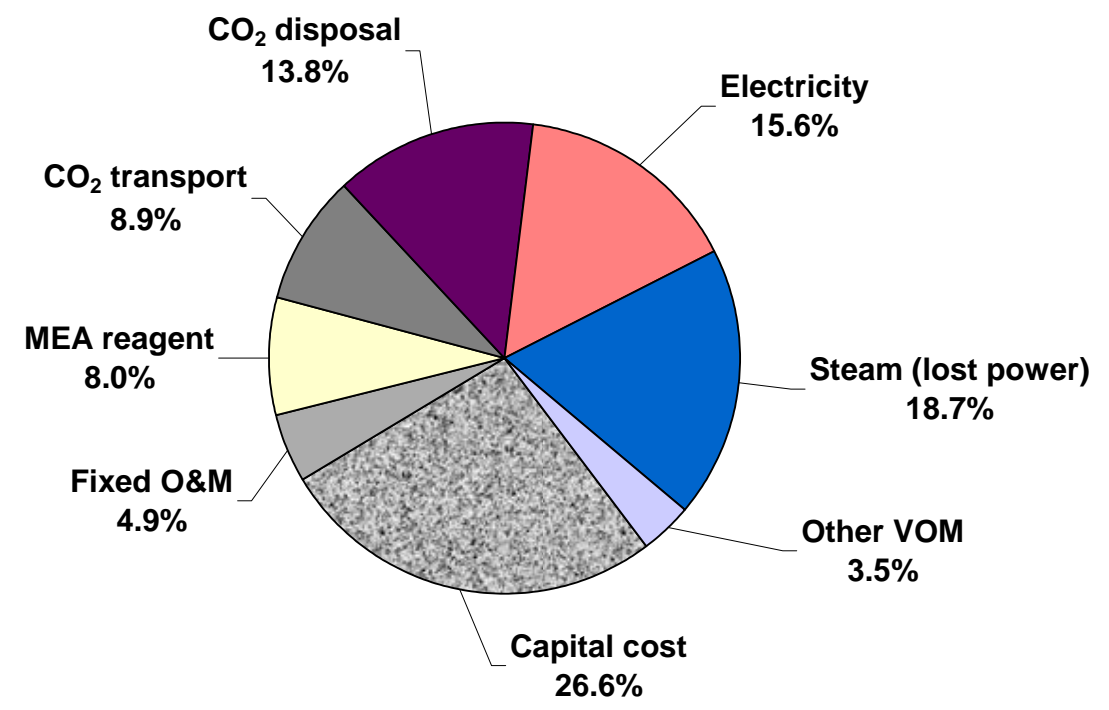

Figure 5.1. Breakup of annualized cost of $\mathrm{CO}_{2}$ capture unit 


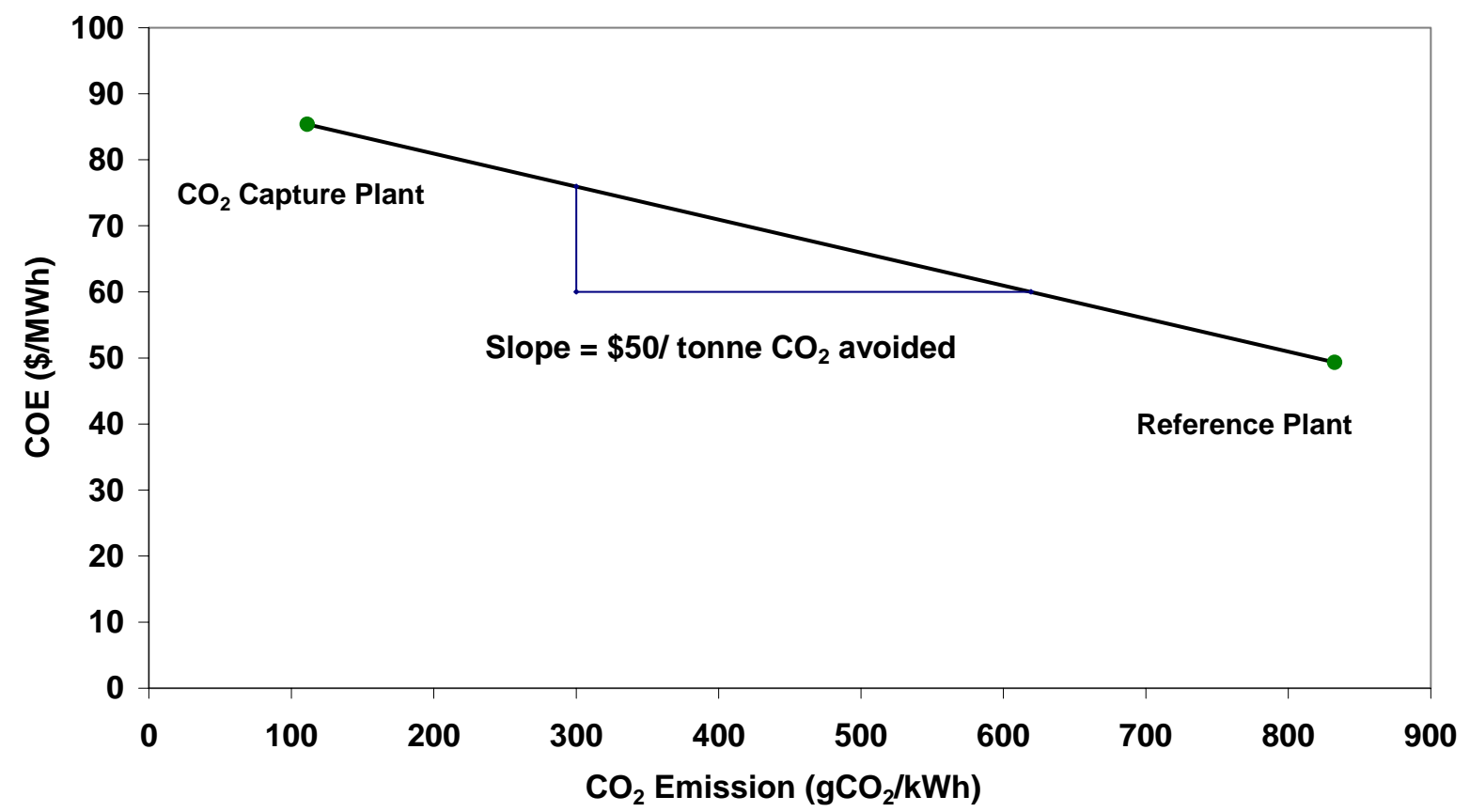

Figure 5.2. Estimation of $\mathrm{CO}_{2}$ avoidance cost for a new low-S coal plant

It may be noted that the $\mathrm{CO}_{2}$ avoidance cost ( $\$ 50 /$ tonne $\mathrm{CO}_{2}$ avoided) is much higher than the $\mathrm{CO}_{2}$ capture cost $\left(\$ 36 /\right.$ tonne $\mathrm{CO}_{2}$ delivered).

\subsubsection{Probabilistic Results}

How much is it likely to cost to avoid $\mathrm{CO}_{2}$ emissions using MEA-based $\mathrm{CO}_{2}$ capture systems from a new pulverized coal (PC) plant in the US today? The question sounds quite simple; however one needs to make a lot of assumptions in order to answer it. Different assumptions about power plant design, coal properties, plant operation and $\mathrm{CO}_{2}$ capture system design can have a significant effect on $\mathrm{CO}_{2}$ mitigation costs, and are responsible for many of the cost differences found in the literature. To systematically characterize the effects of different assumptions, parameters that vary across design studies are represented here by probability distributions, with values selected based on the current literature, experts' opinions and (in some cases) my own judgment. Note that the term "uncertainty" is used loosely here to include parameter variability. More discussion about this issue may be found in Appendix B and Appendix C.

We can set the characteristics of the PC plant (e.g., configuration, heat rate, capacity factor) and that of the fuel used (e.g., coal properties, coal price) in order to define the reference case, i.e. power plant without $\mathrm{CO}_{2}$ capture. Now we want to estimate the cost of $\mathrm{CO}_{2}$ avoidance for this plant using $\mathrm{MEA}-\mathrm{based} \mathrm{CO}_{2}$ capture system. Again, there are many parameters that need to be defined for this system. If we use a 
single value for each of them, we are looking at a unique system configuration with well defined design variables and operating conditions. Thus we get a deterministic cost estimate. However, if we are considering the whole population of potential configurations of MEA-based $\mathrm{CO}_{2}$ capture systems, most of those parameters would rather be represented as ranges of values (probability distributions) in place of the precise values. Various combinations of these parameter values then represent the set of possible configurations of a new commercial $\mathrm{CO}_{2}$ capture system if built today.

Figure 5.3 first shows the effect of considering uncertainties and design variability only in the performance parameters of the amine system (from Table 3.3). The resulting distribution for cost of $\mathrm{CO}_{2}$ avoidance has a 95-percentile range of $\$ 34-54 /$ tonne $\mathrm{CO}_{2}$ avoided. The main contributors to this range are the lean sorbent $\mathrm{CO}_{2}$ loading, sorbent concentration (both of which determine the reboiler duty), and equivalent electrical penalty of the regeneration steam requirement. It may be noted that most of the distributions for the input parameters are triangular, which is also reflected in this result to a certain extent. The tapering tails with low probability generally imply some combinations of design conditions and performance parameter values that are either too idealistic (e.g. high sorbent concentration, high lean loading) or too conservative (e.g. lower sorbent concentration, lower lean loading). For example, as explained earlier, amine systems with high sorbent concentration and high lean loading would have to deal with more severe corrosion problems, making them less practical at this time. Such interactions are not well quantified and, hence, not reflected in the current model. However, R\&D efforts could be targeted to make these potentially "better performing, low cost systems" practical in future. The median value (and its vicinity) represents a more realistic and near-optimal set of design conditions for current commercial systems.

Figure 5.4 shows the effect of uncertainty in $\mathrm{CO}_{2}$ cost model parameters, excluding the costs of the $\mathrm{CO}_{2}$ pipeline transport and geologic storage (see Table 4.3). Also included is the distribution obtained by considering uncertainties only in the cost parameters of the capture system. It shows that the uncertainties in the cost parameters of amine system have a very small effect on the overall distribution of the cost of $\mathrm{CO}_{2}$ avoidance, which is dominated by the variability and uncertainty in design and performance variables. 
Next, we consider the effect of assumptions about the cost of transport and storage/ disposal of $\mathrm{CO}_{2}$ product on the distribution of the cost of $\mathrm{CO}_{2}$ avoidance developed in Figure 5.4. Figure 5.5 shows the effect of considering costs of different options to store or dispose the $\mathrm{CO}_{2}$ product stream. Curve $\mathrm{A}(0)$ represents a case that assumes zero storage cost. Next, curve $\mathrm{B}(5)$ is the case where storage of $\mathrm{CO}_{2}$ stream (e.g., in a geologic formation such as underground saline reservoir) incurs a cost of $\$ 5 /$ tonne $\mathrm{CO}_{2}$. Finally, curve $\mathrm{C}(-18)$ shows a case that assumes a revenue of $\$ 18 /$ tonne $\mathrm{CO}_{2}$ generated through EOR activity, represented as negative cost. The assumption about the cost of disposal of $\mathrm{CO}_{2}$ product has a significant impact on the distribution of $\mathrm{CO}_{2}$ avoidance cost. The 95-percentile ranges for these 3 cases are $\$ 36-60$ (case $\mathrm{A}(0)$ ), $\$ 43-68$ (case $\mathrm{B}(5)$ ) and \$12-31 (case $\mathrm{C}(-18)$ ) per tonne $\mathrm{CO}_{2}$ avoided. These may be compared to the 95-percentile range of $\$ 34-54 /$ tonne $\mathrm{CO}_{2}$ avoided for the case presented earlier in Figure 5.4. So the option $\mathrm{B}(5)$, that assumes an additional cost of $\$ 5 /$ tonne $\mathrm{CO}_{2}$ for storage, not only increases the overall cost of $\mathrm{CO}_{2}$ avoidance (shifting the curve to the right), but also increases the 95-percentile range. On the other hand, option C(-18) leads to lower overall cost of $\mathrm{CO}_{2}$ avoidance (shifting the curve to the left) as well as slightly narrower range.

Figure 5.6 shows the effect of additional uncertainties (or variability) for the assumed power plant efficiency (heat rate), plant capacity factor, fixed charge factor, and coal price (see Table 3.1). These parameter values are identical for the reference plant and capture plant. Here we are now looking at the probability distribution of $\mathrm{CO}_{2}$ mitigation cost for a general population of pulverized coal plants using amine systems. The COE of each plant is strongly affected by assumptions about the base plant parameters. Also, the range in the $\mathrm{CO}_{2}$ avoidance cost increases for all the three options $\mathrm{A}(0), \mathrm{B}(5)$ and $\mathrm{C}(-18)$ to $\$ 33-63 /$ tonne, $\$ 40-71$ and $\$ 8-36 /$ tonne, respectively. 


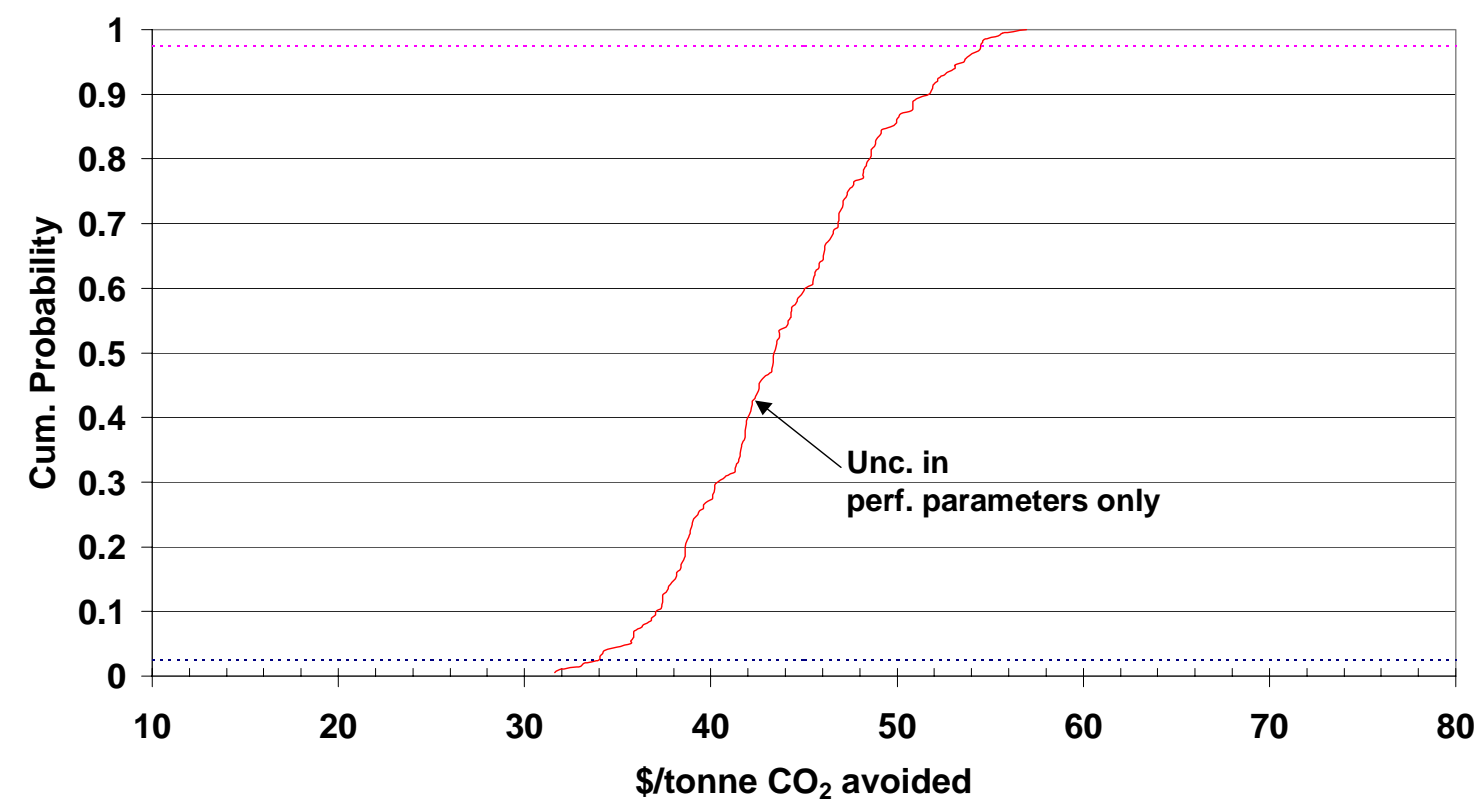

Figure 5.3. Probability distribution for the $\mathrm{CO}_{2}$ avoidance cost, with only the uncertainties in performance parameters of amine system considered.

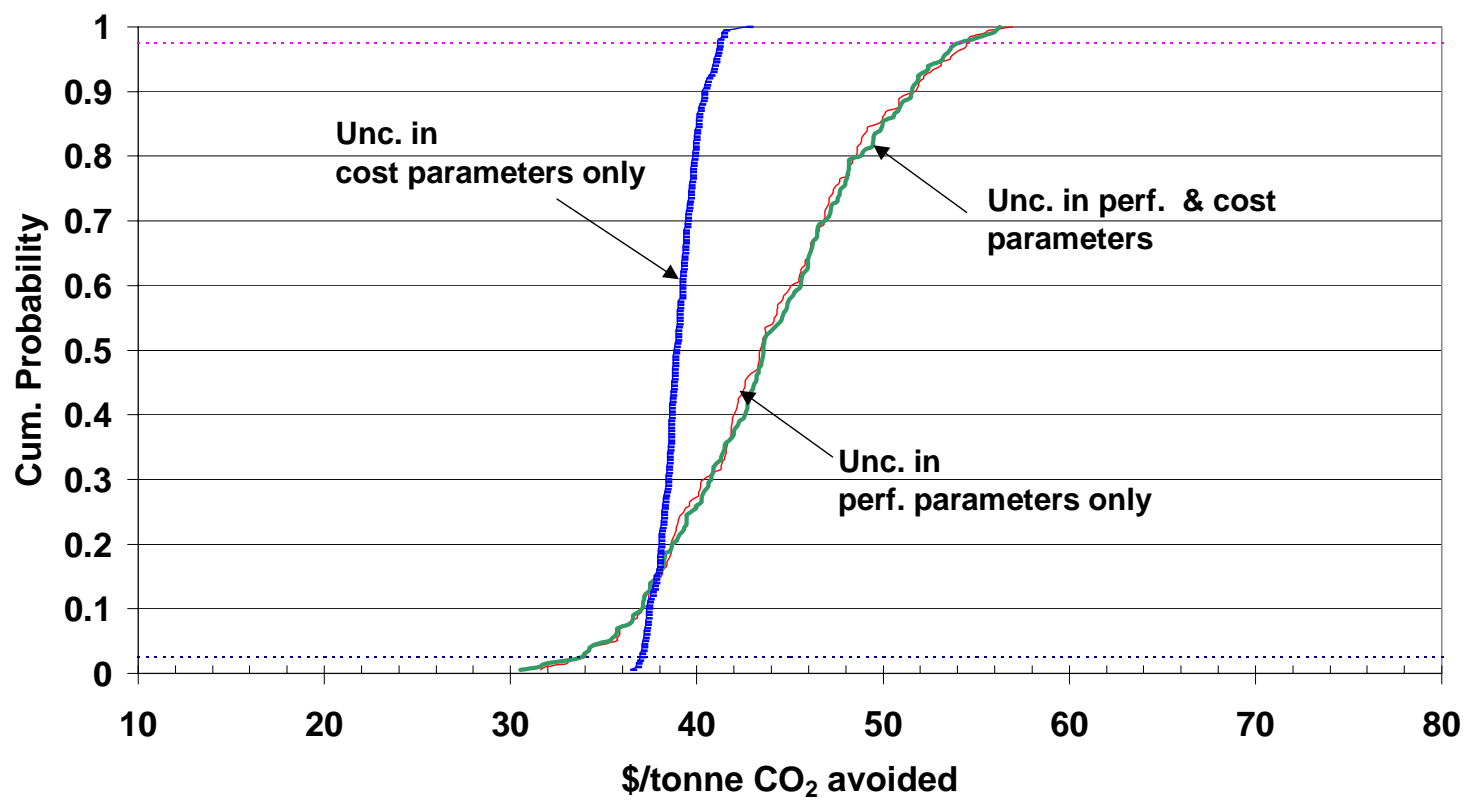

Figure 5.4. Probability distribution for the $\mathrm{CO}_{2}$ avoidance cost, with the uncertainties in performance and cost parameters of amine system considered. 


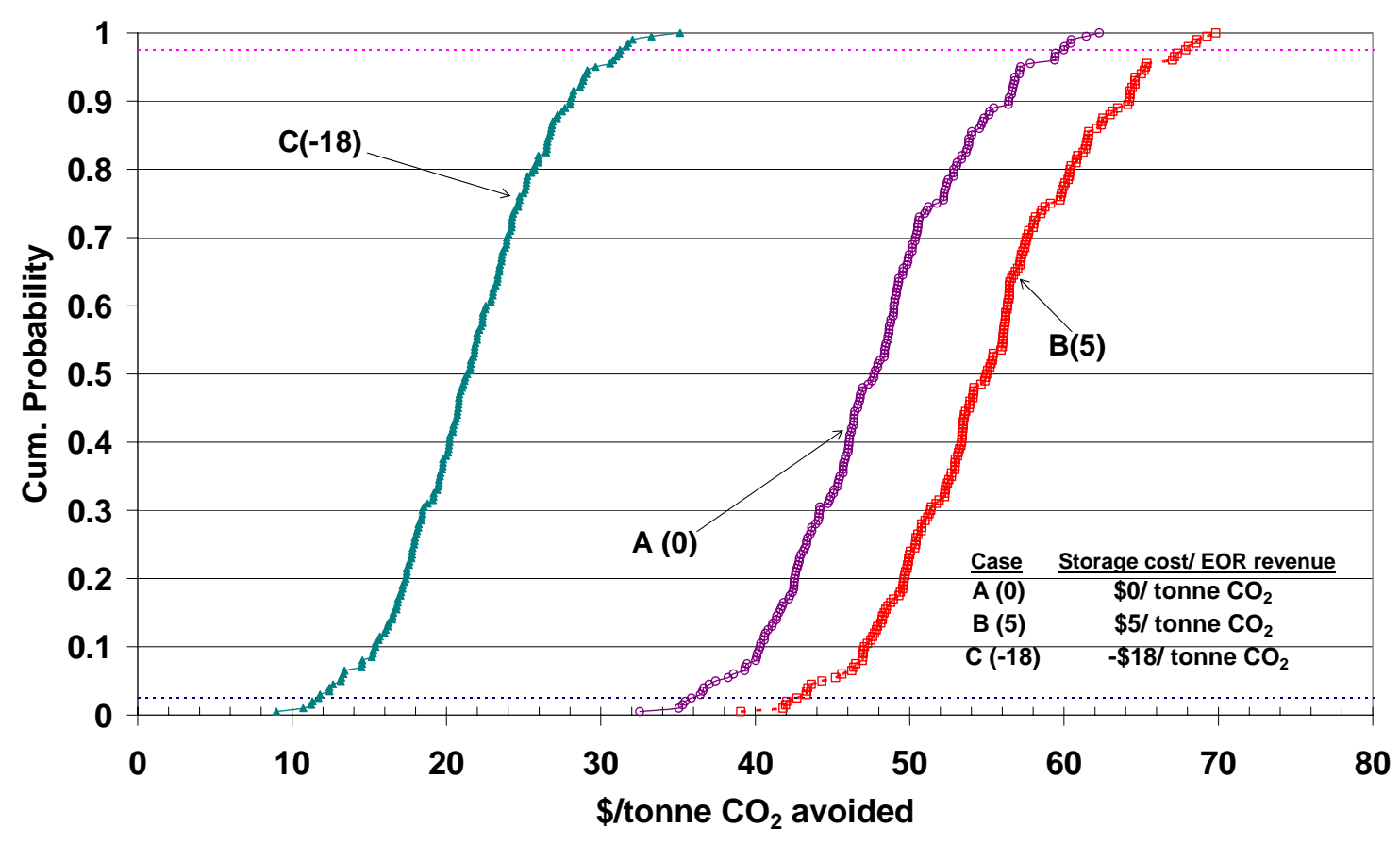

Figure 5.5. Effect of considering three different options for $\mathrm{CO}_{2}$ storage/disposal, assuming either no cost $(\mathrm{A}(0))$, storage cost of $\$ 5 /$ tonne $\mathrm{CO}_{2}(\mathrm{~B}(5))$, or revenue of $\$ 18$ / tonne $\mathrm{CO}_{2}$ from $\mathrm{EOR}(\mathrm{C}(-18))$ ), on the distribution of the $\mathrm{CO}_{2}$ avoidance cost (with uncertainties in amine system parameters).

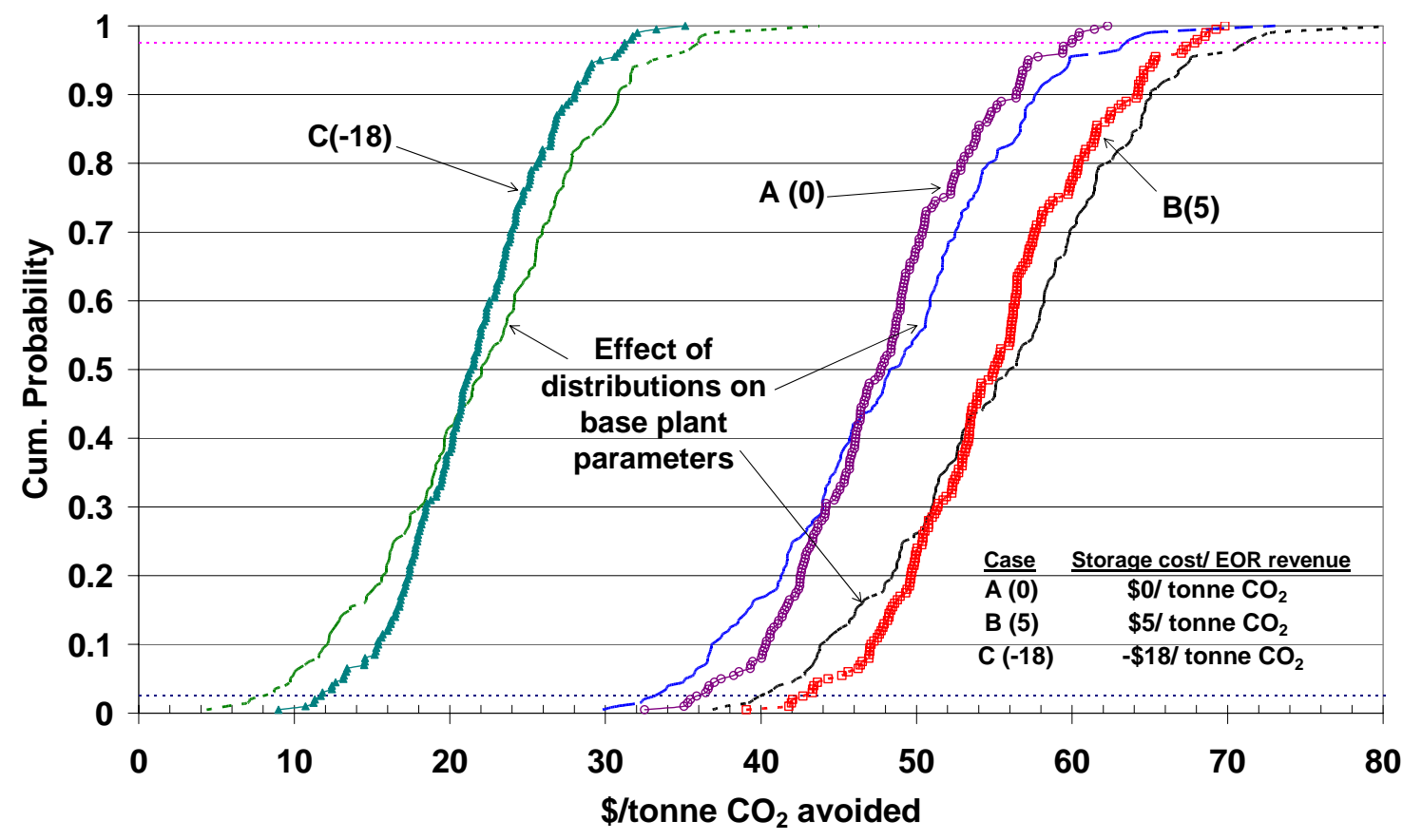

Figure 5.6. Effect of considering uncertainty in base plant parameters (viz. base plant gross cycle heat rate, plant capacity factor, fixed charge factor and coal price) on the distribution of $\mathrm{CO}_{2}$ avoidance cost derived in figure 5.5 . 


\subsection{Applications to $\mathrm{CO}_{2}$ Retrofits}

As discussed earlier, a large number of existing coal-fired power plants may be candidates for $\mathrm{CO}_{2}$ retrofits under a sufficiently stringent climate policy. Because of multi-pollutant interactions, the cost of $\mathrm{CO}_{2}$ mitigation will be affected by policies for other pollutants, especially $\mathrm{SO}_{2}$. Considering that most $(\sim 70 \%)$ of the current coal-based capacity in the U.S. does not have $\mathrm{SO}_{2}$ scrubbers, several cases were modeled to analyze the impacts of adding post-combustion $\mathrm{CO}_{2}$ capture systems to existing coal plants (see Table 5.3).

These cases are based on the analysis of the existing US coal plants database that includes details about 2294 units in as many as 85 fields including plant location, heat rate, plant capacity factor, cola characteristics, and environmental controls (NETL 2002). Two sets of power plants have been identified, viz. those without any sulfur controls and those with wet scrubbers (FGD systems) for $\mathrm{SO}_{2}$ emission control. Some of the key distinguishing features of these sets have been listed in Table 5.4.

Table 5.3. Scenarios for retrofit studies of existing coal-fired power plants

\begin{tabular}{|c|c|c|c|c|c|}
\hline Case & $\begin{array}{c}\text { Base plant } \\
\text { (PC+ESP) }\end{array}$ & $\begin{array}{c}\text { SO } \\
\text { System (FGD) }\end{array}$ & $\begin{array}{c}\mathbf{C O}_{2} \text { Capture } \\
\text { System (MEA) }\end{array}$ & $\begin{array}{c}\text { Auxiliary } \\
\text { NG Boiler }\end{array}$ & $\begin{array}{c}\text { Sec. Steam } \\
\text { Turbine }\end{array}$ \\
\hline \multicolumn{7}{|l|}{ Reference plant without any sulfur control (R) } \\
\hline R & $\checkmark$ & - & - & - & - \\
\hline A1 & $\checkmark$ & - & New & - & - \\
\hline A2 & $\checkmark$ & - & New & New & - \\
\hline A3 & $\checkmark$ & - & New & New & New \\
\hline B1 & $\checkmark$ & New, 99\% & New & - & - \\
\hline B2 & $\checkmark$ & New, 99\% & New & New & - \\
\hline B3 & $\checkmark$ & New, 99\% & New & New & New \\
\hline Reference plant with FGD system (Rf) & \multicolumn{5}{l|}{} \\
\hline Rf & $\checkmark$ & $\checkmark$ & - & - & - \\
\hline C1 & $\checkmark$ & $\checkmark$ & New & - & - \\
\hline C2 & $\checkmark$ & $\checkmark$ & New & New & New \\
\hline C3 & $\checkmark$ & $\checkmark$ & New & - & - \\
\hline D1 & $\checkmark$ & Upgrade, 99\% & New & New & - \\
\hline D2 & $\checkmark$ & Upgrade, 99\% & New & New & New \\
\hline D3 & $\checkmark$ & Upgrade, 99\% & \multicolumn{5}{l}{} \\
\hline
\end{tabular}


Table 5.4 Typical key features of existing coal-fired power plants in U.S.

\begin{tabular}{|l|c|c||}
\hline Characteristic & $\begin{array}{c}\text { Plants without } \\
\text { any sulfur capture }\end{array}$ & $\begin{array}{c}\text { Plants with } \\
\text { FGD systems }\end{array}$ \\
\hline \hline No. of units & 946 & 109 \\
\hline Total nameplate capacity & $232 \mathrm{GW}$ & $51 \mathrm{GW}$ \\
\hline Plant size (gross) & $540 \mathrm{MW}$ & $441 \mathrm{MW}$ \\
\hline Plant capacity factor & $73 \%$ & $76 \%$ \\
\hline Net plant heat rate & $10,150 \mathrm{Btu} / \mathrm{kWh}$ & $10,530 \mathrm{Btu} / \mathrm{kWh}$ \\
\hline Sulfur content in coal & $\sim 0.7 \% \mathrm{w} / \mathrm{w}$ & $\sim 1.1 \% \mathrm{w} / \mathrm{w}$ \\
\hline
\end{tabular}

Table 5.3 lists the various cases modeled using IECM-CS. The first set uses a typical plant without any sulfur control as the reference plant (R). It complies with federal standards for plants constructed before July 1978. Here, one needs to install a sulfur scrubber prior to the amine system during $\mathrm{CO}_{2}$ retrofitting as per the process requirements for an amine system (B1-B3). A case where only an amine-system is added to the reference plant (A1-A3) is without any practical relevance and is included only for the sake of completeness of this analysis, and it reinstates the importance of $\mathrm{SO}_{2}$ control as a pre-requisite for $\mathrm{CO}_{2}$ control using amine system.

The second set of cases uses a typical plant already equipped with an FGD system that meets federal New Source Performance Standards (NSPS) (which in this case is $73 \% \mathrm{SO}_{2}$ removal) as reference (Rf). Here, the $\mathrm{SO}_{2}$ removal efficiency of these systems are just enough to meet the existing emission standards (CFR 1999), however they do emit a substantial amount of $\mathrm{SO}_{2}$ (about $390 \mathrm{ppm}$ ) that maybe undesirable for amine-based $\mathrm{CO}_{2}$ capture system. This plant has the option of upgrading the existing $\mathrm{SO}_{2}$ scrubber in addition to installing an MEA system. It would be preferable to upgrade the existing FGD system to $99 \%$ removal efficiency. Hence, $\mathrm{CO}_{2}$ retrofit options here include 2 alternatives, viz. adding only amine system (C1-C3) and adding amine system with 
upgraded FGD system (D1-D3). The reference plants ( $\mathrm{R} \& \mathrm{Rf}$ ) for these two sets of $\mathrm{CO}_{2}$ capture plant cases are based on the characteristics presented in Table 5.4.

For each $\mathrm{CO}_{2}$ retrofit case, there are three potential configurations in terms of the mode of supplying energy required for the amine based $\mathrm{CO}_{2}$ capture system.

1) Base plant derating: Regeneration steam is extracted from the existing steam cycle of the base plant. Also the electrical energy for pumps, fans and compressors is obtained from the base plant. Hence, the net output of this plant is substantially lower than that of the reference plant.

2) Auxiliary NG boiler: Here, the regeneration steam is provided by adding a new NG-fired boiler so as to avoid steam extraction and subsequent derating of the existing base plant. However, the electrical energy requirement of $\mathrm{CO}_{2}$ capture system is fulfilled through the base plant, and hence the overall output of the capture plant is lower than reference case. It maybe noted that adding a new NGfired boiler to produce only low-pressure steam for sorbent regeneration is not a very practical approach, and is included here only for the sake of completeness of this analysis.

3) Auxiliary NG boiler plus secondary steam turbine: In this case, an auxiliary NGfired boiler is installed to generate high pressure steam that can be passed through a turbine to generate some electricity. The exhaust from the steam turbine is then used to regenerate the sorbent. The electricity generated by this unit is often more than the electrical energy requirement of the $\mathrm{CO}_{2}$ capture unit, and hence the overall power generation of the capture plant may exceed that of the reference plant.

In contrast to the new plant analysis presented earlier, the retrofit scenarios assume, as a bounding case, that all existing capital equipment has been fully amortized. The cost of electricity is then determined only by plant O\&M costs, plus any new capital expenditures for $\mathrm{CO}_{2}$ control. The latter includes the costs of any new sulfur removal systems that are installed to minimize the cost of $\mathrm{CO}_{2}$ avoidance. We may also credit the 
$\mathrm{CO}_{2}$ unit for the market value of additional $\mathrm{SO}_{2}$ allowances that are generated and traded under the national acid rain control program. The remaining plant life is assumed to be at least 15 years. The base capital cost of the $\mathrm{CO}_{2}$ capture unit is the same as that for a greenfield site, but we then consider an additional retrofit cost premium to account for site-specific retrofit difficulties. We also assume higher plant heat rates and energy penalties in providing steam to the MEA system.

\subsubsection{Results for plants without any sulfur control}

The results from the case study of $\mathrm{CO}_{2}$ retrofitting of existing PC plants without any sulfur control have been summarized in Table 5.5. The coal used in this case study was Appalachian low-S bituminous coal with $0.64 \%$ sulfur content and heating value of $13,080 \mathrm{Btu} / \mathrm{lb}$. The delivered price of this coal was assumed to be $\$ 37.1 /$ tonne which is about \$0.96/ MMBtu.

As noted earlier (Rao and Rubin 2002), we find that addition of FGD system along with MEA-based $\mathrm{CO}_{2}$ capture system reduces the overall cost. Cost of electricity and that of $\mathrm{CO}_{2}$ avoidance are substantially lower (almost $50 \%$ or less) in cases B1-B3 (in which FGD system was also installed) as compared to cases A1-A3 (in which only MEA-based $\mathrm{CO}_{2}$ capture system was retrofitted). In cases $\mathrm{A} 1$ and $\mathrm{B} 1$ where steam and electricity required for $\mathrm{CO}_{2}$ capture system is extracted from the base plant, the net plant output goes down to about $55-60 \%$ of the original plant output. This may have serious implications in terms of capacity planning and dispatch. On the other hand, option B3, which seems to be the most economic way (based on COE) to retrofit an existing PC plant with $\mathrm{CO}_{2}$ capture system, leads to additional power generation and is expected to have a matching demand. Although the $\mathrm{CO}_{2}$ capture system is designed to capture $90 \%$ of the $\mathrm{CO}_{2}$ emissions generated by the reference plant, the options using auxiliary NGfired boilers end up avoiding only about $60-65 \%$ of the emissions. In case of a very stringent climate policy, even the $\mathrm{CO}_{2}$ emissions from the newly added NG-fired boilers may have to be captured.

Again, it must be noted that these results are sensitive to the assumptions made about the depreciation of the reference plant, retrofit factors in capital cost estimation, and the price 
of natural gas. Table 5.6 shows the effect of some of these factors on the costs in cases B1 and B3.

Table 5.5. Case study results for plants with FGD systems

\begin{tabular}{|c|c|c|c|c|c|c|c|}
\hline & $\mathbf{R}$ & A1 & A2 & A3 & B1 & B2 & B3 \\
\hline Gross capacity (MW) & 540 & 540 & 540 & 540 & 540 & 540 & 540 \\
\hline Net power generation (MW) & 507.3 & 320.2 & 442.2 & 580.7 & 309.6 & 427.5 & 561.4 \\
\hline Net cycle heat rate $(\mathrm{Btu} / \mathrm{kWh})$ & 10,150 & 16,010 & 16,810 & 13,790 & 16,560 & 17,210 & 14,090 \\
\hline Coal consumption (tonne/hr) & 178.5 & 177.7 & 177.7 & 177.7 & 177.7 & 177.7 & 177.7 \\
\hline NG consumption (tonne/hr) & - & - & 45.96 & 57.44 & - & 44.43 & 55.54 \\
\hline $\mathrm{CO}_{2}$ emission (tonne/ hr) & 469.3 & 46.7 & 170.4 & 201.3 & 46.9 & 166.5 & 196.3 \\
\hline $\mathrm{NO}_{\mathrm{x}}$ emission (tonne/ hr) & 1.34 & 1.32 & 1.58 & 1.65 & 1.32 & 1.57 & 1.64 \\
\hline $\mathrm{SO}_{\mathrm{x}}$ emission $(\mathrm{kg} / \mathrm{hr})$ & 2214 & 11 & 11 & 11 & 0.13 & 0.13 & 0.13 \\
\hline $\mathrm{CO}_{2}$ emission $\left(\mathrm{gCO}_{2} / \mathrm{kWh}\right)$ & 925.1 & 145.9 & 385.3 & 346.7 & 151 & 389.4 & 349.7 \\
\hline $\mathrm{NO}_{\mathrm{x}}$ emission $\left(\mathrm{gNO}_{\mathrm{x}} / \mathrm{kWh}\right)$ & 2.64 & 4.11 & 3.58 & 2.83 & 4.25 & 3.68 & 2.91 \\
\hline $\mathrm{SO}_{\mathrm{x}}$ emission $\left(\mathrm{gNO}_{\mathrm{x}} / \mathrm{kWh}\right)$ & 4.36 & 0.03 & 0.025 & 0.019 & 0.0004 & 0.0003 & 0.0002 \\
\hline FGD waste (tonne/hr) & - & - & - & - & 7.1 & 7.1 & 7.1 \\
\hline Spent MEA sorbent (tonne/hr) & - & 17.1 & 17.1 & 17.1 & 1.4 & 1.4 & 1.4 \\
\hline $\mathrm{CO}_{2}$ product (tonne/ hr) & - & 420.6 & 420.6 & 420.6 & 421.9 & 421.9 & 421.9 \\
\hline $\mathrm{CO}_{2}$ emissions captured $(\%)$ & - & $90 \%$ & $64 \%$ & $57 \%$ & $90 \%$ & $65 \%$ & $58 \%$ \\
\hline $\mathrm{CO}_{2}$ emissions avoided $(\%)$ & - & $84 \%$ & $58 \%$ & $63 \%$ & $84 \%$ & $58 \%$ & $62 \%$ \\
\hline TCR (M\$) & $0^{*}$ & 235.5 & 270.8 & 365.6 & 239.2 & 273.4 & 367.8 \\
\hline $\mathrm{TCR}(\$ / \mathrm{kW})$ & $0^{*}$ & 735.3 & 612.5 & 629.5 & 772.7 & 639.4 & 655.2 \\
\hline $\mathrm{COE}(\$ / \mathrm{MWh})$ & 18.28 & 107.4 & 99.07 & 83.59 & 70.33 & 72.11 & 63.19 \\
\hline$\$ /$ tonne $\mathrm{CO}_{2}$ avoided & - & 114.4 & 149.7 & 112.9 & 67.2 & 100.5 & 78.1 \\
\hline
\end{tabular}

*The existing plant is assumed to be completely depreciated.

In case B1, the cost of electricity and cost of avoidance both increase with either increase in reference plant capital cost (assuming less than 100\% depreciation) or an increase in retrofitting capital cost requirement (assuming retrofit factor $>1$ ). The $\mathrm{CO}_{2}$ avoidance cost may increase as high as $18 \%$ relative to the base case B1 discussed earlier depending upon the particular assumptions presented here in Table 5.6. However, these estimates 
are not affected by natural gas price change, since we are not adding any auxiliary NG boiler in this case.

Table 5.6 Case study results for plants without FGD systems: sensitivity analysis

\begin{tabular}{|l|c|c|c|c|c|}
\hline \multicolumn{1}{|c|}{ Case } & R & \multicolumn{2}{c|}{ B1 } & \multicolumn{2}{c|}{ B3 } \\
\hline & $\begin{array}{c}\mathrm{COE} \\
(\$ / \mathrm{MWh})\end{array}$ & $\begin{array}{c}\mathrm{COE} \\
(\$ / \mathrm{MWh})\end{array}$ & $\begin{array}{c}\$ / \text { tonne } \\
\mathrm{CO}_{2} \text { av. }\end{array}$ & $\begin{array}{c}\mathrm{COE} \\
(\$ / \mathrm{MWh})\end{array}$ & $\begin{array}{c}\text { \$tonne } \\
\mathrm{CO}_{2} \text { av. }\end{array}$ \\
\hline $\begin{array}{l}\text { Nominal case }(100 \% \text { depreciation, } \\
\text { NG price }=\$ 4 / \mathrm{mcf}, \mathrm{rf}=1)\end{array}$ & 18.3 & 70.33 & 67.2 & 63.19 & 78.0 \\
\hline Nominal case $+\mathrm{SO}_{2}$ control credits & 18.3 & 69.26 & 65.8 & 62.60 & 77.0 \\
\hline $80 \%$ depreciation of reference plant & 23.3 & 78.46 & 71.3 & 67.69 & 77.1 \\
\hline 50\% depreciation of reference plant & 30.9 & 90.62 & 77.1 & 74.46 & 75.7 \\
\hline Retrofit factor $(\mathrm{rf})=1.25$ & 18.3 & 75.58 & 74.0 & 67.58 & 85.6 \\
\hline Retrofit factor $(\mathrm{rf})=1.5$ & 18.3 & 79.47 & 79.0 & 71.2 & 91.9 \\
\hline NG price $=\$ 2.5 / \mathrm{mcf}$ & 18.3 & 70.33 & 67.2 & 56.28 & 66.0 \\
\hline NG price $=\$ 5.5 / \mathrm{mcf}$ & 18.3 & 70.33 & 67.2 & 70.09 & 90.0 \\
\hline $80 \%$ deprec., $\mathrm{rf}=1.25, \mathrm{NG}=\$ 2.5 / \mathrm{mcf}$ & 23.3 & 83.71 & 78.0 & 65.19 & 72.8 \\
\hline $80 \%$ deprec., $\mathrm{rf}=1.25, \mathrm{NG}=\$ 4 / \mathrm{mcf}$ & 23.3 & 83.71 & 78.0 & 72.09 & 84.8 \\
\hline $80 \%$ deprec., $\mathrm{rf}=1.25, \mathrm{NG}=\$ 5.5 / \mathrm{mcf}$ & 23.3 & 83.71 & 78.0 & 78.99 & 96.8 \\
\hline
\end{tabular}

In case $\mathrm{B} 3$, although the cost of electricity is lower than case $\mathrm{B} 1$, the $\mathrm{CO}_{2}$ avoidance cost is higher owing to the additional $\mathrm{CO}_{2}$ emissions coming from the auxiliary NG boiler. These costs are highly sensitive to natural gas prices. Changing the natural gas price from a low $\$ 2.5 / \mathrm{mscf}$ to $\$ 5.5 / \mathrm{mscf}$ leads to $25 \%$ increase in COE and $36 \%$ increase in the $\mathrm{CO}_{2}$ avoidance cost. Only at the low natural gas price assumption is option $\mathrm{B} 3$ found to be cheaper than option $\mathrm{B} 1$ in terms of the $\mathrm{CO}_{2}$ avoidance cost. The cost of electricity and cost of avoidance both increase with an increase in retrofitting capital cost requirement when we assume retrofit factor greater than 1. However, assumption about higher capital cost of the reference plant (assuming less than 100\% depreciation) leads to increase in cost of electricity but a nominal decrease in the cost of $\mathrm{CO}_{2}$ avoidance. At $50 \%$ depreciation level, the $\mathrm{CO}_{2}$ avoidance cost was estimated to be about $3 \%$ lower than the base case $\mathrm{B} 3$ (100\% depreciation). The $\mathrm{CO}_{2}$ avoidance cost may increase as high as $44 \%$ 
relative to the base case B1 discussed earlier depending upon the particular assumptions presented in Table 5.6.

\subsubsection{Results for plants with FGD systems}

The results from the case study of $\mathrm{CO}_{2}$ retrofitting of existing PC plants with FGD sulfur control have been summarized in Table 5.7. The coal used in this case study was medium-S bituminous with $1.4 \%$ sulfur content and heating value of $12,180 \mathrm{Btu} / \mathrm{lb}$. The delivered price of this coal was assumed to be $\$ 30.6 /$ tonne which is about $\$ 0.78$ / MMBtu.

Table 5.7. Case study results for plants with FGD systems

\begin{tabular}{|c|c|c|c|c|c|c|c|}
\hline & $\mathrm{R}$ & $\mathrm{C} 1$ & $\mathrm{C} 2$ & $\mathrm{C} 3$ & D1 & D2 & D3 \\
\hline Gross capacity (MW) & 441 & 441 & 441 & 441 & 441 & 441 & 441 \\
\hline Net power generation (MW) & 405.3 & 244.5 & 351.7 & 473.6 & 245.7 & 346.0 & 459.9 \\
\hline Net cycle heat rate $(\mathrm{Btu} / \mathrm{kWh})$ & 10370 & 17130 & 17670 & 14190 & 17040 & 17580 & 14260 \\
\hline Coal consumption (tonne/hr) & 156.6 & 155.9 & 155.9 & 155.9 & 155.9 & & 155.9 \\
\hline NG consumption (tonne/hr) & - & - & 40.43 & 50.54 & - & 37.79 & 47.25 \\
\hline $\mathrm{CO}_{2}$ emission (tonne/ hr) & 400.3 & 39.86 & 148.7 & 175.9 & 40.0 & 141.7 & 167.1 \\
\hline $\mathrm{NO}_{\mathrm{x}}$ emission (tonne/ hr) & 1.175 & 1.155 & 1.386 & 1.444 & 1.155 & 1.372 & 1.425 \\
\hline $\mathrm{SO}_{\mathrm{x}}$ emission $(\mathrm{kg} / \mathrm{hr})$ & 1960 & 9.752 & 9.752 & 9.752 & 0.24 & 0.24 & 0.24 \\
\hline $\mathrm{CO}_{2}$ emission $\left(\mathrm{gCO}_{2} / \mathrm{kWh}\right)$ & 987.8 & 163.0 & 422.8 & 371.4 & 162.8 & 409.6 & 363.4 \\
\hline $\mathrm{NO}_{\mathrm{x}}$ emission $\left(\mathrm{gNO}_{\mathrm{x}} / \mathrm{kWh}\right)$ & 2.9 & 4.7 & 3.94 & 3.05 & 4.7 & 3.96 & 3.1 \\
\hline $\mathrm{SO}_{\mathrm{x}}$ emission $\left(\mathrm{gNO}_{\mathrm{x}} / \mathrm{kWh}\right)$ & 4.84 & 0.04 & 0.03 & 0.02 & 0.001 & 0.0007 & 0.0005 \\
\hline FGD waste (tonne/hr) & 9.8 & 9.8 & 9.8 & 9.8 & 13.18 & 13.18 & 13.18 \\
\hline Spent MEA sorbent (tonne/hr) & - & 14.54 & 14.54 & 14.54 & 1.364 & 1.364 & 1.364 \\
\hline $\mathrm{CO}_{2}$ product (tonne/ hr) & - & 358.8 & 358.8 & 358.8 & 360 & 360 & 360 \\
\hline $\mathrm{CO}_{2}$ emissions captured $(\%)$ & -- & $90 \%$ & $63 \%$ & $56 \%$ & $90 \%$ & $65 \%$ & $58 \%$ \\
\hline $\mathrm{CO}_{2}$ emissions avoided $(\%)$ & -- & $83 \%$ & $57 \%$ & $62 \%$ & $84 \%$ & $59 \%$ & $63 \%$ \\
\hline TCR (M\$) & $0 *$ & 181.3 & 213.1 & 296.5 & 153.1 & 182.7 & 263.3 \\
\hline $\mathrm{TCR}(\$ / \mathrm{kW})$ & $0 *$ & 741.7 & 605.9 & 626.1 & 623.0 & 528.0 & 527.4 \\
\hline $\mathrm{COE}(\$ / \mathrm{MWh})$ & 20.04 & 118.3 & 105.4 & 86.87 & 69.19 & 71.14 & 61.94 \\
\hline$\$ /$ tonne $\mathrm{CO}_{2}$ avoided & - & 119.1 & 151.1 & 108.4 & 59.6 & 88.4 & 67.1 \\
\hline
\end{tabular}

*The existing plant is assumed to be completely depreciated. 
In cases $\mathrm{C} 1$ and $\mathrm{D} 1$ where steam and electricity required for $\mathrm{CO}_{2}$ capture system is extracted from the base plant, the net plant output goes down to about $60 \%$ of the original plant output. On the other hand, options C3 and D3 lead to about 15\% increase in power generation capacity. Again we find that upgrading the FGD system (to $99 \% \mathrm{SO}_{2}$ removal efficiency) along with the addition of MEA-based $\mathrm{CO}_{2}$ capture system does help in reducing the overall cost. Cost of electricity and that of $\mathrm{CO}_{2}$ avoidance are substantially lower (about 30-40\% and 40-50\% lower, respectively) in cases D1-D3 (in which FGD system was upgraded) as compared to cases $\mathrm{C} 1-\mathrm{C} 3$ (in which only MEA-based $\mathrm{CO}_{2}$ capture system was retrofitted).

Again, it must be noted that these results are sensitive to the assumptions made about the depreciation of the reference plant, retrofit factors in capital cost estimation, and the price of natural gas. Table 5.8 shows the effect of some of these factors on the costs in cases D1 and D3.

Table 5.8. Case study results for plants with FGD systems: sensitivity analysis

\begin{tabular}{|l|c|c|c|c|c|}
\hline \multicolumn{1}{|c|}{ Case } & R & \multicolumn{2}{c|}{ D1 } & \multicolumn{2}{c|}{ D3 } \\
\hline & $\begin{array}{c}\mathrm{COE} \\
(\$ / \mathrm{MWh})\end{array}$ & $\begin{array}{c}\mathrm{COE} \\
(\$ / \mathrm{MWh})\end{array}$ & $\begin{array}{c}\$ / \text { tonne } \\
\mathrm{CO}_{2} \text { av. }\end{array}$ & $\begin{array}{c}\mathrm{COE} \\
(\$ / \mathrm{MWh})\end{array}$ & $\begin{array}{c}\text { \$/tonne } \\
\mathrm{CO}_{2} \text { av. }\end{array}$ \\
\hline $\begin{array}{l}\text { Nominal case }(100 \% \text { depreciation, } \\
\left.\text { NG price }=\$ 4 / \mathrm{mcf}_{\mathrm{rf}}=1\right)\end{array}$ & 20.04 & 69.19 & 63.5 & 61.94 & 72.8 \\
\hline Nominal case $+\mathrm{SO}_{2}$ control credits & 20.00 & 68.13 & 62.1 & 61.36 & 71.8 \\
\hline $80 \%$ depreciation of reference plant & 25.88 & 78.74 & 68.3 & 66.97 & 71.4 \\
\hline $50 \%$ depreciation of reference plant & 34.64 & 93.02 & 75.4 & 74.7 & 69.6 \\
\hline Retrofit factor $(\mathrm{rf})=1.25$ & 20.04 & 73.04 & 68.5 & 65.35 & 78.7 \\
\hline Retrofit factor $(\mathrm{rf})=1.5$ & 20.04 & 76.89 & 73.4 & 68.88 & 84.9 \\
\hline NG price $=\$ 2.5 / \mathrm{mcf}$ & 20.04 & 69.19 & 63.5 & 54.77 & 60.4 \\
\hline NG price $=\$ 5.5 / \mathrm{mcf}$ & 20.04 & 69.19 & 63.5 & 69.11 & 85.3 \\
\hline $80 \%$ deprec., $\mathrm{rf}=1.25, \mathrm{NG}=\$ 2.5 / \mathrm{mcf}$ & 25.88 & 82.59 & 73.3 & 63.33 & 65.1 \\
\hline $80 \%$ deprec., $\mathrm{rf}=1.25, \mathrm{NG}=\$ 4 / \mathrm{mcf}$ & 25.88 & 82.59 & 73.3 & 70.5 & 77.5 \\
\hline $80 \%$ deprec., $\mathrm{rf}=1.25, \mathrm{NG}=\$ 5.5 / \mathrm{mcf}$ & 25.88 & 82.59 & 73.3 & 77.67 & 90.0 \\
\hline
\end{tabular}


We observe similar trends in cases C1-C3 Vs D1-D3, as we had noted earlier in cases A1-A3 Vs B1-B3. In general, derating seems to be the cheaper option than adding an auxiliary NG boiler, on the basis of $\mathrm{CO} 2$ avoidance cost. Adding auxiliary NG boiler may be cheaper if the reference plant is not completely depreciated or if NG is available at low price (below $\$ 2.5-3 / \mathrm{mscf}$ ).

\subsubsection{Comparison with other technological options for $\mathrm{CO}_{2}$ retrofitting}

It is also of interest to compare the results obtained for the amine-based $\mathrm{CO}_{2}$ retrofit cases to other possible technological options available to the existing older pulverized coal power plants. In the recent times, IGCC (Integrated Gasification Combined Cycle) has been developed as an alternative technology for coal-based power generation. IGCC repowering of old, less efficient and more polluting pulverized coal plants might be an attractive option that can also facilitate $\mathrm{CO}_{2}$ capture. In a recent case study that we conducted to compare this option, we found that although IGCC re-powering with $\mathrm{CO}_{2}$ capture requires a lot of new capital investment, it might be cheaper in terms of final cost of electricity and $\mathrm{CO}_{2}$ mitigation cost (Chen, Rao et al. 2003). Some of the key assumptions made in this study were as follows:

1) Reference plants are fully amortized and equipped with FGD systems

2) Performance parameters for the reference base plant are based on DOE/NETL's database(NETL 2002)

3) Gross capacity: $270 \mathrm{MW}$

4) Gross plant heat rate: $10,734 \mathrm{Btu} / \mathrm{kWh}$

5) $\mathrm{CO}_{2}$ emission rate: $1004 \mathrm{gCO}_{2} / \mathrm{kW}$

6) Plant capacity factor: $76 \%$

7) $\mathrm{CO}_{2}$ capture efficiency: $90 \%$

8) Financial parameters: Plant life $=20$ years, Interest rate $=9 \%$

9) Amine-based retrofit: Adding 30\% MEA-based $\mathrm{CO}_{2}$ capture system

10) Re-powering: Texaco, $\mathrm{O}_{2}$-blown, quench, GE MS7001F, Selexol-based $\mathrm{CO}_{2}$ capture

11) Identical assumptions have been made about $\mathrm{CO}_{2}$ product compression (2000 psig), transport and storage/disposal costs etc.

In case of amine-based $\mathrm{CO}_{2}$ retrofits, two options were considered, viz. base plant derating (AR1) and adding an auxiliary NG-fired boiler with secondary steam turbine 
(AR2). The case of IGCC re-powering was analyzed using an IGCC performance and cost model developed within the IECM modeling framework (CEES 2003). Again, two options have been considered, viz. Heat recovery re-powering (IR1, using existing steam turbine and land) and site re-powering (IR2, completely new IGCC on the existing land). The key results have been summarized in Table 5.9.

It may be noted that cost of electricity (and hence that of $\mathrm{CO}_{2}$ mitigation) is a strong function of the natural gas price in case of amine-based $\mathrm{CO}_{2}$ capture using auxiliary NG boiler and a turbine. Only at very low natural gas price can this configuration (AR2) compete with IGCC re-powering options (IR1 \& IR2) in terms of the final cost of electricity. However, the later has the lowest $\mathrm{CO}_{2}$ avoidance cost. This observation has also been supported by the probabilistic analysis we conducted for these options.

Table 5.9. Comparison of amine-based $\mathrm{CO}_{2}$ capture and IGCC re-powering options for an existing pulverized coal plant

\begin{tabular}{|c|c|c|c|c|c|}
\hline \multirow{2}{*}{ Parameter } & \multirow{2}{*}{$\begin{array}{c}\text { Reference } \\
\text { plant }\end{array}$} & \multicolumn{2}{|c|}{ Amine-based retrofits } & \multicolumn{2}{|c|}{ IGCC Re-powering } \\
\hline & & AR1 & AR2 & IR1 & IR2 \\
\hline Gross capacity (MW) & 270 & & & & \\
\hline Net capacity (MW) & 248 & 140 & 282 & 590 & 599 \\
\hline $\mathrm{CO}_{2}$ emission $\left(\mathrm{gCO}_{2} / \mathrm{kWh}\right)$ & 1004 & 177 & 369 & 99 & 98 \\
\hline TCR $(\$ /$ kW $)$ & $0 *$ & 837 & 650 & 1493 & 1698 \\
\hline \multicolumn{6}{|l|}{$\mathrm{COE}(\$ / \mathrm{MWh})$} \\
\hline (a) $\$ 4.7 / \mathrm{mcf} \mathrm{NG}$ & \multirow{2}{*}{21} & \multirow{2}{*}{83} & 70 & \multirow{2}{*}{62} & \multirow{2}{*}{67} \\
\hline (a) $\$ 2.4 / \mathrm{mcf} \mathrm{NG}$ & & & 59 & & \\
\hline \multicolumn{6}{|c|}{$\mathrm{CO}_{2}$ avoidance cost ( $\$ /$ tonne $\mathrm{CO}_{2}$ avoided) } \\
\hline (a) $\$ 4.7 / \mathrm{mcf} \mathrm{NG}$ & \multirow[b]{2}{*}{ 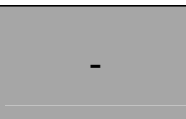 } & \multirow{2}{*}{75} & 77 & \multirow{2}{*}{46} & \multirow{2}{*}{51} \\
\hline a, \$2.4/mcf NG & & & 61 & & \\
\hline
\end{tabular}

*The existing plant is assumed to be completely depreciated.

The cost results ( $\mathrm{COE}$ and cost of $\mathrm{CO}_{2}$ avoidance) of the probabilistic analysis have been presented in Figure 5.7 and Figure 5.8. Under the set of assumptions stated before, amine-retrofit with internal derating of the base plant is clearly the most expensive option. The amine-retrofit option with auxiliary NG-fired boiler and a secondary steam 
turbine may be able to compete with the more efficient IGCC repowering options, only on the basis of cost of electricity. However, since the auxiliary NG boiler generates secondary $\mathrm{CO}_{2}$ emissions, this option is less effective in terms of avoiding $\mathrm{CO}_{2}$ emissions. So, the IGCC repowering options, which are also more energy efficient, are cheaper than the amine-retrofits, in terms of the $\mathrm{CO}_{2}$ avoidance costs. Even though the amine-retrofit with internal derating is much more expensive in terms of the cost of electricity generated than that with auxiliary NG boiler, the probability distribution for the $\mathrm{CO}_{2}$ avoidance cost for these options indicate that there is a small probability that the derating option might be cheaper than adding auxiliary NG boiler, based on the assumptions of this analysis.

\subsection{Discussion of Results}

\subsubsection{Greenfield vs. retrofit applications}

Case studies of new and retrofit application of amine-based $\mathrm{CO}_{2}$ capture system at power plants have been presented in sections 5.1 and 5.2. All these cases have been summarized in Figure 5.9. Here $\mathrm{COE}$ for each case has been plotted against its $\mathrm{CO}_{2}$ emission rate $\left(\mathrm{CO}_{2} / \mathrm{kWh}\right)$. It may be recalled that the slope of the line joining a capture plant with the respective reference plant gives the $\mathrm{CO}_{2}$ avoidance cost for that plant. The new reference and capture plant (discussed earlier in section 5.1.1 and shown in Figure 5.2) has also been included for comparison.

It may be noted that a much higher energy penalty is expected in case of retrofit applications. This makes the cost of avoidance in these cases higher than that in the case of an efficient new plant with $\mathrm{CO}_{2}$ capture. However, since the older plants are assumed to be completely paid of in terms of capital requirement, the retrofit applications are much cheaper than their greenfield counterparts. In the retrofit case, the cost of electricity is essentially based on the operating costs of the base plant and costs associated with the installation and operation of the $\mathrm{CO}_{2}$ capture equipment. Hence, in terms of cost of electricity produced, the retrofit applications are much cheaper than the new pulverized coal plants with $\mathrm{CO}_{2}$ capture. 


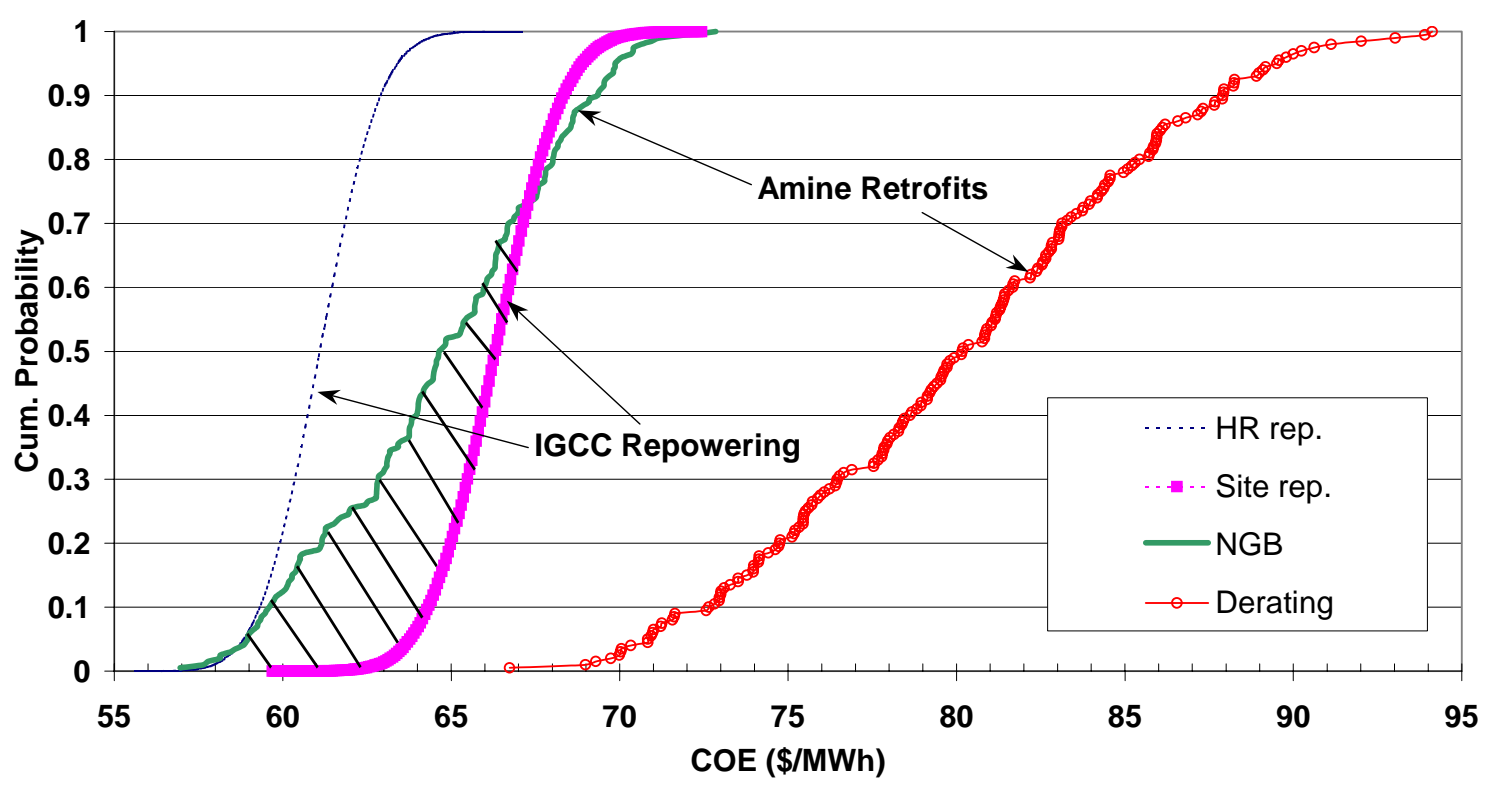

Figure 5.7. Comparison of $\mathrm{CO}_{2}$ retrofitting options: cost of electricity

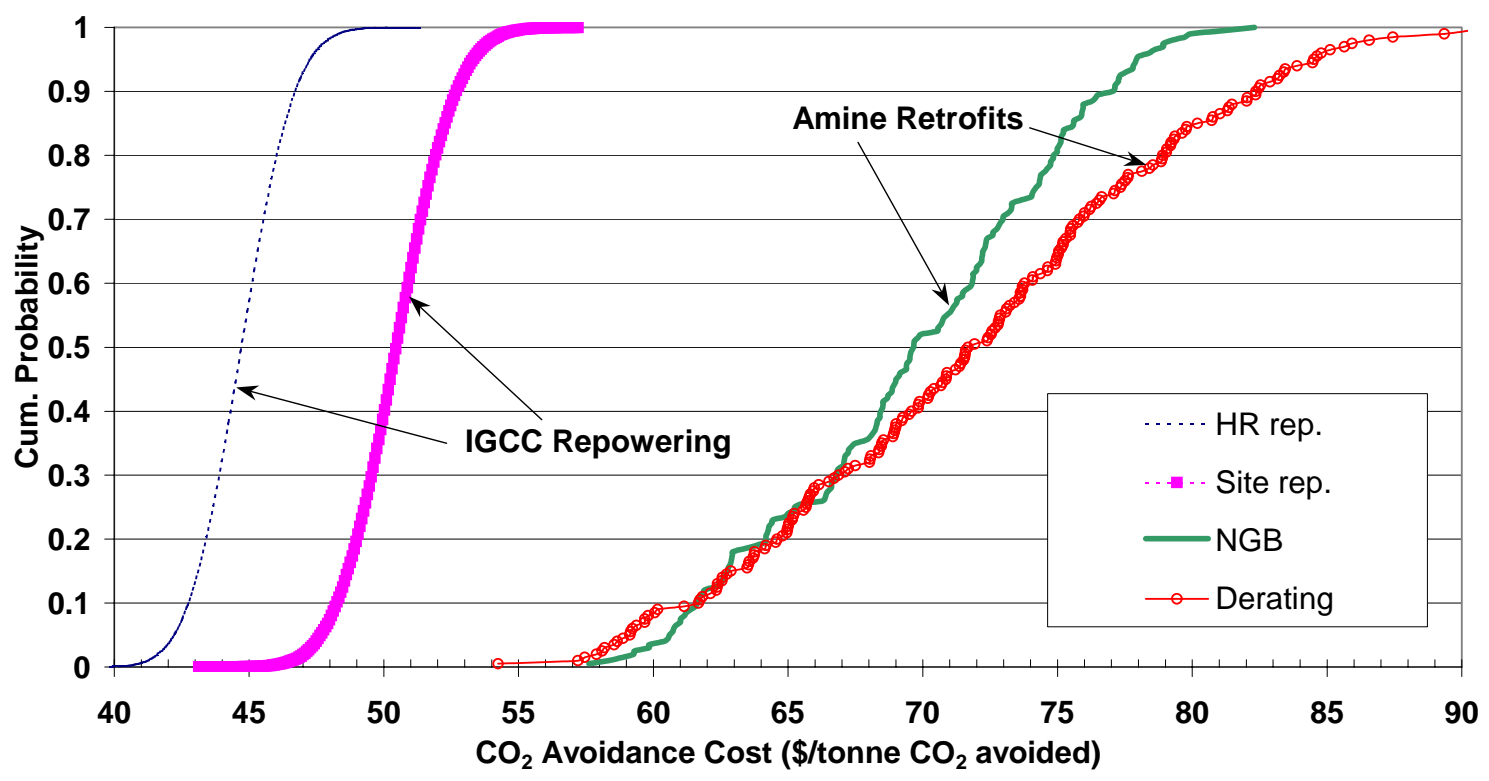

Figure 5.8. Comparison of $\mathrm{CO}_{2}$ retrofitting options: cost of $\mathrm{CO}_{2}$ avoidance

Given a sufficiently stringent $\mathrm{CO}_{2}$ reduction requirement, will utilities invest in new plants with $\mathrm{CO}_{2}$ capture, or will they prefer to spend less for retrofit applications, although it maybe a less effective measure to control $\mathrm{CO}_{2}$ emissions? Will the 
government provide any incentives (subsidies, tax rebates etc.) to any of the power generation options that help in reducing $\mathrm{CO}_{2}$ intensity of electricity sector? Answers to such questions will have a major impact on how the power industry reacts to any future regulation of $\mathrm{CO}_{2}$ emissions and technological choices available.

Similarly, there is another set of issues related to electricity demand and supply management, dispatch dynamics, availability and price of natural gas, evolution of a domestic and/or global market for $\mathrm{CO}_{2}$ trade, access to other (cheaper?) sources of $\mathrm{CO}_{2}$ control credits, role of renewable energy sources (especially wind, biomass etc.) in the total energy mix, and other factors that will play a major role in $\mathrm{CO}_{2}$ mitigation strategies. These factors are beyond the scope of this study, and analysis of such questions requires higher level modeling tools, such as discussed elsewhere (Johnson and Keith 2001; Johnson 2002).

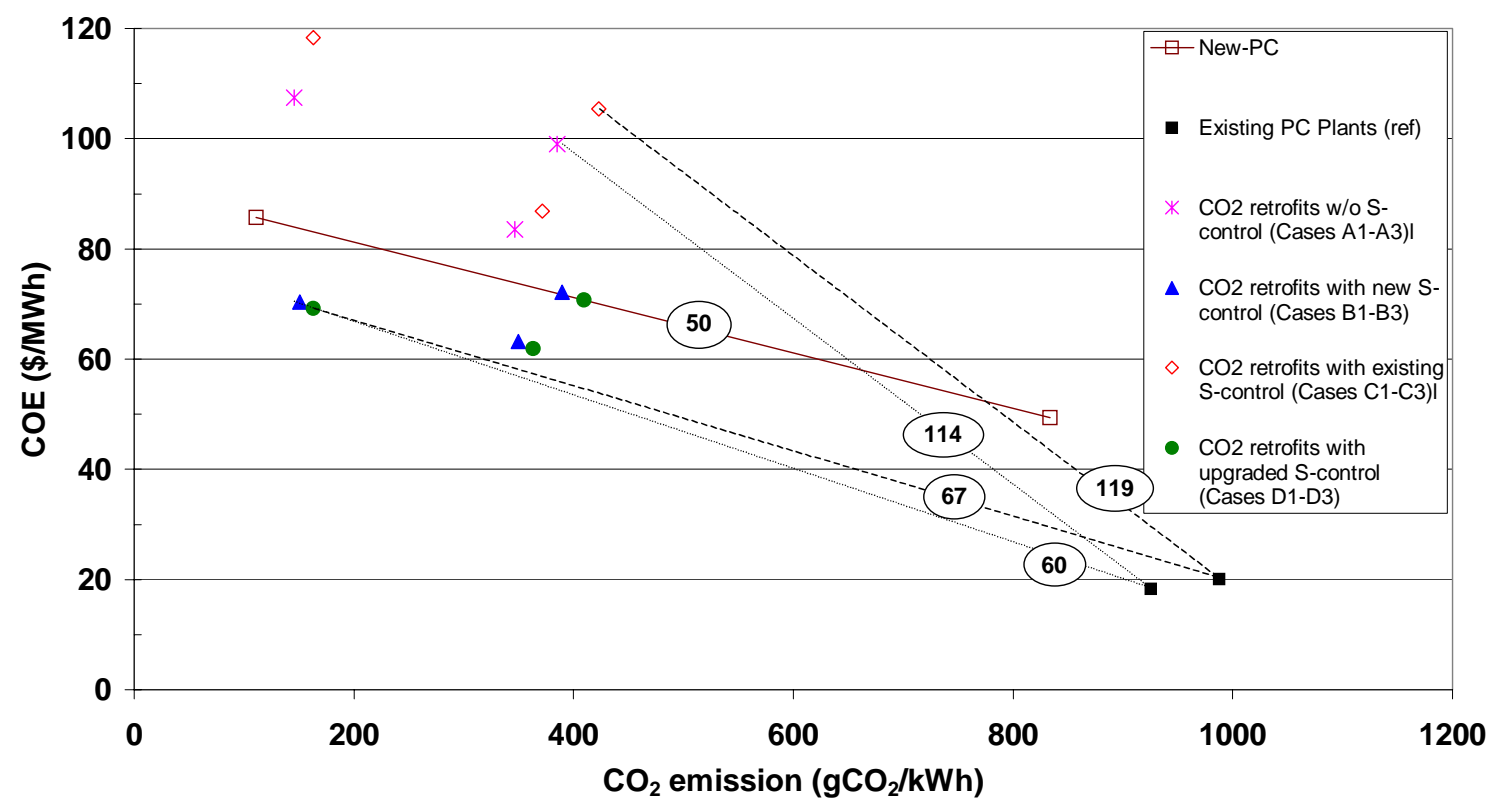

Figure 5.9. Estimation of $\mathrm{CO}_{2}$ avoidance cost for pulverized coal plants: greenfield vs. retrofit application

\subsubsection{Base plant derating vs. Auxiliary NG-fired boiler options}

As we have seen in the case studies of the existing coal plants (sections 5.2.1, 5.2.2), the $\mathrm{CO}_{2}$ capture system configurations with auxiliary NG-fired boiler and steam turbine (B3 
$\&$ D3) have the lowest COEs (for natural gas price $<\$ 5.5 / \mathrm{mscf}$, see Figure 5.10) than the other $\mathrm{CO}_{2}$-retrofitting alternatives in their respective categories. Another advantage of these options is that they increase the net power generation capacity of the plant. So, they are likely to be the preferred applications of this technology. Assuming availability of enough space for this retrofit and that of natural gas fuel, this option may be looked upon as a convenient way to boost the generation capacity of existing older power plants.

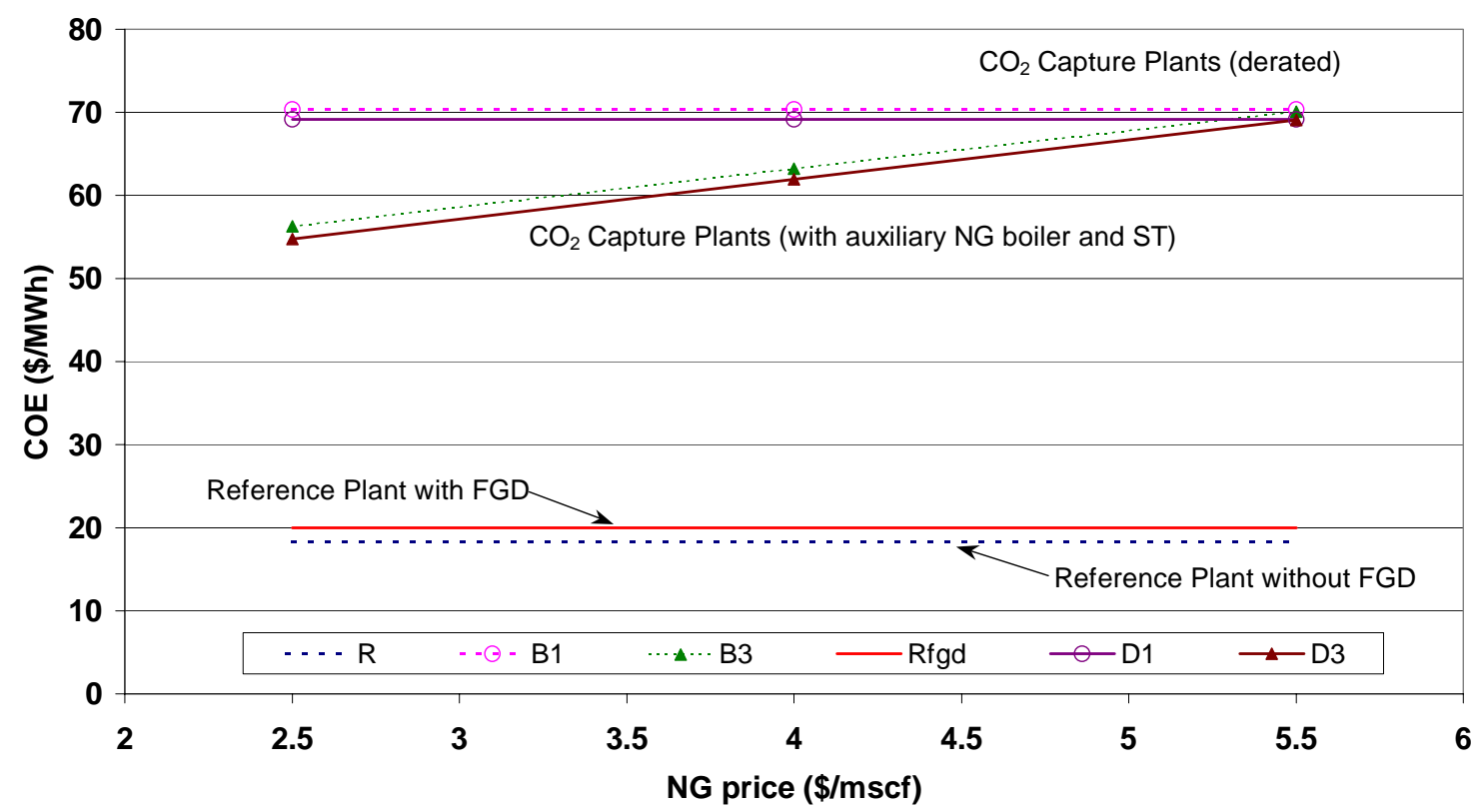

Figure 5.10. Effect of natural gas price on $\mathrm{COE}$ in $\mathrm{CO}_{2}$ retrofit applications

However, their economics is very sensitive to the natural gas price. At the nominal gas price assumption of $\$ 4 / \mathrm{mscf}$, their cost of $\mathrm{CO}_{2}$ avoidance is higher than those estimated for the respective options (B1 \& D1) that derate the base plant; and they can break even only at low gas prices (less than $\$ 2.5-3 / \mathrm{mscf}$ ), as seen in

Figure 5.11. Also, assuming that $\mathrm{CO}_{2}$ emissions from the auxiliary boiler are not controlled, the overall $\mathrm{CO}_{2}$ emissions avoided in these cases is only about $60-65 \%$. Whether this level of $\mathrm{CO}_{2}$ mitigation is acceptable, depends upon the stringency of the $\mathrm{CO}_{2}$ mitigation regulation and other market mechanisms. If new combustion sources are regulated for their emissions of criteria pollutants with concerns about the local pollution, 
then one may also have to account for $\mathrm{NO}_{\mathrm{x}}$-control devices in this option (not accounted for in the present analysis).

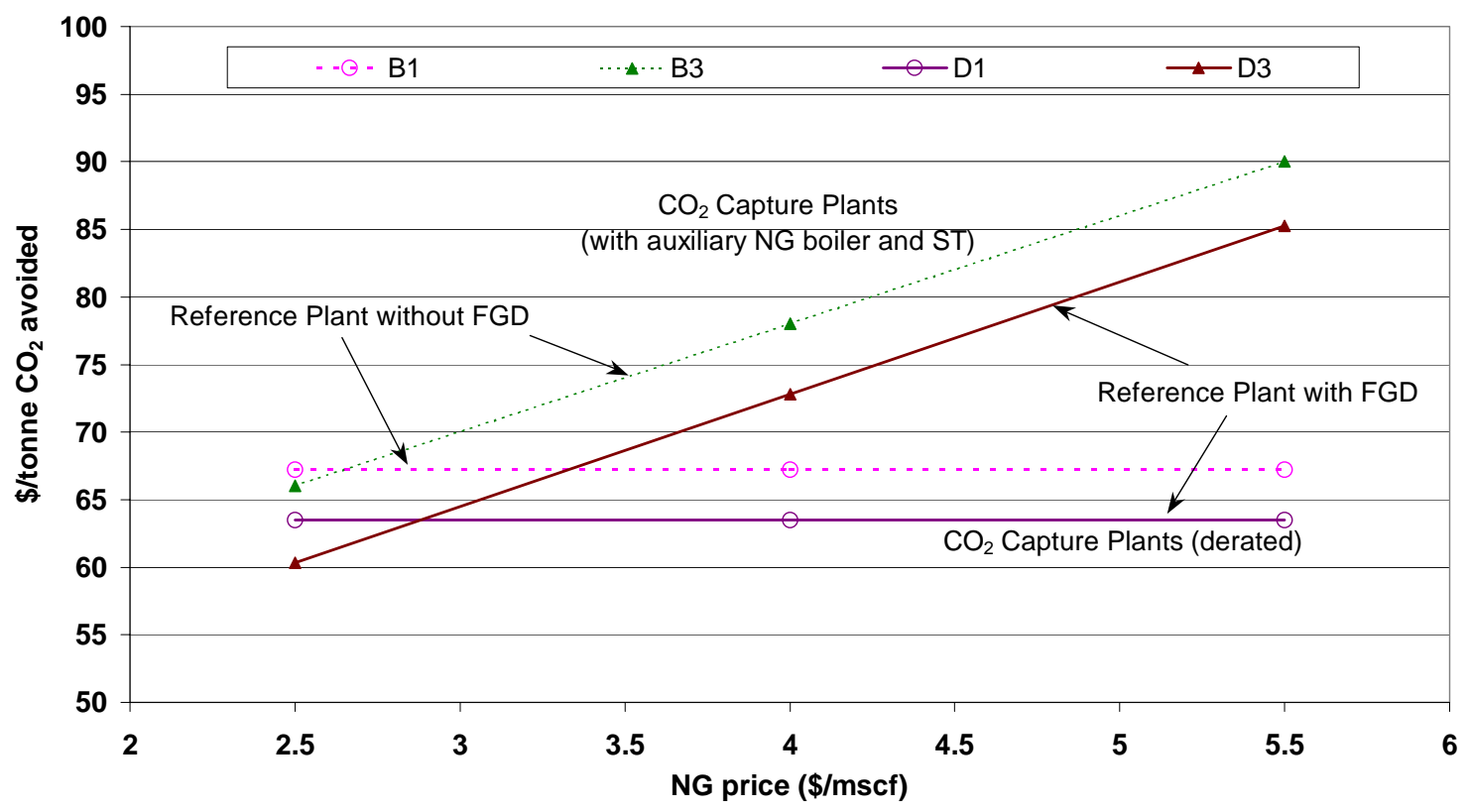

Figure 5.11. Effect of natural gas price on $\mathrm{CO}_{2}$ avoidance cost in $\mathrm{CO}_{2}$ retrofit applications

On the other hand, the $\mathrm{CO}_{2}$ capture options $(\mathrm{B} 1, \mathrm{D} 1)$ that derive their energy requirement from the base plant itself, offer higher levels of $\mathrm{CO}_{2}$ avoidance $(>80 \%)$ and are independent of NG price fluctuations. But these options put a large energy penalty on the reference plant and the net power generation capacity of the plant may decrease by as much as $40 \%$, depending on the site specific design of existing coal-fired units. This may have serious implications in terms of power supply capacity planning and supply management.

\subsection{3. $\mathrm{CO}_{2}$ control from coal-fired vs $\mathrm{NG}$-fired power plants}

The IECM-CS also has a performance and cost model of an NGCC system. Table 5. compares the results from the simulation of a new NGCC system with and without $\mathrm{CO}_{2}$ capture with those from the case study of a coal plant presented earlier in section 5.1.

It may be noted that the capital cost of NGCC plant is quite low $(\$ 521 / \mathrm{kW})$ and even after adding $\mathrm{CO}_{2}$ capture system, it is cheaper than the reference coal plant. This lower 
cost may be attributed to the higher efficiency of combined cycle technology and also to the use of cleaner fuel that cuts down the cost of environmental controls, as compared to a coal plant. NGCC plant with $\mathrm{CO}_{2}$ capture has substantially lower $\mathrm{CO}_{2}$ emissions and produces a smaller stream of concentrated $\mathrm{CO}_{2}$ for storage/ disposal. In comparison with PC plant with $\mathrm{CO}_{2}$ capture, it has much lesser (or no) environmental discharges. Although the $\mathrm{COE}$ from an NGCC plant (even with $\mathrm{CO}_{2}$ capture) is substantially lower than that from a coal plant with $\mathrm{CO}_{2}$ capture, the cost of $\mathrm{CO}_{2}$ avoidance is much higher.

Table 5.10. Comparison of $\mathrm{CO}_{2}$ control from a new NGCC and a new PC plant

\begin{tabular}{|c|c|c|c|c|}
\hline & \multicolumn{2}{|c|}{ NGCC } & \multicolumn{2}{|c|}{ PC } \\
\hline & Ref. & Capture & Ref. & Capture \\
\hline Gross capacity (MW) & 518 & 518 & 500 & 500 \\
\hline Capacity factor (\%) & 75 & 75 & 75 & 75 \\
\hline Fuel cost (\$/GJ) & 3.5 & 3.5 & 1.2 & 1.2 \\
\hline Net power generation (MW) & 518 & 445 & 458 & 341 \\
\hline Net cycle heat rate $(\mathrm{Btu} / \mathrm{kWh})$ & 6,383 & 7,435 & 8,657 & 11,560 \\
\hline Coal consumption (tonne/hr) & - & - & 215.5 & 214.6 \\
\hline NG consumption (tonne/hr) & 65.9 & 65.9 & - & - \\
\hline $\mathrm{CO}_{2}$ emission (tonne/ hr) & 180.8 & 18.1 & 381.3 & 38.0 \\
\hline $\mathrm{CO}_{2}$ emission $\left(\mathrm{gCO}_{2} / \mathrm{kWh}\right)$ & 349.0 & 40.7 & 833.3 & 111.3 \\
\hline FGD waste (tonne/hr) & - & - & 4.2 & 4.3 \\
\hline Spent MEA sorbent (tonne/hr) & - & 0.39 & - & 1.0 \\
\hline $\mathrm{CO}_{2}$ product (tonne/ hr) & - & 163 & - & 342 \\
\hline $\mathrm{CO}_{2}$ emissions captured (\%) & - & 90 & - & 90 \\
\hline $\mathrm{CO}_{2}$ emissions avoided $(\%)$ & - & 88 & - & 87 \\
\hline TCR (M\$) & 270 & 383 & 616 & 760 \\
\hline TCR $(\$ / \mathrm{kW})$ & 521 & 861 & 1345 & 2228 \\
\hline TRR (M\$/yr) & 126 & 179 & 149 & 202 \\
\hline $\mathrm{COE}(\$ / \mathrm{MWh})$ & 36.7 & 61.3 & 49.4 & 89.9 \\
\hline$\$ /$ tonne $\mathrm{CO}_{2}$ avoided & - & 79.2 & - & 56.0 \\
\hline
\end{tabular}

Figure 5.12 plots the $\mathrm{COE}$ and $\mathrm{CO}_{2}$ emission rates for $\mathrm{NGCC}$ plant with and without $\mathrm{CO}_{2}$ capture for various assumptions about natural gas price and plant capacity factor. As 
described earlier, the slope of the line joining a reference plant and a capture plant on this plot gives the $\mathrm{CO}_{2}$ avoidance cost. It appears that even at low gas price $(\$ 2.5 / \mathrm{mscf})$ and high capacity factor (85\%), cost of $\mathrm{CO}_{2}$ avoidance from an NGCC plant is about $30 \%$ higher than that for a PC plant of comparable net power output. However, the COE at such low gas price is even lower than the $\mathrm{COE}$ estimated for a $\mathrm{PC}$ without $\mathrm{CO}_{2}$ control. So, even at low gas prices, controlling $\mathrm{CO}_{2}$ from NGCC plants makes sense only if $\mathrm{COE}$ is the major concern rather than the cost of $\mathrm{CO}_{2}$ mitigation.

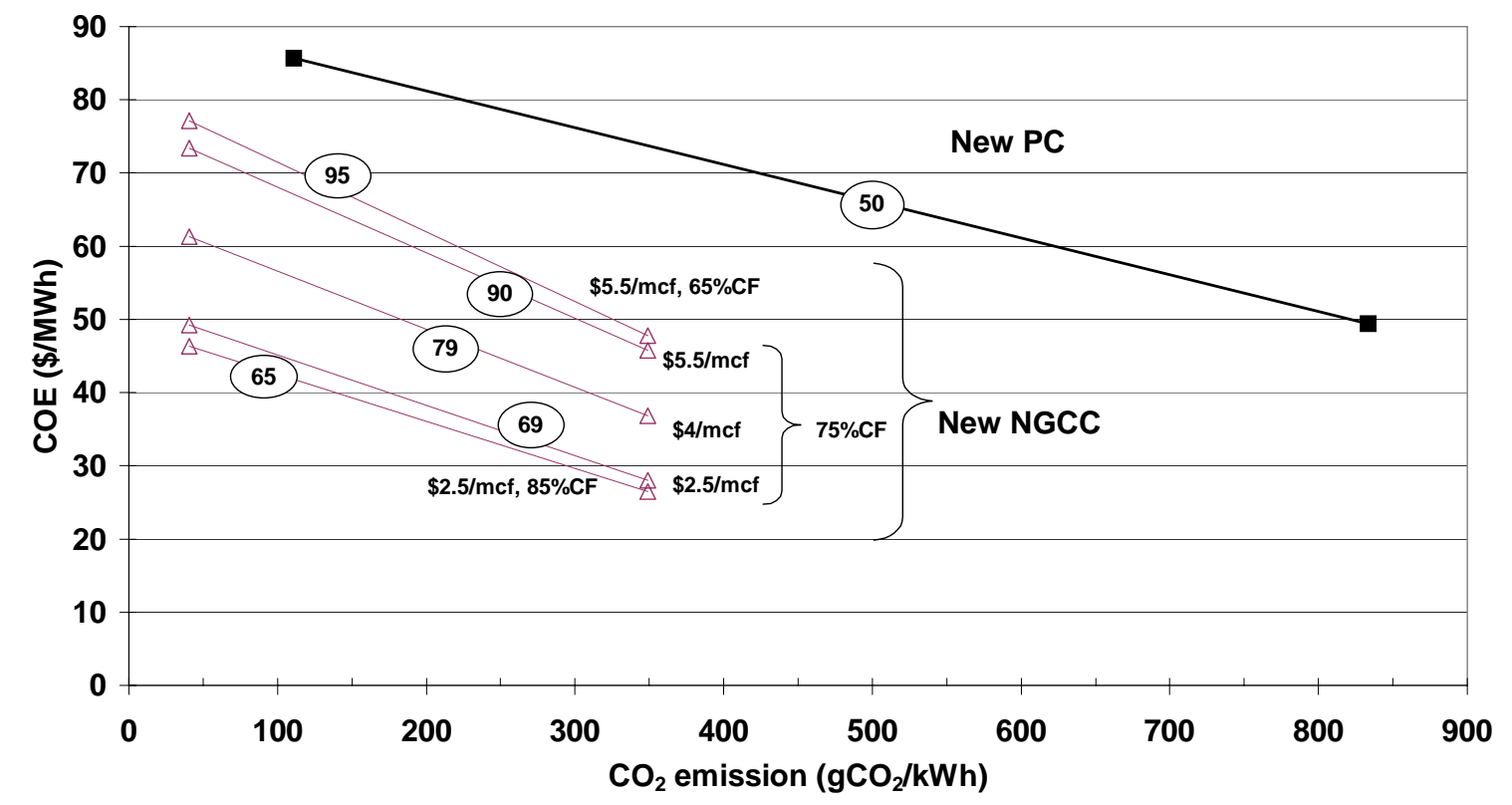

Figure 5.12. Comparison of the cost of $\mathrm{CO}_{2}$ control from a new NGCC and a new PC plant (the numbers in the circles represent the cost of $\mathrm{CO}_{2}$ avoidance)

Natural gas combined cycle (NGCC) is an efficient, cleaner and less capital-intensive technology. This attractive power generation technology is growing in capacity very fast and is expected to grow further in the coming decades (EIA 2000). An NGCC plant produces only about $50 \% \mathrm{CO}_{2}$ per unit of electricity as compared to a conventional coal plant. It might be desirable to capture these emissions under a stringent climate policy, and especially if it is economical (which is not the case, as seen above). Flue gas from an NGCC plant is much cleaner (due to the cleaner fuel) as compared to the flue gas from a coal plant and hence it inflicts less operational difficulties, viz. corrosion and disposal of spent sorbent. Today majority of the $\mathrm{CO}_{2}$ capture plants using amine systems are 
installed to scrub $\mathrm{CO}_{2}$ from gas-fired power plants and hence there is more experience with this application. However, the flue gas from an NGCC plant is less concentrated in $\mathrm{CO}_{2}$ and it takes more energy to regenerate the sorbent per unit of $\mathrm{CO}_{2}$ captured. This partly explains why the cost of $\mathrm{CO}_{2}$ avoidance from these plants was found to be higher than that from a PC plant. Also, the economics of these plants is highly sensitive to the price of natural gas. Often these plants are operated at lower capacity factors as well, which may actually lead to higher avoidance costs. So, based on this analysis, it seems that capture of $\mathrm{CO}_{2}$ from NGCC plants, although technically feasible and favorable, is likely to be more expensive as compared to that from coal plants.

\section{References (Chapter 5)}

CEES (2003). A technical and economic assessment of Selexol-based CO2 capture technology for IGCC power plants, Annual Technical Progress Report prepared by Center for Energy and Environmental Studies, Carnegie Mellon University, Pittsburgh for U.S. Department of Energy, National Energy Technology Laboratory, Morgantown, WV. (Work performed under contract no. DOE/DE-FC26-00NT40935).

CFR (1999). New Source Performance Standards (NSPS). Code of federal regulations, Federal Register. 40CFR, Chapter 1.

Chen, C., A. B. Rao, et al. (2003). Comparative Assessment of $\mathrm{CO}_{2}$ Capture Options for Existing Coal-Fired Power Plants. presented at the 2nd Annual Conference on Carbon Sequestration, May 5-9, 2003, Alexandria, VA, USA.

EIA (2000). Annual Energy Outlook 2001 (with Projections to 2020), A report published by Energy Information Administration, U.S. Department of Energy, Washington, DC.

Johnson, T. L. (2002). Electricity without carbon dioxide: assessing the role of carbon capture and sequestration in US electric markets. Department of Engineering and Public Policy. Pittsburgh, PA 15213, Carnegie Mellon University: 247.

Johnson, T. L. and D. W. Keith (2001). "Electricity from fossil fuels without $\mathrm{CO}_{2}$ emissions: Assessing the costs of carbon dioxide capture and sequestration in U.S. electricity markets." Journal of the Air \& Waste Management Association 51(October): 1452-1459.

NETL (2002). U.S. Coal power plants database, 2000.

Rao, A. B. and E. S. Rubin (2002). "A Technical, Economic, and Environmental Assessment of Amine-based $\mathrm{CO}_{2}$ Capture Technology for Power Plant Greenhouse Gas Control." Environmental Science and Technology 36: 4467-4475. 


\section{R\&D MANAGEMENT: POTENTIAL IMPROVEMENTS AND COST REDUCTION}

Technological innovations in $\mathrm{CO}_{2}$ capture and storage technologies are being pursued worldwide under a variety of private and government-sponsored R\&D programs. While much of this R\&D is directed at novel concepts and potential breakthrough technologies, there are also substantial efforts to improve $\mathrm{CO}_{2}$ capture technologies already in use. As discussed before, amine-based $\mathrm{CO}_{2}$ capture systems have been proposed as one of the solutions for existing as well as new conventional coal-fired power plants. This technology is commercially available today, though it is highly energy intensive and costly, as found in this study and elsewhere (Smelser, Stock et al. 1991; Hendriks 1994; Leci 1996; Simbeck 1998; Desideri and Paolucci 1999; Laboratories 1999; Mariz and al. 1999; Jeremy and Herzog 2000; Parsons Infrastructure \& Technology Group 2002; Rao and Rubin 2002).

Hence, there is a major effort being made to improve amine systems for post-combustion $\mathrm{CO}_{2}$ capture application at power plants. How far can these systems be improved in near future? Technical experts in this field can have a sense about this. As part of this research we sought to quantify the informed judgments of experts to derive uncertainty distributions for various key parameters that will affect the performance and cost of these systems in the future. This is accomplished following the "expert elicitation" protocol widely discussed in the literature and a generally accepted norm to estimate uncertainty when data is lacking (Morgan and Henrion 1990; Frey 1991; Morgan and Keith 1995).

Here we have made an effort to understand what the experts in this field think about

future amine systems. Further, we have used the experts' judgments as input to our model (IECM-CS) to estimate the possible cost reductions in future systems.

\subsection{Methodology}

The following procedure was used to estimate the probability distributions for key performance parameters of future amine systems. 


\subsubsection{Identification of the parameters}

As described before, a model (IECM-CS) has been developed to simulate the performance and cost of post-combustion $\mathrm{CO}_{2}$ capture using amine-based systems at fossil fuel power plants. Analysis of the various parameters helped us identify the key parameters that have a substantial influence on the performance and cost of these systems. Consideration also was given to the clarity of parameter definition, frequency of mention in the literature, and total number of questions to be asked of experts. On this basis, the following set of parameters related to the amine-based $\mathrm{CO}_{2}$ capture system were selected:

- Sorbent concentration (wt \%)

- Sorbent regeneration heat requirement $\left(\mathrm{kJ} / \mathrm{kg} \mathrm{CO}_{2}\right.$ captured)

- Sorbent loss ( $\mathrm{kg} /$ tonne $\mathrm{CO}_{2}$ captured)

- Sorbent cost (US\$/ tonne sorbent)

In addition to these, the following parameters were also included in the questionnaire:

- $\mathrm{CO}_{2}$ compressor efficiency $(\%)$

- Net plant efficiency of the power plant (with and without $\mathrm{CO}_{2}$ capture)

- Total capital requirement (TCR, $\$ / \mathrm{kW}$ ) for the power plant (with and without $\mathrm{CO}_{2}$ capture)

- Cost of electricity (COE, cents/ $\mathrm{kWh}$ ) (with and without $\mathrm{CO}_{2}$ capture)

\subsubsection{Identifying the experts}

Professionals working in the area of amine-based $\mathrm{CO}_{2}$ capture were identified through their authorship of technical papers and participation in International Conferences. Experts were contacted via email and requested to participate in this study. The initial response to this appeal was quite encouraging, and within the time constraints available, final responses were obtained from about a dozen experts. The experts' participation in this study was completely voluntary. There was balanced representation from industry, academia, independent research laboratories and private consultancies. Experts from all over the world participated in this study. The names of these experts have been listed in the acknowledgement section. 

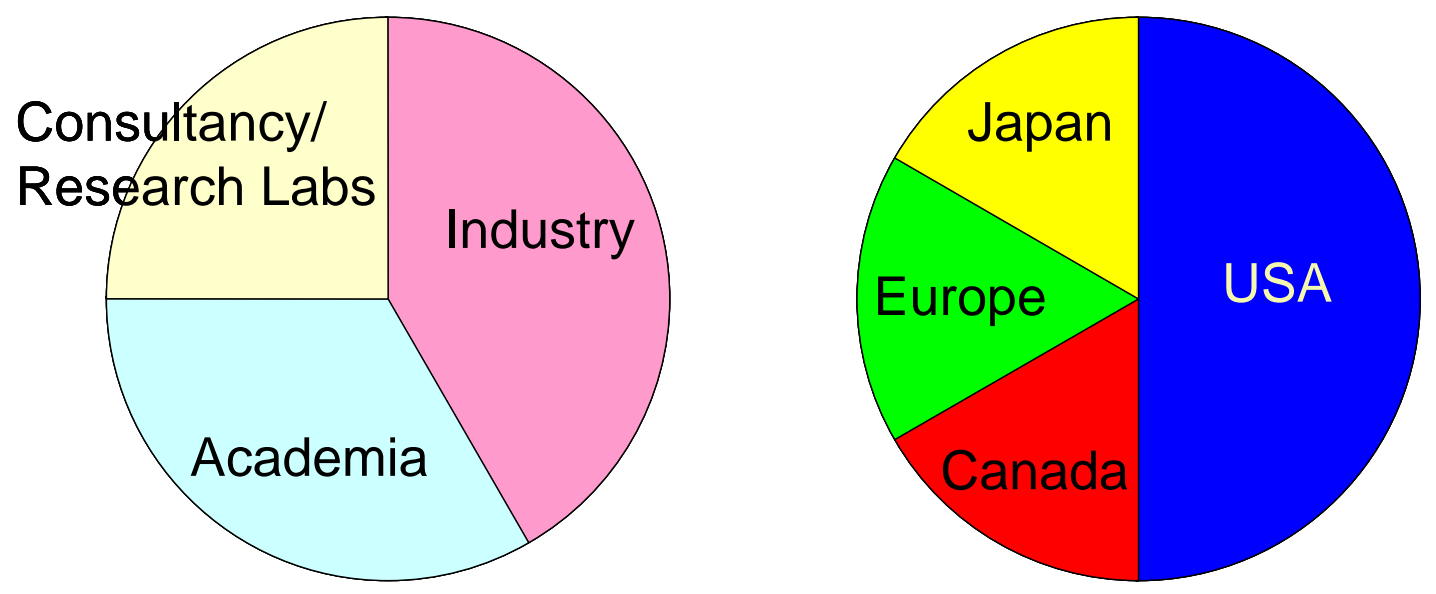

Figure 6.1. Experts' affiliations $(N=12)$

The initial email sent to the potential experts described the purpose of this exercise. Most of the subsequent communication was carried out via email and was supplemented by personal/ telephonic interviews.

\subsubsection{The questionnaire}

Once the key process parameters were identified, a detailed questionnaire was designed (see Appendix E). It included a brief description of the current amine-based $\mathrm{CO}_{2}$ capture systems. This was helpful to set the stage and to ensure consistent use of terminology. Next, it included a checklist of parameters of a current amine-based system. Experts were asked to comment ("OK" or "not OK") on the typical values and ranges of these parameters and to provide replacements if the original numbers were "not OK". This part of the questionnaire helped to know the individual perceptions of the experts about the current systems (baseline). Next, there were detailed questions designed to obtain the uncertainty distributions for the parameters identified above, for a particular scenario of $\mathrm{CO}_{2}$ capture using future amine system at power plants. Assumptions about the size, location, capture efficiency of this system at a new low-sulfur coal-fired power plant for the year 2015 were outlined. The final part of the questionnaire asked the experts to prioritize various research objectives aimed at improving the performance of amine systems and reducing the cost of $\mathrm{CO}_{2}$ capture at power plants. 


\subsubsection{The questionnaire responses}

Responses were obtained from 12 experts over a period of 3 months. Results were processed to plot the uncertainty distributions for various parameters as indicated by all the experts. These compiled results were sent back to all the respondents. This was done in order to avoid any misinterpretation of the responses during data analysis/ units conversion etc. and also to give the experts another chance to review their responses. The respondents were requested to reply within a stipulated timeframe if they wanted to make any changes to their original responses. Only a couple of experts changed their responses during this step. Figure 6.6, Figure 6.7, Figure 6.8, and Figure 6.9 show the final set of distributions for the four parameters derived from the experts' responses about the parameter values for future amine-systems. Some of the data points in these distributions (either $\min / \max$ values or 5-percentile/95-percentile values, whichever were not provided by an expert) have been extrapolated or interpolated on the basis of the rest of the data points provided by the expert.

\subsection{Expert Judgments on Current Amine-based $\mathrm{CO}_{2}$ Capture Systems}

The initial pages of the questionnaire included a checklist of parameters of a current amine-based system. The parameters included were:

- Absorber inlet flue gas pressure

- Temperature of flue gas entering the absorber

- Lean sorbent $\mathrm{CO}_{2}$ loading (mole $\mathrm{CO}_{2} /$ mole sorbent)

- Heat required for sorbent regeneration $(\mathrm{kJ} / \mathrm{kg} \mathrm{CO})$

- Allowable levels of other components in flue gas

- Maximum train size (tonnes $\mathrm{CO}_{2}$ per day)

- Energy required for $\mathrm{CO}_{2}$ compression to $2000 \mathrm{psig}\left(\mathrm{kWh} /\right.$ tonne $\left.\mathrm{CO}_{2}\right)$

Experts were asked to comment ("OK" or "not OK") on the typical values and ranges of these parameters that were provided (based on current IECM-CS defaults) and to provide replacements if the original numbers were "not OK". This part of the questionnaire helped to know the individual perceptions of the experts about the current systems 
(baseline). The last item in this list (related to $\mathrm{CO}_{2}$ compression) was not related to the amine-based $\mathrm{CO}_{2}$ capture system. Nonetheless, some of the experts did respond to this parameter as well. Responses to this parameter were also obtained from a separate questionnaire (containing the same question about $\mathrm{CO}_{2}$ compression energy) that was sent to another set of experts on post-capture processing of $\mathrm{CO}_{2}$.

The responses for these parameters have been summarized in Figure 6.2, Figure 6.3, Figure 6.4, and Figure 6.5. The experts have been represented by letters A, B, C etc. that have been randomly assigned to the experts. The responses to the question on the allowable levels of other components in flue gas have been summarized below:

1) $\mathrm{SO}_{2}(<10 \mathrm{ppmv})$ : Most of the experts believe that a limit of $10 \mathrm{ppmv} \mathrm{SO}_{2}$ is good enough, with few exceptions. One expert suggested that it should be less than 5 ppmv. Another one suggested that the number could lie in the range 2-50 ppmv, and the limit should be decided by the economics of makeup (sorbent requirement) and (spent sorbent) disposal. Lastly, there was an expert who questioned if the suggested limit of 10 ppmv was practical as he believed that even with a caustic polishing, real plants might achieve $\mathrm{SO}_{2}$ levels of about 20 ppmv.

2) $\mathrm{NO}_{2}(<10$ ppmv): Almost all of the experts agreed with this limit, except one person who questioned if this number has any practical basis, and suggested that this number may lie in the range 5-50 ppmv.

3) $\mathrm{O}_{2}(\sim 3.5 \% \mathrm{v} / \mathrm{v})$ : Most of the experts found this limit acceptable. Two experts believed that higher $\mathrm{O}_{2}$ levels could be allowed, one of them quoting $8 \%$ as the limit, another one claiming that the system can work even with $17 \% \mathrm{O}_{2}$, while a third expert said that these systems can work at any oxygen levels although the sorbent degradation economics may worsen at higher levels. 


\subsection{Expert judgments on Future Amine-based $\mathrm{CO}_{2}$ Capture Systems}

The next section of the questionnaire tried to gather technically informed probabilistic judgments about some of the key parameters of a future amine-based $\mathrm{CO}_{2}$ capture system built around the year 2015. The basic assumptions laid out included the following:

- An amine-based plant that treats the flue gas stream from a coal-combustion source which is about $12 \% \mathrm{CO}_{2}$ and which has been pre-treated for removal of $\mathrm{SO}_{\mathrm{x}}$ and $\mathrm{NO}_{\mathrm{x}}$, and removes $90 \%$ of $\mathrm{CO}_{2}$ from the flue gas stream.

- The plant has been optimized for the lowest overall cost of $\mathrm{CO}_{2}$ avoidance (\$/ tonne $\mathrm{CO}_{2}$ avoided), considering both capital and operating costs (including energy costs) over the life of the plant.

- $R \& D$ support for this technology continues to steadily grow at a modest rate through 2015, and includes several new large-scale applications to coal-fired power plants.

The responses to these questions have been processed to obtain the probability distributions presented in Figures 6.6-6.9. It may be seen that there is considerable diversity in these responses, especially in comparison to the distributions for the case of a current commercial amine-based $\mathrm{CO}_{2}$ capture system. Also, not all the experts responded to all the questions.

Most of these responses are optimistic, in the sense they predict an improvement in the parameter value in future systems. For example, consider the sorbent regeneration heat requirement, the parameter that is crucial to the overall energy penalty of this system. Figure 6.10 shows the "best guess" estimates for the current and future systems, as given by each expert. The future estimates are consistently lower than the current ones, implying better performance in the future. 


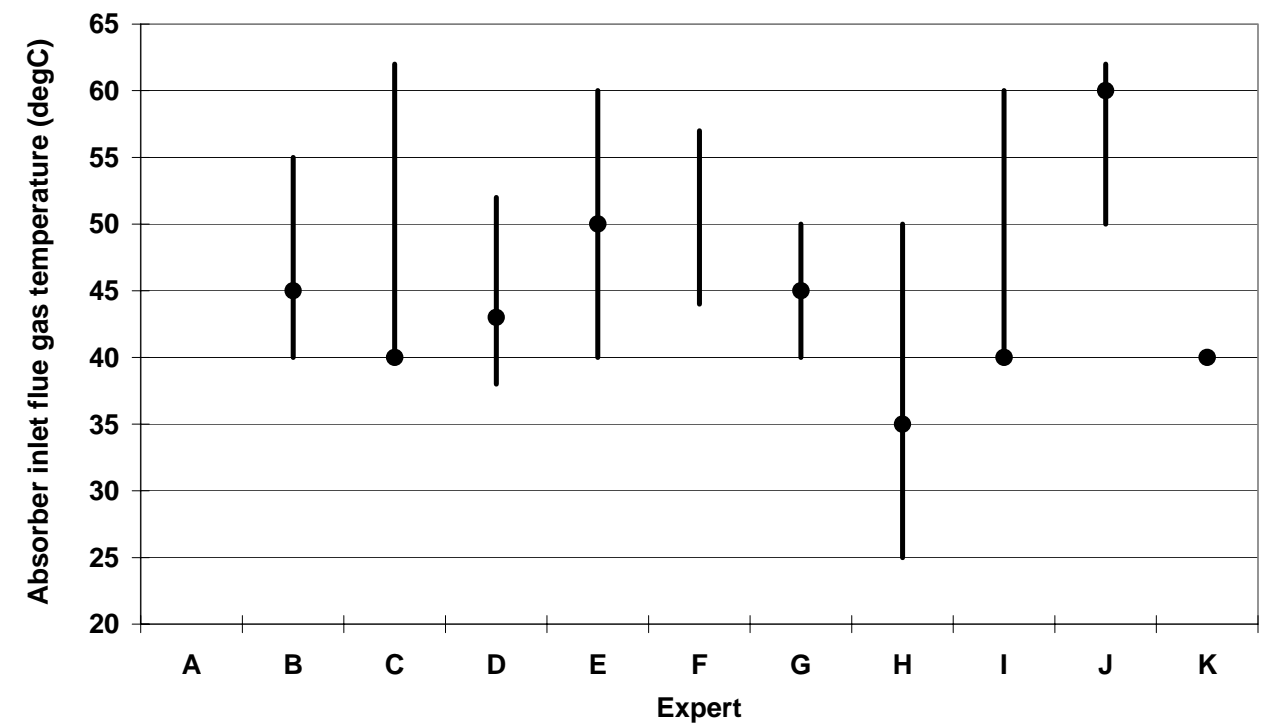

Figure 6.2. Expert judgements on current commercial amine systems for $\mathrm{CO}_{2}$ capture from flue gases: absorber inlet flue gas temperature (deg C)

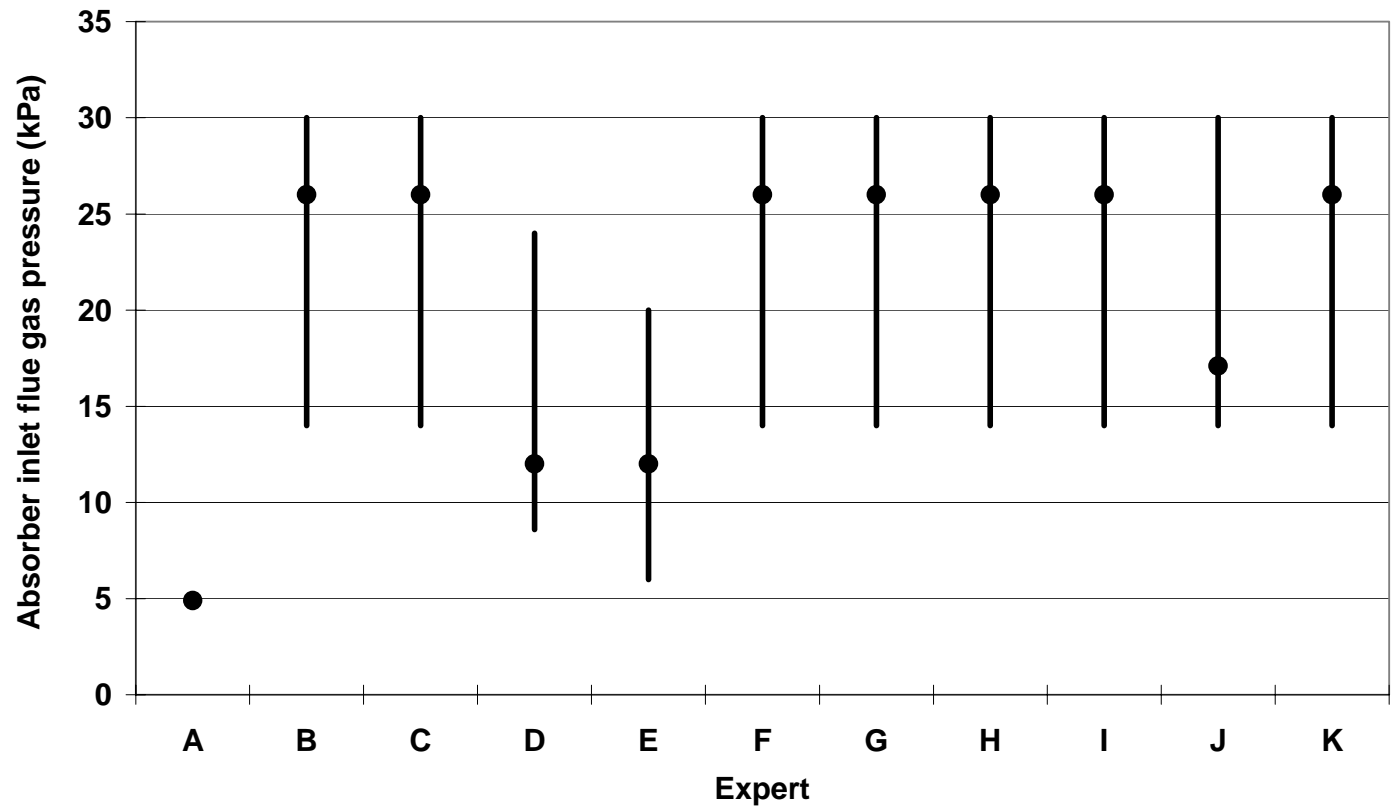

Figure 6.3. Expert judgements on current commercial amine systems for $\mathrm{CO}_{2}$ capture from flue gases: absorber inlet flue gas pressure $(\mathrm{kPa})$ 


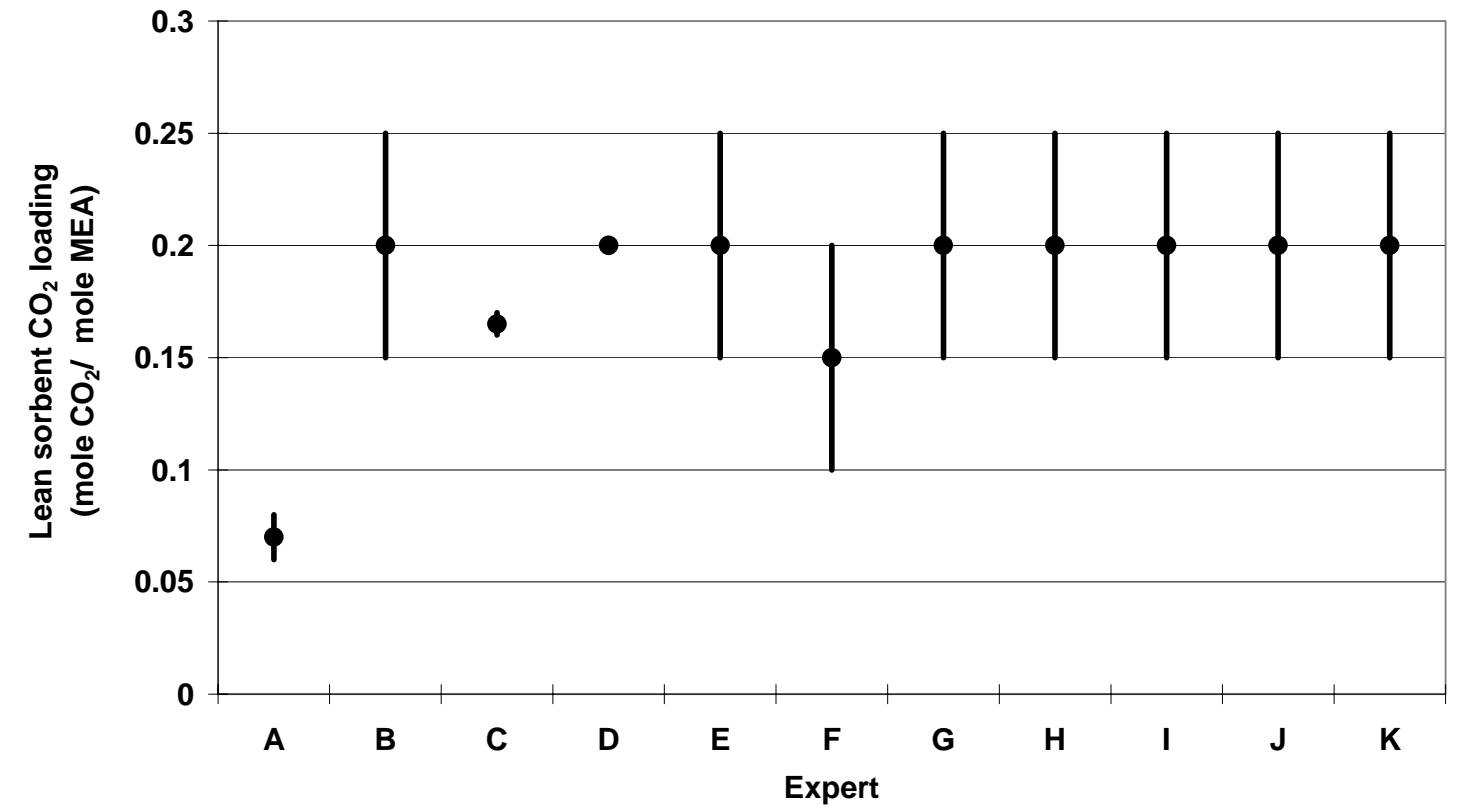

Figure 6.4. Expert on current commercial amine systems for $\mathrm{CO}_{2}$ capture from flue gases: lean sorbent $\mathrm{CO}_{2}$ loading (mole $\mathrm{CO}_{2} /$ mole MEA)

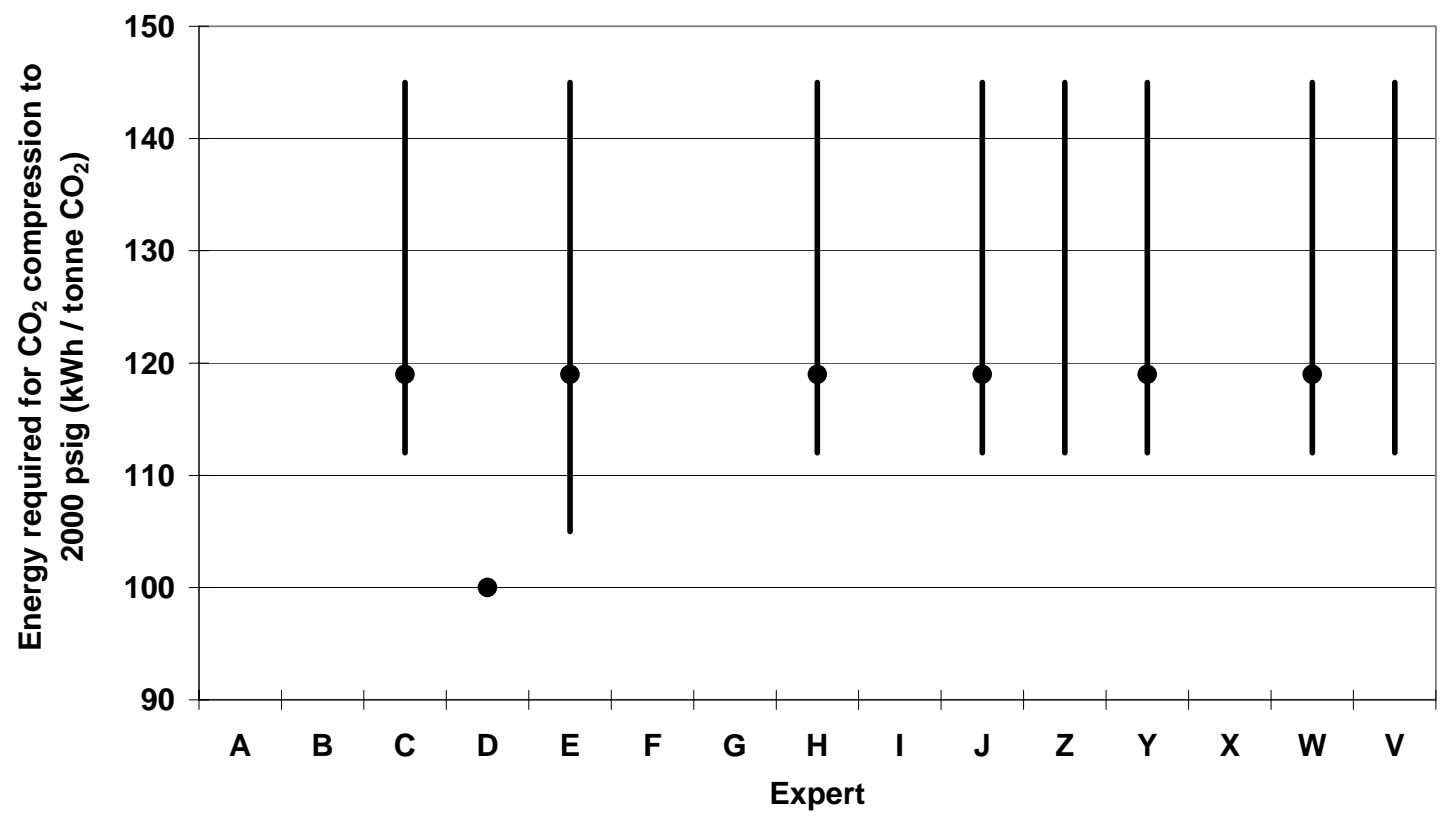

Figure 6.5. Expert on current commercial amine systems for $\mathrm{CO}_{2}$ capture from flue gases: energy required for $\mathrm{CO}_{2}$ compression to $2000 \mathrm{psig}$ ( $\mathrm{kWh} /$ tonne $\mathrm{CO}_{2}$ ) 
The basic motivation behind this study was to understand what experts in this field believe about the possible scope for improvement in the performance of amine-based $\mathrm{CO}_{2}$ capture systems and for reduction in the cost of $\mathrm{CO}_{2}$ capture. From the experts' responses, improvement in the various parameters relative to the current baseline was estimated. The "best guess" judgments gave a nominal/ most probable improvement, while the minimum or maximum values (depending upon the parameter) led to the "most optimistic" estimation of improvement. The relative improvement is calculated as the difference in the current and future estimate expressed as a percentage of the current baseline.

Relative improvement in parameter Z $(\%)=\left(f_{Z}\right) * \frac{(Z)_{\text {future }}-(Z)_{\text {current }}}{(Z)_{\text {current }}} \times 100$

Where, $\quad Z_{\text {future }}=$ Expert's judgment about the value of parameter $Z$ in a future system

$Z_{\text {current }}=$ Current baseline value of parameter $Z$

$f_{Z}=$ Parameter specific multiplier that reflects the "improvement"

$=(+1)$ for $\mathrm{Z}=$ Sorbent concentration

$=(-1)$ for other three parameters viz. sorbent regeneration heat requirement, sorbent loss and sorbent cost

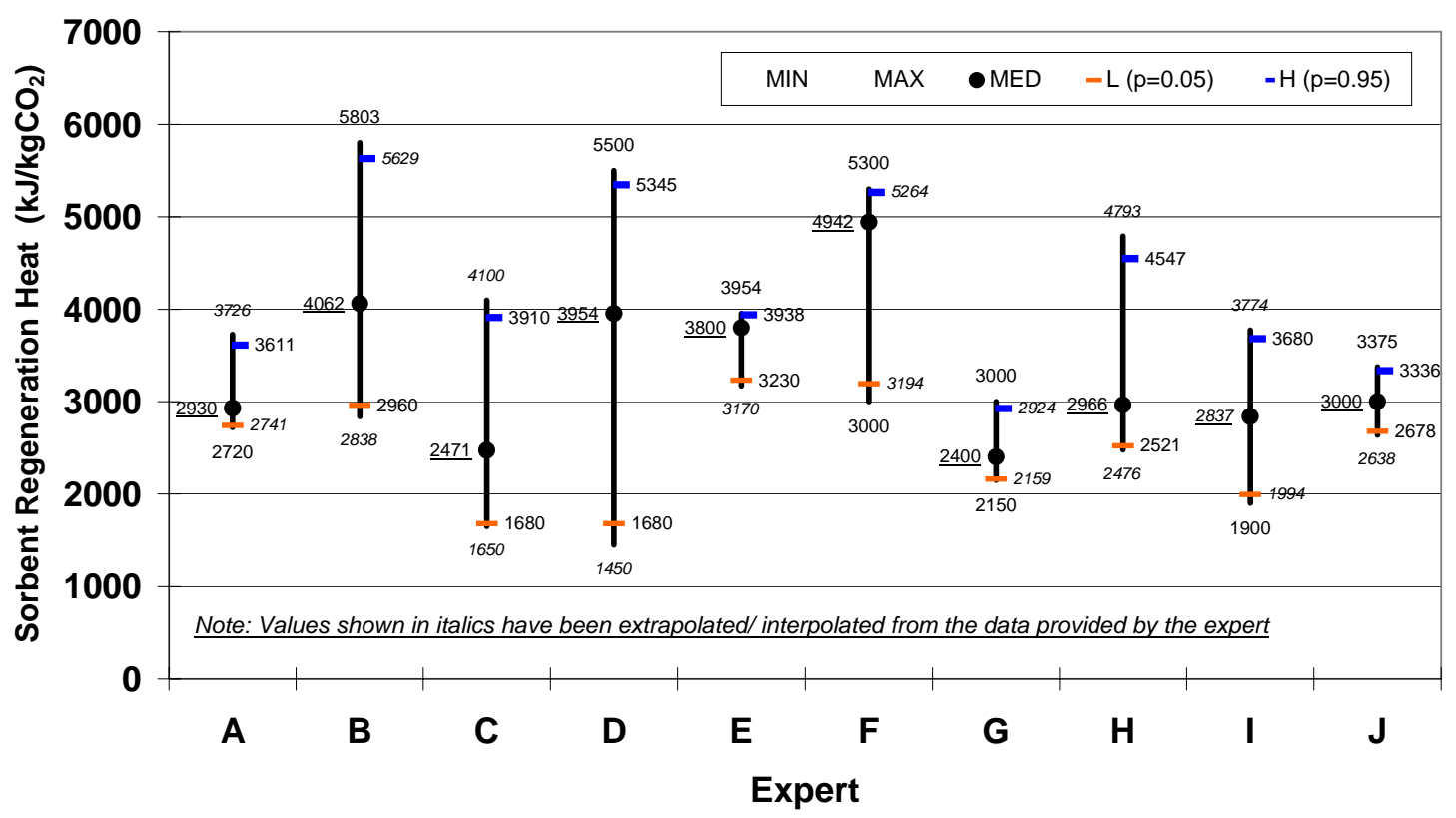

Figure 6.6. Expert judgments on future commercial amine systems: Parameter 1: sorbent regeneration heat $(\mathrm{kJ} / \mathrm{kgCO})$ 


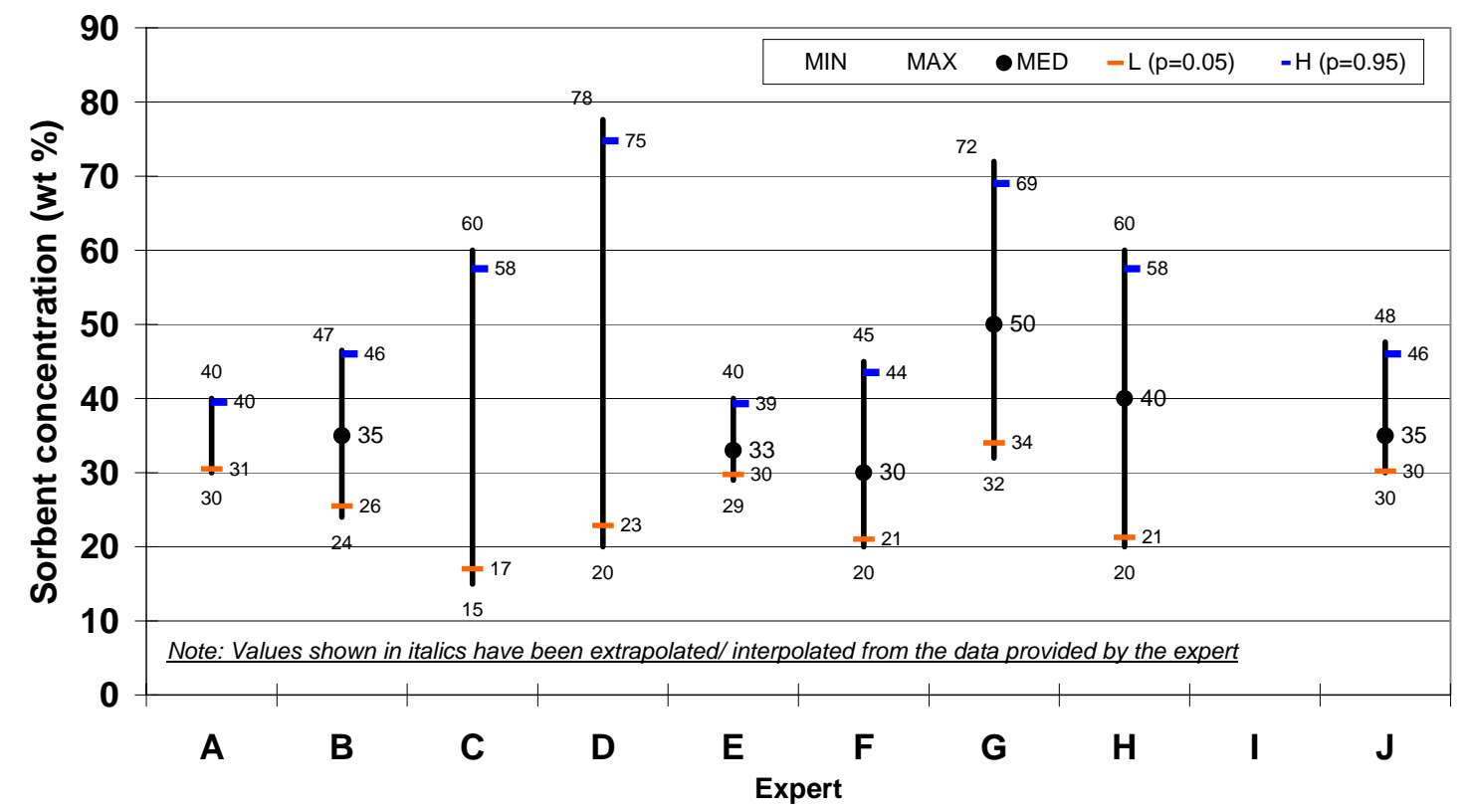

Figure 6.7. Expert judgments on future commercial amine systems: Parameter 2: sorbent concentration ( $\% \mathrm{w} / \mathrm{w})$

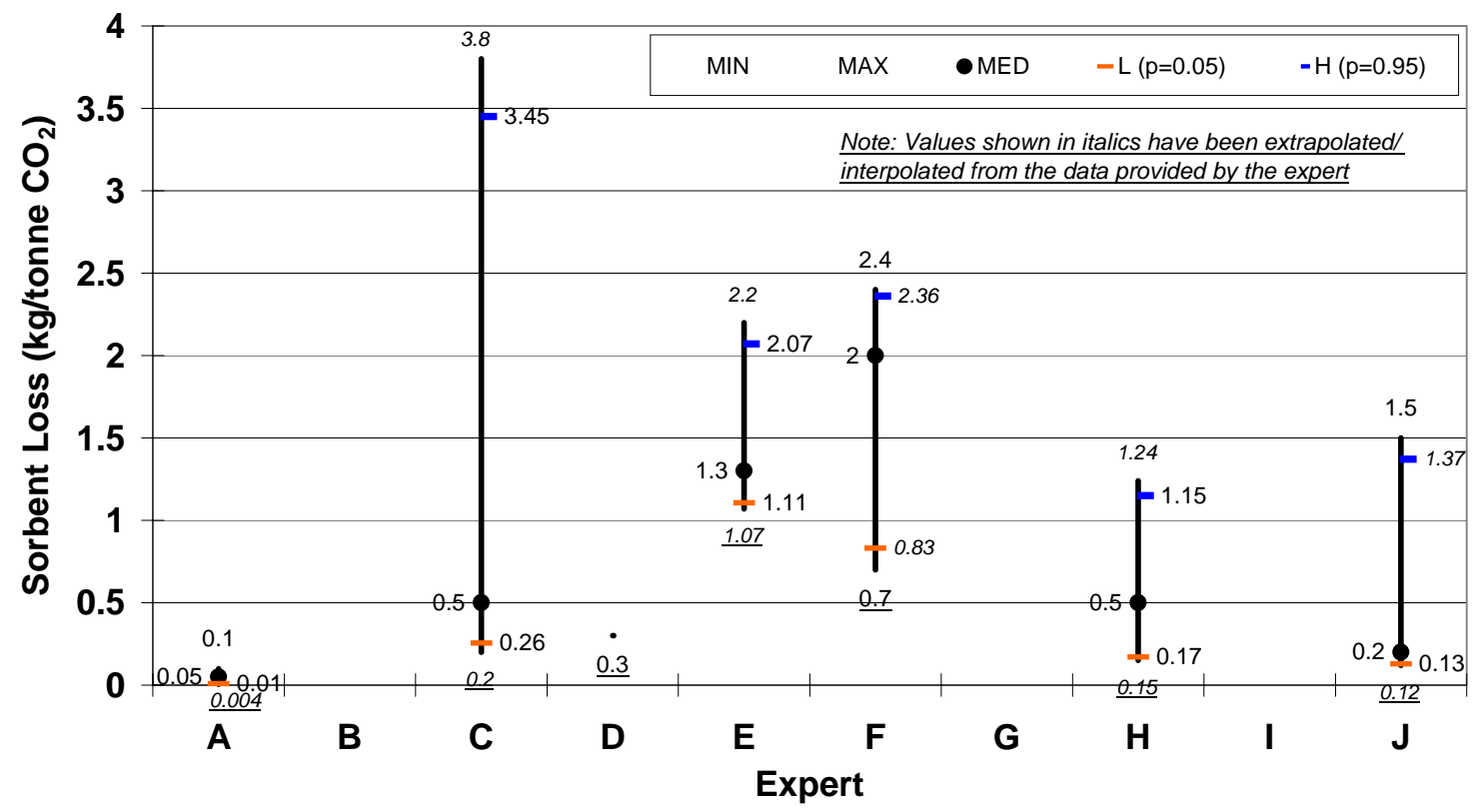

Figure 6.8. Expert judgments on future commercial amine systems: Parameter 3: sorbent loss ( $\mathrm{kg} \mathrm{MEA/tonne} \mathrm{CO}_{2}$ ) 


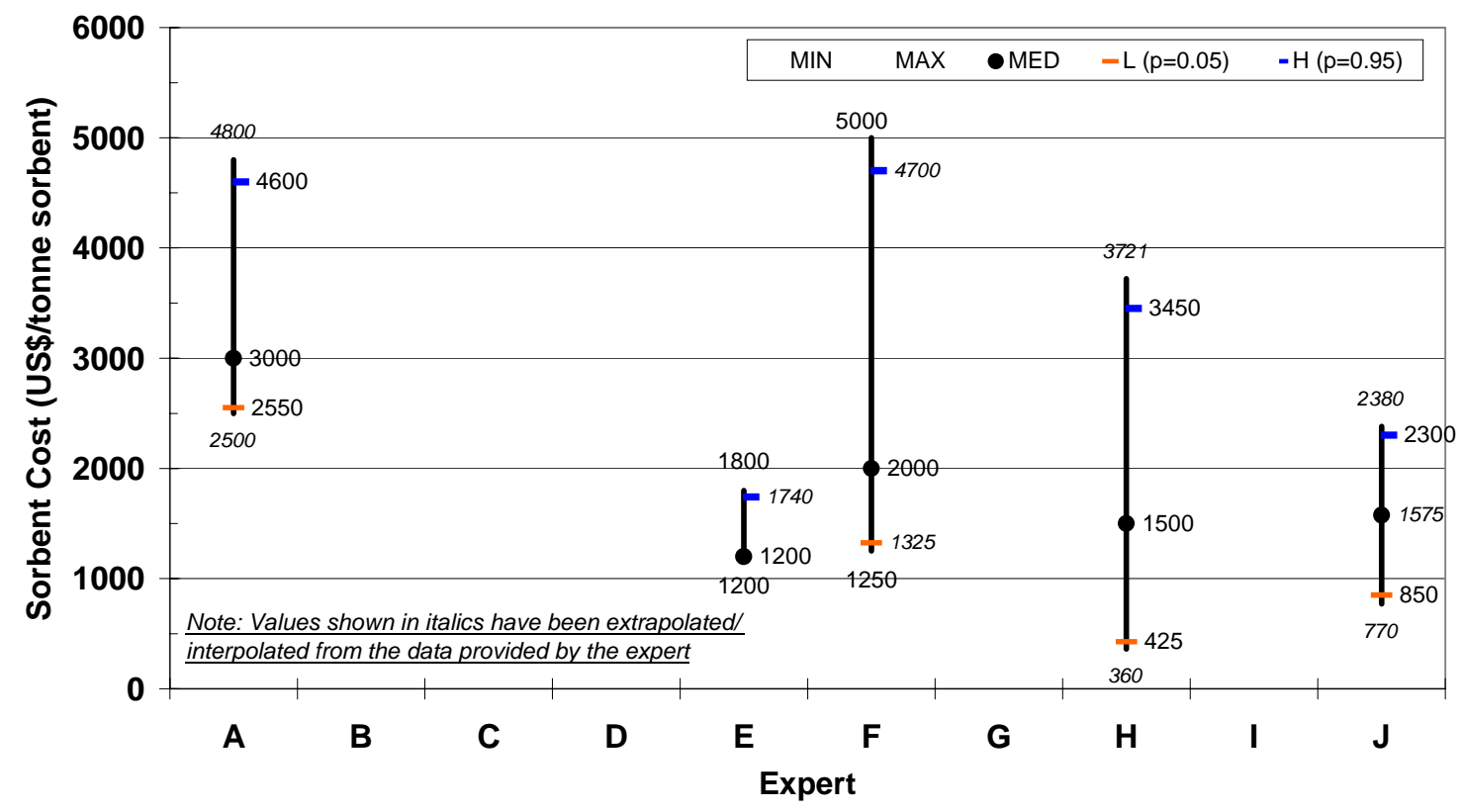

Figure 6.9. Expert judgments on future commercial amine systems: Parameter 4: sorbent loss (US\$/ tonne $\mathrm{CO}_{2}$ )

Based on the "best guess" and "most optimistic" future judgments, the relative improvement is estimated and the results have been summarized in Tables Table 6.1, Table 6.2, Table 6.3, and Table 6.4.

\subsubsection{Parameter 1: Sorbent Regeneration Heat Requirement}

As we have seen before, this parameter determines the energy penalty of the amine system. Figure 6.10 shows the absolute values of this parameter as predicted by the various experts for a future system, compared against their own current baseline "best guess" values. 
It may be noticed that all the experts are optimistic about future improvements in the performance of the amine-based $\mathrm{CO}_{2}$ capture systems and predict that the sorbent regeneration heat requirement would be lower in the future. The best guess estimates predict an improvement in the range of 5 to 40 percent as compared to the experts' individual current estimates. The average improvement is about $23 \%$. The most optimistic judgments are in the range of $15-73 \%$, averaging about a $43 \%$ improvement.

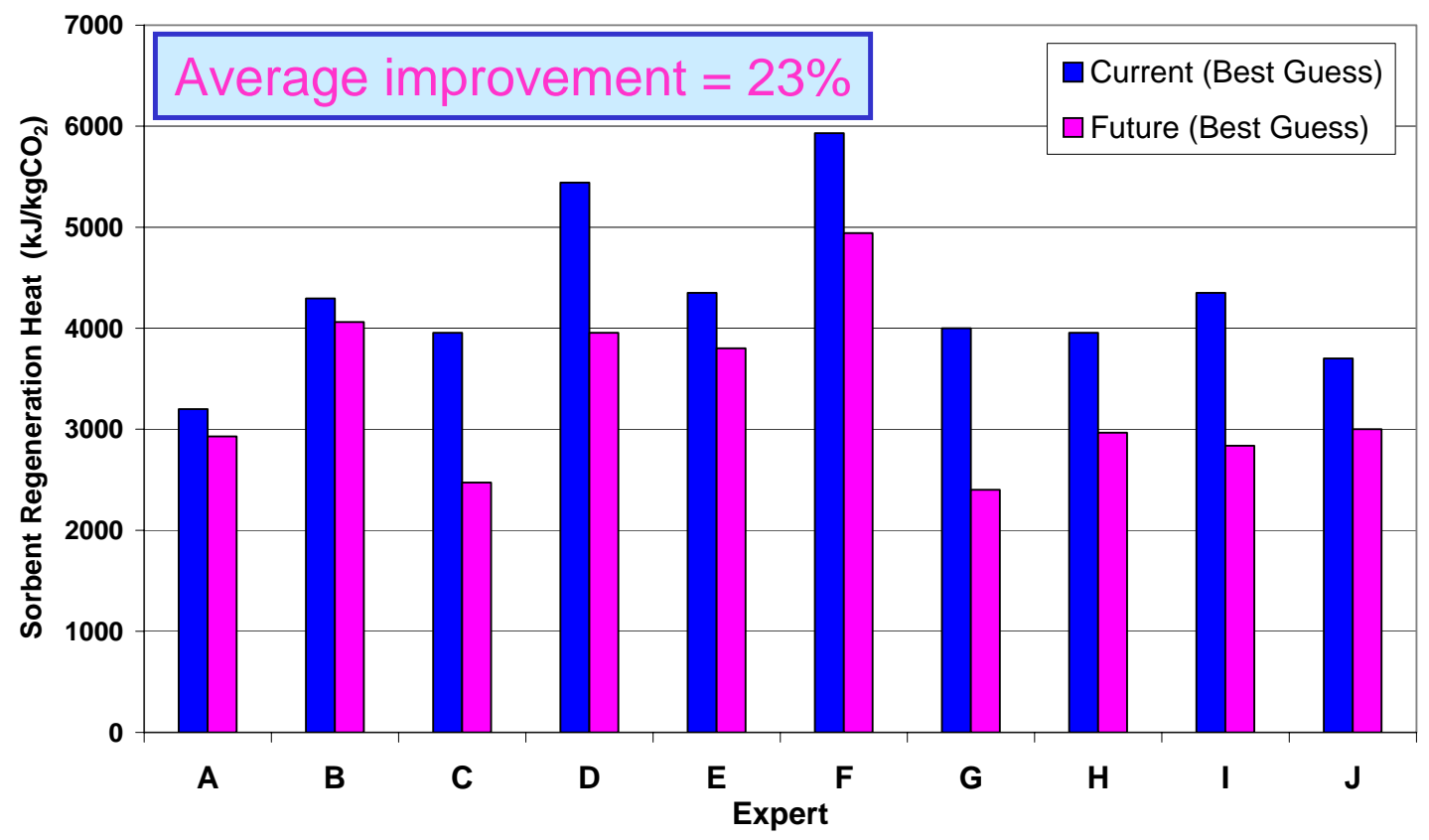

Figure 6.10. Sorbent regeneration heat requirement ( $\left.\mathrm{kj} / \mathrm{kgCO}_{2}\right)$ : current baseline vs. future projections

Table 6.1. Relative improvement in sorbent regeneration heat requirement

\begin{tabular}{||l|c|c||}
\hline \multicolumn{1}{|c|}{ Based on } & Average & Range \\
\hline \hline "Best Guess" future judgments & $23 \%$ & $5 \%-40 \%$ \\
\hline "Most Optimistic" future judgments & $43 \%$ & $15 \%-73 \%$ \\
\hline
\end{tabular}

Experts cited advanced amines, hindered amines, special mixtures of amines, or equipment change as possible strategies to achieve these improvements. A few said they 
had "no idea" as to how the improvements would be achieved, but were optimistic nonetheless that such improvements were achievable.

\subsubsection{Parameter 2: Sorbent Concentration}

Most of the current commercial systems use an amine sorbent concentration of less than or equal to $30 \% \mathrm{w} / \mathrm{w}$. Many experts believe that using a higher concentration of sorbents may help reduce the energy penalty of this system and improve the overall performance. At the same time, there are a few experts who believe that sorbent concentration is a non-issue.

Table 6.2. Relative improvement in sorbent concentration

\begin{tabular}{||l|c|c||}
\hline \multicolumn{1}{|c|}{ Based on } & Average & Range \\
\hline \hline "Best Guess" future judgments & $23 \%$ & $0 \%-67 \%$ \\
\hline "Most Optimistic" future judgments & $80 \%$ & $33 \%-160 \%$ \\
\hline
\end{tabular}

The "best guess" value for sorbent concentration in a future system averaged about 37\% w/w (which is about 23\% improvement over the current baseline), while the most optimistic future judgments average to about $54 \% \mathrm{w} / \mathrm{w}$ (which is $80 \%$ improvement over the current baseline). The main breakthroughs that would enable the use of such higher concentrations are reported to be solving corrosion problem, special additives, corrosion inhibitors, improved metallurgy in the absorber, specially formulated amines, and lower oxygen content in flue gas.

\subsubsection{Parameter 3: Sorbent Loss}

The current baseline for sorbent loss is about $1.5 \mathrm{~kg}$ sorbent lost per tonne of $\mathrm{CO}_{2}$ captured. Although most of the experts believe that the sorbent loss in future amine systems will be lower than that in the current systems, there are few experts who think otherwise. Hence the range of "best guess" estimates of improvement includes a few negative numbers. Across all experts, however, they average out to about a $50 \%$ improvement, i.e., the sorbent losses in future systems are most likely to be about half of the current levels (i.e. about $0.76 \mathrm{~kg}$ sorbent/ tonne $\mathrm{CO}_{2}$ ). The most optimistic judgments averaged across all the experts predict a much lower sorbent loss (on an average, about 
$0.36 \mathrm{~kg}$ sorbent/ tonne $\mathrm{CO}_{2}$ ) in future systems, which is more than a $75 \%$ improvement over the current baseline. The key research requirements that can lead to such performance include better sorbents, better inhibitors, better design and operating conditions, and zero oxygen content in flue gas.

Table 6.3. Relative improvement in sorbent loss

\begin{tabular}{||l|c|c||}
\hline \multicolumn{1}{|c|}{ Based on } & Average & Range \\
\hline \hline "Best Guess" future judgments & $49 \%$ & $(33 \%)-97 \%$ \\
\hline "Most Optimistic" future judgments & $76 \%$ & $29 \%-100 \%$ \\
\hline
\end{tabular}

\subsubsection{Parameter 4: Sorbent Cost}

While the current baseline cost of the amine (MEA) sorbent is about $\$ 1.2 / \mathrm{kg}$ sorbent, the expected improvements in future amine systems may come at a cost premium.

Table 6.4. Relative improvement in sorbent cost

\begin{tabular}{|l|c|c||}
\hline \multicolumn{1}{|c|}{ Based on } & Average & Range \\
\hline \hline "Best Guess" future judgments & $(48 \%)$ & $(140 \%)-4 \%$ \\
\hline "Most Optimistic" future judgments & $3 \%$ & $(100 \%)-71 \%$ \\
\hline
\end{tabular}

Based on the "best guess" estimates of each expert, on average the future sorbents may cost almost 50\% more. The most optimistic judgments estimated that the cost of future sorbents may be almost the same as that of the current sorbents, or even slightly cheaper. According to the various experts, there are several factors that may affect the cost of future sorbents, viz. natural gas (the ultimate feedstock) availability and prices, demand, and generic versus specialized application.

\subsection{Estimation of Future Cost Reductions}

Next, we sought to estimate the process cost reductions that would result from the improvements in future amine systems as envisaged by the various experts. For this purpose, we used the experts' responses about the four parameter values noted above as input to the IECM-CS model. All other model parameters were kept at their default 
values. In these cost estimates, we assumed that the $\mathrm{CO}_{2}$ product is compressed to 2000 psig. The cost of $\mathrm{CO}_{2}$ transport and storage is not included.

The main cost parameters of interest are the capital cost of the $\mathrm{CO}_{2}$ capture system, the incremental cost of electricity and the cost of $\mathrm{CO}_{2}$ avoidance. Table 6.5, Table 6.6, and Table 6.7 give the estimated cost reductions as percentages of the estimates for current systems.

Table 6.5. Expected reduction in capital cost from improvements in four process parameters (relative to current baseline)

\begin{tabular}{||c|c|c||}
\hline \multicolumn{1}{|c|}{ Based on } & Average & Range \\
\hline \hline "Best Guess" future judgments & $6 \%$ & $(2 \%)-9 \%$ \\
\hline "Most Optimistic" future judgments & $16 \%$ & $7 \%-19 \%$ \\
\hline
\end{tabular}

Table 6.6. Expected reduction in incremental COE from improvements in four process parameters (relative to current baseline)

\begin{tabular}{||c|c|c||}
\hline \multicolumn{1}{|c|}{ Based on } & Average & Range \\
\hline \hline "Best Guess" future judgments & $18 \%$ & $(8 \%)-29 \%$ \\
\hline "Most Optimistic" future judgments & $35 \%$ & $20 \%-37 \%$ \\
\hline
\end{tabular}

Table 6.7. Expected reduction in cost of $\mathrm{CO}_{2}$ avoidance from improvements in four process parameters (relative to current baseline)

\begin{tabular}{||l|c|c||}
\hline \multicolumn{1}{|c|}{ Based on } & Average & Range \\
\hline \hline "Best Guess" future judgments & $18 \%$ & $(8 \%)-30 \%$ \\
\hline "Most Optimistic" future judgments & $36 \%$ & $21 \%-38 \%$ \\
\hline
\end{tabular}

It must be noted that these results reflect expert judgments on only 4 parameters of the amine system model. Even in this case, the average expected cost reductions, especially in the incremental cost of electricity and cost of $\mathrm{CO}_{2}$ avoidance, are quite encouraging. There are many more parameters that may help to reduce overall system costs, e.g., better power plant heat integration, improved column designs, shorter construction times and improvements to the base plant characteristics (lower heat rate, lower air leakage etc.). Thus, future amine-based $\mathrm{CO}_{2}$ capture technology may be a more cost effective option to control greenhouse gas emissions from fossil fueled power plants in the next two decades or so. However, future improvements in this technology (and resulting cost reductions) 
will depend a lot on continuing $R \& D$ investments and incentives for deployment resulting from government actions and policies (Taylor and etal 2003).

\subsection{Assessing R\&D Priorities}

In the last section of the questionnaire, (see Appendix E), experts were asked to indicate their R\&D priorities as to reduce the cost of $\mathrm{CO}_{2}$ capture and the cost of $\mathrm{CO}_{2}$ avoidance using amine-based system by year 2015. Items (B1-B13) are related specifically to amine-based systems, while items (A1-A4) are related to the reference plant characteristics and items $(\mathrm{C} 1-\mathrm{C} 3)$ are related to post-capture processing of $\mathrm{CO}_{2}$ product stream. In all, 19 research objectives were listed, and the experts were requested to classify these items into the following three categories:

- High Priority $(\mathrm{H})$

- Medium Priority (M)

- Low Priority (L)

Table 6.8 briefly summarizes the experts' responses to this question. While there were diverse views on the relative importance of each research objective, all the experts agreed (almost unanimously) on the following items as the top priority issues:

1) To develop sorbents with lower regeneration energy requirement [B4]

2) To develop less expensive technologies for $\mathrm{CO}_{2}$ storage/ disposal [C3]

3) To improve heat integration within the power plants (to reduce the energy penalty due to steam extraction for sorbent regeneration) [A4]

4) To develop more efficient power plants (lower heat rate) [A1]

Clearly the topmost priority R\&D objective (ranked "High Priority" by $82 \%$ of the experts) is to develop sorbents having lower regeneration energy requirement. From our 
Table 6.8. Summary of research priorities identified by the experts

\begin{tabular}{|c|c|c|c|c|}
\hline \multirow[b]{2}{*}{ No. } & \multirow[b]{2}{*}{ Research Objective } & \multicolumn{3}{|c|}{$\begin{array}{l}\text { \% of experts who believe that } \\
\text { this item is of: }\end{array}$} \\
\hline & & $\begin{array}{l}\text { High } \\
\text { Priority }\end{array}$ & $\begin{array}{l}\text { Medium } \\
\text { Priority }\end{array}$ & $\begin{array}{c}\text { Low } \\
\text { Priori } \\
\text { y }\end{array}$ \\
\hline A1 & To develop more efficient power plants (lower heat rate) & $50 \%$ & $20 \%$ & $30 \%$ \\
\hline A2 & $\begin{array}{l}\text { To improve boiler designs so that fuel can be burned with } \\
\text { lower excess air (typically for a coal plant, } \sim 20 \% \text { excess } \\
\text { air is used) }\end{array}$ & $40 \%$ & $10 \%$ & $50 \%$ \\
\hline A3 & $\begin{array}{l}\text { To develop more efficient technologies for } \mathrm{SO}_{\mathrm{x}} \text { and } \mathrm{NO}_{\mathrm{x}} \\
\text { control so as to reduce the acidic gas impurities in the } \\
\text { flue gas stream }\end{array}$ & $27 \%$ & $27 \%$ & $45 \%$ \\
\hline A4 & $\begin{array}{l}\text { To improve heat integration within the power plants to } \\
\text { reduce the energy penalty for } \mathrm{CO}_{2} \text { capture due to steam } \\
\text { extraction for sorbent regeneration }\end{array}$ & $55 \%$ & $27 \%$ & $18 \%$ \\
\hline B1 & To develop more efficient fans for flue gas handling & $0 \%$ & $27 \%$ & $73 \%$ \\
\hline B2 & $\begin{array}{l}\text { To develop } \mathrm{CO}_{2} \text { absorbers that can handle higher sorbent } \\
\text { concentrations }\end{array}$ & $36 \%$ & $27 \%$ & $36 \%$ \\
\hline B3 & To develop sorbents with higher $\mathrm{CO}_{2}$ loading capacity & $45 \%$ & $27 \%$ & $27 \%$ \\
\hline B4 & $\begin{array}{l}\text { To develop sorbents with lower regeneration energy } \\
\text { requirement }\end{array}$ & $82 \%$ & $18 \%$ & $0 \%$ \\
\hline B5 & $\begin{array}{l}\text { To develop absorber columns offering lower pressure } \\
\text { drops }\end{array}$ & $18 \%$ & $73 \%$ & $9 \%$ \\
\hline B6 & $\begin{array}{l}\text { To develop absorber columns offering higher } \mathrm{CO}_{2} \\
\text { capture efficiencies }\end{array}$ & $27 \%$ & $27 \%$ & $45 \%$ \\
\hline B7 & To develop more efficient pumps for sorbent circulation & $0 \%$ & $9 \%$ & $91 \%$ \\
\hline B8 & To develop more efficient heat exchanging devices & $0 \%$ & $45 \%$ & $55 \%$ \\
\hline B9 & To reduce the cost of sorbent manufacturing & $9 \%$ & $64 \%$ & $27 \%$ \\
\hline B10 & $\begin{array}{l}\text { To develop sorbents with lower makeup requirements } \\
\text { (less losses) }\end{array}$ & $27 \%$ & $64 \%$ & $9 \%$ \\
\hline B11 & $\begin{array}{l}\text { To develop less expensive technologies for disposal of } \\
\text { spent sorbents }\end{array}$ & $18 \%$ & $45 \%$ & $36 \%$ \\
\hline B12 & $\begin{array}{l}\text { To develop better instrumentation/ automation in the } \mathrm{CO}_{2} \\
\text { capture system so as to reduce the labor requirement }\end{array}$ & $0 \%$ & $18 \%$ & $82 \%$ \\
\hline B13 & $\begin{array}{l}\text { To develop better construction materials so as to reduce } \\
\text { the losses due to corrosion }\end{array}$ & $27 \%$ & $36 \%$ & $36 \%$ \\
\hline $\mathrm{C} 1$ & $\begin{array}{l}\text { To develop more efficient compressors for } \mathrm{CO}_{2} \\
\text { compression }\end{array}$ & $0 \%$ & $60 \%$ & $40 \%$ \\
\hline $\mathrm{C} 2$ & $\begin{array}{l}\text { To develop a transport technology that can handle low- } \\
\text { pressure } \mathrm{CO}_{2} \text { streams }\end{array}$ & $9 \%$ & $18 \%$ & $73 \%$ \\
\hline $\mathrm{C} 3$ & $\begin{array}{l}\text { To develop less expensive technologies for } \mathrm{CO}_{2} \text { storage/ } \\
\text { disposal }\end{array}$ & $64 \%$ & $36 \%$ & $0 \%$ \\
\hline
\end{tabular}

previous analysis, this parameter is crucial in determining the overall energy penalty of this system, and hence affects the overall cost of $\mathrm{CO}_{2}$ avoidance. The other complimentary factor that helps determine the overall energy penalty is the level of heat 
integration between the power plant and the amine system. Significant improvements are required on these fronts so as to make amine-based $\mathrm{CO}_{2}$ capture economically competitive. Researchers around the world have been working in this direction, and the initial results seem to be promising (Mimura, Matsumoto et al. 2000; Iijima and Kamijo 2002; Veawab, Tontiwachwuthikul et al. 2002; Reddy and Roberts 2003).

\section{References (Chapter 6)}

Desideri, U. and A. Paolucci (1999). "Performance modelling of a carbon dioxide removal system for power plants." Energy Conversion and Management 40: 1899-1915.

Frey, H. C. (1991). Probabilistic Modeling of Innovative Clean Coal Technologies: Implications for Technology Evaluation and Research Planning. Department of Engineering and Public Policy. Pittsburgh, PA, USA, Carnegie Mellon University: 605.

Hendriks, C. (1994). Carbon Dioxide Removal from Coal-fired Power Plants. The Netherlands, Kluwer Academic Publishers.

Iijima, M. and T. Kamijo (2002). Flue gas $\mathrm{CO} 2$ recovery and compression cost study for $\mathrm{CO} 2$ enhanced oil recovery. Sixth International Conference on Greenhouse Gas Control Technologies, Kyoto, Japan.

Jeremy, D. and H. J. Herzog (2000). The cost of carbon capture. presented at the Fifth International Conference on Greenhouse Gas Control Technologies, 13-16 August, Cairns, Australia.

Laboratories, A. P. P. (1999). A design study of the application of CO2/O2 combustion technology as retrofit to an existing coal fired boiler. Calgary, Alberta, TransAlta Corp.

Leci, C. L. (1996). "Financial implications on power generation costs resulting from the parasitic effect of $\mathrm{CO} 2$ capture using liquid scrubbing technology from power station flue gases." Energy Conversion and Management 37(6-8): 915-921.

Mariz, C. and e. al. (1999). Recovery of CO2 from flue gases: commercial trends (paper No. 340). the Canadian Society of Chemical Engineers Annual Meeting, Saskatchewan, Canada.

Mimura, T., K. Matsumoto, et al. (2000). Development and application of flue gas carbon dioxide recovery technology. Fifth Greenhouse Gas Control Technologies, Cairns, Australia.

Morgan, M. G. and M. Henrion (1990). Uncertainty: A Guide to Dealing with Uncertainty in Quantitative Risk and Policy Analysis. Cambridge, UK, Cambridge University Press.

Morgan, M. G. and D. W. Keith (1995). "Subjective Judgments by Climate Experts." Environmental Science \& Technology 29(10): 468A-476A. 
Parsons Infrastructure \& Technology Group, I. (2002). Evaluation of fossil fuel power plants with $\mathrm{CO} 2$ recovery. Pittsburgh, PA, USDOE/NETL/EPRI.

Rao, A. B. and E. S. Rubin (2002). "A Technical, Economic, and Environmental Assessment of Amine-based $\mathrm{CO}_{2}$ Capture Technology for Power Plant Greenhouse Gas Control." Environmental Science and Technology 36: 4467-4475.

Reddy, S. and C. A. Roberts (2003). ECONOMINE FGSM Plus: An Enhanced Amine Based $\mathrm{CO}_{2}$ Capture Process. 2nd Annual Conference on Carbon Sequestration, Alexandria, VA, USA.

Simbeck, S. (1998). A portfolio selection approach for power plant CO2 capture, separation and

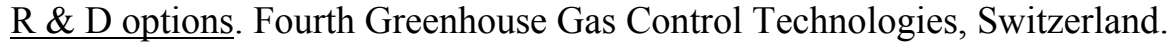

Smelser, S. C., R. M. Stock, et al. (1991). Engineering and economic evaluation of CO2 removal from fossil-fuel-fired power plants, EPRI IE-7365, Volume 1, Project 2999-10, a research project final report prepared by Fluor Daniel Inc., for EPRI and IEA.

Taylor, M. R. and etal (2003). "The influence of government actions on innovative activities in the development of environmental technologies to control sulfur dioxide emissions from stationary sources." submitted to ES\&T.

Taylor, M. R. and etal (2003). "The influence of government actions on innovative activities in the development of environmental technologies to control sulfur dioxide emissions from stationary sources." submitted to ES\&T.

Veawab, A., P. Tontiwachwuthikul, et al. (2002). Performance and cost analysis for CO2 capture from flue gas streams: Absorption and regeneration aspects. Sixth International Conference on Greenhouse Gas Control Technologies, Kyoto, Japan. 


\section{CONCLUDING REMARKS}

The basic objective of this research was to carry out a technical, environmental, and economic assessment of the amine-based $\mathrm{CO}_{2}$ capture technology for greenhouse gas control at power plants. This chapter summarizes the key findings presented in this thesis.

\subsection{Model Development}

Performance and cost model of this technology were developed that served as the analytical tool for this assessment. The performance model was derived using a detailed chemical process simulator together with data obtained from process developers and through personal communication with experts. It was directly linked to the cost model, which used cost data from published literature. Cost estimates for any technology that has not been widely implemented for the application of interest (in this case, large-scale power generation) is inherently subject to uncertainty. Thus, the model has probabilistic capabilities, which allow the user to use uncertainty distributions rather than a single value for various model parameters. In the model applications discussed below, such distributions were based on multiple data sources viz., published literature, technical reports, and experts' technical judgment. Finally, the amine system model was integrated with a larger fossil-fuel based power generation modeling framework (IECM) in order to study multi-pollutant interactions and to assess various $\mathrm{CO}_{2}$ control options on a consistent basis.

\subsection{Model Applications}

Today there is a growing consensus in the global scientific community on the need to control $\mathrm{CO}_{2}$ emissions to mitigate the problem of global climate change problem. Capture and sequestration of $\mathrm{CO}_{2}$ is being proposed as a medium term solution that can allow continued use of conventional fossil-fuel resources over the next century without significantly contributing to global carbon emissions from electric power generation (and, potentially, other industrial sources). Electric utilities, being one of the largest stationary sources of $\mathrm{CO}_{2}$ emissions, are likely to be the early targets in this effort. Thus, the performance and cost model developed in this research were used to study the option of post-combustion $\mathrm{CO}_{2}$ capture from a new PC plant using amine-based system. This can 
serve as a reference against which other $\mathrm{CO}_{2}$ mitigation options could be compared. Also, as we have discussed before, there exists a large existing fleet of coal-fired capacity that may have to be retrofitted under a stringent greenhouse gas control policy. Thus, retrofit applications as well as new plant applications are of interest.

\subsubsection{New Plants}

Using the model, we conducted case studies of a new coal-fired power plant with an amine-based $\mathrm{CO}_{2}$ capture plant. It was concluded that in order to capture $\mathrm{CO}_{2}$ from a pulverized coal plant, a much bigger plant has to be built in order to provide the same net power output as a plant without $\mathrm{CO}_{2}$ capture. This is because approximately $22 \%$ of the gross power generation is consumed by the $\mathrm{CO}_{2}$ capture and compression systems. It was found that a capture plant designed to capture $90 \%$ of the $\mathrm{CO}_{2}$ mass emissions effectively "avoids" about $87 \%$ of the emissions per net $\mathrm{kWh}$ generated because the $\mathrm{CO}_{2}$ capture system itself consumes substantial energy and contributes to $\mathrm{CO}_{2}$ emissions. An amine system also reduces the total emissions of $\mathrm{SO}_{\mathrm{x}}$ and particulates to very low levels. However, the capture plant has higher emission rates of $\mathrm{NO}_{\mathrm{x}}$ and ammonia (per $\mathrm{kWh}$ ). The capture plant uses 33\% more coal to deliver the same amount of electricity, and therefore also has larger amounts of bottom ash, fly ash and FGD waste to be disposed. The capture plant also has to dispose of the spent sorbent (from the amine system) and the $\mathrm{CO}_{2}$ product stream.

The addition of $\mathrm{CO}_{2}$ capture and storage increases the capital requirement as well as the cost of electricity generated. Our case studies showed the capital cost of the new plant to increase by $56 \%$ to $66 \%$ (based on $\$ / \mathrm{kWh}$ ), while the COE increased by about $73 \%$ to $82 \%$ for a plant with $90 \%$ capture. Cost results were found to be sensitive to assumptions about the plant capacity factor and fixed charge factor.

The $\mathrm{CO}_{2}$ avoidance cost was estimated to be about $\$ 50 /$ tonne $\mathrm{CO}_{2}$ avoided. As mentioned earlier, it is higher than the $\mathrm{CO}_{2}$ capture cost $\left(\$ 36 /\right.$ tonne $\mathrm{CO}_{2}$ delivered). This estimate includes about $\$ 8 /$ tonne $\mathrm{CO}_{2}$ captured for transport and storage/ disposal of the concentrated $\mathrm{CO}_{2}$ product stream. From the probabilistic analysis, the $95 \%$ confidence interval was estimated as $\$ 39-71 /$ tonne $\mathrm{CO}_{2}$ avoided. It may be noted that these results 
are within reasonable agreement with some of recent estimates published by other studies (Simbeck and McDonald 2000; Stork Engg Consultancy B.V. 2000; ALSTOM, AEP et al. 2001; Parsons Infrastructure \& Technology Group 2002; Singh, Croiset et al. 2002).

\subsubsection{Retrofit Applications}

Retrofit application of this technology to existing coal-fired plants is another important scenario to be considered. Several cases have been modeled and presented in section 5.2. If the existing plant already has a sulfur-control system, it helps in reducing the cost of $\mathrm{CO}_{2}$ capture using amine-based systems. Optimization studies showed that it is further helpful if the existing sulfur-control system is upgraded to the maximum achievable removal efficiency to minimize $\mathrm{SO}_{2}$ removal in the amine system. It is necessary to add a new, highly efficient sulfur scrubber upstream of amine-based system if one does not already exist at the reference plant. If all the energy required for $\mathrm{CO}_{2}$ capture and compression system is extracted from the base plant ("derating" case), the net output of the plant falls substantially (by almost $40 \%$ ), much more so that at a new plant, because of generally poorer heat integration potential at older plants. Accordingly, the cost of electricity may go up by $150-300 \%$ as compared to that of reference plant, for various cases analyzed. Adding an auxiliary natural-gas-fired boiler (with a secondary steam turbine) may be a lower-cost option for providing auxiliary energy if lower levels of $\mathrm{CO}_{2}$ avoidance $(\sim 60-65 \%)$ are acceptable and if natural gas is available at low price. The flue gas from the auxiliary natural gas boiler is more dilute in $\mathrm{CO}_{2}$ content, so capturing this $\mathrm{CO}_{2}$ is not cost effective. This additional $\mathrm{CO}_{2}$ emission from the auxiliary natural gas boiler reduces overall $\mathrm{CO}_{2}$ avoidance level of the system. The cost of electricity (and that of $\mathrm{CO}_{2}$ avoidance) in this case may be lower than that of the derating case; however, it is very sensitive to the cost and availability of natural gas and assumptions about the retrofit factors. This option might be attractive if the gas price is below $\$ 2.5 / \mathrm{mscf}$.

\subsubsection{Capacity Factor Assumption}

Typically, in a plant-level analysis like this, it is assumed that the $\mathrm{CO}_{2}$ capture plant has the same capacity factor as that assumed for the reference plant (Jeremy and Herzog 2000; Simbeck and McDonald 2000; Stork Engg Consultancy B.V. 2000; ALSTOM, AEP et al. 2001; Parsons Infrastructure \& Technology Group 2002; Singh, E.Croiset et 
al. 2002). The resulting cost difference with $\mathrm{CO}_{2}$ capture then reflects the added cost under identical operating conditions. In practice, however, any number of parameters affecting overall plant costs - such as the capacity factor - may differ in plants with and without a $\mathrm{CO}_{2}$ capture system. Based on dispatch models for two regions of the U.S., recent studies by Johnson and Keith found that the $\mathrm{CO}_{2}$ capture plants would be operated at higher capacity factors (Johnson 2002; Johnson and Keith 2004). However, there are other factors that could discourage the higher utilization of these plants. For example, plants using amine-based $\mathrm{CO}_{2}$ capture system were shown earlier to have $\mathrm{NO}_{\mathrm{x}}$ emission rates that are substantially higher than that for a similar reference plant without $\mathrm{CO}_{2}$ capture (see Table 5.2). This could reduce their use under regional $\mathrm{NO}_{\mathrm{x}}$ caps. In general, the capacity factor of a plant will be determined by a number of factors related to the overall generation mix and the constraints under which plants in a utility system operate. While such factors are clearly important, they are beyond the scope of this thesis. However, the models developed here can be readily used to explore the cost implications of alternative assumptions regarding the capacity factor and other base plant parameters.

\subsection{Expert Elicitations}

The technical judgment of various experts in this field was elicited to explore the potential for reducing the cost of current amine systems. The experts belonged to academia, industries, research laboratories and private consultancies.

\subsubsection{Scope for improvement}

The experts' responses indicate that future amine-based systems can perform significantly better as compared to their current counterparts, given a substantial program of $R \& D$. The average relative improvement in various parameters as predicted by the experts is reported below in Table 7.1. The numbers in the parentheses represent the range of estimates provided by various experts.

Various possible strategies are cited to achieve these improvements. Most experts emphasize development of better sorbents (advanced amines or special mixtures of amines). Other items on the list of suggestions included equipment change, special additives, corrosion inhibitors, improved metallurgy for absorbers, better design and 
operating conditions, and reducing the oxygen levels in the flue gas to zero. All these improvements in performance parameters will help in reducing the future cost of $\mathrm{CO}_{2}$ avoidance.

Table 7.1. Relative improvement in amine-system parameters

\begin{tabular}{|c|c|c|}
\hline \multirow{2}{*}{ Model Parameter } & \multicolumn{2}{|c|}{ Improvement estimates based on } \\
\hline & $\begin{array}{l}\text { "Best Guess" } \\
\text { judgments }\end{array}$ & $\begin{array}{c}\text { "Most Optimistic" } \\
\text { judgments }\end{array}$ \\
\hline $\begin{array}{l}\text { Sorbent Regeneration Heat Requirement } \\
\qquad(\mathrm{kJ} / \mathrm{kg} \mathrm{CO} 2)\end{array}$ & $\begin{array}{c}23 \% \\
(5 \%-40 \%)\end{array}$ & $\begin{array}{c}\mathbf{4 3 \%} \\
(15 \%-73 \%)\end{array}$ \\
\hline $\begin{array}{l}\text { Sorbent Concentration } \\
\text { (wt \%) }\end{array}$ & $\begin{array}{c}23 \% \\
(0 \%-67 \%)\end{array}$ & $\begin{array}{c}\mathbf{8 0 \%} \\
(33 \%-160 \%)\end{array}$ \\
\hline $\begin{array}{c}\text { Sorbent Loss } \\
\left(\mathrm{kg} \mathrm{MEA/tonne} \mathrm{CO}_{2}\right)\end{array}$ & $\begin{array}{c}\mathbf{4 9 \%} \\
((33 \%)-97 \%)\end{array}$ & $\begin{array}{c}\mathbf{7 6 \%} \\
(29 \%-100 \%)\end{array}$ \\
\hline $\begin{array}{c}\text { Sorbent Cost } \\
(\$ / \text { tonne MEA) }\end{array}$ & $\begin{array}{c}(\mathbf{4 8 \%} \mathbf{)} \\
((140 \%)-4 \%)\end{array}$ & $\begin{array}{c}\mathbf{3 \%} \\
((100 \%)-71 \%)\end{array}$ \\
\hline
\end{tabular}

However, the cost of improved sorbents is expected to be substantially higher than current prices, or at best remain at the current level under the most optimistic scenario. Higher sorbent costs were also attributed to natural gas (the ultimate feedstock) availability and prices, demand, and generic versus specialized application.

\subsubsection{Possible future cost reductions}

The experts' responses about the four parameter values for future amine systems (Table 7.1) were used as input to the IECM-CS model along with the other parameter defaults. Table 6.2 gives the results obtained using the average improvement numbers reported in Table 7.1 (in bold), with the range obtained using individual responses given in the parentheses.

It may be noted that all the four parameters under consideration, viz. sorbent regeneration heat requirement, sorbent concentration, sorbent loss and sorbent cost, directly affect the total energy penalty and makeup sorbent requirement cost (the most important variable cost items of this technology). However these parameters only indirectly affect the 
capital cost of this system and hence have less impact on the same. This fact is reflected in the cost reductions that are expected to be lower in case of capital requirement as compared to those in cost of electricity.

Table 7.2. Expected reduction in future cost of $\mathrm{CO}_{2}$ avoidance

\begin{tabular}{|c|c|c||}
\hline \multirow{2}{*}{ Cost Parameter } & \multicolumn{2}{|c|}{ Expected cost reduction based on } \\
\cline { 2 - 3 } & $\begin{array}{c}\text { “Best Guess” } \\
\text { judgments }\end{array}$ & $\begin{array}{c}\text { “Most Optimistic” } \\
\text { judgments }\end{array}$ \\
\hline \hline Total Capital Cost $(\mathrm{M} \$)$ & $\begin{array}{c}\mathbf{6 \%} \\
((2 \%)-9 \%)\end{array}$ & $\begin{array}{c}\mathbf{1 6 \%} \\
(7 \%-19 \%)\end{array}$ \\
\hline Cost of Electricity $(\$ / \mathrm{MWh})$ & $\mathbf{1 8 \%}$ & $\mathbf{3 5 \%}$ \\
$((8 \%)-29 \%)$ & $\mathbf{3 6 \%} \%)$ \\
\hline Cost of $\mathrm{CO}_{2}$ avoidance $\left(\$ /\right.$ tonne $\mathrm{CO}_{2}$ av. $)$ & $(21 \%-38 \%)$ \\
\hline
\end{tabular}

\subsubsection{Probabilistic distribution of current and future costs of $\mathrm{CO}_{2}$ avoidance}

In case of probabilistic runs, most of the experts seem to be optimistic about the potential for substantial cost reductions in future.

Figure 7.1 shows the cumulative probability distributions for avoidance cost based on the four parameter distributions for current and future systems. The average of the experts' responses have been used as input to the model to generate these curves. The distribution for future costs shows substantially lower values than the current cost distribution. The cost difference (cost reduction) maybe accredited to the R\&D inputs being invested to improve this technology. In other words, this cost difference or cost saving maybe looked upon as the benefit of R\&D investments in this technology. From Figure 7.1, the difference in the median values of mitigation cost in current versus future scenario is about $\$ 8 /$ tonne $\mathrm{CO}_{2}$ avoided, or about $20 \%$ of the current value; and the difference between the average value of each distribution is about $\$ 9 /$ tonne $\mathrm{CO}_{2}$ avoided, or about $21 \%$ of the current value. When the distributions are derived for each individual expert, the difference in the median as well as the average values of mitigation cost is found to 
have a range of $\$ 2-9 /$ tonne $\mathrm{CO}_{2}$ avoided, or about $3-24 \%$ of the current value. Based on these measures of the benefit of $\mathrm{R} \& \mathrm{D}$, the wider the application of this technology, the larger the number of tonnes of $\mathrm{CO}_{2}$ avoided and the bigger the overall benefit.

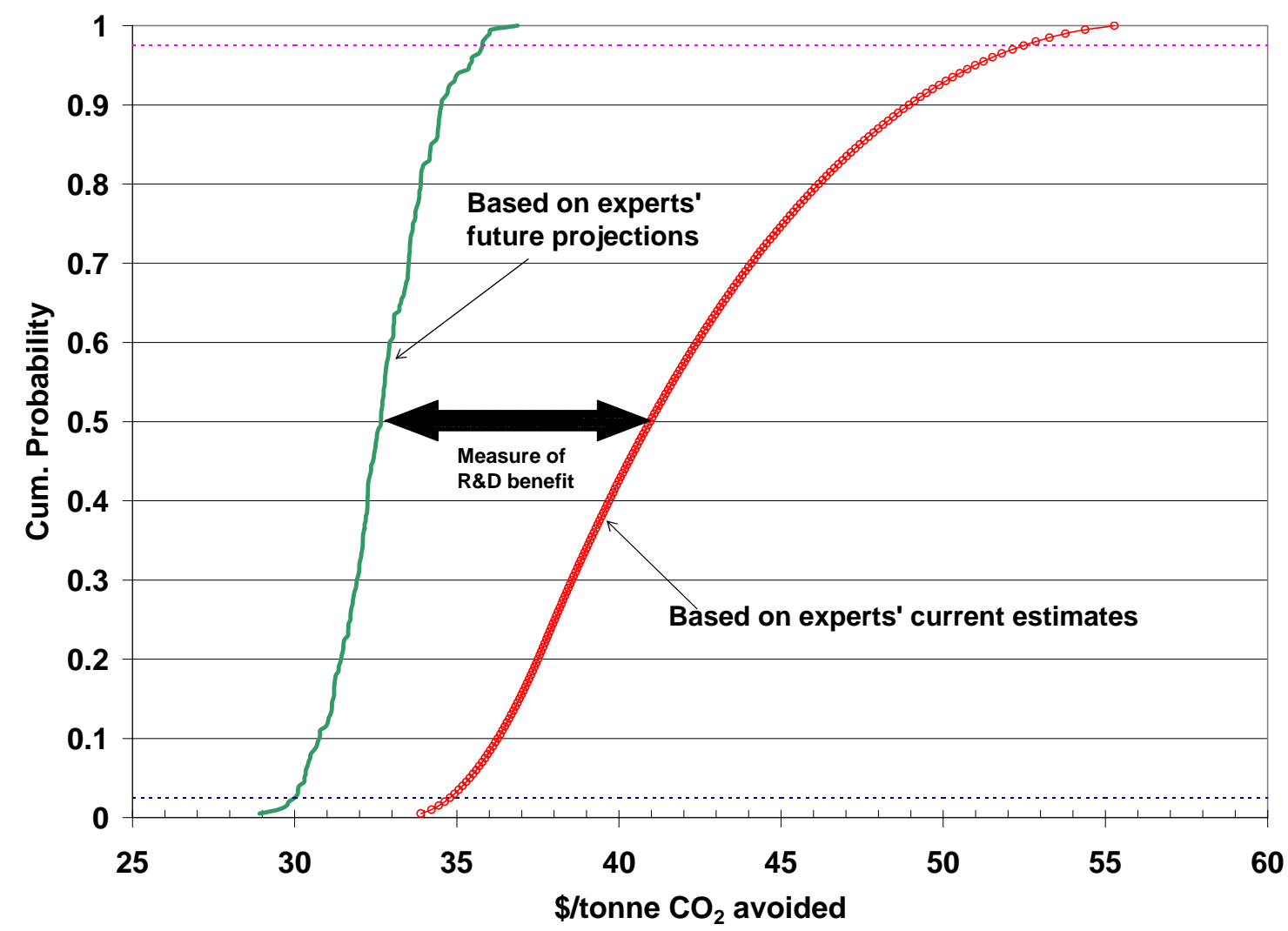

Figure 7.1. Probabilistic estimate of future cost reduction

\subsection{4. $\quad R \& D$ priorities}

Although there were diverse views on the relative importance of various research objectives, all the experts seem to agree (almost unanimously) on the following items as the top priority issues:

5) To develop sorbents with lower regeneration energy requirement [B4]

6) To develop less expensive technologies for $\mathrm{CO}_{2}$ storage/ disposal [C3]

7) To improve heat integration within the power plants (to reduce the energy penalty due to steam extraction for sorbent regeneration) [A4] 
8) To develop more efficient power plants (lower heat rate) [A1]

So, the topmost priority R\&D objective is believed to be to develop sorbents having lower regeneration energy requirement. Together with more effective heat integration schemes, this may be the key to the success of this technology for $\mathrm{CO}_{2}$ capture applications in future. Various researchers around the world have been working in this direction, and the initial results seem to be promising

\subsection{Directions for Future Work}

There are several directions in which this work may be furthered. First, the post-capture $\mathrm{CO}_{2}$ processing modules (for transport, storage or usage of $\mathrm{CO}_{2}$ product) could be developed in more detail. Then, there are also a few areas in the current performance and cost models for amine system that could be improved with the availability of additional data. For example, it is likely that there are correlations between some of the performance and cost parameters (e.g. sorbent concentration, lean sorbent loading and maintenance costs) which are not established in the current model. Also, default values for various parameters for $\mathrm{CO}_{2}$ absorption systems using sorbents other than MEA could be included in the present model.

It would also be useful to elicit appropriate group of experts on other parameters of interest, such as equivalent energy penalty factor $\left(F_{H E}\right)$, direct and indirect capital costs, etc. A more elaborate (and time consuming) protocol for eliciting expert judgments, including greater reliance on face-to-face interviews, also may help in better understanding of their views.

Another direction of future work might be applying the models developed here to address climate policy issues more extensively. For example, a power plant database could be used with the model to assess the feasibility of retrofitting amine-based systems to existing coal plants, the emission reductions that realistically could be achieved through such an effort, and the overall cost of such a program. Another application might be in the form of a case study of a prospective power plant sited in a developing country with site-specific cost data and regulatory framework. This work might also be extended in the direction of modeling other technological options for $\mathrm{CO}_{2}$ capture at power plants, 
including advanced combustion systems using pure oxygen. IECM-CS can provide a consistent framework for comprehensive comparisons of various technological options for carbon sequestration.

\section{References (Chapter 7)}

ALSTOM, AEP, et al. (2001). Engineering feasibility and economics of CO2 capture on an existing coal-fired power plant, Final report prepared by ALSTOM Power Inc., ABB Lummus Global Inc., ALSTOM Power Environmental Systems and American Electric Power (AEP). report no. (PPL-01-CT-09) submitted to Ohio Department of Development, Columbus, OH and US Department of Energy/ NETL, Pittsburgh, PA: 163.

Jeremy, D. and H. J. Herzog (2000). The cost of carbon capture. presented at the Fifth International Conference on Greenhouse Gas Control Technologies, 13-16 August, Cairns, Australia.

Johnson, T. L. (2002). Electricity without carbon dioxide: assessing the role of carbon capture and sequestration in US electric markets. Department of Engineering and Public Policy. Pittsburgh, PA 15213, Carnegie Mellon University: 247.

Johnson, T. L. and D. W. Keith (2004). "Fossil electricity and CO2 sequestration: how natural gas prices, initial conditions and retrofits determine the cost of controlling $\mathrm{CO} 2$ emissions." Energy Policy 32: 367-382.

Parsons Infrastructure \& Technology Group, I. (2002). Evaluation of fossil fuel power plants with $\mathrm{CO} 2$ recovery. Pittsburgh, PA, USDOE/NETL/EPRI.

Simbeck, D. and M. McDonald (2000). Existing coal power plant retrofit $\mathrm{CO}_{2}$ control options analysis. presented at the Fifth International Conference on Greenhouse Gas Control Technologies, 13-16 Augus, Cairns, Australia.

Singh, D., E. Croiset, et al. (2002). Techno-economic study of CO2 capture from an existing coalfired power plant: MEA scrubbing vs. $\mathrm{O} 2 / \mathrm{CO} 2$ recycle combustion. Waterloo, Ontario, Canada: 27.

Singh, D., E.Croiset, et al. (2002). Economics of CO2 capture from a coal-fired power plant - a sensitivity analysis. Sixth Greenhouse Gas Control Technologies, Kyoto, Japan.

Stork Engg Consultancy B.V., A. (2000). Leading options for the capture of CO2 emissions at power stations. UK, IEA GHGRDP. 


\section{APPENDIX A}

\section{POTENTIAL ENVIRONMENTAL IMPACTS OF AMINE SYSTEMS}

Amine system is an "end-of-pipe" treatment. It captures $\mathrm{CO}_{2}$ generated during combustion of a fossil fuel in a power plant. Although an end-of-pipe solution is less desirable than pollution prevention approaches, it is a common practice to reduce environmental impact of industrial activities (Rubin 2001). Often it is observed that the environmental control technologies, especially those based on chemical processes, transfer the pollutants (or their derivatives) from one phase (or part of the ecosystem) to another phase (or part of the ecosystem). Many times, this could result in "pollution transfer" rather than "pollution control." For example, a flue gas desulfurization (FGD) system that controls $\mathrm{SO}_{2}$ emission from a power plant flue gas produces "FGD waste," a solid waste product that needs to be managed later. Many of the wastewater treatment plants separate out contaminants from the water in the form of a solid residue, which again has to be taken care of. In some cases, as in the case of FGD waste, it is possible to make use of this solid product in the manufacture of construction materials. This is the most benign strategy for the environmental protection, and is a good example of industrial ecology. However, this may not be always feasible and some of the solid wastes may have to be disposed of. Depending upon the chemical nature of such solid waste, it is either stored in a landfill or incinerated. Precautions must be taken while disposing the solid waste to avoid creating a new problem in the form of soil pollution, groundwater contamination or air pollution.

In case of MEA-based $\mathrm{CO}_{2}$ capture systems, environmental problems may arise from the spent sorbent slurry discharged from the MEA reclaimer (also called reclaimer bottoms) and the emissions of MEA and ammonia $\left(\mathrm{NH}_{3}\right)$ carried by the treated flue gas. Here is a brief description of these issues. It may be noted that the large concentrated $\mathrm{CO}_{2}$ stream generated as a "byproduct" of this process may have its own set of concerns, and is beyond the scope of this study. 


\section{A. Spent Sorbent or Reclaimer Bottoms}

As mentioned before, MEA degrades under the real operating conditions in a plant. Typically, the degradation products are separated in a reclaimer and disposed of as hazardous waste (Barchas and Davis 1992). The waste is often picked up by a contactor and is incinerated at a permitted facility. This method of waste disposal is expensive and the $\mathrm{CO}_{2}$ capture plant may have to spend substantial amount depending upon the waste quantity.

Recent study undertaken at the National Energy Technology Laboratory (NETL), Pittsburgh, has identified various chemical species that are found in the reclaimer waste samples from an operational $\mathrm{CO}_{2}$ capture plant (Strazisar, Anderson et al. 2003). The major organic compounds which were found in these samples include monoethanolamine $\left(\mathrm{C}_{2} \mathrm{H}_{7} \mathrm{NO}\right.$, MEA), 3-hydroxyethylamino-N-hydroxy-ethyl propanamide $\left(\mathrm{C}_{7} \mathrm{H}_{16} \mathrm{~N}_{2} \mathrm{O}_{3}\right)$, 4-hydroxyethyl-2-piperizinone $\quad\left(\mathrm{C}_{6} \mathrm{H}_{12} \mathrm{~N}_{2} \mathrm{O}_{2}\right), \quad$ 2-hydroxyethylamino-N-hydroxyethyl acetamide $\left(\mathrm{C}_{6} \mathrm{H}_{14} \mathrm{~N}_{2} \mathrm{O}_{3}\right)$, Ammonia $\left(\mathrm{NH}_{3}\right)$, and $\mathrm{N}$-acetylethanolamine $\left(\mathrm{C}_{4} \mathrm{H}_{9} \mathrm{NO}_{2}\right)$ with traces of several other compounds.

The analysis of inorganic species revealed the presence of seven metal cations at a concentration of $0.2 \mathrm{ppm}$ or more. Since sodium carbonate is added to the reclaimer to regenerate MEA, sodium ions dominate all other metal ions (>800 ppm). Other metals (potassium, selenium, arsenic, calcium, iron, aluminum, and zinc) are believed to originate mostly from the coal. Additionally, mercury was found to be present in the reclaimer waste at a concentration of $1 \mathrm{ppb}$. Anion concentrations (chloride, nitrate, fluoride, sulfate, phosphate, bromide) are found to be relatively higher (80-49000 ppm). The amounts of halide anions match the typical halogen concentrations found in coal. This might be the result of "heat-stable-salts" formed by reaction between MEA and mineral acids $(\mathrm{HCl}, \mathrm{HF}, \mathrm{HBr})$ in the flue gas.

This analysis helps to understand why the spent sorbent (reclaimer bottoms) is treated as hazardous waste. From economic as well as environmental perspective, it is preferable for the $\mathrm{CO}_{2}$ capture plant to cleanse the flue gas upstream, before entering the amine 
system, so as to minimize sorbent degradation losses, corrosion problems, makeup sorbent requirement and cost of disposing the spent sorbent.

\section{B. Environmental (Gaseous) Emissions}

a) MEA entrainment with treated flue gas: As mentioned before, MEA is a primary amine with high $\mathrm{pH}$. It is completely soluble in water and is readily biodegradable (Dow 2000). It is a reactive compound and is classified as a corrosive material. So, proper care needs to be taken while handling this compound. Toxic gases and vapors (such as oxides of nitrogen and carbon monoxide) may be released in a fire involving MEA. It can cause irritation and be harmful to gastrointestinal tract, nausea, vomiting and diarrhea when swallowed. It is an irritant to eyes and continued contamination of the eyes could cause permanent injury. MEA is also known to cause mild irritation to skin and repeated or prolonged contact may cause dermatitis. Inhalation of mist or aerosols can produce severe respiratory irritation and cause sore throat, coughing, headache, nausea and vomiting. In terms of chronic effects, lungs may be affected with repeated or prolonged exposure. MEA may have effects on the central nervous system, gastrointestinal tract and kidneys (Deltrex 2000). Several animal studies were conducted by exposing rats and rabbits to MEA vapors of 12-102 ppm for several weeks. Skin was found to be the significant target tissue at relatively high concentration of MEA vapor; other sensitive target tissues include the kidneys and livers (Knaak, Leung et al. 1997). Based on various laboratory tests, it is found to be lacking in genotoxicity.

MEA is not listed as a hazardous air pollutant under the Clean Air Act of 1990 (CAA 1990). The National Institute for Occupational Safety and Health (NIOSH) has established a recommended exposure limit (REL) for MEA of $3 \mathrm{ppm}(8 \mathrm{mg} / \mathrm{m} 3)$ as a time-weighted average for a normal 10-hour workday and a 40-hour workweek and a short-term exposure limit (STEL) of $6 \mathrm{ppm}(15 \mathrm{mg} / \mathrm{m} 3)$ for periods not to exceed 15 minutes (OSHA 1996). These limits are based on the risk of skin, eye and respiratory irritation and narcotic effects. However, no signs or symptoms of chronic exposure to ethanolamine have been reported in humans. MEA has been regarded as hazardous according to the criteria of Worksafe Australia (Deltrex 2000). Based on the $\mathrm{LD}_{50}$ value of $505 \mathrm{mg} / \mathrm{kg}$ body weight (in rabbits exposed by dermal route), MEA is classified as a 
"dangerous" chemical that falls within the European Risk Category 3 (Chemrest 2003). The European Toxicity Risk Code for this chemical is "XI = Irritant" which matches with the hazard category "Harmful/Irritant" assigned by Worksafe Australia (Deltrex 2000). It may be noted that MEA is widely used in various applications such as a dispersant for agricultural products; as a softening agent for hides; as an accelerator in the production of antibiotics, polishes and waving solutions for hair; as a rubber accelerator; an intermediate in the production of emulsifiers, soaps and detergents; and in some hair-care products. In case of MEA-based $\mathrm{CO}_{2}$ capture systems, trace quantities (1-4 ppm) of MEA may be found in the exhaust flue gas at the stack. Under well-mixed atmospheric conditions, it is less likely to pose a risk of direct exposure to human beings. Also, only a small amount of MEA is expected to partition to the atmosphere. It may be removed by reactions with photochemically generated hydroxyl radicals or by precipitation. Thus, relatively low levels of MEA are expected to be present in the atmosphere; and owing to its short half-life, it is unlikely to adversely impact air quality (Davis and Carpenter 1997). However, MEA's complete solubility in water may facilitate its entry into surface and ground water.

According to the Dow Chemical Company (one of the leading manufacturers of MEA), MEA is "practically non-toxic" to aquatic organisms (Dow 1998). It is claimed that as MEA exhibits low (or no) aquatic toxicity, is biodegradable in soil, surface waters and wastewater treatment plants and does not show any tendency to bioaccumulate, the routine manufacture, use and disposal of this compound is not expected to adversely impact the environment (Davis and Carpenter 1997). However, U.S. Environmental Protection Agency has classified MEA as having "moderate" ecological toxicity, based on the effects on aquatic organisms, such as fish and algae (USEPA 2002). This aspect might need further careful investigation before MEA-based $\mathrm{CO}_{2}$ capture systems are installed in large numbers.

It may be noted that the market cost of MEA sorbent lost via leakage or entrainment is likely to be trivial in the overall cost of running the plant. However the externalities and potential risks posed by the same might be significantly higher. In view of the above information, considering the potential health and environmental risks posed by MEA, it is 
important to take special care to avoid MEA losses through entrainment in the absorber and leakage in the circulation piping.

b) Nitrosamines: Another potential problem that may arise from amine-based systems is in the form of "nitrosamines", which are known carcinogens that are formed by a reaction between an amine and a nitrogen oxide. These compounds were found in the samples of the lean sorbent $(2.91 \mu \mathrm{mole} / \mathrm{ml})$. However, detectable amount of nitrosamines was not found in the reclaimer waste, which may be explained in terms of their low boiling point (Strazisar, Anderson et al. 2003). The trace quantities of MEA entrained by the flue gas may combine with nitrogen oxides in the atmosphere (or present in the flue gas itself) and may constitute to health hazards in the surrounding areas.

c) Ammonia: Ammonia $\left(\mathrm{NH}_{3}\right)$ is generated as a dissociation product of MEA. Loss of MEA (and emission of $\mathrm{NH}_{3}$ ) via dissociation route is unavoidable under the normal operating conditions in the amine system. As seen in the case study of a new plant (Section 5.1 \& Appendix D), the emission rate of $\mathrm{NH}_{3}$ from a $\mathrm{CO}_{2}$ capture plant is substantially higher than that from a power plant without $\mathrm{CO}_{2}$ capture system. Emission of ammonia is not regulated under the current air pollution control programs, however, it is a known source of air (and water) pollution (Ashman, Sutton et al. 1998; Howarth, Sharpley et al. 2002). Especially in the polluted urban areas, changes in ammonia emissions have the most significant impact on the total particulate matter levels (Ansari and Pandis 1998). The treated flue gas coming from a capture plant is almost free of $\mathrm{SO}_{2}$, but it contains a significant amount of $\mathrm{NO}_{\mathrm{x}}$. Gaseous emissions of ammonia, $\mathrm{SO}_{2}$ and $\mathrm{NO}_{\mathrm{x}}$ form secondary particulates (ammonium nitrate $\left(\mathrm{NH}_{4} \mathrm{NO}_{3}\right)$ and ammonium sulfates $\left(\mathrm{NH}_{4} \mathrm{HSO}_{4}\right.$ and $\left.\left.\left(\mathrm{NH}_{4}\right)_{2} \mathrm{SO}_{4}\right)\right)$. These compounds are significant part of fine particulate matter $\left(\mathrm{PM}_{2.5}\right)$ in air and can play an important role in visibility impairment and regional haze (Battye, Aneja et al. 2003). Hence, the multi-fold increase in $\mathrm{NH}_{3}$ emissions from a power plant owing to the amine-based $\mathrm{CO}_{2}$ capture system has to be considered carefully in the light of local and regional air pollution issues. The contribution from all the industrial sources combined makes only a small fraction $(0.2 \%$ $9.6 \%$ ) of total ammonia emission inventory that is dominated by emissions from livestock waste and fertilizer application (Battye, Aneja et al. 2003). However, these 
point sources of ammonia, especially in the vicinity of urban centers, can have substantial impact on the particulate matter levels and the air quality in general. So, the severity of

the impacts of the higher ammonia emissions from the capture plant will significantly depend on the land-use pattern around the power plant.

\section{References}

Ansari, A. S. and S. N. Pandis (1998). "Response of inorganic PM to precursor concentrations." Environmental Science and Technology 32(18): 2706-2714.

Ashman, W. A. H., M. A. Sutton, et al. (1998). "Ammonia: emission, atmospheric transport and deposition." New Phytol. 139: 27-48.

Barchas, R. and R. Davis (1992). "The Kerr-McGee/ ABB Lummus Crest technology for the recovery of $\mathrm{CO} 2$ from stack gases." Energy Conversion and Management 33(5-8): 333340.

Battye, W., V. P. Aneja, et al. (2003). "Evaluation and improvement of ammonia emissions inventories." Atmospheric Environment 37: 3873-3883.

CAA (1990). Clean Air Act Amendments. Public Law 101-549, Section 112, Nov. 15, 1990.

Chemrest (2003). Chemical toxicity and risk information: Ethanolamine (http://www.chemrest.com/Toxicity\%20and\%20Risk\%2020Codes/Ethanolamine.htm).

Davis, J. W. and C. L. Carpenter (1997). "Environmental assessment of the alkanolamines." Reviews of Environmental Contamination and Toxicology 149: 87-137.

Deltrex (2000). Monoethanolamine 99\% (http://www.deltex.com.au/products/s133.htm), Deltrex Chemicals, a Division of Campbell Brothers Ltd., Australia).

Dow (1998). Product information: Ethanolamines - Toxicology Overview [Form No. 111-013381198AMS, CH 132-007-E-1198AMS] (wysiwyg://221/http://www.dow.com/alkanolamines/eth/mea.html), The Dow Chemical Company.

Dow (2000). "Specialty Alkanolamines": Product information available at the website of The Dow Chemical Company (www.dow.com/alkanolamines/).

Howarth, R. W., A. Sharpley, et al. (2002). "Sources of nutrient pollution to coastal waters in the United States: Implications for achieving coastal water quality goals." Estuaries 25(4b): 656-676.

Knaak, J. B., H.-W. Leung, et al. (1997). "Toxicology of Mono-, Di-, and Triethanolamine." Reviews of Environmental Contamination and Toxicology 149: 1-86.

OSHA (1996). Occupational safety and health guideline for ethanolamine (http://www.oshaslc.gov/SLTC/healthguidelines/ethanolamine/recognition.html), OSHA (Occupational Safety and Health Administration), U.S. Department of Labor.

Rubin, E. S. (2001). Introduction to engineering and the environment. New York, NY, The McGraw-Hill Companies, Inc.

Strazisar, B. R., R. R. Anderson, et al. (2003). "Degradation pathways for monoethanolamine in a $\mathrm{CO}_{2}$ capture facility." Energy \& Fuels 17(4): 1034-1039.

USEPA (2002). Aqueous and semi-aqueous solvent chemicals: Environmentally preferable choices (http://www.epa.gov/opptintr/solvents/finlchr4.htm), USEPA (U.S.

Environmental Protection Agency). 


\section{APPENDIX B}

\section{CHARACTERIZATION OF UNCERTAINTY AND VARIABILITY}

The term "uncertainty" is a vast umbrella that covers a variety of concepts. Uncertainty often arises from various sources including lack of complete information, conflicting sources of information, variability in a process or an object, linguistic imprecision, and approximations incorporated in a model to simplify the real life situations (Morgan and Henrion 1990). Uncertainty in an empirical quantity is usually expressed using a probability distribution.

Any techno-economic analysis, and especially that of new energy and environmental control technologies that are still in the research phase, involves uncertainties regarding the performance and costs. These uncertainties come from incomplete data and numerous assumptions and approximations built into simulations. Some parameters, especially the cost parameters are influenced by a larger set of factors outside the scope of the particular study, and fluctuations in these quantities may be seen as "inherent randomness" when viewed within this limited focus area. In addition, there may be significant variability in plant or process design assumptions across different studies or organizations.

Uncertainty and variability are often ignored or treated in a limited way using sensitivity analysis. However sensitivity analysis suffers from shortcomings resulting from the difficulty in evaluating the effect of simultaneous variations in several parameters and the lack of insight into the likelihood of obtaining any particular result.

A more robust approach is to represent uncertainties and/or variability in model parameters using probability distributions. Using probabilistic simulations techniques, simultaneous uncertainties in any number of input parameters can be propagated through a model to determine their combined effect on model outputs. The result of a probabilistic simulation includes both the possible range of values for model output parameters and information about the likelihood of obtaining various results. The development of ranges and probability distributions for model input parameters can be 
based on information available in published studies, statistical data analysis and/or the judgments of process engineers with relevant expertise.

One of the distinguishing features of this modeling effort is a probabilistic capability that allows model inputs to be represented by probability distributions rather than single deterministic values. Depending upon the parameter, these distributions reflect the ranges of values reported in the literature, modeling approximations, the evolving nature of the technology, practical considerations in running such plants and variety of plant or process design assumptions. Some of the distributions are also based on an expert elicitation exercise (explained in Chapter 6) in which experts were asked about the nominal values and possible ranges for several important parameters characterizing the performance of current commercial MEA-based systems capturing $90 \% \mathrm{CO}_{2}$ from the flue gas of a typical coal-fired power plant.

While designing an amine-based $\mathrm{CO}_{2}$ capture system for a given flue gas (from a power plant application), there are certain parameters that could be specified independent of others. For example, the sorbent concentration, $\mathrm{CO}_{2}$ capture efficiency target, lean sorbent loading, temperature and pressure of sorbent regeneration steam, $\mathrm{CO}_{2}$ product pressure, etc. Distributions for such parameters reported in different studies essentially represent variability. Experts' technical judgments were useful in defining these distributions.

Probability distributions for parameters such as the efficiencies of fans, pumps or compressors represent the possibility of encountering fluctuations in the performance due to inherent characteristics of these devices in an operating plant. Uncertainties arising from real plant operating conditions and approximations in process simulations are also reflected in the distributions for parameters such as MEA makeup rate, removal efficiencies for other acid gases $\left(\mathrm{SO}_{2}, \mathrm{NO}_{2}\right.$ and $\left.\mathrm{HCl}\right)$, and equivalent energy penalty from extraction of sorbent regeneration steam from the steam cycle of the base plant.

In case of cost parameters such as capital costs, O\&M costs, cost of reagents (including MEA) and cost of disposal, the distributions represent both variability and uncertainty 
arising from disagreement in data sources and inherent characteristics of market mechanism.

Then there are certain process parameters that are interdependent. For example, the $\mathrm{CO}_{2}$ capture efficiency $(\eta)$ is a function of various operating variables of the system for a given column design.

$$
\eta_{\mathrm{CO} 2}=\mathrm{f}\left(\mathrm{y}_{\mathrm{CO} 2}, \mathrm{C}, \mathrm{L} / \mathrm{G}, \phi_{\text {lean }}, \mathrm{T}_{\mathrm{fg}}\right)
$$

where, $\quad \mathrm{y}_{\mathrm{CO} 2}=\mathrm{CO}_{2}$ concentration in the flue gas,

$\mathrm{C}=$ sorbent concentration,

$\mathrm{L} / \mathrm{G}=$ sorbent circulation,

$\phi_{\text {lean }}=$ lean sorbent loading, and

$\mathrm{T}_{\mathrm{fg}}=$ temperatures of the flue gas

In the process model that we have developed here, the $\mathrm{CO}_{2}$ capture efficiency $(\eta)$ is a user-defined parameter (treated as an independent parameter) and sorbent circulation $(\mathrm{L} / \mathrm{G})$ is estimated as:

$$
(\mathrm{L} / \mathrm{G})=\mathrm{f}\left(\mathrm{y}_{\mathrm{CO} 2}, \mathrm{C}, \eta_{\mathrm{CO} 2}, \phi_{\text {lean }}, \mathrm{T}_{\mathrm{fg}}\right)
$$

Similarly, sorbent regeneration heat requirement $(\mathrm{Q} / \mathrm{L})$ is estimated as:

$$
(\mathrm{Q} / \mathrm{L})=\mathrm{f}\left(\mathrm{L} / \mathrm{G}, \phi_{\text {lean }}, \mathrm{C}\right)
$$

Probability distributions for these calculated parameters are not explicitly defined. Instead, the probability distributions on the independent parameters are propagated to these parameters throughout the model.

Table 3.1 (in Chapter 3) lists the probability distributions developed for performance model parameters. Similarly, Table 4.1 (in Chapter 4) lists the probability distributions developed for some of the cost model parameters. These distributions reflect both uncertainty and variability in system designs. These tables have been reproduced here for convenience. Appendix $C$ discusses the basis for these distributions. 
Table 3.3. Amine System Performance Model Parameters and Uncertainties

\begin{tabular}{|c|c|c|c|c|}
\hline $\begin{array}{c}\text { Performance } \\
\text { Parameter }\end{array}$ & Units & $\begin{array}{c}\text { Data } \\
\text { (Range) }\end{array}$ & $\begin{array}{c}\text { Nominal } \\
\text { Value }\end{array}$ & $\begin{array}{c}\text { Unc. Representation } \\
\text { (Distribution Function) }\end{array}$ \\
\hline $\mathrm{CO}_{2}$ removal efficiency & $\%$ & Mostly 90 & 90 & - \\
\hline $\mathrm{SO}_{2}$ removal efficiency & $\%$ & Almost 100 & 99.5 & Uniform $(99,100)$ \\
\hline $\mathrm{NO}_{2}$ removal efficiency & $\%$ & $20-30$ & 25 & Uniform $(20,30)$ \\
\hline $\mathrm{HCl}$ removal efficiency & $\%$ & $90-95$ & 95 & Uniform $(90,95)$ \\
\hline Particulate removal eff. & $\%$ & 50 & 50 & Uniform $(40,60)$ \\
\hline MEA concentration & $\mathrm{wt} \%$ & $15-50$ & 30 & Triangular $(20,30,40)$ \\
\hline $\begin{array}{l}\text { Lean sorbent } \mathrm{CO}_{2} \\
\text { loading }\end{array}$ & $\mathrm{mol} \mathrm{CO}_{2} / \mathrm{mol} \mathrm{MEA}$ & $0.15-0.30$ & 0.2 & Triangular( $(0.1,0.2,0.25)$ \\
\hline Nominal MEA make-up & kg MEA/tonne $\mathrm{CO}_{2}$ & $0.5-3.1$ & 1.5 & Triangular $(0.5,1.5,3.1)$ \\
\hline MEA loss $\left(\mathrm{SO}_{2}\right)$ & $\mathrm{mol} \mathrm{MEA} / \mathrm{mol} \mathrm{SO}_{2}$ & 2 & 2 & - \\
\hline MEA loss $\left(\mathrm{NO}_{2}\right)$ & $\mathrm{mol} \mathrm{MEA} / \mathrm{mol} \mathrm{NO}_{2}$ & 2 & 2 & - \\
\hline MEA loss $(\mathrm{HCl})$ & mol MEA/mol HCl & 1 & 1 & - \\
\hline $\mathrm{NH}_{3}$ generation & $\begin{array}{c}\text { mol } \mathrm{NH}_{3} / \text { mol MEA } \\
\text { oxidized }\end{array}$ & 1 & 1 & - \\
\hline $\begin{array}{l}\text { Caustic consumption in } \\
\text { MEA reclaimer }\end{array}$ & $\mathrm{kg} \mathrm{NaOH} /$ tonneCO ${ }_{2}$ & 0.13 & 0.13 & - \\
\hline Activated carbon use & $\mathrm{kg} \mathrm{C} /$ tonne $\mathrm{CO}_{2}$ & 0.075 & 0.075 & - \\
\hline Gas-phase pressure drop & $\mathrm{kPa}$ & $4-37$ & 14 & Triangular(4,14,37) \\
\hline Fan efficiency & $\%$ & $70-80$ & 75 & Uniform $(70,80)$ \\
\hline Sorbent pumping head & $\mathrm{kPa}$ & 200 & 200 & Triangular(150,200,250) \\
\hline Pump efficiency & $\%$ & $70-80$ & 75 & Uniform $(70,80)$ \\
\hline Equiv. elec. requirement & $\%$ regeneration heat & $9-19$ & $14^{\mathrm{a}}$ & Uniform $(9,19)$ \\
\hline $\mathrm{CO}_{2}$ product pressure & $\mathrm{MPa}$ & $7.58-15.16$ & 13.79 & Triangular( $(7.58,13.79,15.16)$ \\
\hline Compressor efficiency & $\%$ & $75-88$ & 80 & Uniform $(75,88)$ \\
\hline
\end{tabular}

${ }^{\mathrm{a}}$ for retrofit applications, nominal value is 22 . 
Table 4.4. Amine System Cost Model Parameters and Uncertainties

\begin{tabular}{|c|c|c|c|}
\hline $\begin{array}{c}\text { Cost } \\
\text { Parameter }\end{array}$ & Units & $\begin{array}{c}\text { Nominal } \\
\text { Value }\end{array}$ & $\begin{array}{c}\text { Unc. Representation } \\
\text { (Distribution Function) }\end{array}$ \\
\hline Process facilities & M\$ & calc & $* \operatorname{Normal}(1.0,0.1)$ \\
\hline Engineering and home office & $\% \mathrm{PFC}$ & 7 & Triangular $(5,7,10)$ \\
\hline General facilities & $\% \mathrm{PFC}$ & 10 & Triangular $(5,10,15)$ \\
\hline Project contingency & $\% \mathrm{PFC}$ & 15 & Triangular $(10,15,20)$ \\
\hline Process contingency & $\% \mathrm{PFC}$ & 5 & Triangular $(2,5,10)$ \\
\hline Royalty fees & $\% \mathrm{PFC}$ & 0.5 & Triangular $(0,0.5,0.5)$ \\
\hline Startup cost & Month of TOM & 1 & Triangular $(0.5,1,1)$ \\
\hline Inventory cost & $\% \mathrm{TPC}$ & 0.5 & Triangular $(0.4,0.5,0.6)$ \\
\hline Total maintenance cost & $\% \mathrm{TPC}$ & 2.5 & Triangular( $(1,2.5,5)$ \\
\hline Operating labor & Jobs/shift & 2 & Triangular $(1,2,3)$ \\
\hline MEA cost & $\$ /$ tonne MEA & 1200 & Uniform $(1100,1300)$ \\
\hline Waste disposal cost & \$/ tonne waste & 175 & *Normal $(1.0,0.1)$ \\
\hline $\mathrm{CO}_{2}$ transport cost & $\$ /$ tonne $\mathrm{CO}_{2} / \mathrm{km}$ & 0.02 & Triangular $(0.004,0.02,0.08)$ \\
\hline $\mathrm{CO}_{2}$ storage/disposal cost & $\$ /$ tonne $\mathrm{CO}_{2}$ & 5 & Triangular $(0,5,10)$ \\
\hline $\begin{array}{l}\text { Revenue from } \mathrm{CO}_{2} \text { usage in } \\
\text { EOR or } \mathrm{ECBM}^{\mathrm{b}}\end{array}$ & $\$ /$ tonne $\mathrm{CO}_{2}$ & $-10^{\mathrm{a}}$ & Triangular $(-20,-10,0)$ \\
\hline
\end{tabular}

References

Morgan, M. G. and M. Henrion (1990). Uncertainty - A guide to dealing with uncertainty in quantitative risk and policy analysis. Cambridge, UK, Cambridge University Press. 


\section{APPENDIX C}

\section{PROBABILITY DISTRIBUTIONS FOR AMINE SYSTEM MODEL PARAMETERS}

Here we briefly explain the basis for the probability distributions for various model parameters (Table 3.3 and Table 4.3) used in the probabilistic analysis presented in Chapter 5. As mentioned in Appendix B, these distributions take into account the data reported in literature, modeling approximations, and experts' technical judgments (especially when sufficient data is not available).

\section{1. $\mathrm{CO}_{2}$ capture efficiency (\%)}

Almost all the studies report $\mathrm{CO}_{2}$ capture efficiency of $90 \%$. This seems to be the optimum capture level in the MEA-based $\mathrm{CO}_{2}$ capture systems, especially for Fluor Daniel's process using 30\% MEA sorbent. Few studies mention desired capture efficiency of $85 \%$ or less. Only the Kerr-McGee/ ABB Lummus amine process using $20 \%$ MEA sorbent is reported to capture as high as $96 \% \mathrm{CO}_{2}$. Here we use $90 \%$ as the default value for this parameter and do not specify any distribution.

\begin{tabular}{|c|c|}
\hline $\mathbf{C O}_{2}$ capture efficiency (\%) & Reference(s) \\
\hline 80 & {$[1]$} \\
\hline 85 & {$[2],[3]$} \\
\hline $85-90$ & {$[4]$} \\
\hline 90 & {$[5],[6],[7],[8],[9],[10],[11],[12],[13],[14],[15],[16]$,} \\
& {$[17],[18],[19],[20],[21],[22],[23]$} \\
\hline $85-95$ & {$[24],[25]$} \\
\hline 93 & {$[26]$} \\
\hline 96 & {$[27],[28]$} \\
\hline
\end{tabular}

\section{Acid gas removal efficiency of MEA system (\%)}

MEA system designed to remove $\mathrm{CO}_{2}$ from the flue gas also removes other acid gases $\left(\mathrm{SO}_{2}, \mathrm{NO}_{2}\right.$ and $\left.\mathrm{HCl}\right)$ if they are present. Just like other wet scrubber technologies, it also removes sizable portion of the particulate matter in the flue gas. Unfortunately, no data points are available regarding the removal efficiencies of these species in the literature. 
Hence these distributions are solely based on personal communication with technical experts working in this field for a long time [29-31].

- $\mathrm{SO}_{2}$ removal efficiency of MEA system (\%): Uniform(99,100)

- $\mathrm{NO}_{2}$ removal efficiency of MEA system (\%): Uniform(20,30)

- $\mathrm{HCl}$ removal efficiency of MEA system (\%): Uniform(90,95)

- Particulate removal efficiency of MEA system (\%): Uniform(40,60)

\section{MEA concentration ( $\% \mathrm{w} / \mathrm{w})$}

Today the commercially available MEA-based technology supplied by Fluor Daniel uses $30 \%$ MEA sorbent with some inhibitors. Other suppliers who do not use this inhibitor, prefer to use lower MEA concentration in the range of 15-20\%. The highest MEA concentration is reported to be $50 \%$ (an optimization study, not in a real plant). Since the model is based on Fluor Daniel's Econamine FG process, the nominal value of $30 \%$ for MEA concentration is used. Given the higher energy penalty of dilute sorbent systems, it is unlikely that systems with sorbent concentrations less than $20 \%$ will be built today. At the same time, in spite of ongoing R\&D efforts, an optimized amine system with $50 \%$ MEA is far from reality today. Hence a triangular distribution $(20,30,40)$ is used to convey the variability in current commercial system designs.

\begin{tabular}{|c|c|}
\hline MEA concentration (\%) & Reference(s) \\
\hline 12,18 & {$[24],[25]$} \\
\hline 15 & {$[2]$} \\
\hline $15-20$ & {$[32],[19]$} \\
\hline 20 & {$[27],[28],[33]$} \\
\hline $20-30$ & {$[1],[34],[35]$} \\
\hline 30 & {$[6],[8],[9],[12],[14],[33],[13],[4],[36],[37],[17],[24],[26]$} \\
\hline 50 & {$[17]$} \\
\hline
\end{tabular}

\section{Lean sorbent $\mathrm{CO}_{2}$ loading (mole $\mathrm{CO}_{2} /$ mole $\mathrm{MEA}$ )}

The regenerated (or lean) sorbent contains some "left-over" $\mathrm{CO}_{2}$. The level of lean sorbent $\mathrm{CO}_{2}$ loading mainly depends upon the initial $\mathrm{CO}_{2}$ loading in the sorbent and the amount of regeneration heat supplied, or alternatively, the regeneration heat requirement 
depends on the allowable level of lean sorbent loading. The range of values reported in the literature corresponds to variety of designs and operating conditions. Although a higher value translates into lower regeneration heat requirement (and hence lower energy penalty), experts in this technology believe that operating amine system at such conditions will lead to severe corrosion and maintenance problems [29, 30]. Similarly, a very low value like 0.05 implies very high regeneration energy requirement, which may not be economically viable due to the large energy penalty. So, the first $(0.05)$ and the last (0.3) data points have been omitted while characterizing the distribution for this parameter. Here we use a nominal value of 0.2 and a triangular distribution $(0.1,0.2$, 0.25 ) that is based on literature survey as well as experts' opinions.

\begin{tabular}{|c|c|}
\hline $\begin{array}{l}\text { Lean sorbent } \mathrm{CO}_{2} \text { loading } \\
\text { (mole } \mathrm{CO}_{2} / \text { mole MEA) }\end{array}$ & Reference(s) \\
\hline $0.05-.2$ & {$[33]$} \\
\hline 0.1 & [34] \\
\hline 0.16 & [38] \\
\hline 0.2 & {$[39],[40]$} \\
\hline 0.21 & [38] \\
\hline 0.215 & {$[27],[28]$} \\
\hline 0.24 & [17] \\
\hline 0.25 & [17] \\
\hline 0.3 & [2] \\
\hline
\end{tabular}

\section{Nominal MEA makeup (kg MEA/tonne $\mathrm{CO}_{2}$ )}

During the normal operating conditions, MEA is lost through polymerization and oxidation reactions. In order to keep the system continuously running, sorbent makeup is required. Typically this value is reported by various studies in the units of kg MEA per tonnes of $\mathrm{CO}_{2}$ captured. The range of values indicate both variability due to differences in system design and operating conditions as well as uncertainty due to complex chemistry involved. Here we use a nominal value of $1.5 \mathrm{~kg}$ MEA per tonne $\mathrm{CO}_{2}$ captured, with a triangular distribution $(0.5,1.5,3.1)$.

6. 


\begin{tabular}{|c|c|}
\hline $\begin{array}{c}\text { MEA makeup rate } \\
\text { (kg MEA/ tonne } \mathbf{C O}_{2} \text { captured) }\end{array}$ & Reference(s) \\
\hline $0.5-2$ & {$[20]$} \\
\hline 0.7 & {$[28],[14]$} \\
\hline 0.9 & {$[41]$} \\
\hline 1.5 & {$[38],[9],[17]$} \\
\hline 1.6 & {$[42]$} \\
\hline 1.8 & {$[8],[11],[41]$} \\
\hline 3.1 & {$[36]$} \\
\hline
\end{tabular}

\section{Blower pressure head (kPa)}

Blower pressure head $\left(\Delta \mathrm{P}_{\mathrm{fg}}\right)$ is the pressure head that needs to be provided to the flue gas in the blower so that it can overcome the pressure drop in the absorber tower. Typically, it would depend on the absorber design. We find a range of values reported in the literature, as well as indicated by the experts. Based on these numbers, the nominal value for this parameter is taken as 14 . The variability is represented by a triangular distribution covering the range $(4,14,37)$ of all these values.

\begin{tabular}{|c|c|}
\hline Blower pressure head (kPa) & Reference(s) \\
\hline 4 & {$[2]$} \\
\hline 5 & {$[3],[17]$} \\
\hline 12 & {$[28]$} \\
\hline 14 & {$[25],[19]$} \\
\hline 15 & {$[26]$} \\
\hline 17 & {$[6]$} \\
\hline 20 & {$[42],[9]$} \\
\hline 37 & {$[33]$} \\
\hline
\end{tabular}

\section{Sorbent pumping head (kPa)}

Sorbent pumping head $\left(\Delta \mathrm{P}_{\text {sorbent }}\right)$ is the pressure head to be provided to the sorbent using sorbent circulation pumps, so that it can overcome the pressure losses in the absorber and regenerator columns. A typical value is about $200 \mathrm{kPa}$ [17], and a modest range around the same has been assumed to represent the uncertainty coming from lack of sufficient data, triangular(150,200,250). 


\section{Blower and pump efficiency (\%)}

Flue gas blower and sorbent circulation pump are common mechanical devices used in industrial operations. These devices have performance ratings that may vary depending upon the make/type and the operating conditions. Typically, the efficiencies would be around $70-80 \%$. Hence we use a uniform distribution (uniform $(70,80)$ ).

\section{Equivalent electricity generation penalty ( $\%$ of regeneration heat energy)}

As explained before, this factor $\left(\mathrm{F}_{\mathrm{HE}}\right)$ gives the equivalent loss of power generation capacity due to extraction of steam from the power plant steam cycle to meet the sorbent regeneration heat requirement. This factor mainly depends on the design issues such as the quality of steam used for sorbent regeneration, and the level of heat integration between the base plant and the amine system. Recent developments suggest that the energy penalty can be substantially reduced by better heat integration and in-house practices $[43,44]$. Here we use a nominal value of 14 with a uniform distribution $(9,19)$ for a new plant. Retrofit applications are likely to have some constraints on the level of heat integration, and hence have a higher value for this factor. The values obtained from the literature are in the range $(20,25)$, hence we use a nominal value of 22 .

\begin{tabular}{|c|c|}
\hline Equivalent electricity loss (\%) & Reference(s) \\
\hline 9 & {$[17]$} \\
\hline 16 & {$[14],[17]$} \\
\hline 17 & {$[45]$} \\
\hline 19 & {$[6]$} \\
\hline $20-25$ & {$[28],[27]$} \\
\hline 22 & {$[2],[26]$} \\
\hline 25 & {$[3]$} \\
\hline
\end{tabular}

11. $\mathrm{CO}_{2}$ product pressure (psig)

Most of the data sources report the value of $\mathrm{CO}_{2}$ product pressure to be around $2000 \mathrm{psig}$. It is believed that pressure of this magnitude would be sufficient to overcome pressure losses incurred during the transport of $\mathrm{CO}_{2}$ product over a reasonable distance (100 miles or so) without any need for recompression stations to keep the $\mathrm{CO}_{2}$ product in liquefied state. Depending upon the assumptions about the transportation distance and other details, this value may be different. The $\mathrm{CO}_{2}$ product pressure has to be above $1070 \mathrm{psig}$, 
the critical pressure of $\mathrm{CO}_{2}$. Also, the product pressure of 2200 psig is sufficiently high for transport of $\mathrm{CO}_{2}$ via pipelines. So, a triangular distribution has been adopted to reflect this range 1100-2200 with a nominal value of 2000 .

\begin{tabular}{|c|c|}
\hline $\mathbf{C O}_{2}$ product pressure (psig) & Reference(s) \\
\hline 1164 & {$[17]$} \\
\hline 1207 & {$[6]$} \\
\hline 1451 & {$[3],[46]$} \\
\hline 1595 & {$[2]$} \\
\hline 2000 & {$[47],[27],[28],[48],[16],[49]$} \\
\hline 2035 & {$[8],[11],[9]$} \\
\hline 2180 & {$[42],[26]$} \\
\hline 3200 & {$[5]$} \\
\hline
\end{tabular}

\section{Compressor efficiency}

This is the effective efficiency of the compressors used to compress $\mathrm{CO}_{2}$ product to the desirable pressure. As seen from the reported data, the compression efficiencies lie in the range of about $75 \%$ to $88 \%$. Hence the nominal value of compressor efficiency ( $\left.\eta_{\text {comp }}\right)$ is taken as $80 \%$ with a triangular distribution to cover the data range.

\begin{tabular}{|c|c|}
\hline $\mathbf{C O}_{2}$ compression efficiency (\%) & Reference(s) \\
\hline 75 & {$[50]$} \\
\hline $75-85$ & {$[3]$} \\
\hline 85 & {$[17]$} \\
\hline 88 & {$[28]$} \\
\hline
\end{tabular}

It may be noted that probability distributions have not been assigned to some performance parameters such as MEA losses due to acid gases $\left(\mathrm{SO}_{2}, \mathrm{NO}_{2}, \mathrm{HCl}\right)$, ammonia generation and consumption of caustic and activated carbon. These are the parameters that reflect stoichiometry (balance among chemical species).

Next we have discussion about the probability distributions for some cost parameters.

\section{Sorbent cost (\$/ tonne sorbent)}

The cost of the sorbent depends upon various market forces including the cost of natural gas (which is the ultimate feedstock for its manufacture). Hence it is likely to be 
fluctuating. Based on the data and experts' judgments, the nominal value was taken as $\$ 1200$ / tonne sorbent with a uniform distribution $(1000,1300)$.

\begin{tabular}{|c|c|}
\hline $\begin{array}{c}\text { Sorbent cost } \\
\text { (\$/ tonne sorbent) }\end{array}$ & Reference(s) \\
\hline 970 & {$[42]$} \\
\hline 1189 & {$[11]$} \\
\hline 1300 & {$[28]$} \\
\hline 1340 & {$[17,51]$} \\
\hline
\end{tabular}

\section{Total maintenance cost (\% TPC)}

The total maintenance cost is generally specified as a fraction of total plant cost. This parameter depends on some design parameters (e.g. sorbent concentration, column design) as well as the operating conditions (e.g. flue gas characteristics, $\mathrm{CO}_{2}$ loading levels). Hence there is likely to be some variability as well as performance uncertainty reflected in the range of data. Based on the data as well as experts' judgments, $2.5 \%$ was taken as the nominal value with a triangular distribution $(1,2.5,5)$.

\begin{tabular}{|c|c|}
\hline Total maintenance cost (\% TPC) & Reference(s) \\
\hline 1.5 & {$[42]$} \\
\hline 2 & {$[11]$} \\
\hline 2.2 & {$[28]$} \\
\hline 4 & {$[2]$} \\
\hline 4 & {$[17]$} \\
\hline
\end{tabular}

\section{5. $\mathrm{CO}_{2}$ transport cost}

There is a wide range of numbers reported for the cost of transporting the concentrated stream of $\mathrm{CO}_{2}$ product. Most of the variability may be explained in terms of assumptions about the location of the pipeline and design parameters (safety factors, spare capacity etc.). Few representative numbers are listed here. The nominal value is taken as $\$ 0.02$ / $\mathrm{km}$ per tonne $\mathrm{CO}_{2}$, with a triangular distribution $(0.004,0.02,0.08)$. 


\begin{tabular}{|c|c|}
\hline $\begin{array}{c}\mathbf{C O}_{2} \text { transport cost } \\
\left(\mathbf{~} / \mathbf{~ k m} \text { per tonne } \mathbf{C O}_{2}\right)\end{array}$ & Reference(s) \\
\hline $0.004-0.03$ & {$[52]$} \\
\hline $0.01-0.03$ & {$[53]$} \\
\hline $0.01-0.05$ & {$[54]$} \\
\hline 0.012 & {$[55]$} \\
\hline $0.024-0.061$ & {$[34]$} \\
\hline $0.03-0.08$ & {$[56]$} \\
\hline
\end{tabular}

The distributions for other cost parameters (mainly the indirect capital costs) are based on those for other environmental control technologies.

\section{References}

1. Langeland, K. and K. Wilhelmsen, A study of the costs and energy reuirement for carbon dioxide disposal. Energy Conversion and Management, 1993. 34(9-11): p. 807-814.

2. Stork Engg Consultancy B.V., A., Leading options for the capture of CO2 emissions at power stations. 2000, IEA GHGRDP: UK.

3. Bolland, O. and H. Undrum. Removal of CO2 from gas turbine power plants: Evaluation of pre- and postcombustion methods. in Fourth International Conference on Greenhouse Gas Control Technologies, 30 August - 2 September. 1998. Interlaken, Switzerland: Elsevier Science Ltd.

4. Rao, A.D. and W.H. Day, Mitigation of greenhouse gases from gas turbine power plants. Energy Conversion and Management, 1996. 37(6-8): p. 909-914.

5. Slater, M., E. West, and C. Mariz. Carbon dioxide capture from multiple flue gas sources. in Sixth International Conference on Greenhouse Gas Control Technologies. 2002. Kyoto, Japan.

6. Parsons Infrastructure \& Technology Group, I., Evaluation of fossil fuel power plants with CO2 recovery. 2002, USDOE/NETL/EPRI: Pittsburgh, PA.

7. Jeremy, D. and H.J. Herzog. The cost of carbon capture. in presented at the Fifth International Conference on Greenhouse Gas Control Technologies, 13-16 August. 2000. Cairns, Australia. 
8. Chapel, D., J. Ernst, and C. Mariz. Recovery of CO2 from flue gases: commercial trends (paper no. 340). in presented at the Canadian Society of Chemical Engineers Annual Meeting, 4-6 October. 1999. Saskatoon, Saskatchewan, Canada.

9. Desideri, U. and A. Paolucci, Performance modelling of a carbon dioxide removal system for power plants. Energy Conversion and Management, 1999. 40: p. 1899-1915.

10. Simbeck, D. A portfolio selection approach for power plant CO2 capture, separation and $R \& D$ options. in Fourth International Conference on Greenhouse Gas Control Technologies, 30 August - 2 September. 1998. Interlaken, Switzerland: Elsevier Science Ltd.

11. Fluor, Evaluation of technologies for the removal of CO2 from coal fired power plant flue gas. 1998, a report prepared by Fluor Daniel Canada Inc. for TransAlta, November 1998.

12. Desideri, U. and R. Corbelli, CO2 capture in small size cogeneration plants: Technical and economical considerations. Energy Conversion and Management, 1998. 39(9): p. 857-867.

13. Leci, C.L., Development requirements for absorption processes for effective CO2 capture from power plants. Energy Conversion and Management, 1997. 38(Suppl): p. S45-S50.

14. Mimura, T., et al., Development of energy saving technology for flue gas carbon dioxide recovery in power plants by chemical absorption method and steam system. Energy Conversion and Management, 1997. 38(Suppl): p. S57-S62.

15. Leci, C.L., Financial implications on power generation costs resulting from the parasitic effect of CO2 capture using liquid scrubbing technology from power station flue gases. Energy Conversion and Management, 1996. 37(6-8): p. 915921.

16. Tontiwachwuthikul, P.T., W. Kritpiphat, and D. Gelowitz, Carbon dioxide production from co-generation for enhanced oil recovery: An economic evaluation. The J. of Can. Petro. Tech., 1996. 35(6): p. 27-33.

17. Hendriks, C., Carbon Dioxide Removal from Coal-fired Power Plants. 1st ed. 1994, The Netherlands: Kluwer Academic Publishers. 14-223.

18. Riemer, P., H. Audus, and A. Smith, Carbon Dioxide Capture from Power Stations. 1994, a report published by IEA Greenhouse Gas R\&D Programme: UK. 
19. Barchas, R. and R. Davis, The Kerr-McGee/ ABB Lummus Crest technology for the recovery of $\mathrm{CO} 2$ from stack gases. Energy Conversion and Management, 1992. 33(5-8): p. 333-340.

20. Suda, T., et al., Development of flue gas carbon dioxide recovery technology. Energy Conversion and Management, 1992. 33(5-8): p. 317-324.

21. Yagi, T., H. Shibuya, and T. Sasaki, Application of chemical absorption process to CO2 recovery from flue gas generated in power plants. Energy Conversion and Management, 1992. 33(5-8): p. 349-355.

22. Nakayama, S., et al., Pulverized coal combustion in O2/CO2 mixtures on a power plant for CO2 recovery. Energy Conversion and Management, 1992. 33(5-8): p. 379-386.

23. Horn, F.L. and M. Steinburg, An improved carbon dioxide power plant. Energy Progress, 1982. 2(3): p. 154-159.

24. Sander, M.T. and C.L. Mariz, The Fluor Daniel "Econamine FG" process: Past experience and present day focus. Energy Conversion and Management, 1992. 33(5-8): p. 341-348.

25. Wiggins, W.R.J. and R.L. Bixler, Sources, recovery and tranportation of CO2. Energy Progress, 1983. 3(3): p. 132-135.

26. Smelser, S.C., R.M. Stock, and G.J. McCleary, Engineering and economic evaluation of CO2 removal from fossil-fuel-fired power plants. 1991, EPRI IE7365, Volume 1, Project 2999-10, a research project final report prepared by Fluor Daniel Inc., for EPRI and IEA.

27. Marion, J., et al. Engineering feasibility of $\mathrm{CO} 2$ capture on an existing US coalfired power plant. in Twenty Sixth International conf. On coal utilization and fuel systems. 2001. Clearwater, FL.

28. ALSTOM, et al., Engineering feasibility and economics of CO2 capture on an existing coal-fired power plant. 2001, Final report prepared by ALSTOM Power Inc., ABB Lummus Global Inc., ALSTOM Power Environmental Systems and American Electric Power (AEP). report no. (PPL-01-CT-09) submitted to Ohio Department of Development, Columbus, OH and US Department of Energy/ NETL, Pittsburgh, PA. p. 163.

29. Dingman, J.D., personal communication. 2002.

30. Mariz, C., personal communication. 2000-2003.

31. Gupta, J.C., personal communication. 2002. 
32. DuPart, M.S., T.R. Bacon, and D.J. Edwards, Understanding corrosion in alkanolamone gas treating plants. Hydrocarbon Processing, 1993. May: p. 89-94.

33. Kohl, A.L. and R.B. Nielsen, Gas Purification. 5th ed. 1997, Houston, TX: Gulf Publishing Company. 40-277.

34. Anada, H.R., et al., Economics of by-product $\mathrm{CO} 2$ recovery and transportation for EOR. Energy Progress, 1983. 3(4): p. 233-243.

35. Stokes, K.J., Choosing an ammonia plant CO2 removal system for today's conditions. Nitrogen, 1981. 131: p. 35-38.

36. Chakma, A., An energy efficient mixed solvent for the separation of CO2. Energy Conversion and Management, 1995. 36(6-9): p. 427-430.

37. Jou, F., A.E. Mather, and F.D. Otto, The solubility of CO2 in a 30 mass percent monoethanolamine solution. The Canadian Journal of Chemical Engineering, 1995. 73 (Feb): p. 140-147.

38. Guezebroek, F.H., L.H.J.M. Schneider, and G.J.C. Kraaijveld. Exergy analysis of amine-based CO2 removal technology. in Sixth International Conference on Greenhouse Gas Control Technologies. 2002. Kyoto, Japan.

39. Veawab, A., et al. Performance and cost analysis for CO2 capture from flue gas streams: Absorption and regeneration aspects. in Sixth International Conference on Greenhouse Gas Control Technologies. 2002. Kyoto, Japan.

40. Alie, C., et al., Simulating CO2 capture from pulverized coal fired power plants: Assessment of MEA-CO2 capture processes using AspenPlus. 2002, International test network for CO2 capture: Report on 3rd workshop (May 16-17, 2002), Apeldoorn, The Netherlands (Report number PH4/11).

41. Pauley, C.R., P.L. Simiskey, and S. Haigh, $N$-Ren recovers $C O 2$ from flue gas economically. Oil and Gas Journal, 1984. 82(20): p. 87-92.

42. Singh, D., et al., Techno-economic study of CO2 capture from an existing coalfired power plant: MEA scrubbing vs. O2/CO2 recycle combustion. 2002: Waterloo, Ontario, Canada. p. 27.

43. Reddy, S. and C.A. Roberts. ECONOMINE FGSM Plus: An Enhanced Amine Based $\mathrm{CO}_{2}$ Capture Process. in presented at the 2nd Annual Conference on Carbon Sequestration, May 5-9, 2003. 2003. Alexandria, VA, USA.

44. Gibbins, J.R. and R.I. Crane, Scope for reductions in the cost of CO2 capture using flue gas scrubbing with amine solvents, in Draft paper. 2003. p. 27. 
45. Alie, C., et al., Simulatiion of CO2 capture from coal-basd power plants: Supplementary energy management. 2003, International test network for $\mathrm{CO} 2$ capture: Report on 5th workshop (June 2-3, 2002), Carnegie Mellon University, Pittsburgh (Report number PH4/22).

46. Bolland, O. and P. Mathieu, Comparison of two CO2 removal options in combined cycle power plants. Energy Conversion and Management, 1998. 39(1618): p. 1653-1663.

47. Iijima, M. and T. Kamijo. Flue gas CO2 recovery and compression cost study for CO2 enhanced oil recovery. in Sixth International Conference on Greenhouse Gas Control Technologies. 2002. Kyoto, Japan.

48. Simbeck, D. and M. McDonald. Existing coal power plant retrofit $\mathrm{CO}_{2}$ control options analysis. in presented at the Fifth International Conference on Greenhouse Gas Control Technologies, 13-16 Augus. 2000. Cairns, Australia.

49. Clair, J.H. and W.F. Simister, Process to recover $\mathrm{CO} 2$ from flue gas gets first large-scale tryout in Texas. Oil and Gas J., 1983. Feb: p. 109-113.

50. Price, B.C., Processing high CO2 gas. Energy Progress, 1984. 4(3): p. 169-174.

51. Dow, Personal communication with the Marketing Executive, Dow Chemicals. 2003.

52. Herzog, H.J., A Cost model for transport of carbon dioxide, in Draft paper. 2001, MIT.

53. Wallace, D., Capture and storage of CO2: What needs to be done? 2000, IEA.AIE: presented at the COP6, The Hague. p. 9.

54. NOVEM, Potential for CO2 sequestration and enhanced coalbed methane production in the Netherlands. 2001, NOVEM (The Netherlands Agency for Energy and the Environment): Utrecht. p. 105.

55. Bergman, P.D., E.M. Winter, and Z.-Y. Chen, Disposal of power plant CO2 in depleted oil and gas reservoirs in Texas. Energy Conversion and Management, 1997. 38(Suppl): p. S211-S216.

56. Battelle, Carbon dioxide sequestration in saline formations - Engineering and economic assessment final technical report. 2001, NETL, USDOE: Morgantown, WV.p. 93. 


\section{APPENDIX D}

\section{CASE STUDY RESULTS FOR NEW COAL PLANTS}

\section{Assumptions:}

- $\quad$ Base plant (Super critical PC):

o Coal: Wyoming powder river basin, $0.48 \% \mathrm{~S}, 8340 \mathrm{Btu} / \mathrm{lb}, \$ 23.19 \$ /$ tonne

o Heat Rate: Steam cycle HR 6750 Btu/kWh = Gross cycle HR 7923 Btu/kWh

- $\mathrm{SO}_{2}$ control: FGD system using limestone

- $\mathrm{CO}_{2}$ control:

o MEA system using 30\% w/w MEA-based sorbent

o $\mathrm{CO}_{2}$ product compression to $2000 \mathrm{psig}$

o cost for $\mathrm{CO}_{2}$ transport $=\$ 7 /$ tonne $\mathrm{CO}_{2}\left(100\right.$ miles @ $\$ 0.0426 /$ tonne $\mathrm{CO}_{2}$ per $\mathrm{km}$ )

o cost for $\mathrm{CO}_{2}$ disposal $=\$ 5 /$ tonne $\mathrm{CO}_{2}$

- Other details as mentioned in Tables 3.3, 4.2, 4.3 and 5.1.

\begin{tabular}{|l|c|c|c|c|c|}
\hline \multicolumn{1}{|c|}{ Parameter } & $\begin{array}{c}\text { Reference } \\
\text { Plant }\end{array}$ & $\begin{array}{c}\text { Capture } \\
\text { Plant (A) }\end{array}$ & $\begin{array}{c}\text { \% } \\
\text { change }\end{array}$ & $\begin{array}{c}\text { Capture } \\
\text { Plant (B) }\end{array}$ & $\begin{array}{c}\% \\
\text { change }\end{array}$ \\
\hline Model Inputs: & & & & & \\
\hline Gross capacity (MW) & 500 & 500 & $0 \%$ & 670 & $+34 \%$ \\
\hline FGD efficiency (\%) & 95 & 99 & & 99 & \\
\hline $\mathrm{CO}_{2}$ capture efficiency (\%) & - & 90 & & 90 & \\
\hline Energy Penalty: & & & & & \\
\hline Base plant, PC (MW) & 29.3 & 29.3 & $0 \%$ & 39.2 & $+34 \%$ \\
\hline FGD System (MW) & 9.5 & 13.7 & $+43.4 \%$ & 18.3 & $+92 \%$ \\
\hline ESP (MW) & 0.9 & 0.9 & $0 \%$ & 0.9 & $0 \%$ \\
\hline SCR (MW) & 2.7 & 2.7 & $-0.4 \%$ & 3.6 & $+33 \%$ \\
\hline MEA: Regen. heat req. (equiv.) & - & 60.8 & & 81.5 & \\
\hline MEA: fans (MW) & - & 10.4 & & 14.0 & \\
\hline MEA: pumps (MW) & - & 0.9 & & 1.1 & \\
\hline MEA: CO 2 compression (MW) & - & 40.3 & & 54.0 & \\
\hline
\end{tabular}




\begin{tabular}{|c|c|c|c|c|c|}
\hline MEA: total (MW) & - & 112.4 & & 150.6 & \\
\hline Total energy penalty (MW) & 42.4 & 158.4 & $+275 \%$ & 212.6 & $+402 \%$ \\
\hline Parameter & $\begin{array}{c}\text { Reference } \\
\text { Plant }\end{array}$ & $\begin{array}{l}\text { Capture } \\
\text { Plant (A) }\end{array}$ & $\begin{array}{c}\% \\
\text { change }\end{array}$ & $\begin{array}{l}\text { Capture } \\
\text { Plant (B) }\end{array}$ & $\begin{array}{c}\% \\
\text { change }\end{array}$ \\
\hline $\begin{aligned} 7.5 & \text { Plant } \\
& \text { Performance }\end{aligned}$ & & & & & \\
\hline Total energy penalty $\left(\% \mathrm{MW}_{\mathrm{g}}\right)$ & $8.5 \%$ & $31.8 \%$ & $+275 \%$ & $31.7 \%$ & $+275 \%$ \\
\hline Net power generation (MW) & 457.6 & 341.2 & $-26 \%$ & 457.6 & $0 \%$ \\
\hline Net cycle heat rate $(\mathrm{Btu} / \mathrm{kWh})$ & 8,657 & 11,560 & $+34 \%$ & 11,550 & $+33 \%$ \\
\hline Sorbent regen. heat $\left(\mathrm{kJ} / \mathrm{kgCO}_{2}\right)$ & - & 4575 & & 4575 & \\
\hline \multicolumn{6}{|l|}{ Plant Consumption: } \\
\hline Coal consumption (tonne/hr) & 215.5 & 214.6 & $-0.4 \%$ & 287.5 & $+33 \%$ \\
\hline Limestone consumed (tonne/hr) & 2.3 & 2.4 & $+4 \%$ & 3.2 & $+39 \%$ \\
\hline MEA consumed $(\mathrm{kg} / \mathrm{hr})$ & - & 789 & & 1057 & \\
\hline \multicolumn{6}{|l|}{ Emissions/ Discharges: } \\
\hline $\mathrm{CO}_{2}$ emission (tonne/ hr) & 381.3 & 38.0 & $-90 \%$ & 50.9 & $-87 \%$ \\
\hline $\mathrm{NO}_{\mathrm{x}}$ emission $(\mathrm{kg} / \mathrm{hr})$ & 269.5 & 265.0 & $-2 \%$ & 355.2 & $+32 \%$ \\
\hline $\mathrm{SO}_{\mathrm{x}}$ emission $(\mathrm{kg} / \mathrm{hr})$ & 76.4 & 0.11 & $-99.9 \%$ & 0.14 & $-99.8 \%$ \\
\hline $\mathrm{NH}_{3}$ emission $(\mathrm{kg} / \mathrm{hr})$ & 5.4 & 29.8 & $+457 \%$ & 40.0 & $+648 \%$ \\
\hline $\mathrm{CO}_{2}$ emission $\left(\mathrm{gCO}_{2} / \mathrm{kWh}\right)$ & 833.3 & 111.3 & $-87 \%$ & 111.2 & $-87 \%$ \\
\hline $\mathrm{NO}_{\mathrm{x}}$ emission $\left(\mathrm{gNO}_{\mathrm{x}} / \mathrm{kWh}\right)$ & 0.59 & 0.78 & $+32 \%$ & 0.78 & $+32 \%$ \\
\hline $\mathrm{SO}_{\mathrm{x}}$ emission $\left(\mathrm{gNO}_{\mathrm{x}} / \mathrm{kWh}\right)$ & 0.17 & 0.0003 & $-99.8 \%$ & 0.0003 & $-99.8 \%$ \\
\hline Bottom ash (tonne/hr) & 3.5 & 3.5 & $-0.4 \%$ & 4.6 & $+33 \%$ \\
\hline Fly ash (tonne/hr) & 8.0 & 8.0 & $-0.4 \%$ & 10.7 & $+34 \%$ \\
\hline FGD waste (tonne/hr) & 4.2 & 4.3 & $+4 \%$ & 5.8 & $+39 \%$ \\
\hline Spent MEA sorbent (tonne/hr) & - & 1.0 & & 1.4 & \\
\hline $\mathrm{CO}_{2}$ product (tonne/ hr) & - & 342 & & 458 & \\
\hline \multicolumn{6}{|l|}{ Capital costs (TCR, M\$) } \\
\hline $\mathrm{PC}$ & 516.2 & 513.6 & $-0.5 \%$ & 646.0 & $+25 \%$ \\
\hline ESP & 23.5 & 22.1 & $-6 \%$ & 27.8 & $+18 \%$ \\
\hline FGD & 48.3 & 50.1 & $+4 \%$ & 57.6 & $19 \%$ \\
\hline SCR & 27.6 & 27.7 & $+0.2 \%$ & 34.8 & $26 \%$ \\
\hline
\end{tabular}




\begin{tabular}{|c|c|c|c|c|c|}
\hline MEA & - & 146.5 & & 196.6 & \\
\hline Total - TCR (M\$) & 615.7 & 760.1 & $+24 \%$ & 962.8 & $+56 \%$ \\
\hline Normalized TCR $(\$ / \mathrm{kW})$ & 1345 & 2228 & $+66 \%$ & 2104 & $+56 \%$ \\
\hline Parameter & $\begin{array}{c}\text { Reference } \\
\text { Plant }\end{array}$ & $\begin{array}{l}\text { Capture } \\
\text { Plant (A) }\end{array}$ & $\begin{array}{c}\% \\
\text { change }\end{array}$ & $\begin{array}{l}\text { Capture } \\
\text { Plant (B) }\end{array}$ & $\begin{array}{c}\% \\
\text { change }\end{array}$ \\
\hline \multicolumn{6}{|l|}{ O\&M costs $(M \$ / y r)$} \\
\hline $\mathrm{MEA}-\mathrm{FOM}$ & - & 4.1 & & 5.3 & \\
\hline MEA - reagent & - & 6.5 & & 8.7 & \\
\hline MEA - power & - & 13.5 & & 17.2 & \\
\hline MEA - steam (equiv.) & - & 15.9 & & 20.2 & \\
\hline $\mathrm{MEA}-\mathrm{CO}_{2}$ transport & - & 7.2 & & 9.7 & \\
\hline $\mathrm{MEA}-\mathrm{CO}_{2}$ disposal & - & 11.2 & & 15.1 & \\
\hline TOM - MEA & - & 61.3 & & 79.9 & \\
\hline TOM - PC & 44.4 & 13.3 & $-70 \%$ & 17.9 & $-60 \%$ \\
\hline TOM - ESP & 1.6 & 1.6 & $-1.4 \%$ & 1.8 & $+16 \%$ \\
\hline TOM - FGD & 7.5 & 8.6 & $+16 \%$ & 10.2 & $+36 \%$ \\
\hline TOM - SCR & 4.2 & 4.2 & $-0.5 \%$ & 5.5 & $+30 \%$ \\
\hline \multicolumn{6}{|l|}{$\begin{array}{l}\text { 8. OVERALL PLANT } \\
\text { COSTS }\end{array}$} \\
\hline Fuel cost & 32.9 & 32.7 & $-0.4 \%$ & 43.8 & $+34 \%$ \\
\hline TOM - whole plant & 57.6 & 89.1 & $+55 \%$ & 115.2 & $+100 \%$ \\
\hline TRR (M\$/ yr), whole plant & 148.7 & 201.5 & $+36 \%$ & 257.7 & $+73 \%$ \\
\hline $\mathrm{COE}(\$ / \mathrm{MWh})$ & 49.4 & 89.9 & $+82 \%$ & 85.7 & $+73 \%$ \\
\hline$\$ /$ tonne $\mathrm{CO}_{2}$ avoided & - & 56.0 & & 50.2 & \\
\hline$\$ /$ tonne $\mathrm{CO}_{2}$ delivered & - & 40.3 & & 36.2 & \\
\hline
\end{tabular}




\section{APPENDIX E}

\section{QUESTIONNAIRE SENT TO EXPERTS ON AMINE-BASED CO $\mathrm{C}_{2}$ CAPTURE TECHNOLOGY}

Thank you for agreeing to assist us in our work on characterizing the uncertainty and variability associated with the performance of amine-based $\mathrm{CO}_{2}$ capture technology. We plan to use your answers, and those of other experts as inputs to engineering-economic models we have developed to characterize the performance and cost of these systems. While we will acknowledge the experts who have assisted us in this effort, we will not identify any expert with any specific response. If you have questions or concerns please contact me:

By E-mail: abr@cmu.edu, or

By Phone: (412) 268-6826 or (412) 268-6115, or

By Fax: (412) 268-1089 (attn: Anand B. Rao)

Please also send your responses by email or fax, or by regular mail to:

Anand B. Rao

Department of Engineering \& Public Policy

Carnegie Mellon University

Pittsburgh, PA 15213, USA.

Alternatively, if you prefer, I can give you a call and note down your responses.

In the pages that follows we:

1. provide some background on the problem

2. list the key assumptions we are using in our model and ask you to comment on their appropriateness for current systems

3. ask you to make judgments about the likely values of a number of key coefficients for future systems

4. ask you to provide a ranking of various research objectives to reduce the cost of $\mathrm{CO}_{2}$ capture and sequestration from flue gas streams.

We estimate that you may need approximately 40-50 minutes to complete this questionnaire, and hope you will find that acceptable. We really appreciate your time and help, and will be happy to share our findings with you at the end of this study. 


\section{Part 1: Background}

Development of improved technology to capture and sequester the $\mathrm{CO}_{2}$ emitted by power plants using fossil fuels - especially coal - is the subject of major research efforts worldwide. The attraction of this option is that it would allow abundant world resources of fossil fuels to be used for power generation and other applications without contributing significantly to atmospheric emissions of greenhouse gases. The two key barriers to carbon capture and sequestration (CCS), however, are the high cost of current $\mathrm{CO}_{2}$ capture technologies, and uncertainties regarding the technical, economic and political feasibility of $\mathrm{CO}_{2}$ storage options.

As part of the USDOE's Carbon Sequestration Program, we have developed an integrated modeling framework to evaluate the performance and costs of alternative $\mathrm{CO}_{2}$ capture and sequestration technologies for fossil-fueled power plants, in the context of multi-pollutant control requirements. This model (called the IECM-CS) allows for explicit characterization of the uncertainty or variability in any or all model input parameters. One of the purposes of this model is to improve the understanding in both the technical and policy communities about the magnitude of CCS cost and the various factors that affect it. We also want to explore the potential for reducing costs through targeted R\&D.

At this stage, many of the model parameter values and uncertainty distributions have been based on information gathered from the literature. This approach has its limitations owing to the limited availability of data and possibility of inconsistent assumptions across different studies. An alternative method that can allow a more robust analysis of uncertainty and variability involves the development of expert judgments. Here, we want to understand how experts in this field would characterize some of the key parameters that affect the performance and cost of a particular $\mathrm{CO}_{2}$ capture technology, namely, amine-based absorption of $\mathrm{CO}_{2}$ from flue gas. We are interested both in current system designs, and improved future designs that you might envision.

Please briefly review the Supplementary Information on the next two pages to make sure that we use a consistent terminology during this exercise. 


\section{Supplementary Information}

\section{Overview of amine-based $\mathrm{CO}_{2}$ capture systems}

The idea of separating $\mathrm{CO}_{2}$ from flue gas streams started in the 1970 s, not with concern about the greenhouse effect, but as a potentially economic source of $\mathrm{CO}_{2}$, mainly for enhanced oil recovery (EOR) operations. Several commercial $\mathrm{CO}_{2}$ capture plants were constructed in the U.S. in the late 1970s and early 1980s. $\mathrm{CO}_{2}$ was also produced for other industrial applications such as carbonation of brine and production of products like dry ice, urea and beverages. Some of these $\mathrm{CO}_{2}$ capture plants are still in operation today, but all these plants are much smaller than a typical power plant. The first commercial $\mathrm{CO}_{2}$ sequestration facility started in Norway in September 1996 in response to a Norwegian carbon tax. Since then, Statoil has been storing $\mathrm{CO}_{2}$ from the Sleipner West gas field in a sandstone aquifer $1000 \mathrm{~m}$ beneath the North Sea. The international research community is closely monitoring this facility.

All these plants capture $\mathrm{CO}_{2}$ with processes based on chemical absorption using a monoethanolamine (MEA)-based solvent. MEA is an organic chemical belonging to the family of compounds known as amines. It was developed over 60 years ago as a general, non-selective solvent to remove acidic gas impurities (e.g. $\mathrm{H}_{2} \mathrm{~S}, \mathrm{CO}_{2}$ ) from natural gas streams. The process was then adapted to treat flue gas streams for $\mathrm{CO}_{2}$ capture. Fluor Daniel Inc., Dow Chemical Co., Kerr-McGee Chemical Corp. and ABB Lummus Crest Inc., were some of the initial developers of MEA-based technology for $\mathrm{CO}_{2}$ capture. Typically, about $75 \%$ to $90 \%$ of the $\mathrm{CO}_{2}$ is captured using this technology, producing a nearly pure $(>99 \%) \mathrm{CO}_{2}$ product stream.

\section{Process Description}

A continuous scrubbing system is used to separate $\mathrm{CO}_{2}$ from the flue gas stream. The system consists of two main elements: an absorber where $\mathrm{CO}_{2}$ is removed, and a regenerator (or stripper) where $\mathrm{CO}_{2}$ is released (in concentrated form) and the sorbent is recovered. 


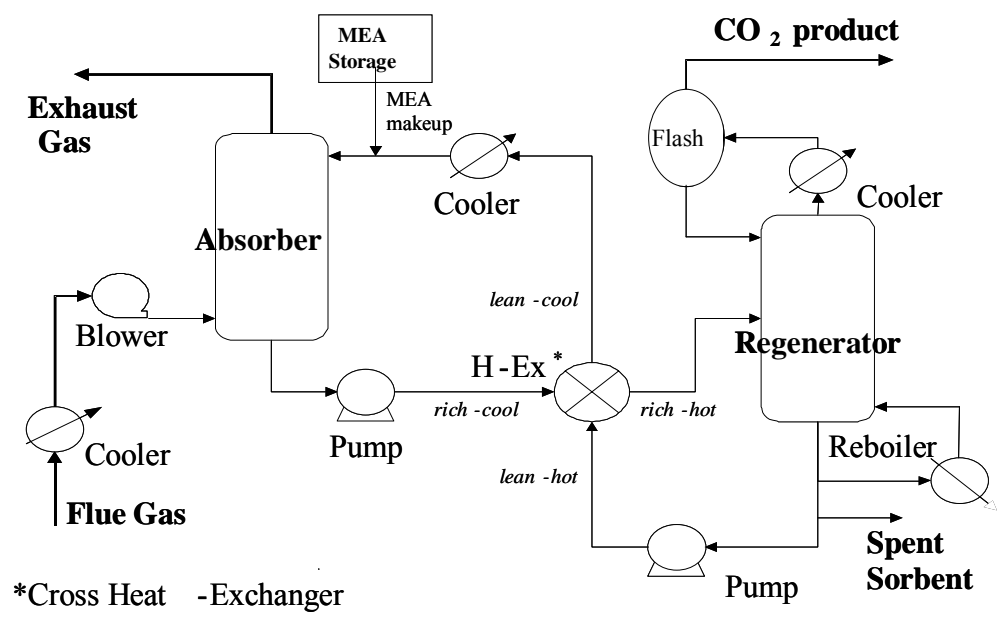

In a power plant application, cooled flue gases flow vertically upwards through the absorber countercurrent to the absorbent (consisting of MEA in a water solution, often with some additives to inhibit corrosion). The MEA reacts chemically with the $\mathrm{CO}_{2}$ in the flue gases to form a weakly bonded compound called carbamate. The scrubbed gases are then washed and vented to the atmosphere. The $\mathrm{CO}_{2}$-rich solution leaves the absorber and passes through a heat exchanger, then is further heated in a reboiler using lowpressure steam. The carbamate formed during absorption is broken down by the application of heat, regenerating the solvent, and producing a concentrated $\mathrm{CO}_{2}$ stream. The hot $\mathrm{CO}_{2}$-lean solvent is then returned to the heat exchanger, where it is cooled, then sent back to the absorber. Some fresh MEA is added to make up for losses incurred in the process. The process chemistry is complex, but the main reactions taking place are:

$\mathrm{CO}_{2}$ Absorption: 2 R-NH$(\mathrm{MEA})+\mathrm{CO}_{2} \rightarrow \mathrm{R}_{2}-\mathrm{NH}_{3}{ }^{+}+\mathrm{R}-\mathrm{NH}-\mathrm{COO}^{-}$(Carbamate)

MEA Regeneration: $\mathrm{R}-\mathrm{NH}-\mathrm{COO}^{-}+\mathrm{R}_{-\mathrm{NH}_{3}}{ }^{+}+$Heat $\rightarrow \mathrm{CO}_{2}+2 \mathrm{R}-\mathrm{NH}_{2}$

Pure MEA (with $\mathrm{R}=\mathrm{HO}-\mathrm{CH}_{2} \mathrm{CH}_{2}$ ) is an "unhindered" amine that forms stable carbamate; hence, only half a mole of $\mathrm{CO}_{2}$ is absorbed per mole of amine, as shown in Reaction (1). For other "hindered" amines (where $\mathrm{R}$ is a bulky group), the carbamate formed is not stable, and an alternate reaction leads to a higher theoretical capacity of one mole of $\mathrm{CO}_{2}$ per mole of amine. But the $\mathrm{CO}_{2}$ uptake rate of hindered amines is very low. Efforts are underway to formulate better solvents by combining favorable properties of these two groups of amines. 
Some of the solvent also is lost during the process because of physical losses, entrainment, vaporization and chemical degradation. All the solvent entering the stripper does not get regenerated. Flue gas impurities, especially oxygen, sulfur dioxide and nitrogen dioxide react with MEA to form heat-stable salts, thus reducing the $\mathrm{CO}_{2}$ absorption capacity of the solvent. However, proprietary inhibitors are available that can render the solvent tolerant to oxygen. Flue gas $\mathrm{NO}_{\mathrm{x}}$ is not a major problem since the predominant form $(\sim 95 \%)$ of total $\mathrm{NO}_{\mathrm{x}}$ in the flue gas is nitric oxide (NO), which does not react with inhibited amines. But $\mathrm{SO}_{2}$ and $\mathrm{NO}_{2}$ do react to degenerate MEA solvent, so very low inlet concentrations of these gases (on the order of $10 \mathrm{ppm}$ ) are desirable to avoid excessive loss of solvent. Since the untreated flue gases of coal-fired power plants typically contain about 700 to $2500 \mathrm{ppm} \mathrm{SO}_{2}$ (plus roughly 10-40 ppm $\mathrm{NO}_{2}$ ), the interaction of $\mathrm{SO}_{2}$ with the $\mathrm{CO}_{2}$ control system is particularly important. The heat-stable salts that are formed in these reactions may be treated in a side-stream MEA reclaimer, which can regenerate some of the MEA. Technologies such as electrodialysis are also being proposed for this purpose.

Finally, corrosion control is very important in amine systems, especially when processing oxygen-containing gases. In order to reduce corrosion rates, corrosion inhibitors, low concentrations of MEA, appropriate materials of construction and mild operating conditions are required.

A key feature of amine systems is the large amount of heat required to regenerate the solvent. This heat is typically drawn from the steam cycle and significantly reduces the net efficiency of the power plant. Substantial electrical energy also is needed to compress the captured $\mathrm{CO}_{2}$ for pipeline transport to a storage site. As shown later in this paper, the overall energy penalty of this process has a major impact on system performance as well as cost.

From a multi-pollutant perspective, there are also important interactions between the $\mathrm{CO}_{2}$ capture system and the control of other air pollutants, especially $\mathrm{SO}_{2}$ and $\mathrm{NO}_{\mathrm{x}}$ emissions. Acid gases like $\mathrm{SO}_{2}$ and $\mathrm{NO}_{2}$ react with MEA to form heat-stable salts that reduce the $\mathrm{CO}_{2}$ absorption capacity of the solvent. Thus, very low concentrations of these gases (on 
the order of $10 \mathrm{ppm}$ ) are desirable to avoid excessive loss of (costly) solvent. The problem is especially acute for $\mathrm{SO}_{2}$ because its concentration in flue gases is typically 700 to $2500 \mathrm{ppm}$ at coal-fired plants. $\mathrm{NO}_{\mathrm{x}}$ is less of a problem because most of the $\mathrm{NO}_{\mathrm{x}}$ is nitric oxide (NO), whereas only $\mathrm{NO}_{2}$ (typically about $5 \%$ of total $\mathrm{NO}_{\mathrm{x}}$ ) is reactive (see Supplementary Information for additional details).

\section{Part 2: Current Amine Systems}

To begin with, please consider a $\mathrm{CO}_{2}$ capture system which:

- uses 30\% (by weight) MEA-based sorbent,

- treats the flue gas stream from a coal-combustion source which has a $\mathrm{CO}_{2}$ concentration of about $12 \%$ (by volume) and which has been pre-treated for removal of $\mathrm{SO}_{\mathrm{x}}$ and $\mathrm{NO}_{\mathrm{x}}$ and cooled to a desired absorber inlet temperature,

- $\quad$ and removes $90 \%$ of $\mathrm{CO}_{2}$ from the flue gas stream.

In the table that follows we have summarized the base case key parameters that we ask you to comment upon. These parameters are intended to characterize the performance of a current commercial system as system described above and in the supplemental information given earlier.

Please indicate whether you find each of these nominal values and ranges to be reasonable. If not, please indicate the value you would prefer and provide us with a brief explanation. If you prefer to use different units than the ones shown here, please indicate clearly the measure that you prefer.

\begin{tabular}{|c|c|c|c|}
\hline Parameter & $\begin{array}{l}\text { Accept- } \\
\text { ability }\end{array}$ & $\begin{array}{l}\text { If not OK, } \\
\text { New Value }\end{array}$ & Explanation of change \\
\hline \multicolumn{4}{|l|}{ Absorber inlet flue gas pressure } \\
\hline $\begin{aligned} \text { Nominal value } & =26 \mathrm{kPa} \text { gauge } \\
& =3.8 \mathrm{psig}\end{aligned}$ & $\begin{array}{l}\text { o OK } \\
\text { o not OK }\end{array}$ & & \\
\hline $\begin{aligned} \text { Range } & =14-30 \mathrm{kPa} \text { gauge } \\
& =2-4.4 \mathrm{psig}\end{aligned}$ & $\begin{array}{l}\text { o OK } \\
\text { o not OK }\end{array}$ & & \\
\hline \multicolumn{4}{|c|}{ Temperature of flue gas entering the absorber } \\
\hline $\begin{aligned} \text { Nominal value } & =60 \operatorname{deg} \mathrm{C} \\
& =140 \mathrm{deg} \mathrm{F}\end{aligned}$ & $\begin{array}{l}\text { o OK } \\
\text { o not OK }\end{array}$ & & \\
\hline $\begin{aligned} \text { Range } & =50-62 \operatorname{deg} \mathrm{C} \\
& =122-144 \operatorname{deg} \mathrm{F}\end{aligned}$ & $\begin{array}{l}\text { o OK } \\
\text { o not OK }\end{array}$ & & \\
\hline \multicolumn{4}{|c|}{ Lean sorbent $\mathrm{CO}_{2}$ loading (mole $\mathrm{CO}_{2} /$ mole sorbent) } \\
\hline $\begin{array}{l}\text { Nominal value }=0.2 \\
=7.3 \times 10^{-3} \mathrm{SCF} \mathrm{CO}_{2} / \text { gal soln }\end{array}$ & $\begin{array}{l}\text { o OK } \\
\text { o not OK }\end{array}$ & & \\
\hline $\begin{array}{l}\text { Range }=0.15-0.25 \\
=5.5-9.1 \times 10^{-3} \mathrm{SCF} \mathrm{CO}_{2} / \mathrm{gal}\end{array}$ & $\begin{array}{l}\text { o OK } \\
\text { o not OK }\end{array}$ & & \\
\hline
\end{tabular}




\begin{tabular}{|c|c|c|c|}
\hline Parameter & $\begin{array}{l}\text { Accept- } \\
\text { ability }\end{array}$ & $\begin{array}{l}\text { If not } O K \text {, } \\
\text { New Value }\end{array}$ & Explanation of change \\
\hline \multicolumn{4}{|c|}{ Heat required for sorbent regeneration $(\mathrm{kJ} / \mathrm{kg} \mathrm{CO}$ ) } \\
\hline Nominal value $=4350$ & o OK & & \\
\hline$=2.2 \mathrm{lb}$ steam $/ \mathrm{lb} \mathrm{CO}_{2}$ & o not OK & & \\
\hline Range $=3500-6000$ & o OK & & \\
\hline$=1.75-3.00 \mathrm{lb} \mathrm{steam} / \mathrm{lb} \mathrm{CO}_{2}$ & o not $\mathrm{OK}$ & & \\
\hline \multicolumn{4}{|c|}{ Allowable levels of other components in flue gas } \\
\hline $\mathrm{SO}_{2}<10 \mathrm{ppmv}$ & $\begin{array}{l}\text { o OK } \\
\text { o not OK }\end{array}$ & & \\
\hline $\mathrm{NO}_{2}<10 \mathrm{ppmv}$ & $\begin{array}{l}\text { o OK } \\
\text { o not OK }\end{array}$ & & \\
\hline $\mathrm{O}_{2} \sim 3.5 \%$ by volume & $\begin{array}{l}\text { o OK } \\
\text { o not OK }\end{array}$ & & \\
\hline \multicolumn{4}{|c|}{ Maximum train size (tonnes $\mathrm{CO}_{2}$ per day) } \\
\hline Nominal value $=5000$ & $\begin{array}{l}\text { o OK } \\
\text { o not OK }\end{array}$ & & \\
\hline \multicolumn{4}{|c|}{ Energy required for $\mathrm{CO}_{2}$ compression to $2000 \mathrm{psig}\left(\mathrm{kWh} /\right.$ tonne $\left.\mathrm{CO}_{2}\right)$} \\
\hline Nominal value $=119$ & $\begin{array}{l}\text { o OK } \\
\text { o not OK }\end{array}$ & & \\
\hline Range $=(112,145)$ & $\begin{array}{l}\text { o OK } \\
\text { o not OK }\end{array}$ & & \\
\hline
\end{tabular}




\section{Part 3: Judgments About Future Amine Systems}

In this section we would like to obtain your technically informed probabilistic judgments about several key parameters of a future amine-based $\mathrm{CO}_{2}$ capture system built around the year 2015. We would like you to consider all of the parameters listed, but feel free to skip any parts that you are not comfortable with. Also, if you prefer to use some different unit of measurement in your answer, please mention it clearly.

In producing your answers please assume that:

- We are still talking about an amine-based plant that treats the flue gas stream from a coalcombustion source, which is about $12 \%, \mathrm{CO}_{2}$ and which has been pre-treated for removal of $\mathrm{SO}_{\mathrm{x}}$ and $\mathrm{NO}_{\mathrm{x}}$, and removes $90 \%$ of $\mathrm{CO}_{2}$ from the flue gas stream.

- The plant has been optimized for the lowest overall cost of $\mathrm{CO}_{2}$ avoidance ( $\$$ / tonne $\mathrm{CO}_{2}$ avoided), considering both capital and operating costs (including energy costs) over the life of the plant.

- $R \& D$ support for this technology continues to steadily grow at a modest rate through 2015, and includes several new large-scale applications to coal-fired power plants. 


\section{Parameter 1: Amine sorbent concentration (wt \%)}

First please give us your highest estimate of the value that you think that amine sorbent concentration might be in state-of-the-art operating plants by 2015 .

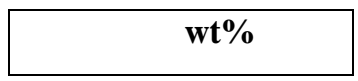

Suppose that we have a clairvoyant who can look ahead to 2015 and tells you that the actual number is 1.15 times this value. Can you think of any plausible story of how that might have happened?

$$
\text { o No o Yes }
$$

If you answered yes, briefly what is the story:

Is there at least a $5 \%$ probability that this story could turn out to be true?

o No o Yes

Next we'd like your lowest estimate of the value that you think that sorbent concentration might be in stateof-the-art operating plants by 2015.

$$
\text { wt } \%
$$

Again, suppose that we have a clairvoyant who can look ahead to 2015 and tells you that the actual number is 0.85 times this value. Can you think of any plausible story of how that might have happened?

$$
\text { o No o Yes }
$$

If you answered yes, briefly what is the story:

Is there at least a $5 \%$ probability that this story could turn out to be true?

$$
\text { o No o Yes }
$$

Finally we'd like your best estimate of the value that you think that average sorbent concentration might be in state-of-the-art operating plants by 2015.

\section{wt \%}

\section{Parameter 2: Unit heat requirement for sorbent regeneration}

First please give us your lowest estimate of the value that you think that sorbent regeneration heat requirement might be in state-of-the-art operating plants by 2015 .

\section{$\mathrm{kJ} / \mathrm{kg} \mathrm{CO}$ captured (or $\quad \mathrm{lb}$ steam/ $\mathrm{lb} \mathrm{CO}_{2}$ captured)}


Suppose that we have a clairvoyant who can look ahead to 2015 and tells you that the actual number is 0.85 times this value. Can you think of any plausible story of how that might have happened?

$$
\text { o No o Yes }
$$

If you answered yes, briefly what is the story:

Is there at least a $5 \%$ probability that this story could turn out to be true?

$$
\text { o No o Yes }
$$

Next we'd like your highest estimate of the value that you think that sorbent regeneration heat requirement might be in state-of-the-art operating plants by 2015 .

\section{$\mathrm{kJ} / \mathrm{kg} \mathrm{CO}$ captured (or $\quad \mathrm{lb}$ steam/ $\mathrm{lb} \mathrm{CO} \mathrm{CO}_{2}$ captured)}

Again, suppose that we have a clairvoyant who can look ahead to 2015 and tells you that the actual number is 1.15 times this value. Can you think of any plausible story of how that might have happened?

$$
\text { o No o Yes }
$$

If you answered yes, briefly what is the story:

Is there at least a $5 \%$ probability that this story could turn out to be true?

$$
\text { o No o Yes }
$$

Finally we'd like your best estimate of the value that you think that sorbent regeneration heat requirement might be in state-of-the-art operating plants by 2015 .

$\mathrm{kJ} / \mathrm{kg} \mathrm{CO}_{2}$ captured (or $\quad \mathrm{lb}$ steam/ $\mathrm{lb} \mathrm{CO}_{2}$ captured)

\section{Parameter 3: Nominal sorbent loss (kg sorbent / tonne $\mathrm{CO}_{2}$ captured)}

First please give us your lowest estimate of the value that you think that nominal sorbent loss might be in state-of-the-art operating plants by 2015.

\section{$\mathrm{kg} /$ tonne $\mathrm{CO}_{2}$ captured}

Suppose that we have a clairvoyant who can look ahead to 2015 and tells you that the actual number is 0.85 times this value. Can you think of any plausible story of how that might have happened?

$$
\text { o No o Yes }
$$

If you answered yes, briefly what is the story:

Is there at least a $5 \%$ probability that this story could turn out to be true? 
o No o Yes

Next we'd like your highest estimate of the value that you think that nominal sorbent loss might be in stateof-the-art operating plants by 2015 .

\section{$\mathrm{kg} /$ tonne $\mathrm{CO}_{2}$ captured}

Again, suppose that we have a clairvoyant who can look ahead to 2015 and tells you that the actual number is 1.15 times this value. Can you think of any plausible story of how that might have happened?

$$
\text { o No o Yes }
$$

If you answered yes, briefly what is the story:

Is there at least a $5 \%$ probability that this story could turn out to be true?

$$
\text { o No o Yes }
$$

Finally we'd like your best estimate of the value that you think that average nominal sorbent loss might be in state-of-the-art operating plants by 2015 .

\section{$\mathrm{kg}$ / tonne $\mathrm{CO}_{2}$ captured}

\section{Parameter 4: Cost of sorbent (US\$ / tonne sorbent)}

First please give us your lowest estimate of the value that you think that unit cost of sorbent might be in state-of-the-art operating plants by 2015.

\section{US\$ / tonne sorbent}

Suppose that we have a clairvoyant who can look ahead to 2015 and tells you that the actual number is 0.85 times this value. Can you think of any plausible story of how that might have happened?

$$
\text { o No o Yes }
$$

If you answered yes, briefly what is the story:

Is there at least a $5 \%$ probability that this story could turn out to be true?

$$
\text { o No o Yes }
$$

Next we'd like your highest estimate of the value that you think that unit cost of sorbent might be in state-of-the-art operating plants by 2015 .

US\$ / tonne sorbent 
Again, suppose that we have a clairvoyant who can look ahead to 2015 and tells you that the actual number is 1.15 times this value. Can you think of any plausible story of how that might have happened?

$$
\text { o No o Yes }
$$

If you answered yes, briefly what is the story:

Is there at least a $5 \%$ probability that this story could turn out to be true?

$$
\text { o No o Yes }
$$

Finally we'd like your best estimate of the value that you think that average unit cost of sorbent might be in state-of-the-art operating plants by 2015 .

\section{US\$ / tonne sorbent}

\section{Parameter 5: Other Sorbents}

The preceding questions have all focused on amine-based sorbents. Today there is much research being directed at developing alternative sorbents for post-combustion $\mathrm{CO}_{2}$ capture from flue gas. How likely do you think it is that by 2015 there will be some other commercially available sorbent (other than advanced amines) that significantly out-performs amine-based sorbents in $\mathrm{CO}_{2}$ capture from flue gas and which is economically competitive? (please mark an $\underline{X}$ on the line)

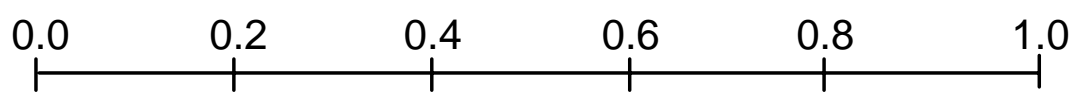

If your answer was less than 0.5 , do you think that some other commercially available and economically competitive sorbent will become available some time after 2015 ?

o No

o Yes in approximately the year 20 
The next page addresses a parameter related to $\mathrm{CO}_{2}$ compression. Please address this too if you can. You may assume that the product $\mathrm{CO}_{2}$ flow rate is about 400 tonne $\mathrm{CO}_{2} / \mathrm{hr}$ (with $99.8 \%$ purity). 


\section{Parameter 6: Energy required for $\mathrm{CO}_{2}$ Compression}

First please give us your highest estimate of the value that you think that energy requirement for $\mathrm{CO}_{2}$ compression might be in state-of-the-art operating plants by 2015 .

$\mathbf{k W h} /$ tonne $\mathrm{CO}_{2}$, or compressor efficiency $=\quad \%$

Suppose that we have a clairvoyant who can look ahead to 2015 and tells you that the actual number is 1.15 times this value. Can you think of any plausible story of how that might have happened?

o No o Yes

If you answered yes, briefly what is the story:

Is there at least a $5 \%$ probability that this story could turn out to be true?

o No o Yes

Next we'd like your lowest estimate of the value that you think that energy requirement for $\mathrm{CO}_{2}$

compression might be in state-of-the-art operating plants by 2015 .

$\mathrm{kWh} /$ tonne $\mathrm{CO}_{2}$, or compressor efficiency =

$\%$

Again, suppose that we have a clairvoyant who can look ahead to 2015 and tells you that the actual number is 0.85 times this value. Can you think of any plausible story of how that might have happened?

o No o Yes

If you answered yes, briefly what is the story:

Is there at least a $5 \%$ probability that this story could turn out to be true?

o No o Yes

Finally we'd like your best estimate of the value that you think that average energy requirement for $\mathrm{CO}_{2}$ compression might be in state-of-the-art operating plants by 2015 .

\begin{tabular}{|ll|}
\hline $\mathrm{kWh} /$ tonne $\mathrm{CO}_{2}$, or compressor efficiency $=$ & $\%$ \\
\hline
\end{tabular}

Part 4: Judgments About Overall Plant Performance and Cost

In the following questions we want your comparative judgments about the overall performance and cost of a state-of-the-art operating coal-fired power plant in the year 2015, with and without amine-based $\mathrm{CO}_{2}$ capture system. Please keep in mind that the terms "reference plant" and "capture plant" refer to the following:

Reference Plant: 
- New low-S coal-fired power plant with environmental control units (ESP, FGD and SCR)

- $\quad$ Net generation $=500 \mathrm{MW}$

\section{Capture Plant:}

- New low-S coal-fired power plant (similar to the reference plant) with environmental control units (ESP, FGD and SCR)

- Amine-based $\mathrm{CO}_{2}$ capture system to capture $90 \% \mathrm{CO}_{2}$ from the power plant flue gas stream

- The product $\mathrm{CO}_{2}$ is compressed to $2000 \mathrm{psig}$ (for transport to a disposal site)

- Net power generation $=500 \mathrm{MW}$

- Costs of $\mathrm{CO}_{2}$ transport and storage are not included

Note: In questions about plant cost, you should also assume:

- A mid-western US plant location

- Total capital requirement (TCR) includes interest during construction and other owner's costs

- The cost of fuel is US\$1.2/GJ (HHV basis)

- Currency is current US dollars (US\$)

Again, feel free to skip any question you're not comfortable expressing a judgment about. 


\section{Parameter 7: Net Plant Efficiency}

Consider the reference plant as described on the previous page.

First please give us your highest estimate of the value that you think that the net efficiency (HHV basis) of this reference plant might be in 2015:

\section{$\%$}

Next we'd like your lowest estimate of the value that you think that the net efficiency (HHV basis) of this reference plant might be in 2015:

$\%$

Finally we'd like your best estimate of the value that you think that average net efficiency (HHV basis) of this reference plant might be in 2015.

\section{$\%$}

Now consider the capture plant as described on the previous page.

First please give us your highest estimate of the value that you think that the net efficiency (HHV basis) of this $\mathrm{CO}_{2}$ capture plant might be in 2015:

\% efficiency points lost, or

$\%$ net efficiency

Next we'd like your lowest estimate of the value that you think that the net efficiency (HHV basis) of this $\mathrm{CO}_{2}$ capture plant might be in 2015 :

$\%$ efficiency points lost, or

$\%$ net efficiency

Finally we'd like your best estimate of the value that you think that average net efficiency (HHV basis) of this $\mathrm{CO}_{2}$ capture plant might be in 2015 .

\% efficiency points lost, or

$\%$ net efficiency 


\section{Parameter 8: Total Capital Requirement}

Consider the reference plant as described on the previous page.

First please give us your highest estimate of the value that you think that the total capital requirement of this reference plant might be in 2015:

\begin{tabular}{|c|}
\hline M (or \\
\hline$\left./ \mathbf{k W}_{\text {net }}\right)$ \\
\hline
\end{tabular}

Next we'd like your lowest estimate of the value that you think that the total capital requirement of this reference plant might be in 2015:

M\$ (or $\quad \$ / \mathbf{k W}_{\text {net }}$ )

Finally we'd like your best estimate of the value that you think that average total capital requirement of this reference plant might be in 2015.

M\$ (or $\$ / \mathbf{k W}_{\text {net }}$ )

Now consider the capture plant as described on the previous page.

First please give us your highest estimate of the value that you think that the total capital requirement of this $\mathrm{CO}_{2}$ capture plant might be in 2015:

M\$ (or $\left.\$ / \mathbf{k W}_{\text {net }}\right)$

Next we'd like your lowest estimate of the value that you think that the total capital requirement of this $\mathrm{CO}_{2}$ capture plant might be in 2015:

M\$ (or $\left.\$ / \mathbf{k W}_{\text {net }}\right)$

Finally we'd like your best estimate of the value that you think that total capital requirement of this $\mathrm{CO}_{2}$ capture plant might be in 2015 .

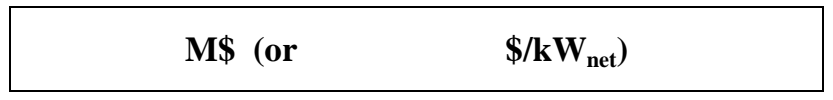

\section{Parameter 9: Cost of Electricity}

Consider the reference plant as described on the previous page.

First please give us your highest estimate of the value that you think that the cost of electricity generated from this reference plant might be in 2015: 


\begin{tabular}{|ll|}
\hline \$MWh (or $\quad$ cents/kWh) \\
\hline
\end{tabular}

Next we'd like your lowest estimate of the value that you think that the cost of electricity generated from this reference plant might be in 2015:

\begin{tabular}{|ll|}
\hline \$MWh (or $\quad$ cents/kWh) \\
\hline
\end{tabular}

Finally we'd like your best estimate of the value that you think that average cost of electricity generated from this reference plant might be in 2015.

\begin{tabular}{|l|l|}
\hline \$MWh (or $\quad$ cents/kWh) \\
\hline
\end{tabular}

\section{Did you have a particular capacity factor (load factor) and capital charge factor (fixed charge factor) in mind for these estimates? If so, what are they:}

\begin{tabular}{|l|l|}
\hline Capacity factor & $\%($ or $\quad$ hours/yr) \\
\hline Capital charge factor & $\%$ (or plant life $=$ yr \& $i=\quad \%)$ \\
\hline
\end{tabular}

Now consider the capture plant as described on the previous page.

First please give us your highest estimate of the value that you think that the cost of electricity generated from this plant might be in 2015:

\begin{tabular}{|ll|}
\hline \$MWh (or & cents/kWh) \\
\hline
\end{tabular}

Next we'd like your lowest estimate of the value that you think that the cost of electricity generated from this $\mathrm{CO}_{2}$ capture plant might be in 2015 :

\begin{tabular}{|ll|}
\hline \$MWh (or & cents/kWh) \\
\hline
\end{tabular}

Finally we'd like your best estimate of the value that you think that cost of electricity generated from this $\mathrm{CO}_{2}$ capture plant might be in 2015 .

\begin{tabular}{|ll|}
\hline \$MWh (or & cents/kWh) \\
\hline
\end{tabular}

Did you have a particular capacity factor (load factor) and capital charge factor (fixed charge factor) in mind for these estimates? If so, what are they:

\begin{tabular}{|l|l|}
\hline Capacity factor & $\%$ (or $\quad$ hours/yr) \\
\hline Capital charge factor & $\%$ (or plant life $=$ yr \& $i=\quad \%)$ \\
\hline
\end{tabular}




\section{Part 5: Research Priorities}

Today there is an ongoing effort worldwide to bring down the cost of $\mathrm{CO}_{2}$ capture and sequestration from flue gas streams. The factors affecting this cost may be categorized as:

a) factors related to the "base" power plant (including other environmental control units)

b) factors related to the amine-based $\mathrm{CO}_{2}$ capture technology

c) factors related to the post-capture processing of the concentrated $\mathrm{CO}_{2}$ stream

On the following pages you will find a list of research objectives that may help reduce the overall cost of $\mathrm{CO}_{2}$ capture and sequestration from flue gas streams. We would like to know how you would prioritize R\&D efforts in these areas.

You may also add more items to this list (items D1, D2 etc.), and take them into consideration while going through the ranking exercise.

Initially, please indicate your priorities as:
H: High priority
M: Medium priority
L: $\underline{\text { Low priority }}$

Finally, please further prioritize your preferences on the same page, by putting a rank/number against each High Priority item, taking into account the potential effectiveness of these factors. (You may give the same rank/number to two or more items if you think they are equally preferred ). Please note that rank 1 indicates the highest priority. 


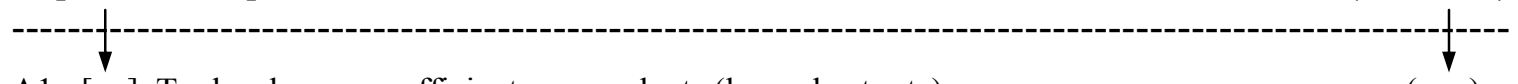

A1: [ ] To develop more efficient power plants (lower heat rate)

A2: [ ] To improve boiler designs so that fuel can be burned with lower excess air (typically for a coal plant, $\sim 20 \%$ excess air is used)

A3: [ ] To develop more efficient technologies for $\mathrm{SO}_{\mathrm{x}}$ and $\mathrm{NO}_{\mathrm{x}}$ control so as to reduce the acidic gas impurities in the flue gas stream

A4: [ ] To improve heat integration within the power plants to reduce the energy penalty for $\mathrm{CO}_{2}$ capture due to steam extraction for sorbent regeneration

B1: [ ] To develop more efficient fans for flue gas handling

B2: [ ] To develop $\mathrm{CO}_{2}$ absorbers that can handle higher sorbent concentrations

B3: [ ] To develop sorbents with higher $\mathrm{CO}_{2}$ loading capacity

B4: [ ] To develop sorbents with lower regeneration energy requirement

B5: [ ] To develop absorber columns offering lower pressure drops

( )

B6: [ ] To develop absorber columns offering higher $\mathrm{CO}_{2}$ capture efficiencies

B7: [ ] To develop more efficient pumps for sorbent circulation

B8: [ ] To develop more efficient heat exchanging devices

( )

B9: [ ] To reduce the cost of sorbent manufacturing

B10:[ ] To develop sorbents with lower makeup requirements (less losses)

B11:[ ] To develop less expensive technologies for disposal of spent sorbents

B12:[ ] To develop better instrumentation/ automation in the $\mathrm{CO}_{2}$ capture system so as to reduce the labor requirement

B13:[ ] To develop better construction materials so as to reduce the losses due to corrosion

C1: [ ] To develop more efficient compressors for $\mathrm{CO}_{2}$ compression

C2: [ ] To develop a transport technology that can handle low-pressure $\mathrm{CO}_{2}$ streams

C3: [ ] To develop less expensive technologies for $\mathrm{CO}_{2}$ storage/ disposal 


\title{
AN INTEGRATED MODELING FRAMEWORK FOR CARBON MANAGEMENT TECHNOLOGIES
}

\author{
Volume 2 - IECM-cs User Manual: \\ Amine-Based $\mathrm{CO}_{2}$ Capture and Storage Systems \\ for Fossil Fuel Power Plant
}

Final Report of

Work Performed Under Contract No.: DE-FC26-00NT40935

Reporting Period Start, October 2000

Reporting Period End, December 2003

Report Submitted, March 2004

to

U.S. Department of Energy

National Energy Technology Laboratory

626 Cochrans Mill Road, P.O. Box 10940

Pittsburgh, Pennsylvania 15236-0940

by

Michael B. Berkenpas

John J. Fry

Karen Kietzke

Constance J. Zaremsky

Edward S. Rubin

Carnegie Mellon University

Center for Energy and Environmental Studies

Department of Engineering and Public Policy

Pittsburgh, PA 15213-3890 


\section{Disclaimer}

This report was prepared as an account of work sponsored by an agency of the United States Government. Neither the United States Government nor any agency thereof, nor any of their employees, makes any warranty, express or implied, or assumes any legal liability or responsibility for the accuracy, completeness, or usefulness of any information, apparatus, product, or process disclosed, or represents that its use would not infringe privately owned rights. Reference herein to any specific commercial product, process, or service by trade name, trademark, manufacturer, or otherwise does not necessarily constitute or imply its endorsement, recommendation, or favoring by the United States Government or any agency thereof. The views and opinions of authors expressed herein do not necessarily state or reflect those of the United States Government or any agency thereof. 


\section{Abstract}

This Integrated Environmental Control Model (IECM) and Interface were developed for the U. S. Department of Energy's National Energy Technology Laboratory (NETL), formerly known as the Federal Energy Technology Center (FETC), under contracts No. DE-AC22-92PC91346, DE-AC21-92MC29094, and DE-FC2600NT40935.

The purpose of the model is to calculate the performance, emissions and cost of employing alternative environmental control methods in a coal-fired power plant. The model consists of a base plant and various control technology modules; these modules may be implemented together in any desired combination.

A Graphical User Interface (GUI) facilitates the configuration of the technologies, entry of data, and retrieval of results. This User Manual gives information on both the interface and the underlying model. It provides detailed descriptions of plant configurations, parameter settings, and result screens. It also describes technical details behind the model's operation and includes an introduction to uncertainty analysis. 



\section{Contents}

Introduction $\quad 1$

The Integrated Environmental Control Model .......................................................................... 1

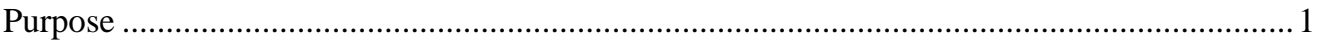

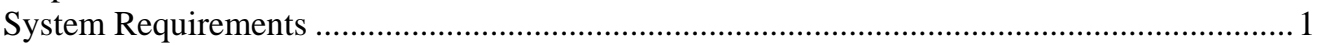

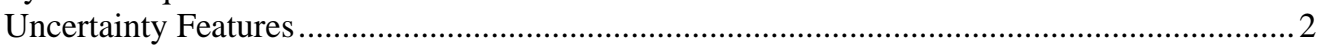

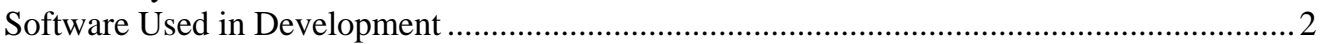

Disclaimer of Warranties and Limitation of Liabilities .............................................................ii

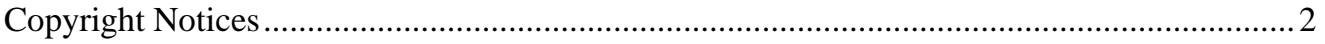

User Documentation and Help 5

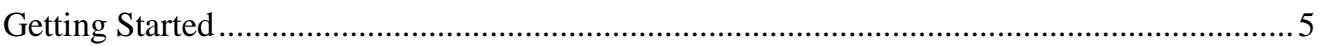

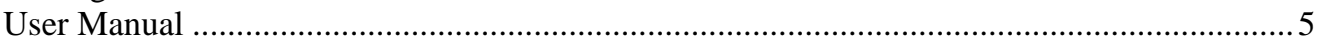

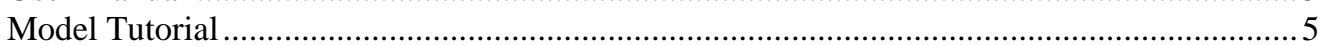

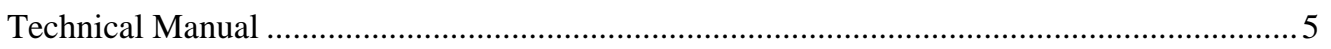

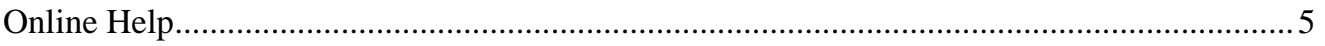

$\begin{array}{ll}\text { Installing the Model - Advanced } & 7\end{array}$

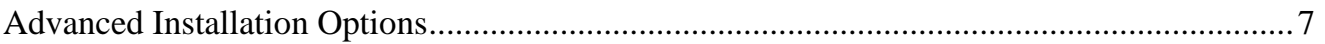

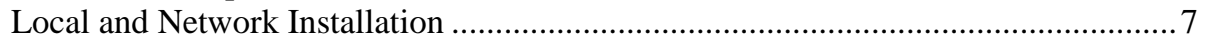

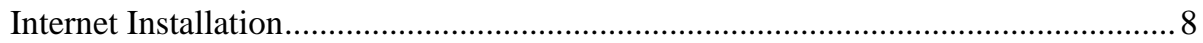

Changing the Destination Location ............................................................................ 10

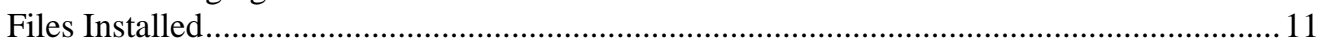

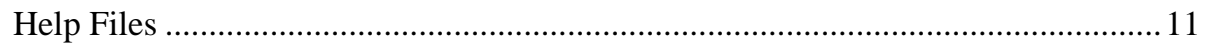

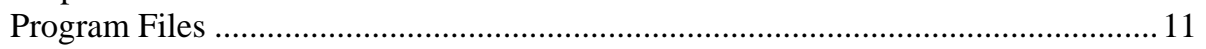

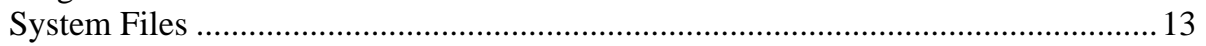

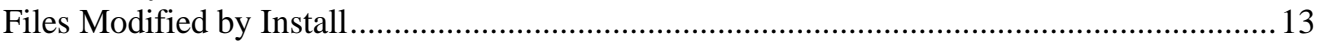

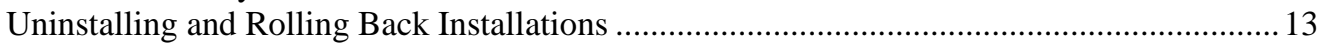

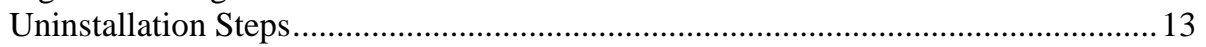

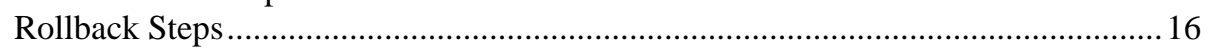

$\begin{array}{lr}\text { Configure Plant } & 19\end{array}$

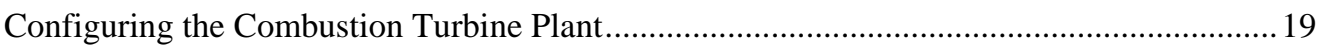

Configuring the Combustion Boiler Plant .................................................................................. 20

$\begin{array}{ll}\text { Overall Plant } & 23\end{array}$

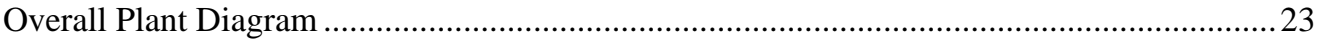

Overall Plant Performance .................................................................................................... 24

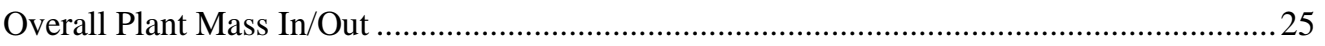

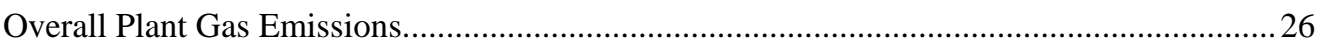

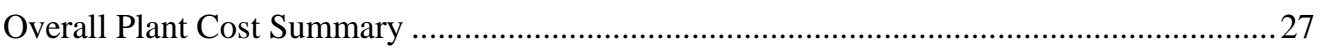




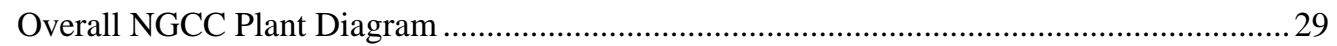

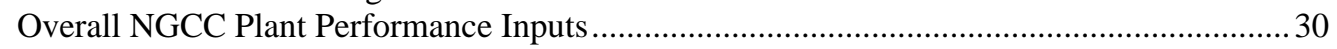

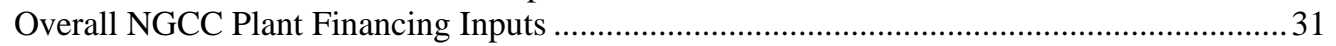

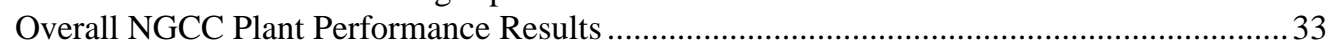

Overall NGCC Plant Mass In/Out Results............................................................................. 34

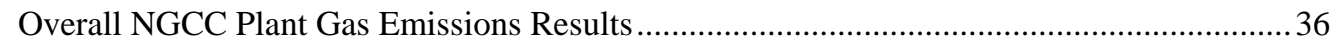

Overall NGCC Plant Cost Summary Results........................................................................... 37

$\begin{array}{ll}\text { Fuel } & 39\end{array}$

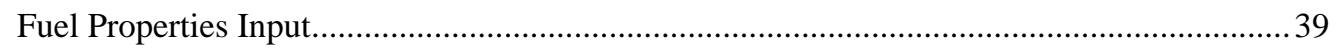

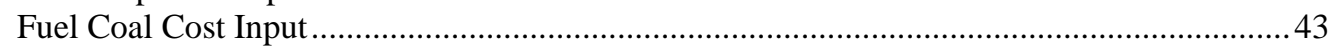

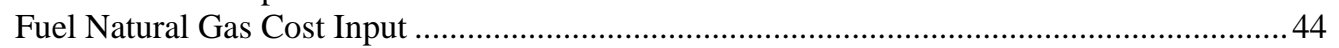

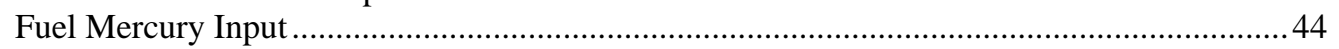

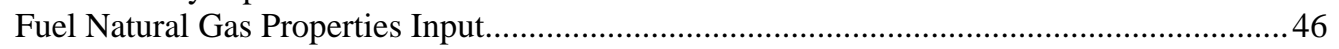

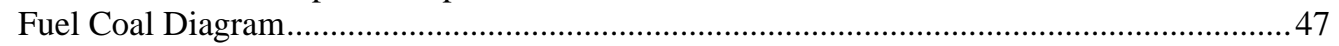

Fuel Natural Gas Diagram ........................................................................................... 48

$\begin{array}{ll}\text { Base Plant } & 49\end{array}$

Base Plant Furnace Factors Inputs ........................................................................................ 49

Base Plant Performance Inputs ............................................................................................. 51

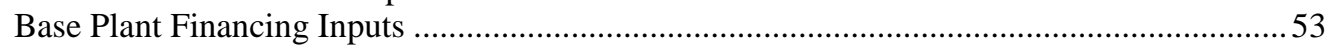

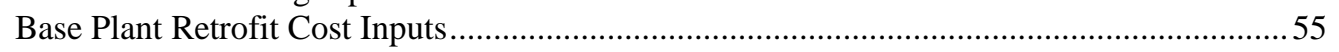

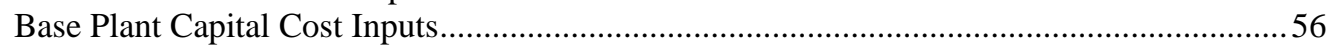

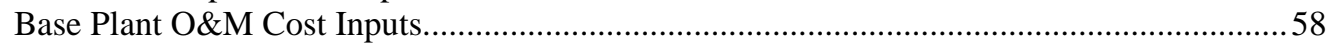

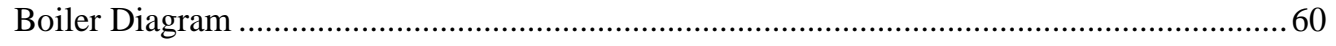

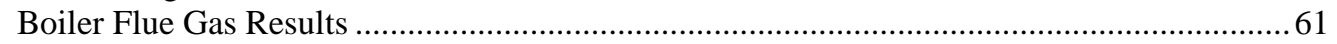

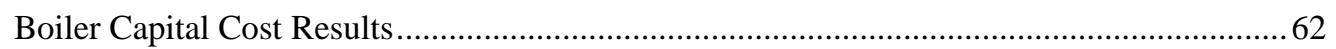

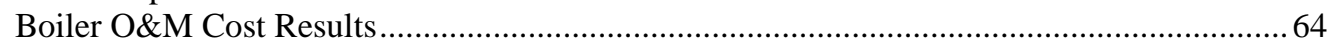

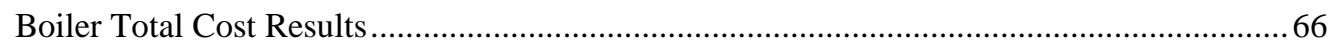

Emission Constraints $\quad 69$

Emission Constraints Performance Inputs ...............................................................................69

Emission Constraints Tax/Credit Inputs ……………............................................................... 71

$\begin{array}{ll}\text { Auxiliary Boiler } & 73\end{array}$

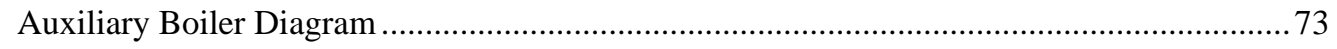

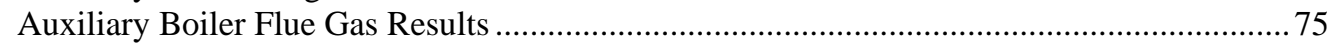

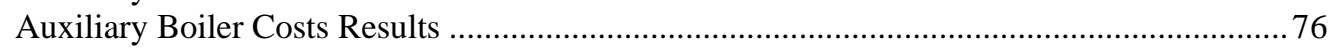

$\begin{array}{ll}\text { Air Preheater } & 77\end{array}$

Air Preheater Diagram .......................................................................................................... 77

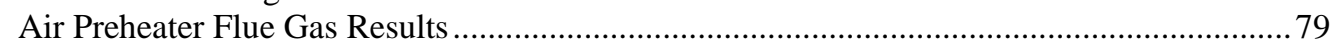

$\begin{array}{ll}\text { In-Furnace Controls } & \mathbf{8 1}\end{array}$

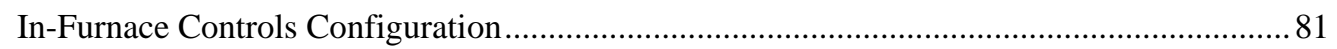

In-Furnace Controls Performance Input ............................................................................... 84

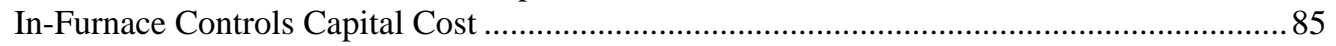

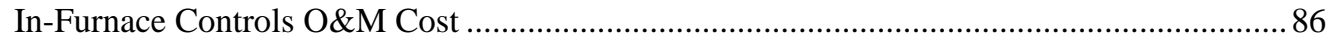

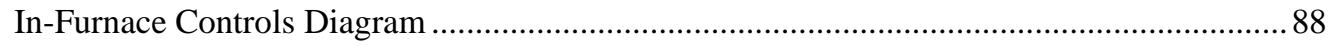

In-Furnace Controls Flue Gas Results .................................................................................... 90 
In-Furnace Controls Capital Cost Results...............................................................................91

In-Furnace Controls O\&M Cost Results..................................................................................... 92

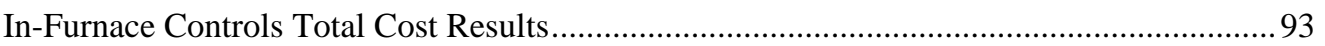

$\begin{array}{ll}\text { Hot-Side SCR } & 95\end{array}$

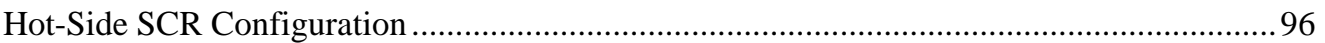

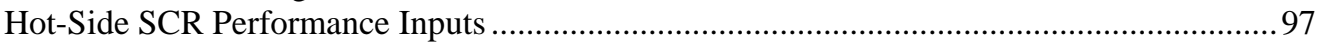

Hot-Side SCR Performance (Continued) .................................................................................... 99

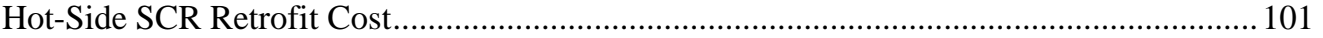

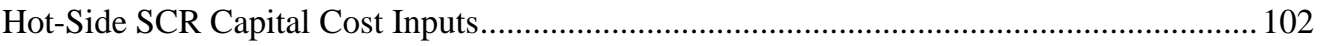

Hot-Side SCR O\&M Cost Inputs....................................................................................... 104

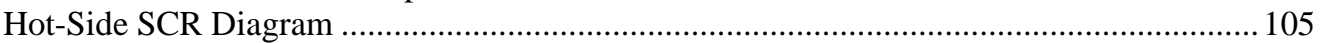

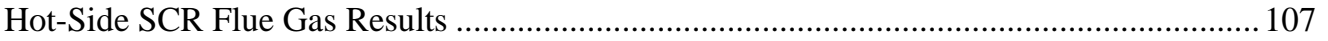

Hot-Side SCR Capital Cost Results ...................................................................................... 108

Hot-Side SCR O\&M Cost Results ......................................................................................110

Hot-Side SCR Total Cost Results ........................................................................................ 112

$\begin{array}{ll}\text { Mercury } & 113\end{array}$

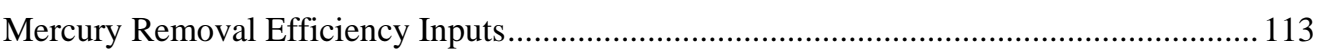

Mercury Carbon (and Water) Injection Inputs............................................................................. 115

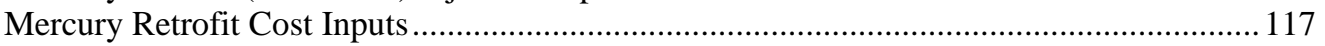

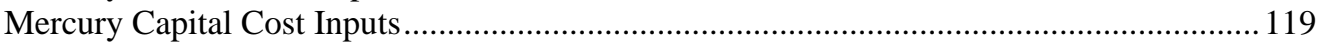

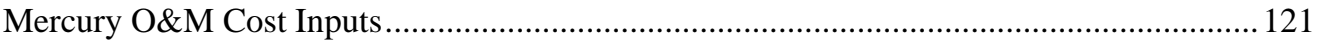

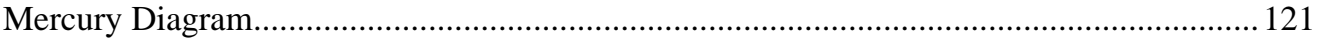

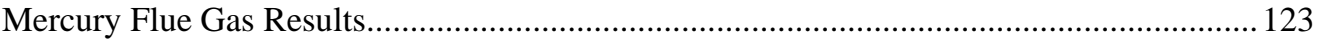

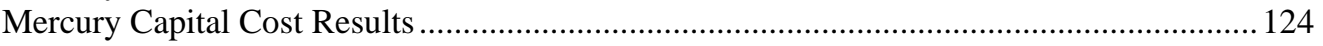

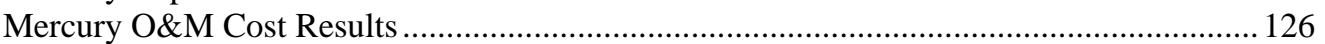

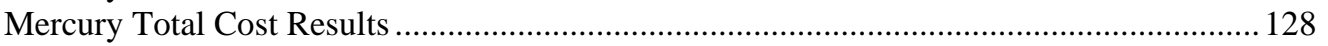

$\begin{array}{ll}\text { Cold-Side ESP } & 131\end{array}$

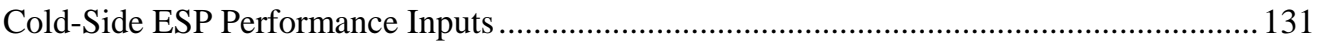

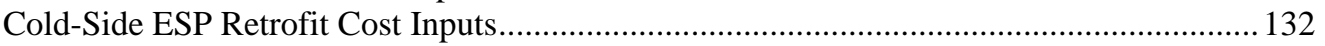

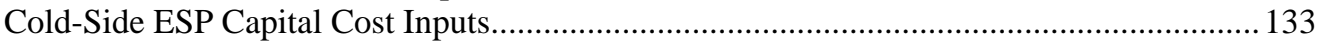

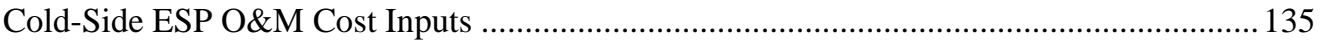

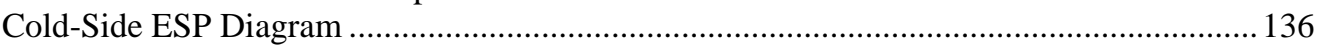

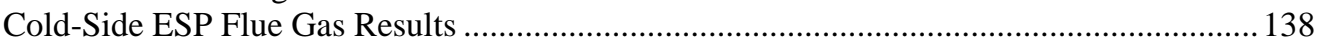

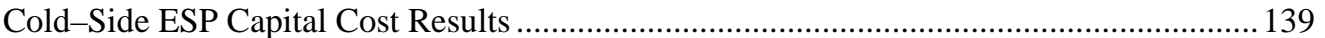

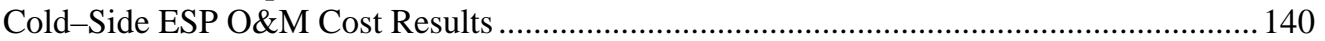

Cold-Side ESP Total Cost Results ......................................................................................... 142

$\begin{array}{ll}\text { Fabic Filter } & 145\end{array}$

Fabric Filter Configuration .............................................................................................. 145

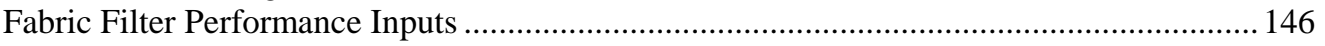

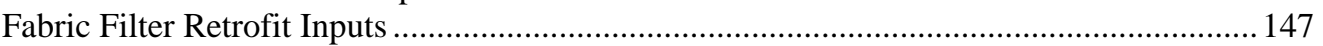

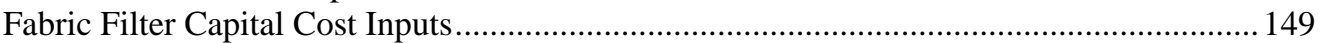

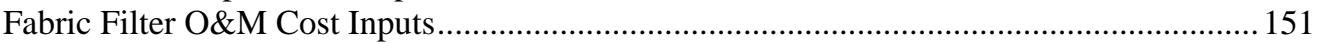

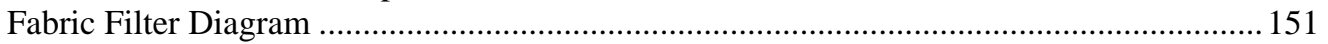

Fabric Filter Flue Gas Results .......................................................................................... 153

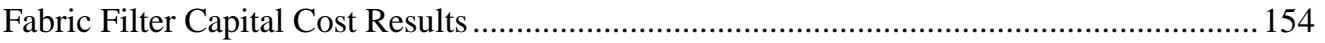

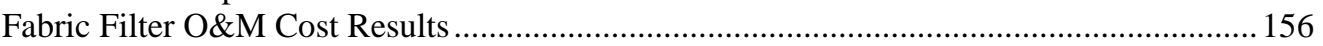

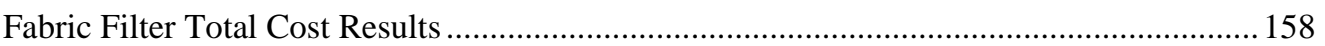

$\begin{array}{ll}\text { Wet FGD } & 161\end{array}$ 


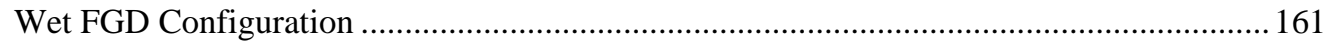

Wet FGD Performance Inputs ............................................................................... 162

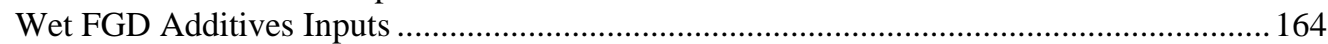

Wet FGD Retrofit Cost Inputs ......................................................................................... 165

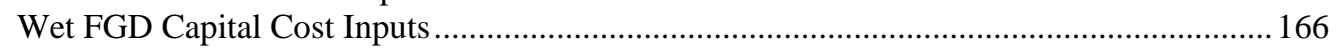

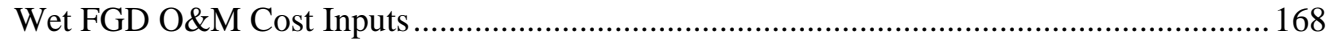

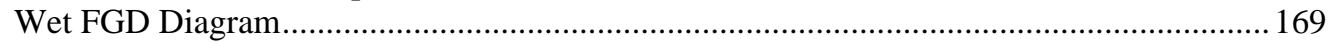

Wet FGD Flue Gas .................................................................................................. 170

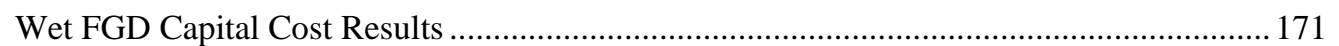

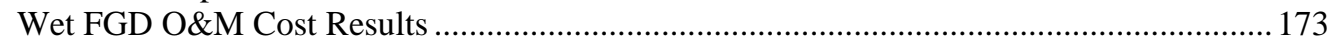

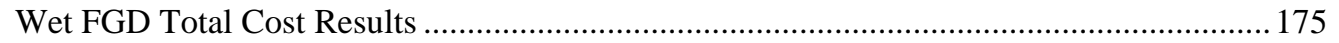

$\begin{array}{ll}\text { Spray Dryer } & 177\end{array}$

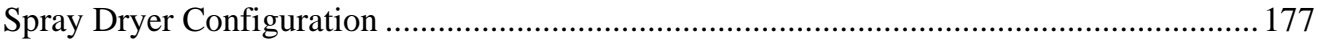

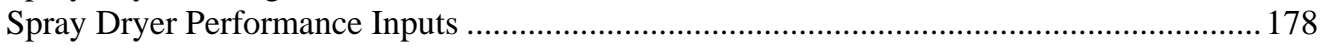

Spray Dryer Retrofit Cost ...................................................................................... 180

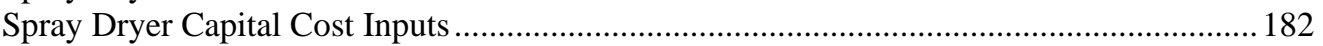

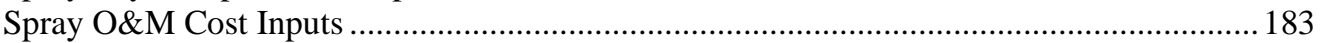

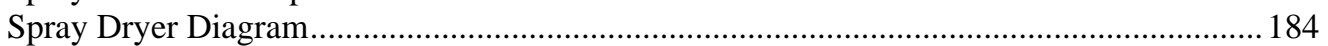

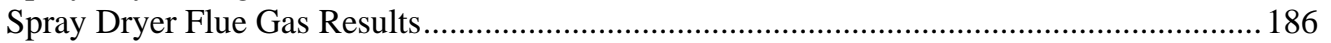

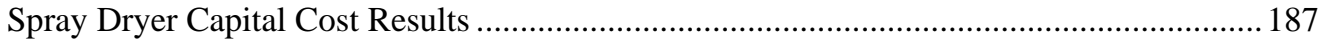

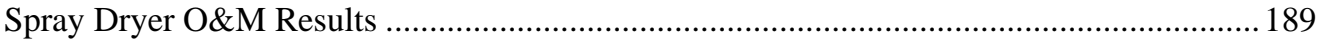

Spray Dryer Total Cost Results ............................................................................. 190

$\begin{array}{ll}\text { Power Block } & 193\end{array}$

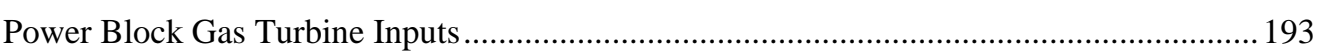

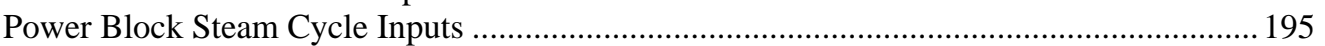

Power Block Emission Factors ........................................................................................ 196

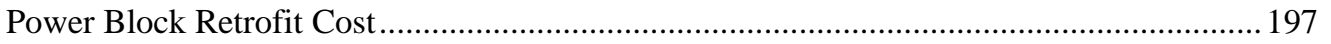

Power Block Capital Cost Inputs ............................................................................... 198

Power Block O\&M Cost Inputs ................................................................................ 199

Power Block HRSG/Steam Diagram ................................................................................ 200

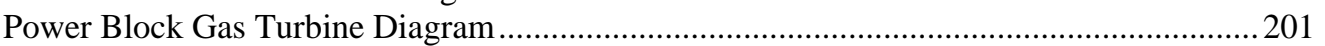

Power Block Syngas Results ........................................................................................ 202

Power Block Flue Gas Results................................................................................... 203

Power Block Capital Cost Results .............................................................................. 204

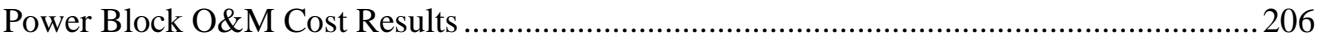

Power Block Total Cost Results ................................................................................. 207

$\begin{array}{ll}\text { Amine System } & 209\end{array}$

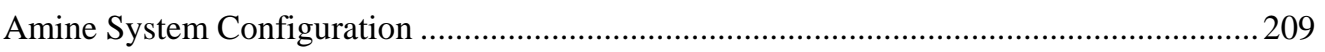

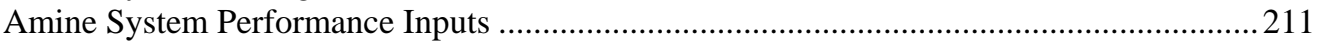

Amine System Capture Inputs ................................................................................... 213

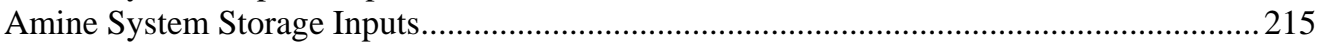

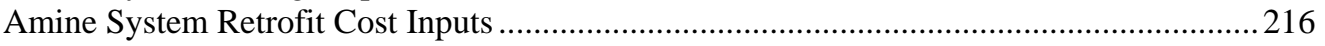

Amine System Capital Cost Inputs ........................................................................... 218

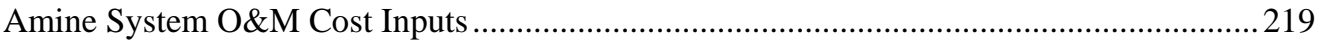

Amine System Diagram.......................................................................................... 221

Amine System Flue Gas Results ..................................................................................... 222

Amine System Capital Cost Results ............................................................................... 223

Amine System O\&M Cost Results .................................................................................. 226

Amine System Total Cost Results ............................................................................ 228

Amine System Cost Factors Results …................................................................... 229 
By Product Management Performance Inputs .................................................................. 233

By Product Management Sequestration Input ............................................................ 234

By Products Management Bottom Ash Pond Diagram.................................................... 234

By Products Management Flue Gas Treatment Diagram .................................................... 235

By Products Management Fly Ash Disposal Diagram ................................................. 237

By Products Management Geological Resevoir Diagram ................................................ 238

Stack

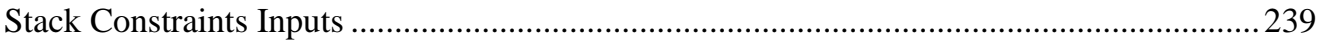

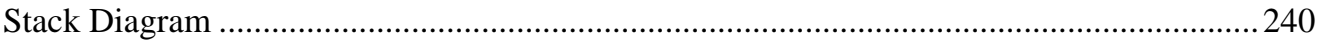

Stack Flue Gas Results ............................................................................................ 241

Units 243

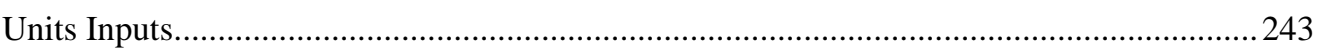

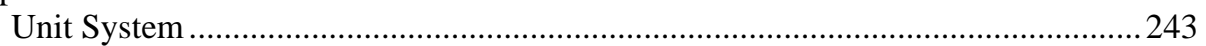

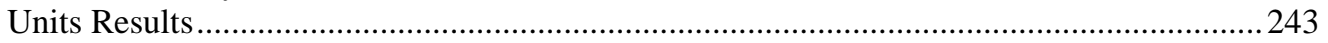

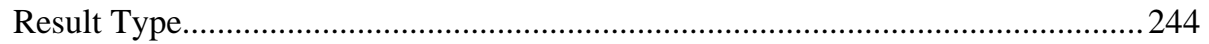

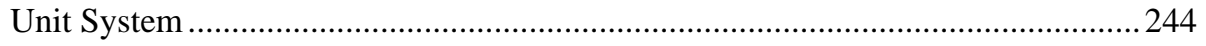

Time Period ..................................................................................................... 244

Performance Table ...................................................................................... 244

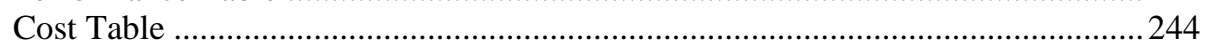

Cost Year......................................................................................................... 244

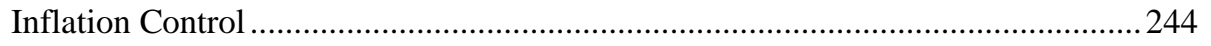

Working with Graphs $\quad 245$

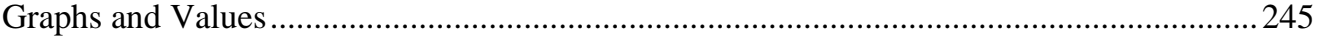

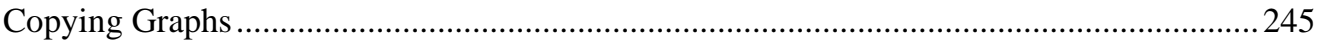

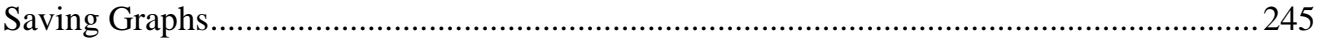

$\begin{array}{ll}\text { Running a Probabilistic Analysis } & 247\end{array}$

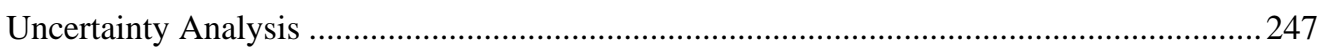

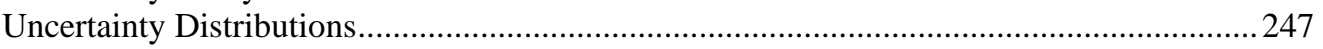

Uncertainty Parameters ................................................................................. 247

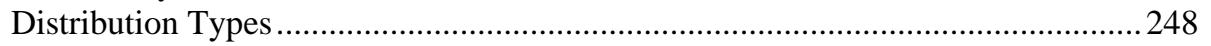

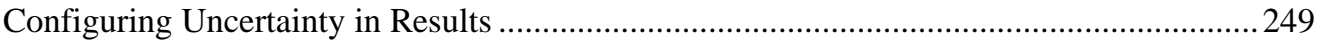

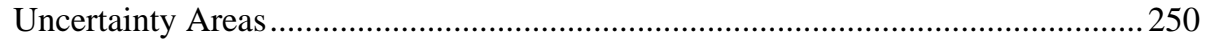

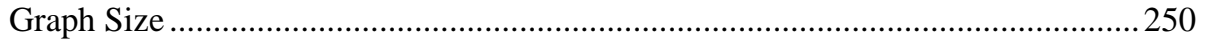

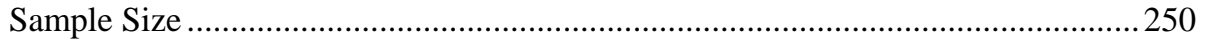

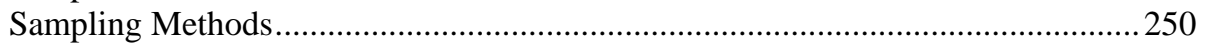

Appendix A - Introduction to Uncertainty Analysis 253

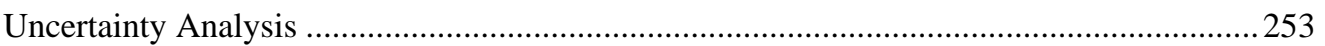

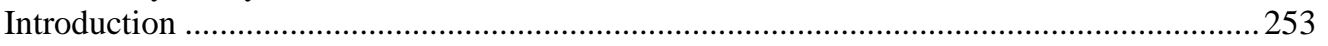

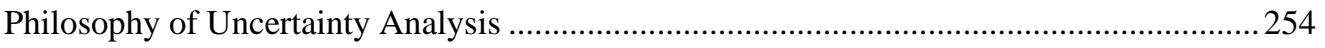

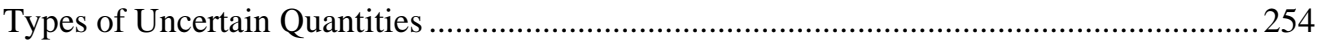

Encoding Uncertainties as Probability Distributions ..................................................... 254

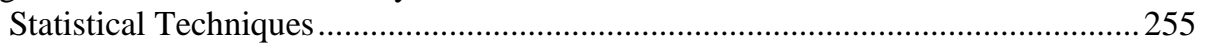

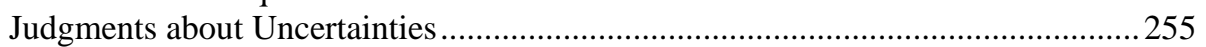

Designing an Elicitation Protocol ....................................................................................... 256

A Non-technical Example.................................................................................... 256 
A Technical Example ……………................................................................................ 257

Appendix B - Technical Support $\quad 259$

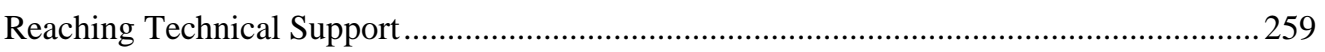

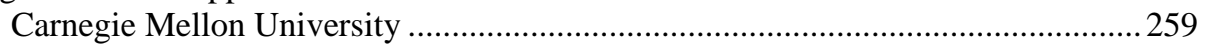




\section{Introduction}

\section{The Integrated Environmental Control Model}

This Integrated Environmental Control Model (IECM) and Interface were developed for the U. S. Department of Energy's National Energy Technology Laboratory (NETL), formerly known as the Federal Energy Technology Center (FETC), under contracts No. DE-AC22-92PC91346, DE-AC21-92MC29094, and DE-FC2600NT40935.

\section{Purpose}

The purpose of the model is to calculate the performance, emissions and cost of employing alternative environmental control methods in a coal-fired power plant. The model consists of a base plant and various control technology modules; these modules may be implemented together in any desired combination.

A Graphical User Interface (GUI) facilitates the configuration of the technologies, entry of data, and retrieval of results.

\section{System Requirements}

The current model requires the following configuration:

- Intel-based computer running Windows 95 (or better) or Windows NT 4.0 (or better) operating system

- Pentium Processor

- any SVGA (or better) display—at a resolution of 800x600 (or more) pixels $^{1}$

- at least 40 Megabytes of free hard disk space

- at least 32 Megabytes of total memory

\footnotetext{
${ }^{1}$ Smaller screen resolution results in the interface screens being scaled smaller. The taskbar, part of the Windows operating system, reduces the useable resolution of the screen if it is always visible. This may force the IECM interface to be scaled down slightly. To avoid this situation, select the "Auto Hide" option of the Taskbar properties in Windows.
} 


\section{Uncertainty Features}

The ability to characterize uncertainties explicitly is a feature unique to this model. As many as one hundred input parameters can be assigned probability distributions. When input parameters are uncertain, an uncertainty distribution of results is returned. Such result distributions give the likelihood of a particular value, in contrast to conventional single-value estimates.

The model can run using single deterministic values or uncertainty distributions. The conventional deterministic form using single values for all input parameters and results may be used, or probabilistic analyses may be run-for instance, to analyze advanced technology costs.

\section{Software Used in Development}

The underlying engineering models are written in Digital Equipment Corporation's Fortran. Fortran runtime libraries are included with the IECM Interface software. This language provides the flexibility to configure many various power plant designs while also providing the power to conduct probabilistic analyses.

All databases are in Microsoft ${ }^{\circledR}$ Access format and may be viewed in Access, as long as they are not changed. This format is a software industry standard and facilitates sharing and updating of information.

To simplify the use of the model, a Graphical User Interface (GUI) has been added. The interface eliminates the need to master the underlying commands normally required for model operation. The interface is written in Microsoft ${ }^{\circledR}$ Visual C++, a standard software development tool for the Windows environment. Visual C++ runtime libraries are included with the IECM Model software and do not need to be licensed separately.

Wise InstallBuilder was used to generate full and upgrade installer programs. This product was chosen based on its flexibility and its support of Visual Basic runtime libraries and Microsoft Data Access Components (MDAC) ${ }^{2}$. The Visual Basic runtime libraries provide the support needed to run the database file compactor program provided with the IECM. MDAC provides the software support needed to link Microsoft ${ }^{\circledR}$ Access data files to the IECM interface program. Wise InstallBuilder provides the VB and MDAC installation as an option, rather than forcing the user to download it from Microsoft and install it prior to installing the IECM.

\section{Copyright Notices}

COMPAQ Visual Fortran 6.5, Copyright @ 2000, Compaq Computer Corporation. All Rights Reserved.

Spread 3.5, Copyright (C) 2001, FarPoint Technologies, Inc. All Rights Reserved.

Tab Pro 3.1, Copyright (c) 1999, FarPoint Technologies, Inc. All Rights Reserved.

Graphics Server 4.5, Copyright (C) 1996, Bits Per Second Ltd. and Pinnacle Publishing, Inc. All Rights Reserved.

\footnotetext{
${ }^{2}$ The current version of MDAC is 2.6. This is installed with the full installer for the IECM. Any update installers provided for upgrading the IECM from a previous version to the current version do not upgrade MDAC unless the user updates MDAC separately.
} 
Microsoft Visual C++ 6.0, Copyright (C) 1992-1997, Microsoft Corporation. All Rights Reserved.

InstallBuilder 8.1, Copyright (C) 1994-2001, Wise Solutions, Inc. All Rights Reserved.

Microsoft Data Access Components 2.6, Copyright @ 2000, Microsoft Corporation. All Rights Reserved.

Integrated Environmental Control Model (IECM) Interface 3.5.0, Copyright (C) 19972001, Carnegie Mellon University. All Rights Reserved.

Integrated Environmental Control Model (IECM) 3.5.0, Copyright (c) 1997-2001, Carnegie Mellon University. All Rights Reserved.

Median Latin Hypercube and Hammersley Sequence Sampling, Copyright ( $~ 1997$, Urmila Diwekar, Carnegie Mellon University. All Rights Reserved.

Graphics Server is a trademark of Bits Per Second Ltd.

Microsoft is a registered trademark; Windows, Windows 95, Windows 98, Windows NT, Windows ME, Windows 2000, and Visual C++ are trademarks of Microsoft Corporation.

Spread $^{\mathrm{TM}}$ is a trademark of FarPoint Technologies, Inc.

Tab Pro ${ }^{\mathrm{TM}}$ is a trademark of FarPoint Technologies, Inc.

DEC, DEC Fortran, and DIGITAL are trademarks of Compaq Computer Corporation. 



\section{User Documentation and Help}

\section{Getting Started}

Getting Started gives step-by-step instructions for installing the model software and using the interface. It describes navigating the model, using the main program areas, working with sessions, and printing or exporting results.

\section{User Manual}

The User Manual gives further information on both the interface and the underlying model. It provides detailed descriptions of plant configurations, parameter settings, and result screens. It also describes technical details behind the model's operation and includes an introduction to uncertainty analysis.

\section{Model Tutorial}

The Model Tutorial is a pictorial introduction to the IECM. It presents a graphical tour of the interface, a case study to follow using the model, and an appendix, which reproduces every screen in the model. These tools help any user to quickly become more familiar with the interface and model.

\section{Technical Manual}

The Technical manual is a detailed engineering description of the technologies and costing assumptions used in the IECM. This manual is not provided by default with the IECM software; however, it can be downloaded with any web browser from http://www.iecm-online.com.

\section{Online Help}

Online help is provided via a Windows Help File containing the text of Getting Started and the User Manual. 


\section{Accessing the IECM Help file:}

If you are not running the IECM interface, click the Help icon inside the IECM folder on the Start menu. This opens the help file to the table of contents.

If you are running the IECM interface, do any one of the following:

- $\quad$ Press the F1 key. The IECM supports context-sensitive help and will open the help file to the topic associated with the item or screen you are viewing.

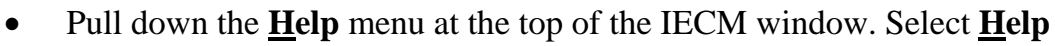
Topics. This opens the help file to the table of contents.

- $\quad$ Click the Context-Sensitive Help icon on the toolbar on the left side of the IECM window. The IECM supports context-sensitive help and will open the help file to the topic associated with the item or screen you are viewing.

- Click the Help Topics icon on the toolbar on the left side of the IECM window. This opens the help file to the table of contents. If this method does not work, try one of the other options above.

The IECM Help File Contents window will display.

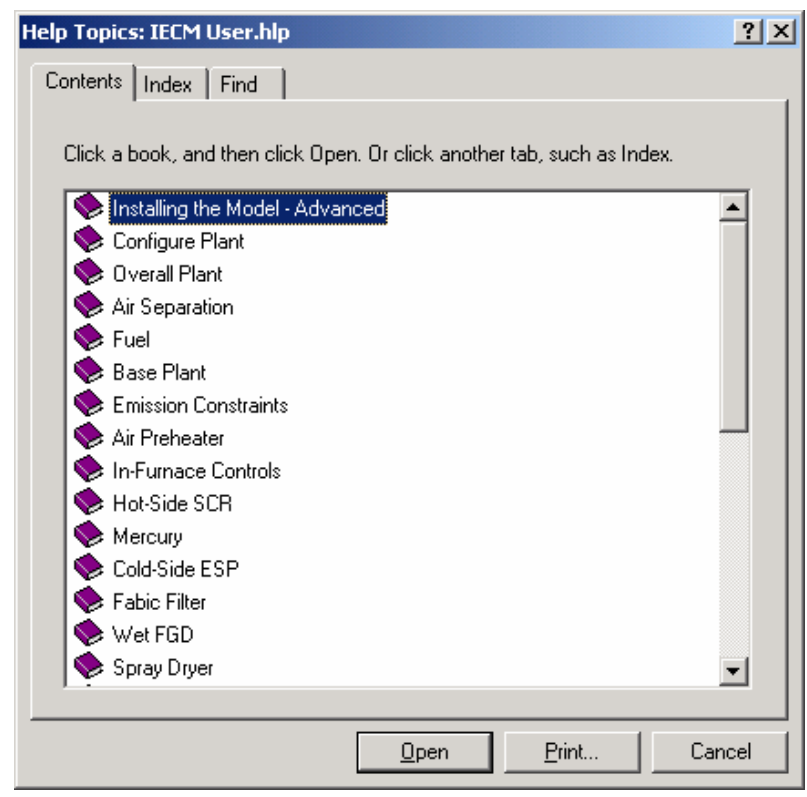

The IECM Help File Topics Window 


\section{Installing the Model - Advanced}

\section{Advanced Installation Options}

Normal installation is described in Getting Started. This section addresses installation from a network drive or the worldwide web. This section also describes advanced features of the installation program and the files installed.

\section{Local and Network Installation}

The Setup program can be run from a local hard drive or a network server. Installing from a hard drive eases the burden of sharing one IECM compact disk (CD) between multiple users. Installing from a network server simplifies the process of installing the entire package on a series of personal computers connected to the network. However, both methods require some familiarity with creating and finding folders and sub-directories on a network hard drive.

\section{NOTE:}

You may also install the interface to a network server. All files will be loaded to the server except the shortcut in the start menu of the local personal computer. The interface will run from the server and all sessions will be saved to the network drive, meaning that others with access to the network drive may change or delete them. Installation to a network server is not currently supported.

\section{Installing the IECM from a Local Hard Drive}

To install from a local hard drive:

1. Copy the contents of the IECM compact disk (CD) disk into one subdirectory or folder on your personal hard drive.

2. On the personal computer, click the Start button.

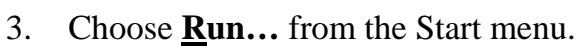

4. Type "X: $\backslash X X X \backslash$ Media $\backslash S E T U P$. EXE" where "X: $\backslash X X X \mid$ " is the drive and directory on your local hard drive to which you copied the files.

The Installation Program will begin. Follow the instructions on the screen.

If you receive an error message while running Setup, restart your computer and run the installation program again. If Setup still returns an error message, call Technical Support. 


\section{Installing the IECM from a Network}

To install from a network hard drive:

1. Copy the contents of the IECM compact disk (CD) disk into one subdirectory or folder on a network hard drive.

2. On the personal computer, click the Start button.

3. Choose Run... from the Start menu.

4. Type "X: $\backslash X X X \backslash$ Media \SETUP . EXE" where "X: $\backslash X X X \mid$ " is the drive and directory on the network hard drive to which you copied the files.

The Installation Program will begin. Follow the instructions on the screen.

If you receive an error message while running Setup, restart your computer and run the installation program again. If Setup still returns an error message, call Technical Support

\section{Internet Installation}

The contents of the IECM CD-ROM are also available on the worldwide web (www.iecm-online.com). The media, documentation and various text files can be downloaded to your local computer or network hard drive.

\section{Downloading the IECM from the worldwide web.}

To download the install software to your computer:

1. Open a web browser program (e.g., Internet Explorer or Netscape).

2. In the "Address" line of the browser, type the following http://www.iecm-online.com. You will see the iecm-online home page.

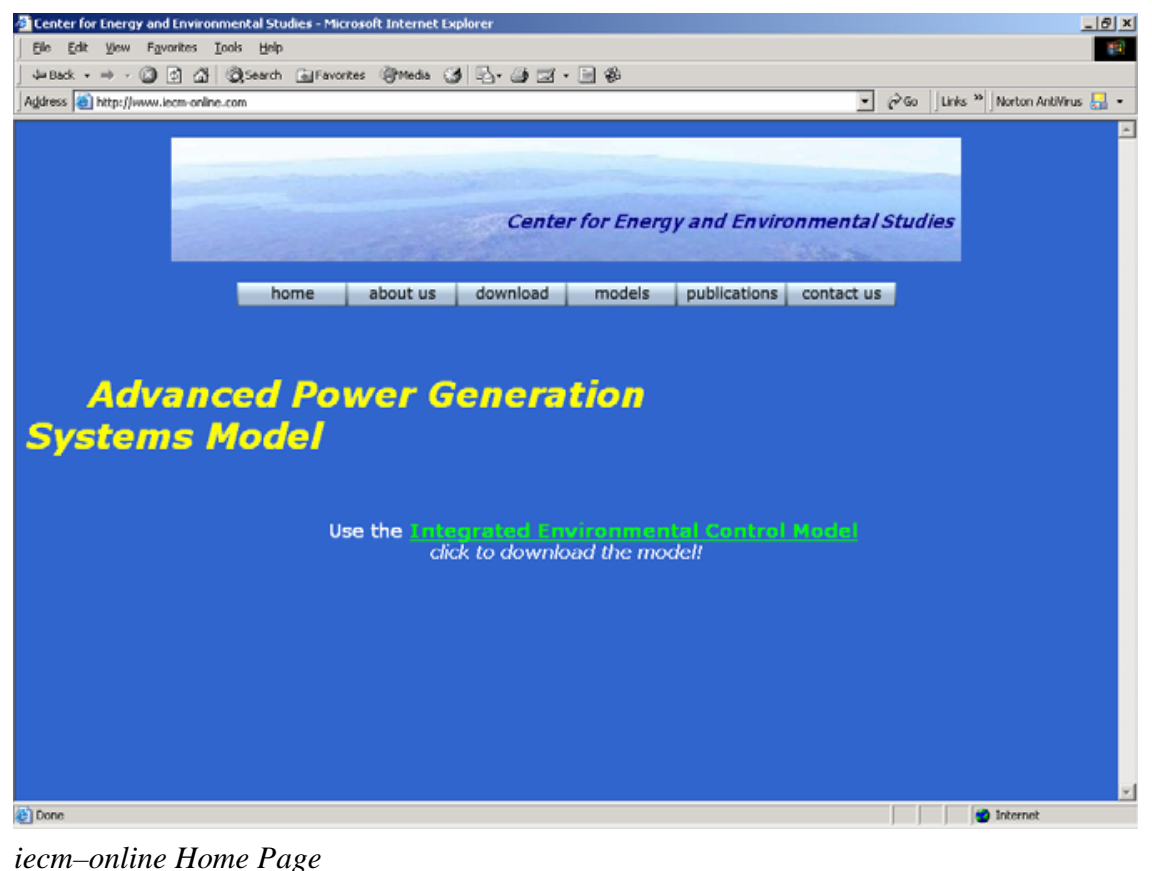

iecm-online Home Page

3. Click on either the button labeled download or the highlighted green text Integrated Environmental Control Model. 


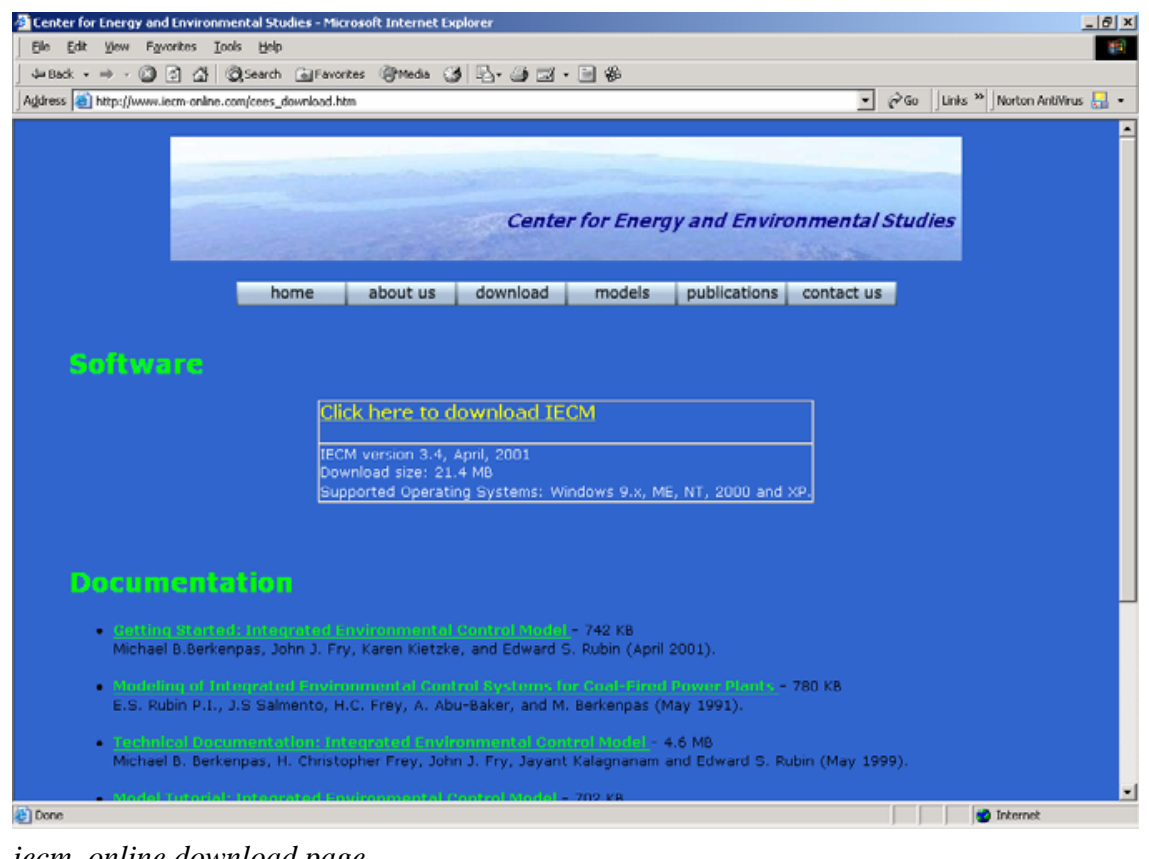

iecm-online download page

4. Click on the the yellow highlighted text Click here to download IECM. A dialog box will appear.

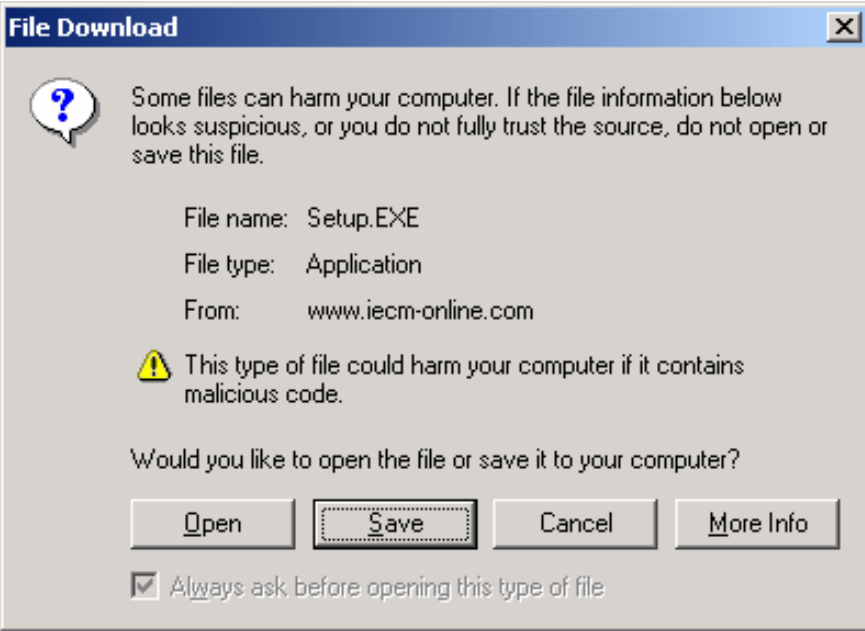

File download dialog box; save the program to disk

5. Click on the Save button.. 


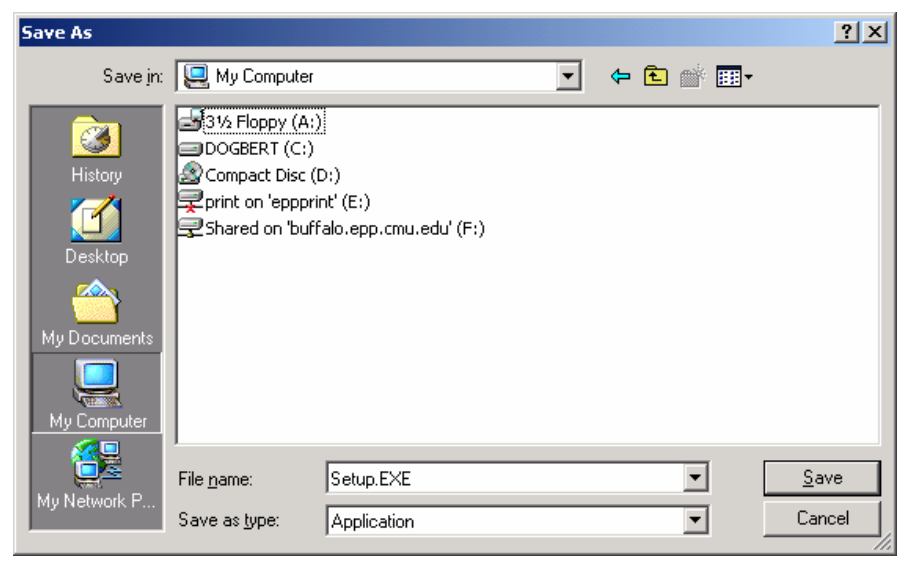

Save file dialog box; use this to select the location to save the program

6. Choose a location to save the setup file and click the "Save" button.

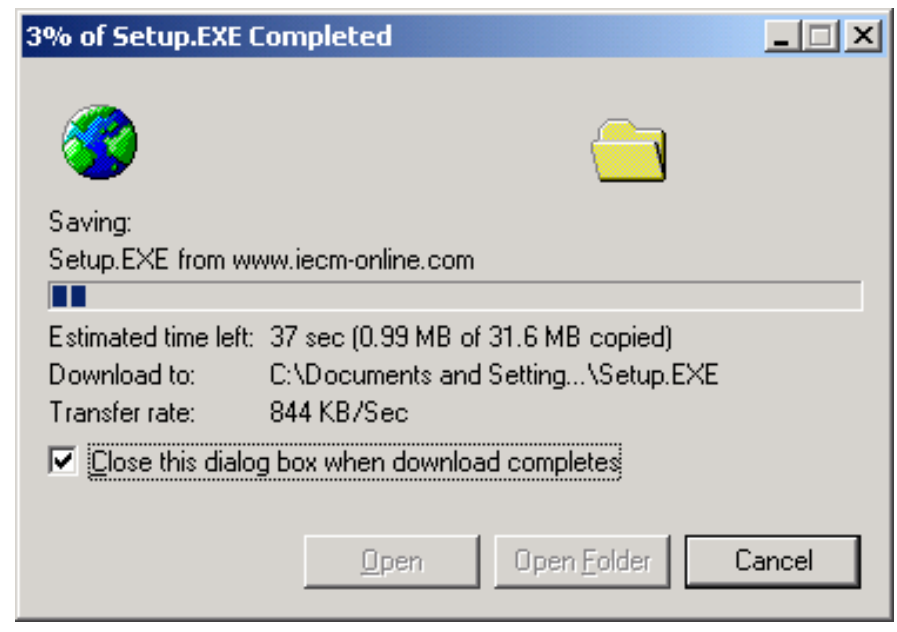

File download progress indicator

The download will begin. Once it is finished, you can proceed to install the IECM software. If you receive an error message while running Setup, restart your computer and run the installation program again. If Setup still returns an error message, call Technical Support

\section{Changing the Destination Location}

The destination location for the IECM software can be changed during the installation process. This is accomplished by clicking the Browse... button on the "Choose Destination Location" screen during the installation process (See the Getting Started manual for a description of the entire installation process). The Browse... button opens the "Select Destination Directory" window which can be used to locate or type the exact destination location for the IECM software. 


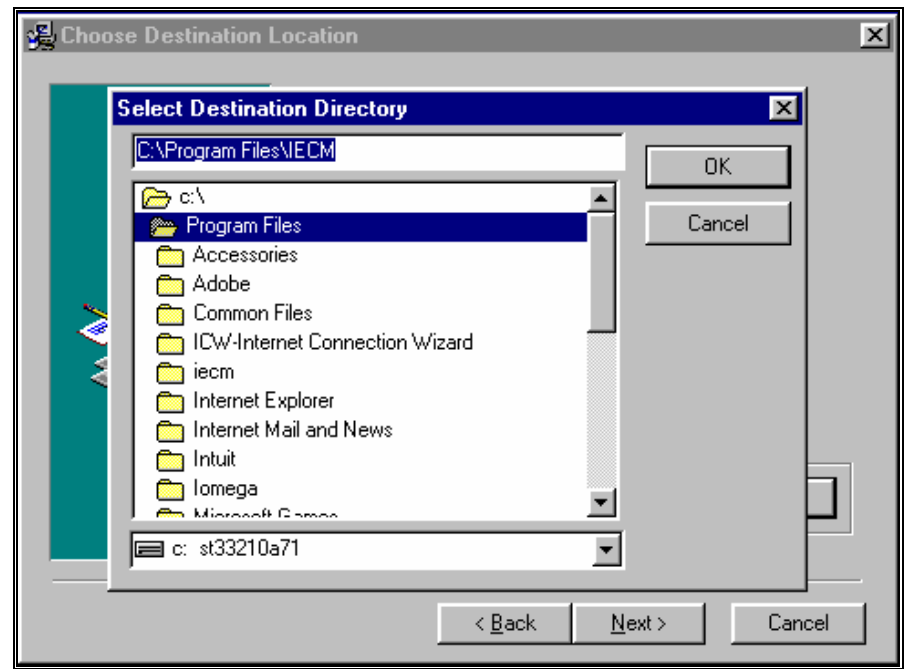

The "Select Destination Directory" window

Select a specific folder in which to install the IECM software by clicking on the folder name to highlight it. Then click the $\mathbf{O K}$ button to return to the "Choose Destination Location" screen.

\section{Files Installed}

This section provides a full list and short description of the files installed by the IECM installer software. The software is divided into three categories.

\section{Help Files}

The following help files are installed by default in the C: \PROGRAM FILES $\backslash I E C M$ - CS directory by the installation program:

\section{C:IPROGRAM FILESIIECM-CSI}

This is the main program directory.

IECMINT . CNT: IECM “Getting Started” online help contents file.

IECMINT . HLP: IECM “Getting Started” online help file.

USER . CNT: IECM “User Manual” online help contents file.

USER . HLP: IECM “User Manual” online help file (linked to IECMINT.HLP).

\section{Program Files}

All applications and their support files specific to the IECM software itself are considered program files. These can be installed into any directory during installation. The folder can be changed from the default location suggested during installation.

\section{C:IPROGRAM FILESIIECM-CSI}

This is the main program directory. The following program files are installed by default in the "C:IProgram FilesIECM" directory by the installation program: 
COMPACT . EXE : Support application that compacts Microsoft Access 97 database files (e.g., the files in the "C:IProgram FileslIECM $\backslash$ Sessdb" directory).

GSW32 . EXE: Graphics Server for Windows 4.5 program file.

GSWAG32 . DLL: Graphics Server for Windows 4.5 support file.

GSWDLL32 . DLL: Graphics Server for Windows 4.5 support file.

[Graphics Server: Graphing Toolkit for Windows. Kent, Washington: Bits Per Second Ltd. And Pinnacle Publishing Co., 1996. http://www.pinpub.com

HISTORY . TXT : History of the IECM software, including features installed and planned.

IECMILIB . DLL: IECM interface support file. It handles all database, uncertainty and model access.

IECMINT . EXE: IECM program file for the interface.

IECMINT . MDB: Microsoft Access 97 template database file.

LHS. DLL: IECM interface support file. It handles all uncertainty sampling.

LICENSE . TXT: IECM license agreement.

MODEL . DLL: IECM interface model support file. It contains all the technology performance and cost modules.

SPR32D35 . DLL: Spread 3.0 support file.

TAB32D30 . DLL: Tab Pro 3.1 support file.

[Spread and Tab Pro for Windows. Morrisville, North Carolina: Far Point Technologies, 1996 and 2000. http://www.fpoint.com]

UNWISE32 . EXE : Uninstaller program. This requires an installer log created during installation.

\section{C:IPROGRAM FILESIIECM-CSIINTDB|}

This directory contains the database files used by the IECM interface. These contain default data used in the interface program. The following files are installed by the installation program:

INTDESC . MDB: Microsoft Access database file. It contains all the descriptions for the IECM interface screens.

MODCOALS. MDB: Microsoft Access database file. It contains the model default coal information.

\section{C:IPROGRAM FILESIIECM-CSISESSDBI}

The "C:IProgram Files\IECM $\backslash S$ essdb" directory contains the database files created by the IECM interface. All user data associated with sessions are stored here. These files are not created by the installation program; rather, they are created by the IECM Interface at runtime if they are not available. This means that user data cannot be overwritten by the installation program. 


\section{System Files}

Several files are installed into the windows system directory. These system files are common to many Windows applications. All of these files are created and distributed freely through Microsoft @ Corporation using their installer packages. These system files are unique to the other IECM components listed above because they are hardwired into the system registry file. In order to maintain consistency with the operating system and stability with the IECM interface, these special system files must be stored in the windows system directory and installed with software installers directly from Microsoft.

A full list of the Microsoft ${ }^{\circledR}$ Corporation files installed is provided in the INSTALLR.TXT file located on the IECM compact disk (CD).

\section{Microsoft Data Access Components}

The use of Microsoft Access database files requires the installation of ODBC drivers and support files from Microsoft. ODBC is a programming interface that enables applications to access data in database management systems that use Structured Query Language (SQL) as a data access standard. The Microsoft MDAC package is included with the IECM installer program as delivered directly from Microsoft and delivers this important functionality. Files are installed into the "C:IWindows System32" directory.

\section{Microsoft Visual Basic 4.0 Runtime}

The components of this package are installed from within the IECM installer as delivered by Microsoft. They provide Microsoft Visual Basic support files and are installed into the "C:IWindows $\backslash$ System32" directory and the "C: $\backslash$ Program Files\Common\Microsoft” directory.

\section{Microsoft MFC 4.2}

The components of this package are installed from within the IECM installer as delivered by Microsoft. They are Microsoft Visual C++ support files delivered under the Microsoft Foundation Class libraries. They are installed into the

"C:IWindows $\mid$ System32" directory.

\section{Files Modified by Install}

Currently no user files are modified when the IECM software is installed. All user files are stored in the "C:IProgram Files\IECM $\backslash$ Sessdb" directory.

\section{Uninstalling and Rolling Back Installations}

The IECM Installation program will uninstall the software or roll back the installation to a previous version.

\section{Uninstallation Steps}

Uninstallation removes the IECM Interface completely from your computer.

\section{Run the Uninstaller}

1. Click the Start button. 
2. Choose Settings, and then $\underline{\text { Control Panel. }}$

3. Double-click Add/Remove Programs in the Control Panel folder.

4. Highlight IECM Interface on the list of installed software.

5. Click the Add/Remove... button.

\section{Select Uninstall Method}

The first screen which displays when the uninstaller is run is the "Select Uninstall Method" screen. It asks you if you want it to remove files automatically or specify which files to delete.

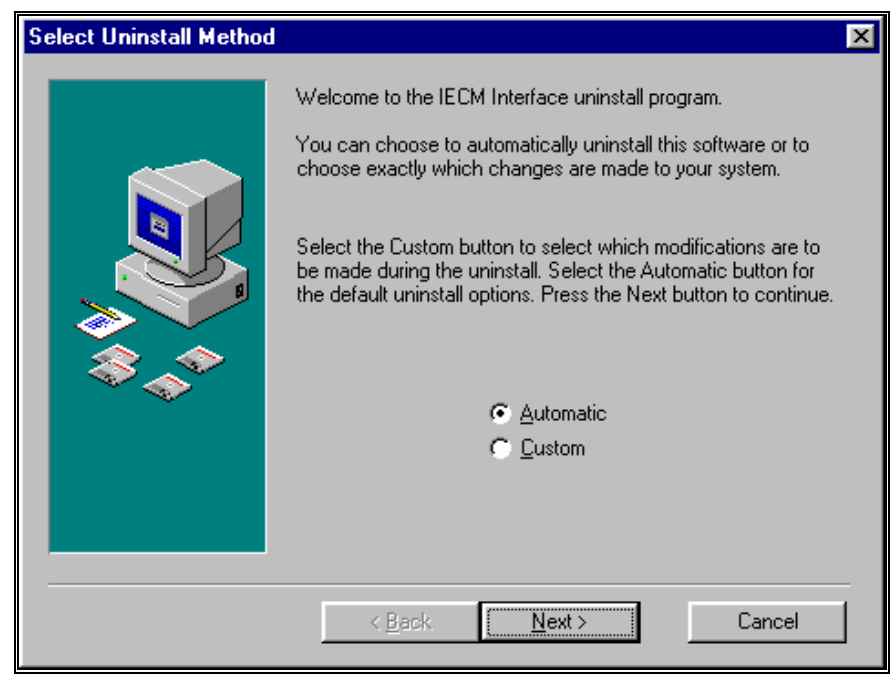

The "Select Uninstall Method" screen.

Click the circle next to $\underline{\text { Automatic }}$ to make automatically uninstall the software, click the circle next to $\underline{\mathbf{C}} \mathbf{u s t o m}$ to choose which files to delete. Click the Next button to continue to the next screen.

\section{Select Files to Remove}

If you have chosen Custom on the "Select Uninstall Method" screen, the "Select Private Files to Remove” screen will display.

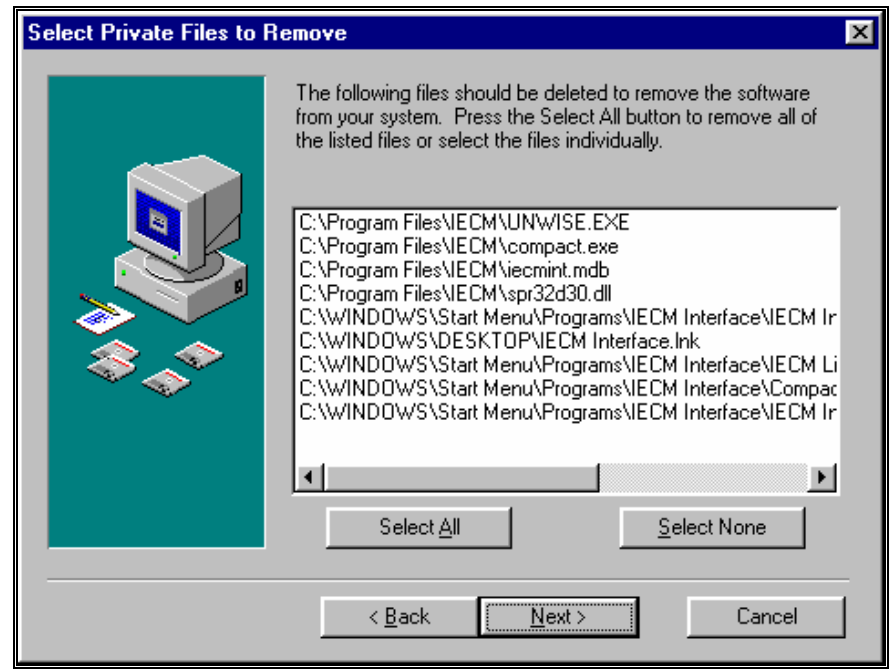


Select files to delete by clicking on their names in the scroll box to highlight them.

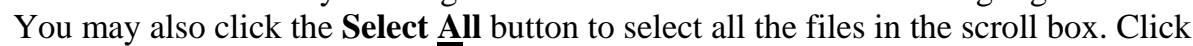
the Next button to continue to the next screen.

\section{Perform Rollback}

The "Perform Rollback" screen asks if you would like to uninstall the software completely, or just to roll back the installation to a previous version.

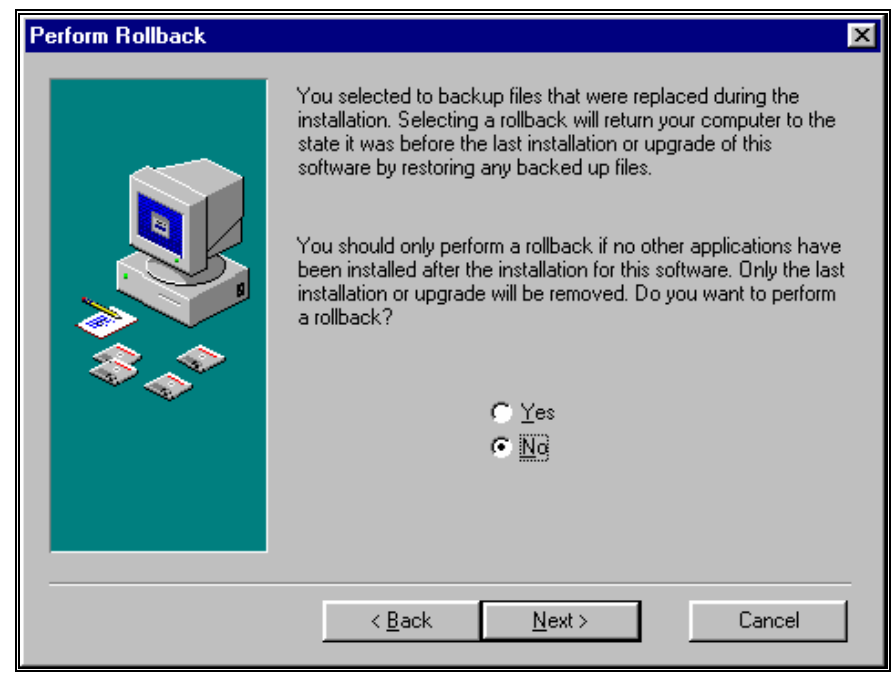

The "Perform Rollback" screen

Click the circle next to $\underline{\text { No }}$ to uninstall the software completely. Click the $\underline{\text { Next }}$ button to continue to the next screen.

\section{Perform Uninstall}

The uninstaller is now ready to perform the uninstall. The "Perform Uninstall" screen allows you to go back one last time to check the options you have chosen for uninstallation.

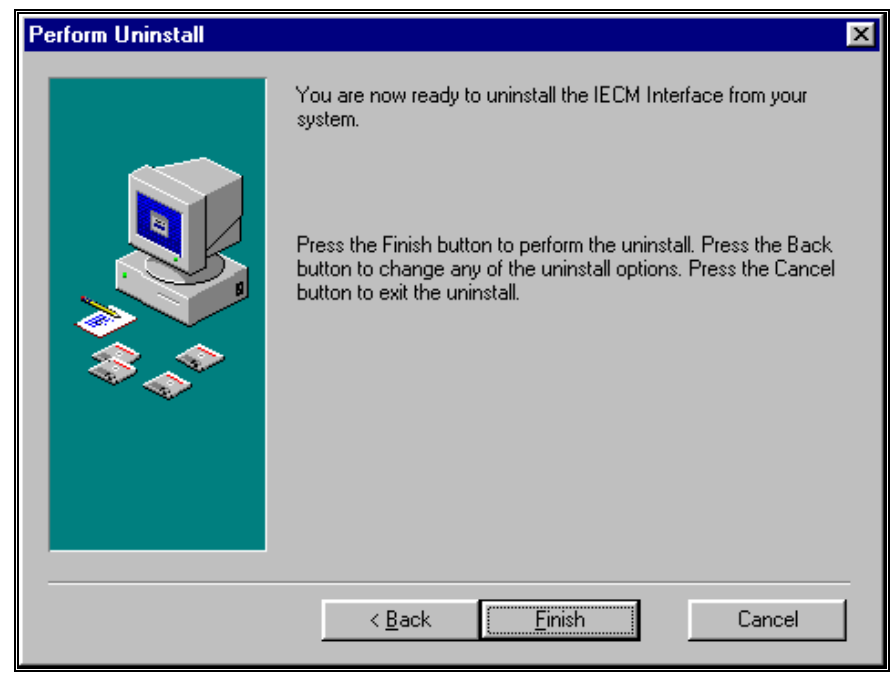

The "Perform Uninstall” screen 
Click the Back button to return to any of the previous screens to check or change the uninstallation options. Click the Next button to continue to uninstall the software.

\section{Rollback Steps}

Instead of completely uninstalling the software, you may rollback the IECM Interface software to the previous installed version. You may only perform a rollback if:

- You have upgraded the IECM Interface software, not just performed a new installation or

- As part of that upgrade, you have backed up the files replaced. See Getting Started for how to backup replaced files as part of an installation.

If you have either not backed up replaced files, or have not upgraded, a rollback will return the software to its previous position, but that condition will be an empty folder.

\section{Run the Uninstaller}

1. Click the Start button.

2. Choose Settings, and then $\underline{\text { Control Panel. }}$

3. Double-click Add/Remove Programs in the Control Panel folder.

4. Highlight IECM Interface on the list of installed software.

5. Click the Add/Remove... button.

\section{Select Uninstall Method}

The first screen which displays when the uninstaller is run is the "Select Uninstall Method" screen. It asks you if you want it to remove files automatically or specify which files to delete.

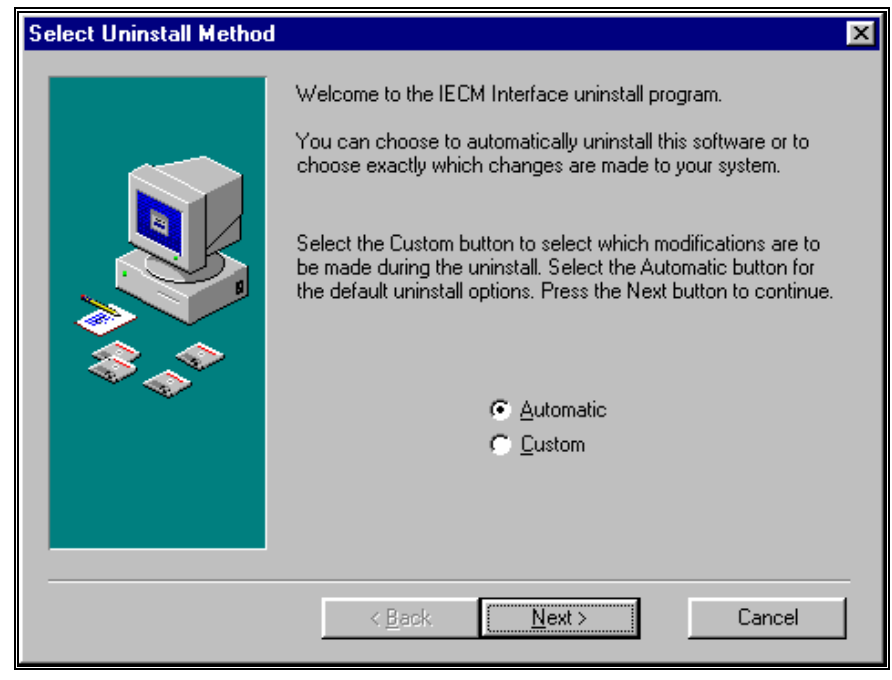

The "Select Uninstall Method" screen.

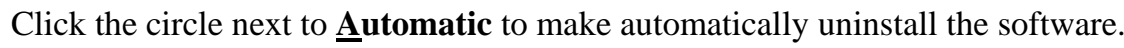
Click the Next button to continue to the next screen. 


\section{Perform Rollback}

The "Perform Rollback" screen asks if you would like to uninstall the software completely, or just to roll back the installation to a previous version.

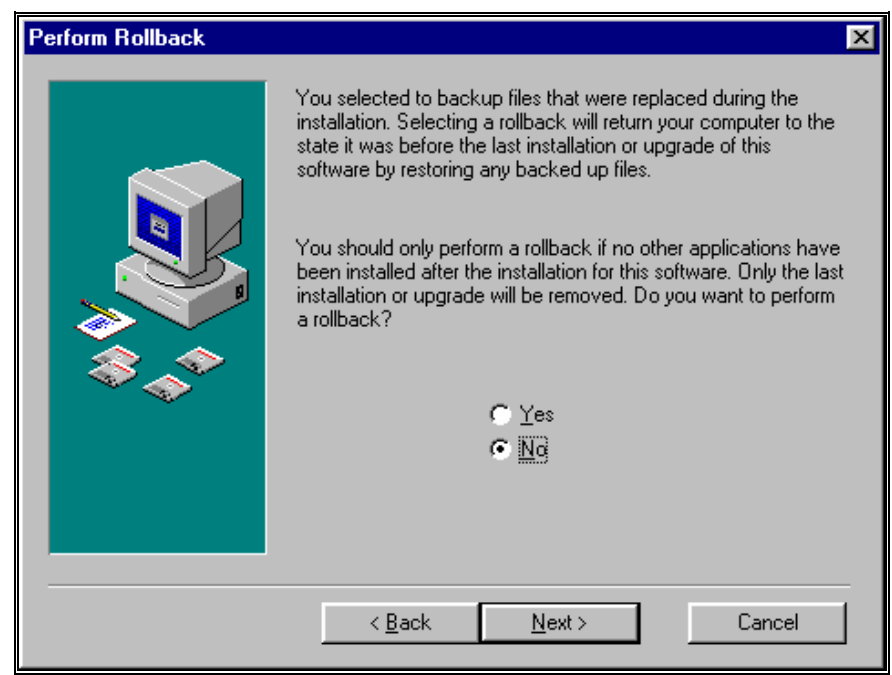

The "Perform Rollback" screen

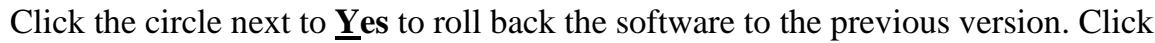
the Next button to continue to the next screen.

\section{Perform Uninstall}

The uninstaller is now ready to perform the rollback. The "Perform Uninstall" screen allows you to go back one last time to check the options you have chosen for the rollback.

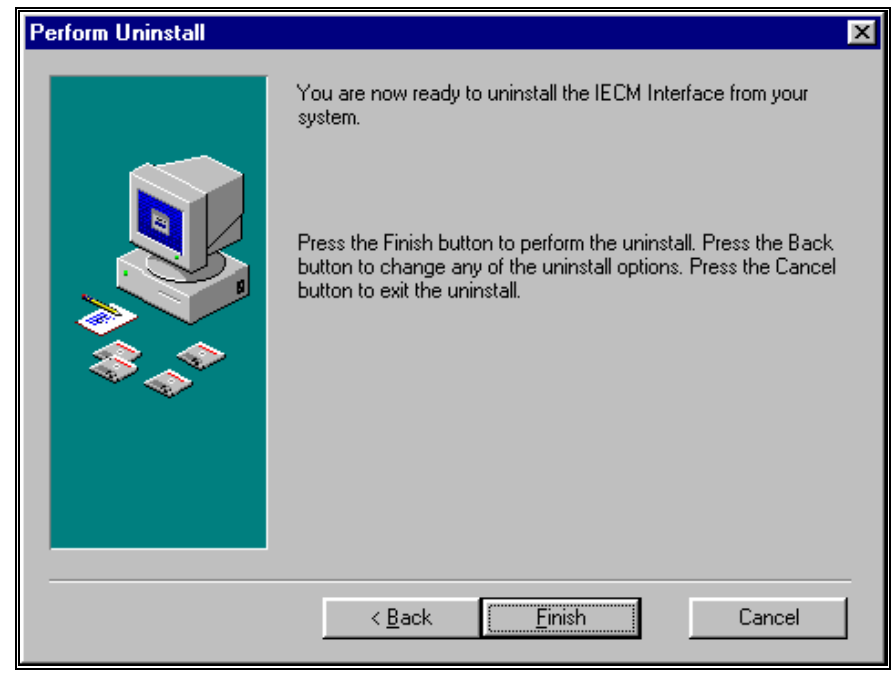

The "Perform Uninstall” screen

Click the Back button to return to any of the previous screens to check or change the rollback options. Click the Next button to continue to roll back the installation of the software to the previous version. 



\section{Configure Plant}

\section{Configuring the Combustion Turbine Plant}

If the user has selected Combustion (Turbine) as the plant type from the New Session pull down menu the NGCC Configuration screen will appear in the Configure Plant program area.

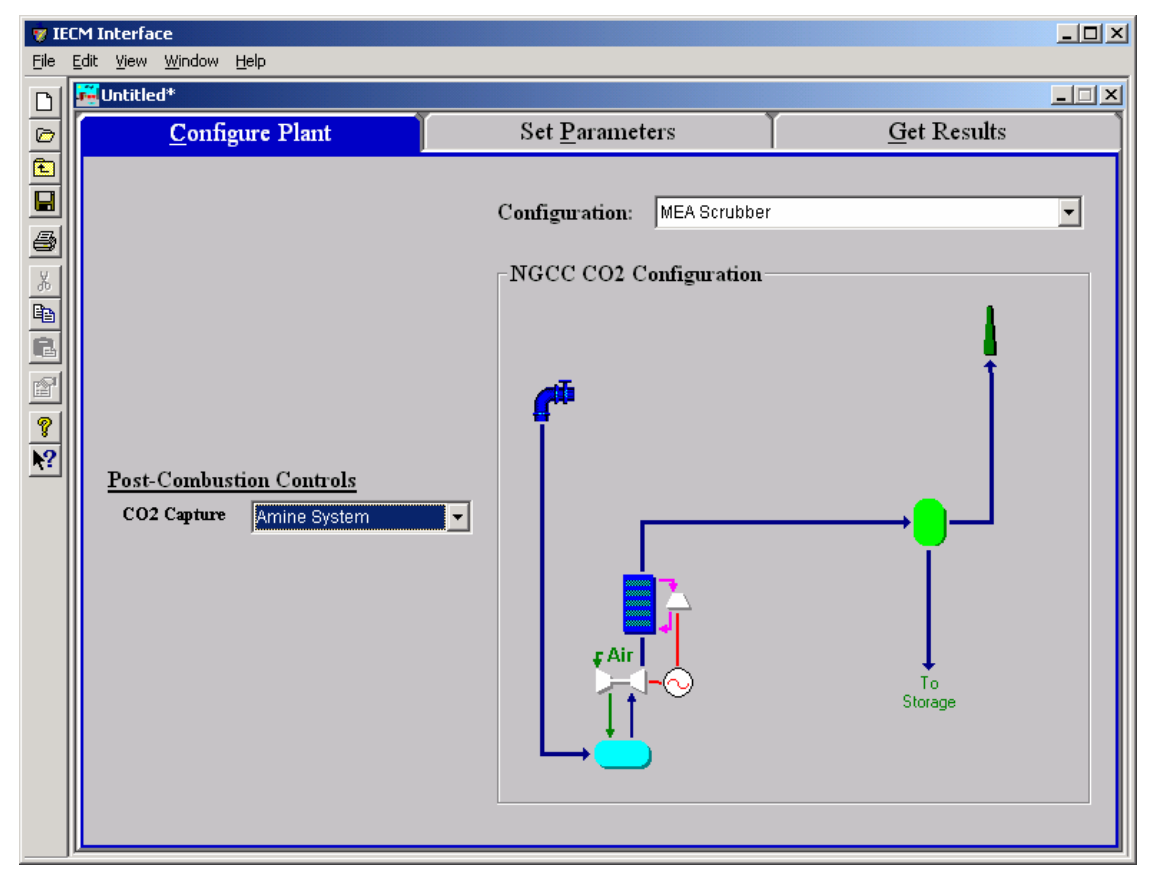

Configure Plant - Combustion (Turbine) input screen.

\section{Post-Combustion Controls}

$\mathbf{C O}_{2}$ Capture: If $\mathbf{C O}_{2}$ Capture is desired it may be added by selecting the Amine System option from the pull down menu. The default is None. 


\section{Configuring the Combustion Boiler Plant}

If the user has selected Combustion (Boiler) as the plant type from the New Session pull down menu the Configuration screen will appear in the Configure Plant program area.

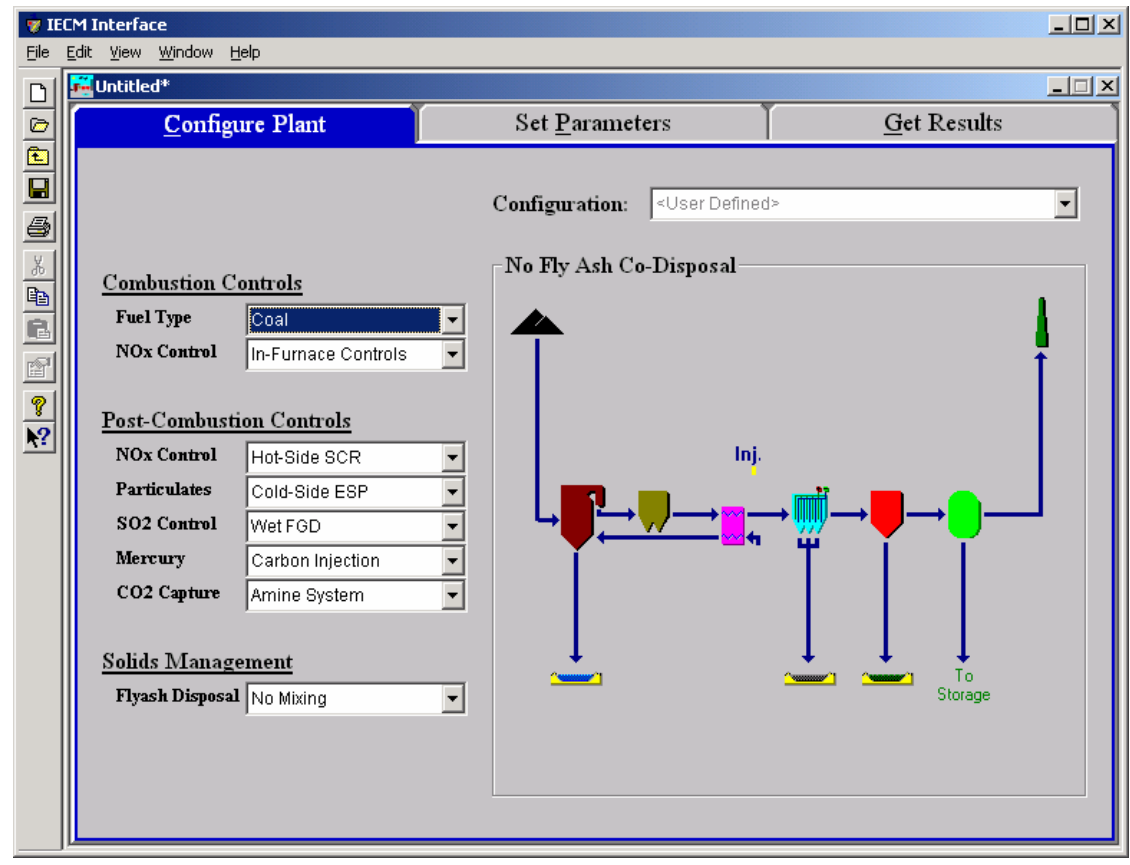

Configure Plant - Combustion (Boiler) input screen

\section{Combustion Controls}

These configuration options determine the type of furnace and any technologies for reducing $\mathrm{NO}_{\mathrm{x}}$ emissions.

Fuel Type: Coal is the fuel that is used by the model.

$\mathrm{NO}_{\mathrm{x}}$ Control: From this configuration screen, you may choose either None or In-Furnace Controls.

In-Furnace $\mathrm{NO}_{\mathrm{x}}$ : Controls include an assortment of options which combine low $\mathrm{NO}_{\mathrm{x}}$ burners (LNB) with overfire air (OFA), selective non-catalytic reduction (SNCR), and natural gas reburning. These options are selected from a pull-down menu in the Set Parameters program

\section{Post-Combustion Controls}

These configuration options determine the presence and type of post-combustion emissions controls.

$\mathrm{NO}_{\mathrm{x}}$ Control: The choices available are None, for no post-combustion $\mathrm{NO}_{\mathrm{x}}$ control, or Hot Side SCR for a Hot-Side Selective Catalytic Reduction technology. The default setting is None. The only postcombustion $\mathrm{NO}_{\mathrm{x}}$ control technology currently included with the IECM is a selective catalytic reduction (SCR) process. Although an SCR technology can be positioned at various points along the flue gas train, the IECM considers only the hot-side, high dust configuration. Hot-side SCR systems are capable of removing up to 80 or 90 percent of the $\mathrm{NO}_{\mathrm{x}}$ emitted from a coal-fired power plant. Although they are not yet 
prevalent in the United States, they are widely used in Europe and Japan. SCR systems affect other systems in the power plant, requiring some modifications to the boiler, air heater and induced draft fan. Other technologies affected but not requiring modifications are the ESP and FGD systems. If In-Furnace Controls is selected under Combustion Controls, a Hot Side SCR may also be selected.

Particulates: The choices available are None for no post-combustion Particulate control, Cold Side ESP for a Cold-Side Electrostatic Precipitator, and four different kinds of Fabric Filters (baghouses):

Reverse Gas Fabric Filter, Reverse Gas Sonic Fabric Filter, Shake \& Deflate Fabric Filter, and Pulse-Jet Fabric Filter. The default setting is None. The None setting is not available when the Mercury technology option is either Carbon Injection or Carbon + Water Injection. This assures the removal of the carbon being injected immediately downstream of the air preheater.

Fabric Filter Types: Fabric filter types are based on the bag cleaning techniques used. Various bag-cleaning techniques influence other process parameters. The choice of the bag cleaning method is usually based on the type of coal used-and therefore the filterability of the ash-and your experience with filtering the particular kind of ash. The particular option you select determines the air to cloth ratio, bag life, bag length, power requirements, pressure drop, capital costs and O\&M costs. There are four available bag filter types:

Reverse Gas Fabric Filter: Uses an off-line bag cleaning technique in which an auxiliary fan forces a relatively gentle flow of filtered flue gas backwards through the bags causing them to partially collapse and dislodge the dustcake. Over $90 \%$ of baghouses in U. S. utilities use reverse-gas cleaning.

Reverse Gas Sonic Fabric Filter: Uses a variation of Reverse Gas cleaning in which low frequency pneumatic horns sound simultaneously with the flow of reverse gas to add energy to the dustcake removal process.

Shake \& Deflate Fabric Filter: Uses a method for off-line cleaning in which the bags are mechanically shaken immediately after or while a small quantity of filtered gas is forced back to relax the bags. The amount of filtered gas used is smaller than that used in Reverse Gas cleaning.

Pulse-Jet Fabric Filter: Uses a method for on-line cleaning in which pulses of compressed air are blown down inside and through the bags to remove dustcake while the bags are filtering flue gas. Wire support cages are used to prevent bag collapse during filtration and ash is collected outside of the bags.

$\mathbf{S O}_{2}$ Control: The choices available are None for no post-combustion $\mathrm{SO}_{2}$ control, Wet FGD for a Wet Flue Gas Desulfurization technology (Lime or Limestone_-this is selected in the Set Parameters-SO2 control-1. Configuration screen), or a Lime Spray Dryer. Choice of a Lime Spray Dryer places this technology before the Particulate control technology in the plant design and diagrams. The default setting is None. 
Mercury: There are three choices available for Mercury control. The options provided are None, Carbon Injection, and Carbon + Water Injection. The default option is None. Although some mercury removal is accomplished naturally in a power plant, carbon injection is provided as a technology to supplement the natural removal to reach higher removals. It is believed that some mercury is captured or trapped in ash and is removed with bottom ash and fly ash. Mercury removal is accomplished by injecting fine particles of activated carbon into the flue gas after the air preheater. Because the removal increases with lower flue gas temperatures, water and carbon injection is added as a second technology option. The Carbon Injection, and Carbon + Water Injection options are only available when a cold-side ESP or fabric filter are selected. This is because a particulate device is required to remove the mercury-laden ash and activated carbon.

$\mathbf{C O}_{2}$ Capture: If $\mathbf{C O}_{2}$ Capture is desired it may be added by selecting the Amine System option from the pull down menu. The default is None.

\section{Solids Management}

Flyash Disposal: This configuration setting determines how flyash is disposed. Fly ash collected from a particulate removal system is typically combined with other solid waste streams if other waste streams exist. The waste disposal option has little effect on the rest of the IECM. The choices are Mixed w/FGD Wastes to dispose of flyash with FGD wastes, and Mixed w/ Bottom Ash to dispose of flyash with bottom ash (e.g. in the pond) or No Mixing. 


\section{Overall Plant}

\section{Overall Plant Diagram}

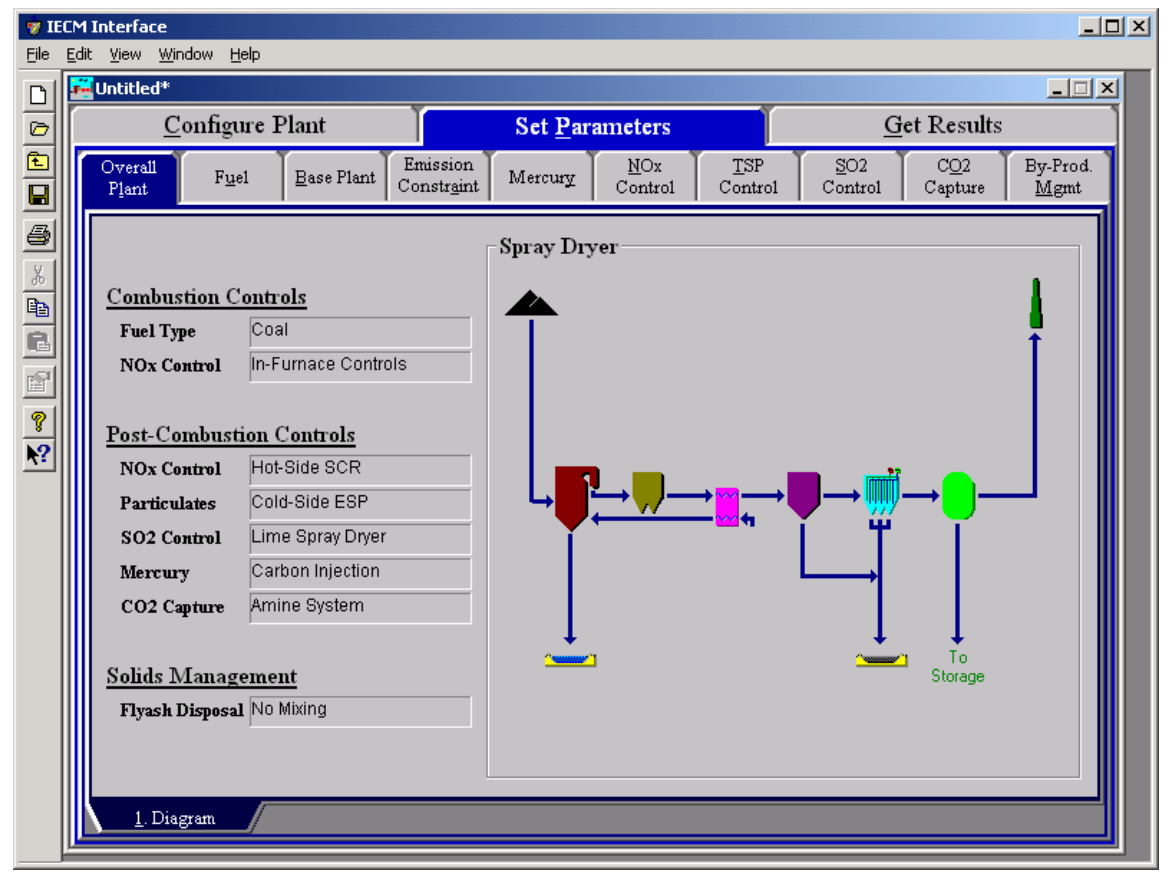

Overall Plant - Diagram result screen.

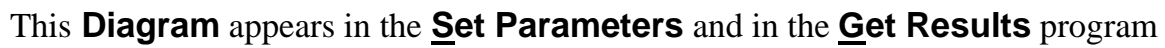
area. The screen displays the plant configuration settings on the left side of the page and a diagram of the plant as configured at the right of the page. No results are displayed on this screen. 


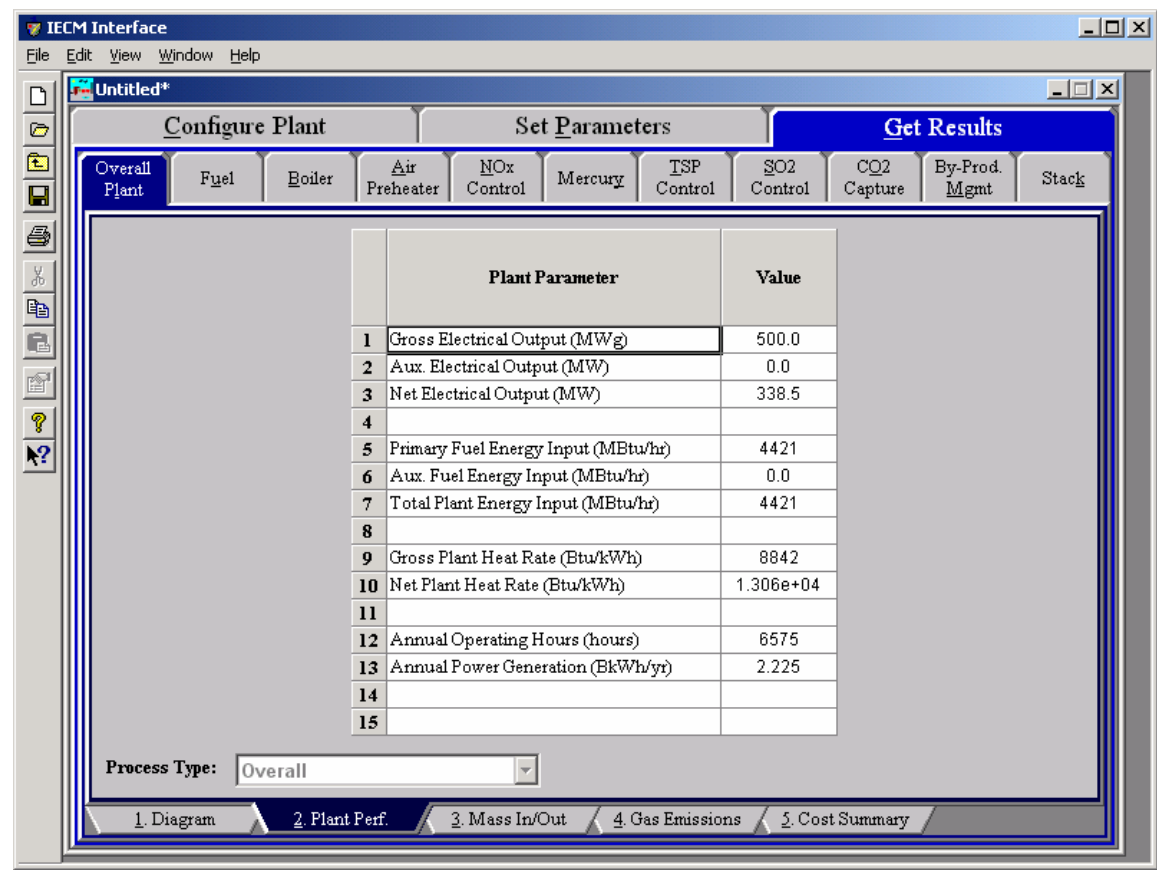

Overall Plant - Plant Perf. result screen.

The Plant Perf. result screen displays performance results for the plant as a whole. Values for the major input and outputs of the power plant are given. Each result is described briefly below.

Gross Electrical Output: This is the gross output of the generator in megawatts (MWg). The value does not include auxiliary power requirements. The model uses this information to calculate key mass flow rates. The value is an input parameter.

Aux. Electrical Output: If an auxiliary natural gas boiler is used to provide steam and power for the Amine System, this is the additional electricity that it produces.

Net Electrical Output: This is the net plant capacity, which is the gross plant capacity minus the losses due to plant equipment and pollution equipment (energy penalties).

Gross Cycle Heat Rate: This is the heat rate of the gross cycle including the effects of the boiler efficiency. This is considered the gross heat rate.

Net Cycle Heat Rate: This is the net heat rate, which includes the effect of plant equipment and pollution control equipment.

Fuel Energy Input: This is the fuel energy input for the plant on an hourly basis (maximum capacity).

Annual Operating Hours: This is the number of hours per year that the plant is in operation. If a plant runs 24 hours per day, seven days per week, with no outages, the calculation is 24 hours * 365 days. or 8,760 hours/year.

Annual Power Generation: This is the net annual MWh production of the plant. The capacity factor is used in determining its value. 


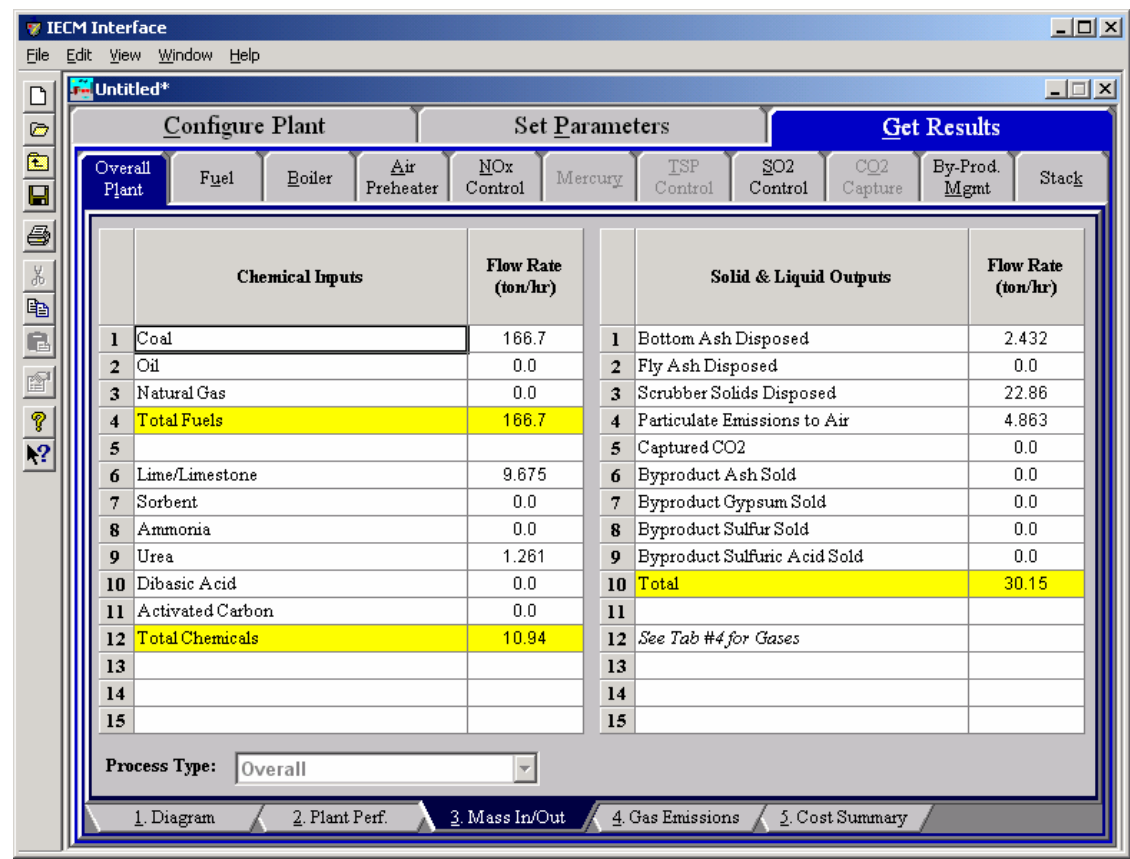

Overall Plant - Mass In/Out result screen.

The Mass In/Out result screen displays the flow rates of fuels and chemicals into the plant and solid and liquid flow rates out of the plant. Each result is described briefly below.

\section{Input Flow Rates}

Coal: Total mass of coal entering the boiler on a wet basis.

Oil: Total mass of oil used in the power plant

Natural Gas: : Total mass of natural gas used in the power plant

Total Fuels: This is the total fuel mass entering the power plant. This result is highlighted in yellow.

Lime/Limestone: Total mass of this reagent used in the power plant on a wet basis.

Sorbent: Total mass of sorbent used in the power plant.

Ammonia: : Total mass of ammonia used in the power plant.

Urea: Total mass of urea used in the power plant. Urea is the reagent used to reduce $\mathrm{NO}_{\mathrm{x}}$ in the SNCR technology.

Dibasic Acid: Total mass of dibasic acid used in the power plant.

Activated Carbon: Total mass of activated carbon injected in the power plant.

Total Chemicals: This is the total reagent mass entering the power plant. This result is highlighted in yellow.

\section{Output Flow Rates}

Bottom Ash Disposed: Total mass of bottom ash collected in the power plant on a dry basis. 
Fly Ash Disposed: Total mass of fly ash collected in the power plant on a dry basis.

Scrubber Solids Disposed: Total mass of scrubber solid wastes collected in the power plant on a dry basis.

Particulate Emissions to Air: Total mass of particulates emitted to the air from the power plant.

\section{Captured CO2:}

Byproduct Ash Sold: : Total mass of ash (bottom and fly ash) sold in commerce as a by-product on a dry basis.

Byproduct Gypsum Sold: : Total mass of flue gas treatment solids sold in commerce as a by-product on a dry basis.

Byproduct Sulfur Sold: Total mass of elemental sulfur recovered from flue gas and sold in commerce as a by-product on a dry basis.

Byproduct Sulfuric Acid Sold: Total mass of sulfuric acid recovered from the flue gas and sold in commerce as a by-product.

Total: This is the total wet solid mass exiting the power plant. This result is highlighted in yellow.

\section{Overall Plant Gas Emissions}

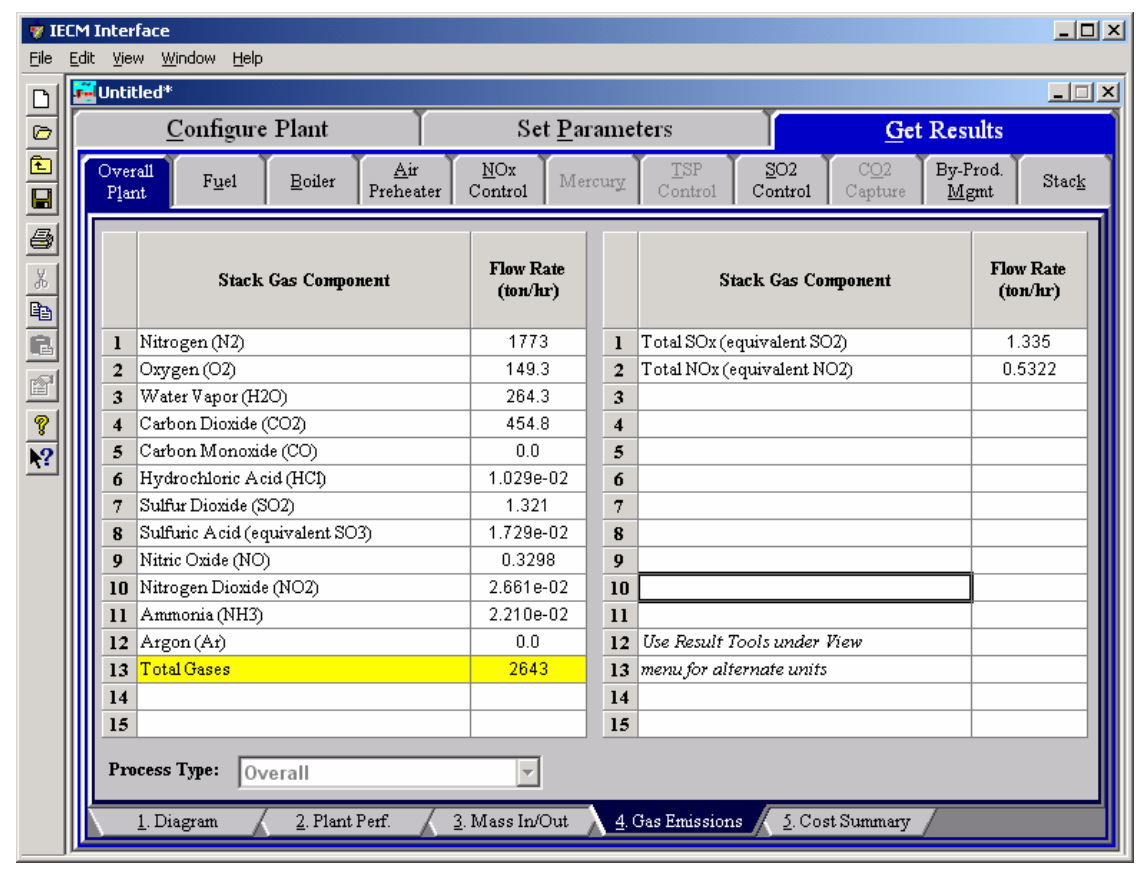

Overall Plant - Gas Emissions result screen.

The Gas Emissions result screen displays values for the major flows of gas out of the power plant Each result is described briefly below.

\section{StackGas Components}

Nitrogen $\left(\mathbf{N}_{\mathbf{2}}\right)$ : Total mass of emitted nitrogen.

Oxygen $\left(\mathbf{O}_{2}\right)$ : Total mass of emitted oxygen. 
Water Vapor $\left(\mathrm{H}_{2} \mathrm{O}\right)$ : Total mass of water vapor.

Carbon Dioxide $\left(\mathrm{CO}_{2}\right)$ : Total mass of carbon dioxide.

Carbon Monoxide (CO): Total mass of carbon monoxide.

Hydrochloric Acid (HCl): Total mass of hydrochloric acid.

Sulfur Dioxide $\left(\mathrm{SO}_{2}\right)$ : Total mass of sulfur dioxide.

Sulfuric Acid (equivalent $\mathbf{S O}_{3}$ ): Total mass of sulfuric acid.

Nitric Oxide (NO): Total mass of nitric oxide.

Nitrogen Dioxide $\left(\mathrm{NO}_{2}\right)$ : Total mass of nitrogen dioxide.

Ammonia $\left(\mathrm{NH}_{3}\right)$ : Total mass of ammonia.

Total Gases: Total flow rate of all gases. This result is highlighted in yellow.

Total $\mathrm{SO}_{\mathrm{x}}$ (equivalent $\mathrm{SO}_{2}$ ): Total mass of all sulfur oxides. This is the sum of the $\mathrm{SO}_{2}$ and $\mathrm{SO}_{3}$ above.

Total $\mathrm{NO}_{\mathbf{x}}$ (equivalent $\mathrm{NO}_{2}$ ): Total mass of all nitrogen oxides. This is the sum of the $\mathrm{NO}$ and $\mathrm{NO}_{2}$ above.

\section{Overall Plant Cost Summary}

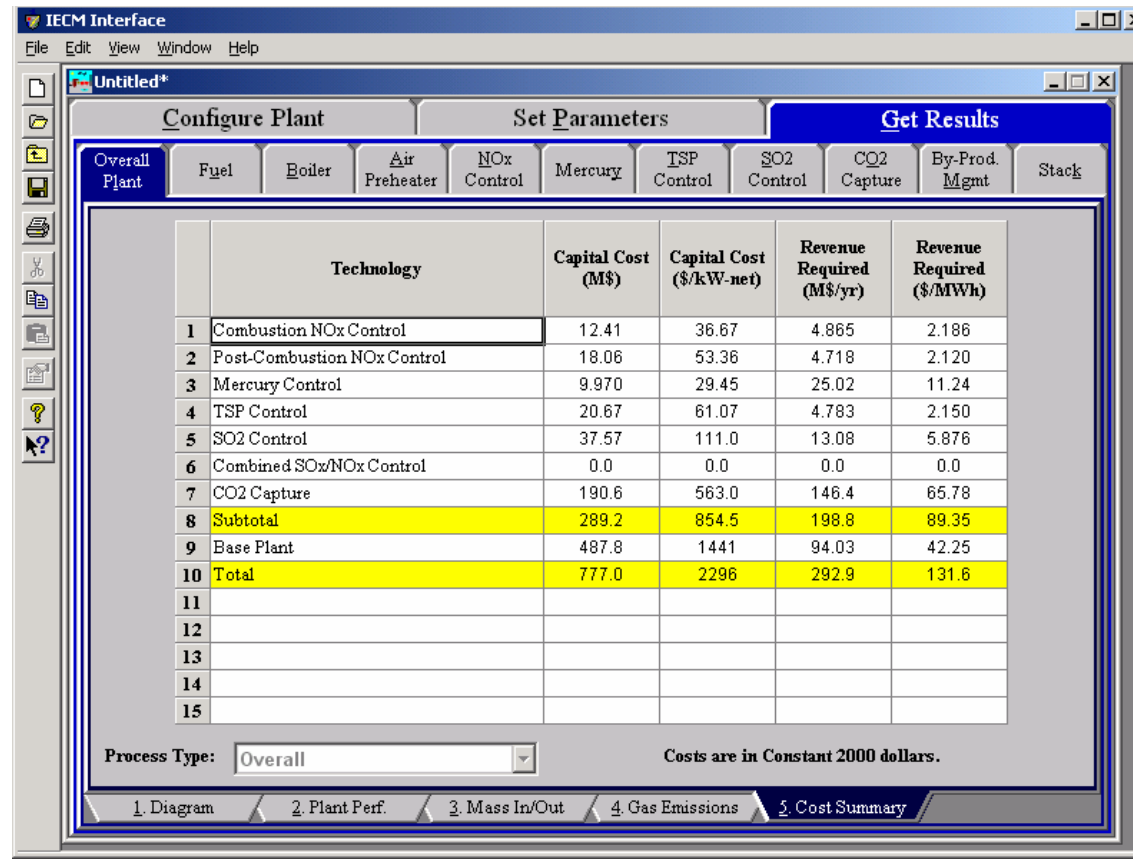

Overall Plant Cost Summary result screen.

The Cost Summary result screen displays costs associated with the power plant as a whole. Each technology (row) is described briefly below.

Combustion $\mathrm{NO}_{\mathrm{x}}$ Control: The total cost of the In-Furnace $\mathrm{NO}_{\mathrm{x}}$ controls used.

Post-Combustion $\mathrm{NO}_{\mathrm{x}}$ Control: The total cost of all the PostCombustion $\mathrm{NO}_{\mathrm{x}}$ removal modules used. 
Mercury Control: The total cost of all the mercury control modules used.

TSP Control: The total cost of all the conventional particulate removal modules used.

$\mathbf{S O}_{2}$ Control: The total cost of all the $\mathrm{SO}_{2}$ conventional removal modules used.

Combined $\mathrm{SO}_{\mathrm{x}} / \mathrm{NO}_{\mathrm{x}}$ : The total cost of all the combined $\mathrm{SO}_{\mathrm{x}} / \mathrm{NO}_{\mathrm{x}}$ advanced removal modules used.

Subtotal: This is the cost of the conventional and advanced abatement technology modules alone. This is the total abatement cost. The subtotal is highlighted in yellow.

Base Plant: The total cost of the base plant without consideration of any abatement technologies. This can be used to compare with other power plant types.

Total: This is the total cost of the entire power plant. This result is highlighted in yellow.

Each cost category (column) is described briefly below.

Capital Cost: The total capital requirement (TCR). This is the money that is placed (capitalized) on the books of the utility on the service date. The total cost includes the total plant investment plus capitalized plant startup. Escalation and allowance for funds used during construction (AFUDC) are also included. The capital cost is given on both a total and an annualized basis.

Revenue Required: Amount of money that must be collected from customers to compensate a utility for all expenditures in capital, goods, and services. The revenue requirement is equal to the carrying charges plus expenses. The revenue required is given on both an annualized and a net energy output basis. 


\section{Overall NGCC Plant}

\section{Overall NGCC Plant Diagram}

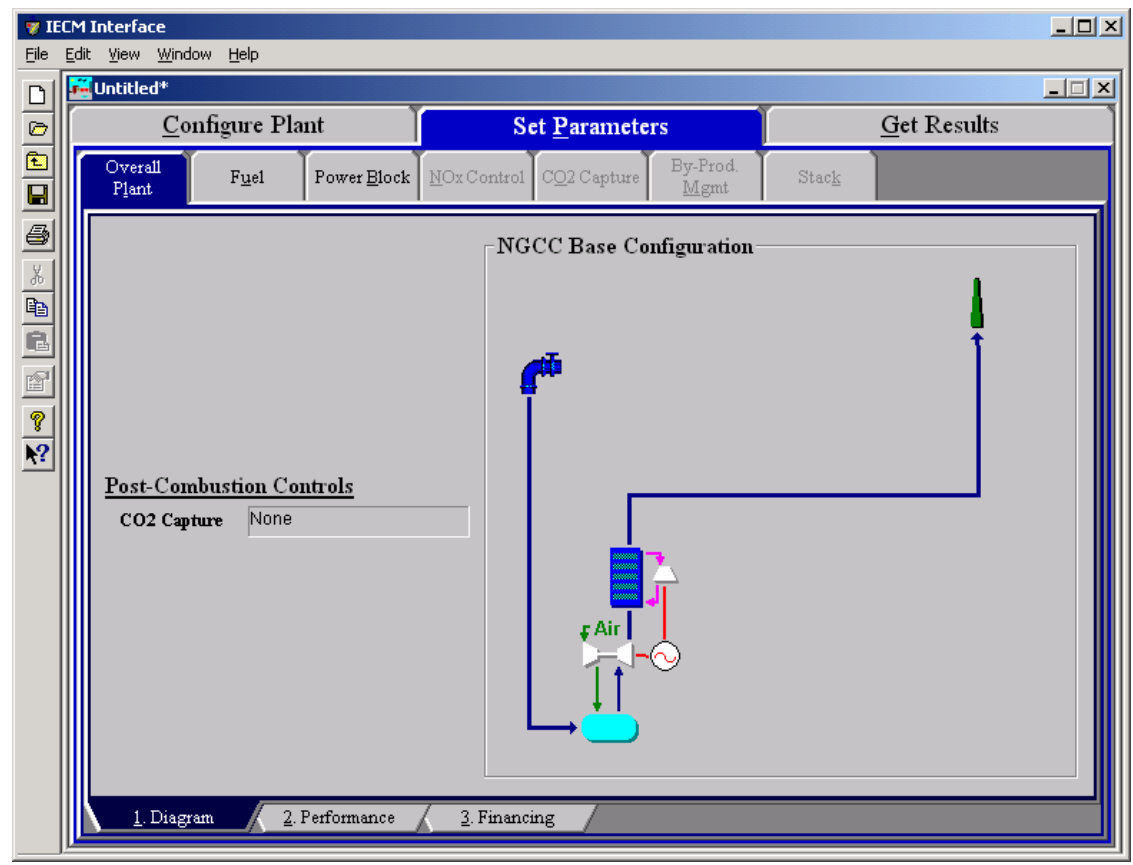

Overall NGCC Plant - Diagram input screen

The Overall NGCC Plant Diagram appears in the Set Parameters and in the Get Results program area. The screen displays the plant configuration settings on the left side of the page and a diagram of the plant as configured at the right of the page. No results are displayed on this screen. 


\section{Overall NGCC Plant Performance Inputs}

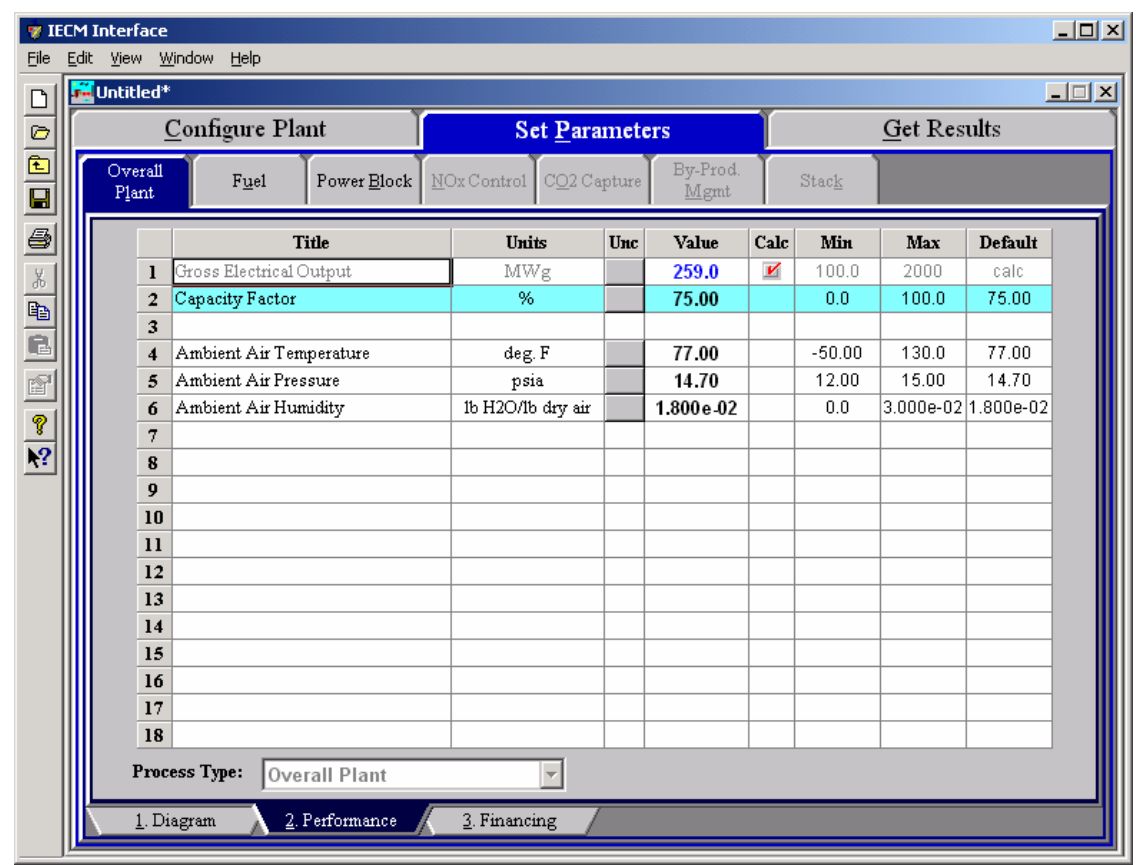

Overall NGCC Plant - Performance input screen.

The Natural Gas Combined Cycle input performance parameters are described below:

Gross Electrical Output: This is the gross output of the generator in megawatts $\left(\mathrm{MW}_{\mathrm{g}}\right)$. The value does not include auxiliary power requirements. The model uses this information to calculate key mass flow rates.

Capacity Factor: This is an annual average value, representing the percent of equivalent full load operation during a year. The capacity factor is used to calculate annual average emissions and materials flows.

Ambient Air Temperature: This is the temperature of the ambient plant air.

Ambient Air Pressure: This is the pressure of the ambient plant air.

Ambient Air Humidity: This is the humidity of the ambient plant air. 


\section{Overall NGCC Plant Financing Inputs}

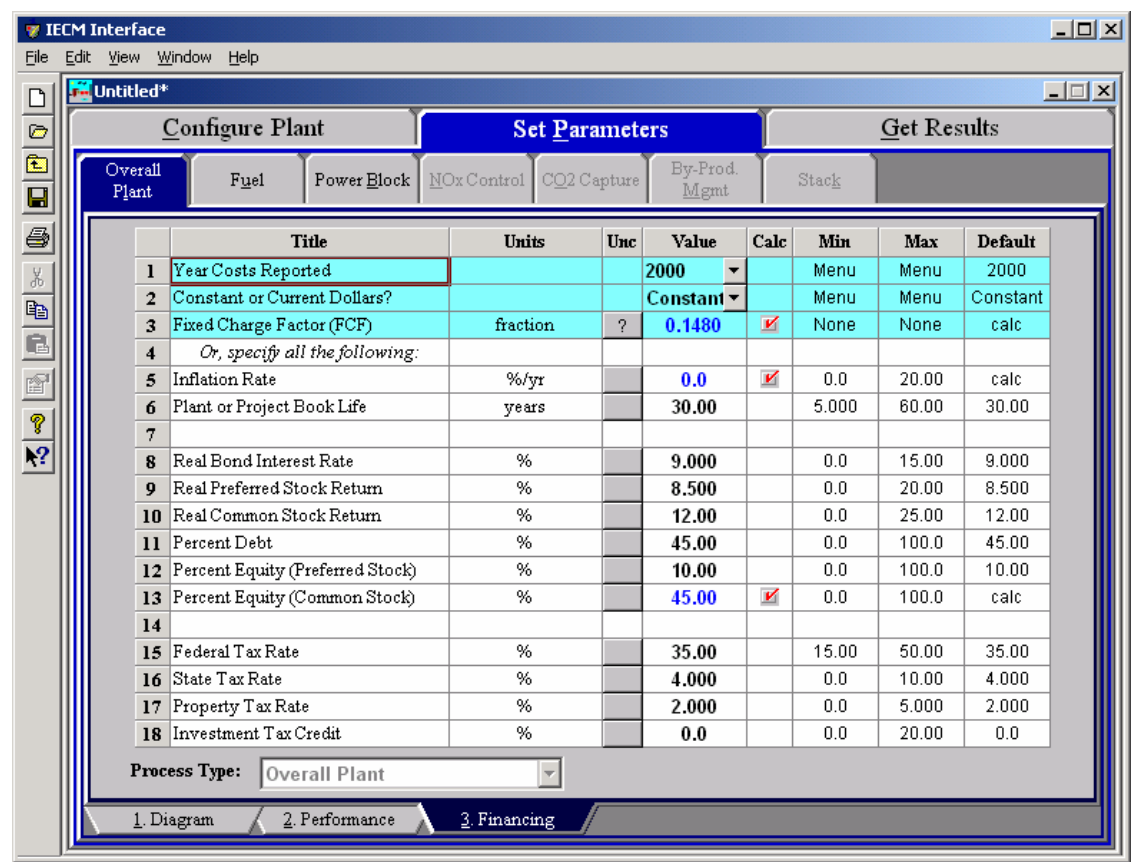

Overall NGCC Plant - Financing input screen.

This screen describes the factors required to determine the carrying charge for all capital investments. The carrying charge is defined as the revenue required to pay for any capital investment. The total charge can also be expressed as a levelized cost factor or fixed charge factor. The fixed charge factor is a function of many items. You have the option of specifying the fixed charge factor directly, or allowing it to be calculated from the other input quantities below it on the financial input screen.

The first four inputs are highlighted in blue. Each parameter is described briefly below.

Year Costs Reported: This is the year in which all costs are given or displayed, both in the input screens and the results. A cost index is used by the IECM to scale all costs to the cost year specified by this parameter.

Constant or Current Dollars: Constant dollar analysis does not include the affect of inflation, although real escalation is included. Current dollar analysis includes inflation and real escalation. This choice allows you to choose the mode of analysis for the entire IECM economics.

Fixed Charge Factor (FCF): The fixed charge factor is one of the most important parameters in the IECM. It determines the revenue required to finance the power plant based on the capital expenditures. Put another way, it is a levelized factor which accounts for the revenue per dollar of total plant cost that must be collected from customers in order to pay the carrying charges on that capital investment.

One may specify a Fixed Charge Factor, or fill in the following inputs and the model will calculate the FCF based on them: 
Inflation Rate: This is the rise in price levels caused by an increase in the available currency and credit without a proportionate increase in available goods or services. It does not include real escalation.

Plant or Project Book Life: This is the years of service expected from a capital investment. It is also the period over which an investment is recovered through book depreciation.

Real Bond Interest Rate: This is a debt security associated with a loan or mortgage. It is the most secure form of security but the lowest in its return.

Real Preferred Stock Return: This equity security is the second most speculative type and pays the second highest rate of return. The holder of the stock is a part owner of the company.

Real Common Stock Return: This is the most speculative type of equity security sold by a utility and pays the highest relative return. The holder of the stock is a part owner of the company.

Percent Debt: This is the percent of the total capitalization that is associated with debt money. This includes loans and mortgage bonds.

Percent Equity (Preferred Stock): This is the percent of the total capitalization that is associated with the sale of preferred stock.

Percent Equity (Common Stock): This value is the remainder of the capitalization, calculated as $100 \%$ minus the percent debt, minus the percent equity in preferred stock.

Federal Tax Rate: This is the federal tax rate. It is used to calculate the amount of taxes paid and deferred.

State Tax Rate: This is the state tax rate. It is used to calculate the amount of taxes paid and deferred.

Property Tax Rate: The property tax rate, or ad valorem, is used to calculate the carrying charge.

Investment Tax Credit: This is an immediate reduction in income taxes equal to a percentage of the installed cost of a new capital investment. It is zero by default. It is used to set the initial balance and the book depreciation. 


\section{Overall NGCC Plant Performance Results}

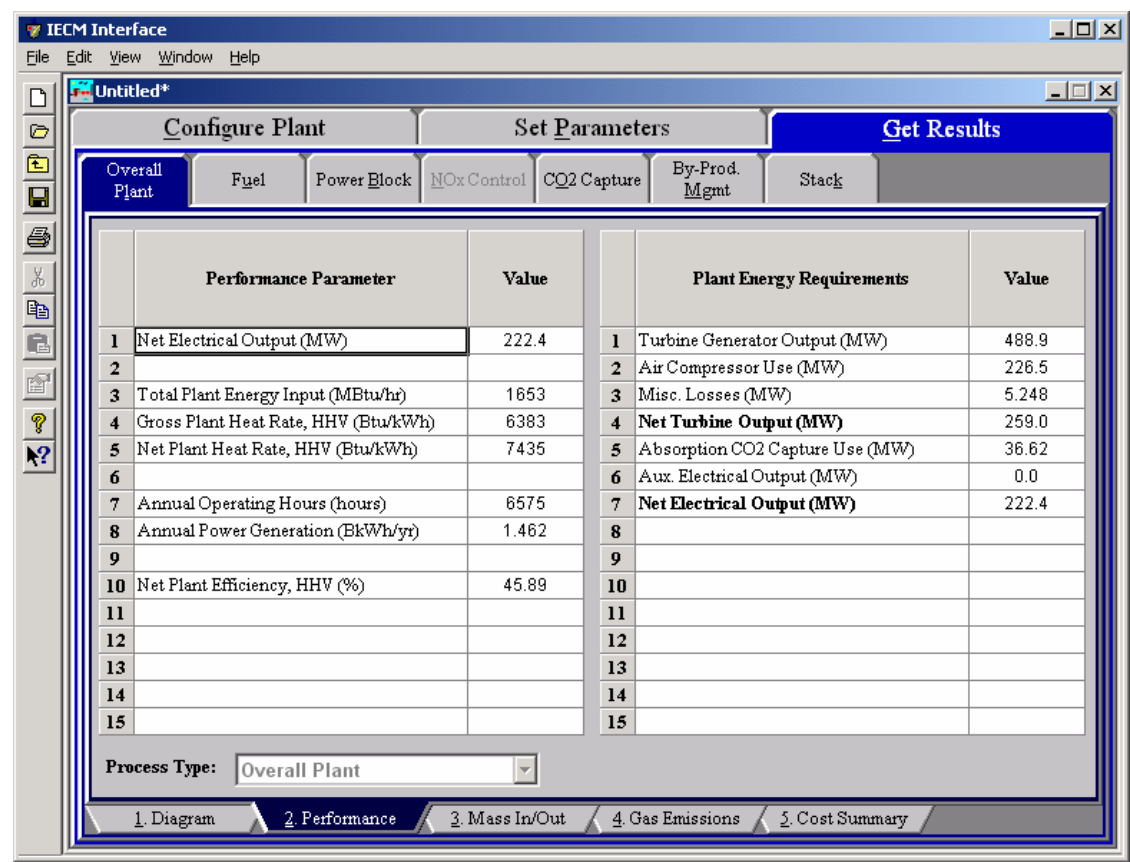

Overall NGCC Plant - Performance results screen.

\section{Performance Parameter}

Net Electrical Output: This is the net plant capacity, which is the gross plant capacity minus the losses due to plant equipment and pollution equipment (energy penalties).

Total Plant Energy Input: The fuel energy input to the plant on an hourly basis (maximum capacity).

Gross Plant Heat Rate, HHV: This is the gross heat rate of the entire plant.

Net Plant Heat Rate, HHV: This is the net heat rate of the entire plant (including aux power produced) which includes the effect of plant equipment and pollution control equipment.

Annual Operating Hours: This is the number of hours per year that the plant is in operation. If a plant runs 24 hours per day, seven days per week, with no outages, the calculation is 24 hours * 365 days. or 8,760 hours/year.

Annual Power Generation: This is the net annual MWh production of the plant. The capacity factor is used in determining its value.

Net Plant Efficiency, HHV: This is the net efficiency of the entire plant.

\section{Plant Energy Requirements}

Turbine Generator Output: This is the energy generated by the turbine.

Air Compressor Use: The energy required to operate the air compressor.

Misc. Losses: This variable accounts for any turbine electricity losses that are not incorporated into the lossed due to air compressor use. 
Net Turbine Output: This if the net energy generated by the turbine. This is the gross output of the turbine minus the energy required by the air compressor and any miscellaneous losses.

*Absorption $\mathrm{CO}_{2}$ Capture Use: If a $\mathbf{C O}_{2}$ Capture system is in use, this is the energy required to operate the system.

*Aux. Electrical Output: If an auxiliary natural gas boiler is used to provide steam and power for the Amine System, this is the additional electricity that it produces

Net Electrical Output: This is the net plant capacity, which is the gross plant capacity minus the losses due to plant equipment and pollution equipment (energy penalties).

\section{Overall NGCC Plant Mass In/Out Results}

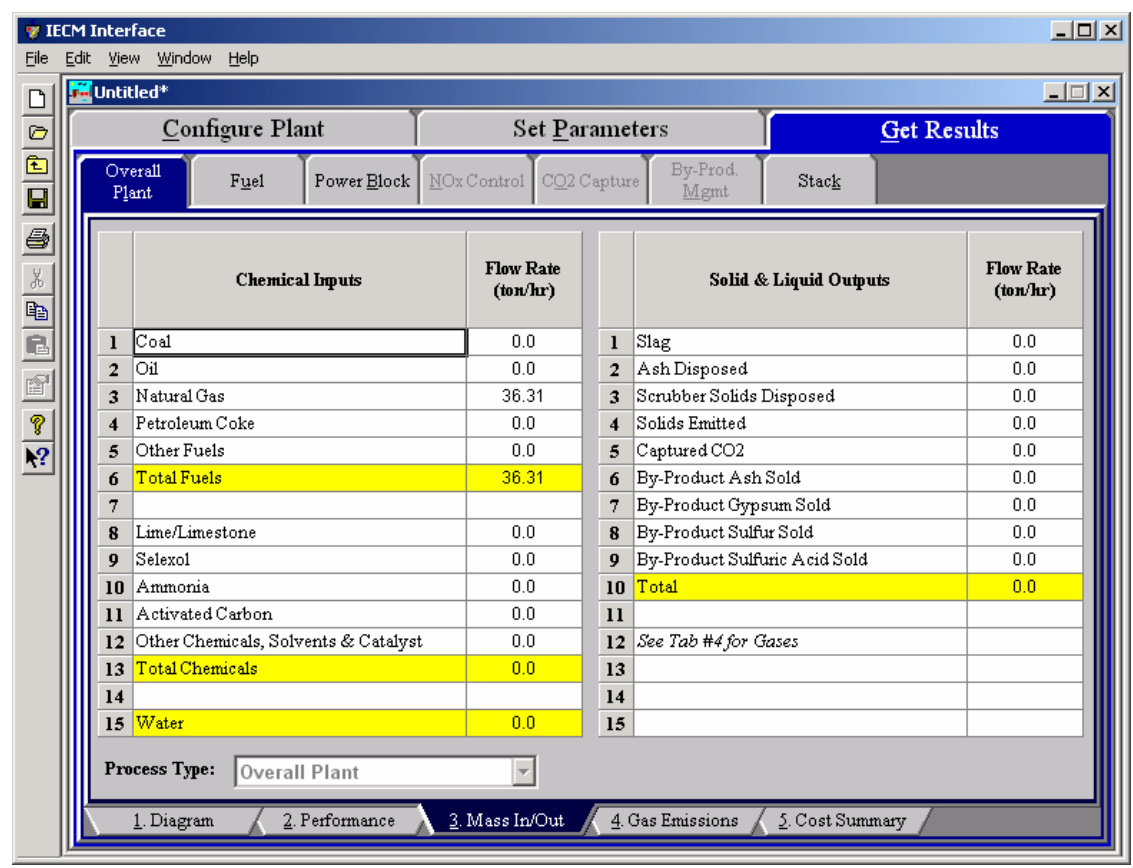

Overall NGCC Plant - Mass In/Out results screen.

\section{Chemical Inputs}

Coal: Flow rate of coal used in the power plant.

Oil:Flow rate of oil used in the power plant

Natural Gas: : Flow rate of natural gas used in the power plant

Petroleum Coke: Total mass of petroleum coke used in the power plant

Other Fuels: Flow rate of other fuels used in the power plant

Total Fuels: This is theFlow rate of fuel entering the power plant. This result is highlighted in yellow.

Lime/Limestone: Total mass of this reagent used in the power plant on a wet basis.

Selexol:Total mass of selexol sorbent used in the power plant 
Ammonia: : Total mass of ammonia used in the power plant.

Activated Carbon: Flow rate of activated carbon injected in the power plant.

Other Chemicals, Solvents \& Catalyst: Flow rate of other chemicals, solvents and catalysts used in the power plant.

Total Chemicals: Flow rate of reagent entering the power plant. This result is highlighted in yellow.

Water: Flow rate of water used in the power plant.

\section{Solid \& Liquid Outputs}

Slag: Flow rate of slag from the power plant on a dry basis.

Ash Disposed: Flow rate of ash from the power plant on a dry basis.

Scrubber Solids Disposed: Flow rate of scrubber treatment solid wastes from the power plant on a dry basis.

Solids Emitted: Flow rate of solids emitted from the plant.

Captured $\mathrm{CO}_{2}$ : Flow rate of the captured $\mathrm{CO}_{2}$.

Byproduct Ash Sold: : Flow rate of ash (bottom and fly ash) sold in commerce as a by-product on a dry basis.

Byproduct Gypsum Sold: : Flow rate of flue gas treatment solids sold in commerce as a by-product on a dry basis.

Byproduct Sulfur Sold: Flow rate of elemental sulfur recovered from flue gas and sold in commerce as a by-product on a dry basis.

Byproduct Sulfuric Acid Sold: Total mass of sulfuric acid recovered from the flue gas and sold in commerce as a by-product.

Total: This is the total wet solid mass exiting the power plant. This result is highlighted in yellow. 


\section{Overall NGCC Plant Gas Emissions Results}

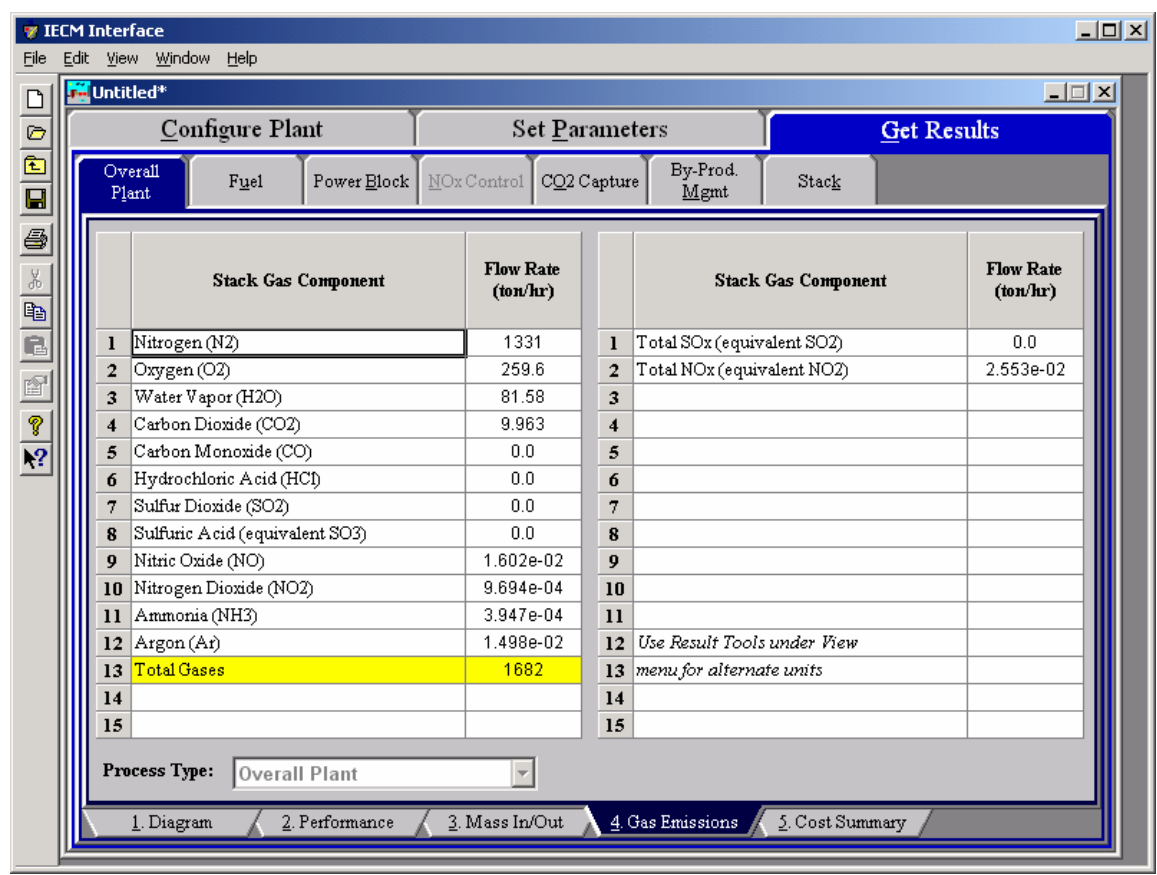

Overall NGCC Plant - Gas Emissions result screen.

\section{Stack Gas Component}

Each result is described briefly below:

Nitrogen $\left(\mathbf{N}_{2}\right)$ : Total mass of nitrogen.

Oxygen $\left(\mathrm{O}_{2}\right)$ : Total mass of oxygen.

Water Vapor $\left(\mathrm{H}_{2} \mathrm{O}\right)$ : Total mass of water vapor.

Carbon Dioxide $\left(\mathrm{CO}_{2}\right)$ : Total mass of carbon dioxide.

Carbon Monoxide (CO): Total mass of carbon monoxide.

Hydrochloric Acid (HCl): Total mass of hydrochloric acid.

Sulfur Dioxide $\left(\mathrm{SO}_{2}\right)$ : Total mass of sulfur dioxide.

Sulfuric Acid (equivalent $\mathrm{SO}_{3}$ ): Total mass of sulfuric acid.

Nitric Oxide (NO): Total mass of nitric oxide.

Nitrogen Dioxide $\left(\mathrm{NO}_{2}\right)$ : Total mass of nitrogen dioxide.

Ammonia $\left(\mathrm{NH}_{3}\right)$ : Total mass of ammonia.

Total Gases: Total of the individual components listed above. This item is highlighted in yellow.

Total $\mathrm{SO}_{\mathbf{x}}$ (equivalent $\mathrm{SO}_{2}$ ): Total mass of $\mathrm{SO}_{\mathrm{x}}$ as equivalent $\mathrm{SO}_{2}$.

Total $\mathrm{NO}_{\mathbf{x}}$ (equivalent $\mathrm{NO}_{2}$ ): Total mass of $\mathrm{NO}_{\mathrm{x}}$ as equivalent $\mathrm{NO}_{2}$. 


\section{Overall NGCC Plant Cost Summary Results}

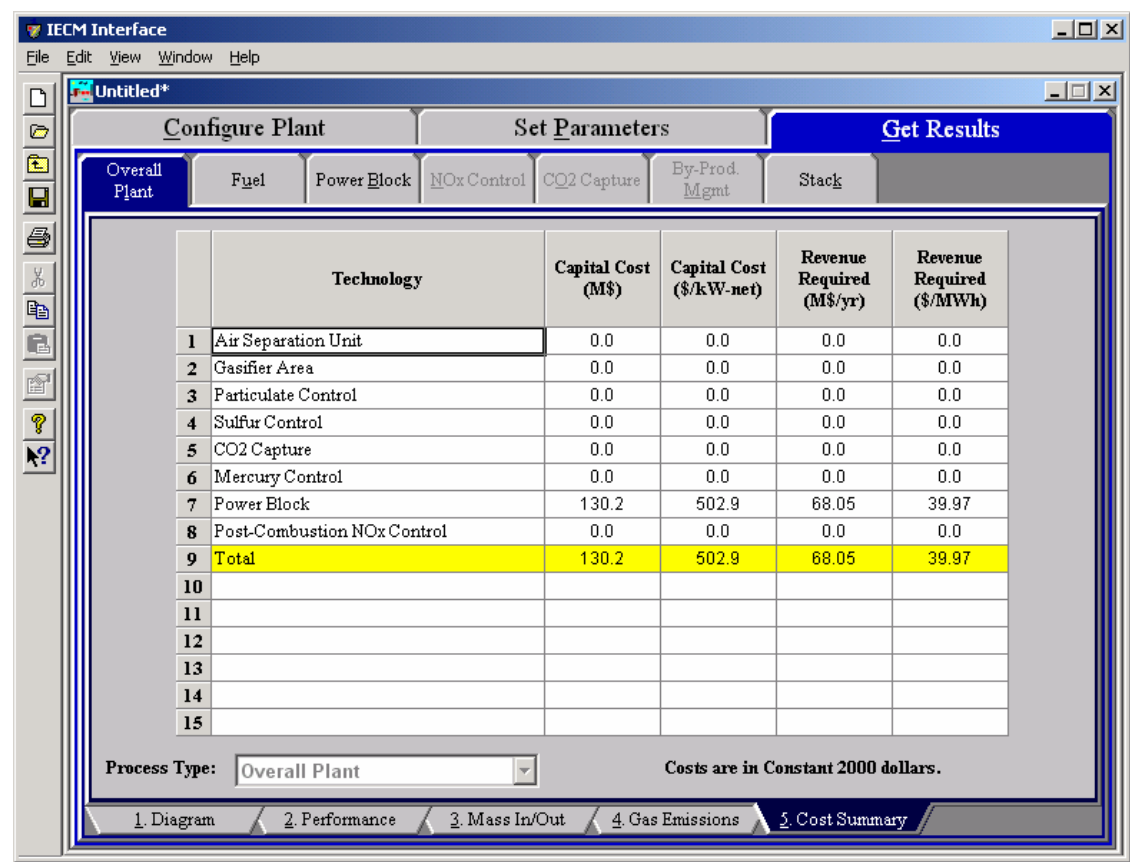

Overall NGCC Plant - Cost Summary results screen.

\section{Technology}

Air Separation Unit: This is the capital cost for the Air Separation process area of the plant.

Gasifier Area:This is the capital cost for the Gasifier process area of the plant.

Particulate Control: This is the capital cost for the equipment that performs Particulate Capture in the plant.

Sulfur Control: This is the capital cost for the equipment that performs Sulfur Capture in the plant.

$\mathbf{C O}_{2}$ Capture: This is the capital cost for the equipment that performs $\mathbf{C O}_{2}$ Capture in the plant.

Mercury Control:This is the capital cost for the Air Separation process area of the plant.

Power Block: This is the capital cost for the Power Block process area of the plant.

Post-Combustion $\mathrm{NO}_{\mathrm{x}}$ Control:This is the capital cost for the equipment that performs Post-Combustion $\mathrm{NO}_{\mathrm{x}}$ Control in the plant.

Total:This is the Sum of all of the above capital costs for all of the process areas in the plant. 



\section{Fuel}

The screens associated with the Fuel Technology Navigation Tab display and define the composition and cost of the fuels used in the plant

\section{Fuel Properties Input}

The selection of the particular coal model default, cleaned, saved externally, or userspecified and its ultimate and ash properties are selected and editable on the Properties input screen.

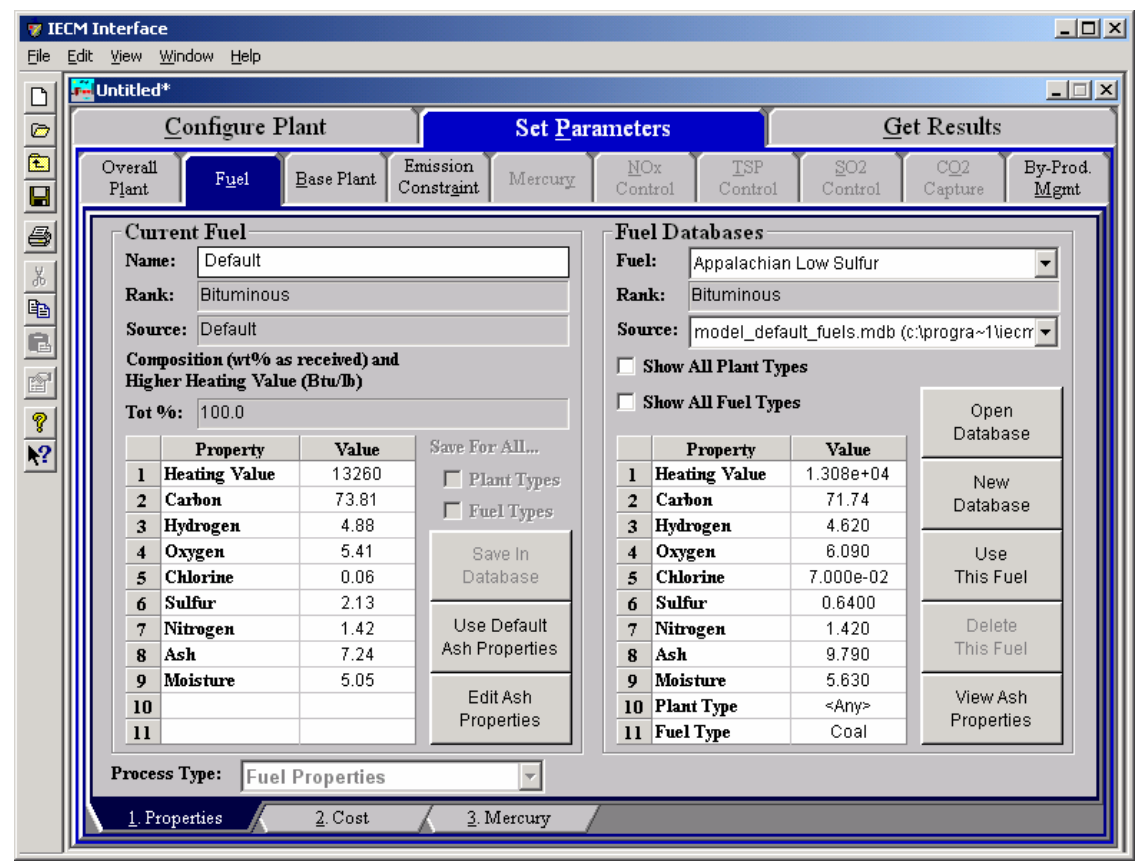

Fuel - Properties input screen.

There are two panes on the Fuel Properties input screen: one for the composition and higher heating value properties of the Current Fuel, the other for properites of the fuels in the Fuel Databases. The Current Fuel is the fuel for which the model will conduct its calculations. The IECM interface currently supports only one fuel selection per session. The Fuel Databases pane displays the properties for other selectable fuels. From this screen, you may choose a fuel from the model defaults, enter a user-defined fuel, or choose a previously saved user-defined fuel. Properties 
of existing fuels may be modified and new fuels may be created and saved to user specified databases. A full suite of buttons have been provided to make the selection and management of the fuel properties easier.

Both the Current Fuel pane and the Fuel Databases pane display the following information: for a fuel.

Name This is the name of the fuel, it may be the trade name or a unique identifier supplied by the user.

Rank: The rank of a coal refers to the degree of coalification endured by the organic matter. It is estimated by measuring the moisture content, specific energy, reflectance of vitrinite or volatile matter (these are known as rank parameters

Source: The model provides the values for default fuel properties, these can be used "as is" or modified and used. Modified fuels maybe stored in a new database or an existing database. Source displays the database file from which the data was retrieved, or indicates that the data has been enetered by the user.

Fuel Properties: The property value spreadsheet is used to display the heating value and content of carbon, hydrogen, oxygen, chlorine, sulfur, nitrogen, ash, and moisture are specified on a weight percent basis for coal fuels. The data can be edited only in the Current Coal pane. The fuel composition is used in a combustion equation to calculate the flue gas composition in the furnace. The heating value is used to calculate the mass flow rate of fuel. Property data also determines the fuel rank (bituminous, subbituminous, or lignite). This, in turn, determines the default values of several boiler parameters. The editable fuel properties are:

Heating Value: This is the higher heating value of the fuel in Btu/lb.

Carbon: The weight percent of carbon in the fuel on a wet basis.

Hydrogen: This is the weight percent of hydrogen in the fuel on a wet basis.

Oxygen: This is the weight percent of oxygen in the fuel on a wet basis.

Chlorine: This is the weight percent of chlorine in the fuel on a wet basis.

Sulfur: This is the weight percent of sulfur in the fuel on a wet basis.

Nitrogen: This is the weight percent of nitrogen in the fuel on a wet basis.

Ash: This is the weight percent of ash in the fuel on a wet basis.

Moisture: This is the weight percent of moisture in the fuel on a wet basis.

Ash Properties: The property value spreadsheet is also used to display the oxide content of the ash in coal on a percent of total ash basis. The data can be edited only in the Current Fuel pane. The ash content is used to determine the resistivity of the ash. This, in turn, determines the specific collection area (SCA) of the cold-side ESP. The editable ash properties are:

$\mathrm{SiO}_{2}$ : The percent by wieght of silicon dioxide in the ash. 
$\mathrm{Al}_{2} \mathbf{O}_{3}$ : The percent by wieght of Aluminum Oxide in the ash.

$\mathrm{Fe}_{2} \mathrm{O}_{3}$ : The percent by wieght of ferric oxide in the ash.

CaO: The percent by wieght of calcium oxide in the ash.

MgO: The percent by wieght of magnesium oxide in the ash.

$\mathrm{Na}_{2} \mathrm{O}$ : The percent by wieght of sodium oxide in the ash.

$\mathrm{K}_{2} \mathrm{O}$ : The percent by wieght of potassium oxide in the ash.

$\mathrm{TiO}_{2}$ : The percent by wieght of titanium dioxide in the ash.

$\mathrm{MnO}_{2}$ : The percent by wieght of manganese dioxide in the ash.

$\mathbf{P}_{2} \mathbf{O}_{5}$ : The percent by wieght of phosphorus pentoxide in the ash.

$\mathrm{SO}_{3}$ : The percent by wieght of sulfur trioxide in the ash.

\section{Selecting a Fuel}

The Current Fuel pane displays the fuel that is in use by the model. The Fuel Databases pane initially displays the first default fuel in the model's default database. To make the fuel that is displayed in the Fuel Database pane the fuel to be used by the model, press the Use this Fuel button. The fuel will then be displayed in the Current Fuel pane. To view the ash properties, press the View Ash Properties button in the Fuels Database , the ash properties are displayed and the button that was pressed, labeled View Ash Properties has changed to View Fuel Properties. This button toggles between View Ash Properties and View Fuel Properties. To find other fuels:

Select a Different Fuel in the Open Database: Select the pull down menu on the text box labeled Fuel:. The list of fuels in the database is displayed another fuel can be chosen.

Select a Different Open Database: Select the pull down menu on the text box labeled Source:. The list of other open databases is displayed.

Open Another Fuel Database: When pressed the button labeled Open Database will display the Windows Open screen. All files with .fdb extension will be displayed. .fdb is the default extension for the Fuel Databases files. Select a file and press the Open button.

\section{Modifiying a Fuel}

The fuel values that are displayed in the Current Fuel pane may be modified. Put the cursor into the cell containing the value of the property to be edited and enter the new value. To edit the ash properties of the current fuel; press the Edit Ash Properties button in the Current Fuel pane, the ash properties are displayed and the button that was pressed, labeled Edit Ash Properties has changed to Edit Fuel Properties. This button toggles between Edit Ash Properties and Edit Fuel Properties. . The ash properties may be edited in the same way as the fuel properties. Place the cursor in the value of the property to be modified and enter the new value. The model will run using the fuel that is displayed in the Current Fuel pane.

\section{Saving a Modified Fuel}

A fuel that has been modified may be saved to any user specified fuel database except the default database, model_default_fuels.mdb. Use the Save in Database button to save the modified fuel, displayed in the Current Fuel pane to the database that is displayed in the Source text box. If the default database, model_default_fuels.mdb is displayed in the text box titled Source, the Save 
in Database button will be grayed out, not active. Activate the Save in Database, by opening another database or creating a new database.

\section{Deleting a Fuel}

A fuel that is displayed in the Fuel Databases pane, may be deleted using the Delete this Fuel button, if it is not a model default fuel. Fuels in the model default database, model_default_fuels.mdb, cannot be deleted.

\section{Open Database}

Press the Open Database button on the Fuels Database pane and the Windows Open Screen will appear. A valid fuel database file as an .fdb extension. Click on the database file to open and press the Open button. The Fuels Database displays the first fuel in the selected database and the Source: text box displays the full path and file name of the database that has just been opened.

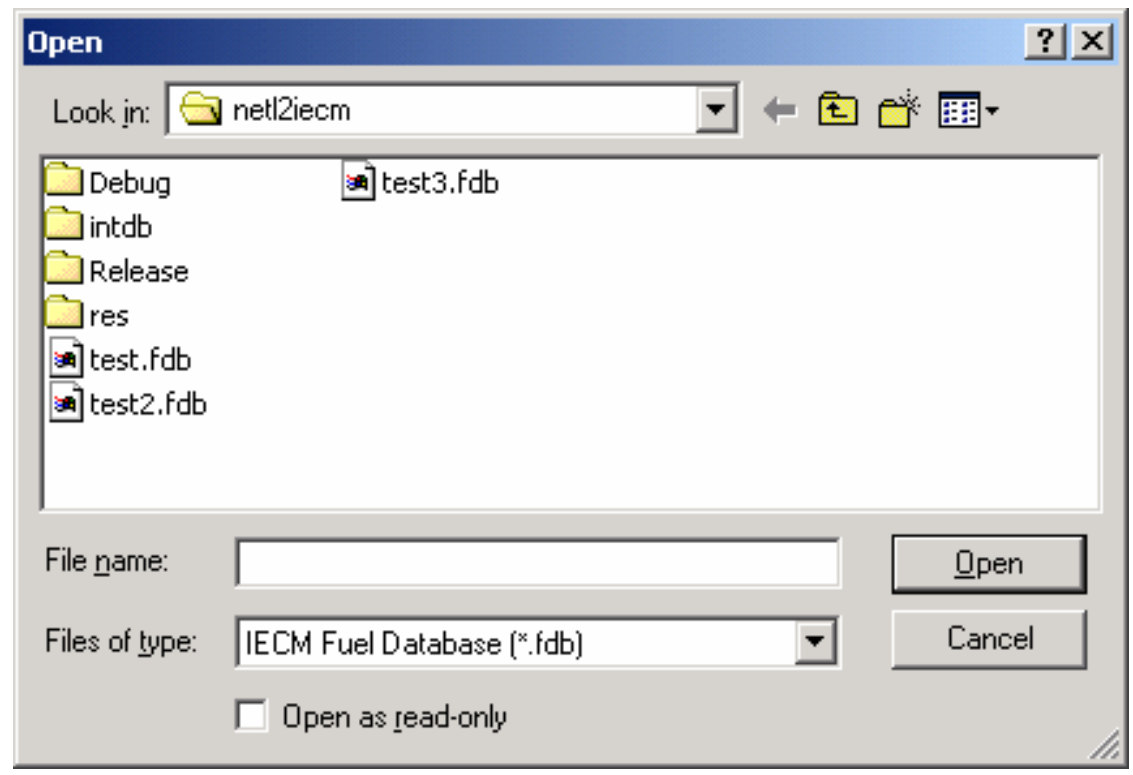

Fuels - Windows Open screen.

\section{New Database}

Press the New Database button on the Fuels Database pane and the Windows Save As Screen will appear. Type in the name of the new database file into the File name: text box. All fuel database files have an .fdb extension. Press the Save button. The Source: text box displays the full path and file name of the new database and all other fuel values in the Fuels Database pane will be blank 


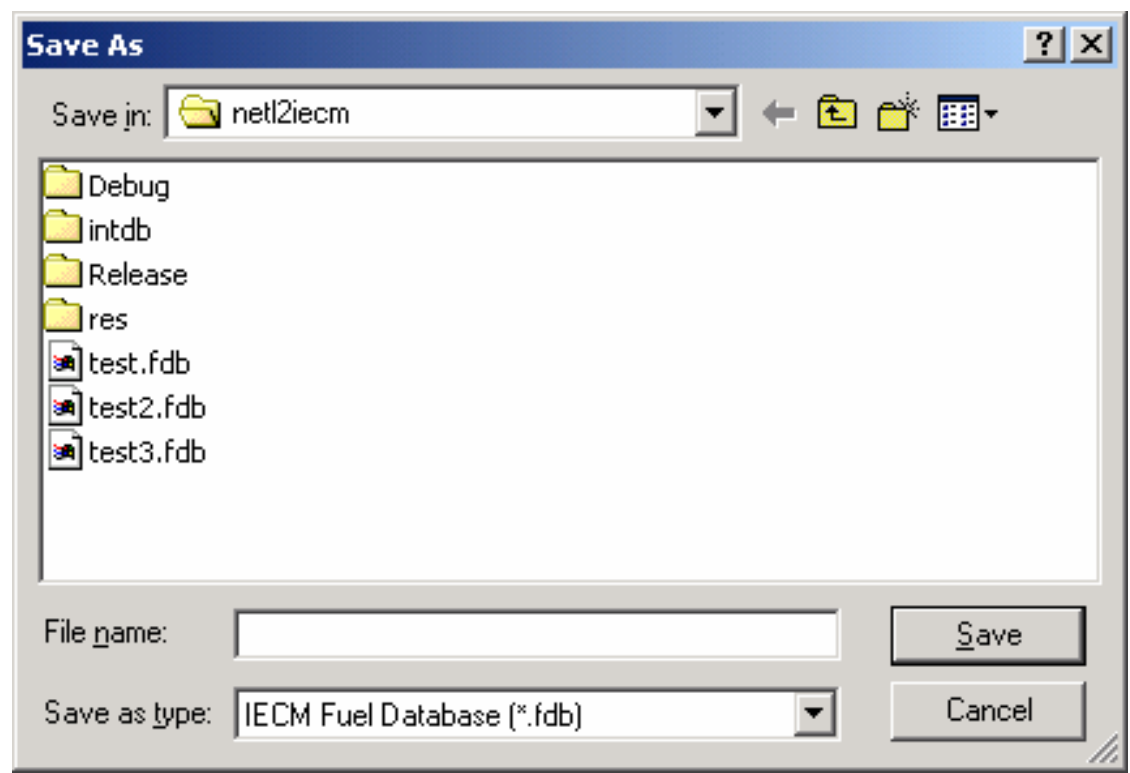

Fuels - Windows Save As screen.

\section{Fuel Coal Cost Input}

The cost of the cleaned coal, transportation costs, and other miscellaneous coal costs are accessed on the Cost input screen.

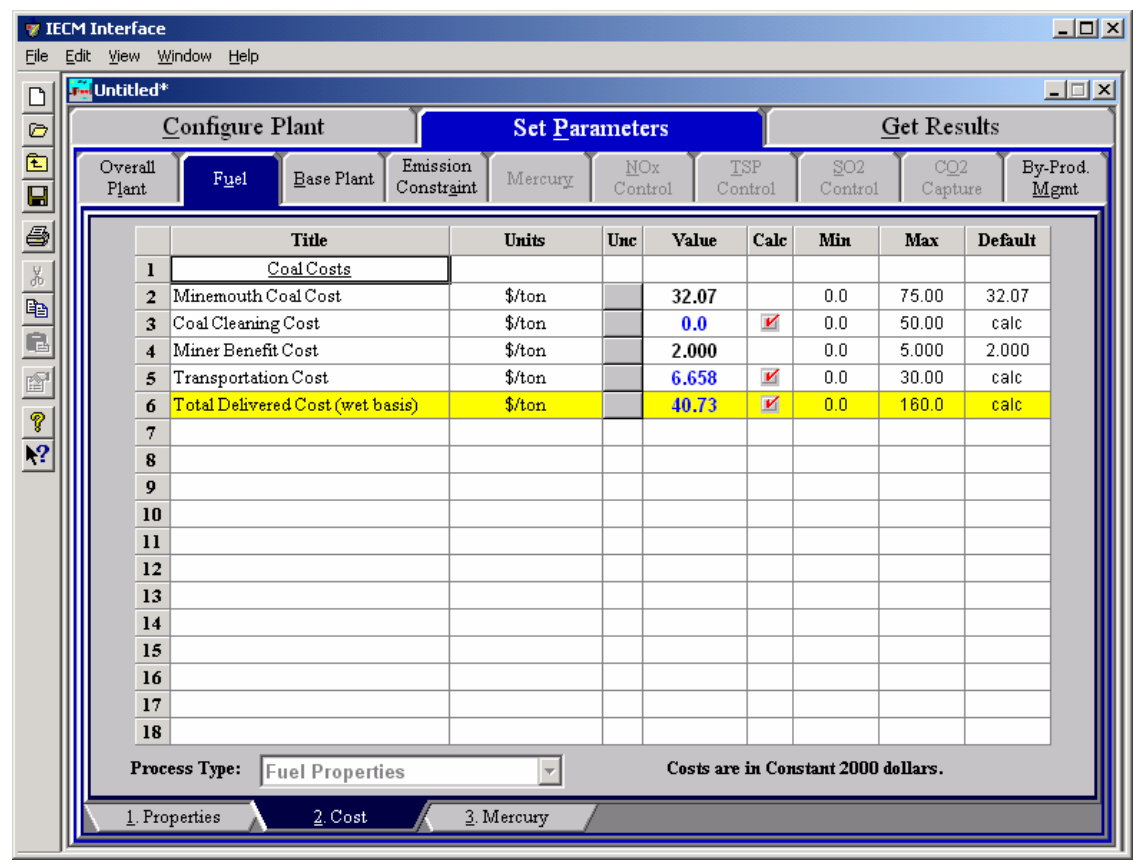

Fuel - Coal Cost input screen.

Each parameter is described briefly below.

Minemouth Coal Cost: This is the cost of the delivered coal in dollars per wet ton. It does not include any cleaning costs. 
Coal Cleaning Cost: This is the cost of cleaning the coal. The default value is calculated. It is zero if the coal was not cleaned.

Miner Benefit Cost: This is the cost associated with the miner pension and benefit fund. It is expressed on a wet ton basis.

Transportation Cost: This is the transportation cost associated with the coal used by the power plant. It is scaled by the cost index and expressed on a wet basis.

Total Delivered Cost (as-fired): This is the total cost of delivered coal on a wet ton basis. It is simply the sum of all the quantities listed above. This input is highlighted in yellow.

\section{Fuel Natural Gas Cost Input}

If the user has configured the plant to use a Natural Gas fueled turbine the Natural Gas Cost input screen will be displayed.

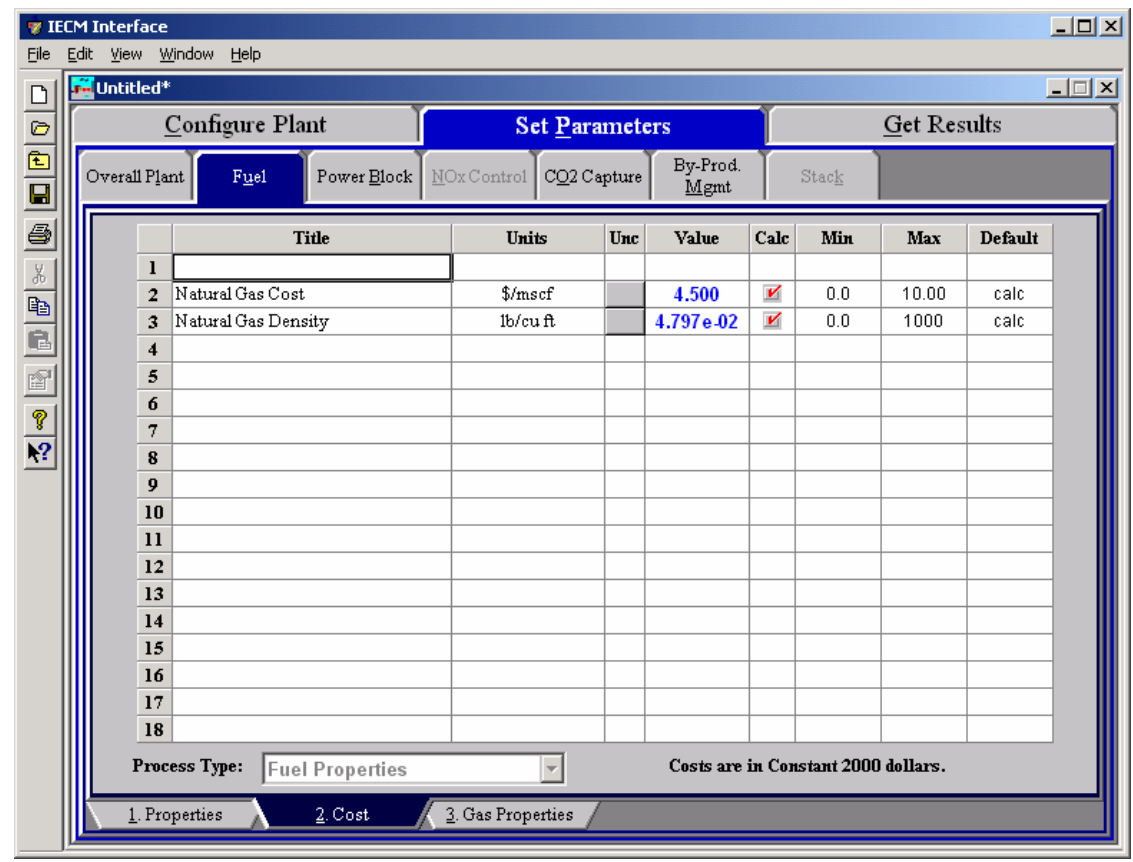

Fuel - Natural Gas Cost Input Screen

Each parameter is described briefly below.

Natural Gas Cost: This is the cost of the delivered natural gas in millions of dollars per cubic foot.

Natural Gas Density: This the density of the natural gas in pounds per cubic foot.

\section{Fuel Mercury Input}

The concentration of mercury in the as-fired coal and speciation of mercury after combustion are entered on the Mercury input screen 


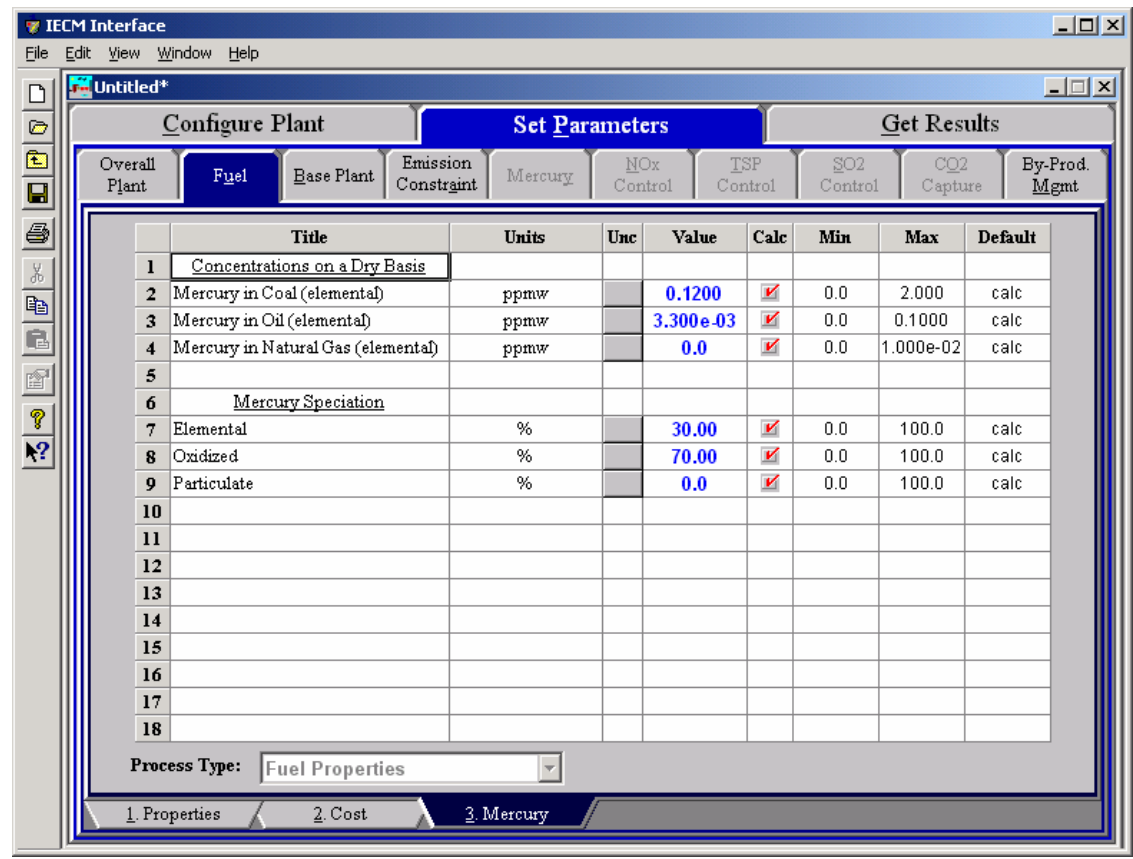

Fuel - Mercury input screen.

Each parameter is described briefly below:

\section{Concentration on a Dry Basis}

Trace elements found in fuels are typically measured and reported as a mass concentration given on a dry basis. The IECM uses this concentration in conjunction with the fuel flow rate and fuel moisture to determine the mass flow rate. Currently Mercury is the only trace species tracked in the IECM.

Mercury in Coal (elemental): This input parameter specifies the mass concentration of total mercury in the coal given on a dry basis. The mercury concentration should be given on an elemental basis, not on a mercury compound basis. The default value is a function of the coal rank.

Mercury in Oil (elemental): This input parameter specifies the mass concentration of total mercury in the oil. The mercury concentration should be given on an elemental basis, not on a mercury compound basis.

Mercury in Natural Gas (elemental): This input parameter specifies the mass concentration of total mercury in the natural gas. The mercury concentration should be given on an elemental basis, not on a mercury compound basis.

\section{Mercury Speciation}

Once the fuel is combusted, the mercury can be identified in primarily two chemical states: elemental $\left(\mathrm{Hg}^{0}\right)$ and oxidized $\left(\mathrm{Hg}^{+2}\right)$. Although mercury can alternatively be reported as particulate or gas phase, the IECM assumes Mercury is reported on an elemental and oxidized basis.

Elemental: This is the percent of total mercury that is in an elemental state $\left(\mathrm{Hg}^{0}\right)$ after combustion. Elemental mercury is typically unreactive and passes through a power plant. The default value is a function of the coal rank. 
Oxidized: This is the percent of total mercury that is in an oxidized state $\left(\mathrm{Hg}^{+2}\right)$ after combustion. Oxidized mercury is very reactive and typically forms mercury compounds. The default value is a function of the coal rank.

Particulate: This parameter is not currently used in the IECM. It's value is set to force the sum of the speciation types to be $100 \%$.

\section{Fuel Natural Gas Properties Input}

If the user has configured the plant to have $\mathbf{C O 2}$ Capture and added an Auxiliary Natural Gas Boiler, or if the user has configured the plant to use a Natural Gas fueled turbine then the Natural Gas Properties input screen will also be available.

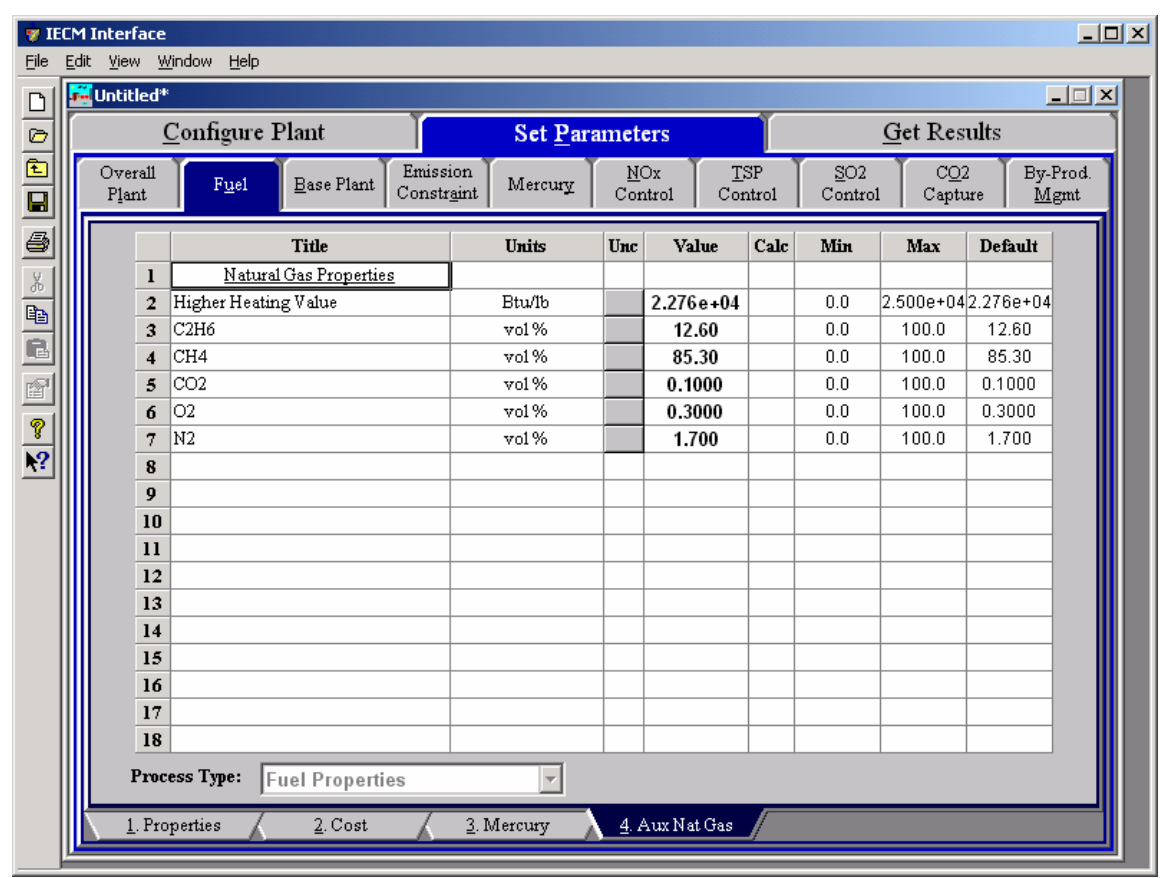

Fuel - Natural Gas input screen.

The Natural Gas Properties input screen displays and allows the user to update the fuel properties of Natural Gas.

\section{Natural Gas Properties}

Higher Heating Value: Higher heating value (HHV) is the thermal energy produced in Btu/lb of fuel (wet) from completely burning the fuel to produce carbon dioxide and liquid water. The latent heat of condensation is included in the value.

Ethane $\left(\mathrm{C}_{2} \mathrm{H}_{6}\right)$ : The volume, by percent, of ethane in the natural gas.

Methane $\left(\mathrm{CH}_{4}\right)$ : The volume, by percent, of methane in the natural gas.

Carbon Dioxide $\left(\mathrm{CO}_{2}\right)$ :The volume, by percent, of carbon dioxide in the natural gas.

Oxygen $\left(\mathrm{O}_{2}\right)$ :The volume, by percent, of oxygen in the natural gas.

Nitrogen $\left(\mathrm{N}_{2}\right)$ : The volume, by percent, of nitrogen in the natural gas. 


\section{Fuel Coal Diagram}

The FuelTechnology Navigation Tab in the Get Results program area contains the Diagram result screen. It displays the properties set up in the Fuel Properties input screens of the of the Set Parameters program area.

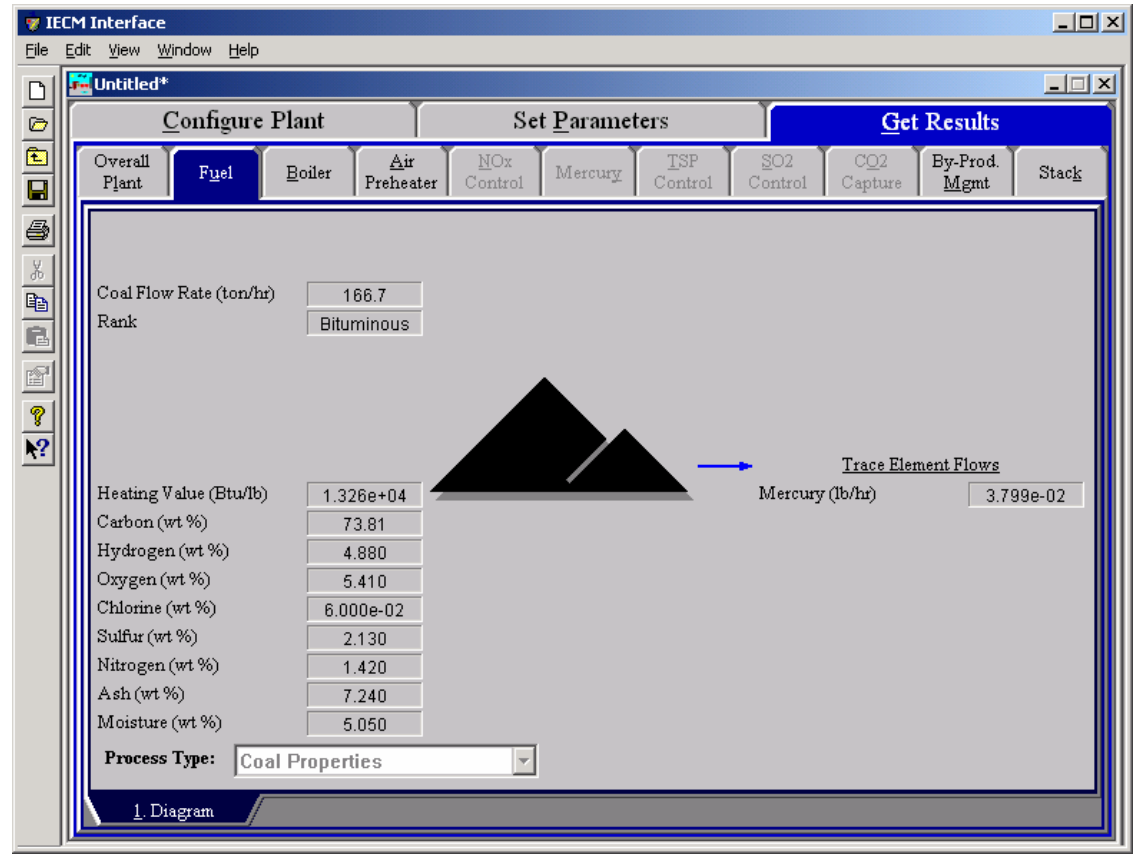

Fuel - Diagram result screen for coal.

The Coal Diagram result screen displays fuel composition and flow rate information, which is described briefly below.

Coal Flow Rate: Coal flow rate into the boiler on a wet basis. Waste products removed prior to the burners are not considered here.

Rank: The rank of the coal based on the higher heating value. This is primarily determined by the higher heating value and to a lesser degree by the sulfur and ash content.

Heating Value: Higher heating value (HHV) is the thermal energy produced in Btu/lb of fuel (wet) from completely burning the fuel to produce carbon dioxide and liquid water. The latent heat of condensation is included in the value.

Carbon: The carbon content of the coal by weight on an elemental and wet basis.

Hydrogen: The hydrogen content of the coal by weight on an elemental (H) and wet basis.

Oxygen: The oxygen content of the coal by weight on an elemental (O) and wet basis.

Chlorine: The chlorine content of the coal by weight on an elemental (Cl) and wet basis.

Sulfur: The sulfur content of the coal by weight on an elemental (S) and wet basis. 
Nitrogen: The nitrogen content of the coal by weight on an elemental $(\mathrm{N})$ and wet basis.

Ash: The ash content of the coal by weight on a wet basis.

Moisture: The inherent moisture content of the coal by weight.

\section{Trace Element Flows}

Trace elements are now supported in the IECM. The mass flow rate is reported in units of pounds per unit of time. All values reflect the elemental mass flow rate.

Mercury: This is the elemental mercury flow rate in coal.

\section{Fuel Natural Gas Diagram}

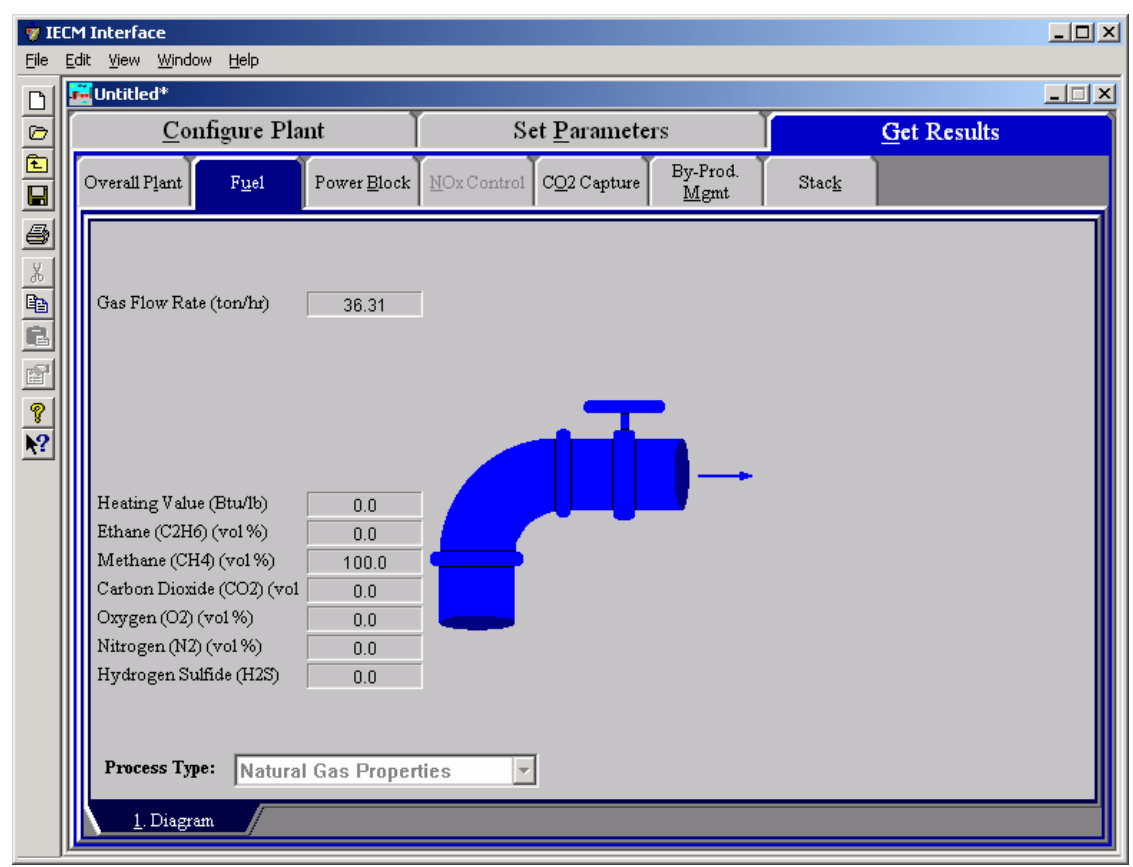

Fuel - Diagram result screen for natural gas.

The Natural Gas Diagram result screen displays fuel composition and flow rate information, which is described briefly below.

Gas Flow Rate: The natural gas flow rate to the turbine.

Heating Value: Higher heating value (HHV) is the thermal energy produced in Btu/lb of fuel.

Ethane $\left(\mathrm{C}_{2} \mathrm{H}_{6}\right)$ : The volume, by percent, of ethane in the natural gas.

Methane $\left(\mathrm{CH}_{4}\right)$ : The volume, by percent, of methane in the natural gas.

Carbon Dioxide $\left(\mathrm{CO}_{2}\right)$ : The volume, by percent, of carbon dioxide in the natural gas.

Oxygen $\left(\mathrm{O}_{2}\right)$ : The volume, by percent, of oxygen in the natural gas.

Nitrogen $\left(\mathrm{N}_{2}\right)$ : The volume, by percent, of nitrogen in the natural gas.

Hydrogen Sulfide $\left(\mathrm{H}_{2} \mathrm{~S}\right)$ : The volume, by percent, of hydrogen sulfide in the natural gas. 


\section{Base Plant}

The Base Plant Technology Navigation Tab screens display and define the performance and costs directly associated with the power plant, particularly the boiler. Pre-combustion and post-combustion control technologies are not considered part of the Base Plant.

\section{Base Plant Furnace Factors Inputs}

Inputs for the furnace factors that effect the major flow rates and concentrations of the gas and solids streams are entered on the Furnace Factors input screen.

This screen accepts inputs for the flue gas and ash products emitted from the boiler into the flue gas and ash streams. Factors in emissions include: incomplete combustion and thermodynamic equilibrium between gas species associated with the combustion products.

This screen's inputs are needed to calculate boiler efficiency and air pollutant emissions. The emission of carbon, ash, sulfur and nitrogen are specified by the United States Government's Environmental Protection Agency's (EPA) compilation of emission factors. Also included from the compilation are the incomplete transfer percentages of solid and gaseous forms of these substances.

This screen is available for all plant configurations. 


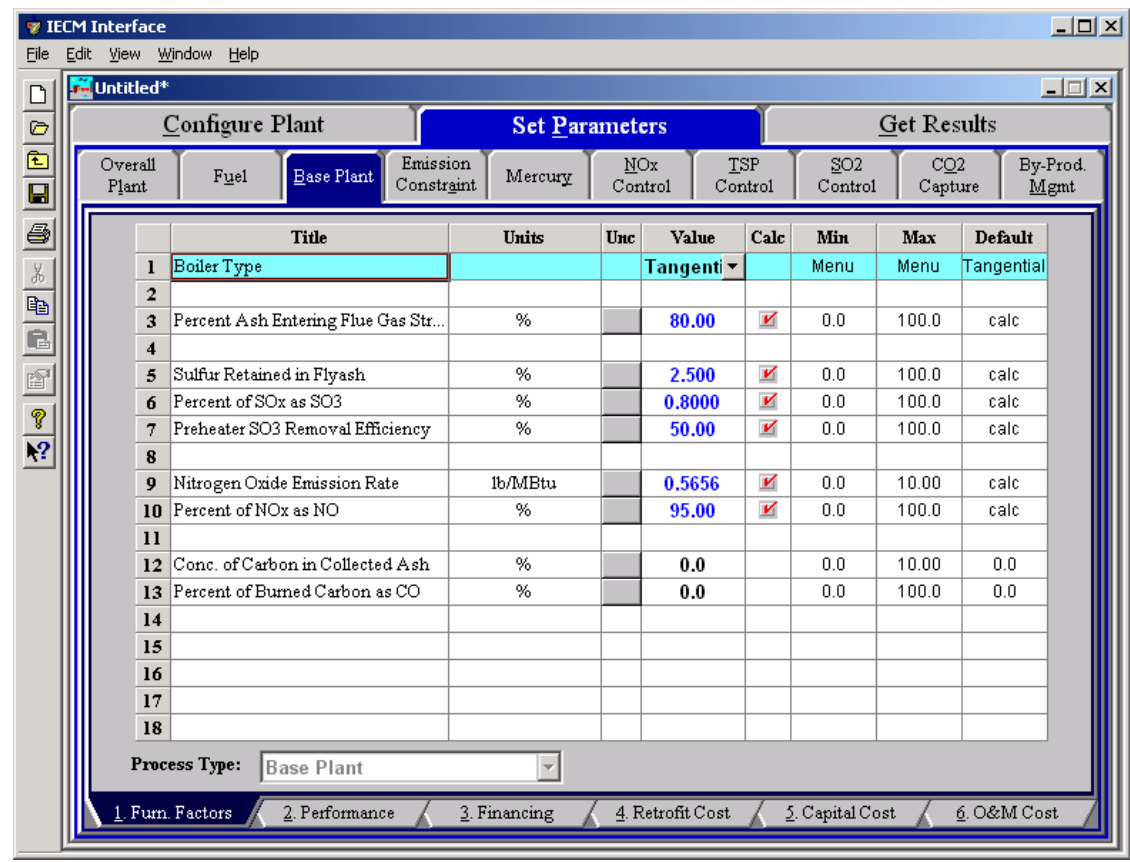

Base Plant - Furn. Factors input screen.

Each parameter is described briefly below:

Boiler Type: The choices available are Tangential, Wall, and Cyclone. The default setting is Tangential. The firing method affects model default parameters for nitrogen oxide emissions and the partitioning of fly ash and bottom ash in the furnace.

Percent Ash Entering Flue Gas Stream: The default values for this parameter are a function of the fuel and boiler types and are based on the AP-42 EPA emission factors. Ash not entering the flue gas stream is assumed to be removed as bottom ash. This is also referred to as the overhead ash fraction.

Sulfur Retained in Flyash: This parameter gives the percent of total sulfur input to the boiler that is retained in the flyash stream of a coalfired power plant. The default values are a function of the selected boiler type and the coal rank as specified by the AP-42 EPA compilation of emission factors.

Percent of $\mathrm{SO}_{\mathrm{x}}$ as $\mathrm{SO}_{3:}$ This parameter quantifies the sulfur species in the flue gas stream. Sulfur not converted to $\mathrm{SO}_{2}$ is assumed to be converted to $\mathrm{SO}_{3}$. The default value is based on emission factors derived by Southern Company ${ }^{3}$ and are a function of the selected coal.

Preheater $\mathrm{SO}_{3}$ Removal Efficiency: Sulfuric acid $\left(\mathrm{H}_{2} \mathrm{SO}_{4}\right)$ is created downstream of the boiler by the reaction of $\mathrm{SO}_{3}$ with $\mathrm{H}_{2} \mathrm{O}$. A percent of the sulfuric acid is condensed on particulates in the preheater and removed from the flue gas. This parameter specifies the amount of $\mathrm{SO}_{3}$ removed from the flue gas in the preheater as a function of the coal rank. The default value is based on data provided by Southern Company Services. ${ }^{4}$

${ }^{3}$ Hardman, R., R. Stacy, et al. (1998). Estimating Total Sulfuric Acid Emissions from Coal-FIred Power Plants, Southern Company Services.

4 Ibid. 
Nitrogen Oxide Emission Rate: This parameter establishes the level of $\mathrm{NO}_{\mathrm{x}}$ emissions from the boiler. The default values reflect the AP-42 EPA emission factors. It is a function of boiler firing method and the coal rank. The model calculates this value and expresses it in pounds of equivalent $\mathrm{NO}_{2}$ per ton of coal.

Percent of $\mathrm{NO}_{\mathbf{x}}$ as NO: This parameter establishes the level of nitric oxide (NO) in the flue gas stream. The remainder of the total $\mathrm{NO}_{\mathrm{x}}$ emissions is assumed to be nitrogen dioxide $\left(\mathrm{NO}_{2}\right)$. The default parameters reflect the AP-42 EPA emission factors and are dependent on the fuel type.

Conc. of Carbon in Collected Ash: This parameter accounts for retention of carbon in the fly ash and bottom ash. The amount of carbon in the collected ash streams is typically known. It is used to calculate the total unburned carbon in coal, boiler efficiency and flue gas composition.

Percent of Burned Carbon as CO: This parameter accounts for any incomplete combustion in the furnace, and is used to calculate boiler efficiency and flue gas composition. The remainder is assumed to be $\mathrm{CO}_{2}$ or unburned carbon.

\section{Base Plant Performance Inputs}

Inputs for the major flow rates and concentrations of the gas and solids streams are entered on the Performance input screen

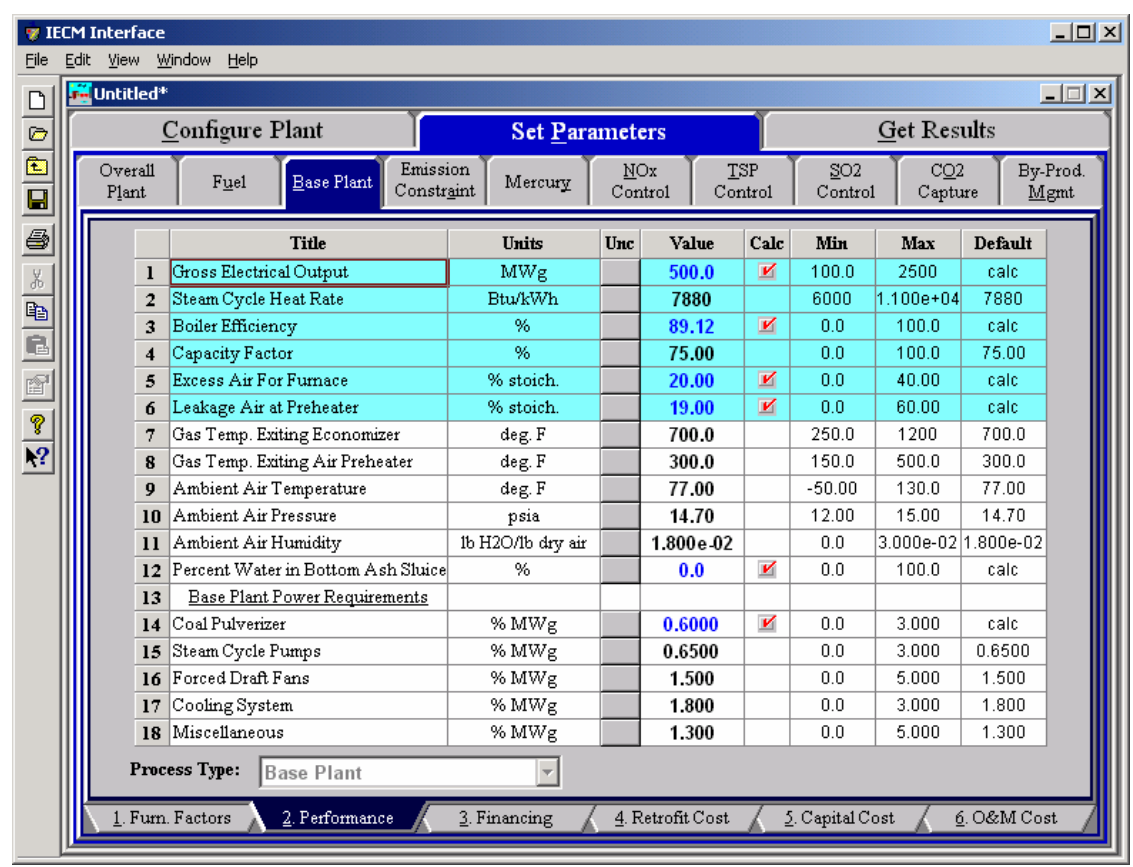

Base Plant-Performance input screen.

The first six inputs are highlighted in blue. Each parameter is described briefly below.

Gross Electrical Output:This is the gross output of the generator in megawatts $\left(\mathrm{MW}_{\mathrm{g}}\right)$. The value does not include auxiliary power 
requirements. The model uses this information to calculate key mass flow rates.

Steam Cycle Heat Rate: This is the gross amount of energy in steam needed to produce a kilowatt-hour $(\mathrm{kWh})$ of electricity at the generator. This variable does not consider auxiliary power requirements. This heat rate, plus the boiler efficiency, is used to figure out the overall plant performance (i.e., the gross cycle heat rate).

Boiler Efficiency: This is the percentage of fuel input energy transferred to steam in the boiler. The model default is to calculate the boiler efficiency using standard algorithms described in the literature. The efficiency is a function of energy losses due to inefficient heat transfer across the preheater, latent heat of evaporation, incomplete combustion, radiation losses, and unaccounted losses.

Capacity Factor: This is an annual average value, representing the percent of equivalent full load operation during a year. The capacity factor is used to calculate annual average emissions and materials flows.

Excess Air for Furnace: This is the excess theoretical air used for combustion. It is added to the stoichiometric air requirement calculated by the model. The value is calculated and based on the fuel type and boiler type.

Leakage Air at Preheater: This is the additional excess air introduced because of leakage into the system at or beyond the air preheater. It is based on the stoichiometric air required for combustion. The leakage air increases the total gas volume downstream of the air preheater.

Gas Temperature Exiting Economizer: This is the temperature of the flue gas exiting the economizer. The temperature is used in the calculation of the flue gas volume and air preheater performance.

Gas Temperature Exiting Air Preheater: This is the temperature of the flue gas exiting the air preheater. The temperature is used in the calculation of the flue gas volume and air preheater performance.

Ambient Air Temperature: This is the inlet temperature of the ambient combustion air prior to entering the preheater. The model presumes an annual average temperature. Inlet air temperature affects the boiler energy balance and efficiency. It provides a reference point for the calculation of pressure throughout the system. Currently, the model cannot have temperatures below $77^{\circ} \mathrm{F}$.

Ambient Air Pressure: This is the absolute pressure of the air inlet stream to the boiler. The air pressure is used to convert flue gas molar flow rates to volume flow rates.

Ambient Air Humidity: This is the water content of the inlet combustion air. This value is used in calculating the total water vapor content of the flue gas stream. The value is referred to as the specific humidity ratio, expressed as a ratio of the water mass to the dry air mass.

Percent Water in Bottom Ash Sluice: : This is the water content of the collected bottom ash. Bottom ash is assumed to be sluiced with water and dry otherwise, it may be combined with fly ash and then disposed.

\section{Base Plant Power Requirements}


These parameters specify the electrical energy requirements of pulverizers, steam pumps, forced draft fans, cooling system equipment (fans and pumps), and other miscellaneous equipment excluding gas cleanup systems. These energy requirements or penalties are expressed as a percent of a gross plant capacity and are used to calculate the net plant performance.

Coal Pulverizer: This is the energy needed to run the coal pulverizers prior to the coal being blown into the boiler. It is also referred to as an energy penalty to the base plant. The value is calculated and based on the fuel type. It is expressed as a percentage of the gross plant capacity.

Steam Cycle Pumps: This is the energy needed to operate the pumps in the steam cycle. It is also referred to as an energy penalty to the base plant. It is expressed as a percentage of the gross plant capacity.

Forced Draft Fans: This is the energy required for the forced draft fans and primary air fan expressed as a percentage of the gross plant capacity. It is also referred to as an energy penalty for the base plant.

Cooling System: This is the energy needed to run the pumps and other equipment for the water cooling system. It is expressed as a percentage of the gross plant capacity. It is also referred to as a base plant energy penalty.

Miscellaneous: This is the energy used by any other miscellaneous equipment in the base plant, not including equipment used for pollution control equipment. It is expressed as a percentage of the gross plant capacity. It is also referred to as a base plant energy penalty.

\section{Base Plant Financing Inputs}

Inputs for the financing costs of the base plant itself are entered on the Financing input screen.

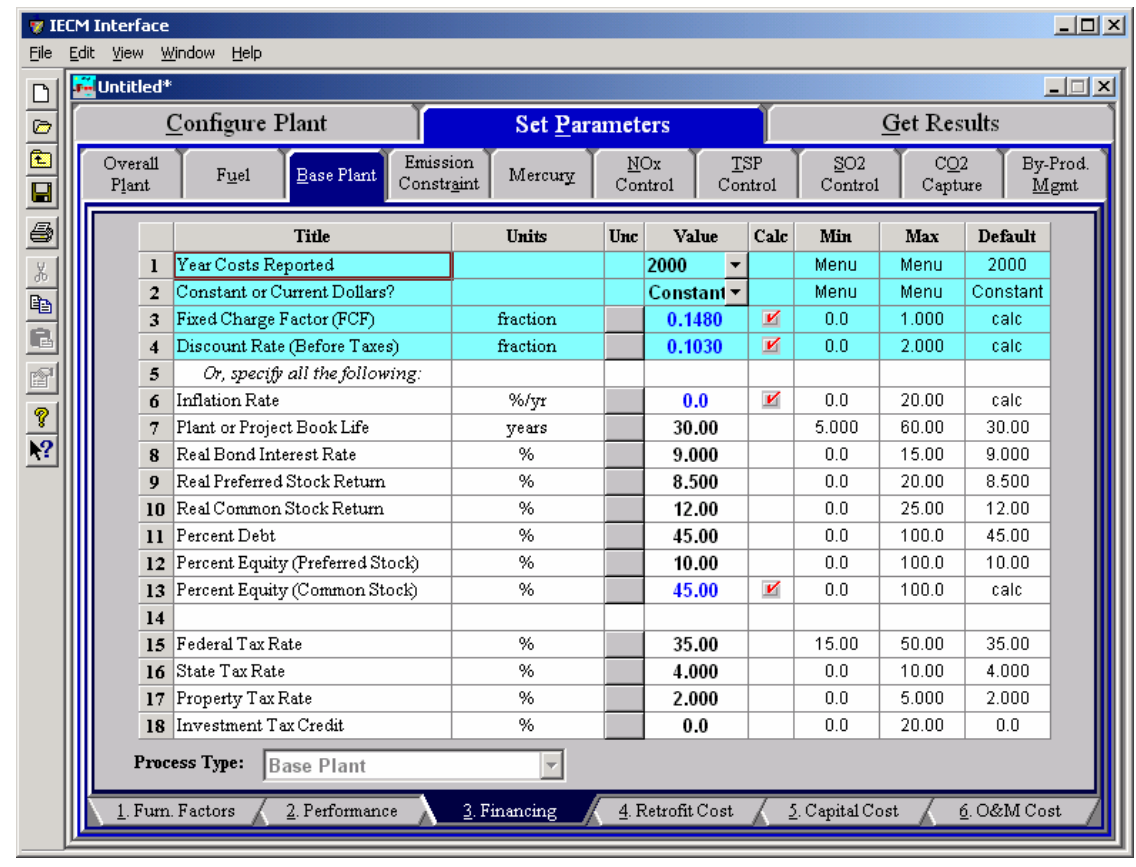

Base Plant-Financing input screen. 
This screen describes the factors required to determine the carrying charge for all capital investments. The carrying charge is defined as the revenue required to pay for any capital investment. The total charge can also be expressed as a levelized cost factor or fixed charge factor. The fixed charge factor is a function of many items. You have the option of specifying the fixed charge factor directly, or allowing it to be calculated from the other input quantities below it on the financial input screen.

The first four inputs are highlighted in blue. Each parameter is described briefly below.

Year Costs Reported: This is the year in which all costs are given or displayed, both in the input screens and the results. A cost index is used by the IECM to scale all costs to the cost year specified by this parameter.

Constant or Current Dollars: Constant dollar analysis does not include the affect of inflation, although real escalation is included. Current dollar analysis includes inflation and real escalation. This choice allows you to choose the mode of analysis for the entire IECM economics.

Fixed Charge Factor (FCF): The fixed charge factor is one of the most important parameters in the IECM. It determines the revenue required to finance the power plant based on the capital expenditures. Put another way, it is a levelized factor which accounts for the revenue per dollar of total plant cost that must be collected from customers in order to pay the carrying charges on that capital investment.

\section{Discount Rate (Before Taxes):}

One may specify a Fixed Charge Factor, or fill in the following inputs and the model will calculate the FCF based on them:

Inflation Rate: This is the rise in price levels caused by an increase in the available currency and credit without a proportionate increase in available goods or services. It does not include real escalation.

Plant or Project Book Life: This is the years of service expected from a capital investment. It is also the period over which an investment is recovered through book depreciation.

Real Bond Interest Rate: This is a debt security associated with a loan or mortgage. It is the most secure form of security but the lowest in its return.

Real Preferred Stock Return: This equity security is the second most speculative type and pays the second highest rate of return. The holder of the stock is a part owner of the company.

Real Common Stock Return: This is the most speculative type of equity security sold by a utility and pays the highest relative return. The holder of the stock is a part owner of the company.

Percent Debt: This is the percent of the total capitalization that is associated with debt money. This includes loans and mortgage bonds.

Percent Equity (Preferred Stock): This is the percent of the total capitalization that is associated with the sale of preferred stock.

Percent Equity (Common Stock): This value is the remainder of the capitalization, calculated as $100 \%$ minus the percent debt, minus the percent equity in preferred stock. 
Federal Tax Rate: This is the federal tax rate. It is used to calculate the amount of taxes paid and deferred.

State Tax Rate: This is the state tax rate. It is used to calculate the amount of taxes paid and deferred.

Property Tax Rate: The property tax rate, or ad valorem, is used to calculate the carrying charge.

Investment Tax Credit: This is an immediate reduction in income taxes equal to a percentage of the installed cost of a new capital investment. It is zero by default. It is used to set the initial balance and the book depreciation.

\section{Base Plant Retrofit Cost Inputs}

Inputs for the capital costs of modifications to process areas of the base plant itself are entered on the Retrofit Cost input screen

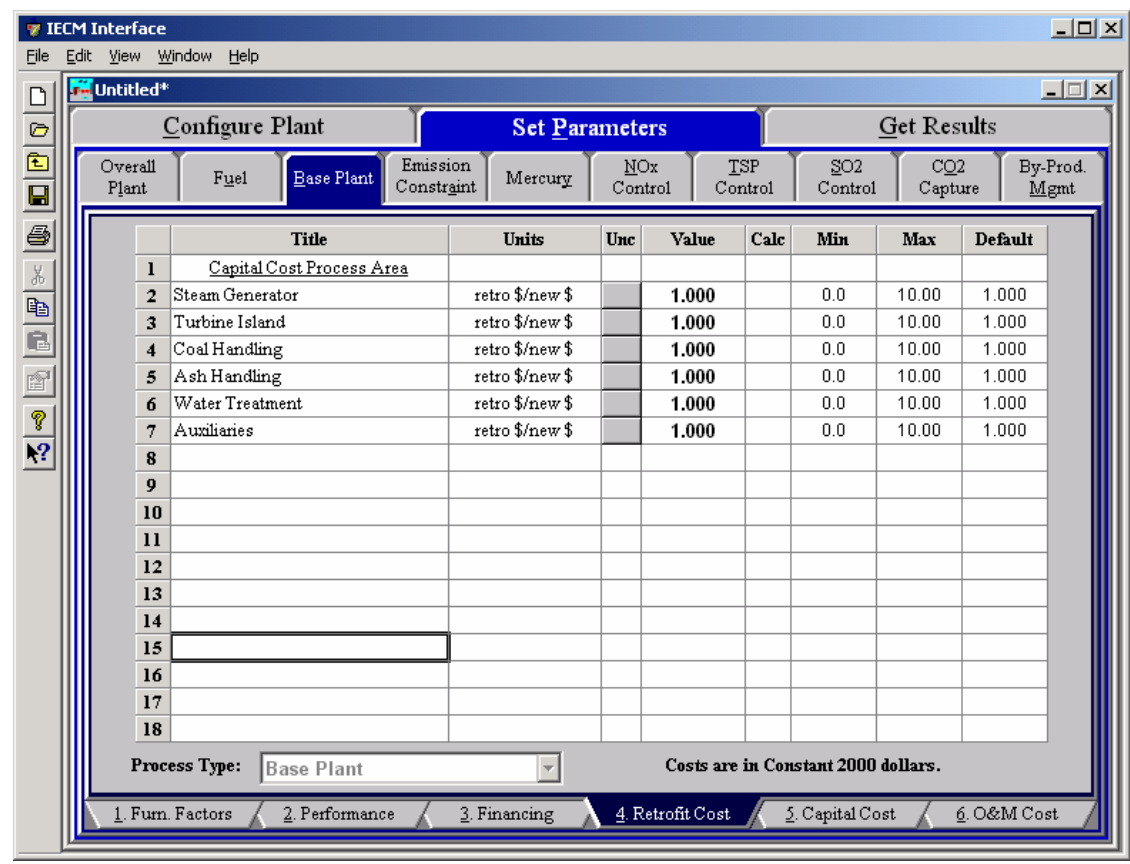

Base Plant-Retrofit Cost input screen.

The retrofit cost factor of each process is a multiplicative cost adjustment, which considers the cost of retrofitted capital equipment relative to similar equipment installed in a new plant. These factors affect the capital costs directly and the operating and maintenance costs indirectly.

Direct capital costs for each process area are calculated in the IECM. These calculations are reduced form equations derived from more sophisticated models and reports. The sum of the direct capital costs associated with each process area is defined as the process facilities capital (PFC). The retrofit cost factor provided for each of the process areas can be used as a tool for adjusting the anticipated costs and uncertainties across the process area separate from the other areas.

Uncertainty can be applied to the retrofit cost factor for each process area in each technology. Thus, uncertainty can be applied as a general factor across an entire process area, rather than as a specific uncertainty for the particular cost on the capital 
or O\&M input screens. Any uncertainty applied to a process area through the retrofit cost factor compounds any uncertainties specified later in the capital and O\&M cost input parameter screens.

Each Capital Cost Process Area is described briefly below.

Steam Generator: This area accounts for the steam cycle equipment and pumps.

Turbine Island: This area accounts for the turbine island and associated pumps.

Coal Handling: This area accounts for the mechanical collection and transport equipment of coal in the plant.

Ash Handling: This area accounts for the mechanical collection and transport of ash in the plant.

Water Treatment: This area accounts for the pumps, tanks, and transport equipment used for water treatment.

Auxiliaries: Any miscellaneous auxiliary equipment is treated in this process area.

\section{Base Plant Capital Cost Inputs}

Inputs for the capital costs of the base plant itself are entered on the Capital Cost input screen.

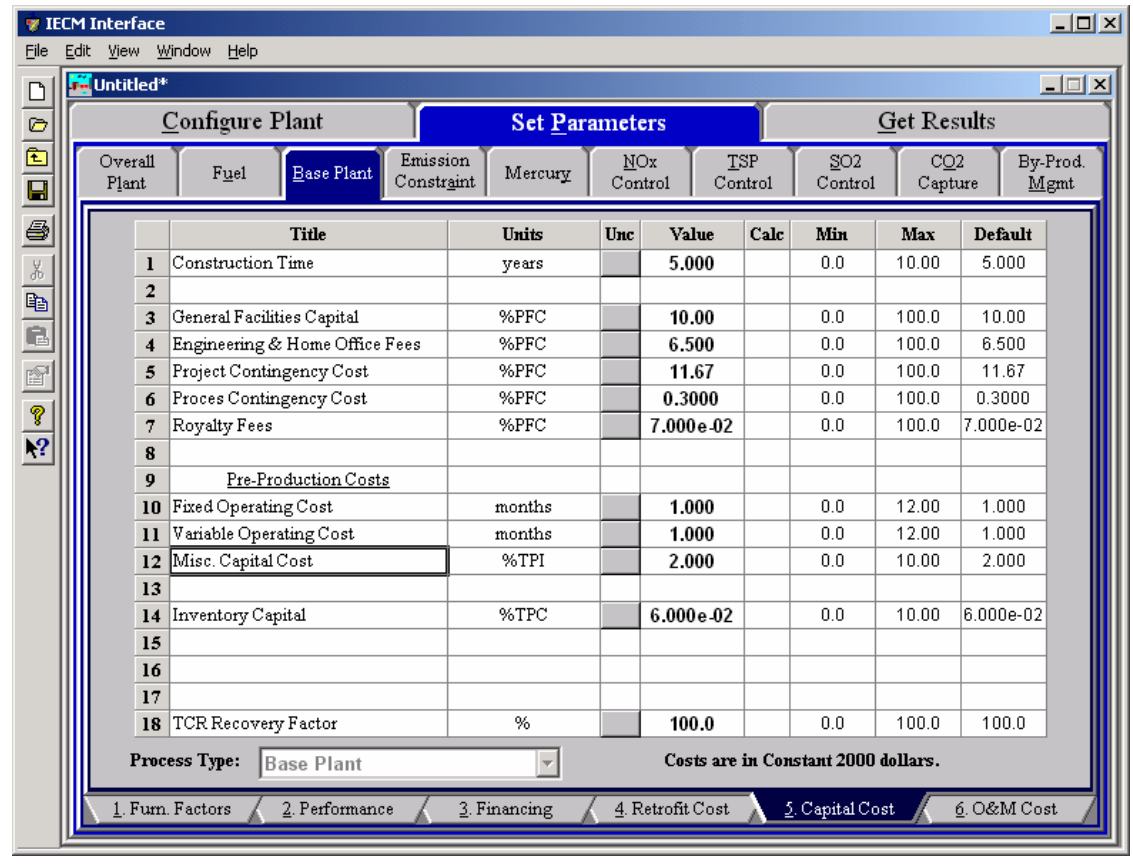

Base Plant-Capital Cost input screen

The necessary capital cost input parameters associated with the base plant are on this input screen. The capital cost parameters and terminology used in the IECM are based on the methodologies developed by the Electric Power Research Institute (EPRI). They have prepared a Technical Assessment Guide (TAG) in order to provide a consistent basis for reporting cost and revenues associated with the electric power industry. This system of reporting is used by a wide audience, including 
energy engineers, researchers, planners, and managers. The IECM has been developed around this TAG system so that costs associated with various technologies can be compared directly on a consistent basis and communicated in the language used by the audience listed above.

Total Plant Cost (TPC): Total Plant Cost is the sum of the process facilities capital, general facilities capital, engineering and home office fees, and the contingencies (project and process). This is considered the cost on an instantaneous basis (overnight), and expressed in December dollars of a reference year.

Direct Capital Costs: Direct capital costs for each process area are calculated in the IECM. These calculations are reduced form equations derived from more sophisticated models and reports. The sum of the direct capital costs associated with each process area is defined as the process facilities capital (PFC). This is the basis for all other capital cost parameters.

Process Facilities Capital: The process facilities capital for the technology is the total constructed cost of all on-site processing and generating units, including all direct and indirect construction costs. All sales taxes and freight costs are included where applicable implicitly. These direct capital costs are generally calculated by the IECM and not presented directly on input screens. However, when important input variables are required for these calculations, they are listed at the top of the input screen.

\section{Indirect Capital Costs}

Costs that are indirectly applied to the technology are based on the process facilities cost. Each of the cost factors below is expressed as a percentage of the process facilities cost, and is entered on this screen. Each parameter is described briefly below.

Construction Time: This is the idealized construction period in years. It is used to determine the allowance for funds used during construction (AFUDC).

General Facilities Capital (GFC): The general facilities include construction costs of roads, office buildings, shops, laboratories, etc. Sales taxes and freight costs are included implicitly. The cost typically ranges from 5-20\%.

Engineering \& Home Office Fees: The engineering \& home office fees are a percent of total direct capital cost. This is an overhead fee paid to the architect/engineering company. These fees typically range from 7$15 \%$.

Project Contingency Cost: This is factor covering the cost of additional equipment or other costs resulting from a more detailed design. Higher contingency factors will be applied to simplified or preliminary designs and lower factors to detailed or finalized designs.

Process Contingency Cost: This quantifies the design uncertainty and cost of a commercial-scale system. This is generally applied on an areaby-area basis. Higher contingency factors are applied to new regeneration systems tested at a pilot plant and lower factors to full-size or commercial systems.

Royalty Fees: Royalty charges may apply to some portions of generating units incorporating new proprietary technologies. 


\section{Pre-Production Costs}

These costs consider the operator training, equipment checkout, major changes in unit equipment, extra maintenance, and inefficient use of fuel or other materials during start-up. These are typically applied to O\&M costs over a specified period of time (months).

Fixed Operating Cost: Time period of fixed operating costs (operating and maintenance labor, administrative and support labor, and maintenance materials) used for plant startup.

Variable Operating Cost: Time period of variable operating costs at full capacity (chemicals, water, and other consumables, and waste disposal changes) used for plant startup. Full capacity estimates of the variable operating costs will assume operations at $100 \%$ load.

Misc. Capital Cost: This is a percent of total plant investment (sum of TPC and AFUDC) to cover expected changes to equipment to bring the system up to full capacity.

Inventory Capital: Percent of the total direct capital for raw material supply based on $100 \%$ capacity during a 60 day period. These materials are considered storage. The inventory capital includes fuels, consumables, by-products, and spare parts. This is typically $0.5 \%$.

TCR Recovery Factor: The actual total capital required (TCR) to finance the base plant as a percent of the TCR of a new power plant. This value is $100 \%$ for a new plant and may be set as low as $0 \%$ for a base plant that has been paid off.

\section{Base Plant O\&M Cost Inputs}

Inputs for the operation and maintenance costs of the base plant itself are entered on the O\&M Cost input screen.

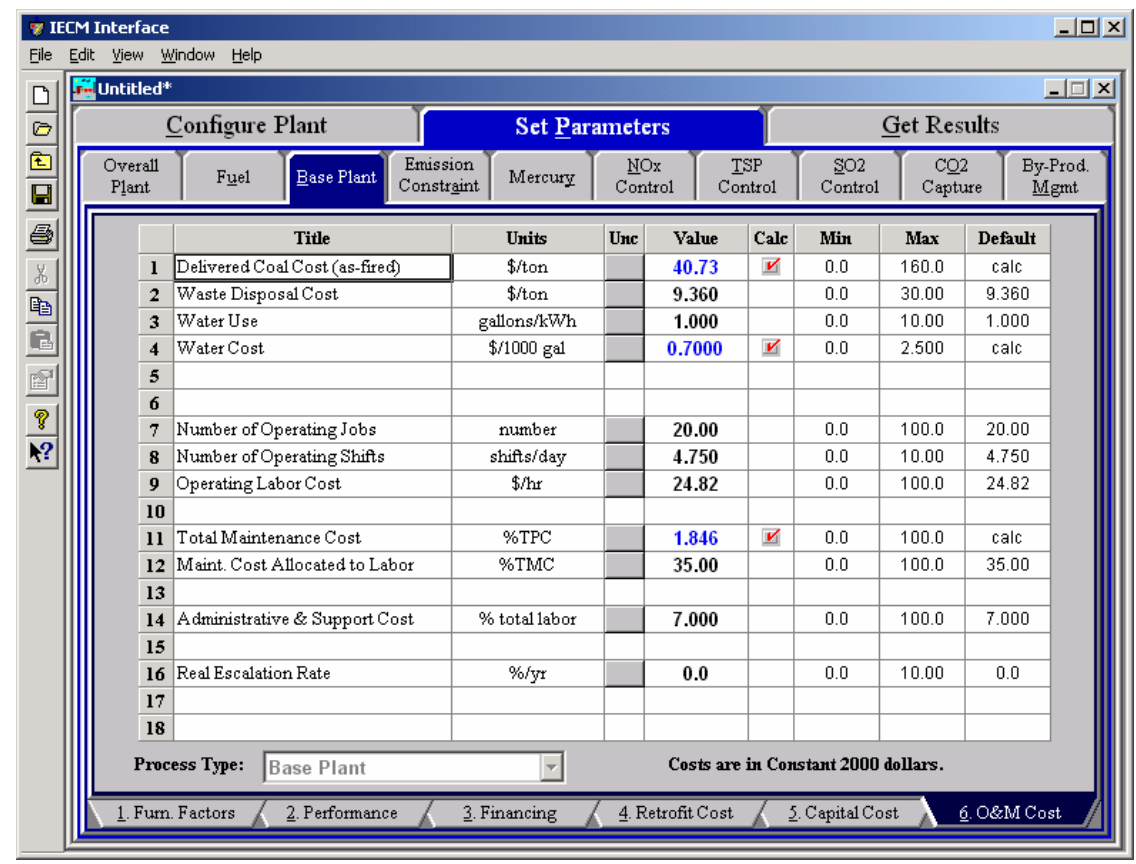

Base Plant-O\&M Cost input screen 
The EPRI TAG method of categorization has been used for operating and maintenance costs screens. It provides a consistent basis of reporting for a wider audience of users.

O\&M costs are expressed on an average annual basis. The costs are broken down into two categories: variable and fixed. Variable costs include the costs of reagents, chemicals, water, and other materials consumed during plant operation. Fixed costs are associated with labor and overhead charges. All operating costs are subject to inflation.

The base plant considers a more detailed breakdown for the costs associated with the fuel. Together they characterize the fuel costs. Each parameter is described briefly below.

Delivered Coal Cost (as-fired): This is the cost of the delivered coal in dollars per wet ton. The value is calculated by the IECM from the particular regional coal selected. It does not include any cleaning costs.

Waste Disposal Cost: This is the bottom ash disposal cost for the base plant.

Water Use: This is the water used by the base plant.

Water Cost: This is the water cost as used for the base plant.

Base Plant Electricity Cost: This is the unit cost of electricity for the base plant alone, without utility considerations.

\section{Operating Labor}

Operating labor is based on the number of personnel required to operate the plant per shift. The number of shifts should be specified.

Number of Operating Jobs: This is the total number of operating jobs that are required to operate the plant per eight-hour shift.

Number of Operating Shifts: This is the total number of equivalent operating shifts in the plant per day. The number takes into consideration paid time off and weekend work ( 3 shifts/day * 7 days/5 day week * 52 weeks/(52 weeks -6 weeks PTO $)=4.75$ equiv. Shifts/day)

Operating Labor Cost: The hourly cost of labor is specified in the base plant O\&M cost screen. The same value is used throughout the other technologies.

\section{Total Maintenance Costs}

The annual maintenance costs are tied to the capital cost of the technology and the maintenance labor. The parameters below provide the necessary information to determine the total maintenance cost as broken down by the material and labor components.

Total Maintenance Cost: This is the annual maintenance cost as a percentage of the total plant cost. Maintenance cost estimates can be developed separately for each process area.

Maint. Cost Allocated to Labor: Maintenance cost allocated to labor as a percentage of the total maintenance cost.

\section{Overhead Charges}

The only overhead charge in the EPRI TAG is associated with a charge for administrative and support. General materials are not included in this. 
Administrative \& Support Cost: This is the percent of the total operating and maintenance labor associated with administrative and support labor.

\section{Other Factors}

Real escalation rates are typically applied to variable O\&M costs that do not change at the same rate as those provided by cost indices used in the IECM. Real escalation rates adjust the costs relative to the cost index adjustments from year to year.

Real Escalation Rate: This is the annual rate of increase of an expenditure due to factors such as resource depletion, increased demand, and improvements in design, manufacturing or construction techniques (negative rate). The real escalation rate does not include inflation.

\section{Boiler Diagram}

The Diagram result screen displays an icon for the Boiler and values for major flows in and out of it.

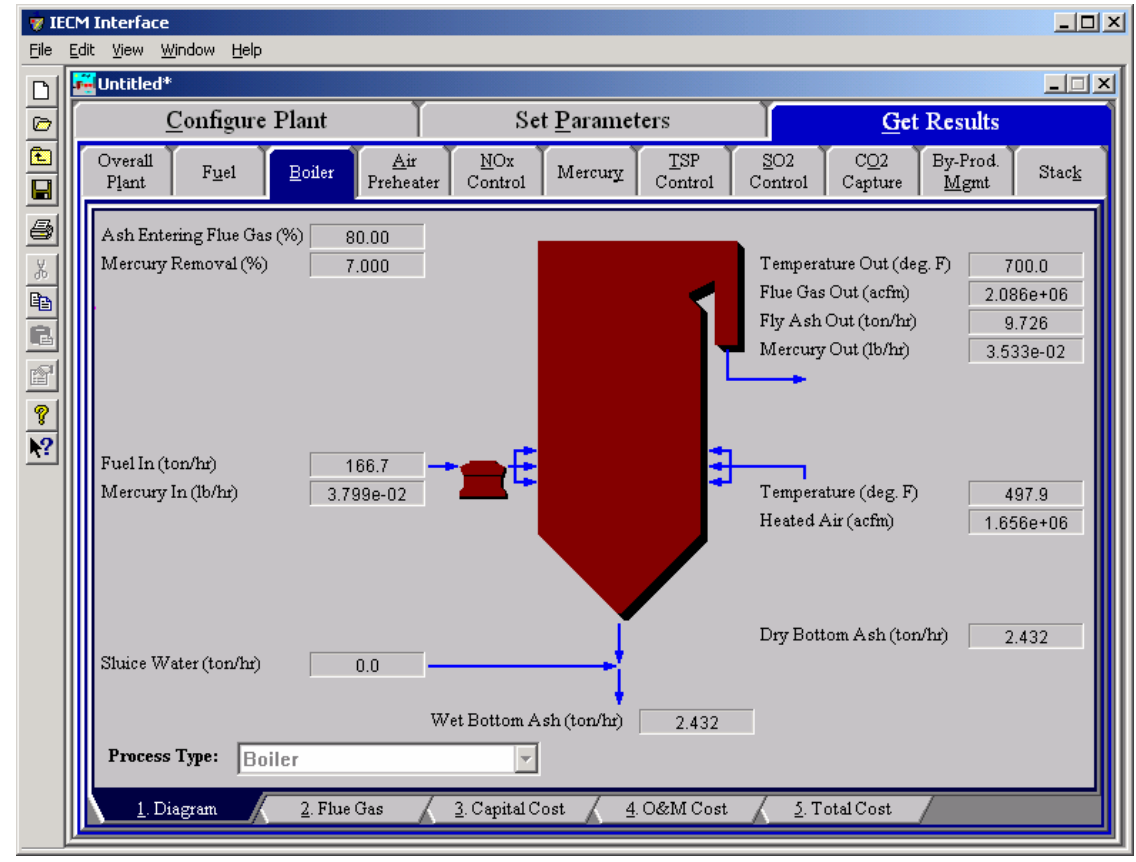

Boiler-Diagram result screen

Each result is described briefly below in flow order (not from top to bottom and left to right as they display on the screen).

\section{Fuel Entering Boiler}

Wet Fuel: Fuel flow rate into the boiler on a wet basis. Waste products removed prior to the burners are not considered here.

Mercury In: This is the mass flow rate of total mercury entering the boiler. The mass reflects the molecular weight of elemental mercury.

\section{Boiler Performance}


Ash Entering Flue Gas: Percent of the ash in coal exiting the boiler in the flue gas.

Mercury Removal: Percent of the total mercury in coal removed from the boiler in the bottom ash.

\section{Air Entering Boiler}

Temperature: Heated air temperature measured at the burners. This is generally determined by the combustion air temperature exiting the air preheater.

Heated Air: Volumetric flow rate of the air at the burners, based on the air temperature at the burners and atmospheric pressure.

\section{Flue Gas Exiting the Economizer}

Temperature Out: Temperature of the flue gas at the exit of the economizer.

Flue Gas Out: Volumetric flow rate of the flue gas at the exit of the economizer, based on the temperature at the exit of the economizer and atmospheric pressure.

Fly Ash Out: Total solids mass flow rate in the flue gas at the exit of the economizer. This includes ash, unburned carbon and unburned sulfur.

Mercury Out: Total mass of mercury exiting the economizer. The value is a sum of all the forms of mercury (elemental, oxidized, and particulate).

\section{Bottom Ash}

Sluice Water: Water added to the dry bottom ash. This water is added for transportation purposes.

Dry Bottom Ash: Total solids mass flow rate of the bottom ash. This includes ash, unburned carbon and unburned sulfur. The value is given on a dry basis.

Wet Bottom Ash: Total solids mass flow rate of the bottom ash for waste management. This includes dry bottom ash and sluice water. The value is given on a wet basis.

\section{Boiler Flue Gas Results}

The Flue Gas result screen displays a table of quantities of flue gas components entering the boiler in heated air and exiting the boiler in the flue gas. For each component, quantities are given in both moles and mass per hour. 


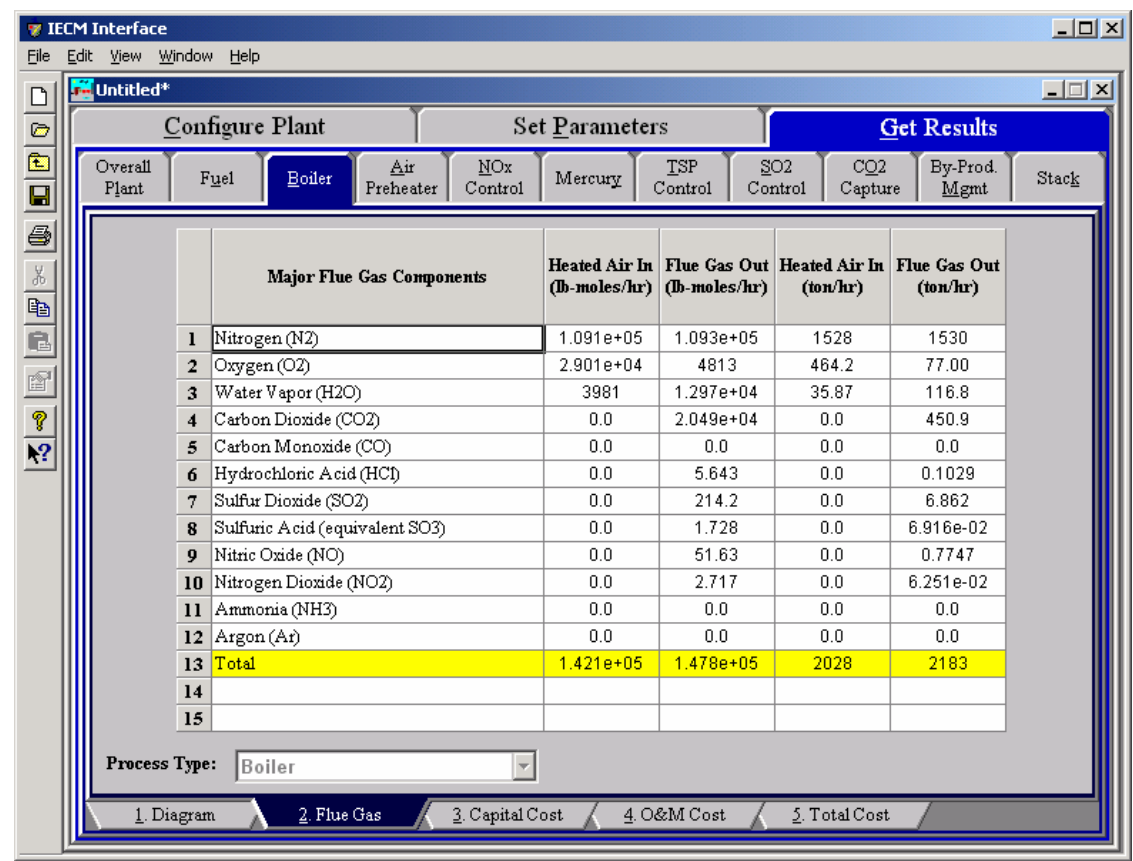

Boiler-Flue Gas result screen

Each result is described briefly below.

Nitrogen $\left(\mathbf{N}_{\mathbf{2}}\right)$ : Total mass of nitrogen.

Oxygen $\left(\mathrm{O}_{2}\right)$ : Total mass of oxygen.

Water Vapor $\left(\mathrm{H}_{\mathbf{2}} \mathrm{O}\right)$ : Total mass of water vapor.

Carbon Dioxide $\left(\mathrm{CO}_{2}\right)$ : Total mass of carbon dioxide.

Carbon Monoxide (CO): Total mass of carbon monoxide.

Hydrochloric Acid (HCl): Total mass of hydrochloric acid.

Sulfur Dioxide $\left(\mathbf{S O}_{\mathbf{2}}\right)$ : Total mass of sulfur dioxide.

Sulfuric Acid (equivalent $\mathbf{S O}_{3}$ ): Total mass of sulfuric acid.

Nitric Oxide (NO): Total mass of nitric oxide.

Nitrogen Dioxide $\left(\mathrm{NO}_{2}\right)$ : Total mass of nitrogen dioxide.

Ammonia $\left(\mathrm{NH}_{3}\right)$ : Total mass of ammonia.

Argon (Ar): Total mass of argon.

Total: Total of the individual components listed above. This item is highlighted in yellow.

\section{Boiler Capital Cost Results}

The Capital Cost result screen displays tables for the direct and indirect capital costs related to the Boiler 


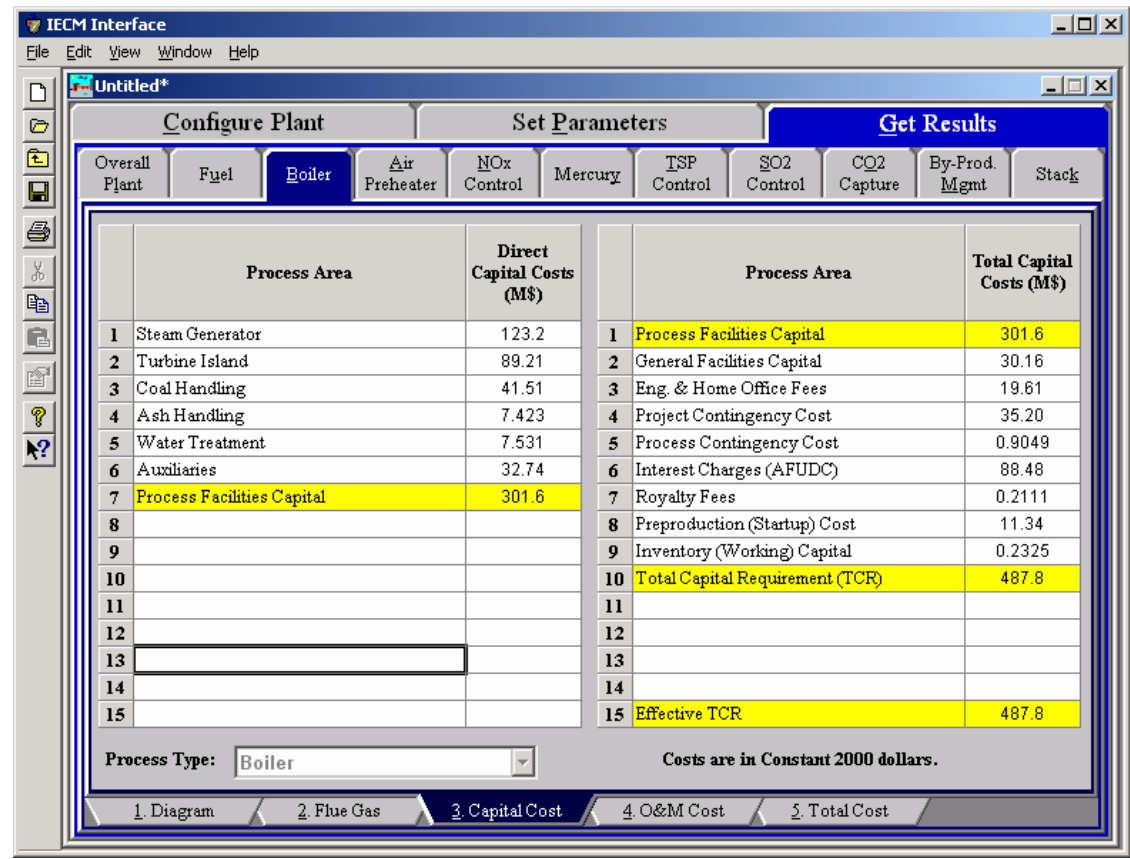

Boiler-Capital Cost result screen

Each result is described briefly below.

\section{Direct Capital Costs}

The direct capital costs described here apply to the "base power plant" without any of the environmental control options that are separately modeled in the IECM. While the purpose of the IECM is to model the cost and performance of emission control systems, costs for the base plant are also needed to properly account for precombustion control options that increase the cost of fuel, and affect the characteristics or performance of the base plant. Base plant costs are also needed to calculate the internal cost of electricity which determines pollution control energy costs.

Each process area direct capital cost is a reduced-form model based on regression analysis of data collected from several reports and analyses. They are described in general below. The primary factors in the model that effect the capital cost of the base plant are the plant size, the coal rank, and the geographic location of the plant.

Steam Generator: This area accounts for the steam cycle equipment and pumps.

Turbine Island: This area accounts for the turbine island and associated pumps.

Coal Handling: This area accounts for the mechanical collection and transport equipment of coal in the plant.

Ash Handling: This area accounts for the mechanical collection and transport of ash in the plant.

Water Treatment: This area accounts for the pumps, tanks, and transport equipment used for water treatment.

Auxiliaries: Any miscellaneous auxiliary equipment is treated in this process area. 
Process Facilities Capital: The process facilities capital is the total constructed cost of all on-site processing and generating units listed above, including all direct and indirect construction costs. All sales taxes and freight costs are included where applicable implicitly. This result is highlighted in yellow.

\section{Total Capital Costs}

Process Facilities Capital: See definition above. This result is highlighted in yellow.

General Facilities Capital: The general facilities include construction costs of roads, office buildings, shops, laboratories, etc. Sales taxes and freight costs are included implicitly.

Eng. \& Home Office Fees: The engineering \& home office fees are a percent of total direct capital cost. This is an overhead fee paid to the architect/engineering company.

Project Contingency Cost: Capital cost contingency factor covering the cost of additional equipment or other costs that would result from a more detailed design of a definitive project at the actual site.

Process Contingency Cost: Capital cost contingency factor applied to a new technology in an effort to quantify the uncertainty in the technical performance and cost of the commercial-scale equipment.

Interest Charges (AFUDC): Allowance for funds used during construction, also referred to as interest during construction, is the time value of the money used during construction and is based on an interest rate equal to the before-tax weighted cost of capital. This interest is compounded on an annual basis (end of year) during the construction period for all funds spent during the year or previous years.

Royalty Fees: Royalty charges may apply to some portions of generating units incorporating new proprietary technologies.

Preproduction (Startup) Cost: These costs consider the operator training, equipment checkout, major changes in unit equipment, extra maintenance, and inefficient use of fuel or other materials during startup.

Inventory (Working) Capital: The raw material supply based on $100 \%$ capacity during a 60 day period. These materials are considered storage. The inventory capital includes fuels, consumables, byproducts, and spare parts.

Total Capital Requirement (TCR): Money that is placed (capitalized) on the books of the utility on the service date. TCR includes all the items above. This result is highlighted in yellow.

Effective TCR: The TCR of the base plant that is used in determining the total power plant cost. The effective TCR is determined by the "TCR Recovery Factor” for the base plant.

\section{Boiler O\&M Cost Results}

The O\&M Cost result screen displays tables for the variable and fixed operation and maintenance costs involved with the base plant. The variable O\&M costs are calculated from the variable costs for fuel, water consumption and bottom ash 
disposal (from the furnace). The fixed O\&M costs are based on maintenance and labor costs.

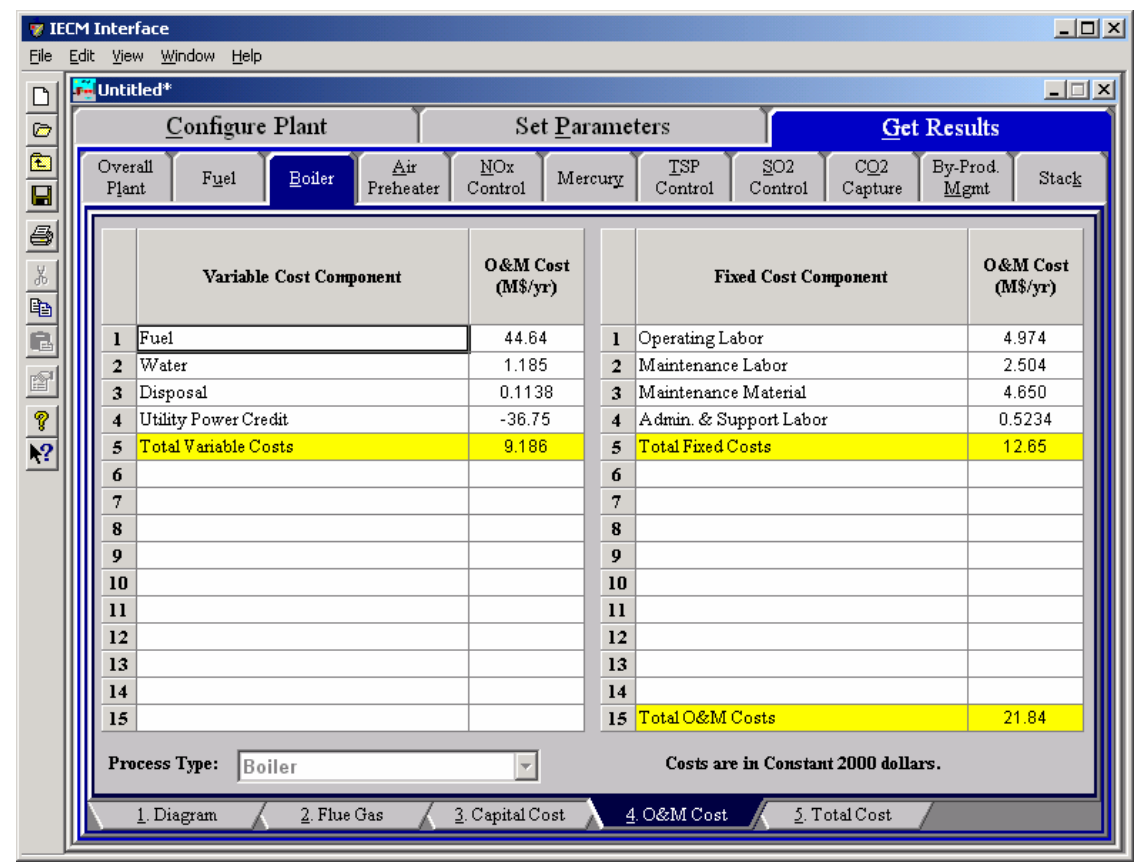

The Boiler-O\&M Cost result screen

Each result is described briefly below.

\section{Variable Cost Components}

Variable operating costs and consumables are directly proportional to the amount of kilowatts produced and are referred to as incremental costs. All the costs are subject to inflation.

Fuel: The total cost of as-fired fuel. Minemouth cost, coal cleaning costs and transportation costs are all included.

Water: The total cost of water consumed by the base plant for direct or reheat use.

Disposal: The total cost of bottom ash disposal. The value is given on a wet ash basis. This does not consider by-product ash sold in commerce.

Total Variable Costs: This is the sum of all the variable O\&M costs listed above. This result is highlighted in yellow.

\section{Fixed Cost Components}

Fixed operating costs are essentially independent of actual capacity factor, number of hours of operation, or amount of kilowatts produced. All the costs are subject to inflation.

Operating Labor: Operating labor cost is based on the operating labor rate, the number of personnel required to operate the plant per eighthour shift, and the average number of shifts per day over 40 hours per week and 52 weeks.

Maintenance Labor: The maintenance labor is determined as a fraction of the total maintenance cost. 
Maintenance Material: The cost of maintenance material is the remainder of the total maintenance cost, considering the fraction associated with maintenance labor.

Admin. \& Support Labor: The administrative and support labor is the only overhead charge. It is taken as a fraction of the total operating and maintenance labor costs.

Total Fixed Costs: This is the sum of all the fixed O\&M costs listed above. This result is highlighted in yellow.

Total O\&M Costs: This is the sum of the total variable and total fixed O\&M costs. It is used to determine the base plant total revenue requirement. This result is highlighted in yellow.

\section{Boiler Total Cost Results}

The Total Cost result screen displays a table which totals the annual fixed, variable, operations and maintenance, and capital costs associated with the boiler.

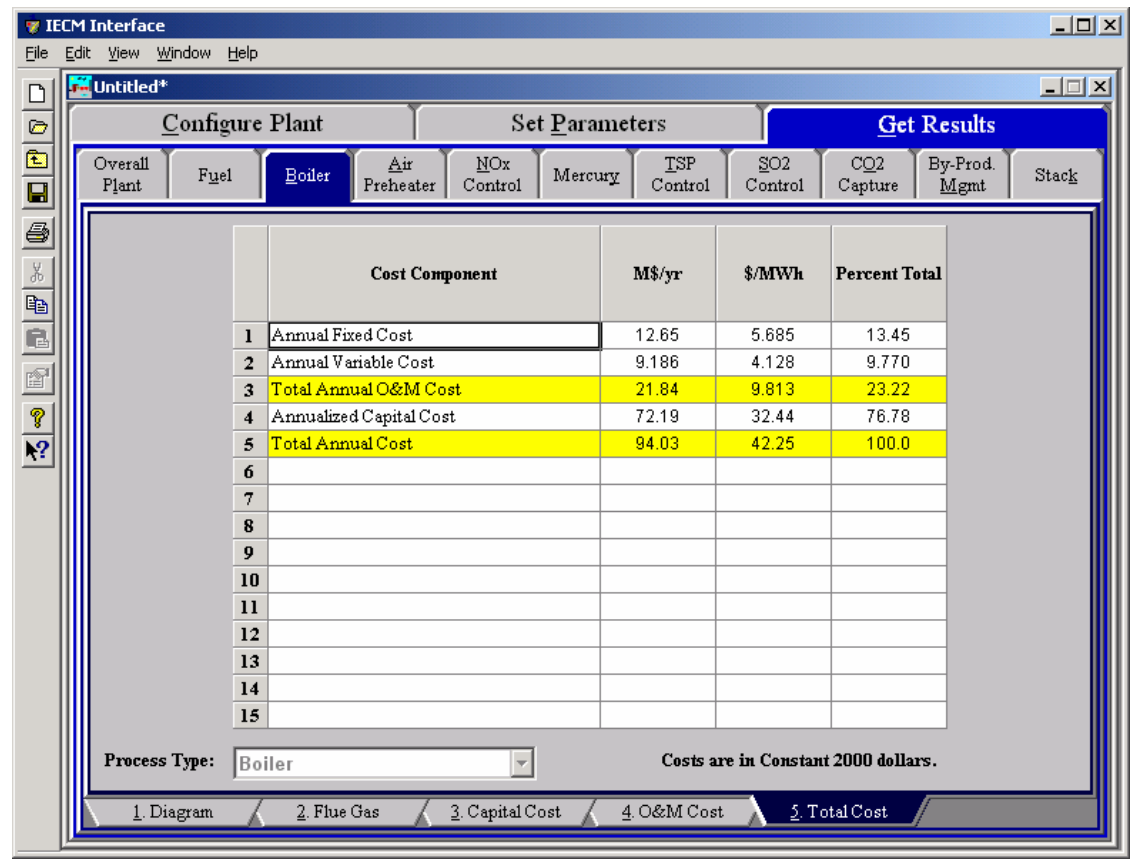

Boiler-Total Cost result screen

Each result is described briefly below.

Annual Fixed Cost: The operating and maintenance fixed costs are given as an annual total. This number includes all maintenance materials and all labor costs.

Annual Variable Cost: The operating and maintenance variables costs are given as an annual total. This includes all reagent, chemical, steam, and power costs.

Total Annual O\&M Cost: This is the sum of the annual fixed and variable operating and maintenance costs above. This result is highlighted in yellow. 
Annualized Capital Cost: This is the total capital cost expressed on an annualized basis, taking into consideration the levelized carrying charge factor, or fixed charge factor, over the entire book life.

Total Annual Cost: The total annual cost is the sum of the total annual $\mathrm{O} \& \mathrm{M}$ cost and annualized capital cost items above. This result is highlighted in yellow. 



\section{Emission Constraints}

\section{Emission Constraints Performance Inputs}

The Emission Constraints Technology Navigation Tab in the Set Parameters program area contains the Performance input screen.

This screen accepts input for the allowable emission limits for sulfur dioxide, nitrogen oxides and particulate matter. The default values reflect current United States New Source Performance Standards (NSPS), which are applicable to all units constructed since 1978.

You may override the default values for any of these parameters. $\mathrm{SO}_{2}$ emission limits are calculated by the model based on the NSPS limits that are a function of the sulfur content of the coal.

The emission constraints are used to calculate the removal efficiencies of control systems for $\mathrm{SO}_{2}, \mathrm{NO}_{\mathrm{x}}$, and particulate matter needed to comply with the specified emission constraints. As discussed later, however, user-specified values for control technology performance may cause the plant to over-comply or under-comply with the emission constraints specified in this screen.

This screen is available for all plant configurations. 


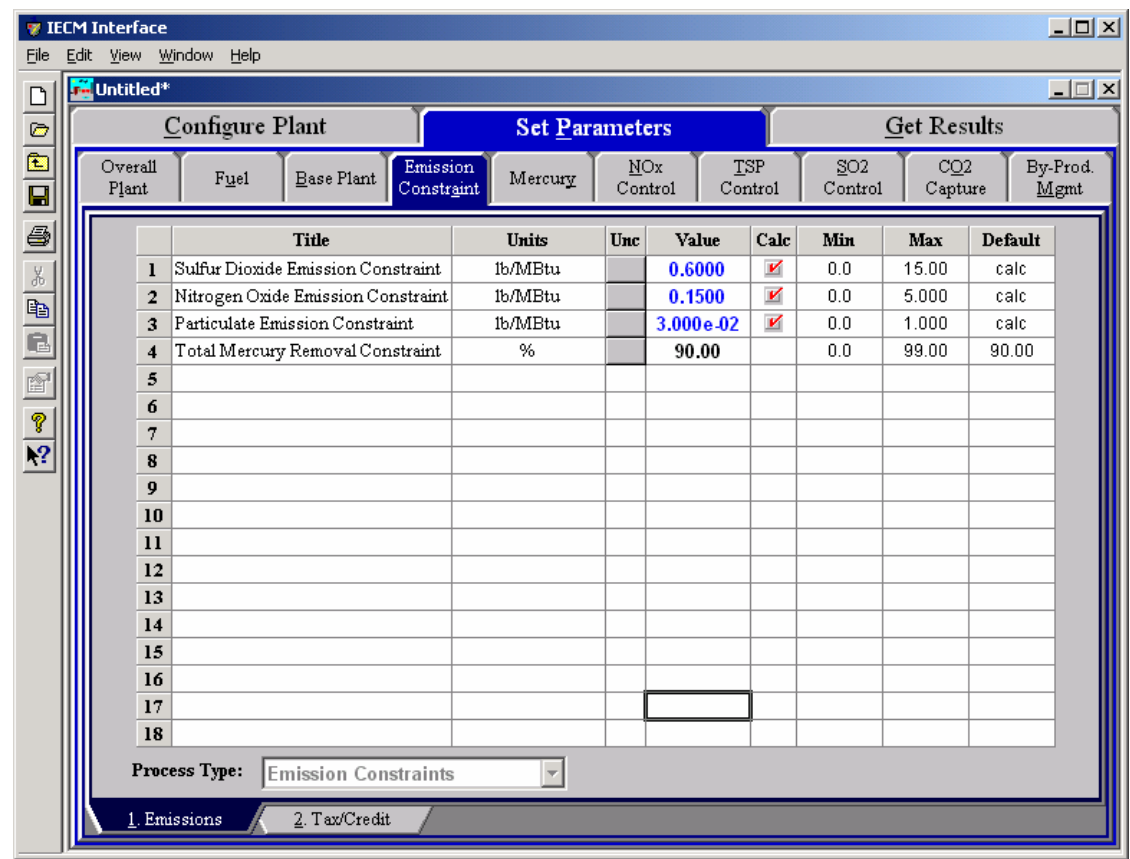

Emission Constraints - Performance input screen.

Each parameter is described briefly below.

Sulfur Dioxide Emission Limit: The emission constraint is defined by the 1979 revised NSPS. The calculated value is determined by the potential emission of the raw coal, minus the amount of sulfur retained in the ash streams. The emission limit is dependent on the fuel type and is used to determine the removal efficiency of $\mathrm{SO}_{\mathrm{x}}$ control systems.

Nitrogen Oxide Emission Constraint: The combined emissions of $\mathrm{NO}_{2}$ and $\mathrm{NO}_{3}$ of present power plants are constrained by NSPS standards. The limit is a function of the coal rank and fuel type and is used to determine the removal efficiency of $\mathrm{NO}_{\mathrm{x}}$ control systems.

Particulate Emission Constraint: The emission constraint of the total suspended particulates is defined by the NSPS standards of 1978 . The limit is a function of the fuel type and is used to determine the removal efficiency of particulate control systems.

Total Mercury Removal Constraint: The emission constraint of total after the economizer. Mercury removed in the furnace due to bottom ash removal is not considered in this constraint. The limit determines the removal efficiency of the particulate control systems. 


\section{Emission Constraints Tax/Credit Inputs}

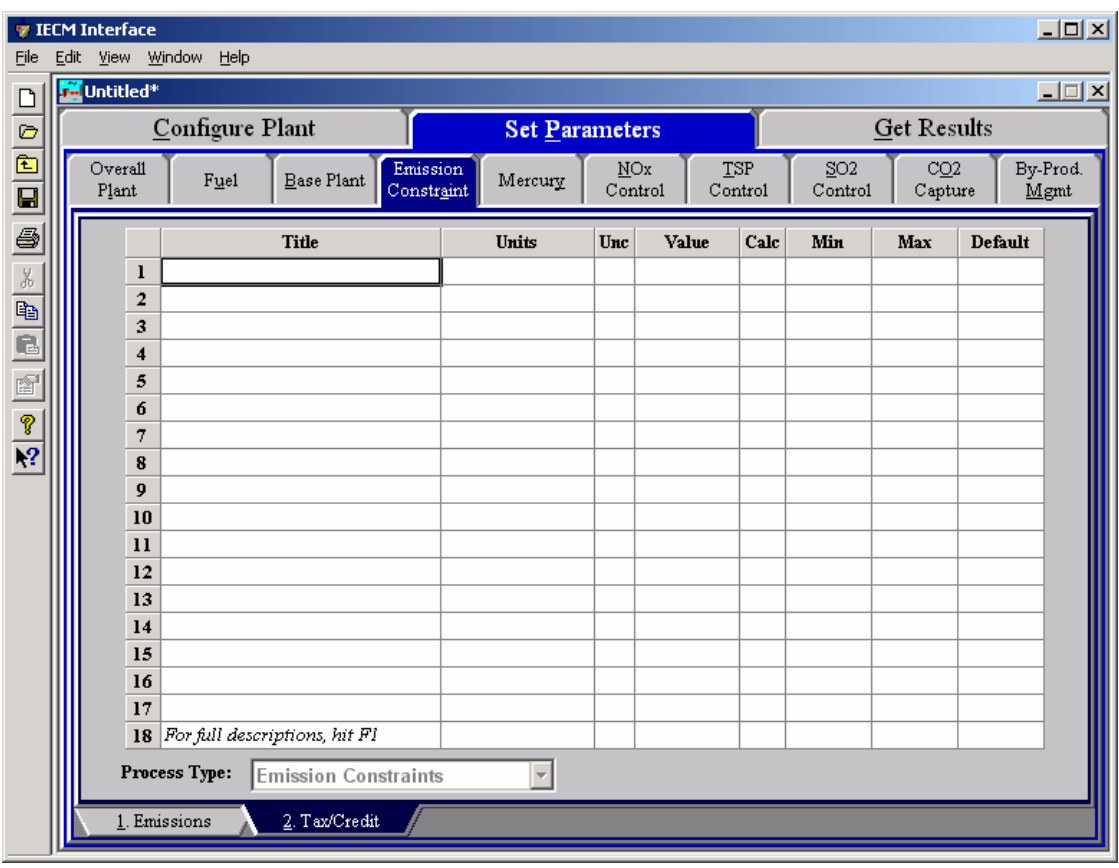

Emission Constraints Tax/Credit input screen. 



\section{Auxiliary Boiler}

The $\underline{\mathbf{C O}}_{2}$ Technology Navigation Tab in the Get Results program area contains result screens that display the flow rates and temperatures of substances through an Auxiliary Boiler.

If you have added an Auxiliary Boiler from the Set Parameters program area of the $\underline{\mathbf{C O}}_{2}$ Capture Configuration Input Screen then you may view either the Amine System or the Aux. Boiler using the pull down menu labeled Proces Type. Switch between the two sets of screens by using the Process Type pull-down menu at the bottom of the screen.

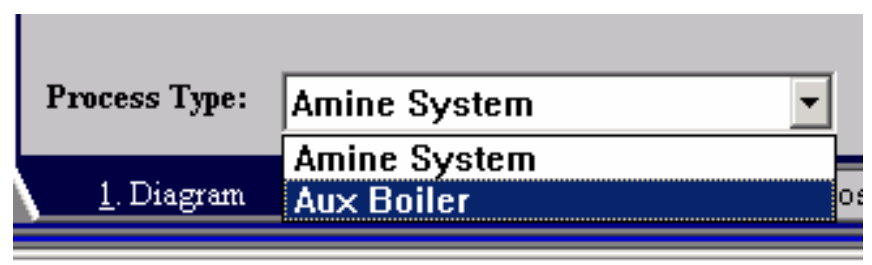

Auxiliary Boiler - Process Type

\section{Auxiliary Boiler Diagram}

The Diagram result screen displays an icon for the Boiler and values for major flows in and out of it. 


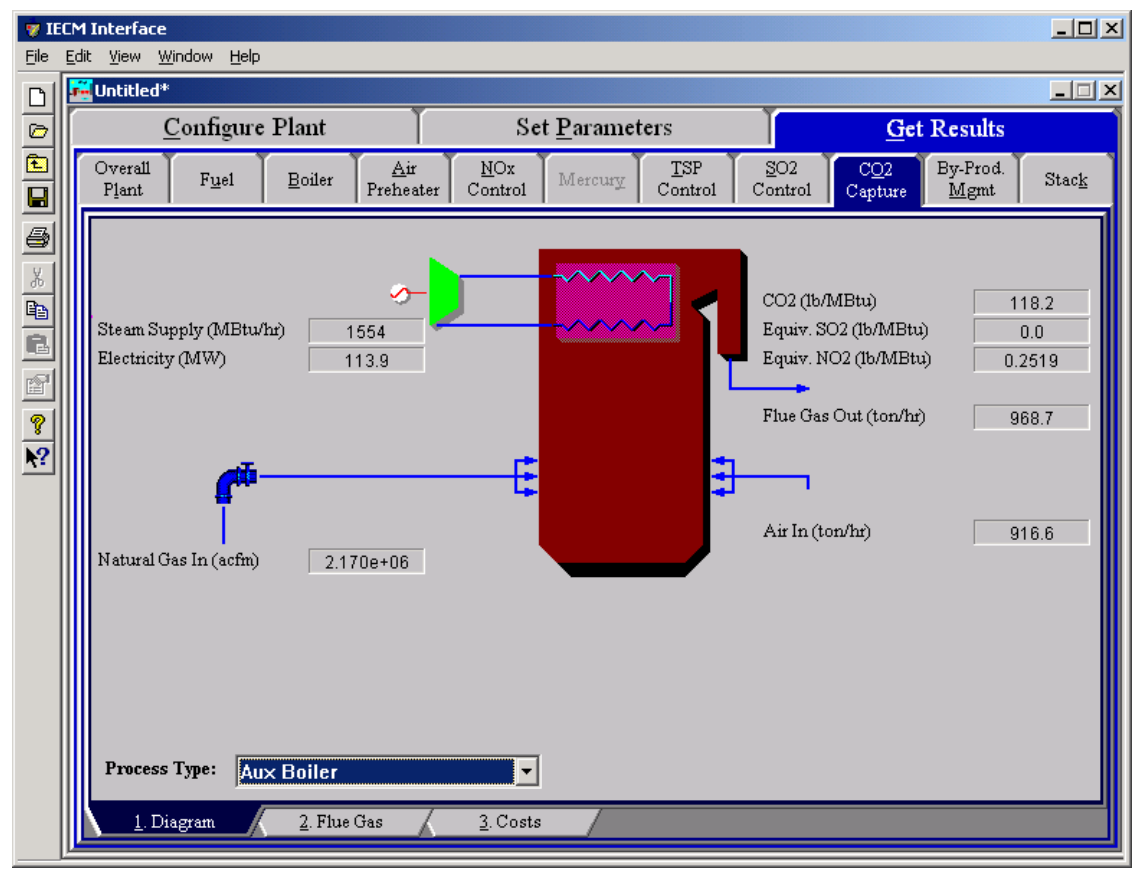

Auxiliary Boiler - Diagram.

Each result is described briefly below in flow order (not from top to bottom and left to right as they display on the screen).

\section{Fuel Entering Auxiliary Boiler}

Natural Gas In: Natural gas flow rate into the boiler.

\section{Air Entering Auxiliary Boiler}

Air In: Volumetric flow rate of the air at the burners, based on the air temperature at the burners and atmospheric pressure.

\section{Auxiliary Boiler Performance}

Steam Supply: The flow rate of steam produced by the auxiliary boiler.

Electricity: Electricity produced by the steam turbine that is powered by the auxiliary boiler.

\section{Flue Gas Exiting the Auxiliary Boiler}

Flue Gas Out: Volumetric flow rate of the flue gas at the exit of the auxiliary boiler.

$\mathrm{CO}_{2}$ : This is the amount of $\mathrm{CO}_{2}$ produced by the auxiliary boiler.

Equiv. $\mathrm{SO}_{2}$ : This is the amount of equivalent $\mathrm{SO}_{2}$ produced by the auxiliary boiler.

Equiv. $\mathrm{NO}_{2}$ : This is the amount of equivalent $\mathrm{NO} 2$ produced by the auxiliary boiler. 


\section{Auxiliary Boiler Flue Gas Results}

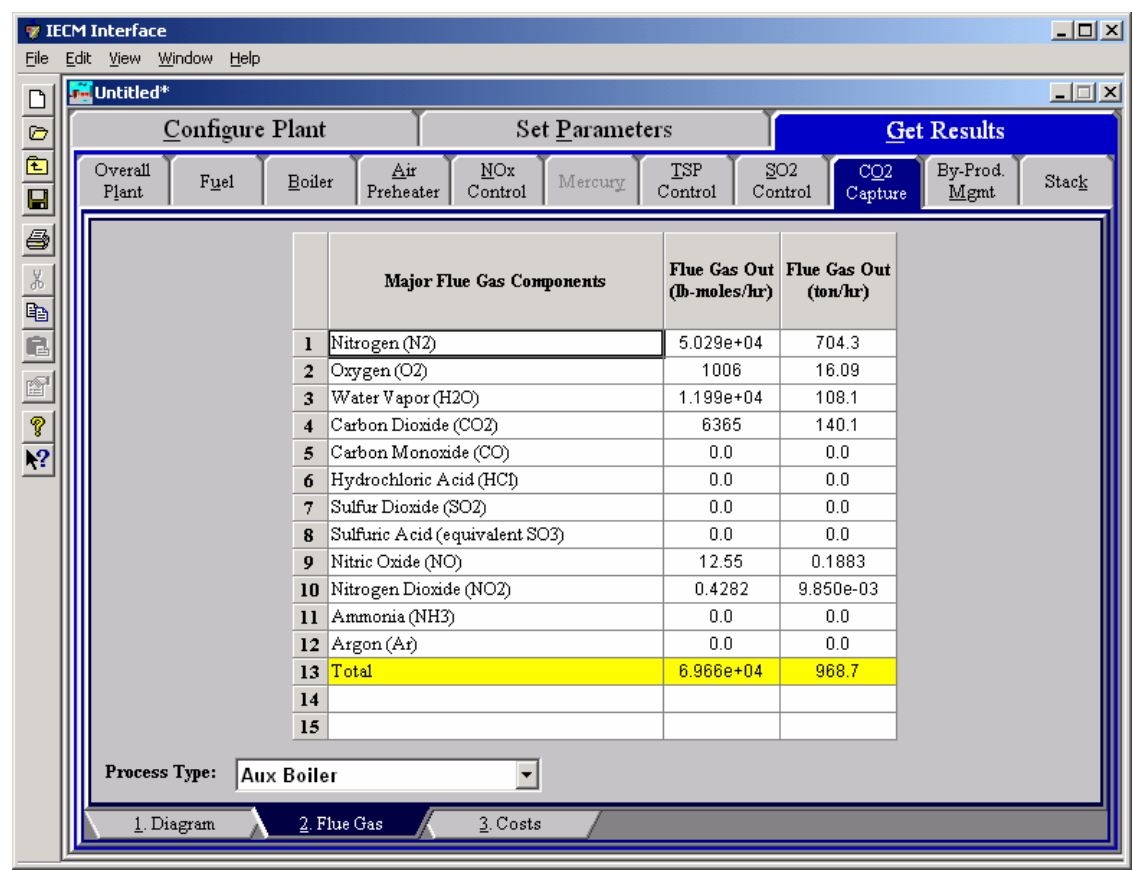

Auxiliary Boiler - Flue Gas result screen

\section{Major Flue Gas Components}

Each result is described briefly below:

Nitrogen $\left(\mathbf{N}_{\mathbf{2}}\right)$ : Total mass of nitrogen.

Oxygen $\left(\mathrm{O}_{2}\right)$ : Total mass of oxygen.

Water Vapor $\left(\mathbf{H}_{2} \mathbf{O}\right)$ : Total mass of water vapor.

Carbon Dioxide $\left(\mathrm{CO}_{2}\right)$ : Total mass of carbon dioxide.

Carbon Monoxide (CO): Total mass of carbon monoxide.

Hydrochloric Acid (HCl): Total mass of hydrochloric acid.

Sulfur Dioxide $\left(\mathbf{S O}_{2}\right)$ : Total mass of sulfur dioxide.

Sulfuric Acid (equivalent $\mathbf{S O}_{3}$ ): Total mass of sulfuric acid.

Nitric Oxide (NO): Total mass of nitric oxide.

Nitrogen Dioxide $\left(\mathrm{NO}_{2}\right)$ : Total mass of nitrogen dioxide.

Ammonia $\left(\mathrm{NH}_{3}\right)$ : Total mass of ammonia.

Argon (Ar): Total mass of argon.

Total: Total of the individual components listed above. This item is highlighted in yellow. 


\section{Auxiliary Boiler Costs Results}

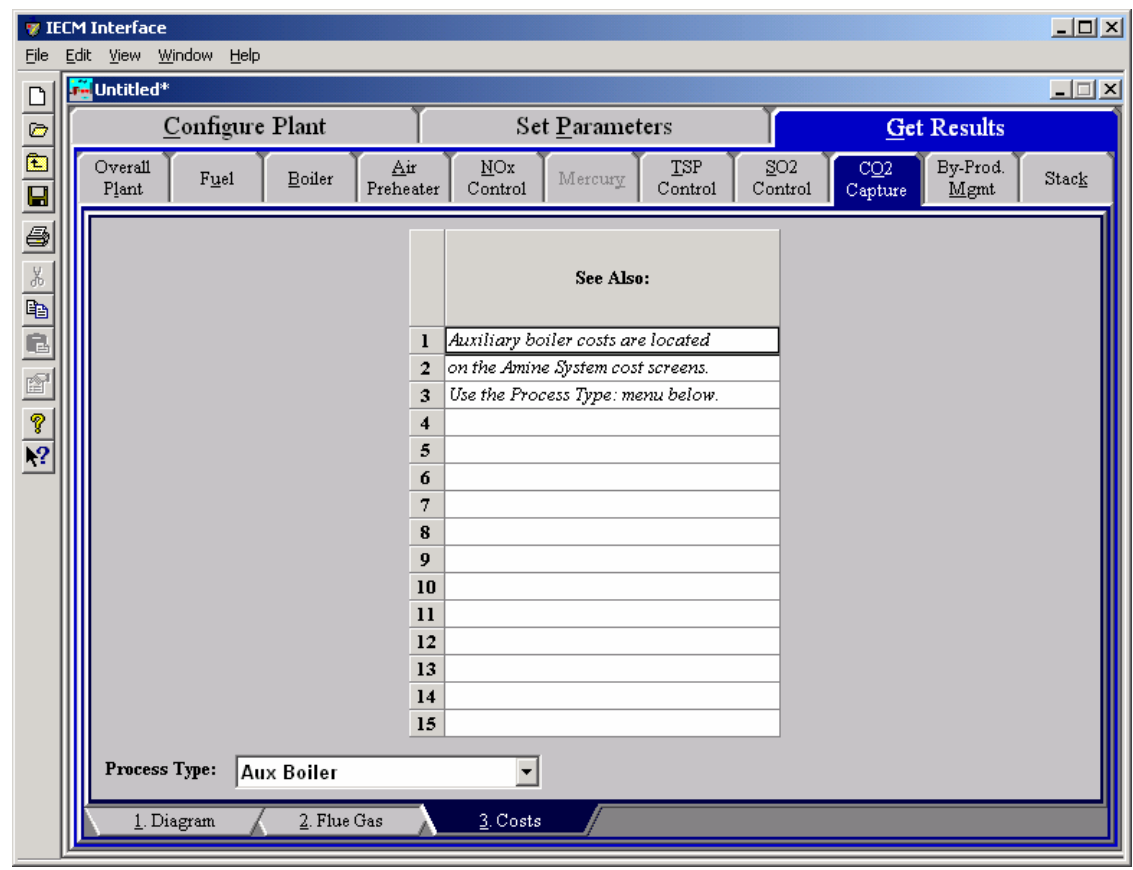

Aux. Boiler-Costs result screen.

Auxiliary Boiler costs are displayed by the Amine System cost screens. View these by selecting the Amine System from the Process Type menu on the bottom of the screen. 


\section{Air Preheater}

The Air Preheater Technology Navigation Tab in the Get Results program area contains result screens that display the flow rates and temperatures of substances through the air preheater.

\section{Air Preheater Diagram}

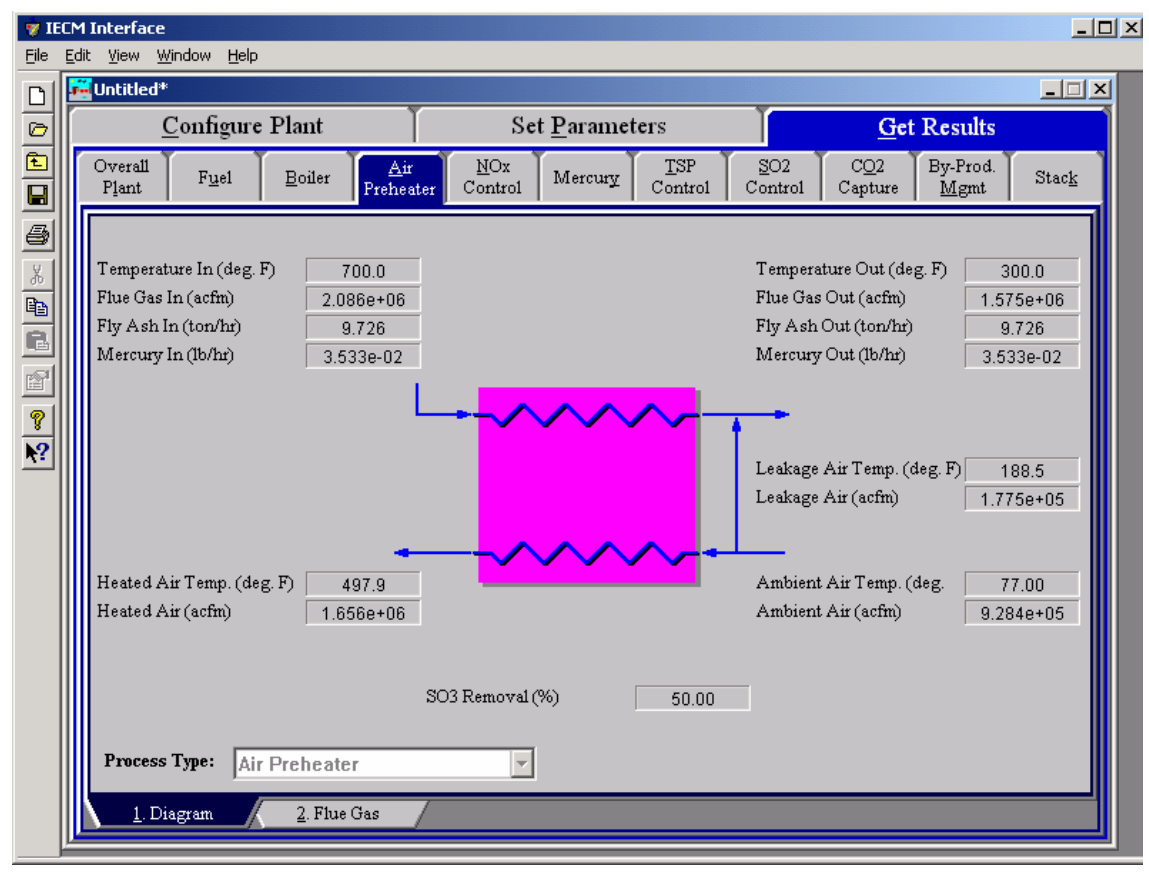

Air Preheater - Diagram.

The Diagram result screen displays an icon for the Air Preheater and values for major flows in and out of it. Each result is described briefly below in flow order (not from top to bottom and left to right as they display on the screen).

\section{Atmospheric Air Entering Preheater}

Ambient Air Temp: Temperature of the atmospheric air entering the induced-draft fan.

Ambient Air: Volumetric flow rate of air entering the induced-draft fan, based on the atmospheric air temperature and atmospheric pressure. 


\section{Heated Air Exiting Preheater}

Heated Air Temp: Heated combustion air temperature exiting the air preheater. This is a complicated function of the heat content and temperatures of the flue gas.

Heated Air: Volumetric flow rate of the combustion air exiting the air preheater, based on the combustion air temperature and atmospheric pressure.

\section{Leakage Air}

Leakage Air Temp: Temperature of the atmospheric air leaking across the air preheater into the flue gas. This is determined by the leakage parameter on the base plant performance input screen.

Leakage Air: Volumetric flow rate of the atmospheric air leaking across the air preheater into the flue gas. This is based on the leakage temperature and atmospheric pressure.

\section{Flue Gas Entering Preheater}

Temperature In: Temperature of the flue gas entering the air preheater. This is determined by the flue gas outlet temperature of the module upstream of the air preheater (e.g., the boiler economizer).

Flue Gas In: Volumetric flow rate of the flue gas entering the air preheater, based on the flue gas inlet temperature and atmospheric pressure.

Fly Ash In: Total solids mass flow rate in the flue gas entering the air preheater. This is determined by the solids exiting the module upstream of the air preheater (e.g., the boiler economizer).

Mercury In: Total mass of mercury entering the air preheater in the flue gas. The value is a sum of all the forms of mercury (elemental, oxidized, and particulate).

\section{Air Preheater Performance}

$\mathrm{SO}_{3}$ Removal: Percent of the $\mathrm{SO}_{3}$ removed from the flue gas.

\section{Cooled Flue Gas Exiting Preheater}

Temperature Out: Temperature of the flue gas exiting the air preheater. This is determined by the parameter on the base plant performance input screen.

Flue Gas Out: Volumetric flow rate of the flue gas exiting the air preheater, based on the flue gas exit temperature and atmospheric pressure.

Fly Ash Out: Total solids mass flow rate in the flue gas exiting the air preheater. This is a function of the percent ash entering the flue gas (furnace emissions input parameter) and the ash content of the fuel.

Mercury Out: Total mass of mercury exiting the air preheater in the flue gas. The value is a sum of all the forms of mercury (elemental, oxidized, and particulate). 


\section{Air Preheater Flue Gas Results}

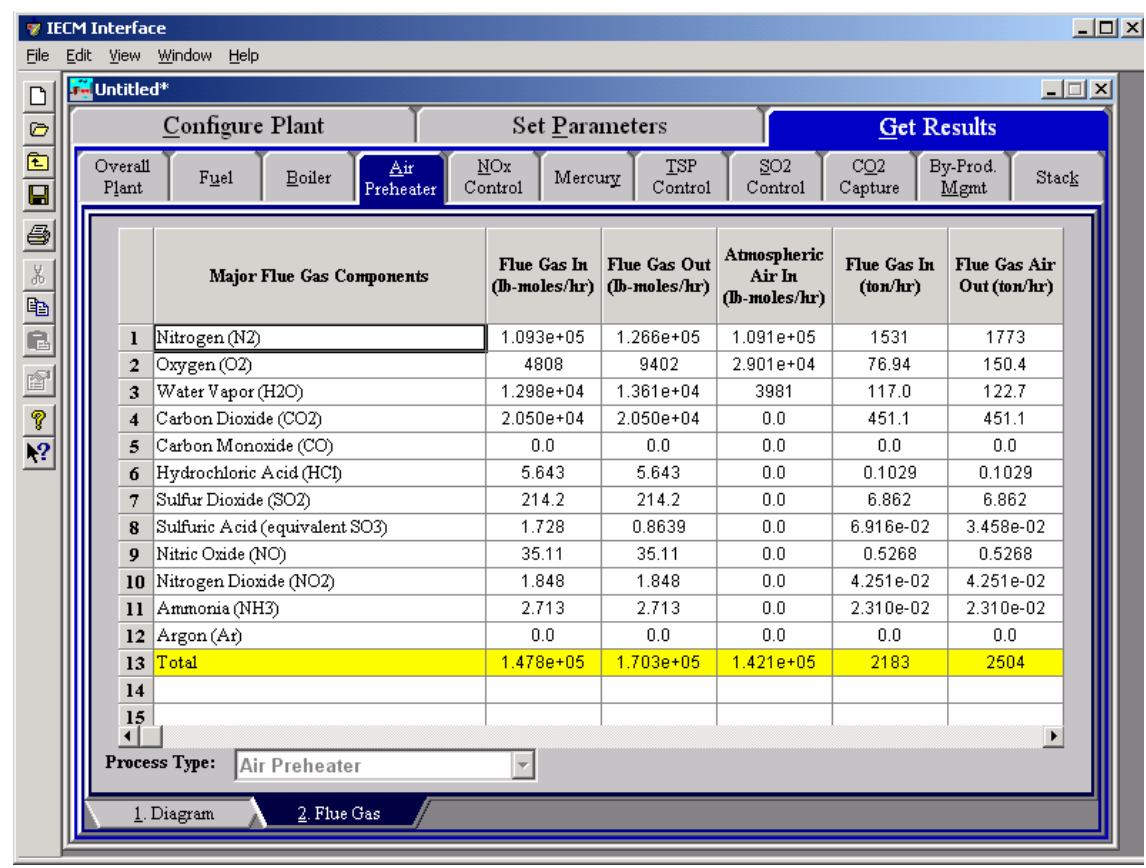

Air Preheater - Flue Gas result screen.

\section{Major Flue Gas Components}

The Flue Gas result screen displays a table of quantities of flue gas components entering and exiting the air preheater. For each component entering and exiting in flue gas, values are given in both moles and mass per hour. For each component entering in atmospheric air, values are given in moles per hour. Each result is described briefly below.

Nitrogen $\left(\mathrm{N}_{2}\right)$ : Total mass of nitrogen.

Oxygen $\left(\mathrm{O}_{2}\right)$ : Total mass of oxygen.

Water Vapor $\left(\mathrm{H}_{2} \mathrm{O}\right)$ : Total mass of water vapor.

Carbon Dioxide $\left(\mathrm{CO}_{2}\right)$ : Total mass of carbon dioxide.

Carbon Monoxide (CO): Total mass of carbon monoxide.

Hydrochloric Acid $(\mathbf{H C l})$ : Total mass of hydrochloric acid.

Sulfur Dioxide $\left(\mathrm{SO}_{2}\right)$ : Total mass of sulfur dioxide.

Sulfuric Acid (equivalent $\mathbf{S O}_{3}$ ): Total mass of sulfuric acid.

Nitric Oxide (NO): Total mass of nitric oxide.

Nitrogen Dioxide $\left(\mathrm{NO}_{2}\right)$ : Total mass of nitrogen dioxide.

Ammonia (NH3): Total mass of ammonia.

Argon (Ar): Total mass of argon.

Total: Total of the individual components listed above. This item is highlighted in yellow. 



\section{In-Furnace Controls}

The $\mathbf{N O}_{\mathbf{x}}$ Control Technology Navigation Tab contains screens that address combustion or post-combustion air pollution technologies for Nitrogen Oxides.

These screens are available if the In-Furnace Controls has been selected for $\mathrm{NO}_{\mathrm{x}}$ control under Combustion Controls. If you have selected both In-Furnace Controls and a Hot-Side SCR for $\mathrm{NO}_{\mathrm{x}}$ control, you may switch between the two sets of screens that configure these technologies by using the Process Type pull-down menu at the bottom of the screen

\begin{tabular}{|l|l||}
\hline Process Type: & Hot-Side SCR \\
& In-Furnace Controls \\
\hline & Hot-Side SCR \\
\hline \hline
\end{tabular}

The Process Type pull-down menu

\section{In-Furnace Controls Configuration}

Inputs for configuring the $\mathrm{NO}_{\mathrm{x}}$ Control technology are entered on the Config input screen. Each parameter is described briefly below. 


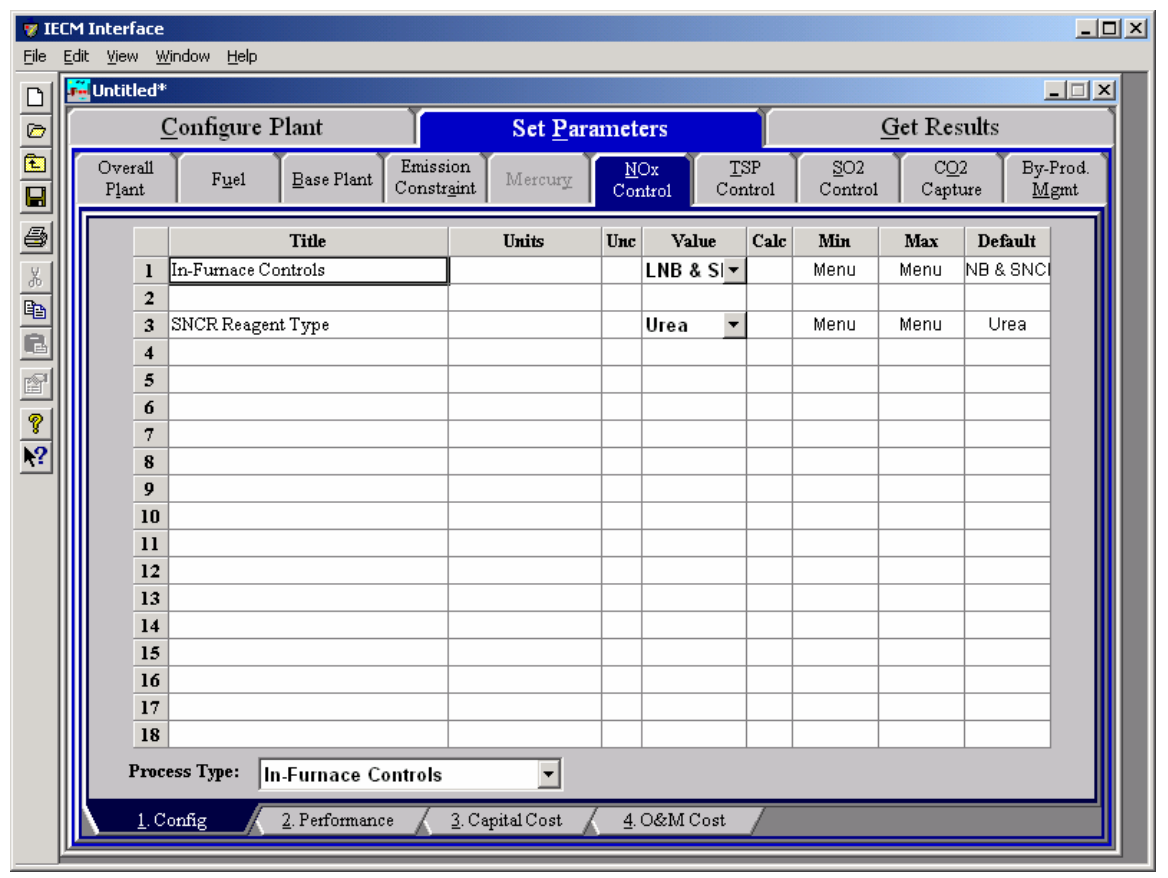

In - Furnace Controls - Config input screen.

\section{In - Furnace Controls}

This pull-down menu chooses what type of in-furnace $\mathrm{NO}_{\mathrm{x}}$ controls are used. These technologies reduce $\mathrm{NO}_{\mathrm{x}}$ between the primary fuel injection into the furnace and the economizer. These can be used in the combinations given in addition to the SCR. The low $\mathrm{NO}_{\mathrm{x}}$ burner options are not displayed when a cyclone boiler is configured. The full list of choices is:

LNB - Low $\mathrm{NO}_{\mathrm{x}}$ burners are a combustion $\mathrm{NO}_{\mathrm{x}}$ control. These burners replace the upper coal nozzle of the standard two-nozzle cell burner with a secondary air port. The lower burner coal nozzle is enlarged to the same fuel input capacity as the two standard coal nozzles. The LNB operates on the principle of staged combustion to reduce $\mathrm{NO}_{\mathrm{x}}$ emissions. Approximately 70\% of the total air (primary, secondary, and excess air) is supplied through or around the coal-feed nozzle. The remainder of the air is directed to the upper port of each cell to complete the combustion process. The fuel-bound nitrogen compounds are converted to nitrogen gas, and the reduced flame temperature minimizes the formation of thermal $\mathrm{NO}_{\mathrm{x}}$. The net effect of this technology is greater than $50 \%$ reduction in $\mathrm{NO}_{\mathrm{x}}$ formation with no boiler pressure part changes and no impact on boiler operation or performance. Low $\mathrm{NO}_{\mathrm{x}}$ burners are not available for cyclone boilers.

LNB \& OFA - Low $\mathrm{NO}_{x}$ burners (see above) with overfire air is another combustion $\mathrm{NO}_{\mathrm{x}}$ reduction method. Overfire air is an enhancement to LNB to reduce $\mathrm{NO}_{\mathrm{x}}$ formation by further separating the air injection locations. An addition of approximately $10 \% \mathrm{NO}_{\mathrm{x}}$ is reduced by the addition of OFA. A portion of the secondary air used by LNB is diverted to injection ports located above the primary combustion zone, reducing available oxygen in the primary combustion zone. Overfire air in the IECM refers to separated OFA for both wall and tangential-fired boilers. This option is not supported for cyclone boilers. 
Gas Reburn - Gas reburn is a post-combustion $\mathrm{NO}_{\mathrm{x}}$ reduction method. Gas reburn substitutes up to one-fourth of the heat input of coal with natural gas, reducing the $\mathrm{NO}_{\mathrm{x}}$ up to $60 \%$ as a function of the amount of reburn. The natural gas is injected above the primary combustion zone to create a reducing zone. Reburn has been shown to be effective for wall and tangential-fired boilers and more recently for cyclone boilers.

SNCR - Selective non-catalytic reduction is a post-combustion $\mathrm{NO}_{\mathrm{x}}$ reduction method. This process removes $\mathrm{NO}_{\mathrm{x}}$ from flue gas by injecting one of two nitrogen-based reagents, ammonia or urea, in the presence of oxygen to form nitrogen and water vapor. Optimum removal is achieved in a temperature window of 1600-2000 F. Although the technology is very simple, the narrow temperature window provides the primary challenge. Ammonia slip and ash contamination are additional concerns that must be considered with SNCR.

LNB \& SNCR - Low $\mathrm{NO}_{\mathrm{x}}$ burners can be used in conjunction with SNCR to achieve very high $\mathrm{NO}_{\mathrm{x}}$ removals. Both technologies are described in detail above.

If a Tangential or Wall Furnace Type have been selected in $\underline{\text { Configure Plant, then }}$ all five options will display. If you have selected a Cyclone Furnace type, then only Gas Reburn and SNCR will display.

The default for Tangential and Wall furnaces is LNB \& SNCR. The default for a Cyclone furnace is Gas Reburn.

\section{SNCR Reagent Type}

Only displayed when SNCR or LNB \& SNCR have been selected in the In-Furnace Controls pull-down menu. Nitrogen-based reagent injection is used in an SNCR to reduce $\mathrm{NO}_{\mathrm{x}}$ in the presence of oxygen to form nitrogen and water vapor. The reagent choices are:

Urea - Urea $\left(\mathrm{CO}\left(\mathrm{NH}_{2}\right)_{2}\right)$ is typically diluted to a $15-20 \%$ concentration with water. Urea has the advantage of safety and ease of storage and handling. Urea is the default reagent used in the IECM.

Ammonia - Ammonia can be supplied in two forms: anhydrous $\left(\mathrm{NH}_{3}\right)$ and aqueous $\left(\mathrm{NH}_{4} \mathrm{OH}\right)$. The IECM considers only anhydrous ammonia. Ammonia may be an advantage when using an SNCR in conjunction with an SCR system. 


\section{In-Furnace Controls Performance Input}

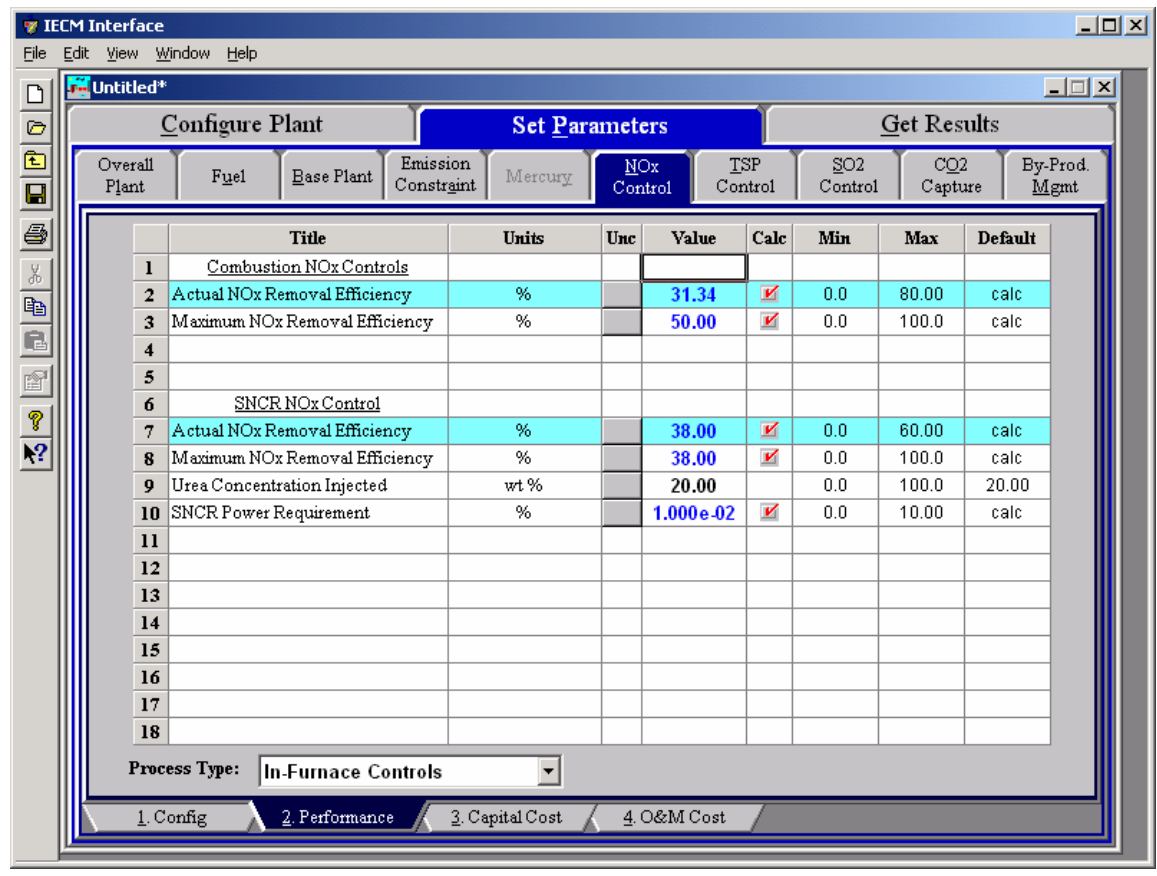

In - Furnace Controls - Performance input screen.

Inputs for the performance of the In-Furnace Controls $\mathrm{NO}_{\mathrm{x}}$ control technology are entered on the on the Performance input screen. Combustion NOx Controls These inputs will display if any combustion technology is used in the option selected in the In-Furnace Controls pull-down menu. This includes the LNB, LNB + OFA, Gas Reburn, and the LNB + SNCR options.

Actual NOx Removal Efficiency: This is the $\mathrm{NO}_{\mathrm{x}}$ removal efficiency of the LNB, LNB + OFA, and Gas Reburn options, and the LNB removal portion of the LNB + SNCR option. The percent reduction of $\mathrm{NO}_{\mathrm{x}}$ is calculated by comparing the actual $\mathrm{NO}_{\mathrm{x}}$ emission to the uncontrolled $\mathrm{NO}_{\mathrm{x}}$ emission. The removal is a function of the InFurnace Control type selected in the pull-down menu, the boiler type, and the maximum removal efficiency (below). Note: that the removal is not a function of the $\mathrm{NO}_{\mathrm{x}}$ emission constraint. This input is highlighted in blue.

Maximum NOx Removal Efficiency: The maximum removal efficiency of $\mathrm{NO}_{\mathrm{x}}$ sets the upper bound for the actual $\mathrm{NO}_{\mathrm{x}}$ removal efficiency (above). The maximum removal is a function of the InFurnace control type and the boiler type.

Natural Gas Heat Input: This input will only display if Gas Reburn is selected in the In-Furnace Controls pull-down menu. The flow rate of natural gas injected is determined by this input on a Btu heat input basis.

\section{SNCR NOx Control}

These inputs will only display if SNCR or LNB \& SNCR is selected in the InFurnace Controls pull-down menu. 
Actual NOx Removal Efficiency: The actual $\mathrm{NO}_{\mathrm{x}}$ removal efficiency is a function of the maximum $\mathrm{NO}_{\mathrm{x}}$ removal efficiency (below) and the $\mathrm{NO}_{\mathrm{x}}$ emission constraint. This input is highlighted in blue.

Maximum NOx Removal Efficiency: The maximum removal efficiency is calculated as a function of the gross electrical output. Because of difficulty mixing the reagent in the flue gas for larger boilers, the maximum efficiency decreases with increasing plant size.

Urea Concentration Injected: Urea is typically injected as a liquid diluted by water. This parameter defines the amount of water used to dilute the urea prior to injection.

SNCR Power Requirement: As mentioned above, the power requirement for the SNCR is a function of gross electrical output of the power plant. The value is determined by the need for tank heaters when urea reagent is used.

\section{In-Furnace Controls Capital Cost}

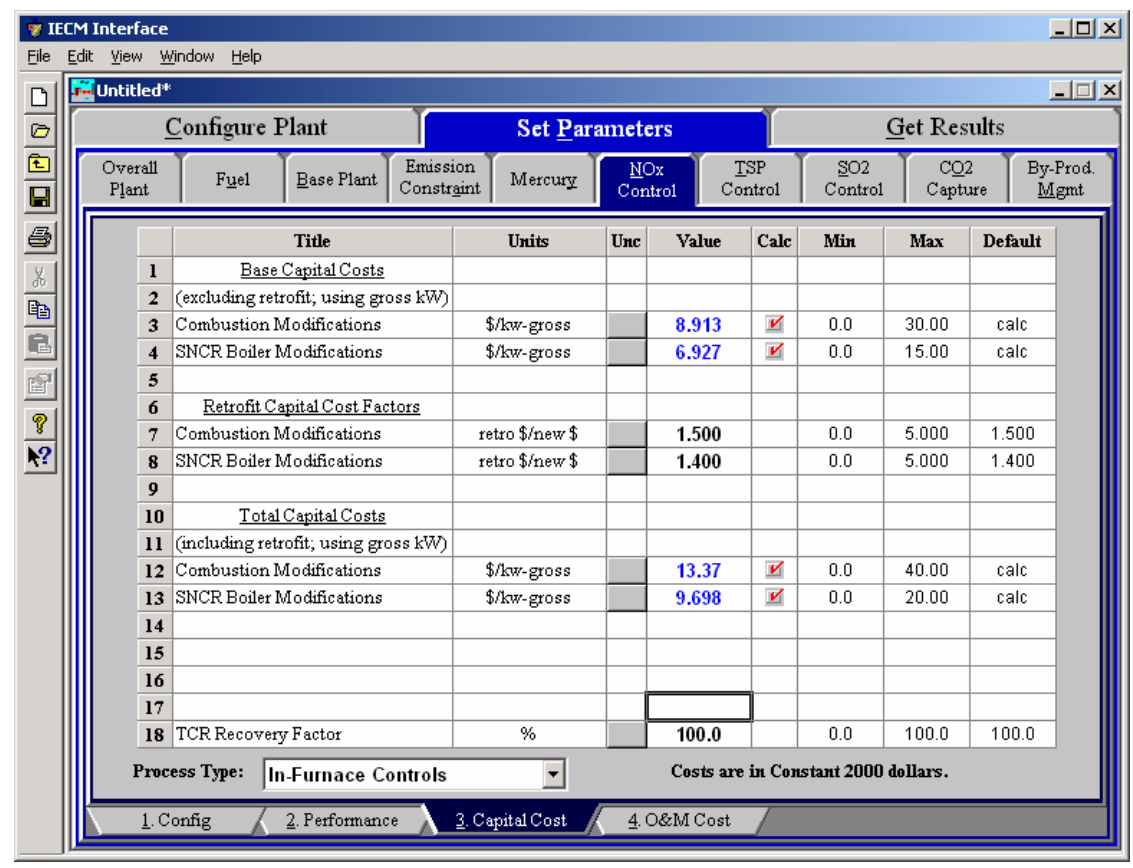

In-Furnace Controls - Capital Cost input screen.

The Combustion Modifications inputs will not display if SNCR is selected in the In-Furnace Controls pull-down menu. The SNCR Boiler Modifications inputs will only display if SNCR or LNB \& SNCR is selected

\section{Base Capital Costs}

The base capital costs_(excluding retrofit, using gross KW) specify the total base capital costs, not considering any retrofit factors. No detailed information about direct or indirect costs is given. The costs are given as a total in units of dollars per gross kilowatt.

Combustion Modifications: This is the base capital cost of the LNB, LNB + OFA, and Gas Reburn options, and the LNB removal portion 
of the LNB + SNCR option. This parameter is not shown when one of these options is not selected.

SNCR Boiler Modifications: This specifies the total base capital cost for the SNCR boiler $\mathrm{NO}_{\mathrm{x}}$ removal equipment alone. This parameter is not shown when one of the SNCR options is not selected.

\section{Retrofit Capital Cost Factors}

Retrofit cost factors allow you to differentiate between the base cost of purchasing the capital equipment and the actual cost incurred. These factors vary from unit to unit.

Combustion Modifications: This is the retrofit cost factor for the LNB, LNB + OFA, and Gas Reburn options, and the LNB removal portion of the LNB + SNCR option. This parameter is not shown when one of these options is not selected

SNCR Boiler Modifications: This is the retrofit cost factor for the SNCR option alone. This parameter is not shown when one of the SNCR options is not selected

\section{Total Capital Costs (including retrofit, using gross KW):}

Combustion Modifications: This is the total capital cost of the LNB, LNB + OFA, and Gas Reburn options, and the LNB removal portion of the LNB + SNCR option. This combines the base capital cost with the retrofit cost factor. This parameter is not shown when one of these options is not selected.

SNCR Boiler Modifications: This specifies the total capital cost for the SNCR boiler $\mathrm{NO}_{\mathrm{x}}$ removal equipment alone. This parameter is not shown when one of the SNCR options is not selected

TCR Recovery Factor: The actual total capital required (TCR) as a percent of the TCR in a new power plant. This value is $100 \%$ for a new installation and may be set as low as $0 \%$ for in-furnace controls that has been paid off.

\section{In-Furnace Controls O\&M Cost}

Inputs for the operation and maintenance costs of the In-Furnace Controls O\&M $\mathrm{CosNO}_{\mathrm{x}}$ control technology are entered on the $\underline{6}$. O\&M Cost input screen for the Hot-Side SCR, and the 4. O\&M Cost input screen for In-Furnace Controls. Each parameter is described briefly below. 


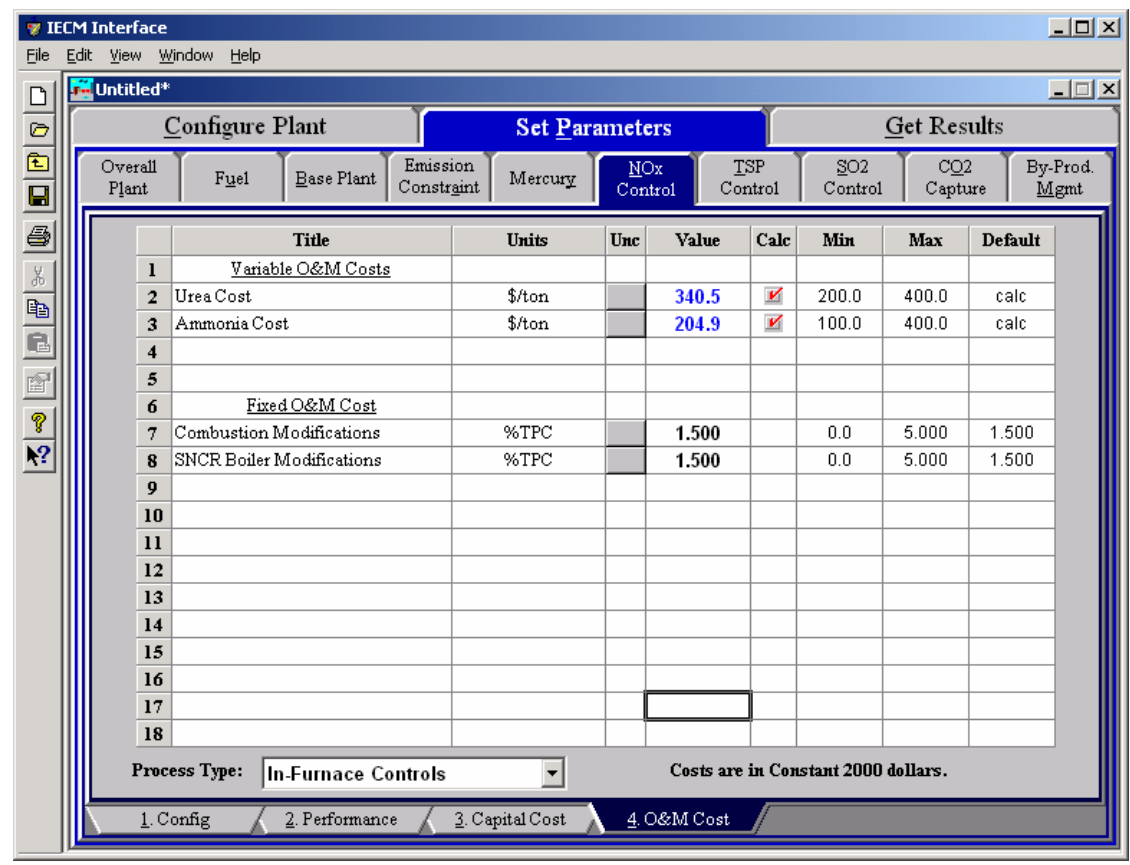

In-Furnace Controls - O\&M Cost input screen.

Each parameter is described briefly below.

\section{Variable O\&M Costs}

Urea Cost: This is the cost of urea used for any of the SNCR options. This input will only display if SNCR or LNB \& SNCR is selected in the InFurnace Controls pull-down menu

Ammonia Cost: This is the cost of ammonia used for any of the SNCR options. This input will only display if SNCR or LNB \& SNCR is selected in the In-Furnace Controls pull-down menu

Natural Gas Cost: This is the cost of natural gas used for the Gas

Reburn option. This input will only display if Gas Reburn is selected

\section{Fixed O\&M Cost}

Fixed O\&M costs are given as a total cost, rather than itemized costs broken down by individual maintenance and labor costs. The results are given as a percent of the total capital cost.

Combustion Modifications: This is the total fixed operating and maintenance cost for boiler $\mathrm{NO}_{\mathrm{x}}$ modifications made in the combustion zone (LNB, OFA, natural gas reburn). This parameter is not shown if one of these options is not selected.

SNCR Boiler ModificationsVariable O\&M Costs: This is the total fixed O\&M cost for the SNCR equipment alone. This input is not shown if one of the SNCR options is not selected. 


\section{In-Furnace Controls Diagram}

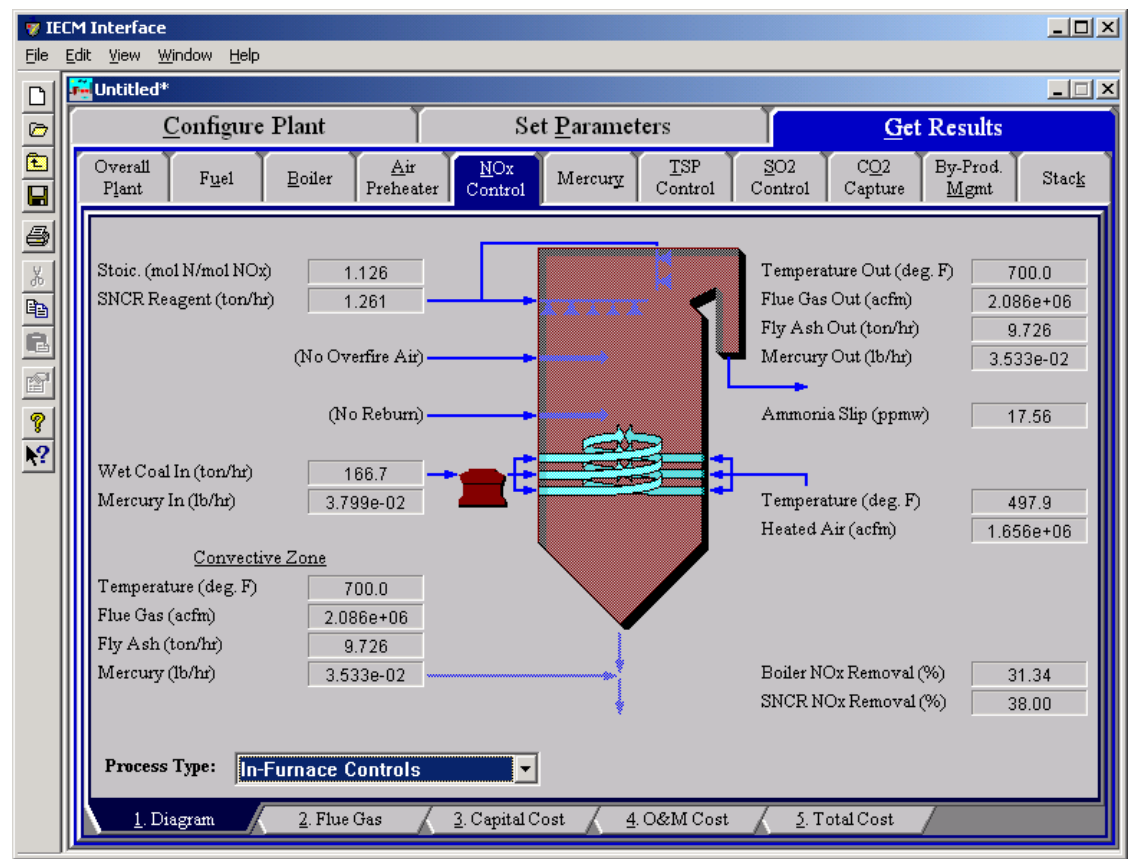

In-Furnace Controls - Diagram

The Diagram result screen displays an icon for the In-Furnace Controls $\mathrm{NO}_{\mathrm{x}}$ technology selected and values for major flows in and out of it.

\section{Fuel Entering Boiler}

Wet Coal In: Fuel flow rate into the boiler on a wet basis. Waste products removed prior to the burners are not considered here.

Mercury In: This is the mass flow rate of total mercury entering the boiler. The mass reflects the molecular weight of elemental mercury.

\section{Air Entering Boiler}

Temperature: Heated air temperature measured at the burners. This is generally determined by the combustion air temperature exiting the air preheater.

Heated Air: Volumetric flow rate of the air at the burners, based on the air temperature at the burners and atmospheric pressure.

\section{Flue Gas Exiting Convective Zone}

This the area of the furnace between the combustion zone and the SNCR (if present). Changes in the flue gas after combustion due to in-furnace combustion $\mathrm{NO}_{\mathrm{x}}$ controls are reflected here.

Temperature: Temperature of the flue gas exiting the convective zone.

Flue Gas: Volumetric flow rate of the flue gas exiting the convective zone, based on the temperature exiting the convective zone and atmospheric pressure.

Fly Ash: Total solids mass flow rate in the flue gas exiting the convective zone. This includes ash, unburned carbon and unburned sulfur. 
Mercury: Total mass of mercury in the flue gas exiting the convective zone. The value is a sum of all the forms of mercury (elemental, oxidized, and particulate).

\section{Flue Gas Exiting the Economizer}

Temperature Out: Temperature of the flue gas at the exit of the economizer.

Flue Gas Out: Volumetric flow rate of the flue gas at the exit of the economizer, based on the temperature at the exit of the economizer and atmospheric pressure.

Fly Ash Out: Total solids mass flow rate in the flue gas at the exit of the economizer. This includes ash, unburned carbon and unburned sulfur.

Mercury Out: Total mass of mercury in the flue gas exiting the economizer. The value is a sum of all the forms of mercury (elemental, oxidized, and particulate).

\section{Gas Reburn}

Reburn Gas: This is the flow rate of natural gas into the boiler. This result will only display if Gas Reburn is selected in the In-Furnace Controls pull-down menu

\section{SNCR}

The SNCR is located in the upper portion of the boiler. Several parameters are reported as a summary. These results will only display if SNCR or LNB \& SNCR is selected in the In-Furnace Controls pull-down menu.

Stoic.: This is the actual reagent stoichiometry used in the SNCR. Note that urea has double the moles of nitrogen relative to that of ammonia.

SNCR Reagent: This is the mass flow rate of reagent (urea or ammonia) injected by the SNCR into the boiler. Note that water used to dilute the urea is included in this flow rate.

Ammonia Slip: This is the concentration of ammonia leaving the SNCR technology in the flue gas. 


\section{In-Furnace Controls Flue Gas Results}

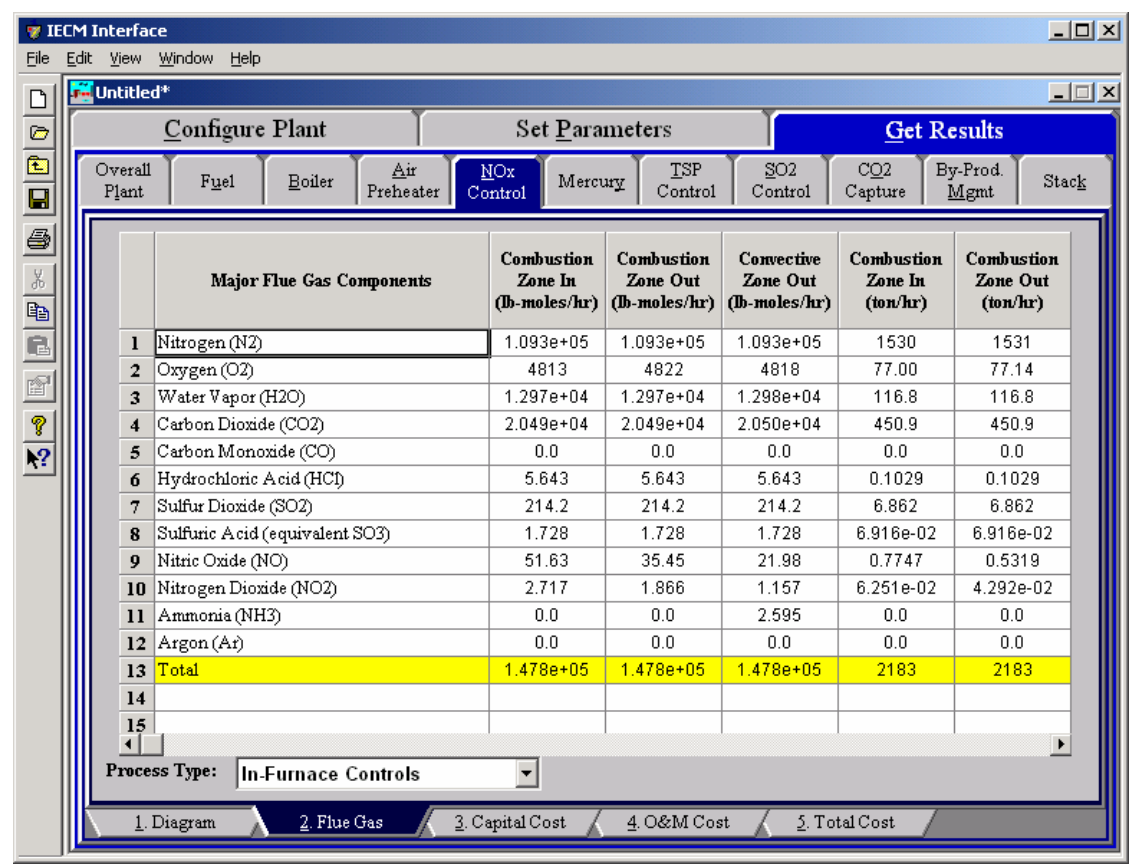

In-Furnace Controls - Flue Gas result screen.

The Flue Gas result screen for In-Furnace Controls displays a table of quantities of gas components entering and exiting the combustion zone. For each component, quantities are given in both moles and mass per hour. It also displays quantities of gas components exiting the convective zone in moles per hour. Each result is described briefly below.

\section{Major Flue Gas Components}

Nitrogen $\left(\mathbf{N}_{\mathbf{2}}\right)$ : Total mass of nitrogen.

Oxygen $\left(\mathrm{O}_{2}\right)$ : Total mass of oxygen.

Water Vapor $\left(\mathrm{H}_{2} \mathrm{O}\right)$ : Total mass of water vapor.

Carbon Dioxide $\left(\mathrm{CO}_{2}\right)$ : Total mass of carbon dioxide.

Carbon Monoxide (CO): Total mass of carbon monoxide.

Hydrochloric Acid ( $\mathrm{HCl})$ : Total mass of hydrochloric acid.

Sulfur Dioxide $\left(\mathrm{SO}_{2}\right)$ : Total mass of sulfur dioxide.

Sulfuric Acid (equivalent $\mathrm{SO}_{3}$ ): Total mass of sulfuric acid.

Nitric Oxide (NO): Total mass of nitric oxide.

Nitrogen Dioxide $\left(\mathrm{NO}_{2}\right)$ : Total mass of nitrogen dioxide.

Ammonia $\left(\mathrm{NH}_{3}\right)$ : Total mass of ammonia.

Argon (Ar): Total mass of argon

Total: Total of the individual components listed above. This item is highlighted in yellow. 


\section{In-Furnace Controls Capital Cost Results}

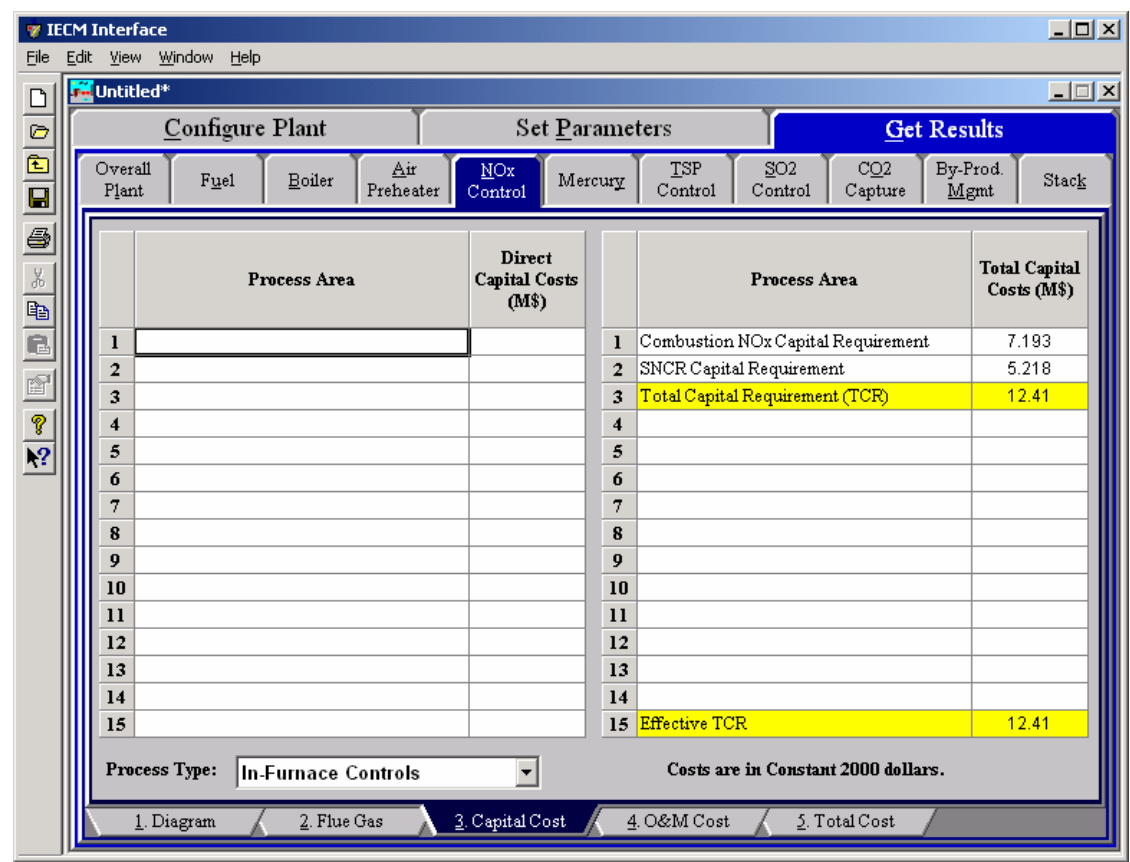

In-Furnace Controls - Capital Cost result screen.

The Capital Cost result screen displays tables for the direct and indirect capital costs related to the In-Furnace Controls $\mathrm{NO}_{\mathrm{x}}$ control technology.

\section{Total Capital Costs}

Combustion $\mathrm{NO}_{\mathrm{x}}$ Capital Requirement: The total capital costs, including retrofit costs, for the LNB, OFA, and gas reburn technologies are included here. A zero is displayed when none of these technologies are installed.

SNCR Capital Requirement: The total capital costs, including retrofit costs, for the SNCR technology is included here. A zero is displayed when an SNCR is not installed.

Total Capital Requirement: Sum of the above.

Effective TCR: The TCR of the retrofit $\mathrm{NO}_{\mathrm{x}}$ controls that is used in determining the total power plant cost. The effective TCR is determined by the "TCR Recovery Factor" for the hot-side SCR. 


\section{In-Furnace Controls O\&M Cost Results}

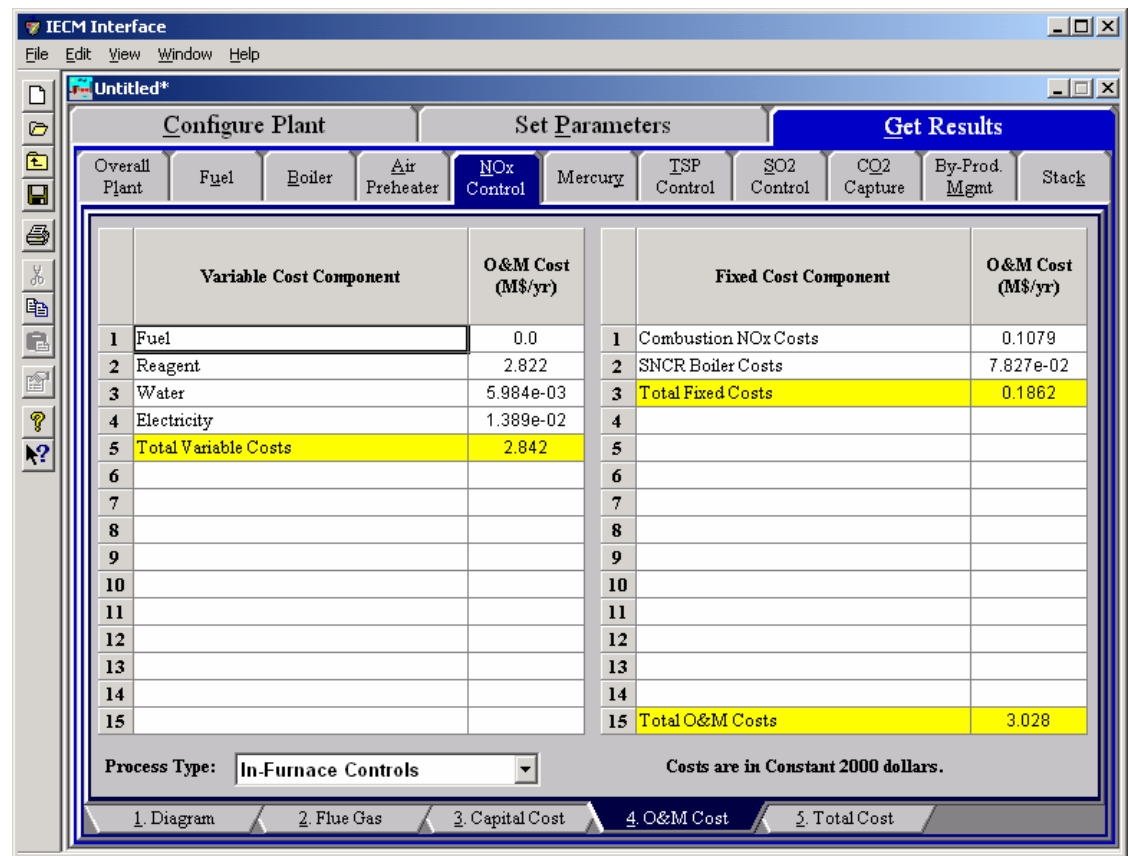

In-Furnace Controls- O\&M Cost result screen.

The O\&M Cost result screen displays tables for the variable and fixed operation and maintenance costs involved with the In-Furnace Controls $\mathrm{NO}_{\mathrm{x}}$ control technology. Each result is described briefly below

\section{Variable Cost Components}

Variable operating costs and consumables are directly proportional to the amount of kilowatts produced and are referred to as incremental costs. All the costs are subject to inflation.

Reagent: The total reagent costs (urea and ammonia) are included here.

Water: This is the cost of the water used to dilute the urea for the SNCR.

Power: This is the power used for the pumps to move reagents and water in the SNCR.

Total Variable Costs: This is the sum of all the variable O\&M costs listed above. This result is highlighted in yellow.

\section{Fixed Cost Components}

Fixed operating costs are essentially independent of actual capacity factor, number of hours of operation, or amount of kilowatts produced. All the costs are subject to inflation.

Combustion $\mathrm{NO}_{\mathrm{x}}$ Costs: This is the fixed O\&M costs associated with the LNB, OFA, and gas reburn systems.

SNCR Boiler Costs: This is the fixed O\&M costs associated with the SNCR system.

Total Fixed Costs: This is the sum of all the fixed O\&M costs listed above. This result is highlighted in yellow. 
Total O\&M Costs: This is the sum of the total variable and total fixed O\&M costs. It is used to determine the base plant total revenue requirement. This result is highlighted in yellow.

\section{In-Furnace Controls Total Cost Results}

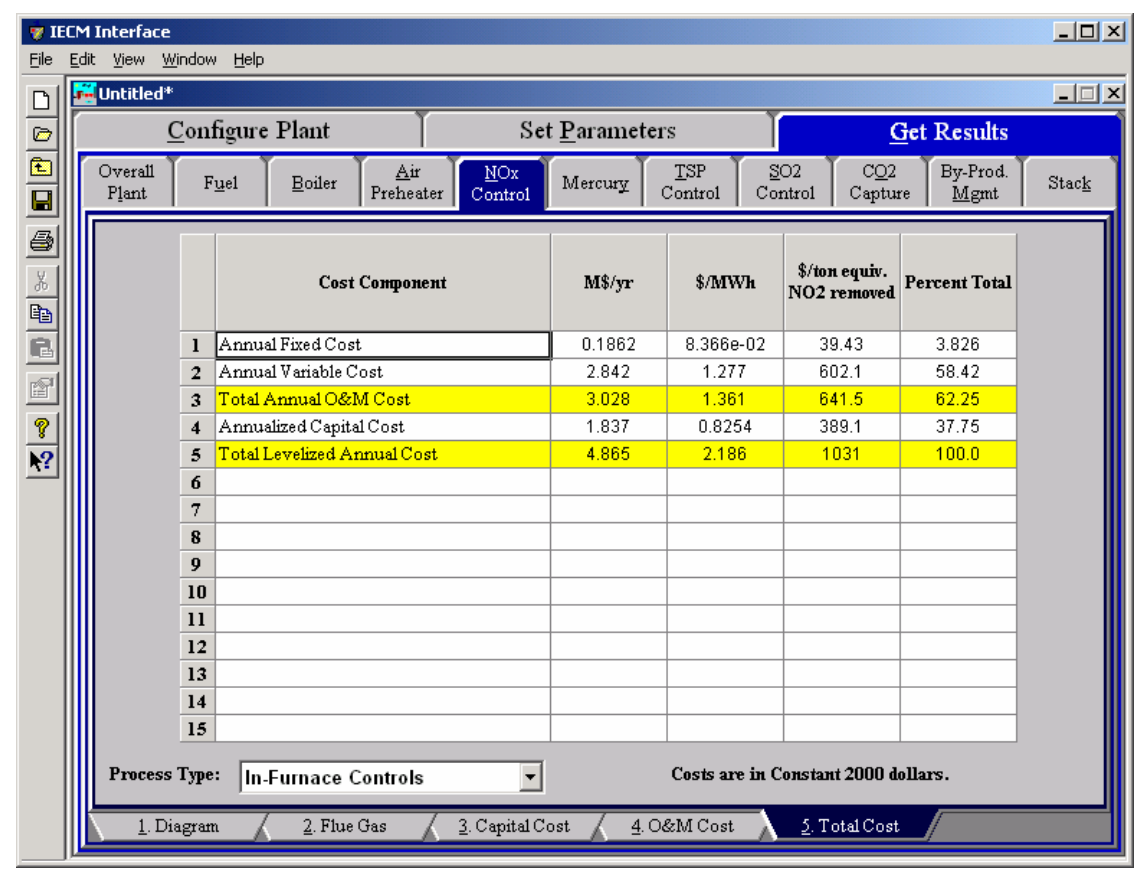

In-Furnace Controls - Total Cost result screen

The Total Cost result screen displays a table which totals the annual fixed, variable, operations and maintenance, and capital costs associated with the In-Furnace

Controls $\mathrm{NO}_{\mathrm{x}}$ Control technology. Each result is described briefly below. Note that all costs expressed in $\$$ /ton of $\mathrm{NO}_{2}$ removed assume tons of equivalent $\mathrm{NO}_{2}$.

Annual Fixed Cost: The operating and maintenance fixed costs are given as an annual total. This number includes all maintenance materials and all labor costs.

Annual Variable Cost: The operating and maintenance variables costs are given as an annual total. This includes all reagent, chemical, steam, and power costs.

Total Annual O\&M Cost: This is the sum of the annual fixed and variable operating and maintenance costs above. This result is highlighted in yellow.

Annualized Capital Cost: This is the total capital cost expressed on an annualized basis, taking into consideration the levelized carrying charge factor, or fixed charge factor, over the entire book life.

Total Levelized Annual Cost: The total annual cost is the sum of the total annual O\&M cost and annualized capital cost items above. This result is highlighted in yellow. 



\section{Hot-Side SCR}

The $\mathbf{N O}_{\mathbf{x}}$ Control Technology Navigation Tab contains screens that address combustion or post-combustion air pollution technologies for Nitrogen Oxides.

If you have selected a Hot-Side SCR, there will be six input screens and therefore six Input Navigation Tabs. If you have selected In-Furnace Controls, there will be four input screens and therefore four Input Navigation Tabs.

These input screens are only available if a Hot-Side SCR has been selected under

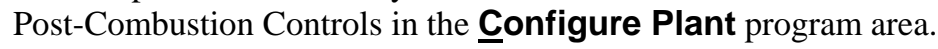

If you have selected both In-Furnace Controls and a Hot-Side SCR for $\mathrm{NO}_{\mathrm{x}}$ control, you may switch between the two sets of screens that configure these technologies by using the Process Type pull-down menu at the bottom of the screen.

\begin{tabular}{|l|l||}
\hline Process Type: & Hot-Side SCR \\
& In-Furnace Controls \\
& Hot-Side SCR \\
\hline \hline
\end{tabular}

The Process Type pull-down menu 


\section{Hot-Side SCR Configuration}

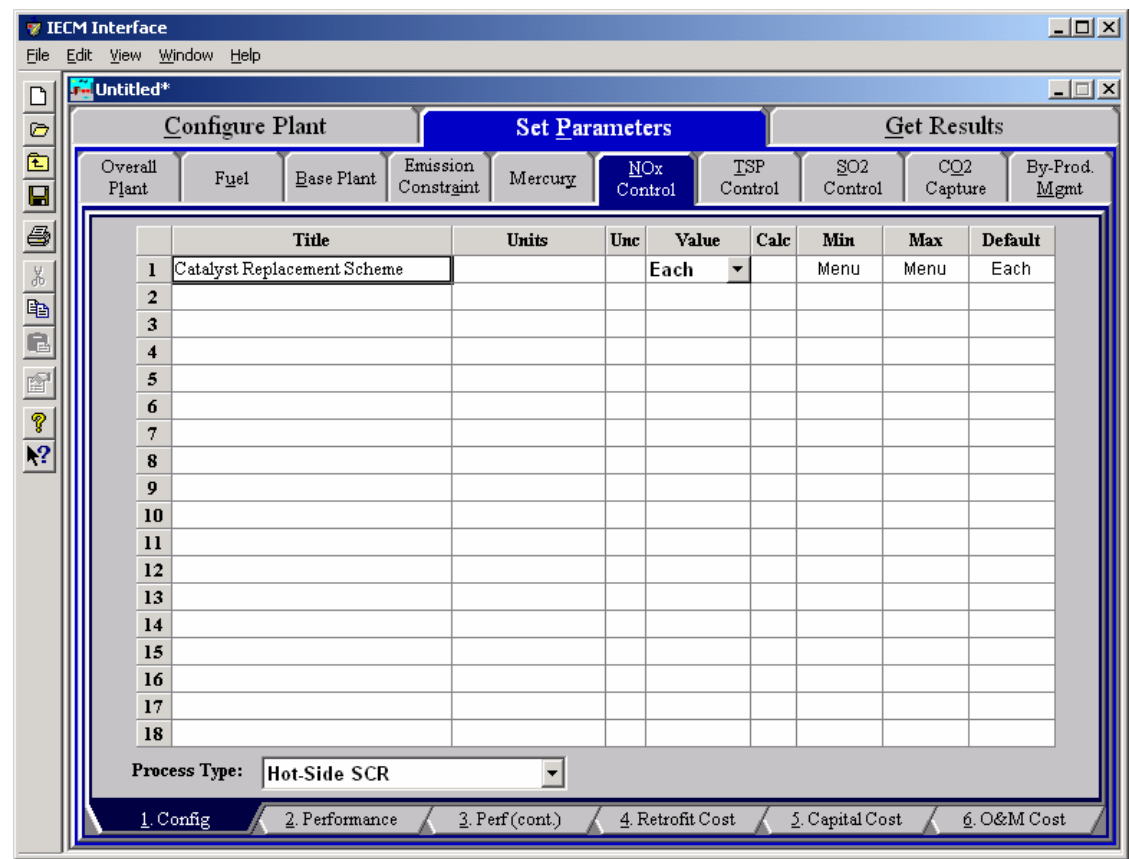

Hot-Side SCR - Config. input screen.

Inputs for configuring the Hot-Side SCR $\mathrm{NO}_{\mathrm{x}}$ Control technology are entered on the Config input screen. Each parameter is described briefly below.

Catalyst Replacement Scheme: Catalyst is installed in the SCR as a series of layers. These activity or effectiveness of these layers decreases with time due to fouling and poisoning. The layers are replaced with clean layers on a regular basis in one of two ways: all at once or one layer at time (staggered). The selection of the replacement scheme involves trade-offs between capital and annual costs via the initial catalyst requirement and the replacement interval. More specifically:

Each - Individual Layers. Replacing individual layers sequentially, rather than simultaneously, increases the effective catalyst life for a given volume of catalyst, decreasing the replacement interval. This reduces the O\&M cost relative to simultaneous replacement.The default setting is Each.

All - All Layers: Simultaneous replacement may lead to a smaller initial catalyst volume to achieve the same design activity as a sequential replacement scheme. This reduces the capital cost but increases the O\&M cost. 


\section{Hot-Side SCR Performance Inputs}

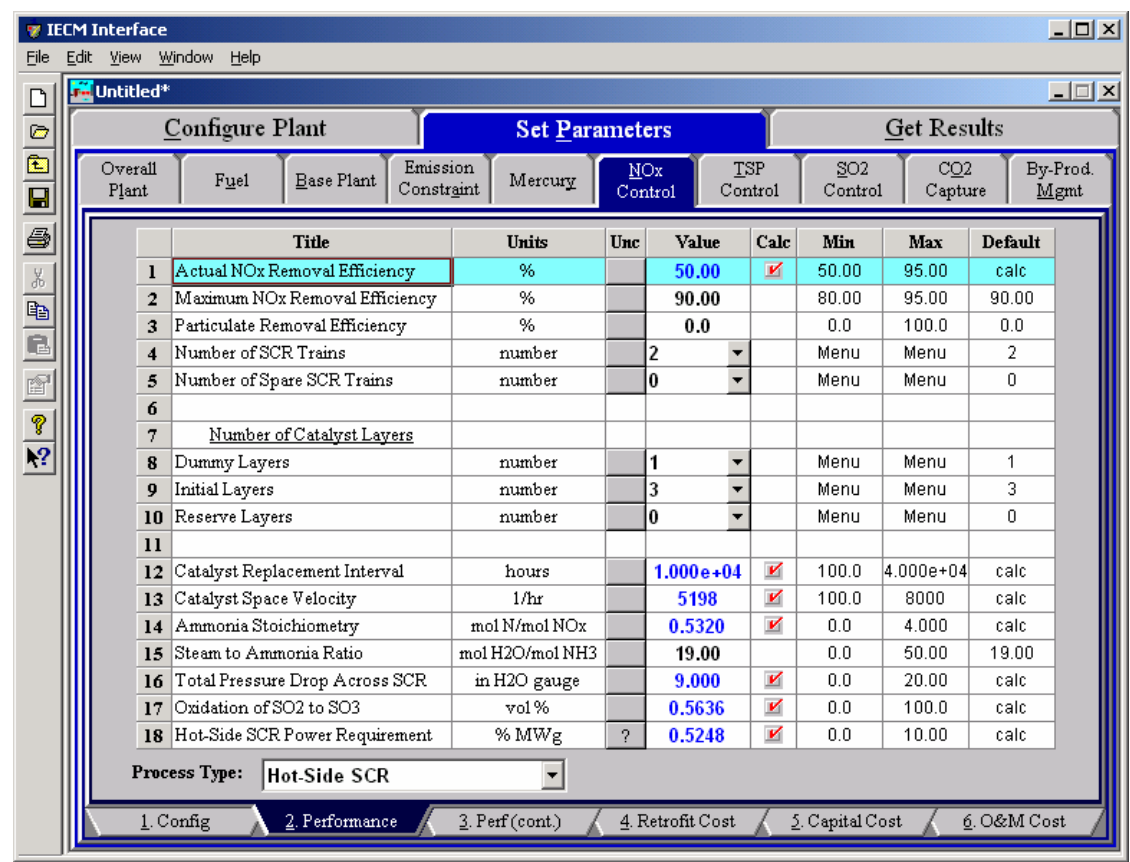

Hot-Side SCR - Performance input screen.

Inputs for the performance of the Hot-Side SCR $\mathrm{NO}_{\mathrm{x}}$ control technology are entered on the on the Performance input screen. Each parameter is described briefly below.

Actual $\mathrm{NO}_{\mathrm{x}}$ Removal Efficiency: The actual removal efficiency is dependent on the minimum and maximum removal efficiencies of the SCR and the emission constraint for $\mathrm{NO}_{\mathrm{x}}$. The model assumes a minimum removal of $50 \%$. The actual removal is set to match the constraint, if feasible. It is possible that the SCR may under or over comply with the emission constraint. This input is highlighted in blue.

Maximum $\mathrm{NO}_{\mathrm{x}}$ Removal Efficiency: This parameter specifies the maximum efficiency possible for the absorber on an annual average basis. The value is used as a limit in calculating the actual $\mathrm{NO}_{\mathrm{x}}$ removal efficiency for compliance.

Particulate Removal Efficiency: The ash in the high dust gas entering the SCR collects on the catalyst layers and causes fouling. Ash removal is not a design goal; rather, it is a reality which is taken into consideration by this parameter.

Number of SCR Trains: This is the total number of SCR equipment trains. It is used primarily to calculate the capital costs. The value must be an integer.

Number of Spare SCR Trains: This is the total number of spare SCR equipment trains. It is used primarily to calculate capital costs. The value must be an integer.

Number of Catalyst Layers: The total number of catalyst layers is a sum of the dummy, initial and spares used. All catalyst layer types are of equal dimensions, geometry, and catalyst formulation. You specify each value; the value must be an integer. The catalyst layer types and 
quantities are combined with pressure drop information to determine the auxiliary power requirements and the capital cost of the SCR technology. A layer may be interpreted as either a full layer (e.g., typically 1 meter deep), or a half layer (e.g., typically 0.5 meters deep) to represent alternative SCR catalyst replacement schemes. There is a limit of 8 total initial and reserve layers.

Dummy Layers: This is the number of dummy catalyst layers. The value must be an integer. A dummy layer corrects the flow distribution. It is used to calculate the total pressure drop across the SCR and the auxiliary power requirements.

Initial Layers: This is the number of initial active catalyst layers. The value must be an integer. Three layers are installed initially. It is used to calculate the total pressure drop across the SCR and the auxiliary power requirements.

Reserve Layers: This is the number of reserve or extra catalyst layers. These are available for later catalyst additions. The value must be an integer. It is used to calculate the total pressure drop across the SCR and the auxiliary power requirements.

Catalyst Replacement Interval: This parameter calculates the operating hour interval between catalyst replacements. The interval is determined by the decision to replace all at once or each of them separately after each interval. Currently, the model is not set up to replace two half layers simultaneously.

Catalyst Space Velocity: The calculated space velocity is determined by several factors, including many of the reference parameters in the next Section. The space velocity is used to determine the catalyst volume required.

Ammonia Stoichiometry: This is the molar stoichiometry ratio of ammonia to $\mathrm{NO}_{\mathrm{x}}$ entering the SCR device. The calculated quantity is based on an assumed $\mathrm{NO}_{\mathrm{x}}$ removal reaction stoichiometry of 1:1 for both $\mathrm{NO}$ and $\mathrm{NO}_{2}$, and a specified ammonia slip. It affects the amount of ammonia used and the amount of $\mathrm{NO}_{\mathrm{x}}$ converted to moisture.

Steam to Ammonia Ratio: The molar ratio of steam to ammonia is used to determine the amount of steam injected to vaporize the ammonia. The value assumes the steam is saturated at 450 degrees Fahrenheit and the ammonia is diluted to 5 volume percent of the injected gas.

Total Pressure Drop Across SCR: The total is determined from the individual pressure drops due to air preheater deposits, the active catalyst layers, the dummy catalyst layers, the ammonia injection system and the duct work. It is used to calculate the total pressure drop across the SCR and the auxiliary power requirements.

Oxidation of $\mathrm{SO}_{2}$ to $\mathrm{SO}_{3}$ : The oxidation rate is calculated for a high sulfur catalyst and affects the flue gas composition. It uses the space velocity and the inlet temperature. The $\mathrm{SO}_{3}$ produced acts as an ashconditioning agent if an ESP is used downstream.

SCR Energy Penalty: The default calculation of auxiliary power is based on the additional pressure drop, electricity to operate pumps and compressors, and equivalent energy for steam consumed. It is expressed as a percent of the gross plant capacity. 


\section{Hot-Side SCR Performance (Continued)}

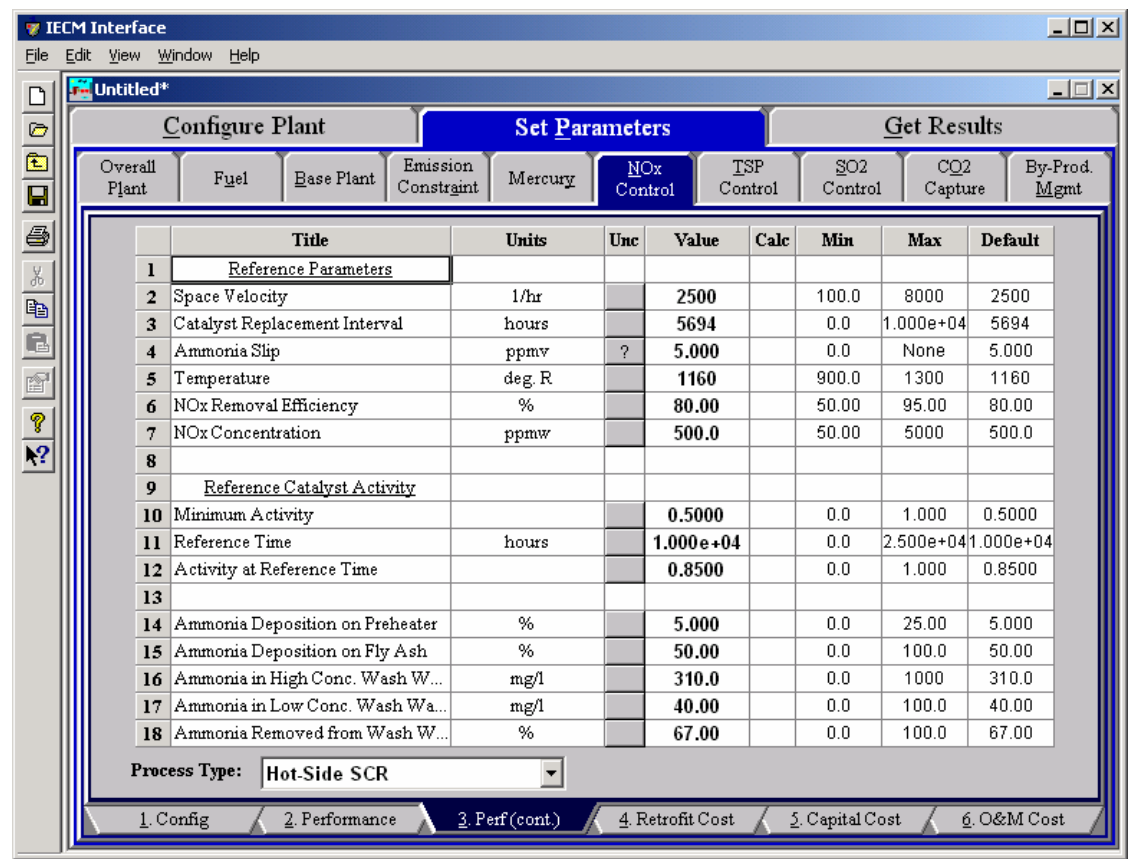

Hot-Side SCR - Perf.(cont.) input screen

The Hot-Side SCR system has additional inputs for performance entered on the Perf (Cont.) input screen. Many of the calculated quantities on the Performance screen are determined by the reference parameters described below.

\section{Reference Parameters}

The first set of reference parameters is primarily used to determine the actual space velocity. The values are used with actual operating conditions through a series of correction factors in the IECM. If you set the actual space velocity displayed on the Performance screen, this set of input parameters is not used by the IECM and does not have to be set.

Space Velocity: This is the reference space velocity for a high dust system. It is used to calculate the actual space velocity.

Catalyst Replacement Interval: This is the reference operating life in hours associated with the reference space velocity for the high dust catalyst. It is used to calculate the actual space velocity.

Ammonia Slip: Ammonia slip accounts for the ammonia passing through the reactor unchanged and further downstream. The value is based on an 80 percent or lower $\mathrm{NO}_{\mathrm{x}}$ removal efficiency. It is used in calculating the ammonia stoichiometry and actual space velocity.

Temperature: This is the operating temperature associated with the reference space velocity. It is used to determine the actual space velocity.

$\mathrm{NO}_{\mathrm{x}}$ Removal Efficiency: This is the $\mathrm{NO}_{\mathrm{x}}$ removal efficiency associated with the reference design specifications for the SCR system. It is used to determine the actual space velocity. 
$\mathrm{NO}_{\mathrm{x}}$ Concentration: This is the inlet $\mathrm{NO}_{\mathrm{x}}$ concentration associated with the reference design specifications for the SCR system. It is used to determine the actual space velocity

\section{Reference Catalyst Activity}

Catalyst activity decreases with operating time due to plugging and catalyst poisoning. The loss is a complex function of the catalyst formulation and geometry, the operating conditions associated with the flue gas, including temperature and composition, and the loading and composition of the fly ash. This complex function is represented by an exponential decay formula in the IECM. The following parameters are used to determine the reference catalyst activity, assuming the initial activity has a value of unity:

Minimum Activity: The minimum activity is a lower limit for catalyst activity decay. The actual activity approaches this value over a long period of time.

Reference Time: This is the time that corresponds to a particular activity known for the catalyst. It is used to determine a decay rate constant.

Activity at Reference Time: A second activity reference point is needed to determine the activity decay rate. The activity should correspond to the reference time specified. It is used to determine a decay rate constant.

Ammonia Deposition on Preheater: This is the percent of the ammonia slip that is deposited as ammonium salts in the air preheater. It is treated like a partition coefficient.

\section{Ammonia Parameters}

Ammonia Deposition on Fly Ash: This is the percent of the ammonia slip that is absorbed onto the fly ash. It is treated like a partition coefficient. This is important for high dust systems.

Ammonia in High Conc. Wash Water: The ammonia that deposits in the air preheater is periodically removed by washing. It is initially highly concentrated and requires denitrification pretreatment prior to regular treatment. This is the average concentration in that stream.

Ammonia in Low Conc. Wash Water: The ammonia that deposits in the air preheater is periodically removed by washing. The concentration is initially high, but gradually decreases. This is the average concentration of the low concentration stream.

Ammonia Removed from Wash Water: The ammonia that deposits in the air preheater is periodically removed by washing. This is the average amount of ammonia removed from the high and low concentrated streams. 


\section{Hot-Side SCR Retrofit Cost}

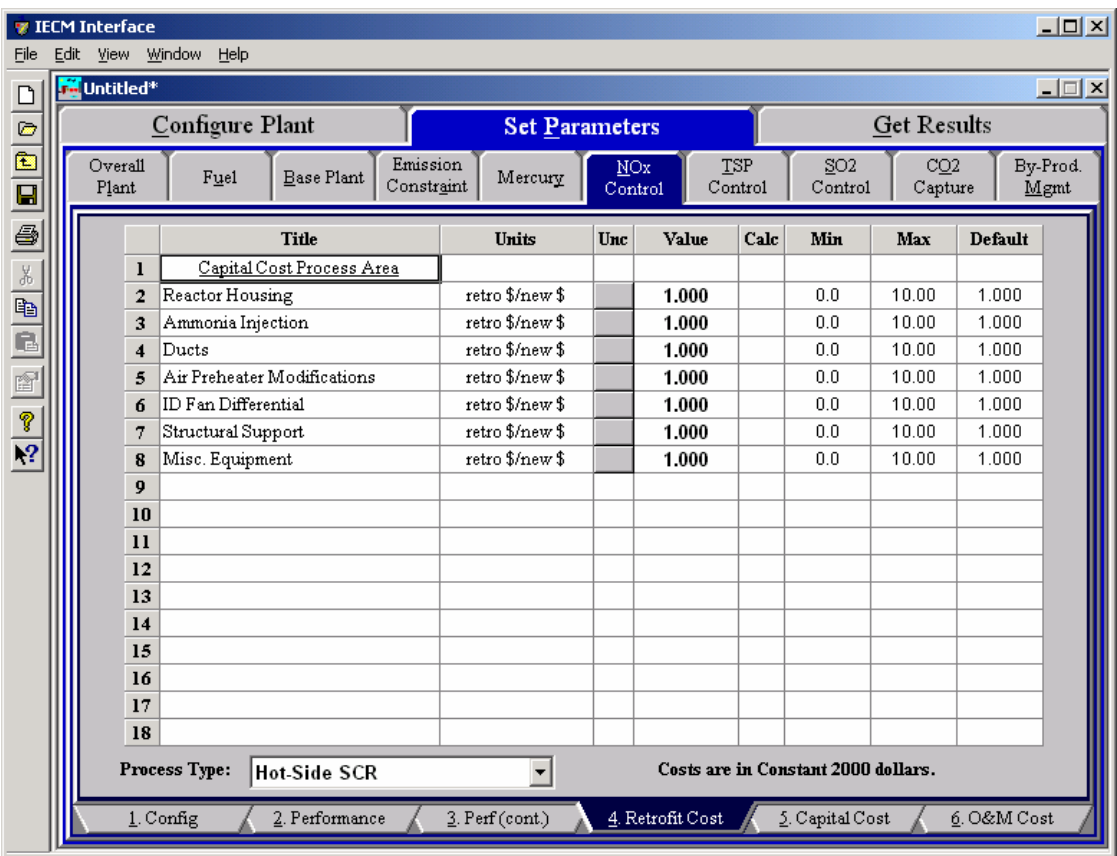

Hot-Side SCR - Retrofit Cost input screen.

The Hot-Side SCR system has inputs for the capital costs of modifications to process areas necessary to implement the technology entered on the Retrofit Cost input screen.

The retrofit cost factor of each process is a multiplicative cost adjustment, which considers the cost of retrofitted capital equipment relative to similar equipment installed in a new plant. These factors affect the capital costs directly and the operating and maintenance costs indirectly.

Direct capital costs for each process area are calculated in the IECM. These calculations are reduced form equations derived from more sophisticated models and reports. The sum of the direct capital costs associated with each process area is defined as the process facilities capital (PFC). The retrofit cost factor provided for each of the process areas can be used as a tool for adjusting the anticipated costs and uncertainties across the process area separate from the other areas.

Uncertainty can be applied to the retrofit cost factor for each process area in each technology. Thus, uncertainty can be applied as a general factor across an entire process area, rather than as a specific uncertainty for the particular cost on the capital or O\&M input screens. Any uncertainty applied to a process area through the retrofit cost factor compounds any uncertainties specified later in the capital and O\&M cost input parameter screens. Each parameter is described briefly below.

\section{Capital Cost Process Area}

Reactor Housing: The reactor housing costs include carbon steel reactor vessel with six inches of mineral wool insulation, vessel internals and supports, steam sootblowers, reactor crane and hoist, installation, labor, foundations, structures, piping, and electrical equipment.

Ammonia Injection: The ammonia unloading, storage, and supply system includes a storage vessel with a seven day capacity, an ammonia 
vaporizer, mixer, injection grid, ductwork, dampers, and a truck unloading station.

Ducts: The ductwork includes economizer bypass and outlet ducts, SCR inlet and outlet ducts, SCR and economizer control dampers, air preheater inlet plenum, various expansion joints in the ductwork, and air preheater cross-over ducting.

Air Preheater Modifications: Thicker and smoother material is used for the heat transfer surfaces in the preheater. A larger motor is provided for the heat exchanger. High pressure steam soot blowers and water wash spray nozzles are also added.

ID Fan Differential: The ID fans must be sized to deal with the increased flue gas pressure drop resulting from the additional ductwork and the SCR reactor.

Structural Support: The costs of this area are related primarily to the structural support required for the SCR reactor housing, ductwork, and air preheater.

Misc. Equipment: This area includes the capital costs incurred for ash handling addition, water treatment addition, and flow modeling for a hot-side SCR system.

\section{Hot-Side SCR Capital Cost Inputs}

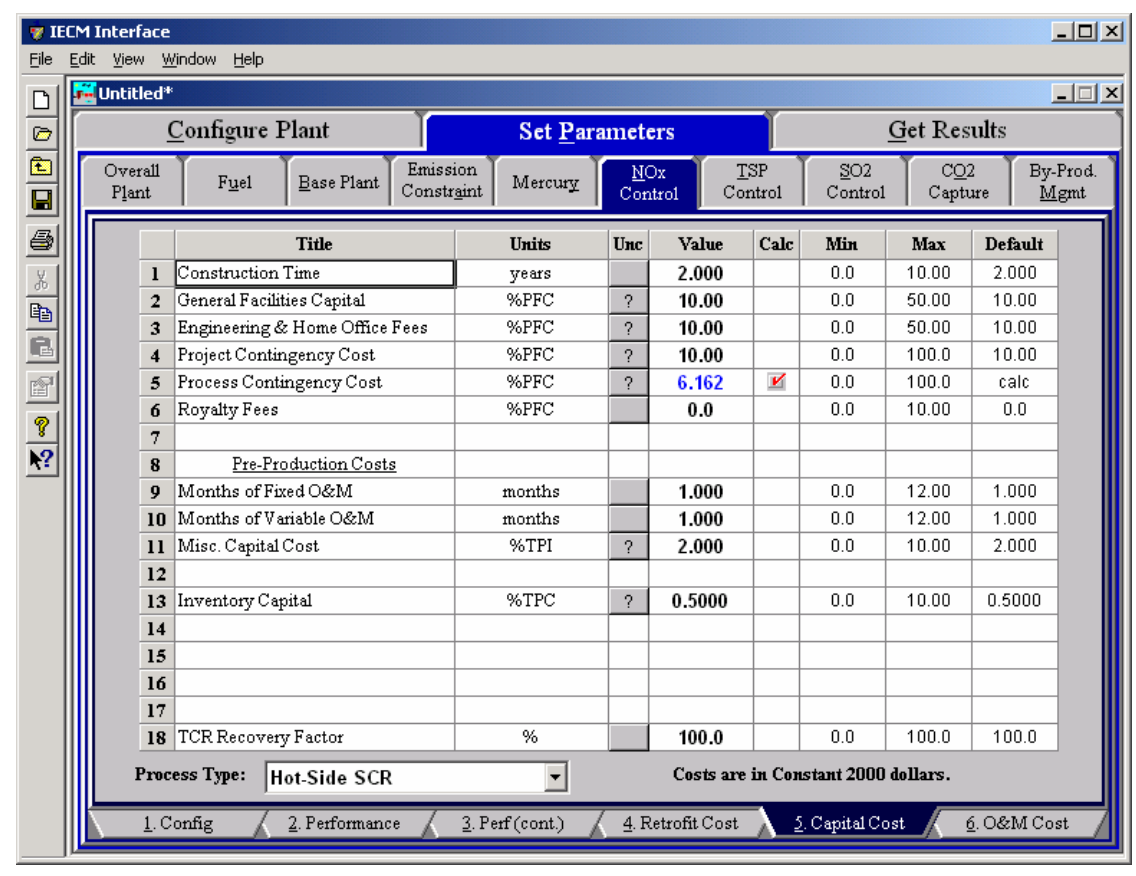

Hot-Side SCR - Capital Cost input screen.

Inputs for the capital costs of the Hot-Side SCR $\mathrm{NO}_{\mathrm{x}}$ control technology are entered on the Capital Cost screen for the Hot-Side SCR, and the Capital Cost input screen for In-Furnace Controls. Each parameter is described briefly below.

Construction Time: This is the idealized construction period in years. It is used to determine the allowance for funds used during construction (AFUDC). 
General Facilities Capital (GFC): The general facilities include construction costs of roads, office buildings, shops, laboratories, etc. Sales taxes and freight costs are included implicitly. The cost typically ranges from $5-20 \%$.

Engineering \& Home Office Fees: The engineering \& home office fees are a percent of total direct capital cost. This is an overhead fee paid to the architect/engineering company. These fees typically range from 7$15 \%$.

Project Contingency Cost: This is factor covering the cost of additional equipment or other costs resulting from a more detailed design. Higher contingency factors will be applied to simplified or preliminary designs and lower factors to detailed or finalized designs.

Process Contingency Cost: This quantifies the design uncertainty and cost of a commercial-scale system. This is generally applied on an areaby-area basis. Higher contingency factors are applied to new regeneration systems tested at a pilot plant and lower factors to full-size or commercial systems.

Royalty Fees: Royalty charges may apply to some portions of generating units incorporating new proprietary technologies.

Pre-Production Costs: These costs consider the operator training, equipment checkout, major changes in unit equipment, extra maintenance, and inefficient use of fuel or other materials during startup. These are typically applied to the O\&M costs over a specified period of time (months). The two time periods for fixed and variable O\&M costs are described below with the addition of a miscellaneous capital cost factor.

Months of Fixed O\&M: Time period of fixed operating costs used for preproduction to cover training, testing, major changes in equipment, and inefficiencies in start-up. This includes operating, maintenance, administrative and support labor. It also considers maintenance materials.

Months of Variable O\&M: Time period of variable operating costs used for preproduction to cover chemicals, water, consumables, and solid disposal charges in start-up, assuming 100\% load. This excludes any fuels.

Misc. Capital Costs: This is a percent of total plant investment (sum of TPC and AFUDC) to cover expected changes to equipment to bring the system up to full capacity.

Inventory Capital: Percent of the total direct capital for raw material supply based on $100 \%$ capacity during a 60 day period. These materials are considered storage. The inventory capital includes fuels, consumables, by-products, and spare parts. This is typically $0.5 \%$.

TCR Recovery Factor: The actual total capital required (TCR) as a percent of the TCR in a new power plant. This value is $100 \%$ for a new installation and may be set as low as $0 \%$ for a hot-side SCR that has been paid off. 


\section{Hot-Side SCR O\&M Cost Inputs}

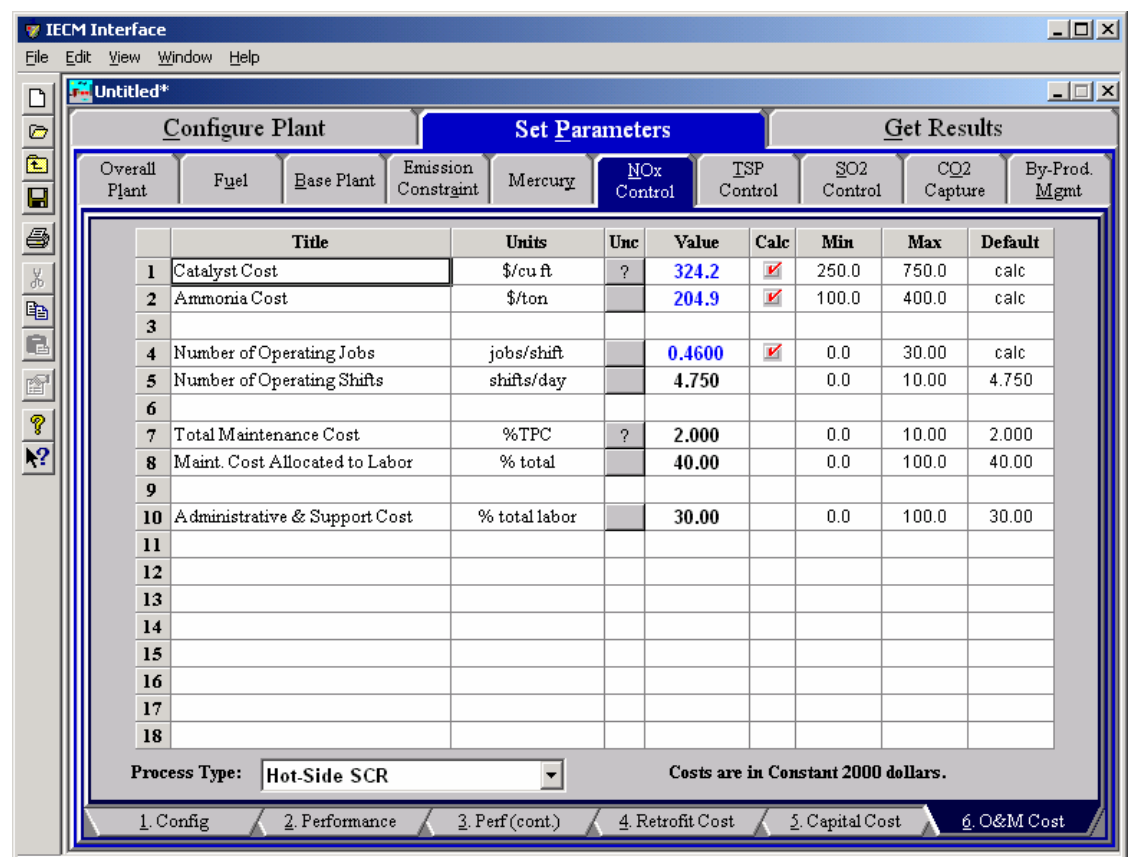

Hot-Side SCR - O\&M Cost input screen.

Inputs for the operation and maintenance costs of the Hot-Side SCR $\mathrm{NO}_{\mathrm{x}}$ control technology are entered on the $\mathbf{O} \mathbf{\&}$ Cost input screen. Each parameter is described briefly below.

Catalyst Cost: This is the cost of the catalyst used for the SCR technology.

Ammonia Cost: This is the cost of the ammonia used for the SCR technology.

Number of Operating Jobs: This is the total number of operating jobs that are required to operate the plant per eight-hour shift.

Number of Operating Shifts: This is the total number of equivalent operating shifts in the plant per day. The number takes into consideration paid time off and weekend work ( 3 shifts/day * 7 days/5 day week $* 52$ weeks $/(52$ weeks -6 weeks PTO $)=4.75$ equiv. Shifts/day)

Total Maintenance Cost: This is the annual maintenance cost as a percentage of the total plant cost. Maintenance cost estimates can be developed separately for each process area.

Maint. Cost Allocated to Labor: Maintenance cost allocated to labor as a percentage of the total maintenance cost.

Administrative \& Support Cost: This is the percent of the total operating and maintenance labor associated with administrative and support labor. 


\section{Hot-Side SCR Diagram}

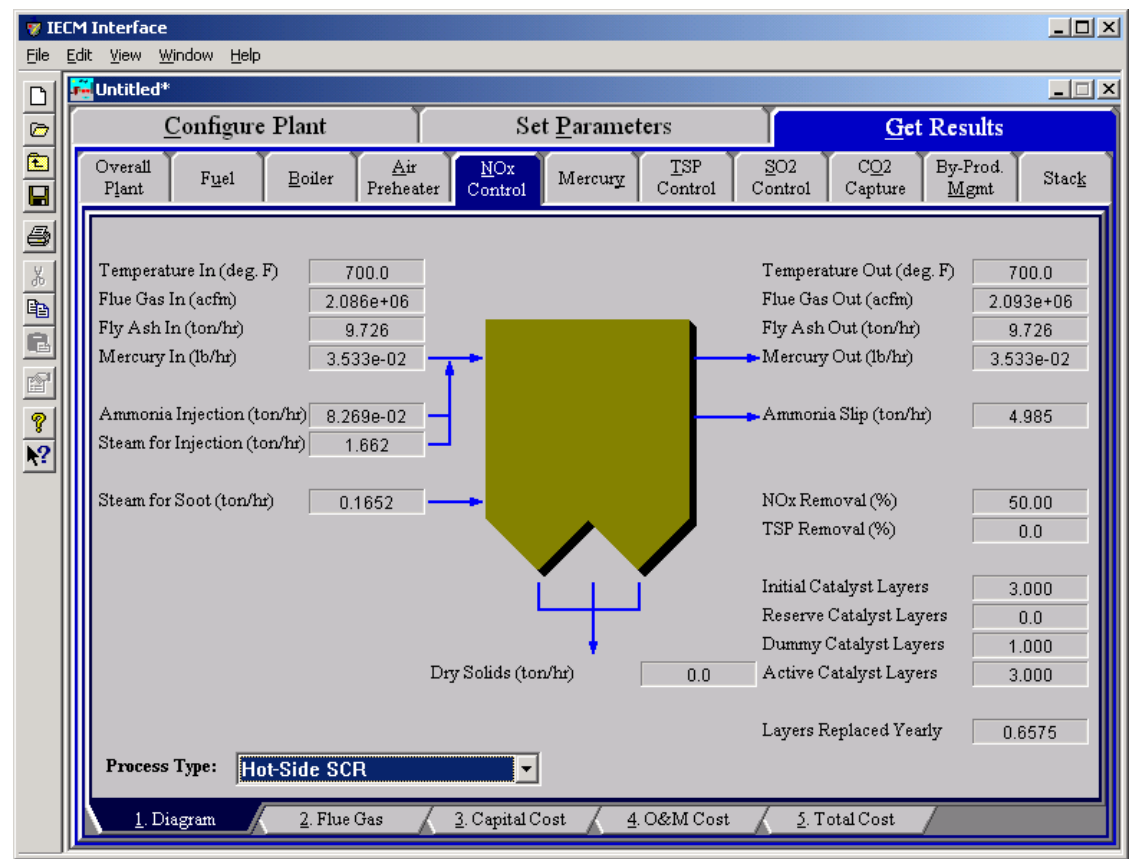

Hot-Side SCR - Diagram result screen.

The Diagram result screen displays an icon for the Hot-Side SCR $\mathrm{NO}_{x}$ technology selected and values for major flows in and out of it.

\section{Reagent}

Ammonia Injection: The total mass flow rate of ammonia injected into the SCR. This is a function of the $\mathrm{NO}_{\mathrm{x}}$ concentration in the flue gas and the ammonia stoichiometric performance input value.

Steam for Injection: The total mass flow rate of steam into the SCR. This is the amount of steam added to the SCR to vaporize and transport ammonia into the inlet gas stream. This is determined by the steam to ammonia ratio input value and the ammonia injection.

\section{Catalyst}

Steam for Soot: This is the amount of steam blown into the hot-side SCR to remove soot buildup on the catalyst layers. The soot blowing steam is assumed to be directly proportional to catalyst volume.

Initial Catalyst Layers: This is the number of initial active catalyst layers. Three layers are installed initially. It is used to calculate the total pressure drop across the SCR and the auxiliary power requirements. This is set by the input parameter.

Reserve Catalyst Layers: This is the number of reserve or extra catalyst layers. These are available for later catalyst additions. It is used to calculate the total pressure drop across the SCR and the auxiliary power requirements. This is set by the input parameter.

Dummy Catalyst Layers: This is the number of dummy catalyst layers. A dummy layer corrects the flow distribution. It is used to calculate the total pressure drop across the SCR and the auxiliary power requirements. This is set by the input parameter. 
Active Catalyst Layers: This is the number of initial active catalyst layers. Three layers are installed initially. It is used to calculate the total pressure drop across the SCR and the auxiliary power requirements. It is equal to the number of initial and reserve catalyst layers.

Layers Replaced Yearly: Average catalyst layer replacement rate per year. This assumes that all catalyst layers are of equal depth.

\section{Flue Gas Entering SCR}

Temperature In: Temperature of the flue gas entering the SCR. This is determined by the flue gas outlet temperature of the module upstream of the SCR (e.g., the boiler economizer)

Flue Gas In: Volumetric flow rate of flue gas entering the SCR, based on the flue gas temperature entering the SCR and atmospheric pressure.

Fly Ash In: Total solids mass flow rate in the flue gas entering the SCR. This is determined by the solids exiting from the module upstream of the SCR (e.g., the boiler economizer).

Mercury In: Total mass of mercury entering the hot-side SCR in the flue gas. The value is a sum of all the forms of mercury (elemental, oxidized, and particulate).

\section{Flue Gas Exiting SCR}

Temperature Out: Temperature of the flue gas exiting the SCR. The model currently does not alter this temperature through the SCR.

Flue Gas Out: Volumetric flow rate of the flue gas exiting the SCR, based on the flue gas temperature exiting the SCR and atmospheric pressure.

Fly Ash Out: Total solids mass flow rate in the flue gas exiting the SCR. This is a function of the ash removal parameter on the SCR performance input screen.

Ammonia Slip: Total mass flow rate of ammonia that is unreacted and exits the SCR in the flue gas stream. This is a function if the ammonia injection flow rate, $\mathrm{NO}_{\mathrm{x}}$ concentration in the flue gas, and $\mathrm{NO}_{\mathrm{x}}$ removal efficiency.

Mercury Out: Total mass of mercury exiting the hot-side SCR in the flue gas. The value is a sum of all the forms of mercury (elemental, oxidized, and particulate).

\section{SCR Performance}

$\mathrm{NO}_{\mathrm{x}}$ Removal: Actual removal efficiency of $\mathrm{NO}_{\mathrm{x}}$ in the SCR. This is a function of the minimum (50\%) and maximum removal efficiencies (SCR performance input parameter) and the emission constraint for $\mathrm{NO}_{\mathrm{x}}$ (emission constraints input parameter). It is possible that the SCR may over or under-comply with the emission constraint.

TSP Removal: Actual particulate removal efficiency in the SCR. This is set by the SCR input parameter.

\section{Collected Solids}

Dry Solids: Total solids mass flow rate of solids removed from the SCR. This is a function of the solids content in the flue gas and the particulate removal efficiency of the SCR. 


\section{Hot-Side SCR Flue Gas Results}

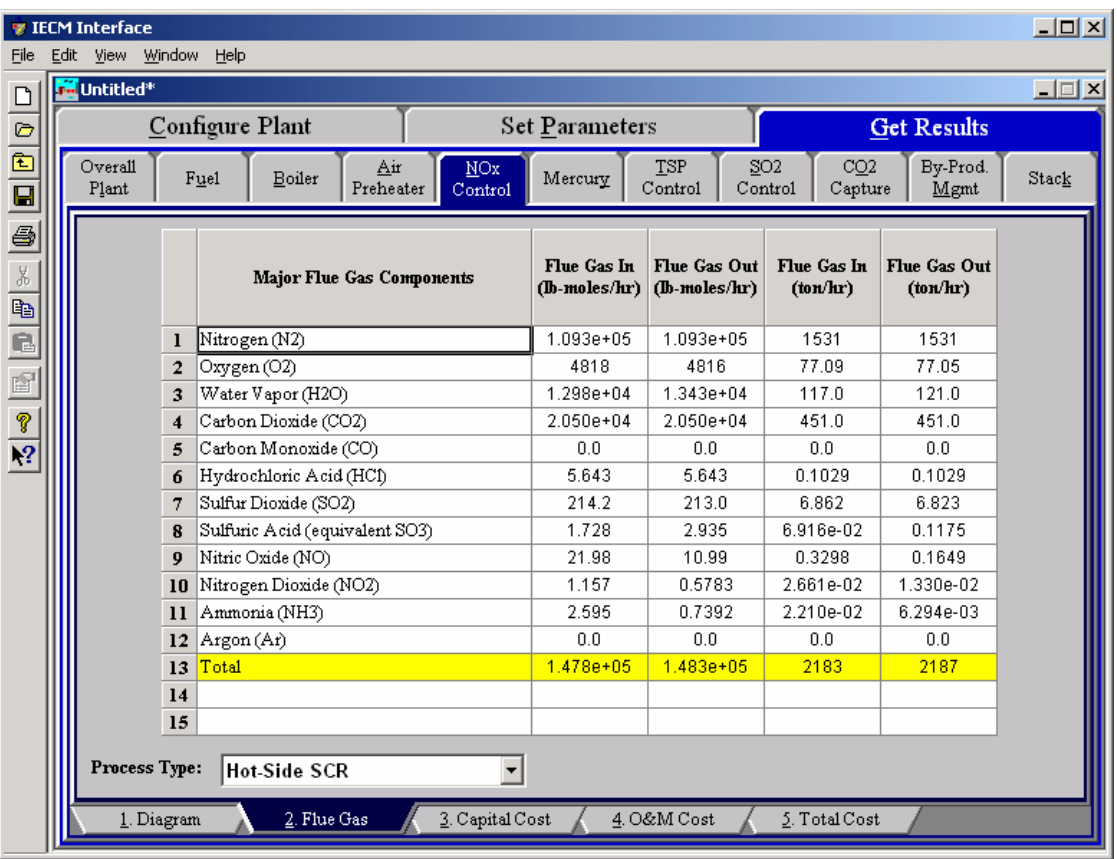

Hot-Side SCR - Flue Gas result screen.

\section{Major Flue Gas Components}

The Flue Gas result screen for the Hot-Side SCR displays a table of quantities of flue gas components entering and exiting the SCR. For each component, quantities are given in both moles and mass per hour. Each result is described briefly below.

Nitrogen $\left(\mathrm{N}_{2}\right)$ : Total mass of nitrogen.

Oxygen $\left(\mathrm{O}_{2}\right)$ : Total mass of oxygen.

Water Vapor $\left(\mathrm{H}_{2} \mathrm{O}\right)$ : Total mass of water vapor.

Carbon Dioxide $\left(\mathrm{CO}_{2}\right)$ : Total mass of carbon dioxide.

Carbon Monoxide (CO): Total mass of carbon monoxide.

Hydrochloric Acid (HCl): Total mass of hydrochloric acid.

Sulfur Dioxide $\left(\mathrm{SO}_{2}\right)$ : Total mass of sulfur dioxide.

Sulfuric Acid (equivalent $\mathbf{S O}_{3}$ ): Total mass of sulfuric acid.

Nitric Oxide (NO): Total mass of nitric oxide.

Nitrogen Dioxide $\left(\mathrm{NO}_{2}\right)$ : Total mass of nitrogen dioxide.

Ammonia $\left(\mathrm{NH}_{3}\right)$ : Total mass of ammonia.

Argon (Ar): Total mass of argon

Total: Total of the individual components listed above. This item is

highlighted in yellow. 


\section{Hot-Side SCR Capital Cost Results}

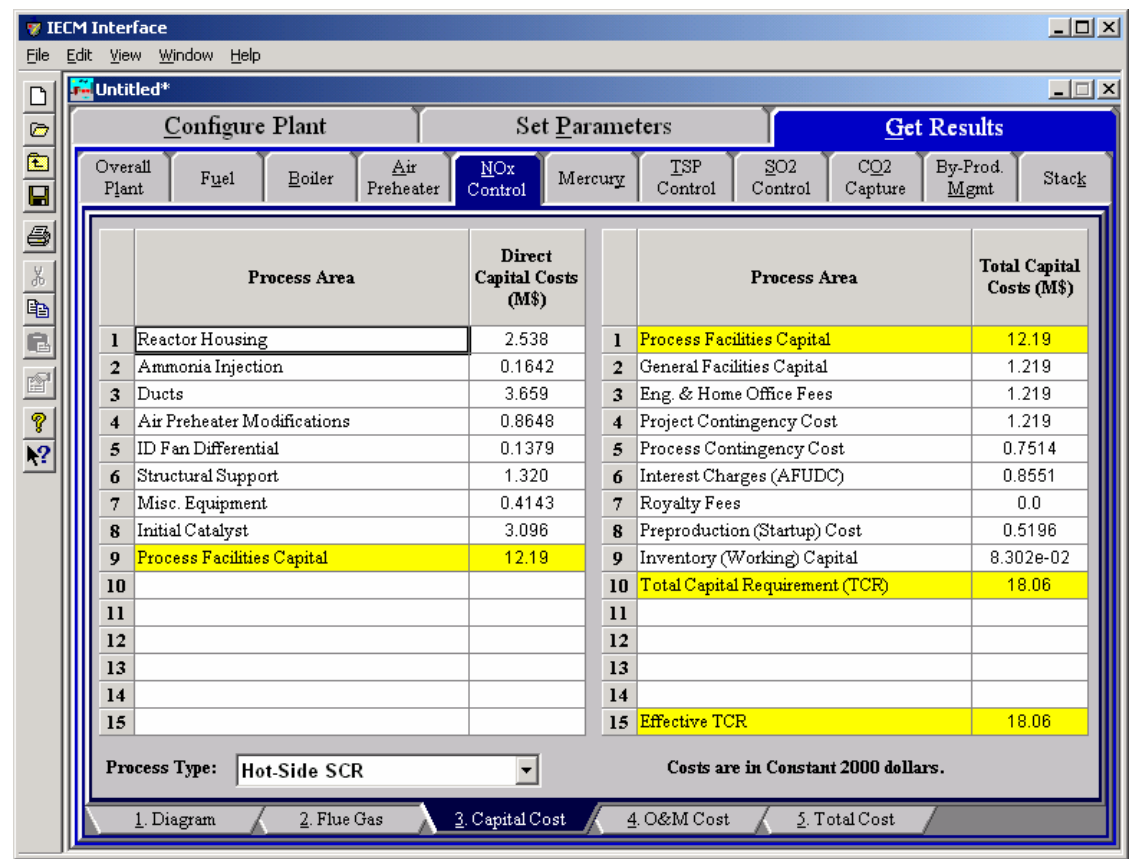

Hot-Side SCR - Capital Cost result screen.

The Capital Cost result screen displays tables for the direct and indirect capital costs related to the Hot-Side SCR NO described briefly below:

\section{Direct Capital Costs}

Each process area direct capital cost is a reduced-form model based on regression analysis of data collected from several reports and analyses of hot-side SCR units. They are described in general with specific model parameters that effect them described in particular.

Reactor Housing: The reactor housing costs include carbon steel reactor vessel with six inches of mineral wool insulation, vessel internals and supports, steam soot blowers, reactor crane and hoist, installation labor, foundations, structures, piping, and electrical equipment. The costs are a function of the number of vessels, including spares, and the volume of catalyst required. Catalyst costs are excluded.

Ammonia Injection: The ammonia unloading, storage, and supply system includes a storage vessel with a seven day capacity, an ammonia vaporizer, mixer, injection grid, ductwork, dampers, and a truck unloading station. The costs are a function of the ammonia injected.

Ducts: The ductwork includes economizer bypass and outlet ducts, SCR inlet and outlet ducts, SCR and economizer control dampers, air preheater inlet plenum, various expansion joints in the ductwork, and air preheater cross-over ducting. The costs are a function of the flue gas flow rate through the SCR.

Air Preheater Modifications: Thicker and smoother material is used for the heat transfer surfaces in the preheater. A larger motor is provided for the heat exchanger. High pressure steam soot blowers and water wash spray nozzles are also added. The costs are a function of the 
number of operating vessels, and the heat transfer efficiency of the air preheater (UA product).

ID Fan Differential: The ID fans must be sized to deal with the increased flue gas pressure drop resulting from the additional ductwork and the SCR reactor. The costs are a function of the flue gas flow rate and pressure drop across the SCR.

Structural Support: The costs of this area are related primarily to the structural support required for the SCR reactor housing, ductwork, and air preheater. The costs are a function of the reactor housing costs, duct costs and air preheater modification costs above.

Misc. Equipment: This area includes the capital costs incurred for ash handling addition, water treatment addition, and flow modeling for a hot-side SCR system. The costs are a function of the gross plant capacity.

Initial Catalyst: The cost of the initial catalyst charge is included in the total direct cost, because it is such a large and integral part of the SCR system. The costs are a function of the initial catalyst charge.

Process Facilities Capital: The process facilities capital is the total constructed cost of all on-site processing and generating units listed above, including all direct and indirect construction costs. All sales taxes and freight costs are included where applicable implicitly. This result is highlighted in yellow.

\section{Total Capital Costs}

Process Facilities Capital: (see definition above)

General Facilities Capital: The general facilities include construction costs of roads, office buildings, shops, laboratories, etc. Sales taxes and freight costs are included implicitly.

Eng. \& Home Office Fees: The engineering \& home office fees are a percent of total direct capital cost. This is an overhead fee paid to the architect/engineering company.

Project Contingency Cost: Capital cost contingency factor covering the cost of additional equipment or other costs that would result from a more detailed design of a definitive project at the actual site.

Process Contingency Cost: Capital cost contingency factor applied to a new technology in an effort to quantify the uncertainty in the technical performance and cost of the commercial-scale equipment.

Interest Charges (AFUDC): Allowance for funds used during construction, also referred to as interest during construction, is the time value of the money used during construction and is based on an interest rate equal to the before-tax weighted cost of capital. This interest is compounded on an annual basis (end of year) during the construction period for all funds spent during the year or previous years.

Royalty Fees: Royalty charges may apply to some portions of generating units incorporating new proprietary technologies.

Preproduction (Startup) Cost: These costs consider the operator training, equipment checkout, major changes in unit equipment, extra maintenance, and inefficient use of fuel or other materials during startup. 
Inventory (Working) Capital: The raw material supply based on $100 \%$ capacity during a 60 day period. These materials are considered storage. The inventory capital includes fuels, consumables, byproducts, and spare parts.

Total Capital Requirement (TCR): Money that is placed (capitalized) on the books of the utility on the service date. TCR includes all the items above. This result is highlighted in yellow.

Effective TCR: The TCR of the hot-side SCR that is used in determining the total power plant cost. The effective TCR is determined by the “TCR Recovery Factor” for the hot-side SCR.

\section{Hot-Side SCR O\&M Cost Results}

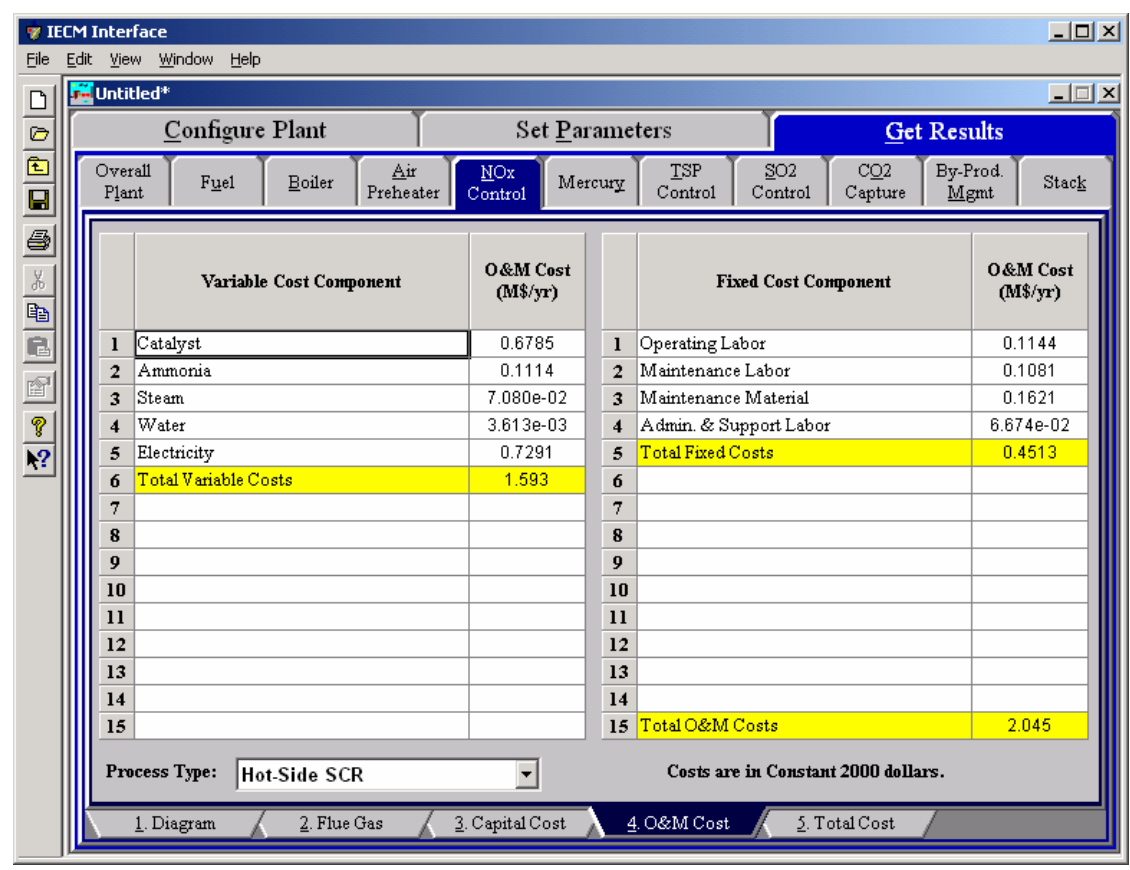

Hot-Side SCR - O\&M Cost result screen.

The O\&M Cost result screen displays tables for the variable and fixed operation and maintenance costs involved with the Hot Side SCR $\mathrm{NO}_{\mathrm{x}}$ control technology. Each result is described briefly below:

\section{Variable Cost Components}

Variable operating costs and consumables are directly proportional to the amount of kilowatts produced and are referred to as incremental costs. All the costs are subject to inflation.

Catalyst: Replacement catalyst cost per year for the hot-side SCR. This is a function of the number of catalyst layers, the number of layers replaced each year, and the catalyst space velocity (all three are performance input parameters).

Ammonia: Ammonia reagent cost per year for the hot-side SCR. This is a function of the concentration of $\mathrm{NO}_{\mathrm{x}}$ in the flue gas and the ammonia mass flow rate. 
Steam: Annual cost of steam used for ammonia vaporization and ammonia injection. This is a function of the steam to ammonia ratio (performance input parameter) and the ammonia mass flow rate.

Water: Cost of water used to wash ammonia that deposits in the air preheater. This is a function of the efficiency and concentration of ammonia removed by wash water performance input parameters and the amount of ammonia salts deposited on the air preheater.

Electricity: Cost of electricity consumption of the hot-side SCR. This is a function of the gross plant capacity and the SCR energy penalty performance input parameter.

Total Variable Costs: This is the sum of all the variable O\&M costs listed above. This result is highlighted in yellow.

\section{Fixed Cost Components}

Fixed operating costs are essentially independent of actual capacity factor, number of hours of operation, or amount of kilowatts produced. All the costs are subject to inflation.

Operating Labor: Operating labor cost is based on the operating labor rate, the number of personnel required to operate the plant per eighthour shift, and the average number of shifts per day over 40 hours per week and 52 weeks.

Maintenance Labor: The maintenance labor is determined as a fraction of the total maintenance cost.

Maintenance Material: The cost of maintenance material is the remainder of the total maintenance cost, considering the fraction associated with maintenance labor.

Admin. \& Support Labor: The administrative and support labor is the only overhead charge. It is taken as a fraction of the total operating and maintenance labor costs.

Total Fixed Costs: This is the sum of all the fixed O\&M costs listed above. This result is highlighted in yellow.

Total O\&M Costs: This is the sum of the total variable and total fixed O\&M costs. It is used to determine the base plant total revenue requirement. This result is highlighted in yellow. 


\section{Hot-Side SCR Total Cost Results}

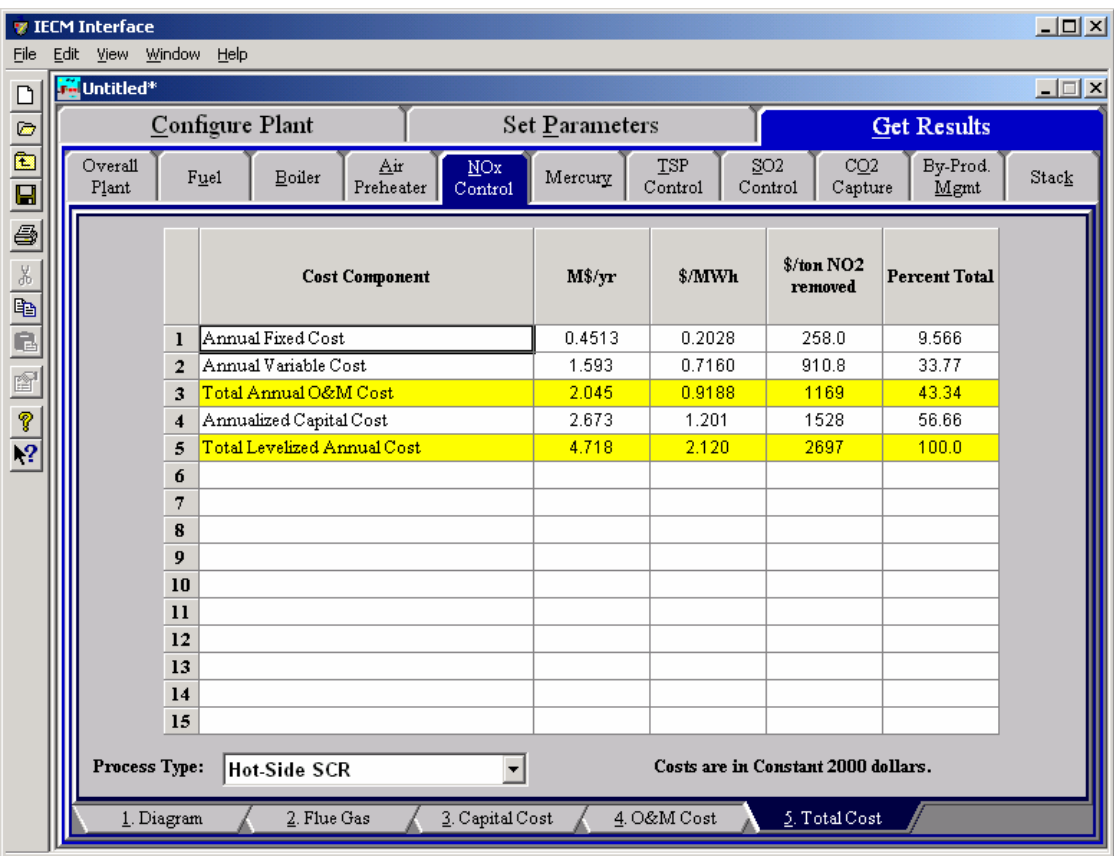

Hot-Side SCR - Total Cost result screen.

The Total Cost result screen displays a table which totals the annual fixed, variable, operations and maintenance, and capital costs associated with the Hot-Side SCR $\mathrm{NO}_{\mathrm{x}}$ Control technology. Note: that all costs expressed in \$/ton of $\mathrm{NO}_{2}$ removed assume tons of equivalent $\mathrm{NO}_{2}$. Each result is described briefly below.

\section{Cost Component}

Annual Fixed Cost: The operating and maintenance fixed costs are given as an annual total. This number includes all maintenance materials and all labor costs.

Annual Variable Cost: The operating and maintenance variables costs are given as an annual total. This includes all reagent, chemical, steam, and power costs.

Total Annual O\&M Cost: This is the sum of the annual fixed and variable operating and maintenance costs above. This result is highlighted in yellow.

Annualized Capital Cost: This is the total capital cost expressed on an annualized basis, taking into consideration the levelized carrying charge factor, or fixed charge factor, over the entire book life.

Total Levelized Annual Cost: The total annual cost is the sum of the total annual O\&M cost and annualized capital cost items above. This result is highlighted in yellow. 


\section{Mercury}

Mercury Control is a Technology Navigation Tab in the Set Parameters and in the Get Results program area. These screens define and display results for the performance and costs directly associated with the removal of mercury from each technology in the power plant Pre-combustion and post-combustion control technologies are all considered. Special consideration is given to flue gas conditioning used to enhance mercury removal. Water and activated carbon injection are currently considered as conditioning agents.

\section{Mercury Removal Efficiency Inputs}

Inputs for the removal of the speciated mercury from the flue gas stream are entered on the Removal Eff. input screen.

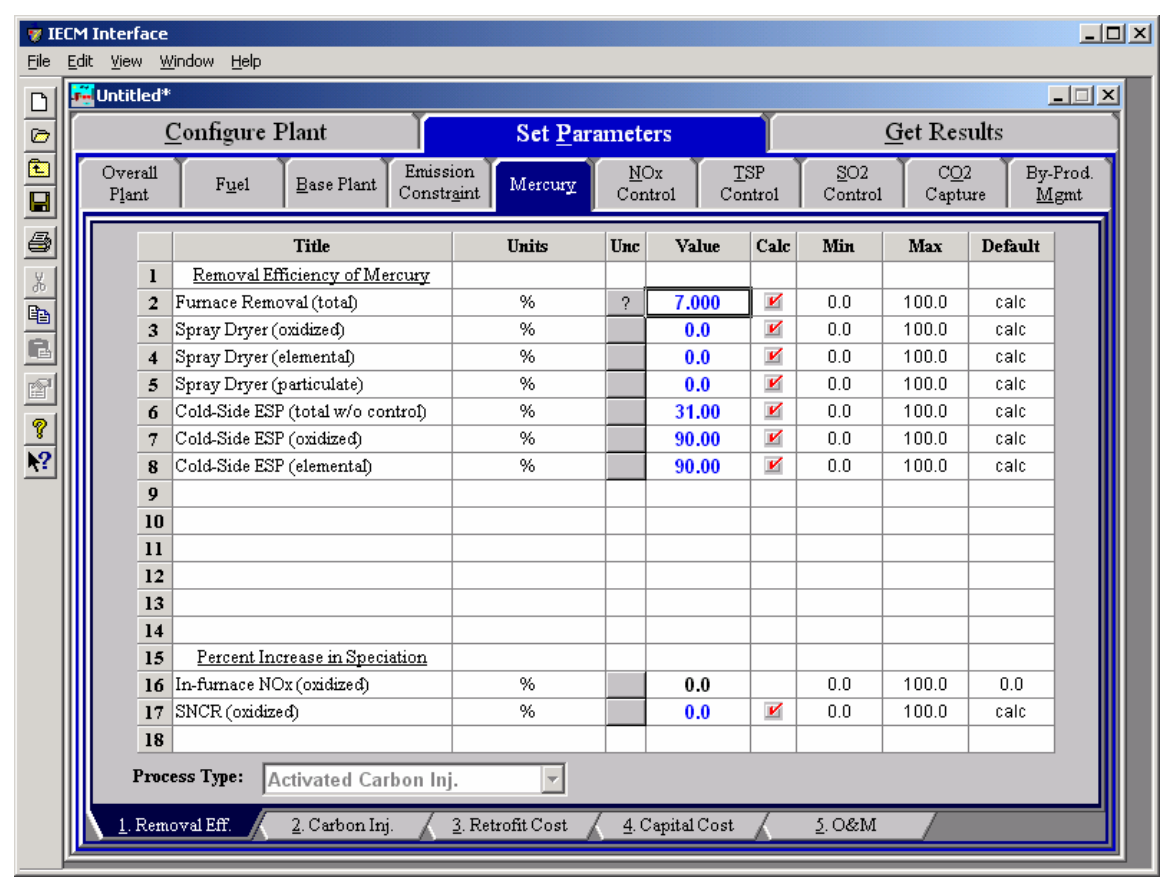

Mercury - Removal Efficiency input screen.

Each parameter is described briefly below.

\section{Removal Efficiency of Mercury}


The removal of mercury for each control technology configured is given as a percent of the total entering the control technology. The user is given the opportunity to specify the removal separately for each speciation type. Control technologies not currently configured are hidden.

Furnace Removal (total): Mercury present in ash is removed from the furnace through the removal of bottom ash. The speciation is not known, so the removal is specified as a total removal. The mercury removed in bottom ash is not credited toward the required removal to meet the mercury emission constraint.

\section{Fabric Filter}

Fabric Filter (total w/o control): Mercury present in ash is removed from the fabric filter through the removal of captured fly ash. The speciation is not known, so the removal is specified as a total removal. The value shown is determined without regard to particular mercury control methods. It has a substantial effect on the amount of activated carbon needed to meet the required removal of mercury.

Fabric Filter (oxidized): The fabric filter typically removes some mercury without adding a specific mercury control technology. This mercury is present in the ash and is removed with the collected ash. When a mercury control technology is added, the removal is enhanced. The default value is set to meet the overall removal efficiency constraint, with consideration given to the mercury removed by flue gas desulfurization and elemental mercury oxidized in a $\mathrm{NO}_{\mathrm{x}}$ control technology. The lower limit is set by the removal efficiency of ash alone as specified by "Fabric Filter (total w/o control)" specified above.

Fabric Filter (elemental): Elemental mercury is assumed to be removed with the same efficiency as the removal of oxidized mercury specified above.

\section{Cold - Side ESP}

Cold-Side ESP (total w/o control): Mercury present in ash is removed from the cold-side ESP through the removal of captured fly ash. The speciation is not known, so the removal is specified as a total removal. The value shown is determined without regard to particular mercury control methods. It has a substantial effect on the amount of activated carbon needed to meet the required removal of mercury.

Cold-Side ESP (oxidized): The cold-side ESP typically removes some mercury without adding a specific mercury control technology. This mercury is present in the ash and is removed with the collected ash. When a mercury control technology is added, the removal is enhanced. The default value is set to meet the overall removal efficiency constraint, with consideration given to the mercury removed by flue gas desulfurization and elemental mercury oxidized in a $\mathrm{NO}_{\mathrm{x}}$ control technology. The lower limit is set by the removal efficiency of ash alone as specified by "Cold-Side ESP (total w/o control)" specified above.

Cold-Side ESP (elemental): Elemental mercury is assumed to be removed with the same efficiency as the removal of oxidized mercury specified above.

\section{Wet FGD}


Wet FGD (oxidized): The wet lime/limestone FGD typically removes all the oxidized mercury due to its' high solubility in water.

Wet FGD (elemental): Elemental mercury is assumed to pass through the wet lime/limestone FGD. It is assumed that elemental mercury is present in the flue gas and is unreactive.

\section{Spray Dryer}

Spray Dryer (oxidized): Oxidized mercury is assumed to pass through the lime spray dryer. Although soluble in water, moisture injected into the spray dryer evaporates, resulting in the mercury remaining in the flue gas. The default value is zero.

Spray Dryer (elemental): Elemental mercury is assumed to pass through the lime spray dryer. It is assumed that elemental mercury is present in the flue gas and is unreactive.

\section{Percent Increase in Speciation}

Although $\mathrm{NO}_{\mathrm{x}}$ control technologies do not remove mercury from the flue gas, they can change the mercury from one form to another. This is particularly true when catalysts are present. In this case, elemental mercury is converted to oxidized mercury. The parameters in this section define the percent increase in oxidized mercury across the control technology.

In-furnace $\mathrm{NO}_{\mathrm{x}}$ (oxidized): Low $\mathrm{NO}_{\mathrm{x}}$ burners with or without overfire air and gas reburn can effect the amount of oxidized mercury. At present, there is insufficient information available to specify a default value. The default is set to zero.

SNCR (oxidized): An SNCR can effect the amount of oxidized mercury. However, there is insufficient information available to specify a default value. The default is set to zero.

Hot-Side SCR (oxidized): Hot-side SCR as a control technology chances elemental mercury to oxidized mercury. It is believed that the catalyst is responsible for this shift in speciation. The default value is a function of the coal rank. .

\section{Mercury Carbon (and Water) Injection Inputs}

Inputs for activated carbon and water injected into the flue gas are entered on the Carbon Inj. input screen. Water can be optionally added to reduce the flue gas temperature and enhance the effect of the carbon on removing mercury. Note that the actual removal of the carbon and mercury are accomplished in particulate and flue gas desulfurization control technologies downstream 


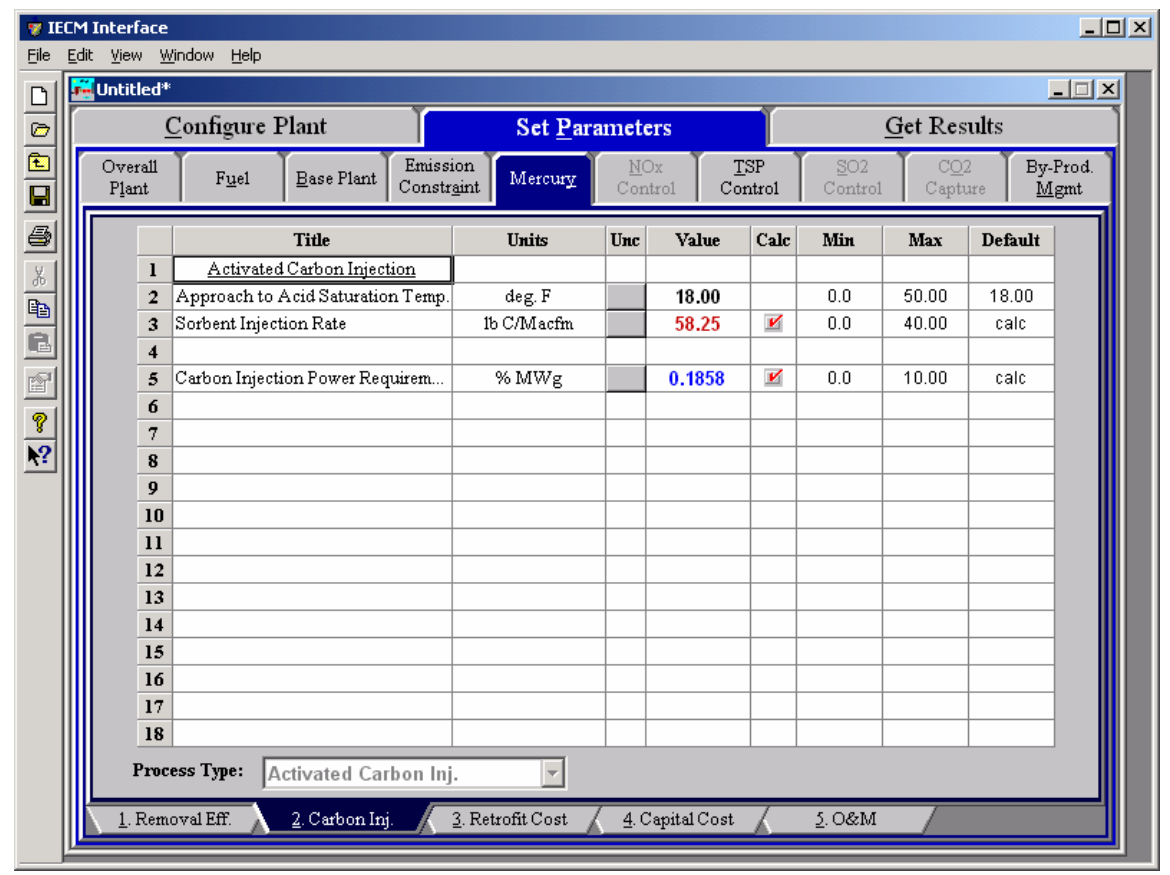

Mercury - Removal Efficiency input screen.

Each parameter is described briefly below.

\section{Activated Carbon Injection}

Injection of water to reduce the flue gas temperature and activated carbon to enhance mercury removal are the only control technologies presently incorporated into the IECM.

Approach to Acid Saturation Temperature: When water is selected to be injected with the activated carbon this parameter appears on the Removal Efficiency input screen. It is important to keep the flue gas temperature above the sulfuric acid dew point temperature. This avoids condensation of acid on equipment. This parameter determines the amount of water injected into the flue gas. If the approach is above the actual temperature, the temperature is dropped to be the approach above the dew point. The dew point is a function of the $\mathrm{SO}_{3}$ and $\mathrm{H}_{2} \mathrm{O}$ content in the flue gas and the pressure of the flue gas.

Sorbent Injection Rate: The flue gas temperature, the mercury removal efficiency in the particulate device, the coal rank, and the mercury removal efficiency without control, determines the injection rate of activated carbon into the flue gas. Mercury removal due to the ash removed in a cold-side ESP or fabric filter in the absence of enhanced mercury control methods is specified in the input screen. The default value is most sensitive to the flue gas temperature and the mercury removal efficiency without control.

Carbon Injection Power Requirement: The power required for the water and carbon injection system is a function of carbon injection rate, the water injection rate, and the flue gas flow rate. This assumes the addition of a fan in the flue gas to balance the pressure drop. The default value is calculated as the ratio of the actual energy consumption by the gross electrical output of the power plant. 


\section{Mercury Retrofit Cost Inputs}

Inputs for the capital costs of modifications to process areas of the activated carbon and water injection system are entered on the Retrofit Cost input screen.

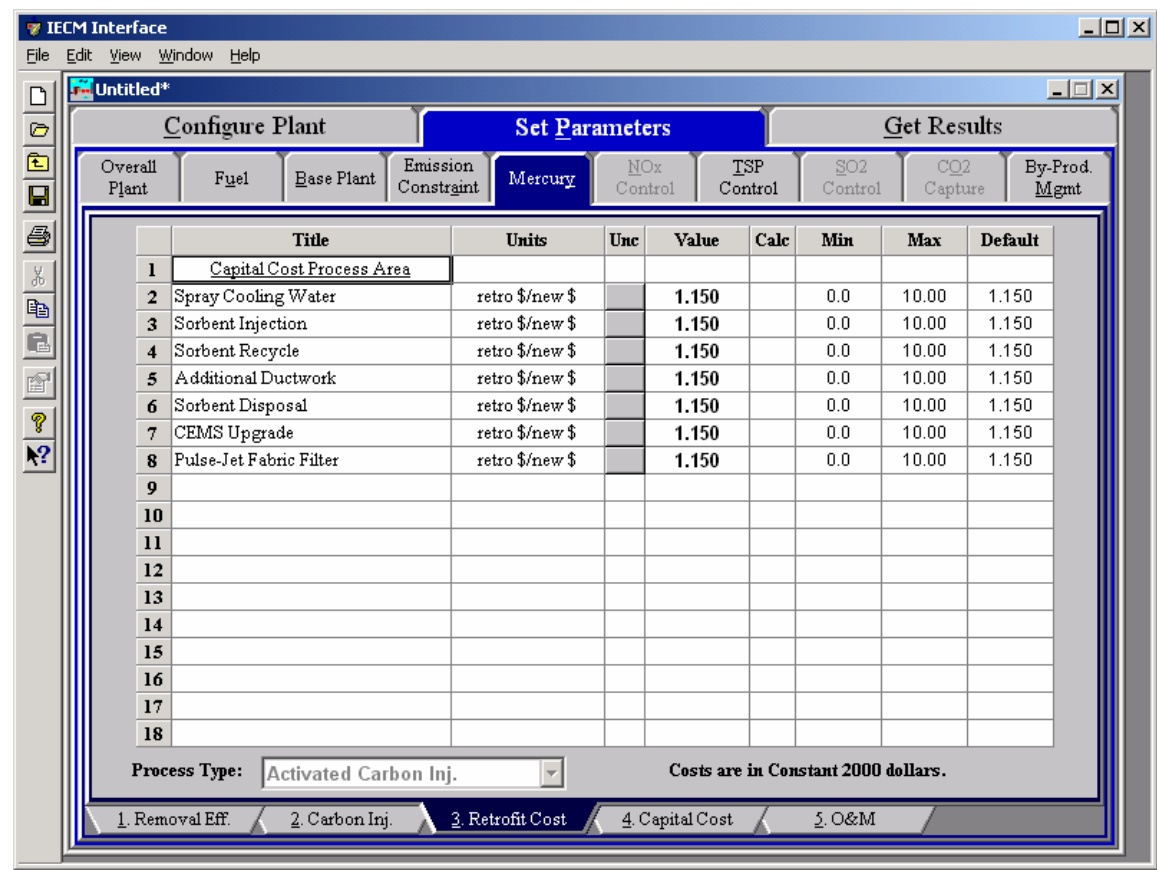

Mercury - Retrofit Cost input screen.

The retrofit cost factor of each process is a multiplicative cost adjustment, which considers the cost of retrofitted capital equipment relative to similar equipment installed in a new plant. These factors affect the capital costs directly and the operating and maintenance costs indirectly.

Direct capital costs for each process area are calculated in the IECM. These calculations are reduced form equations derived from more sophisticated models and reports. The sum of the direct capital costs associated with each process area is defined as the process facilities capital (PFC). The retrofit cost factor provided for each of the process areas can be used as a tool for adjusting the anticipated costs and uncertainties across the process area separate from the other areas.

Uncertainty can be applied to the retrofit cost factor for each process area in each technology. Thus, uncertainty can be applied as a general factor across an entire process area, rather than as a specific uncertainty for the particular cost on the capital or O\&M input screens. Any uncertainty applied to a process area through the retrofit cost factor compounds any uncertainties specified later in the capital and O\&M cost input parameter screens.

Each parameter is described briefly below. Although the user cannot set the capital cost directly, the descriptions below include the key parameters used to determine the capital cost itself. The input parameters on this screen adjust this capital cost as calculated in the IECM.

\section{Capital Cost Process Area}

Spray Cooling Water: This capital cost area represents the materials and equipment necessary to inject water into the flue gas duct for the purpose of cooling the flue gas to a prerequisite temperature. 
Equipment includes water storage tanks, pumps, transport piping, injection grid with nozzles, and a control system. The direct capital cost is a function of the water flow rate.

Sorbent Injection: This capital cost area represents the materials and equipment necessary to deliver the activated carbon into the flue gas. Equipment includes silo pneumatic loading system, storage silos, hoppers, blowers, transport piping, and a control system. The direct capital cost is a function of the sorbent flow rate.

Sorbent Recycle: This capital cost area represents the materials and equipment necessary to recycle ash and activated carbon from the particulate collector back into the duct injection point. The purpose is to create a equilibrium state where the carbon is reintroduced to improve performance. Equipment includes hoppers, blowers, transport piping, and a control system. The direct capital cost is a function of the recycle rate of ash and spent sorbent.

NOTE: Sorbent recycling is a feature to be added in a future version of the IECM.

Additional Ductwork: This capital cost area represents materials and equipment for ductwork necessary beyond the other process areas. Extra ductwork may be required for difficult retrofit installations.

NOTE: Future versions of the IECM will include parameters to determine a capital cost for this area. The current version assumes no additional ductwork.

Sorbent Disposal: This capital cost area represents materials and equipment required to house and dispose the collected sorbent. Equipment includes hoppers, blowers, transport piping, and a control system. This is in excess of existing hoppers, tanks, and piping used for existing particulate collectors. The direct capital cost is determined by the incremental increase in collected solids in the particulate collector.

CEMS Upgrade: This capital cost area represents materials and equipment required to install a continuous emissions monitoring system (CEMS) upgrade. The direct capital cost is determined by the net electrical output of the power plant.

Pulse-Jet Fabric Filter: This capital costs area represents an upgrade to an existing cold-side ESP, where one section at the back end of the unit is replaced with a pulse-jet fabric filter. This can be considered a pseudo-COHPAC. Equipment includes pulse-jet FF, filter bags, ductwork, dampers, and MCCs, instrumentation and PLC controls for baghouse operation. Equipment excludes ash removal system, power distribution and power supply, and distributed control system. The direct capital cost is a function of the flue gas flow rate and the air to cloth ratio of the fabric filter.

NOTE: The IECM currently does not support multiple particulate devices in the same configuration nor a modified cold-side ESP. 


\section{Mercury Capital Cost Inputs}

Inputs for the capital costs of the activated carbon and water injection system are entered on the Capital Cost input screen.

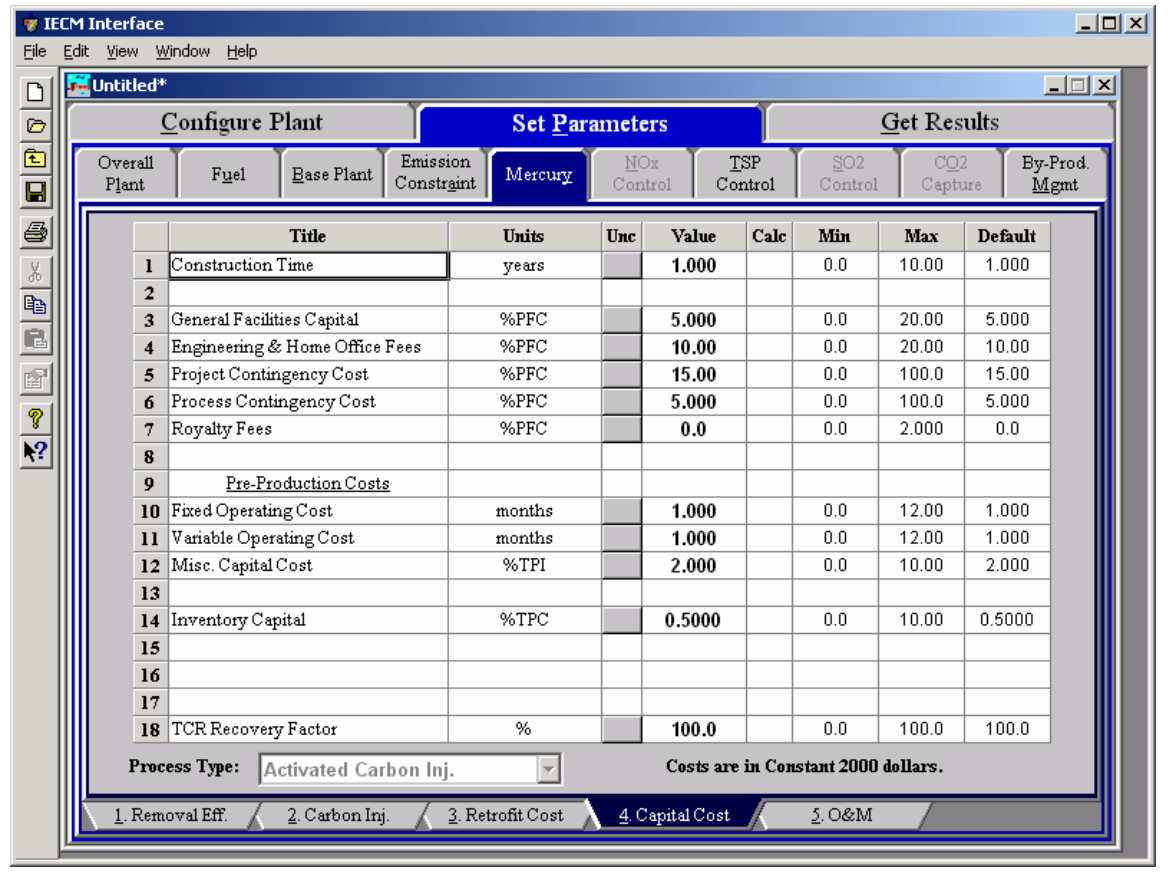

Mercury - Capital Cost input screen.

The necessary capital cost input parameters associated with the base plant are on this input screen. The capital cost parameters and terminology used in the IECM are based on the methodologies developed by the Electric Power Research Institute (EPRI). They have prepared a Technical Assessment Guide (TAG) in order to provide a consistent basis for reporting cost and revenues associated with the electric power industry. This system of reporting is used by a wide audience, including energy engineers, researchers, planners, and managers. The IECM has been developed around this TAG system so that costs associated with various technologies can be compared directly on a consistent basis and communicated in the language used by the audience listed above.

Total Plant Cost (TPC) is the sum of the process facilities capital, general facilities capital, engineering and home office fees, and the contingencies (project and process). This is considered the cost on an instantaneous basis (overnight), and expressed in December dollars of a reference year.

Direct Capital Costs: Direct capital costs for each process area are calculated in the IECM. These calculations are reduced form equations derived from more sophisticated models and reports. The sum of the direct capital costs associated with each process area is defined as the process facilities capital (PFC). This is the basis for all other capital cost parameters.

The process facilities capital for the technology is the total constructed cost of all onsite processing and generating units, including all direct and indirect construction costs. All sales taxes and freight costs are included where applicable implicitly. These direct capital costs are generally calculated by the IECM and not presented directly on input screens. However, when important input variables are required for these calculations, they are listed at the top of the input screen. 
Indirect Capital Costs: Costs that are indirectly applied to the technology are based on the process facilities cost. Each of the cost factors below is expressed as a percentage of the process facilities cost, and is entered on this screen. Each parameter is described briefly below.

Construction Time: This is the idealized construction period in years. It is used to determine the allowance for funds used during construction (AFUDC).

General Facilities Capital (GFC): The general facilities include construction costs of roads, office buildings, shops, laboratories, etc. Sales taxes and freight costs are included implicitly. The cost typically ranges from $5-20 \%$.

Engineering \& Home Office Fees: The engineering \& home office fees are a percent of total direct capital cost. This is an overhead fee paid to the architect/engineering company. These fees typically range from 7$15 \%$.

Project Contingency Cost: This is factor covering the cost of additional equipment or other costs resulting from a more detailed design. Higher contingency factors will be applied to simplified or preliminary designs and lower factors to detailed or finalized designs.

Process Contingency Cost: This quantifies the design uncertainty and cost of a commercial-scale system. This is generally applied on an areaby-area basis. Higher contingency factors are applied to new regeneration systems tested at a pilot plant and lower factors to full-size or commercial systems.

Royalty Fees: Royalty charges may apply to some portions of generating units incorporating new proprietary technologies.

\section{Pre-Production Costs}

These costs consider the operator training, equipment checkout, major changes in unit equipment, extra maintenance, and inefficient use of fuel or other materials during start-up. These are typically applied to O\&M costs over a specified period of time (months).

Fixed Operating Cost: Time period of fixed operating costs (operating and maintenance labor, administrative and support labor, and maintenance materials) used for plant startup.

Variable Operating Cost: Time period of variable operating costs at full capacity (chemicals, water, and other consumables, and waste disposal changes) used for plant startup. Full capacity estimates of the variable operating costs will assume operations at $100 \%$ load.

Misc. Capital Cost: This is a percent of total plant investment (sum of TPC and AFUDC) to cover expected changes to equipment to bring the system up to full capacity.

Inventory Capital: Percent of the total direct capital for raw material supply based on $100 \%$ capacity during a 60 day period. These materials are considered storage. The inventory capital includes fuels, consumables, by-products, and spare parts. This is typically $0.5 \%$.

TCR Recovery Factor: The actual total capital required (TCR) as a percent of the TCR in a new power plant. This value is $100 \%$ for a new installation and may be set as low as $0 \%$ for an activated carbon and water injection system that has been paid off. 


\section{Mercury O\&M Cost Inputs}

Inputs for the operation and maintenance costs of the mercury control technology are entered on the $\mathbf{O} \& \mathbf{M}$ cost input screen.

Activated Carbon Cost (w. shipping): This is the cost for the activated carbon, including the cost of shipping.

Disposal Cost: This is the disposal cost for the particulate control system. It is assumed that the ash is not hazardous, therefore can be disposed with the collected fly ash.

Number of Operating Jobs: This is the total number of operating jobs that are required to operate the plant per eight-hour shift.

Number of Operating Shifts: This is the total number of equivalent operating shifts in the plant per day. The number takes into consideration paid time off and weekend work ( 3 shifts/day * 7 days/5 day week * 52 weeks/(52 weeks -6 weeks PTO $)=4.75$ equiv. Shifts/day)

Total Maintenance Cost: This is the annual maintenance cost as a percentage of the total plant cost. Maintenance cost estimates can be developed separately for each process area.

Maint. Cost Allocated to Labor: Maintenance cost allocated to labor as a percentage of the total maintenance cost.

Administrative \& Support Cost: This is the percent of the total operating and maintenance labor associated with administrative and support labor.

\section{Mercury Diagram}

The Diagram result screen displays an icon for the water and carbon injection systems, both part of the overall mercury control option and values for major flows in and out of it 


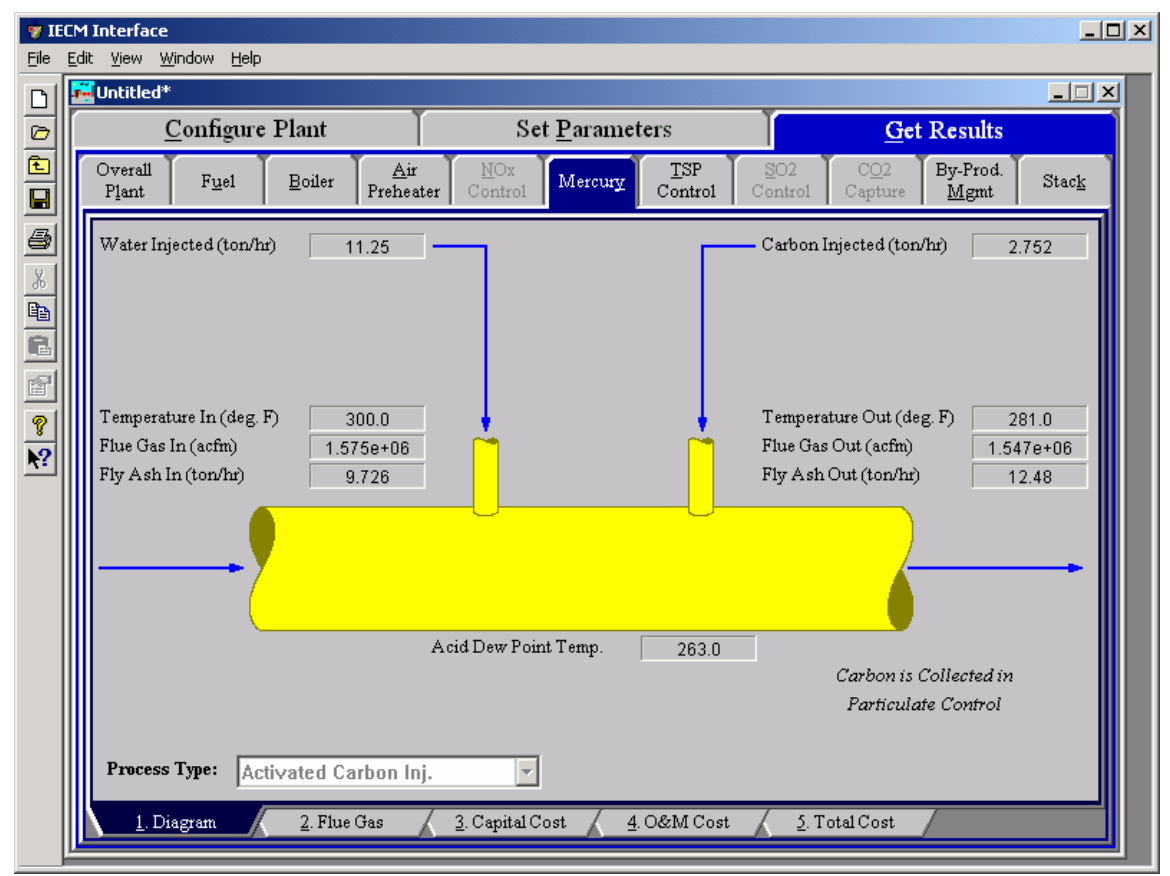

Mercury-Diagram result screen

Each result is described briefly below in flow order (not from top to bottom and left to right as they display on the screen).

\section{Flue Gas Prior to Injection}

Temperature In: Temperature of the flue gas prior to flue gas conditioning.

Flue Gas In: Volumetric flow rate of the flue gas prior to flue gas conditioning, based on the temperature prior to flue gas conditioning and atmospheric pressure.

Fly Ash In: Total solids mass flow rate in the flue gas prior to flue gas conditioning. This includes ash, unburned carbon and unburned sulfur.

\section{Flue Gas After Injection}

Temperature Out: Temperature of the flue gas after flue gas conditioning. This should be above the acid dew point temperature at the bottom of the screen.

Flue Gas Out: Volumetric flow rate of the flue gas after flue gas conditioning, based on the temperature after flue gas conditioning and atmospheric pressure.

Fly Ash Out: Total solids mass flow rate in the flue gas after flue gas conditioning. This includes ash, unburned carbon, activated carbon, and unburned sulfur.

Acid Dew Point: This is the temperature that $\mathrm{H}_{2} \mathrm{SO}_{4}$ vapor condenses into the liquid phase. .

\section{Flue Gas Conditioning}

Water Injected: Water added to the flue gas to reduce the temperature No water is injected if water injection is not specified in the configuration 
or the inlet temperature is within the approach to saturation relative to the acid dew point.

Carbon Injected: Total activated carbon mass flow rate injected into the flue gas.

NOTE: Carbon injected into the flue gas is collected downstream in the particulate control device (e.g., the cold-side ESP).

\section{Mercury Flue Gas Results}

The Flue Gas result screen displays a table of quantities of flue gas components entering and exiting the flue gas conditioning area. For each component, quantities are given in both moles and mass per hour.

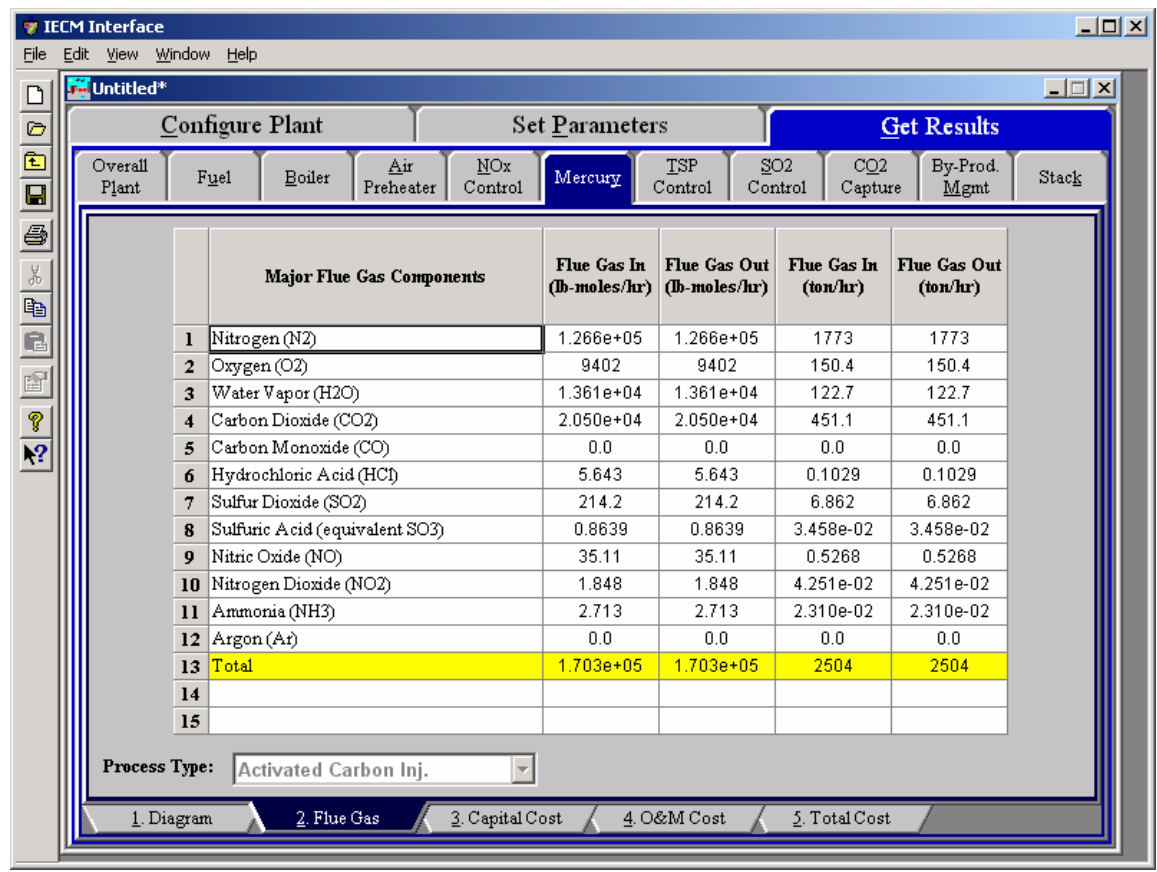

Mercury - Flue Gas result screen.

\section{Major Flue Gas Components}

Each result is described briefly below.

Nitrogen $\left(\mathbf{N}_{2}\right)$ : Total mass of nitrogen.

Oxygen $\left(\mathrm{O}_{2}\right)$ : Total mass of oxygen.

Water Vapor $\left(\mathrm{H}_{2} \mathrm{O}\right)$ : Total mass of water vapor.

Carbon Dioxide $\left(\mathrm{CO}_{2}\right)$ : Total mass of carbon dioxide.

Carbon Monoxide (CO): Total mass of carbon monoxide.

Hydrochloric Acid (HCl): Total mass of hydrochloric acid.

Sulfur Dioxide $\left(\mathrm{SO}_{2}\right)$ : Total mass of sulfur dioxide.

Sulfuric Acid (equivalent $\mathbf{S O}_{3}$ ): Total mass of sulfuric acid.

Nitric Oxide (NO): Total mass of nitric oxide. 
Nitrogen Dioxide $\left(\mathrm{NO}_{2}\right)$ : Total mass of nitrogen dioxide.

Ammonia (NH3): Total mass of ammonia.

Argon (Ar): Total mass of argon.

Total: Total of the individual components listed above. This item is highlighted in yellow.

\section{Mercury Capital Cost Results}

The Capital Cost result screen displays tables for the direct and indirect capital costs related to the water and carbon injection systems, both part of the overall mercury control option.

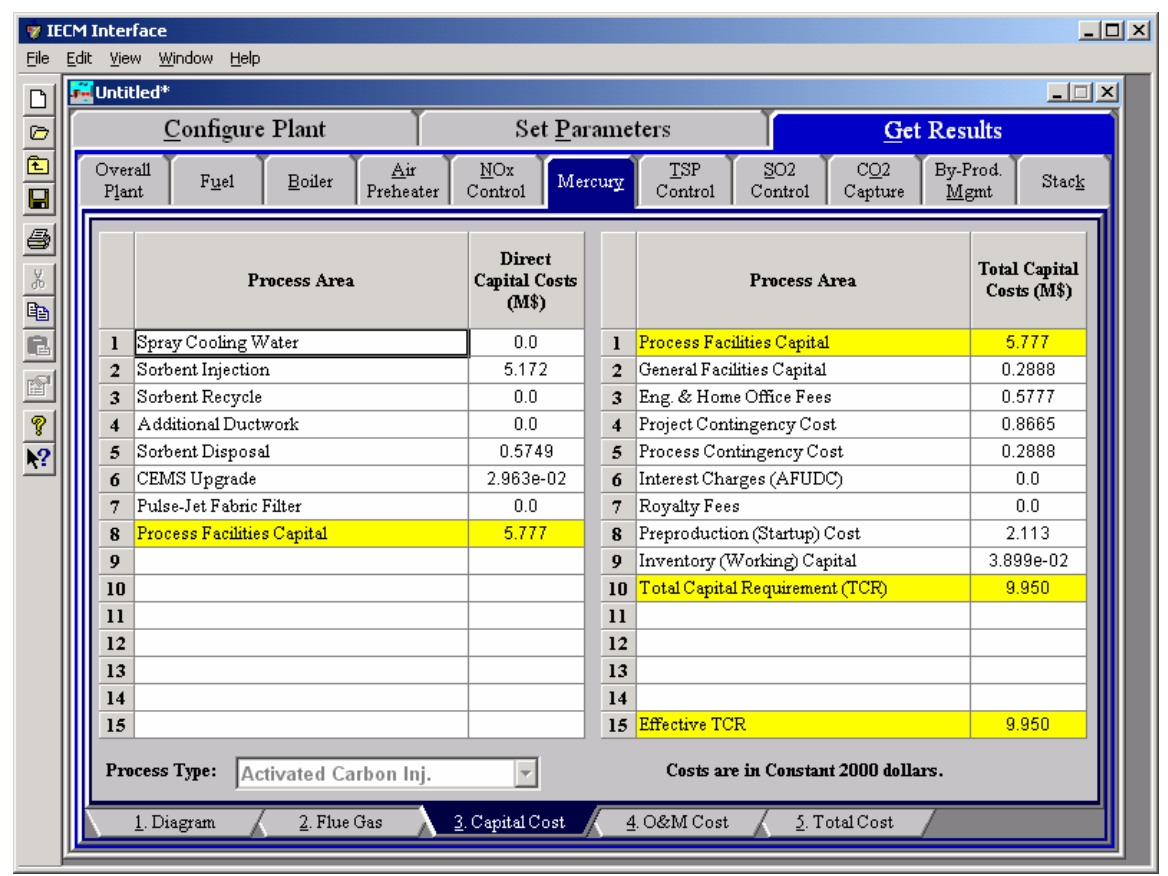

Mercury - Capital Cost result screen.

Each result is described briefly below.

\section{Direct Capital Costs}

The direct capital costs described here apply to the various mercury control equipment added to the power plant. These controls may physically be part of other control technologies, but have their particular capital costs considered here.

Each process area direct capital cost is a reduced-form model based on regression analysis of data collected from several reports and analyses. They are described in general below. The primary factors in the model that effect the capital cost of the base plant are the plant size, the amount of water injected, the amount of activated carbon injected, and the sulfur and moisture content of the coal.

Spray Cooling Water: This capital cost area represents the materials and equipment necessary to inject water into the flue gas duct for the purpose of cooling the flue gas to a prerequisite temperature. Equipment includes water storage tanks, pumps, transport piping, injection grid with nozzles, and a control system. The direct capital cost is a function of the water flow rate. 
Sorbent Injection: This capital cost area represents the materials and equipment necessary to deliver the activated carbon into the flue gas. Equipment includes silo pneumatic loading system, storage silos, hoppers, blowers, transport piping, and a control system. The direct capital cost is a function of the sorbent flow rate.

Sorbent Recycle: This capital cost area represents the materials and equipment necessary to recycle ash and activated carbon from the particulate collector back into the duct injection point. The purpose is to create a equilibrium state where the carbon is reintroduced to improve performance. Equipment includes hoppers, blowers, transport piping, and a control system. The direct capital cost is a function of the recycle rate of ash and spent sorbent.

NOTE: Sorbent recycling is a feature to be added in a future version of the IECM.

Additional Ductwork: This capital cost area represents materials and equipment for ductwork necessary beyond the other process areas. Extra ductwork may be required for difficult retrofit installations.

NOTE: Future versions of the IECM will include parameters to determine a capital cost for this area. The current version assumes no additional ductwork.

Sorbent Disposal: This capital cost area represents materials and equipment required to house and dispose the collected sorbent. Equipment includes hoppers, blowers, transport piping, and a control system. This is in excess of existing hoppers, tanks, and piping used for existing particulate collectors. The direct capital cost is determined by the incremental increase in collected solids in the particulate collector.

CEMS Upgrade: This capital cost area represents materials and equipment required to install a continuous emissions monitoring system (CEMS) upgrade. The direct capital cost is determined by the net electrical output of the power plant.

Pulse-Jet Fabric Filter: This capital costs area represents an upgrade to an existing cold-side ESP, where one section at the back end of the unit is replaced with a pulse-jet fabric filter. This can be considered a pseudo-COHPAC. Equipment includes pulse-jet FF, filter bags, ductwork, dampers, and MCCs, instrumentation and PLC controls for baghouse operation. Equipment excludes ash removal system, power distribution and power supply, and distributed control system. The direct capital cost is a function of the flue gas flow rate and the air to cloth ratio of the fabric filter.

NOTE: The IECM currently does not support multiple particulate devices in the same configuration nor a modified cold-side ESP.

Process Facilities Capital: The process facilities capital is the total constructed cost of all on-site processing and generating units listed above, including all direct and indirect construction costs. All sales taxes and freight costs are included where applicable implicitly. This result is highlighted in yellow.

Total Capital Costs 
Process Facilities Capital: See definition above. This result is highlighted in yellow.

General Facilities Capital: The general facilities include construction costs of roads, office buildings, shops, laboratories, etc. Sales taxes and freight costs are included implicitly.

Eng. \& Home Office Fees: The engineering \& home office fees are a percent of total direct capital cost. This is an overhead fee paid to the architect/engineering company.

Project Contingency Cost: Capital cost contingency factor covering the cost of additional equipment or other costs that would result from a more detailed design of a definitive project at the actual site.

Process Contingency Cost: Capital cost contingency factor applied to a new technology in an effort to quantify the uncertainty in the technical performance and cost of the commercial-scale equipment.

Interest Charges (AFUDC): Allowance for funds used during construction, also referred to as interest during construction, is the time value of the money used during construction and is based on an interest rate equal to the before-tax weighted cost of capital. This interest is compounded on an annual basis (end of year) during the construction period for all funds spent during the year or previous years.

Royalty Fees: Royalty charges may apply to some portions of generating units incorporating new proprietary technologies.

Preproduction (Startup) Cost: These costs consider the operator training, equipment checkout, major changes in unit equipment, extra maintenance, and inefficient use of fuel or other materials during startup.

Inventory (Working) Capital: The raw material supply based on $100 \%$ capacity during a 60 day period. These materials are considered storage. The inventory capital includes fuels, consumables, byproducts, and spare parts.

Total Capital Requirement (TCR): Money that is placed (capitalized) on the books of the utility on the service date. TCR includes all the items above. This result is highlighted in yellow.

Effective TCR: The TCR of the water and carbon injection controls that is used in determining the total power plant cost. The effective TCR is determined by the "TCR Recovery Factor" for the water and carbon injection system.

\section{Mercury O\&M Cost Results}

The O\&M Cost result screen displays tables for the variable and fixed operation and maintenance costs related to the water and carbon injection systems, both part of the overall mercury control option. The variable O\&M costs are calculated from the variable costs for carbon, water consumption and fly ash disposal (from the particulate control device). The fixed O\&M costs are based on maintenance and labor costs 


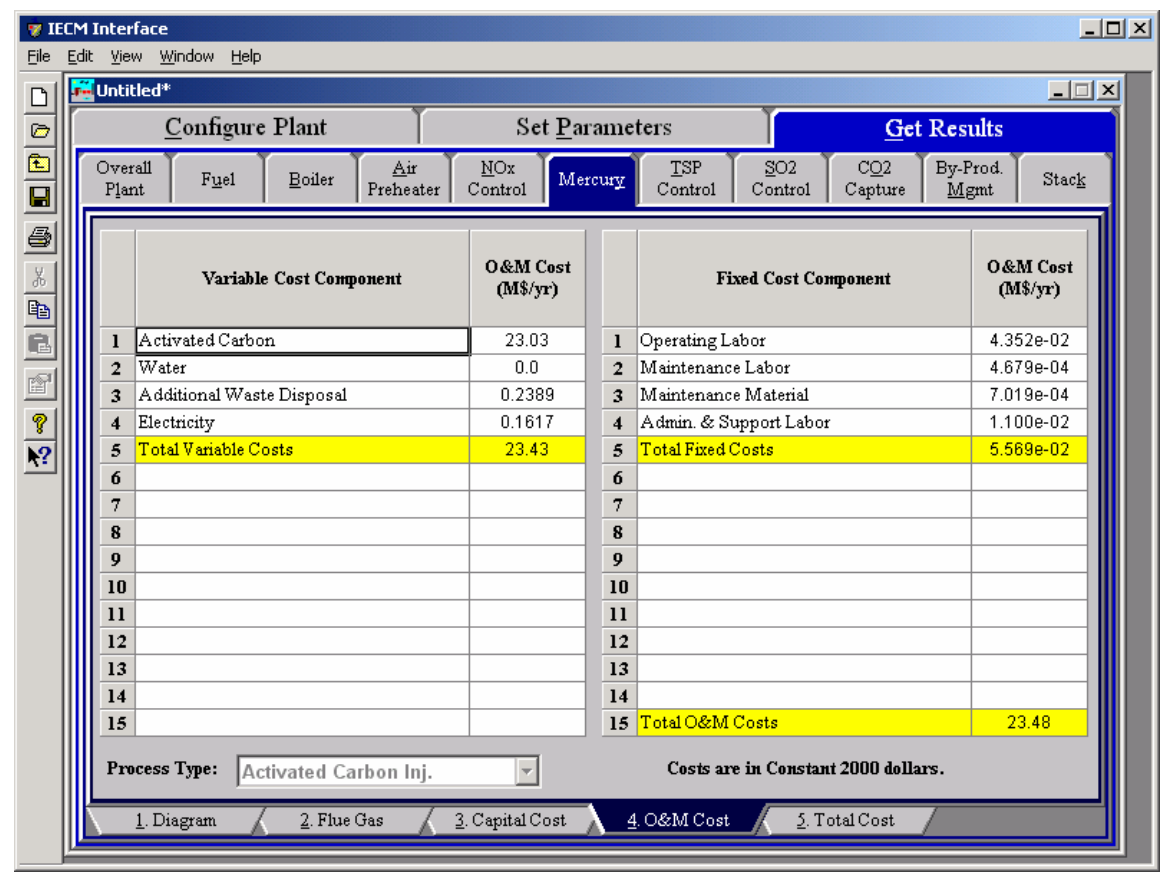

Mercury - O\&M Cost result screen.

Each result is described briefly below.

\section{Variable Cost Components}

Variable operating costs and consumables are directly proportional to the amount of kilowatts produced and are referred to as incremental costs. All the costs are subject to inflation.

Activated Carbon: This is the activated carbon cost for flue gas conditioning.

Water: This is the water cost for flue gas conditioning.

Additional Waste Disposal: This is the solid disposal cost per year for the flue gas conditioning. Only the removal of carbon from the particulate device is considered here.

Electricity: This is the power utilization cost per year for the flue gas conditioning.

Total Variable Costs: This is the sum of all the variable O\&M costs listed above. This result is highlighted in yellow.

\section{Fixed Cost Components}

Fixed operating costs are essentially independent of actual capacity factor, number of hours of operation, or amount of kilowatts produced. All the costs are subject to inflation.

Operating Labor: Operating labor cost is based on the operating labor rate, the number of personnel required to operate the plant per eighthour shift, and the average number of shifts per day over 40 hours per week and 52 weeks.

Maintenance Labor: The maintenance labor is determined as a fraction of the total maintenance cost. 
Maintenance Material: The cost of maintenance material is the remainder of the total maintenance cost, considering the fraction associated with maintenance labor.

Admin. \& Support Labor: The administrative and support labor is the only overhead charge. It is taken as a fraction of the total operating and maintenance labor costs.

Total Fixed Costs: This is the sum of all the fixed O\&M costs listed above. This result is highlighted in yellow.

Total O\&M Costs: This is the sum of the total variable and total fixed O\&M costs. It is used to determine the base plant total revenue requirement. This result is highlighted in yellow.

\section{Mercury Total Cost Results}

The Total Cost result screen displays a table which totals the annual fixed, variable, operations and maintenance, and capital costs related to the water and carbon injection systems, both part of the overall mercury control option.

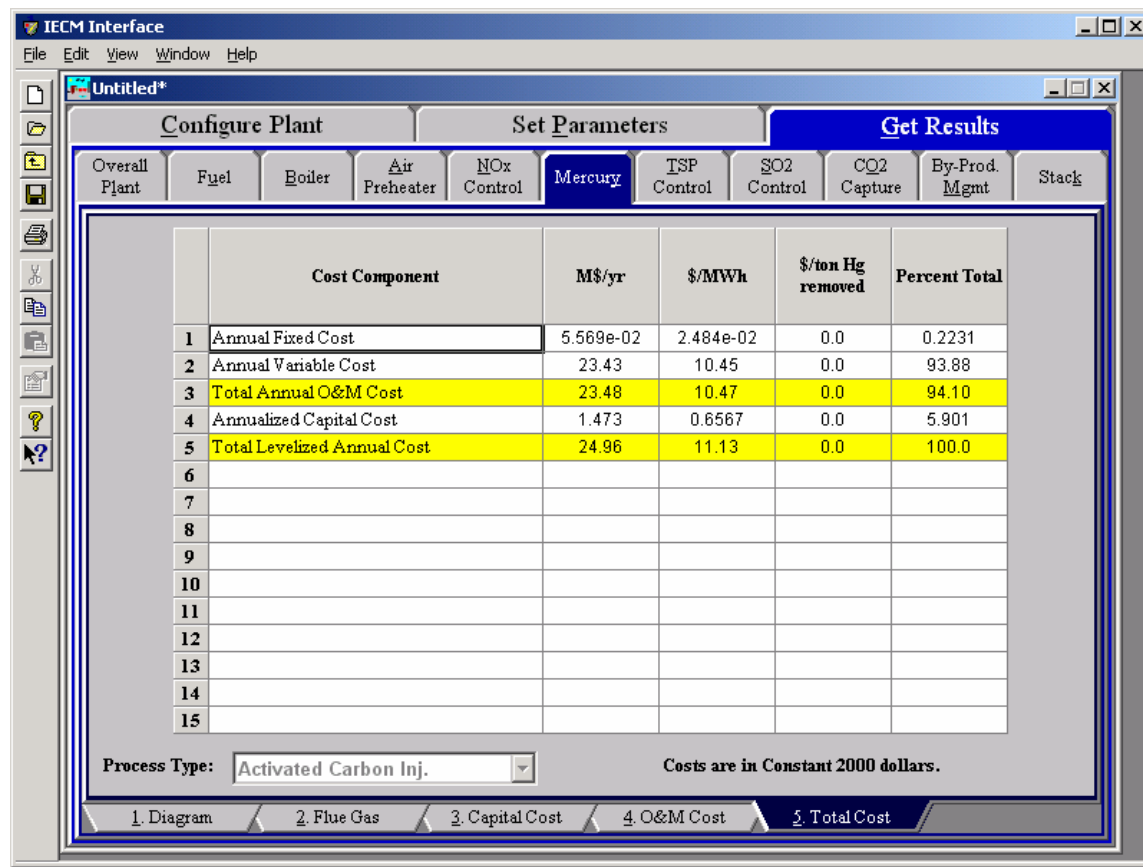

Mercury - Total Cost result screen.

Each result is described briefly below.

\section{Cost Component}

Annual Fixed Cost: The operating and maintenance fixed costs are given as an annual total. This number includes all maintenance materials and all labor costs.

Annual Variable Cost: The operating and maintenance variables costs are given as an annual total. This includes all reagent, chemical, steam, and power costs. 
Total Annual O\&M Cost: This is the sum of the annual fixed and variable operating and maintenance costs above. This result is highlighted in yellow.

Annualized Capital Cost: This is the total capital cost expressed on an annualized basis, taking into consideration the levelized carrying charge factor, or fixed charge factor, over the entire book life.

Total Levelized Annual Cost: The total annual cost is the sum of the total annual O\&M cost and annualized capital cost items above. This result is highlighted in yellow. 



\section{Cold-Side ESP}

The TSP Control. Technology Navigation screens define and display flows and costs related to the particulate control technology. These screens are available only if the Cold-Side ESP TSP control technology is selected.

\section{Cold-Side ESP Performance Inputs}

Inputs for the performance of the Cold-Side ESP TSP control technology are entered on the Performance input screen. Many of the parameters are calculated by the IECM. Each parameter is described briefly below.

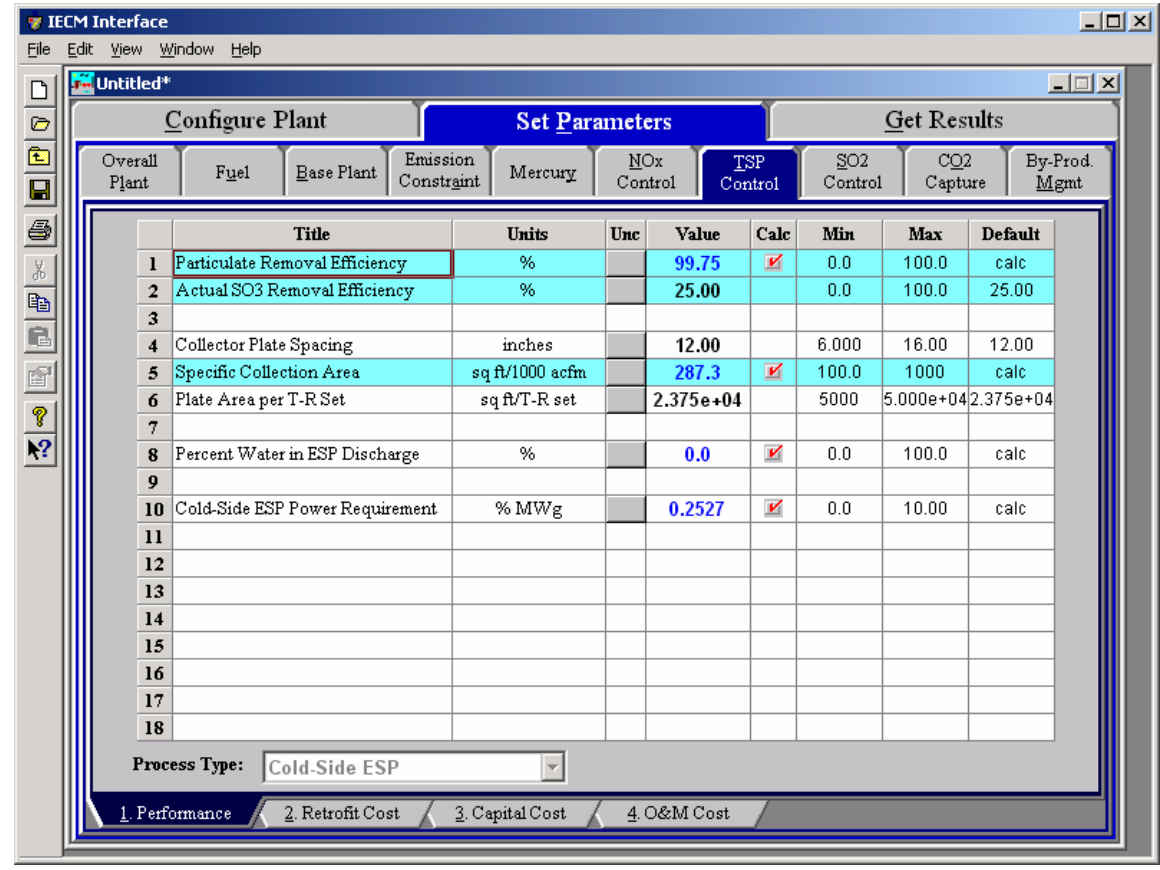

Cold-Side ESP - Performance input screen.

ESPs consist of a series of parallel plates with rows of electrodes in between them and carry a high voltage of opposite polarity. As the particle laden flue gas enters the unit, the particles are charged by the electrodes and is attracted to the plates. At controlled intervals the plates are rapped which shakes the dust to a hopper below. However, some of the dust is re-entrained and carried to the next zone or out of the 
stack. Most ESPs use rigid collecting plates with shielded air pockets (baffles) through which ash falls into the hoppers after rapping.

The major design parameters which can significantly impact the total system capital cost are gas flow volume (which depends on the generating unit size), SCA, the collecting plate area per transformer-rectifier (T-R) set and the spacing between collector plates.

Particulate Removal Efficiency: The calculated value determines the removal efficiency needed to comply with the specified particulate emission limit set earlier. This efficiency then determines the mass of particulate matter removed in the collector.

Actual $\mathrm{SO}_{3}$ Removal Efficiency: The default value is taken from the removal efficiency reported by Southern Company. ${ }^{5}$ This efficiency then determines the mass of $\mathrm{SO}_{3}$ removed from the flue gas in the collector.

Collector Plate Spacing: The collector plate spacing is typically 12 inches. The spacing is used to determine the specific collection area.

Specific Collection Area: The specific collection area (SCA) is the ratio of the total plate area and flue gas volume. It sizes the ESP. The value is calculated from the removal efficiency, plate spacing, and the drift velocity. It is used to determine the capital cost and the total collection area required.

Plate Area per T-R Set: This is the total surface area of one T-R set of plates. It is used to determine the total number of T-R sets needed and the capital costs.

Percent Water in ESP Discharge: This is the water content of the collected fly ash. Fly ash disposed with bottom ash is assumed to be sluiced with water and dry otherwise. The occluded water in wet fly ash is difficult to remove, resulting in a rather high water content when the fly ash is mixed with bottom ash.

Cold-Side ESP Power Requirement: The default calculation is based on the T-R set power consumption with estimates for auxiliary energy requirements and electro-mechanical efficiencies of fan motors. The T$\mathrm{R}$ set power consumption is a function of removal efficiency.

\section{Cold-Side ESP Retrofit Cost Inputs}

Inputs for the capital costs of modifications to process areas to implement the Particulate control technology are entered on the Retrofit Cost input screen. All costs are in constant 1996 dollars.

The retrofit cost factor of each process is a multiplicative cost adjustment, which considers the cost of retrofitted capital equipment relative to similar equipment installed in a new plant. These factors affect the capital costs directly and the operating and maintenance costs indirectly.

Direct capital costs for each process area are calculated in the IECM. These calculations are reduced form equations derived from more sophisticated models and reports. The sum of the direct capital costs associated with each process area is defined as the process facilities capital (PFC). The retrofit cost factor provided for

${ }^{5}$ Ibid. 
each of the process areas can be used as a tool for adjusting the anticipated costs and uncertainties across the process area separate from the other areas.

Uncertainty can be applied to the retrofit cost factor for each process area in each technology. Thus, uncertainty can be applied as a general factor across an entire process area, rather than as a specific uncertainty for the particular cost on the capital or O\&M input screens. Any uncertainty applied to a process area through the retrofit cost factor compounds any uncertainties specified later in the capital and O\&M cost input parameter screens.

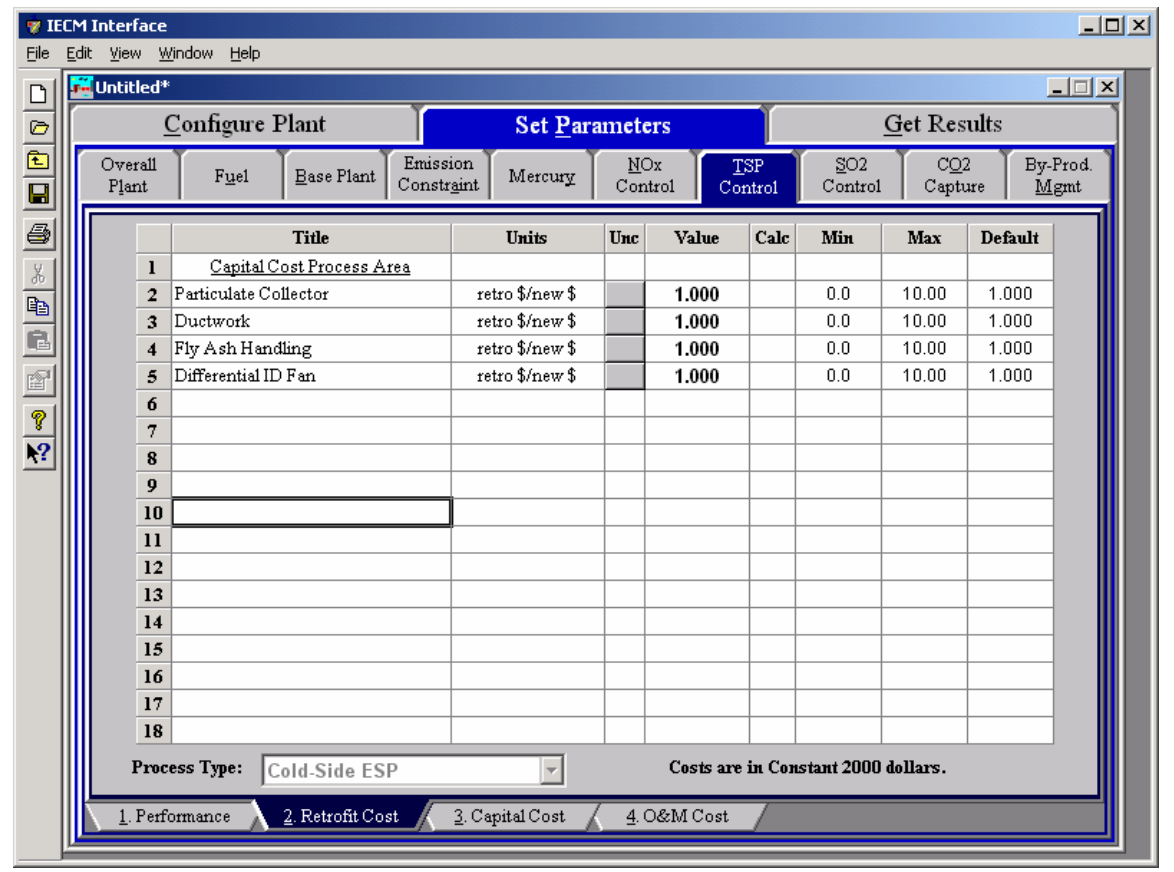

Cold-Side ESP - Retrofit Cost input screen.

Each parameter is described briefly below.

\section{Capital Cost Process Area}

Particulate Collector: This area covers the material and labor, flange to flange, for the equipment and labor cost for installation of the entire collection system.

Ductwork: This area includes the material and labor for the ductwork needed to distribute flue gas to the inlet flange, and from the outlet flange to a common duct leading to the suction side of the ID fan.

Fly Ash Handling: The complete fly ash handling cost includes the conveyor system and ash storage silos.

Differential ID Fan: The complete cost of the ID fan and motor due to the pressure loss that results from particulate collectors.

\section{Cold-Side ESP Capital Cost Inputs}

Inputs for the capital costs of particulate control technology are entered on the Capital Cost input screen. 


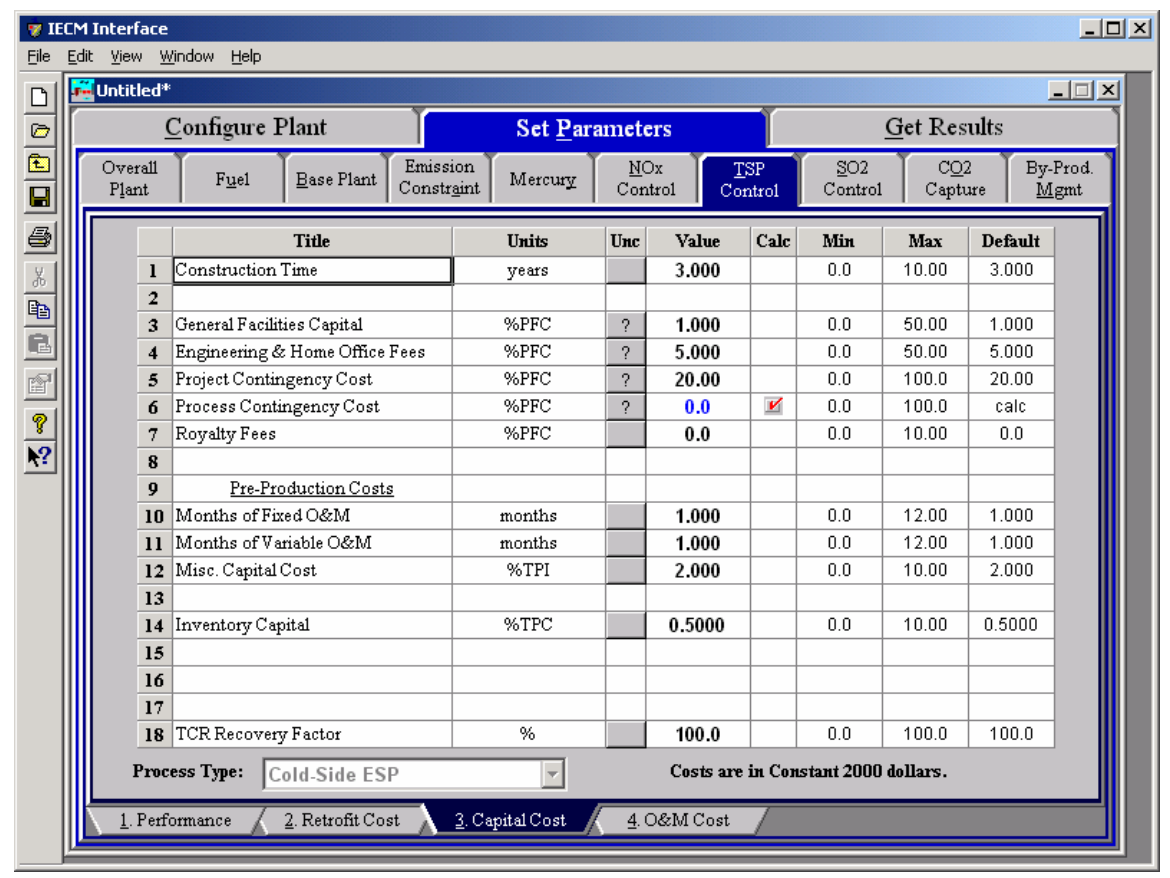

Cold-Side ESP - Capital Cost input screen.

The necessary capital cost input parameters associated with the electrostatic precipitator control technology are shown on this input screen.

Indirect Capital Costs: Costs that are indirectly applied to the technology are based on the process facilities cost. Each of the cost factors below is expressed as a percentage of the process facilities cost, and is entered on this screen. Each parameter is described briefly below.

Construction Time: This is the idealized construction period in years. It is used to determine the allowance for funds used during construction (AFUDC).

General Facilities Capital (GFC): The general facilities include construction costs of roads, office buildings, shops, laboratories, etc. Sales taxes and freight costs are included implicitly. The cost typically ranges from $5-20 \%$.

Engineering \& Home Office Fees: The engineering \& home office fees are a percent of total direct capital cost. This is an overhead fee paid to the architect/engineering company. These fees typically range from 7$15 \%$.

Project Contingency Cost: This is factor covering the cost of additional equipment or other costs resulting from a more detailed design. Higher contingency factors will be applied to simplified or preliminary designs and lower factors to detailed or finalized designs.

Process Contingency Cost: This quantifies the design uncertainty and cost of a commercial-scale system. This is generally applied on an areaby-area basis. Higher contingency factors are applied to new regeneration systems tested at a pilot plant and lower factors to full-size or commercial systems.

Royalty Fees: Royalty charges may apply to some portions of generating units incorporating new proprietary technologies. 


\section{Pre-Production Costs}

These costs consider the operator training, equipment checkout, major changes in unit equipment, extra maintenance, and inefficient use of fuel or other materials during start-up. These are typically applied to the O\&M costs over a specified period of time (months)The two time periods for fixed and variable O\&M costs are described below with the addition of a miscellaneous capital cost factor.

Months of Fixed O\&M: Time period of fixed operating costs used for preproduction to cover training, testing, major changes in equipment, and inefficiencies in start-up. This includes operating, maintenance, administrative and support labor. It also considers maintenance materials.

Months of Variable O\&M: Time period of variable operating costs used for preproduction to cover chemicals, water, consumables, and solid disposal charges in start-up, assuming $100 \%$ load. This excludes any fuels.

Misc. Capital Cost: This is a percent of total plant investment (sum of TPC and AFUDC) to cover expected changes to equipment to bring the system up to full capacity.

Inventory Capital: Percent of the total direct capital for raw material supply based on $100 \%$ capacity during a 60 day period. These materials are considered storage. The inventory capital includes fuels, consumables, by-products, and spare parts. This is typically $0.5 \%$.

TCR Recovery Factor: The actual total capital required (TCR) as a percent of the TCR in a new power plant. This value is $100 \%$ for a new installation and may be set as low as $0 \%$ for a cold-side ESP that has been paid off.

\section{Cold-Side ESP O\&M Cost Inputs}

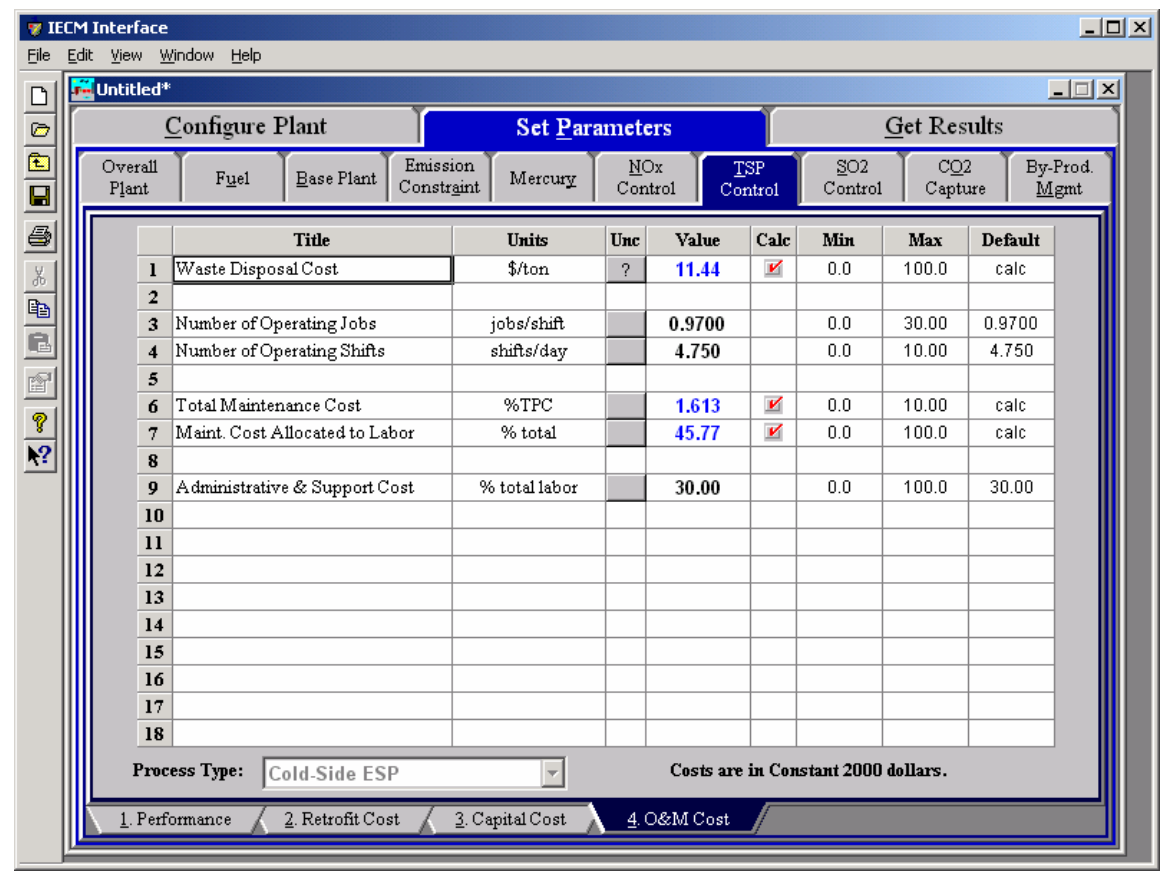

Cold-Side ESP - O\&M Cost screen input. 
Inputs for the operation and maintenance costs of the particulate control technology are entered on this screen.

Waste Disposal Cost: This is the disposal cost for the particulate control system.

Number of Operating Jobs: This is the total number of operating jobs that are required to operate the plant per eight-hour shift.

Number of Operating Shifts: This is the total number of equivalent operating shifts in the plant per day. The number takes into consideration paid time off and weekend work ( 3 shifts/day * 7 days/5 day week * 52 weeks/(52 weeks -6 weeks PTO $)=4.75$ equiv. Shifts/day)

Total Maintenance Cost: This is the annual maintenance cost as a percentage of the total plant cost. Maintenance cost estimates can be developed separately for each process area.

Maint. Cost Allocated to Labor: Maintenance cost allocated to labor as a percentage of the total maintenance cost.

Administrative \& Support Cost: This is the percent of the total operating and maintenance labor associated with administrative and support labor.

\section{Cold-Side ESP Diagram}

The Diagram result screen displays an icon for the particulate control technology selected and values for major flows in and out of it.

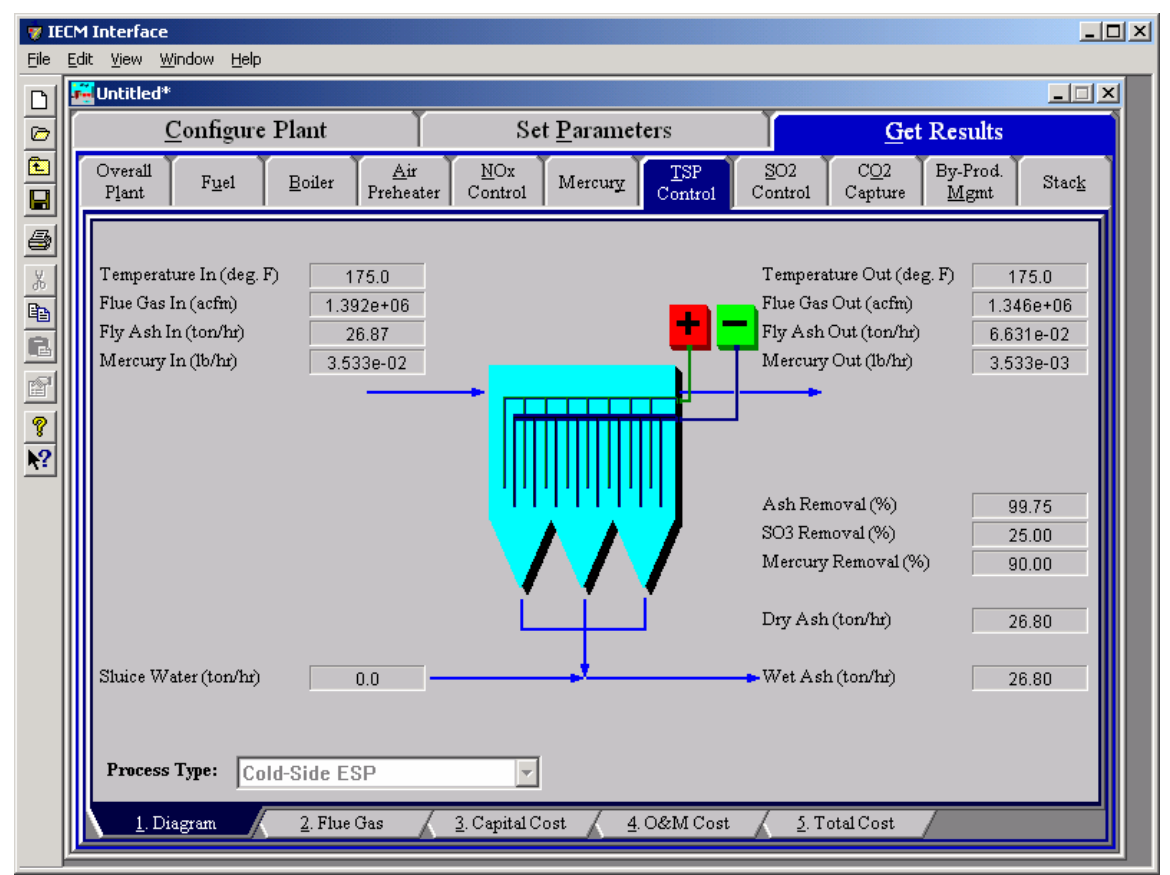

Cold-Side ESP - Diagram

Each result is described briefly below:

Flue Gas Entering ESP 
Temperature In: Temperature of the flue gas entering the particulate control technology. This is determined by the flue gas outlet temperature of the module upstream of the air preheater (e.g., the air preheater).

Flue Gas In: Volumetric flow rate of the flue gas entering the particulate control technology, based on the flue gas inlet temperature and atmospheric pressure.

Fly Ash In: Total solids mass flow rate in the flue gas entering the air preheater. This is determined by the solids exiting the module upstream of the particulate control technology (e.g., the air preheater).

Mercury In: Total mass of mercury entering the particulate control technology. The value is a sum of all the forms of mercury (elemental, oxidized, and particulate).

\section{Flue Gas Exiting ESP}

Temperature Out: Temperature of the flue gas exiting the particulate control technology. The model currently does not alter this temperature through the particulate control technology.

Flue Gas Out: Volumetric flow rate of the flue gas exiting the particulate control technology, based on the flue gas exit temperature and atmospheric pressure.

Fly Ash Out: Total solids mass flow rate in the flue gas exiting the particulate control technology. This is a function of the ash content of the inlet flue gas and the ash removal efficiency performance input parameter.

Mercury Out: Total mass of mercury exiting the particulate control technology. The value is a sum of all the forms of mercury (elemental, oxidized, and particulate).

\section{ESP Performance}

Ash Removal: Ash removal efficiency of the particulate control technology. This is a function of the ash emission constraint and the inlet ash mass flow rate.

$\mathrm{SO}_{3}$ Removal: Percent of $\mathrm{SO}_{3}$ in the flue gas removed from the particulate control technology. The $\mathrm{SO}_{3}$ is assumed to combine with $\mathrm{H}_{2} \mathrm{O}$ and leave with the ash solids as a sulfate (in the form of $\mathrm{H}_{2} \mathrm{SO}_{4}$ ).

Mercury Removal: Percent of the total mercury removed from the particulate control technology. The value reflects a weighted average based on the particular species of mercury present (elemental, oxidized, and particulate).

\section{Collected Fly Ash}

Dry Ash: Total mass flow rate of the solids removed from the ESP. This is a function of the solids content in the flue gas and the particulate removal efficiency of the ESP. The value is given on a dry basis.

Sluice Water: Water added to the dry fly ash. This water is added for transportation purposes.

Wet Ash: Total mass flow rate of the solids removed for waste management. This includes dry fly ash and sluice water. The value is given on a wet basis. 


\section{Cold-Side ESP Flue Gas Results}

The Flue Gas result screen displays a table of quantities of flue gas components entering and exiting the Particulate Control Technology. For each component, quantities are given in both moles and mass per hour.

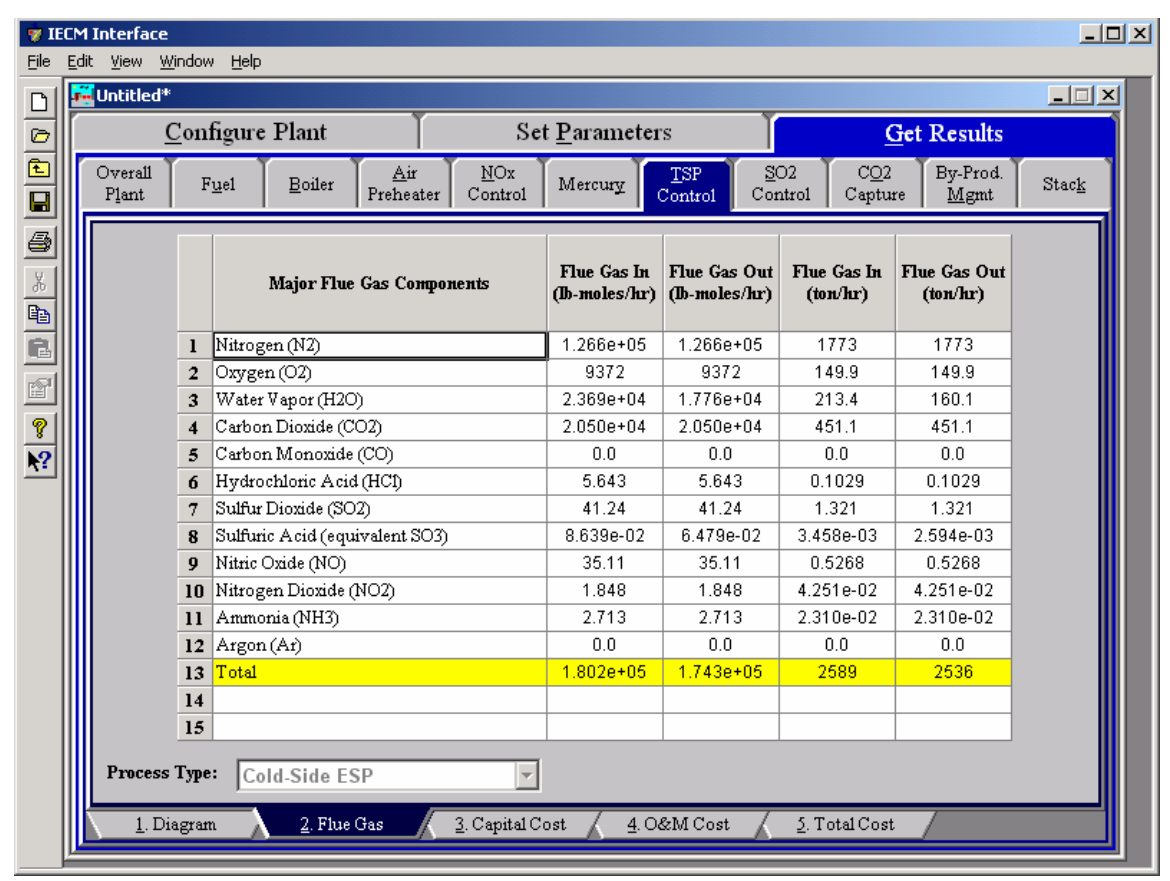

Cold-Side ESP - Flue Gas results screen.

Each result is described briefly below:

\section{Major Flue Gas Components}

Nitrogen $\left(\mathrm{N}_{2}\right)$ : Total mass of nitrogen.

Oxygen $\left(\mathrm{O}_{2}\right)$ : Total mass of oxygen.

Water Vapor $\left(\mathrm{H}_{2} \mathrm{O}\right)$ : Total mass of water vapor.

Carbon Dioxide $\left(\mathrm{CO}_{2}\right)$ : Total mass of carbon dioxide.

Carbon Monoxide (CO): Total mass of carbon monoxide.

Hydrochloric Acid ( $\mathrm{HCl})$ : Total mass of hydrochloric acid.

Sulfur Dioxide $\left(\mathrm{SO}_{2}\right)$ : Total mass of sulfur dioxide.

Sulfuric Acid (equivalent $\mathbf{S O}_{3}$ ): Total mass of sulfuric acid.

Nitric Oxide (NO): Total mass of nitric oxide.

Nitrogen Dioxide $\left(\mathrm{NO}_{2}\right)$ : Total mass of nitrogen dioxide.

Ammonia (NH3): Total mass of Ammonia.

Argon (Ar): Total mass of Argon.

Total: Total of the individual components listed above. This item is highlighted in yellow. 


\section{Cold-Side ESP Capital Cost Results}

The Capital Cost result screen displays tables for the direct and indirect capital costs related to the particulate control technology.

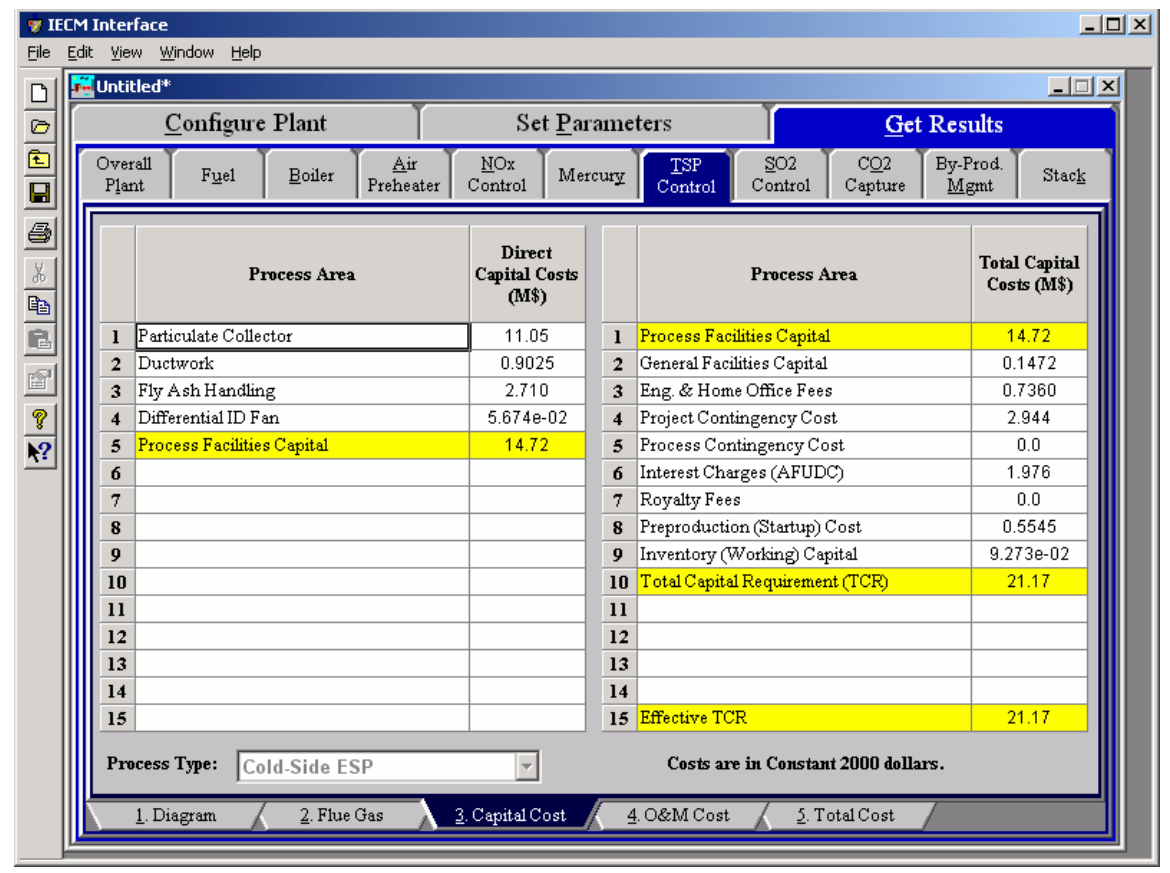

Cold-Side ESP — Capital Costs results screen.

\section{Direct Capital Costs}

Each process area direct capital cost is a reduced-form model based on regression analysis of data collected from several reports and analyses of particulate control technology units. They are described in general below. The primary factors in the model that effect the capital costs of the cold-side ESP are the specific and total collection areas of the T-R plate sets, and the flue gas flow rate through the ESP. The primary model factors that effect the capital costs of the fabric filter are the fabric filter type, the air to cloth ratio, the number of bags and compartments, and the flue gas flow rate through the fabric filter.

Collector: This area covers the material and labor, flange to flange, for the equipment and labor cost for installation of the entire collection system.

Ductwork: This area includes the material and labor for the ductwork needed to distribute flue gas to the inlet flange, and from the outlet flange to a common duct leading to the suction side of the ID fan.

Fly Ash Handling: The complete fly ash handling cost includes the conveyor system and ash storage silos.

Differential ID Fan: The complete cost of the ID fan and motor due to the pressure loss that results from particulate collectors.

Process Facilities Capital: The process facilities capital is the total constructed cost of all on-site processing and generating units listed above, including all direct and indirect construction costs. All sales taxes and freight costs are included where applicable implicitly. This result is highlighted in yellow. 


\section{Total Capital Costs}

Process Facilities Capital: (see definition above)

General Facilities Capital: The general facilities include construction costs of roads, office buildings, shops, laboratories, etc. Sales taxes and freight costs are included implicitly.

Eng. \& Home Office Fees: The engineering \& home office fees are a percent of total direct capital cost. This is an overhead fee paid to the architect/engineering company.

Project Contingency Cost: Capital cost contingency factor covering the cost of additional equipment or other costs that would result from a more detailed design of a definitive project at the actual site.

Process Contingency Cost: Capital cost contingency factor applied to a new technology in an effort to quantify the uncertainty in the technical performance and cost of the commercial-scale equipment.

Interest Charges (AFUDC): Allowance for funds used during construction, also referred to as interest during construction, is the time value of the money used during construction and is based on an interest rate equal to the before-tax weighted cost of capital. This interest is compounded on an annual basis (end of year) during the construction period for all funds spent during the year or previous years.

Royalty Fees: Royalty charges may apply to some portions of generating units incorporating new proprietary technologies.

Preproduction (Startup) Cost: These costs consider the operator training, equipment checkout, major changes in unit equipment, extra maintenance, and inefficient use of fuel or other materials during startup.

Inventory (Working) Capital: The raw material supply based on $100 \%$ capacity during a 60 day period. These materials are considered storage. The inventory capital includes fuels, consumables, byproducts, and spare parts.

Total Capital Requirement (TCR): Money that is placed (capitalized) on the books of the utility on the service date. TCR includes all the items above. This result is highlighted in yellow.

Effective TCR: The TCR of the cold-side ESP that is used in determining the total power plant cost. The effective TCR is determined by the TCR Recovery Factor for the cold-side ESP.

\section{Cold-Side ESP O\&M Cost Results}

The O\&M Cost result screen displays tables for the variable and fixed operation and maintenance costs involved with the Cold-Side ESP TSP particulate control technology. 


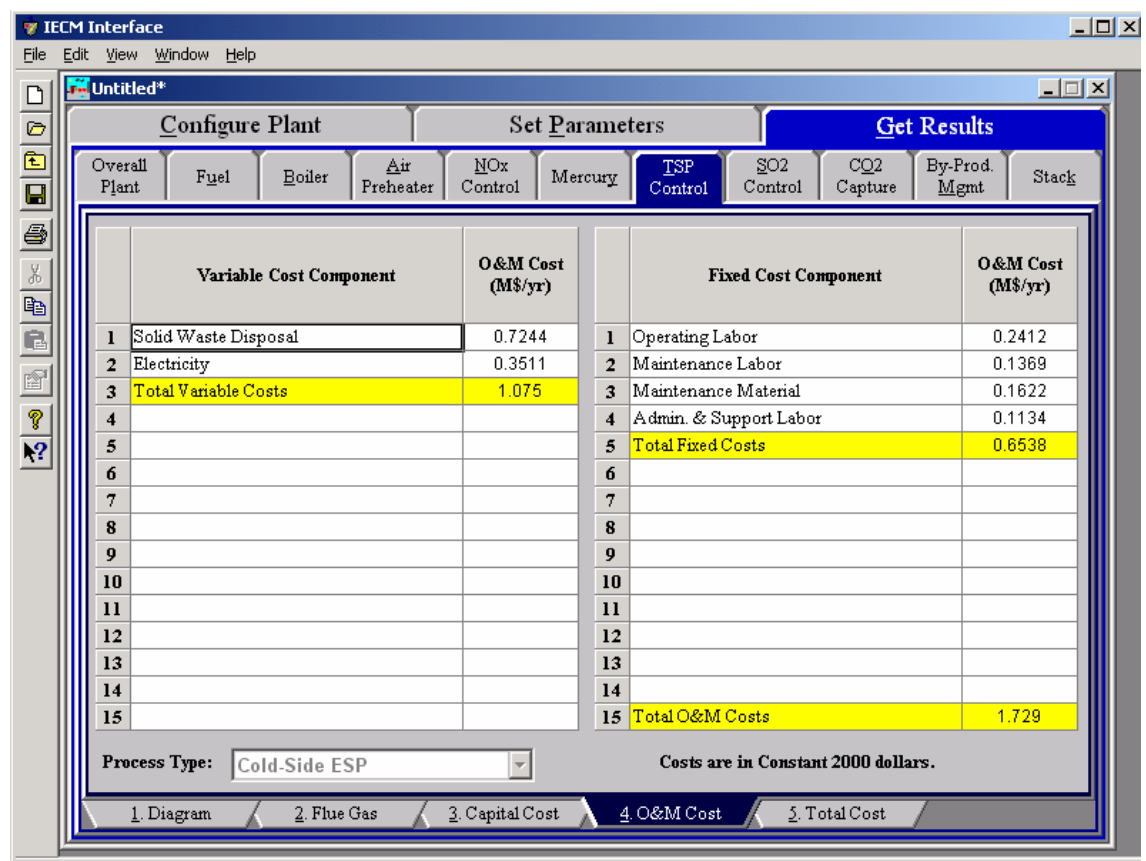

Cold-Side ESP - O\&M Cost result screen.

Each result is described briefly below

\section{Variable Cost Component}

Variable operating costs and consumables are directly proportional to the amount of kilowatts produced and are referred to as incremental costs. All the costs are subject to inflation.

Solid Waste Disposal: Total cost to dispose the collected fly ash. This does not consider by-product ash sold in commerce.

Power: Cost of power consumption of the particulate control technology. This is a function of the flue gas flow rate, ash removal efficiency and the type of coal (ash properties).

Total Variable Costs: This is the sum of all the variable O\&M costs listed above. This result is highlighted in yellow.

\section{Fixed Cost Components}

Fixed operating costs are essentially independent of actual capacity factor, number of hours of operation, or amount of kilowatts produced. All the costs are subject to inflation.

Operating Labor: Operating labor cost is based on the operating labor rate, the number of personnel required to operate the plant per eighthour shift, and the average number of shifts per day over 40 hours per week and 52 weeks.

Maintenance Labor: The maintenance labor is determined as a fraction of the total maintenance cost.

Maintenance Material: The cost of maintenance material is the remainder of the total maintenance cost, considering the fraction associated with maintenance labor. 
Admin. \& Support Labor: The administrative and support labor is the only overhead charge. It is taken as a fraction of the total operating and maintenance labor costs.

Total Fixed Costs: This is the sum of all the fixed O\&M costs listed above. This result is highlighted in yellow.

Total O\&M Costs: This is the sum of the total variable and total fixed O\&M costs. It is used to determine the base plant total revenue requirement. This result is highlighted in yellow.

\section{Cold-Side ESP Total Cost Results}

The Total Cost result screen displays a table which totals the annual fixed, variable, operations and maintenance, and capital costs associated with the Cold-Side ESP TSP Control technology.

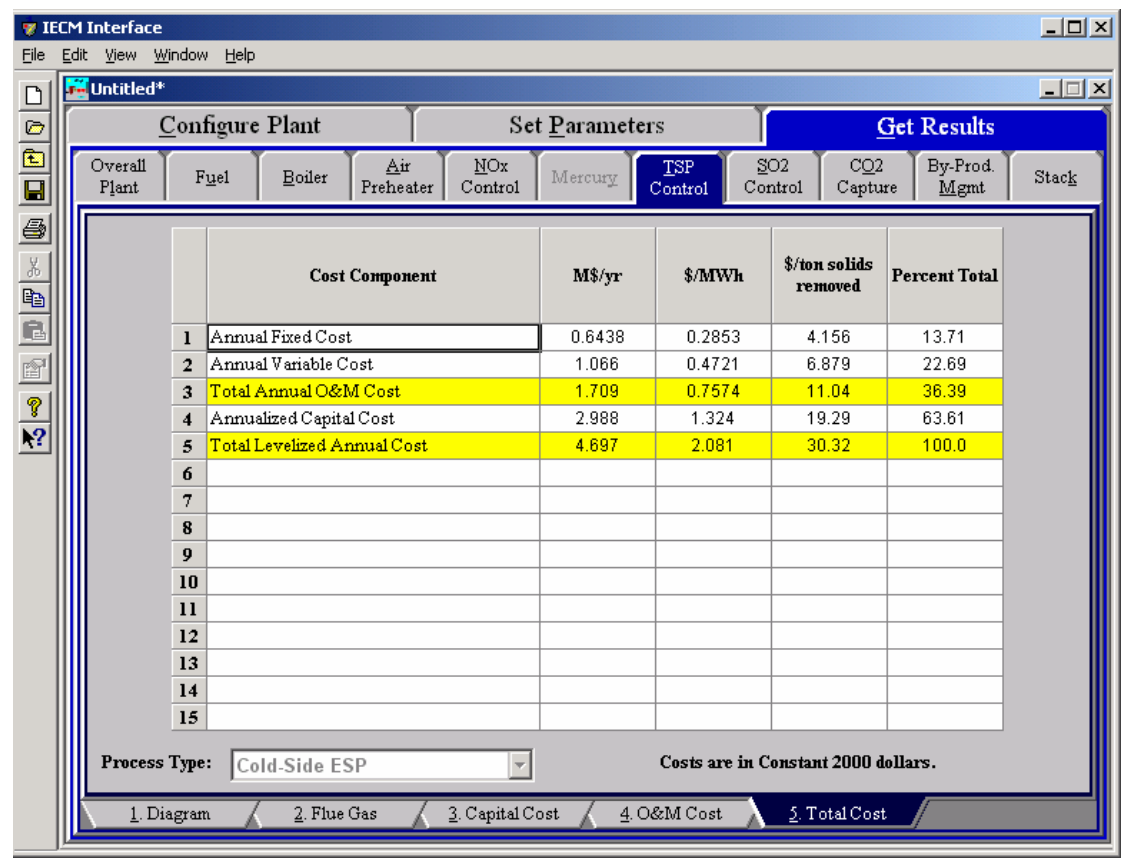

Cold-Side ESP - Total Cost result screen.

Each result is described briefly below.

\section{Cost Component}

Annual Fixed Cost: The operating and maintenance fixed costs are given as an annual total. This number includes all maintenance materials and all labor costs.

Annual Variable Cost: The operating and maintenance variables costs are given as an annual total. This includes all reagent, chemical, steam, and power costs.

Total Annual O\&M Cost: This is the sum of the annual fixed and variable operating and maintenance costs above. This result is highlighted in yellow. 
Annualized Capital Cost: This is the total capital cost expressed on an annualized basis, taking into consideration the levelized carrying charge factor, or fixed charge factor, over the entire book life.

Total Levelized Annual Cost: The total annual cost is the sum of the total annual O\&M cost and annualized capital cost items above. This result is highlighted in yellow. 



\section{Fabic Filter}

The TSPControl Technology Navigation Tab contains screens that design and display flows and costs related to the particulate control technology.

\section{Fabric Filter Configuration}

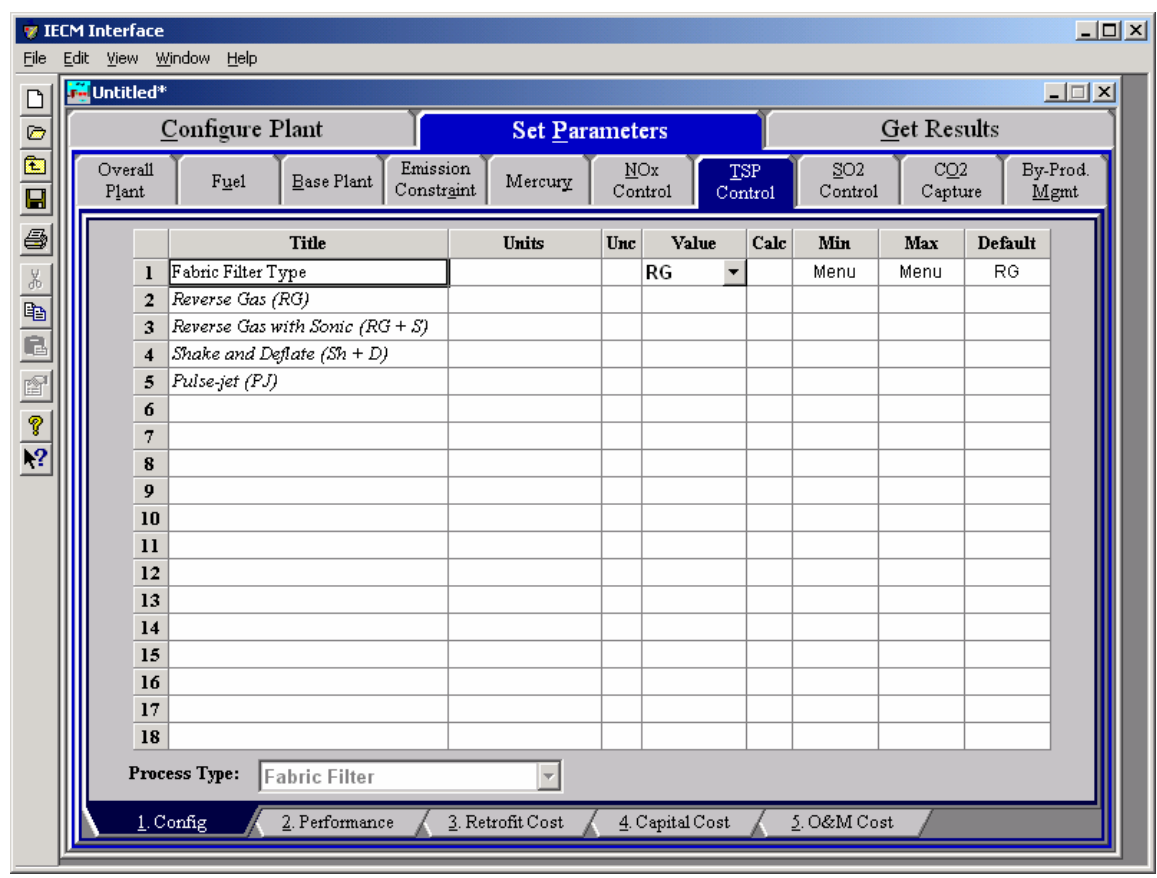

Fabric Filter - Configuration input screen.

\section{Fabric Filter Type}

Fabric filters consist of a large number of long tubular filter bags arranged in parallel flow paths. As the ash-laden flue gas passes through these filters, much of the particulate matter is removed. Ash accumulated on the bags is removed periodically by various methods of cleaning. Choose the cleaning method in the Config. input screen. The available methods are:

- $\quad$ Reverse Gas (RG)

- Reverse Gas with Sonic (RG + S)

- $\quad$ Shake and Deflate $(\mathrm{Sh}+\mathrm{D})$ 


\section{Fabric Filter Performance Inputs}

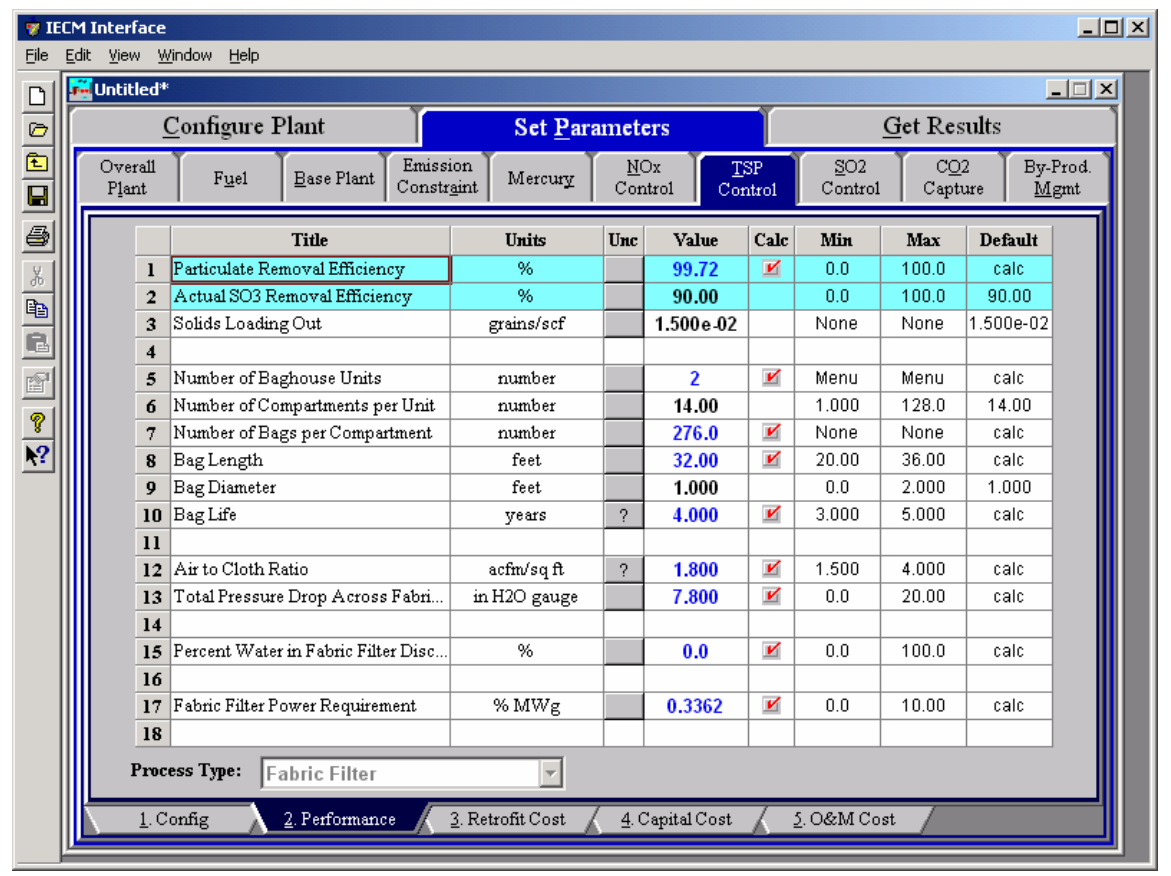

Fabric Filter - Performance input screen.

The baghouse system is very efficient in removing particulate matter from the flue gas. It's model design is simple, requiring few parameters to characterize its effects on the overall performance of the plant. For properly designed fabric filters, the size of the system is independent of the removal efficiency.

Although the performance is determined by very few parameters, there are several design parameters necessary to determine the cost. These factors are also determined in this section. The major design parameters that can significantly impact the total system cost of the fabric filter are gas flow volume (which depends on the generating unit size), A/C ratio, the flange-to-flange pressure drop in the baghouse and the bag life.

Particulate Removal Efficiency: The calculated removal is set to comply with the particulate emission limit set earlier. The mass removed is then determined. If you select a spray dryer, the particulate removal efficiency applies to the combined mass of flyash and sulfurladen wastes. This input is highlighted in blue.

Actual $\mathrm{SO}_{3}$ Removal Efficiency: The default value is taken from the removal efficiency reported by Southern Company. ${ }^{6}$ This efficiency then determines the mass of $\mathrm{SO}_{3}$ removed from the flue gas in the collector.

Solids Loading Out: This is the fabric filter output loading. It is an average value based on typical fabric filter units. The value is used to determine the particulate removal efficiency.

${ }^{6}$ Ibid. 
Number of Baghouse Units: This is the number of baghouse units. The value is based on the gross plant size. The value must be an integer. Each unit contains several compartments. It is used to calculate the capital cost of the baghouse.

Number of Compartments per Unit: This parameter specifies the average number of compartments used per baghouse unit. It is used to calculate the capital cost of the baghouse.

Number of Bags per Compartment: The number of individual bags per compartment is calculated by comparing the required bag surface area to the bag dimensions and the total number of compartments. It is used to calculate the capital cost of the baghouse.

Bag Length: Bag length generally fall into two size categories: 30-36 ft or $20-22 \mathrm{ft}$ in length. It is based on the fabric filter type and used to calculate the capital cost of the baghouse.

Bag Diameter: Bags are generally between 2/3 and 1 foot in diameter. The value is based on the fabric filter type and used to calculate the capital cost of the baghouse.

Bag Life: Bag life is typically between 3-5 years. The bag life values are dependent on the fabric filter type and are used to calculate the cost of the baghouse.

Air to Cloth Ratio: The Air to Cloth ratio is the most important baghouse parameter. It is the ratio of volumetric flue gas flow rate and total bag cloth area. The calculated value is a function of fabric filter type. It is used to determine the cost and energy use of the baghouse.

Total Pressure Drop across Fabric Filter: Baghouse pressure drop (flange-to-flange) is caused by pressure losses in gas flow as it moves through the bag fabric and dust cake. Typical values range from 6 to 8 in. $\mathrm{H}_{2} \mathrm{O}$ and depend on the baghouse type selected. The value affects the power consumption.

Percent Water in Fabric Filter Discharge: This is the water content of the collected fly ash. Fly ash disposed with bottom ash is assumed to be sluiced with water and dry otherwise. The occluded water in wet fly ash is difficult to remove, resulting in a rather high water content when the fly ash is mixed with bottom ash.

Fabric Filter Power Requirement: The default calculation is based on the air-to-cloth ratio and the flue gas flow rate. The energy accounts for the auxiliary energy requirements and electro-mechanical efficiencies of fan motors.

\section{Fabric Filter Retrofit Inputs}

Inputs for the capital costs of modifications to process areas to implement the Particulate control technology are entered on the Retrofit Cost input screen. All costs are in constant 1996 dollars.

The retrofit cost factor of each process is a multiplicative cost adjustment, which considers the cost of retrofitted capital equipment relative to similar equipment installed in a new plant. These factors affect the capital costs directly and the operating and maintenance costs indirectly. 
Direct capital costs for each process area are calculated in the IECM. These calculations are reduced form equations derived from more sophisticated models and reports. The sum of the direct capital costs associated with each process area is defined as the process facilities capital (PFC). The retrofit cost factor provided for each of the process areas can be used as a tool for adjusting the anticipated costs and uncertainties across the process area separate from the other areas.

Uncertainty can be applied to the retrofit cost factor for each process area in each technology. Thus, uncertainty can be applied as a general factor across an entire process area, rather than as a specific uncertainty for the particular cost on the capital or O\&M input screens. Any uncertainty applied to a process area through the retrofit cost factor compounds any uncertainties specified later in the capital and O\&M cost input parameter screens.

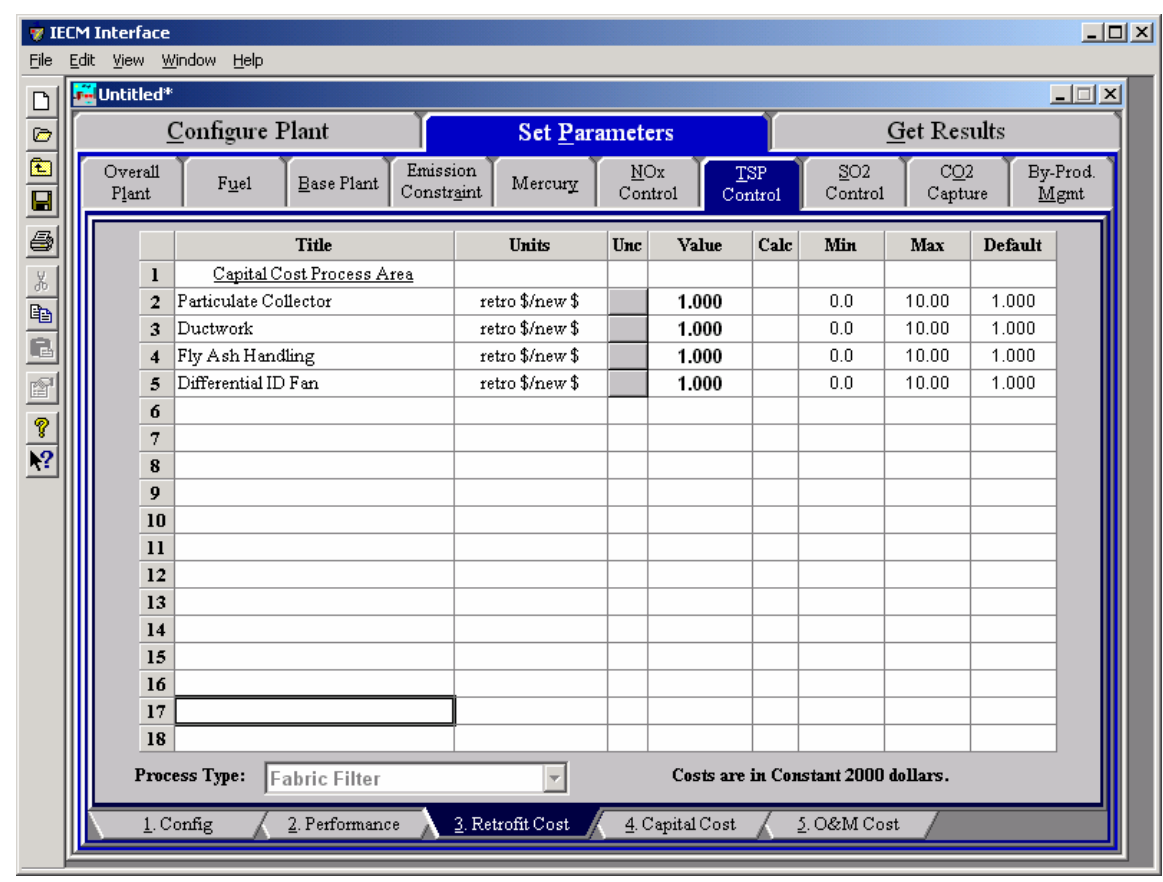

Fabric Filter - Retrofit Cost input screen.

\section{Capital Cost Process Area}

Particulate Collector: This is the cost for the collecting equipment, based on actual vendor prices. Included in the cost are the mechanical equipment and labor, particulate removal system, alternate cleaning system, gas conditioning system, structural supports, electrical, and instrumentation.

Ductwork: This is the cost of all the mechanical, electrical, and supports of the ductwork to and from the collector.

Fly Ash Handling: This is the cost of all the mechanical, conveyors, storage, and electrical portions of the ash handling system. The costs are based on actual vendor prices.

Differential ID Fan: This area includes the additional cost of the ID fan and the motor due to the pressure loss that results from the particulate collectors. Also included are the erection, piping, electrical, and foundation costs. 


\section{Fabric Filter Capital Cost Inputs}

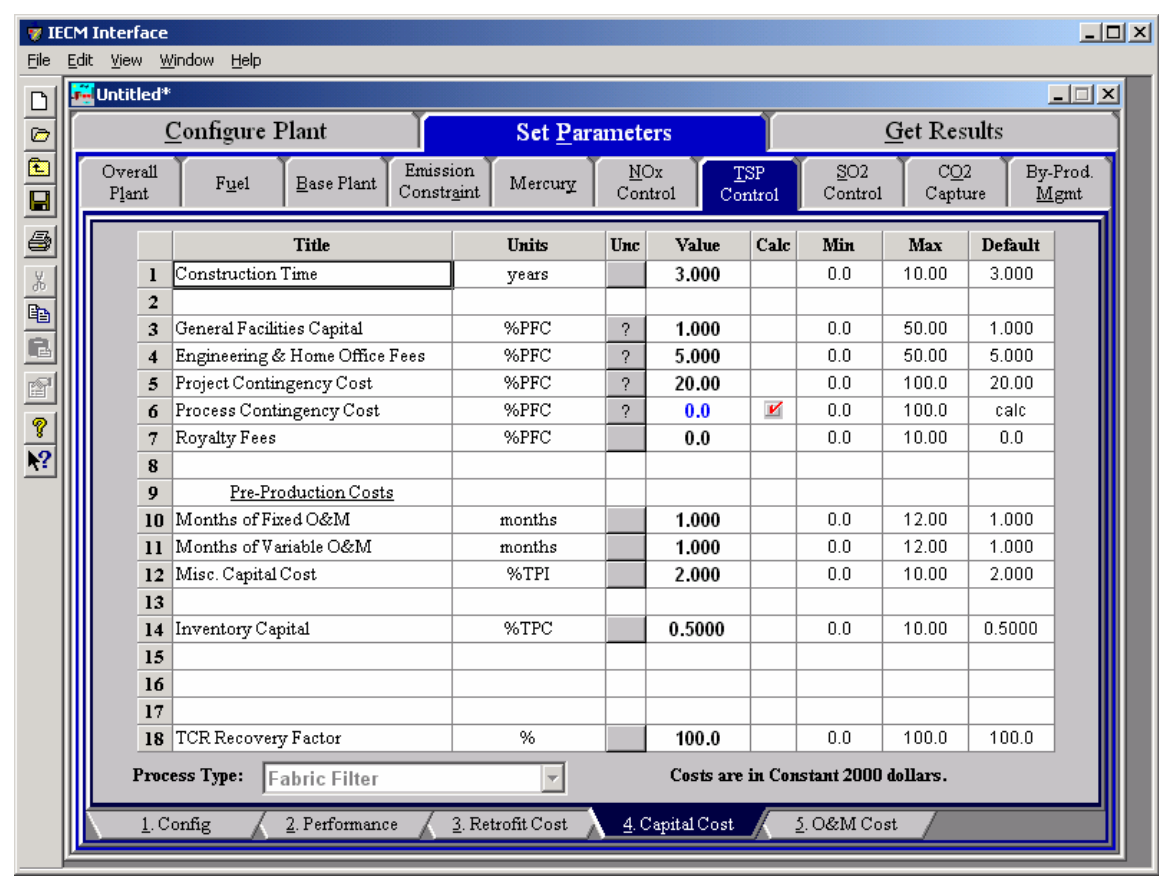

Fabric Filter - Capital Cost input screen.

The necessary capital cost input parameters associated with the fabric filter control technology are shown on this input screen (no distinction is made between the various types of fabric filtersEach parameter is described briefly below.

Indirect Capital Costs: Costs that are indirectly applied to the technology are based on the process facilities cost. Each of the cost factors below is expressed as a percentage of the process facilities cost, and is entered on this screen. Each parameter is described briefly below.

Construction Time: This is the idealized construction period in years. It is used to determine the allowance for funds used during construction (AFUDC).

General Facilities Capital (GFC): The general facilities include construction costs of roads, office buildings, shops, laboratories, etc. Sales taxes and freight costs are included implicitly. The cost typically ranges from $5-20 \%$.

Engineering \& Home Office Fees: The engineering \& home office fees are a percent of total direct capital cost. This is an overhead fee paid to the architect/engineering company. These fees typically range from 7$15 \%$.

Project Contingency Cost: This is factor covering the cost of additional equipment or other costs resulting from a more detailed design. Higher contingency factors will be applied to simplified or preliminary designs and lower factors to detailed or finalized designs.

Process Contingency Cost: This quantifies the design uncertainty and cost of a commercial-scale system. This is generally applied on an areaby-area basis. Higher contingency factors are applied to new 
regeneration systems tested at a pilot plant and lower factors to full-size or commercial systems.

Royalty Fees: Royalty charges may apply to some portions of generating units incorporating new proprietary technologies.

\section{Pre-Production Costs}

These costs consider the operator training, equipment checkout, major changes in unit equipment, extra maintenance, and inefficient use of fuel or other materials during start-up. These are typically applied to the O\&M costs over a specified period of time (months)The two time periods for fixed and variable O\&M costs are described below with the addition of a miscellaneous capital cost factor.

Months of Fixed O\&M: Time period of fixed operating costs used for preproduction to cover training, testing, major changes in equipment, and inefficiencies in start-up. This includes operating, maintenance, administrative and support labor. It also considers maintenance materials.

Months of Variable O\&M: Time period of variable operating costs used for preproduction to cover chemicals, water, consumables, and solid disposal charges in start-up, assuming $100 \%$ load. This excludes any fuels.

Misc. Capital Costs: This is a percent of total plant investment (sum of TPC and AFUDC) to cover expected changes to equipment to bring the system up to full capacity.

Inventory Capital: Percent of the total direct capital for raw material supply based on $100 \%$ capacity during a 60 day period. These materials are considered storage. The inventory capital includes fuels, consumables, by-products, and spare parts. This is typically $0.5 \%$.

TCR Recovery Factor: The actual total capital required (TCR) as a percent of the TCR in a new power plant. This value is $100 \%$ for a new installation and may be set as low as $0 \%$ for a fabric filter that has been paid off. 


\section{Fabric Filter O\&M Cost Inputs}

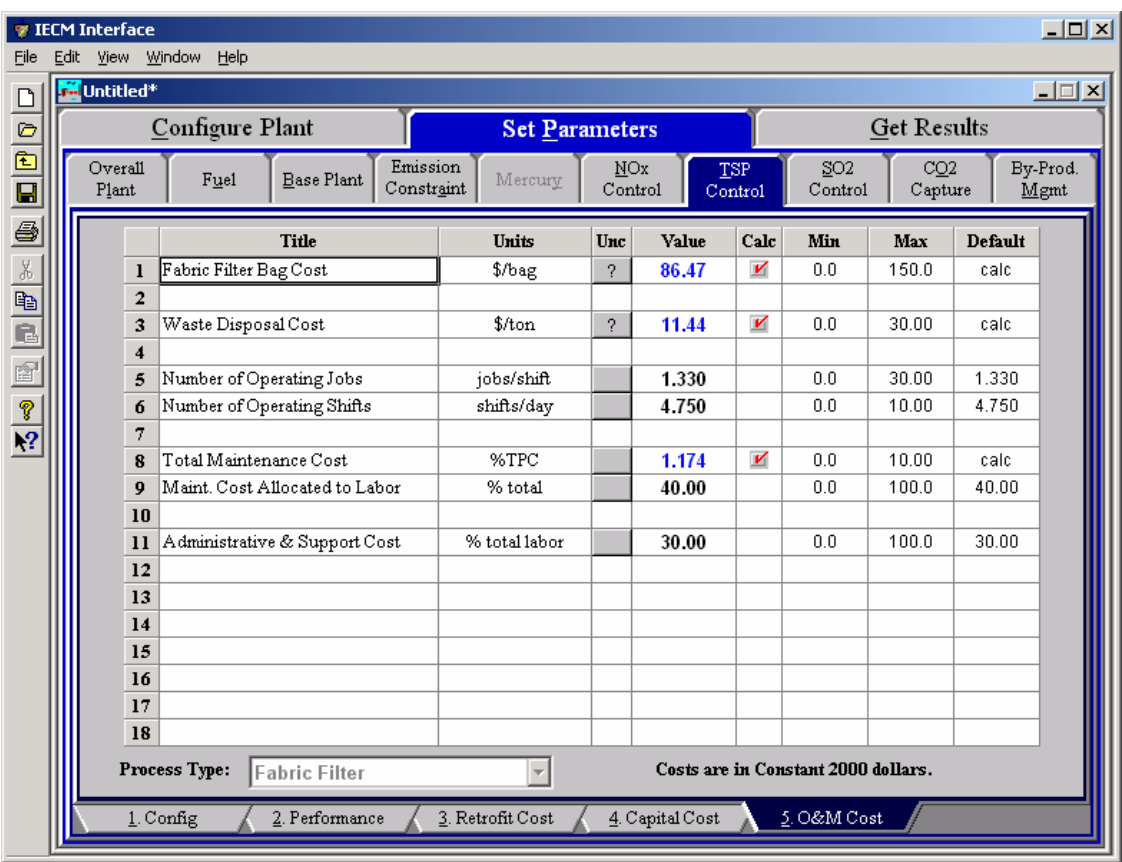

Fabric Filter - O\&M Cost input screen.

Fabric Filter Bag Cost: This is the cost of a fabric filter bag as used for the fabric filter technology.

Waste Disposal Cost: This is the disposal cost for the particulate control system.

Number of Operating Jobs: This is the total number of operating jobs that are required to operate the plant per eight-hour shift.

Number of Operating Shifts: This is the total number of equivalent operating shifts in the plant per day. The number takes into consideration paid time off and weekend work ( 3 shifts/day * 7 days/5 day week $* 52$ weeks $/(52$ weeks -6 weeks PTO $)=4.75$ equiv. Shifts/day)

Total Maintenance Cost: This is the annual maintenance cost as a percentage of the total plant cost. Maintenance cost estimates can be developed separately for each process area.

Maint. Cost Allocated to Labor: Maintenance cost allocated to labor as a percentage of the total maintenance cost.

Administrative \& Support Cost: This is the percent of the total operating and maintenance labor associated with administrative and support labor.

\section{Fabric Filter Diagram}

The Diagram result screen displays an icon for the Fabric Filter particulate control technology selected and values for major flows in and out of it. 


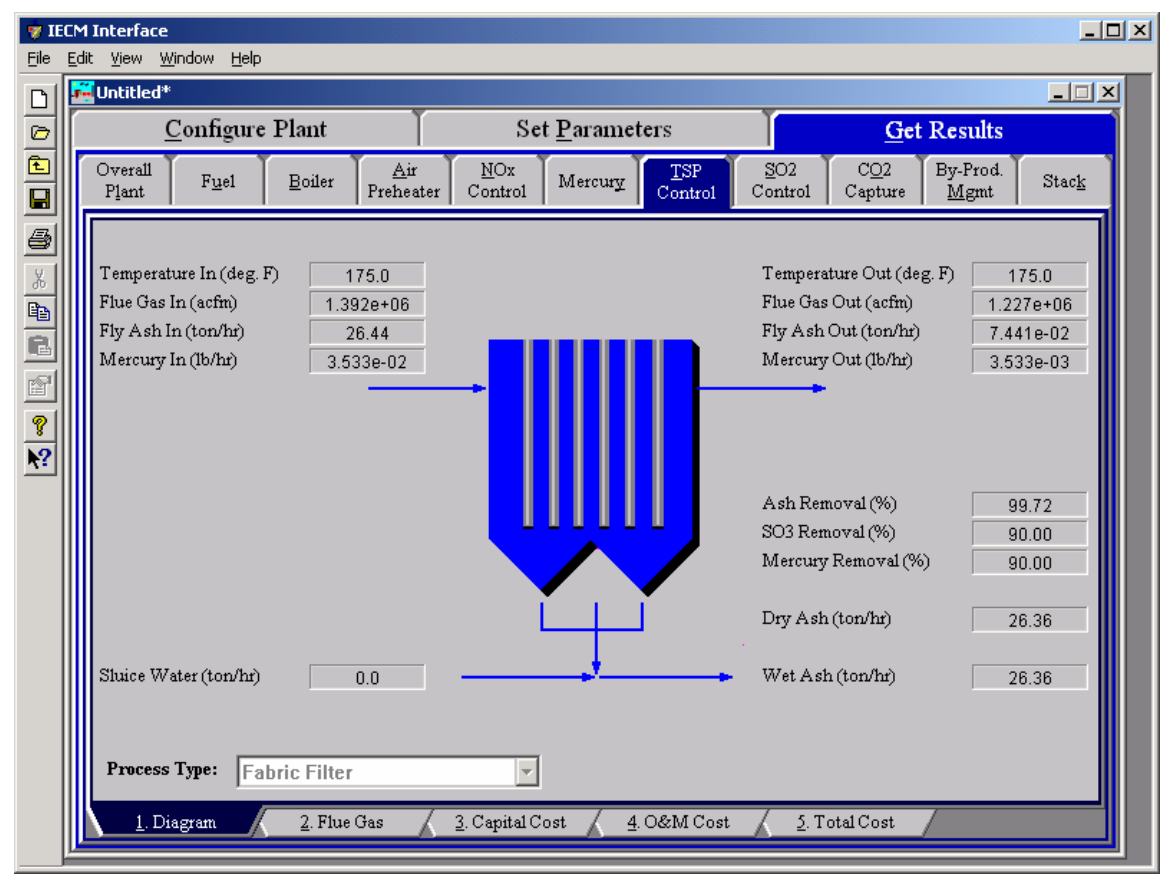

Fabric Filter - Diagram

Each result is described briefly below

\section{Flue Gas Entering Filter}

Temperature In: Temperature of the flue gas entering the particulate control technology. This is determined by the flue gas outlet temperature of the module upstream of the air preheater (e.g., the air preheater).

Flue Gas In: Volumetric flow rate of the flue gas entering the particulate control technology, based on the flue gas inlet temperature and atmospheric pressure.

Fly Ash In: Total solids mass flow rate in the flue gas entering the air preheater. This is determined by the solids exiting the module upstream of the particulate control technology (e.g., the air preheater).

Mercury In: Total mass of mercury entering the particulate control technology. The value is a sum of all the forms of mercury (elemental, oxidized, and particulate).

\section{Flue Gas Exiting Filter}

Temperature Out: Temperature of the flue gas exiting the particulate control technology. The model currently does not alter this temperature through the particulate control technology.

Flue Gas Out: Volumetric flow rate of the flue gas exiting the particulate control technology, based on the flue gas exit temperature and atmospheric pressure.

Fly Ash Out: Total solids mass flow rate in the flue gas exiting the particulate control technology. This is a function of the ash content of the inlet flue gas and the ash removal efficiency performance input parameter. 
Mercury Out: Total mass of mercury exiting the particulate control technology. The value is a sum of all the forms of mercury (elemental, oxidized, and particulate).

\section{Fabric Filter Performance}

Ash Removal: Ash removal efficiency of the fabric filter technology. This is a function of the ash emission constraint and the inlet ash mass flow rate.

$\mathrm{SO}_{3}$ Removal: Percent of $\mathrm{SO}_{3}$ in the flue gas removed from the particulate control technology. The $\mathrm{SO}_{3}$ is assumed to combine with $\mathrm{H}_{2} \mathrm{O}$ and leave with the ash solids as a sulfate (in the form of $\mathrm{H}_{2} \mathrm{SO}_{4}$ ).

Mercury Removal: Percent of the total mercury removed from the particulate control technology. The value reflects a weighted average based on the particular species of mercury present (elemental, oxidized, and particulate).

\section{Collected Fly Ash}

Dry Ash: Total mass flow rate of the solids removed from the fabric filter. This is a function of the solids content in the flue gas and the particulate removal efficiency of the fabric filter. The value is given on a dry basis.

Sluice Water: Water added to the dry fly ash. This water is added for transportation purposes.

Wet Ash: Total mass flow rate of the solids removed for waste management. This includes dry fly ash and sluice water. The value is given on a wet basis.

\section{Fabric Filter Flue Gas Results}

The Flue Gas result screen displays a table of quantities of flue gas components entering and exiting the Particulate Control Technology. For each component, quantities are given in both moles and mass per hour. 


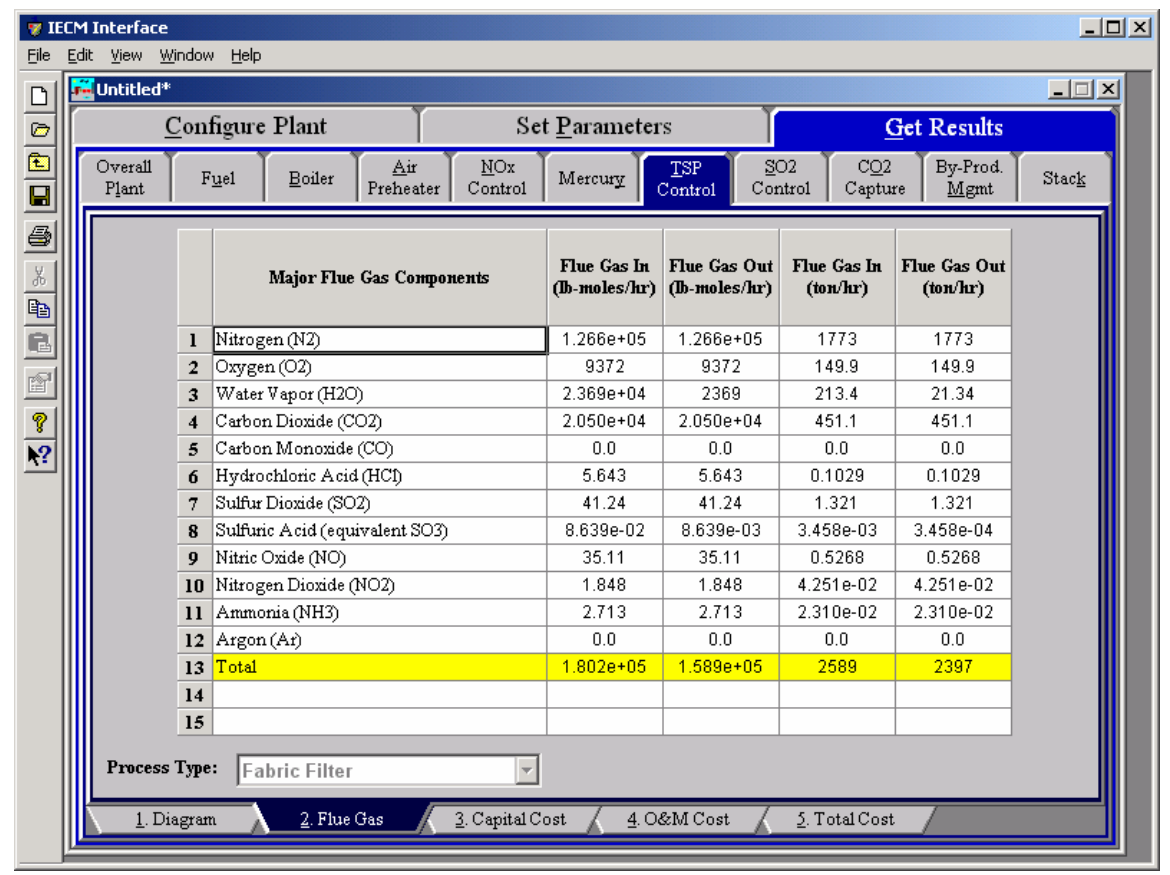

Fabric Filter - Flue Gas result screen.

Each result is described briefly below

Nitrogen $\left(\mathbf{N}_{\mathbf{2}}\right)$ : Total mass of nitrogen.

Oxygen $\left(\mathrm{O}_{2}\right)$ : Total mass of oxygen.

Water Vapor $\left(\mathbf{H}_{2} \mathbf{O}\right)$ : Total mass of water vapor.

Carbon Dioxide $\left(\mathrm{CO}_{2}\right)$ : Total mass of carbon dioxide.

Carbon Monoxide (CO): Total mass of carbon monoxide.

Hydrochloric Acid (HCl): Total mass of hydrochloric acid.

Sulfur Dioxide $\left(\mathbf{S O}_{\mathbf{2}}\right)$ : Total mass of sulfur dioxide.

Sulfuric Acid (equivalent $\mathbf{S O}_{3}$ ): Total mass of sulfuric acid.

Nitric Oxide (NO): Total mass of nitric oxide.

Nitrogen Dioxide $\left(\mathrm{NO}_{2}\right)$ : Total mass of nitrogen dioxide.

Ammonia (NH3):Total mass of ammonia.

Argon (Ar): Total mass of argon.

Total: Total of the individual components listed above. This item is highlighted in yellow.

\section{Fabric Filter Capital Cost Results}

The Capital Cost result screen displays tables for the direct and indirect capital costs related to the particulate control technology. 


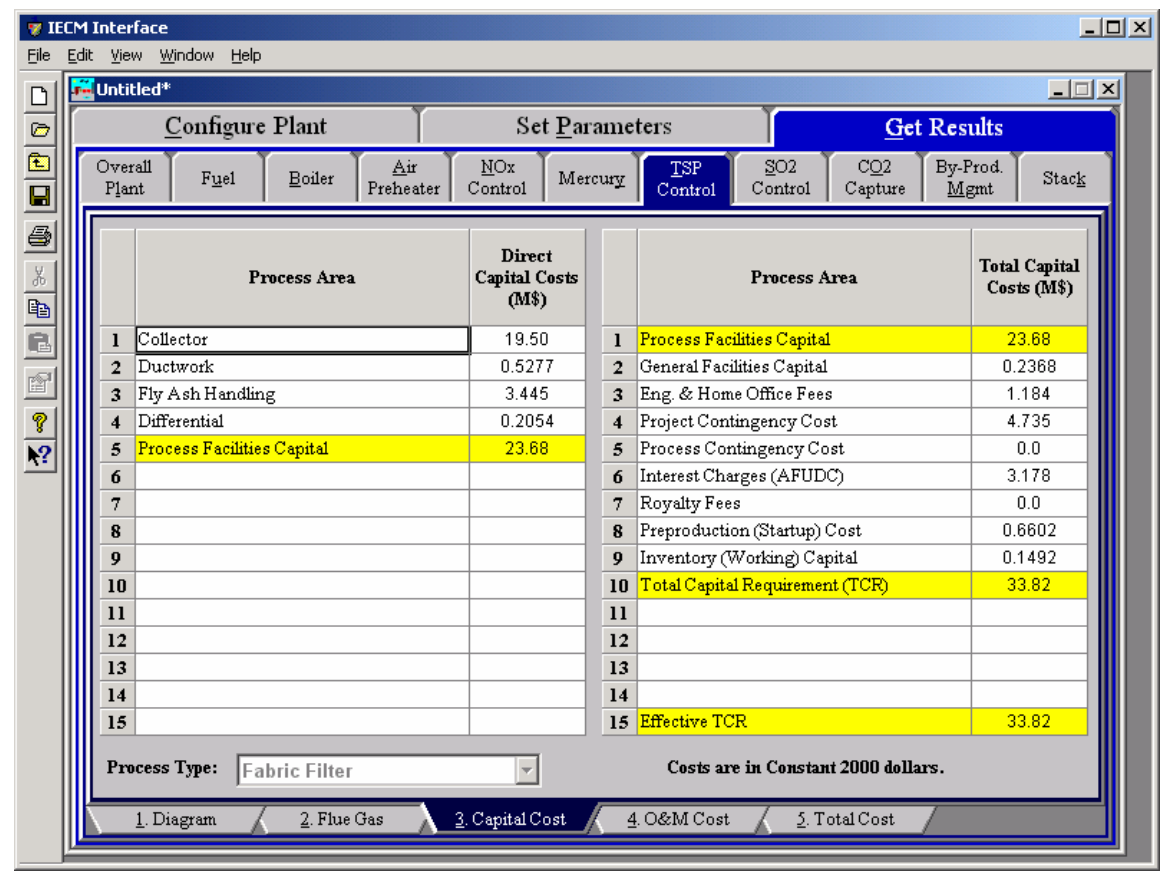

Fabric Filter - Capital Cost result screen.

Each result is described briefly below

\section{Direct Capital Costs}

Each process area direct capital cost is a reduced-form model based on regression analysis of data collected from several reports and analyses of particulate control technology units. They are described in general below. The primary factors in the model that effect the capital costs of the cold-side ESP are the specific and total collection areas of the T-R plate sets, and the flue gas flow rate through the ESP. The primary model factors that effect the capital costs of the fabric filter are the fabric filter type, the air to cloth ratio, the number of bags and compartments, and the flue gas flow rate through the fabric filter.

Collector: This area covers the material and labor, flange to flange, for the equipment and labor cost for installation of the entire collection system.

Ductwork: This area includes the material and labor for the ductwork needed to distribute flue gas to the inlet flange, and from the outlet flange to a common duct leading to the suction side of the ID fan.

Fly Ash Handling: The complete fly ash handling cost includes the conveyor system and ash storage silos.

Differential: The complete cost of the ID fan and motor due to the pressure loss that results from particulate collectors.

Process Facilities Capital: The process facilities capital is the total constructed cost of all on-site processing and generating units listed above, including all direct and indirect construction costs. All sales taxes and freight costs are included where applicable implicitly. This result is highlighted in yellow.

Total Capital Costs

Process Facilities Capital: (see definition above) 
General Facilities Capital: The general facilities include construction costs of roads, office buildings, shops, laboratories, etc. Sales taxes and freight costs are included implicitly.

Eng. \& Home Office Fees: The engineering \& home office fees are a percent of total direct capital cost. This is an overhead fee paid to the architect/engineering company.

Project Contingency Cost: Capital cost contingency factor covering the cost of additional equipment or other costs that would result from a more detailed design of a definitive project at the actual site.

Process Contingency Cost: Capital cost contingency factor applied to a new technology in an effort to quantify the uncertainty in the technical performance and cost of the commercial-scale equipment.

Interest Charges (AFUDC): Allowance for funds used during construction, also referred to as interest during construction, is the time value of the money used during construction and is based on an interest rate equal to the before-tax weighted cost of capital. This interest is compounded on an annual basis (end of year) during the construction period for all funds spent during the year or previous years.

Royalty Fees: Royalty charges may apply to some portions of generating units incorporating new proprietary technologies.

Preproduction (Startup) Cost: These costs consider the operator training, equipment checkout, major changes in unit equipment, extra maintenance, and inefficient use of fuel or other materials during startup.

Inventory (Working) Capital: The raw material supply based on $100 \%$ capacity during a 60 day period. These materials are considered storage. The inventory capital includes fuels, consumables, byproducts, and spare parts.

Total Capital Requirement (TCR): Money that is placed (capitalized) on the books of the utility on the service date. TCR includes all the items above. This result is highlighted in yellow.

Effective TCR: The TCR of the fabric filter that is used in determining the total power plant cost. The effective TCR is determined by the "TCR Recovery Factor” for the fabric filter.

\section{Fabric Filter O\&M Cost Results}

The O\&M Cost result screen displays tables for the variable and fixed operation and maintenance costs involved with the particulate control technology. 


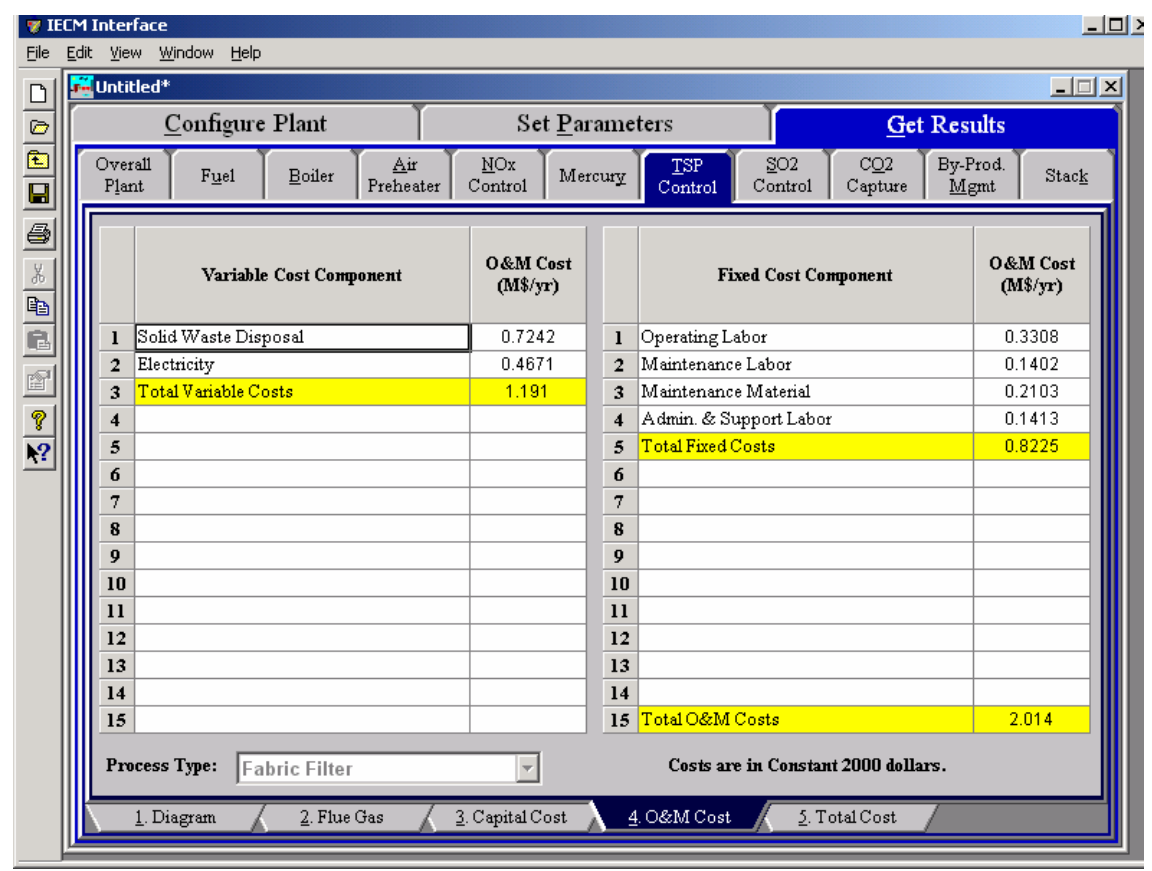

Fabric Filter - O\&M Cost result screen.

Each result is described briefly below:

\section{Variable Cost Component}

Variable operating costs and consumables are directly proportional to the amount of kilowatts produced and are referred to as incremental costs. All the costs are subject to inflation.

Solid Waste Disposal: Total cost to dispose the collected fly ash. This does not consider by-product ash sold in commerce.

Electricity: Cost of power consumption of the particulate control technology. This is a function of the flue gas flow rate, ash removal efficiency and the type of coal (ash properties).

Total Variable Costs: This is the sum of all the variable O\&M costs listed above. This result is highlighted in yellow.

\section{Fixed Cost Component}

Fixed operating costs are essentially independent of actual capacity factor, number of hours of operation, or amount of kilowatts produced. All the costs are subject to inflation.

Operating Labor: Operating labor cost is based on the operating labor rate, the number of personnel required to operate the plant per eighthour shift, and the average number of shifts per day over 40 hours per week and 52 weeks.

Maintenance Labor: The maintenance labor is determined as a fraction of the total maintenance cost.

Maintenance Material: The cost of maintenance material is the remainder of the total maintenance cost, considering the fraction associated with maintenance labor. 
Admin. \& Support Labor: The administrative and support labor is the only overhead charge. It is taken as a fraction of the total operating and maintenance labor costs.

Total Fixed Costs: This is the sum of all the fixed O\&M costs listed above. This result is highlighted in yellow.

Total O\&M Costs: This is the sum of the total variable and total fixed O\&M costs. It is used to determine the base plant total revenue requirement. This result is highlighted in yellow.

\section{Fabric Filter Total Cost Results}

The Total Cost result screen displays a table which totals the annual fixed, variable, operations and maintenance, and capital costs associated with the Particulate Control technology. The result categories are the same for both the Cold-Side ESP and the Fabric Filter

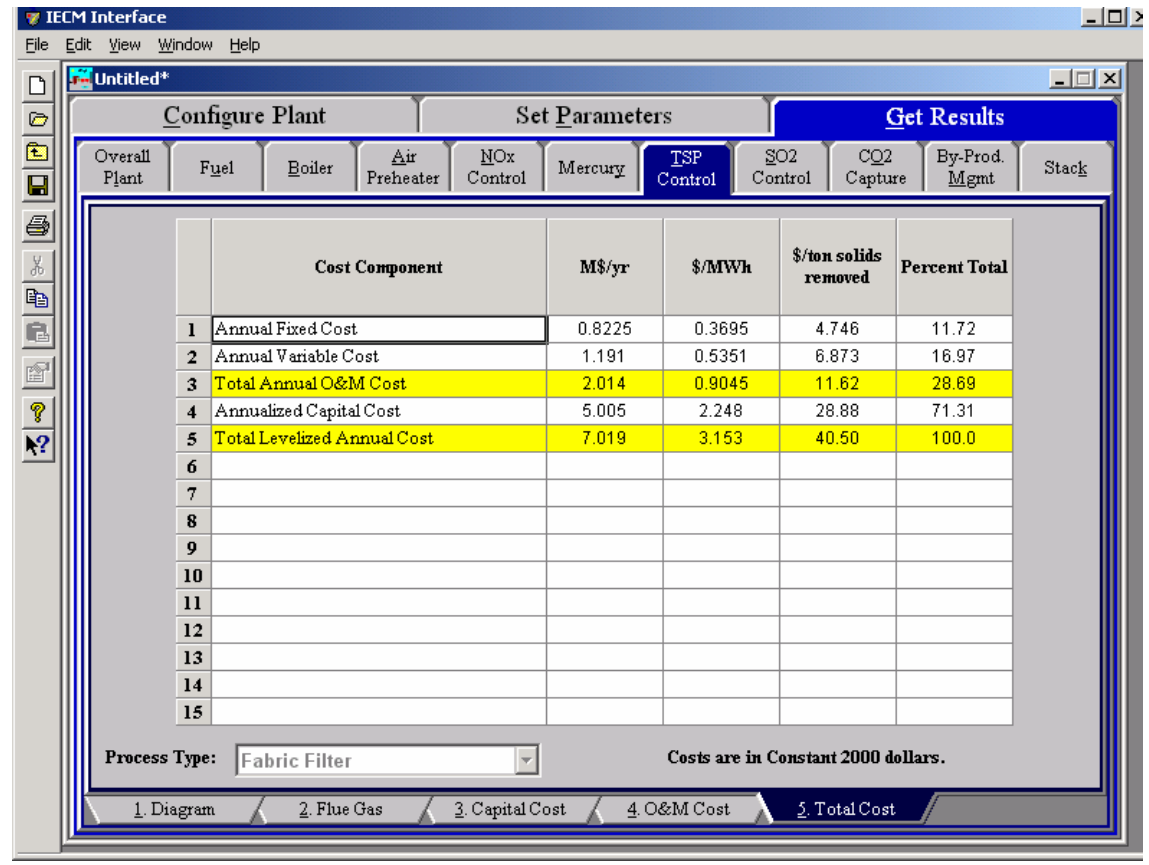

Fabric Filter - Total Cost result screen.

Each result is described briefly below.

\section{Cost Component}

Annual Fixed Cost: The operating and maintenance fixed costs are given as an annual total. This number includes all maintenance materials and all labor costs.

Annual Variable Cost: The operating and maintenance variables costs are given as an annual total. This includes all reagent, chemical, steam, and power costs.

Total Annual O\&M Cost: This is the sum of the annual fixed and variable operating and maintenance costs above. This result is highlighted in yellow. 
Annualized Capital Cost: This is the total capital cost expressed on an annualized basis, taking into consideration the levelized carrying charge factor, or fixed charge factor, over the entire book life.

Total Annual Cost: The total annual cost is the sum of the total annual O\&M cost and annualized capital cost items above. This result is highlighted in yellow. 



\section{Wet FGD}

The So2 2 Control Technology Navigation contains screens that address postcombustion air pollution technologies for Sulfur Dioxide. The model includes options for a Wet FGD. The screens are available if this $\mathrm{SO}_{2}$ control technology has

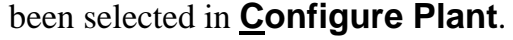

\section{Wet FGD Configuration}

Inputs for configuration of the Wet $\mathrm{FGD} \mathrm{SO}_{2}$ control technology are entered on the Config input screen.

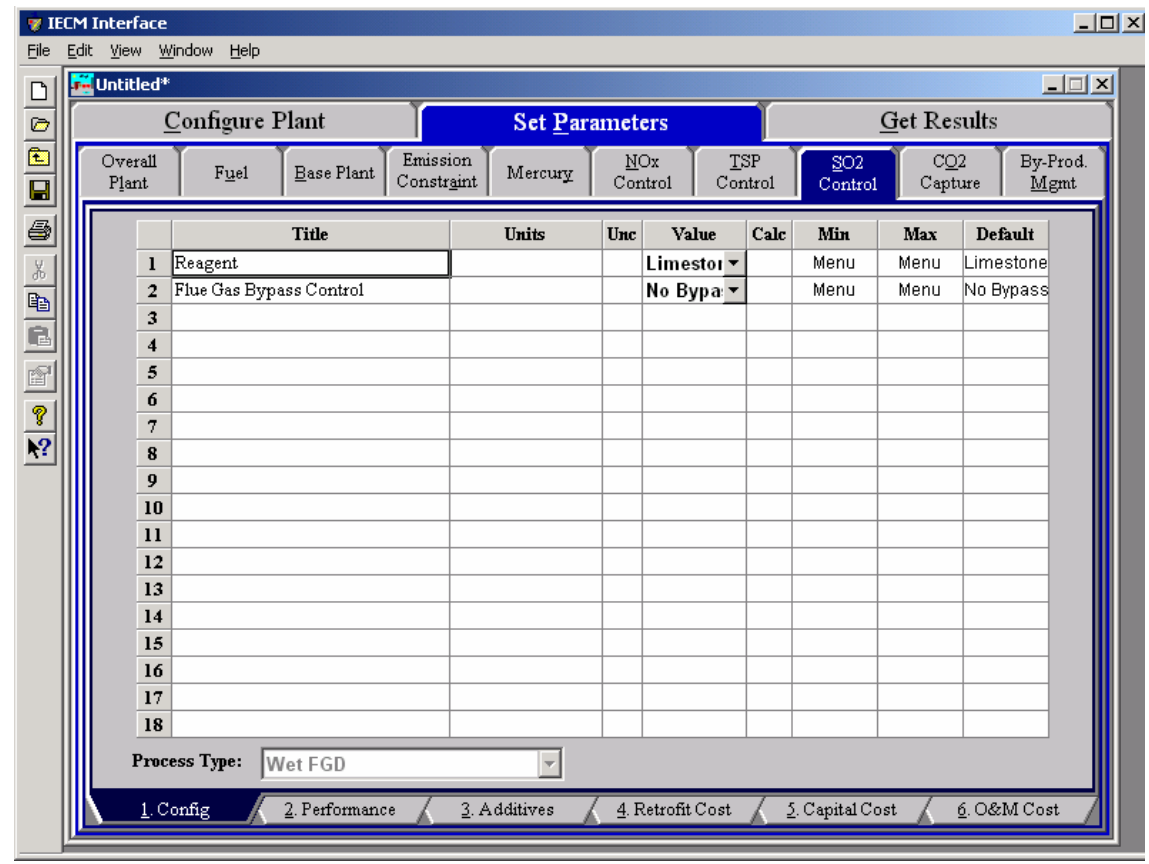

Wet FGD - Config. input screen.

Each parameter is described briefly below.

\section{Reagent}

For Wet FGD systems, the choice of reagent affects nearly all of the performance and economic parameters of the FGD. Three choices are available: 
Limestone: Limestone with Forced Oxidation-A limestone slurry is used in an open spray tower with in-situ oxidation to remove $\mathrm{SO}_{2}$ and form a gypsum sludge. The main advantages as compared to conventional systems are easier dewatering, more economical disposal of scrubber products, and decreased scaling on tower walls.

Limestone with Additivess: Limestone with Dibasic Acid AdditiveDibasic acid (DBA) is added to the Limestone to act as a buffer/catalyst in the open spray tower. The main advantages are increased $\mathrm{SO}_{2}$ removal and decreased liquid to gas ratio.

Lime: Magnesium Enhanced Lime System-A magnesium sulfite and lime slurry (maglime) is used to remove $\mathrm{SO}_{2}$ and form a precipitate high in calcium sulfite. The high alkalinity of the maglime slurry allows very high $\mathrm{SO}_{2}$ removal. However, the reagent cost is also higher and solid waste is not easily disposed.

\section{Flue Gas Bypass Control}

Popup selection menu that controls whether or not a portion of the inlet flue gas may bypass the scrubber and recombine with the treated flue gas. Bypass allows the scrubber to operate at full efficiency while allowing some of the flue gas to go untreated.

\section{Wet FGD Performance Inputs}

Inputs for performance of the Wet FGD $\mathrm{SO}_{2}$ control technology are entered on the Performance input screen. Each parameter is described briefly below.

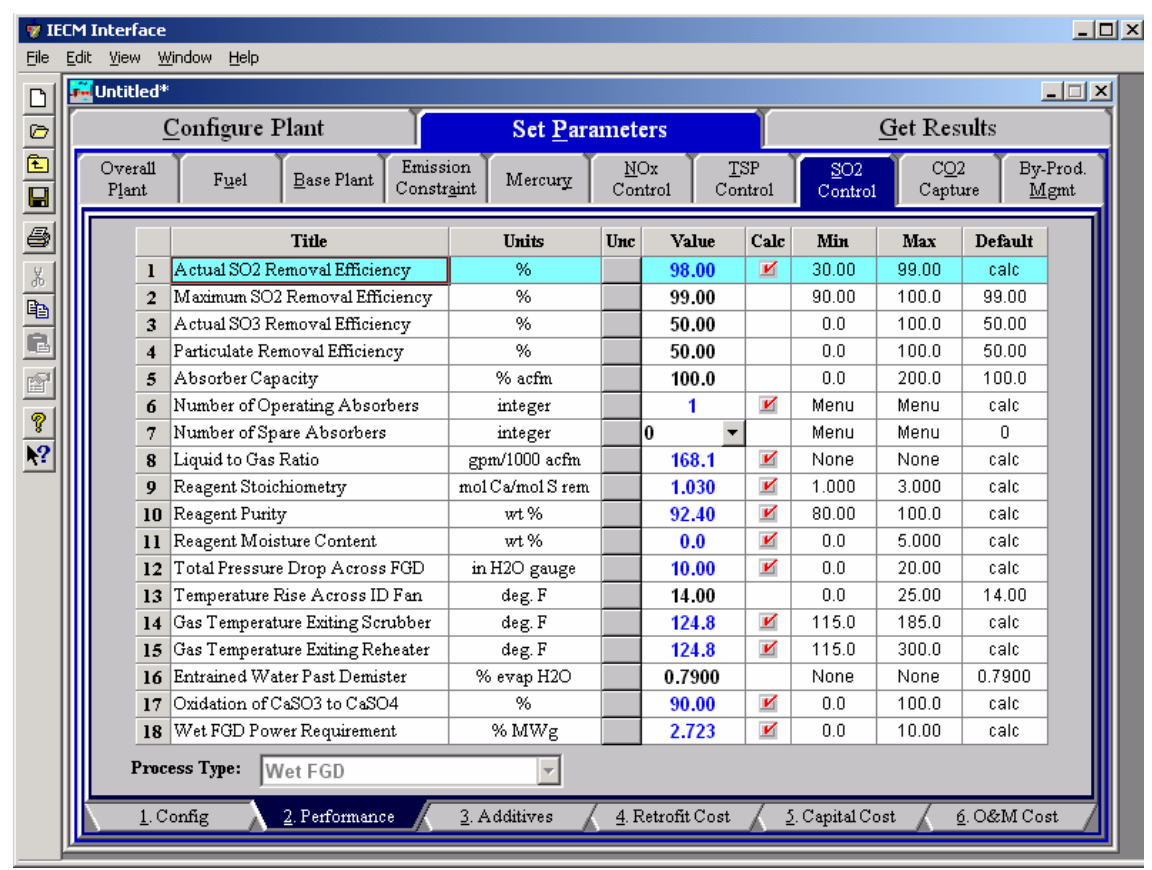

Wet FGD - Performance input screen.

Actual $\mathrm{SO}_{2}$ Removal Efficiency: This is the annual average $\mathrm{SO}_{2}$ removal efficiency achieved in the absorber. The calculated value assumes compliance with the $\mathrm{SO}_{2}$ emission limit specified earlier, if 
possible. The efficiency is used to determine the liquid to gas ratio and emissions. This input is highlighted in blue.

Maximum $\mathrm{SO}_{2}$ Removal Efficiency: This parameters specifies the maximum efficiency possible for the absorber on an annual average basis. The value is used as a limit in calculating the actual $\mathrm{SO}_{2}$ removal efficiency for compliance.

Actual $\mathrm{SO}_{3}$ Removal Efficiency: The default value is taken from the removal efficiency reported by Southern Company. ${ }^{7}$ This efficiency then determines the mass of $\mathrm{SO}_{3}$ removed from the flue gas in the scrubber.

Particulate Removal Efficiency: This is the percent removal of particulate matter entering the FGD system from the upstream particulate collector. Particulate collectors are designed to comply with the specified particulate emission limit. This is additional particulate removal.

Absorber Capacity: This is the percent of the flue gas treated by each operating absorber. This value is used to determine the number of operating absorbers and the capital costs.

Number of Operating Absorbers: This is the number of operating scrubber towers. The number is determined by the absorber capacity and is used to calculate the capital costs. The value must be an integer.

Number of Spare Absorbers: This is the total number of spare absorber vessels. It is used primarily to calculate capital costs. The value must be an integer.

Liquid to Gas Ratio: The design of spray towers for high efficiency is achieved by using high liquid-to-gas $(\mathrm{L} / \mathrm{G})$ ratios. The calculated value is a function of the reagent type, the removal efficiency, and stoichiometry. It determines the energy requirement and capital cost.

Reagent Stoichiometry: This is the moles of calcium per mole of sulfur removed from the absorber. The stoichiometry is calculated as a function of the reagent type. It is used to determine the liquid to gas ratio, reagent usage, reagent waste, and capital cost.

Reagent Purity: This is the percent of the reagent that is lime $(\mathrm{CaO})$ or limestone $\left(\mathrm{CaCO}_{3}\right)$. The calculated value is a function of the reagent type. This parameter determines the waste solids produced and the reagent needed to remove the necessary $\mathrm{SO}_{2}$.

Reagent Moisture Content: This is the moisture content of the reagent. The remaining reagent impurities are assumed to be inert substances such as silicon dioxide (sand). This parameter is used to determine the waste solids produced.

Total Pressure Drop across FGD: This is the total pressure drop across the FGD vessel prior to the reheater. This is used in the calculations of the energy requirements (or energy penalty) and thermodynamic properties of the flue gas.

Temperature Rise Across ID Fan: An induced draft (ID) fan is assumed to be located upstream of the FGD system. The fan raises the temperature of the flue gas due to dissipation of electro-mechanical energy.

\footnotetext{
${ }^{7}$ Ibid.
} 
Gas Temperature Exiting Scrubber: A thermodynamic equation is used to calculate this equilibrium flue gas temperature exiting the scrubber. The gas is assumed to be saturated with water at the exiting temperature and pressure. The value determines the water evaporated in the scrubber.

Gas Temperature Exiting Reheater: This is the desired temperature of flue gas after the reheater. It is assumed to be equal to the stack gas exit temperature. If scrubber bypass is employed, reheat requirements are reduced or eliminated. It determines the reheat energy required.

Entrained Water Past Demister: This is a liquid water entrained in the flue gas leaving the demister expressed as a percentage of the total water evaporated in the absorber.

Oxidation of $\mathrm{CaSO}_{3}$ to $\mathrm{CaSO}_{4}$ : This parameter determines the mixture of chemical species (calcium sulfite and calcium sulfate) in the solid waste stream. The default values depend on the selection of forced or natural oxidation.

Wet FGD Power Requirement: This is the equivalent electrical output of thermal (steam) energy used for reheat, plus the actual electrical output energy required for pumps and booster fans.

\section{Wet FGD Additives Inputs}

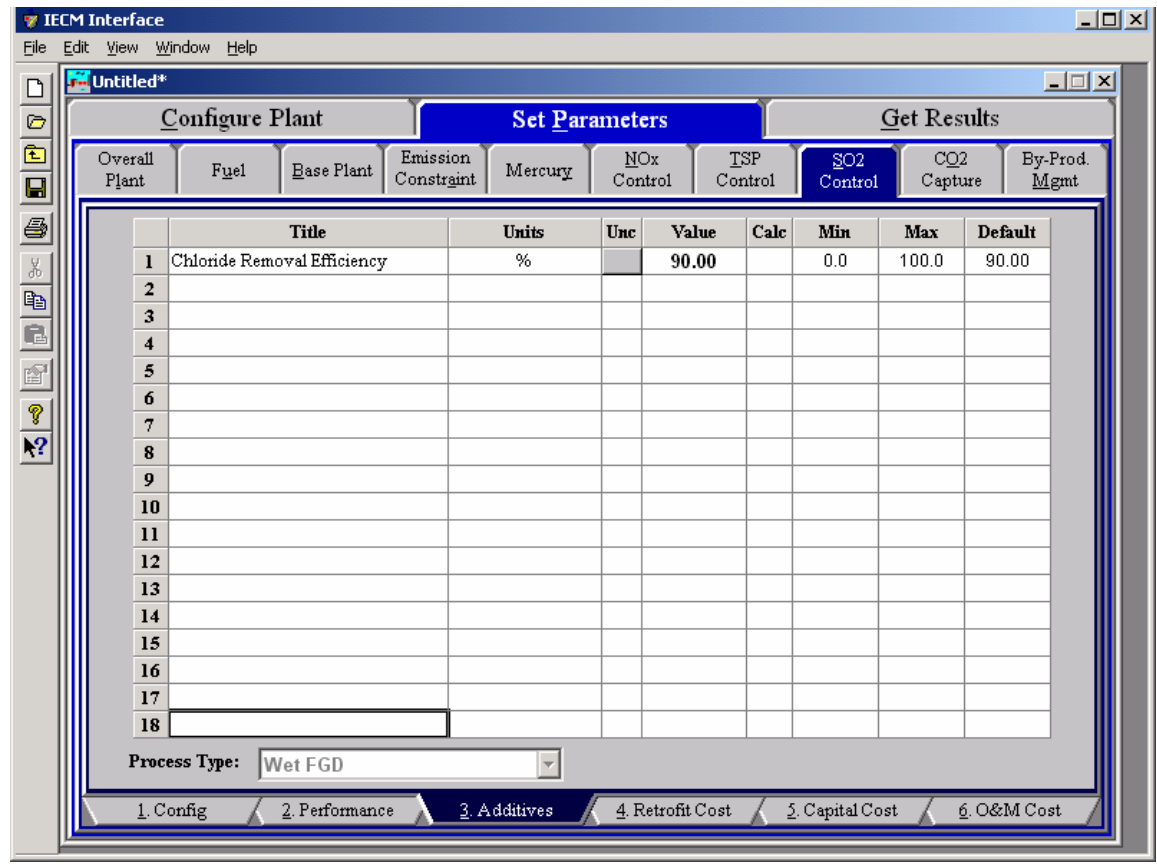

Wet FGD - Additives input screen.

The parameter is described briefly below.

Chloride Removal Efficiency: Chlorides in the flue gas inlet stream are removed by the lime/limestone slurry. This parameter determines the amount of chlorides removed. 


\section{Wet FGD Retrofit Cost Inputs}

Inputs for capital costs of modifications to process areas to implement the $\mathrm{SO}_{2}$ control technology are entered on the Retrofit Cost input screen for the Wet FGD system. All costs are in constant 1996 dollars.

The retrofit cost factor of each process is a multiplicative cost adjustment which considers the cost of retrofitted capital equipment relative to similar equipment installed in a new plant. These factors affect the capital costs directly and the operating and maintenance costs indirectly.

Direct capital costs for each process area are calculated in the IECM. These calculations are reduced form equations derived from more sophisticated models and reports. The sum of the direct capital costs associated with each process area is defined as the process facilities capital (PFC). The retrofit cost factor provided for each of the process areas can be used as a tool for adjusting the anticipated costs and uncertainties across the process area separate from the other areas.

Uncertainty can be applied to the retrofit cost factor for each process area in each technology. Thus, uncertainty can be applied as a general factor across an entire process area, rather than as a specific uncertainty for the particular cost on the capital or O\&M input screens. Any uncertainty applied to a process area through the retrofit cost factor compounds any uncertainties specified later in the capital and O\&M cost input parameter screens.

Each parameter is described briefly below.

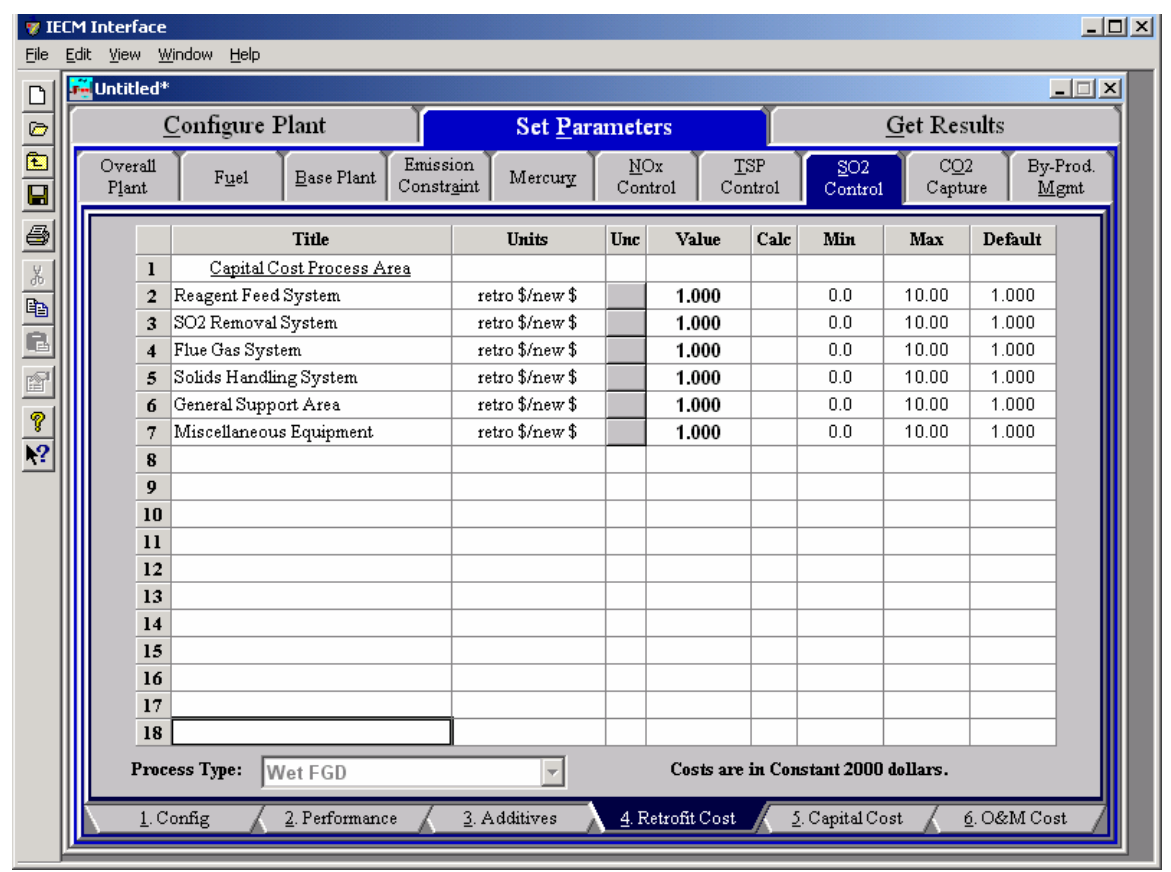

Wet FGD - Retrofit Cost input screen.

\section{Capital Cost Process Area}

Reagent Feed System: This area includes all equipment for storage, handling and preparation of raw materials, reagents, and additives used.

$\mathbf{S O}_{2}$ Removal System: This area deals with the cost of equipment for $\mathrm{SO}_{2}$ scrubbing, such as absorption tower, recirculation pumps, and other equipment. 
Flue Gas System: This area treats the cost of the duct work and fans required for flue gas distribution to $\mathrm{SO}_{2}$ system, plus gas reheat equipment.

Solids Handling System: This area includes the cost of the equipment for fixation, treatment, and transportation of all sludge/dry solids materials produced by scrubbing.

General Support Area: The cost associated with the equipment required to support FGD system operation such as makeup water and instrument air are treated here.

Miscellaneous Equipment: Any miscellaneous equipment is treated in this process area.

\section{Wet FGD Capital Cost Inputs}

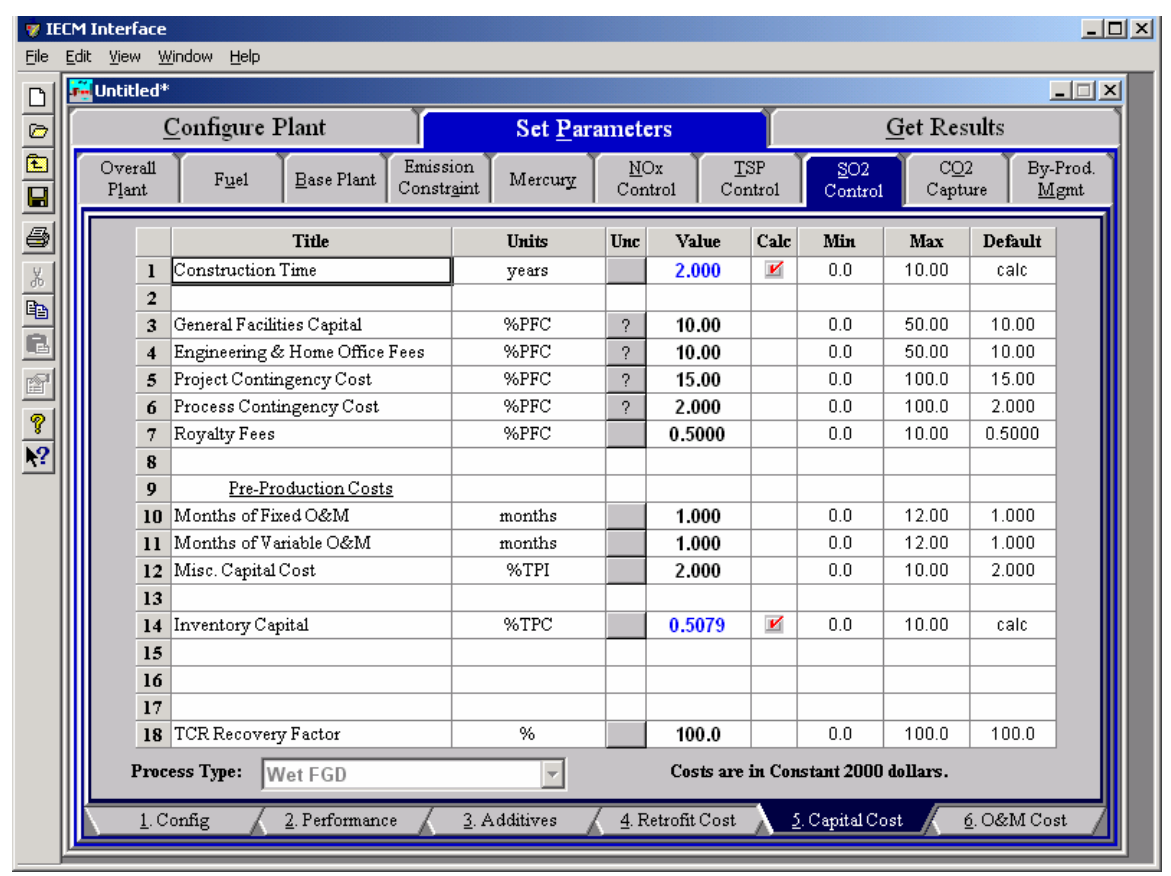

Wet FGD - Capital Cost input screen.

Each parameter is described briefly below

Construction Time: This is the idealized construction period in years. It is used to determine the allowance for funds used during construction (AFUDC).

General Facilities Capital (GFC): The general facilities include construction costs of roads, office buildings, shops, laboratories, etc. Sales taxes and freight costs are included implicitly. The cost typically ranges from $5-20 \%$.

Engineering \& Home Office Fees: The engineering \& home office fees are a percent of total direct capital cost. This is an overhead fee paid to the architect/engineering company. These fees typically range from 7$15 \%$. 
Project Contingency Cost: This is factor covering the cost of additional equipment or other costs resulting from a more detailed design. Higher contingency factors will be applied to simplified or preliminary designs and lower factors to detailed or finalized designs.

Process Contingency Cost: This quantifies the design uncertainty and cost of a commercial-scale system. This is generally applied on an areaby-area basis. Higher contingency factors are applied to new regeneration systems tested at a pilot plant and lower factors to full-size or commercial systems.

Royalty Fees: Royalty charges may apply to some portions of generating units incorporating new proprietary technologies.

\section{Pre-Production Costs:}

These costs consider the operator training, equipment checkout, major changes in unit equipment, extra maintenance, and inefficient use of fuel or other materials during start-up. These are typically applied to the O\&M costs over a specified period of time (months). The two time periods for fixed and variable O\&M costs are described below with the addition of a miscellaneous capital cost factor.

Months of Fixed O\&M: Time period of fixed operating costs used for preproduction to cover training, testing, major changes in equipment, and inefficiencies in start-up. This includes operating, maintenance, administrative and support labor. It also considers maintenance materials.

Months of Variable O\&M: Time period of variable operating costs used for preproduction to cover chemicals, water, consumables, and solid disposal charges in start-up, assuming $100 \%$ load. This excludes any fuels.

Misc. Capital Cost: This is a percent of total plant investment (sum of TPC and AFUDC) to cover expected changes to equipment to bring the system up to full capacity.

Inventory Capital: Percent of the total direct capital for raw material supply based on $100 \%$ capacity during a 60 day period. These materials are considered storage. The inventory capital includes fuels, consumables, by-products, and spare parts. This is typically $0.5 \%$.

TCR Recovery Factor: The actual total capital required (TCR) as a percent of the TCR in a new power plant. This value is $100 \%$ for a new installation and may be set as low as $0 \%$ for a wet FGD that has been paid off. 


\section{Wet FGD O\&M Cost Inputs}

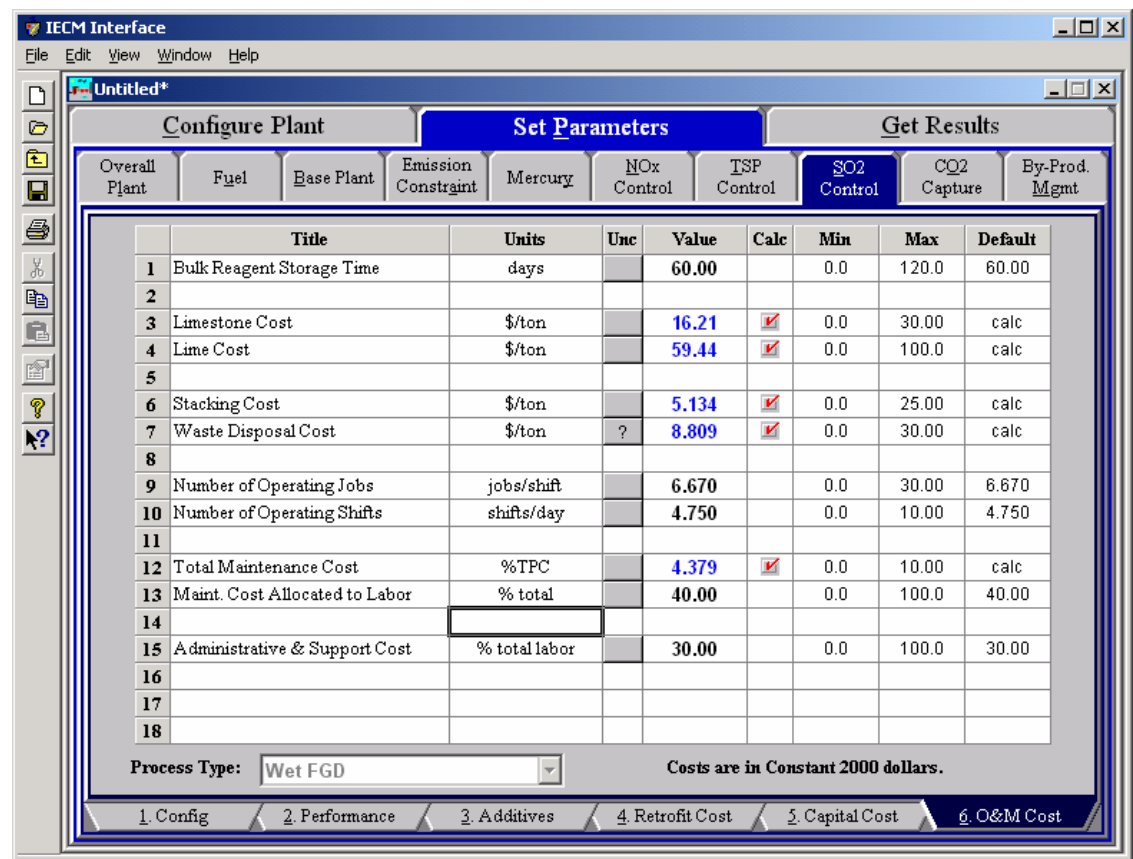

Wet FGD - O\&M Cost input screen

Each parameter is described briefly below

Bulk Reagent Storage Time: This is the number of days of bulk storage of reagent. This factor is used to determine the inventory capital cost.

Limestone Cost: This is the cost of Limestone for the Wet FGD system.

Lime Cost: This is the cost of Lime for the Wet FGD or Lime Spray Dryer system.

Dibasic Acid Cost: This is the cost of Dibasic acid for the Wet FGD system.

Stacking Cost: This is the stacking cost as used for the Wet FGD system.

Waste Disposal Cost: This is the sludge disposal cost for the FGD system.

Number of Operating Jobs: This is the total number of operating jobs that are required to operate the plant per eight-hour shift.

Number of Operating Shifts: This is the total number of equivalent operating shifts in the plant per day. The number takes into consideration paid time off and weekend work ( 3 shifts/day * 7 days/5 day week * 52 weeks $/(52$ weeks -6 weeks PTO $)=4.75$ equiv. Shifts/day)

Total Maintenance Cost: This is the annual maintenance cost as a percentage of the total plant cost. Maintenance cost estimates can be developed separately for each process area.

Maint. Cost Allocated to Labor: Maintenance cost allocated to labor as a percentage of the total maintenance cost. 
Administrative \& Support Cost: This is the percent of the total operating and maintenance labor associated with administrative and support labor.

\section{Wet FGD Diagram}

The Diagram result screen displays an icon for theWet FGD $\mathrm{SO}_{2}$ control technology selected and values for major flows in and out of it.

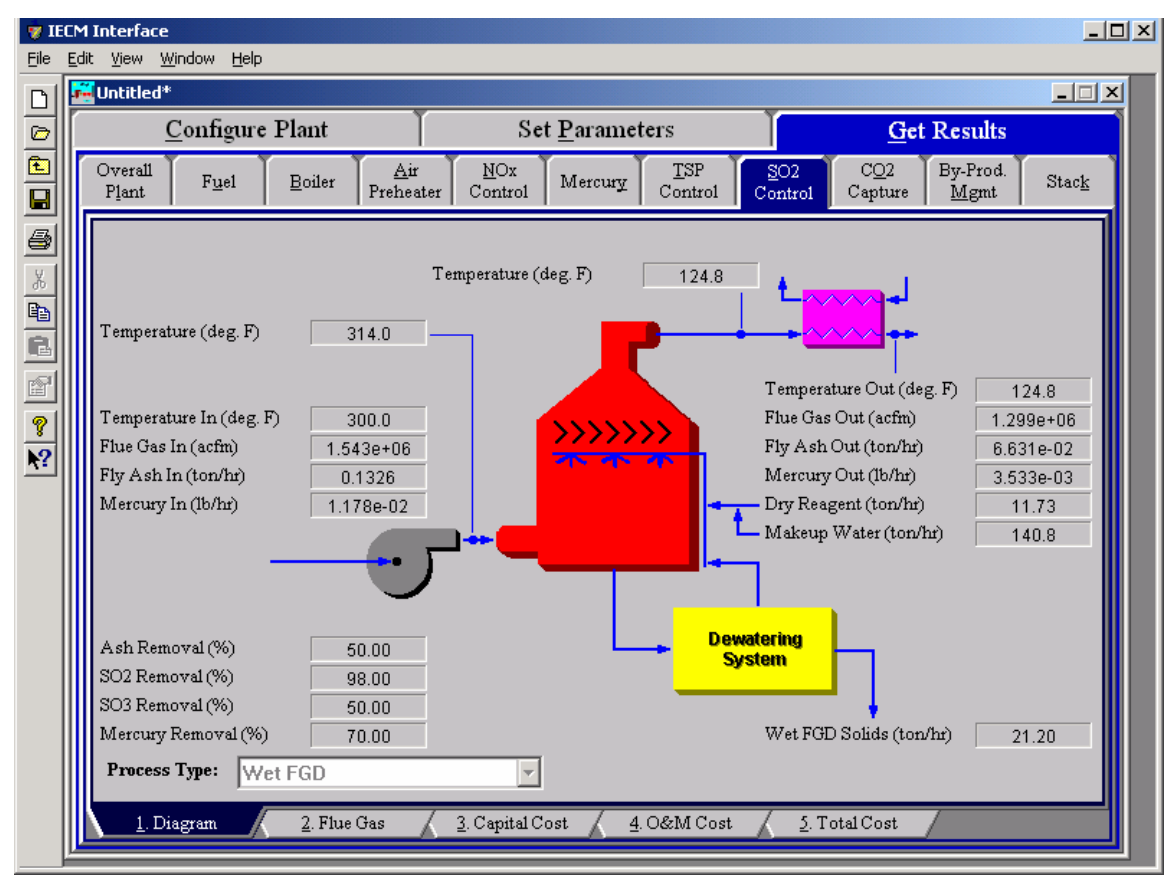

Wet FGD - Diagram.

Each result is described briefly below.

\section{Reagent}

Dry Reagent: The total mass flow rate of lime, limestone or limestone with dibasic acid injected into the scrubber. This is a function of the $\mathrm{SO}_{2}$ removal efficiency, the reagent purity and the reagent stoichiometric (all performance input parameters).

Makeup Water: Water needed to replace the evaporated water in the reagent sluice circulation stream.

\section{Flue Gas Entering FGD}

Temperature In: Temperature of the flue gas entering the scrubber. This is determined by the flue gas outlet temperature of the module upstream of the scrubber (e.g., a particulate removal technology).

Flue Gas In: Volumetric flow rate of flue gas entering the scrubber, based on the flue gas temperature entering the scrubber and atmospheric pressure.

Fly Ash In: Total solids mass flow rate in the flue gas entering the scrubber. This is determined by the solids exiting from the module upstream of the scrubber (e.g., a particulate removal technology). 
Mercury In: Total mass of mercury entering the scrubber. The value is a sum of all the forms of mercury (elemental, oxidized, and particulate).

Temperature: Temperature of the flue gas entering the scrubber after the forced draft fan. This is determined by the flue gas inlet temperature of the FGD and the temperature rise across ID fan input parameter.

\section{Flue Gas Exiting FGD}

Temperature: Temperature of the flue gas immediately on exiting the scrubber, prior to any flue gas bypass remixing and prior to reheating.

Temperature Out: Temperature of the flue gas exiting the scrubber. This is a function of flue gas bypass, saturation temperature, reheater and the flue gas component concentrations.

Flue Gas Out: Volumetric flow rate of the flue gas exiting the scrubber after the reheater, based on the flue gas temperature exiting the scrubber and atmospheric pressure.

Fly Ash Out: Total solids mass flow rate in the flue gas exiting the scrubber after the reheater. This is a function of the ash removal and flue gas bypass input parameters.

Mercury Out: Total mass of mercury exiting the scrubber after the reheater. The value is a sum of all the forms of mercury (elemental, oxidized, and particulate).

\section{FGD Performance}

Ash Removal: Actual particulate removal efficiency in the scrubber. This is set by the scrubber ash removal input parameter.

$\mathbf{S O}_{2}$ Removal: Actual removal efficiency of $\mathrm{SO}_{2}$ in the scrubber. This is a function of the maximum removal efficiency (scrubber performance input parameter) and the emission constraint for $\mathrm{SO}_{2}$ (emission constraints input parameter). It is possible that the scrubber may over or under-comply with the emission constraint.

$\mathrm{SO}_{3}$ Removal: Percent of $\mathrm{SO}_{3}$ in the flue gas removed from the scrubber. The $\mathrm{SO}_{3}$ is assumed to combine with $\mathrm{H}_{2} \mathrm{O}$ and leave with the ash solids or sluice water as a sulfate (in the form of $\mathrm{H}_{2} \mathrm{SO}_{4}$ ).

Mercury Removal: Percent of the total mercury removed from the scrubber. The value reflects a weighted average based on the particular species of mercury present (elemental, oxidized, and particulate).

\section{Collected Solids}

Wet FGD Solids: Total solids mass flow rate of solids removed from the scrubber. This is a function of the solids content in the flue gas and the particulate removal efficiency of the scrubber. The solids are shown on a wet basis.

\section{Wet FGD Flue Gas}

The Flue Gas result screen displays a table of quantities of flue gas components entering and exiting the Wet FGD SO${ }_{2}$ Control Technology. For each component, quantities are given in both moles and mass per hour. 


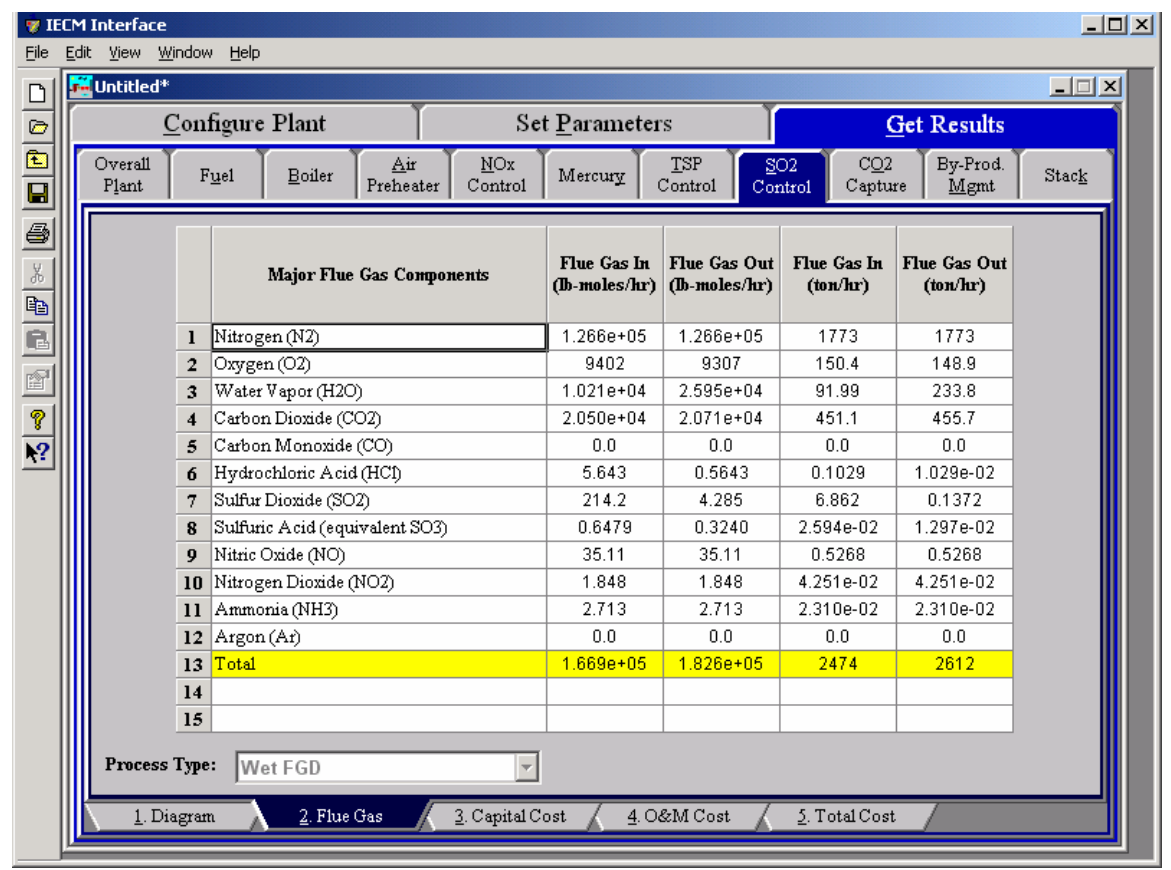

Wet FGD - Flue Gas result screen.

Each result is described briefly below

\section{Major Flue Gas Component}

Nitrogen $\left(\mathbf{N}_{2}\right)$ : Total mass of nitrogen.

Oxygen $\left(\mathrm{O}_{2}\right)$ : Total mass of oxygen.

Water Vapor $\left(\mathrm{H}_{2} \mathrm{O}\right)$ : Total mass of water vapor.

Carbon Dioxide $\left(\mathrm{CO}_{2}\right)$ : Total mass of carbon dioxide.

Carbon Monoxide (CO): Total mass of carbon monoxide.

Hydrochloric Acid ( $\mathrm{HCl})$ : Total mass of hydrochloric acid.

Sulfur Dioxide $\left(\mathrm{SO}_{2}\right)$ : Total mass of sulfur dioxide.

Sulfuric Acid (equivalent $\mathrm{SO}_{3}$ ): Total mass of sulfuric acid.

Nitric Oxide (NO): Total mass of nitric oxide.

Nitrogen Dioxide $\left(\mathrm{NO}_{2}\right)$ : Total mass of nitrogen dioxide.

Ammonia (NH3): Total mass of ammonia.

Argon (Ar): Total mass of argon.

Total: Total of the individual components listed above. This item is highlighted in yellow.

\section{Wet FGD Capital Cost Results}

The Capital Cost result screen displays tables for the direct and indirect capital costs related to the $\mathrm{SO}_{2}$ control technology. 


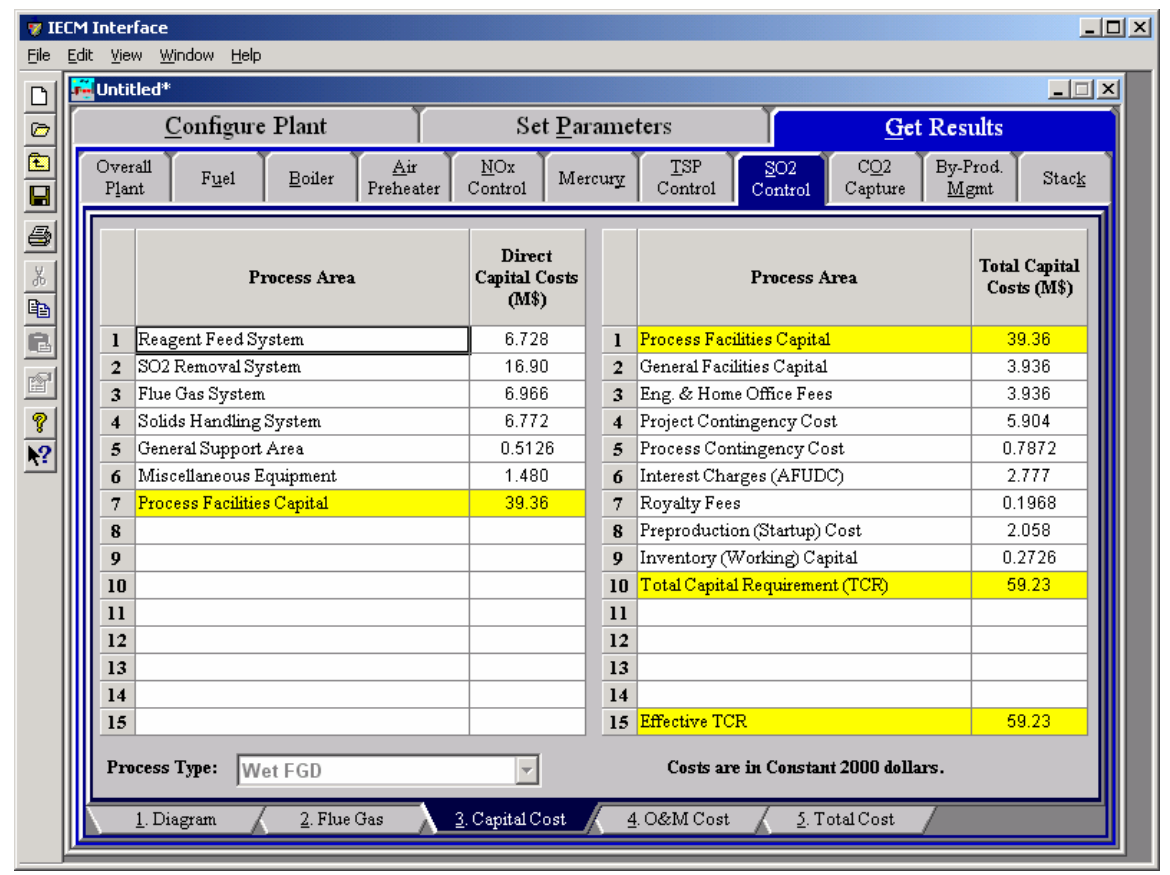

Wet FGD - Capital Cost result screen.

Each result is described briefly below

\section{Direct Capital Costs}

Each process area direct capital cost is a reduced-form model based on regression analysis of data collected from several reports and analyses of particulate control technology units. They are described in general below. The primary factors in the model that effect the capital costs of the scrubbers are the flue gas flow rate through the scrubber, the composition of the flue gas, the reagent stoichiometry, and the reagent flow rate.

Reagent Feed System: This area includes all equipment for storage, handling and preparation of raw materials, reagents, and additives used.

$\mathrm{SO}_{2}$ Removal System: This area deals with the cost of equipment for $\mathrm{SO}_{2}$ scrubbing, such as absorption tower, recirculation pumps, and other equipment.

Flue Gas System: This area treats the cost of the duct work and fans required for flue gas distribution to $\mathrm{SO}_{2}$ system, plus gas reheat equipment.

Solids Handling System: This area includes the cost of the equipment for fixation, treatment, and transportation of all sludge/dry solids materials produced by scrubbing.

General Support Area: The cost associated with the equipment required to support FGD system operation such as makeup water and instrument air are treated here.

Miscellaneous Equipment: Any miscellaneous equipment is treated in this process area.

Process Facilities Capital: The process facilities capital is the total constructed cost of all on-site processing and generating units listed above, including all direct and indirect construction costs. All sales 
taxes and freight costs are included where applicable implicitly. This result is highlighted in yellow.

\section{Total Capital Costs}

Process Facilities Capital: (see definition above)

General Facilities Capital: The general facilities include construction costs of roads, office buildings, shops, laboratories, etc. Sales taxes and freight costs are included implicitly.

Eng. \& Home Office Fees: The engineering \& home office fees are a percent of total direct capital cost. This is an overhead fee paid to the architect/engineering company.

Project Contingency Cost: Capital cost contingency factor covering the cost of additional equipment or other costs that would result from a more detailed design of a definitive project at the actual site.

Process Contingency Cost: Capital cost contingency factor applied to a new technology in an effort to quantify the uncertainty in the technical performance and cost of the commercial-scale equipment.

Interest Charges (AFUDC): Allowance for funds used during construction, also referred to as interest during construction, is the time value of the money used during construction and is based on an interest rate equal to the before-tax weighted cost of capital. This interest is compounded on an annual basis (end of year) during the construction period for all funds spent during the year or previous years.

Royalty Fees: Royalty charges may apply to some portions of generating units incorporating new proprietary technologies.

Preproduction (Startup) Cost: These costs consider the operator training, equipment checkout, major changes in unit equipment, extra maintenance, and inefficient use of fuel or other materials during startup.

Inventory (Working) Capital: The raw material supply based on $100 \%$ capacity during a 60 day period. These materials are considered storage. The inventory capital includes fuels, consumables, byproducts, and spare parts.

Total Capital Requirement (TCR): Money that is placed (capitalized) on the books of the utility on the service date. TCR includes all the items above. This result is highlighted in yellow.

Effective TCR: The TCR of the wet FGD that is used in determining the total power plant cost. The effective TCR is determined by the "TCR Recovery Factor" for the wet FGD.

\section{Wet FGD O\&M Cost Results}

The O\&M Cost result screen displays tables for the variable and fixed operation and maintenance costs involved with the $\mathrm{SO}_{2}$ control technology. 


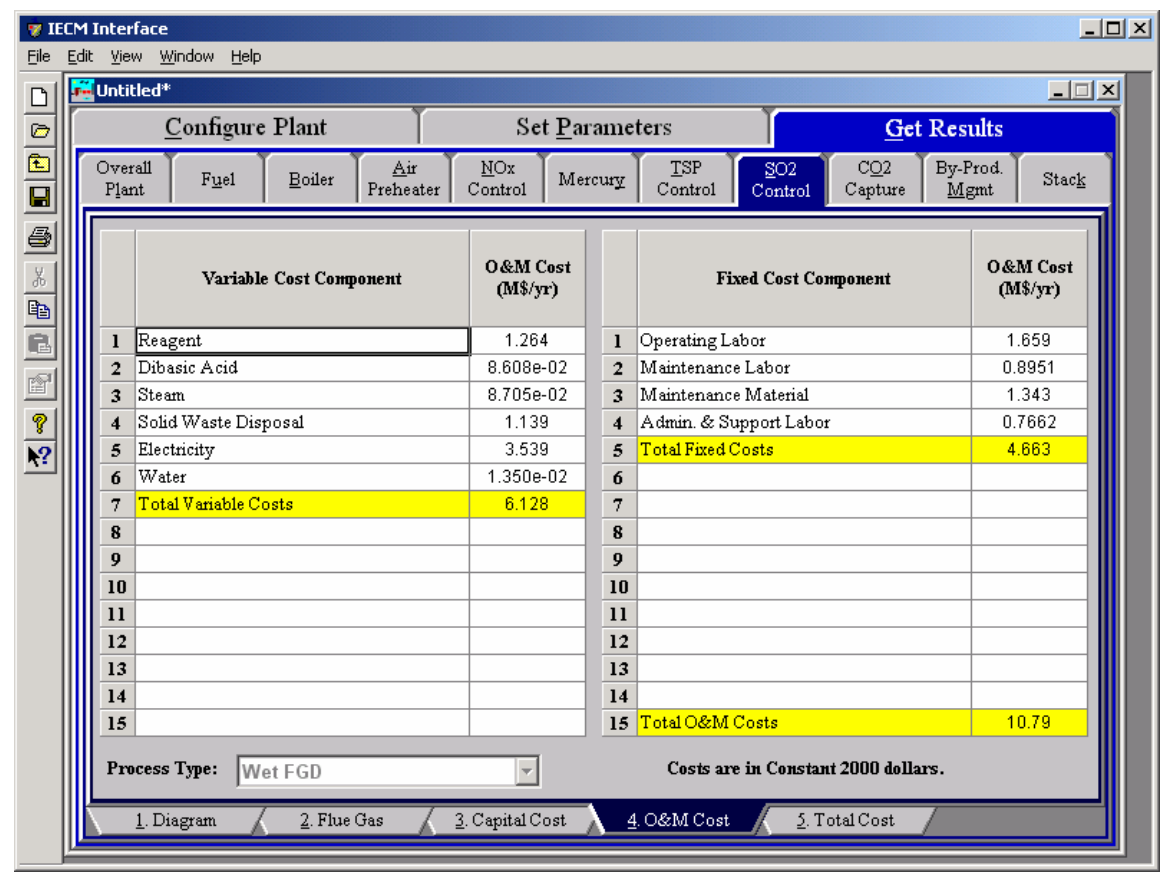

Wet FGD - O\&M Cost result screen.

Each result is described briefly below

\section{Variable Cost Components}

Variable operating costs and consumables are directly proportional to the amount of kilowatts produced and are referred to as incremental costs. All the costs are subject to inflation.

Reagent: The total mass flow rate of lime or limestone injected into the scrubber on a wet basis. This is a function of the $\mathrm{SO}_{2}$ concentration in the flue gas and the reagent stoichiometric performance input value.

Dibasic Acid: If limestone with additives, LS w/ Add. has been selected in the reagent pull down menu on the Wet FGD - Config. input screen, then this component is displayed. This is the total mass flow rate of dibasic acid added to reagent. It is determined by $\mathrm{SO}_{2}$ concentration in the flue gas and the dibasic acid stoichiometric performance input parameter.

Steam: Annual cost of steam used for direct or reheat use in the scrubber. This is a function of the steam heat rate, reheat energy requirement and gross plant capacity.

Solid Waste Disposal: Total cost to dispose the collected flue gas waste solids. This does not consider by-product gypsum sold in commerce.

Electricity: Cost of power consumption of the scrubber. This is a function of the gross plant capacity and the scrubber energy penalty performance input parameter.

Water: Cost of water for reagent sluice in the scrubber. This is a function of the liquid to gas ratio performance input parameter for the wet FGD. The cost is a function of the flue gas flow rate and the slurry recycle ratio performance input parameter for the spray dryer. 
Total Variable Costs: This is the sum of all the variable O\&M costs listed above. This result is highlighted in yellow.

\section{Fixed Cost Components}

Fixed operating costs are essentially independent of actual capacity factor, number of hours of operation, or amount of kilowatts produced. All the costs are subject to inflation.

Operating Labor: Operating labor cost is based on the operating labor rate, the number of personnel required to operate the plant per eighthour shift, and the average number of shifts per day over 40 hours per week and 52 weeks.

Maintenance Labor: The maintenance labor is determined as a fraction of the total maintenance cost.

Maintenance Material: The cost of maintenance material is the remainder of the total maintenance cost, considering the fraction associated with maintenance labor.

Admin. \& Support Labor: The administrative and support labor is the only overhead charge. It is taken as a fraction of the total operating and maintenance labor costs.

Total Fixed Costs: This is the sum of all the fixed O\&M costs listed above. This result is highlighted in yellow.

Total O\&M Costs: This is the sum of the total variable and total fixed O\&M costs. It is used to determine the base plant total revenue requirement. This result is highlighted in yellow.

\section{Wet FGD Total Cost Results}

The Total Cost result screen displays a table which totals the annual fixed, variable, operations and maintenance, and capital costs associated with the $\mathrm{SO}_{2}$ control technology. The result categories are the same for both the Wet FGD and the Lime Spray Dryer. 


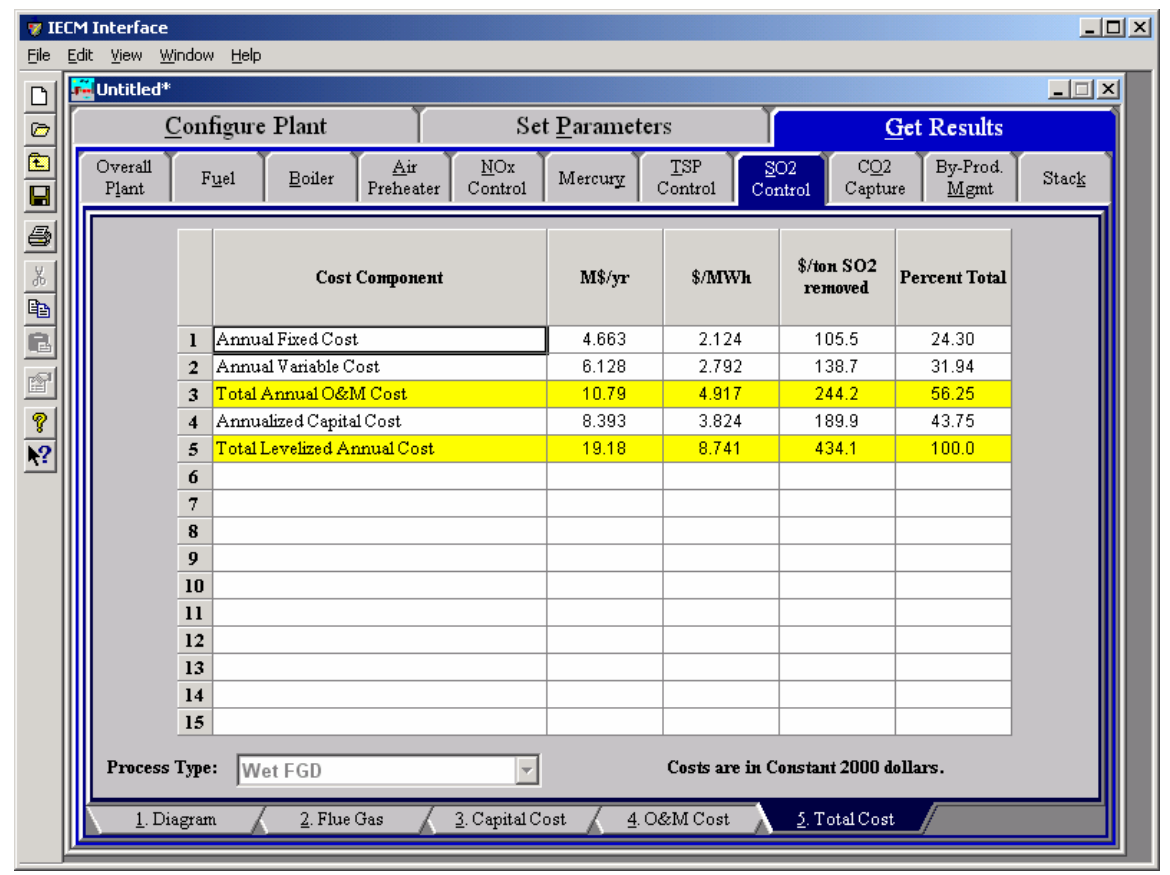

Wet FGD - Total Cost result screen.

\section{Cost Component}

Each result is described briefly below.

Annual Fixed Cost: The operating and maintenance fixed costs are given as an annual total. This number includes all maintenance materials and all labor costs.

Annual Variable Cost: The operating and maintenance variables costs are given as an annual total. This includes all reagent, chemical, steam, and power costs.

Total Annual O\&M Cost: This is the sum of the annual fixed and variable operating and maintenance costs above. This result is highlighted in yellow.

Annualized Capital Cost: This is the total capital cost expressed on an annualized basis, taking into consideration the levelized carrying charge factor, or fixed charge factor, over the entire book life.

Total Levelized Annual Cost: The total annual cost is the sum of the total annual O\&M cost and annualized capital cost items above. This result is highlighted in yellow. 


\section{Spray Dryer}

The So2 2 Control Technology Navigation Tab contains screens that address postcombustion air pollution technologies for Sulfur Dioxide. The model includes options for a Lime Spray Dryer. A spray dryer is sometimes used instead of a wet scrubber because it provides simpler waste disposal and can be installed with lower capital costs. These screens are available if the Lime Spray Dryer $\mathrm{SO}_{2}$ control technology has been selected in $\underline{\text { Configure Plant. }}$

\section{Spray Dryer Configuration}

Inputs for configuration of the Lime Spray Dryer $\mathrm{SO}_{2}$ control technology are entered on the Config input screen

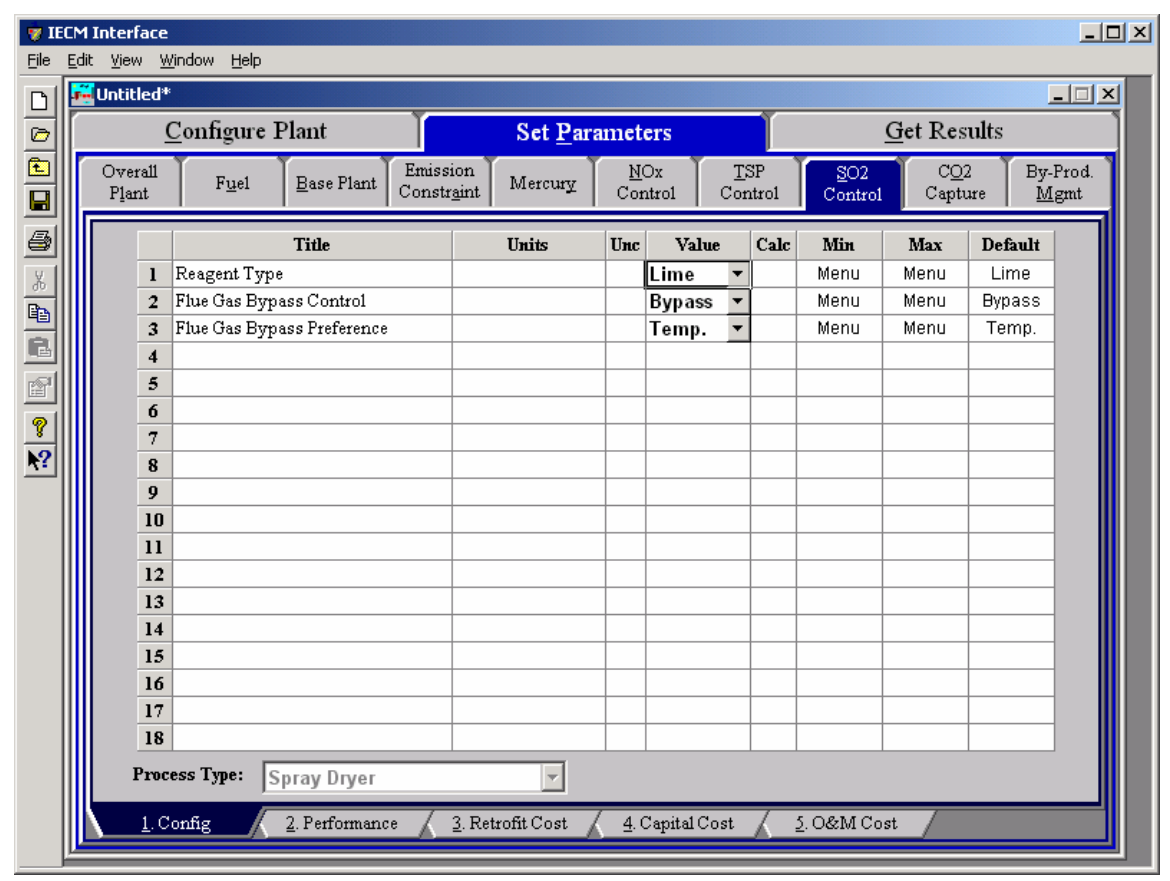

Spray Dryer - Config. input screen.

Each parameter is described briefly below.

Reagent: For the Lime Spray Dryer the only option is Lime. 
Lime: Magnesium Enhanced Lime System-A magnesium sulfite and lime slurry (maglime) is used to remove $\mathrm{SO}_{2}$ and form a precipitate high in calcium sulfite. The high alkalinity of the maglime slurry allows very high $\mathrm{SO}_{2}$ removal. However, the reagent cost is also higher and solid waste is not easily disposed.

Flue Gas Bypass Control: Popup selection menu that controls whether or not a portion of the inlet flue gas may bypass the scrubber and recombine with the treated flue gas. Bypass allows the scrubber to operate at full efficiency while allowing some of the flue gas to go untreated.

Flue Gas Bypass Preference: (Spray dryer only) Popup selection menu that controls whether the bypass is determined by the exit temperature or based on the maximum efficiency. If exit temperature is selected, the flue gas bypass amount is based on the amount of bypass required to raise the exit temperature from the scrubber to the desired stack temperature. If maximum efficiency is selected, the flue gas bypass amount is based on the overall required $\mathrm{SO}_{\mathrm{x}}$ removal efficiency.

\section{Spray Dryer Performance Inputs}

Inputs for performance of the Lime Spray Dryer $\mathrm{SO}_{2}$ control technology are entered on the Performance input screen.

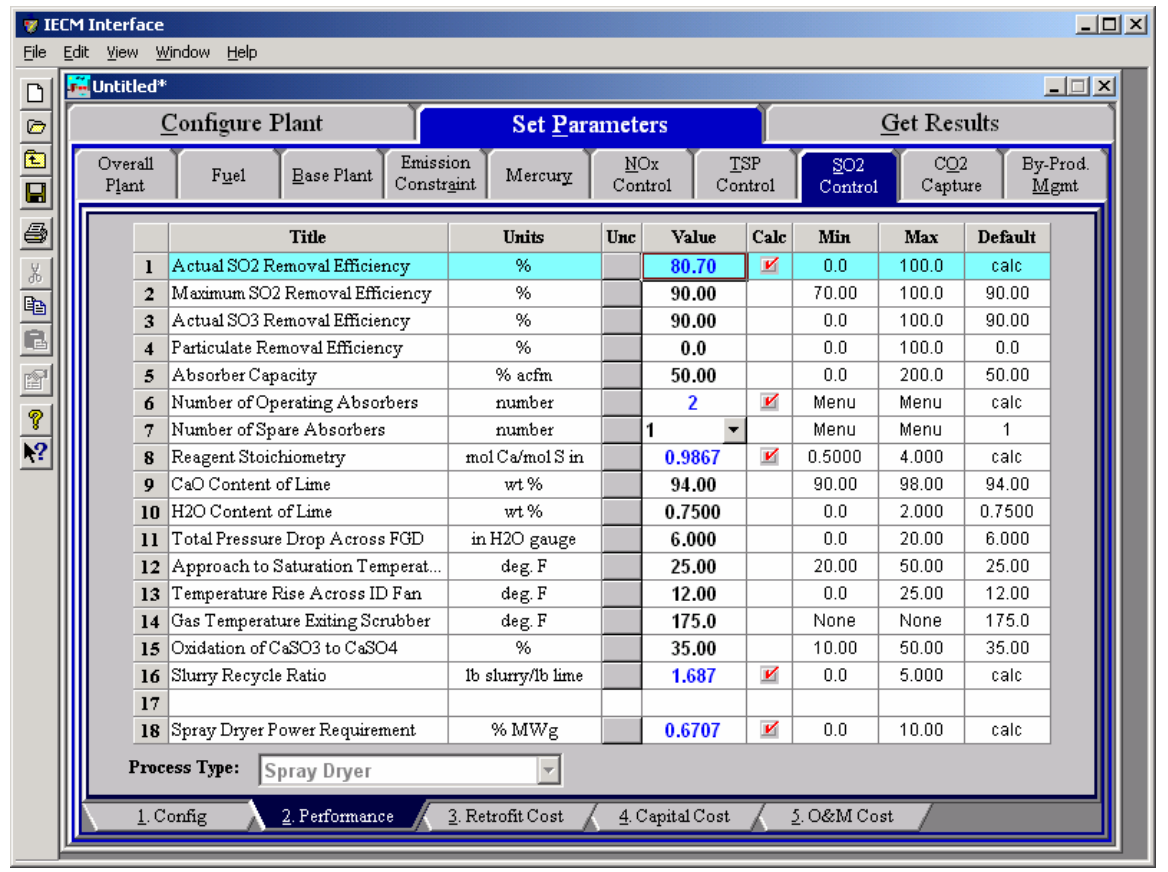

Spray Dryer - Performance input screen.

In a Lime Spray Dryer, an atomized spray of a mixture of lime slurry and recycled solids is brought into contact with the hot flue gas. The water in the slurry evaporates leaving dry reaction products and flyash, which drops out of the scrubber. A particulate control device such as a baghouse is also used to remove the rest of the dry products from the flue gas before releasing it. The $\mathrm{SO}_{2}$ removal efficiency is the total of $\mathrm{SO}_{2}$ removed in the scrubber and the baghouse. 
Many lime spray dryer input parameters are similar to those defined above for wet lime/limestone systems. Each parameter is described briefly below.

Actual $\mathrm{SO}_{2}$ Removal Efficiency: This is the annual average $\mathrm{SO}_{2}$ removal efficiency achieved in the absorber. The calculated default value assumes compliance with the $\mathrm{SO}_{2}$ emission limit specified earlier, if possible. The default value reflects other model parameter values, including the sulfur retained in bottom ash. This input is highlighted in blue.

Maximum $\mathrm{SO}_{2}$ Removal Efficiency: This parameters specifies the maximum efficiency possible for the absorber on an annual average basis. The value is used as a limit in calculating the actual $\mathrm{SO}_{2}$ removal efficiency for compliance.

Actual $\mathrm{SO}_{3}$ Removal Efficiency: The default value is taken from the removal efficiency reported by Southern Company. This efficiency then determines the mass of $\mathrm{SO}_{3}$ removed from the flue gas in the scrubber.

Particulate Removal Efficiency: Ash and particulate matter are assumed to be removed by a separate particulate removal device, such as a fabric filter. However, this parameters is provided for conditions where particulates are removed directly from the scrubber.

Absorber Capacity: This is the percent of the flue gas treated by each operating absorber. This value is used to determine the number of operating absorbers and the capital costs.

Number of Operating Absorbers: This is the number of operating scrubber towers. The number is determined by the absorber capacity and is used to calculate the capital costs. The value must be an integer.

Number of Spare Absorbers: This is the total number of spare absorber vessels. It is used primarily to calculate capital costs. The value must be an integer.

Reagent Stoichiometry: This is the moles of calcium per mole of sulfur into the absorber. The stoichiometry is calculated as a function of the required $\mathrm{SO}_{2}$ removal efficiency, inlet flue gas temperature, inlet sulfur concentration, and approach to saturation temperature.

CaO Content of Lime: This is the percent of reagent that is pure lime $(\mathrm{CaO})$. This parameter determines the waste solids produced and the reagent mass requirements, given the stoichiometry needed for $\mathrm{SO}_{2}$ removal.

$\mathrm{H}_{2} \mathrm{O}$ Content of Lime: This is the moisture content of the lime $(\mathrm{CaO})$. The remaining reagent impurities are assumed to be inert substances such as silicon dioxide (sand). This parameter is used to determine the waste solids produced.

Total Pressure Drop Across FGD: This is the total pressure drop across the spray dryer vessel prior to the reheater. This is used in the calculations of the energy requirements (or energy penalty) and thermodynamic properties of the flue gas.

Approach to Saturation Temperature: This defines the gas temperature exiting the absorber. The approach is the increment over the water saturation temperature at the exit pressure. As the approach to saturation temperature increases, the evaporation time decreases thereby decreasing removal efficiency. 
Temperature Rise Across ID Fan: An induced draft (ID) fan is assumed to be located upstream of the FGD system. The fan raises the temperature of the flue gas due to dissipation of electro-mechanical energy.

Gas Temperature Exiting Scrubber: A thermodynamic equation is used to calculate this equilibrium flue gas temperature exiting the scrubber. The gas is assumed to be saturated with water at the exiting temperature and pressure. The value determines the water evaporated in the scrubber.

Oxidation of $\mathrm{CaSO}_{3}$ to $\mathrm{CaSO}_{4}$ : This parameter determines the mixture of the two chemical species in the solid waste stream.

Slurry Recycle Ratio: An atomized spray of a mixture of lime slurry and recycled solids is brought into contact with the hot flue gas. This parameter specifies the amount of solid waste recycled and lime slurry used. It is calculated from the sulfur content of the coal.

Spray Dryer Power Requirement: This is the equivalent electrical output of thermal (steam) energy used for reheat, plus the actual electrical output energy required for pumps and booster fans.

\section{Spray Dryer Retrofit Cost}

Inputs for capital costs of modifications to process areas to implement the $\mathrm{SO}_{2}$ control technology are entered on the Retrofit Cost input screen. All costs are in constant 2000 dollars.

The retrofit cost factor of each process is a multiplicative cost adjustment which considers the cost of retrofitted capital equipment relative to similar equipment installed in a new plant. These factors affect the capital costs directly and the operating and maintenance costs indirectly.

Direct capital costs for each process area are calculated in the IECM. These calculations are reduced form equations derived from more sophisticated models and reports. The sum of the direct capital costs associated with each process area is defined as the process facilities capital (PFC). The retrofit cost factor provided for each of the process areas can be used as a tool for adjusting the anticipated costs and uncertainties across the process area separate from the other areas.

Uncertainty can be applied to the retrofit cost factor for each process area in each technology. Thus, uncertainty can be applied as a general factor across an entire process area, rather than as a specific uncertainty for the particular cost on the capital or O\&M input screens. Any uncertainty applied to a process area through the retrofit cost factor compounds any uncertainties specified later in the capital and O\&M cost input parameter screens. 


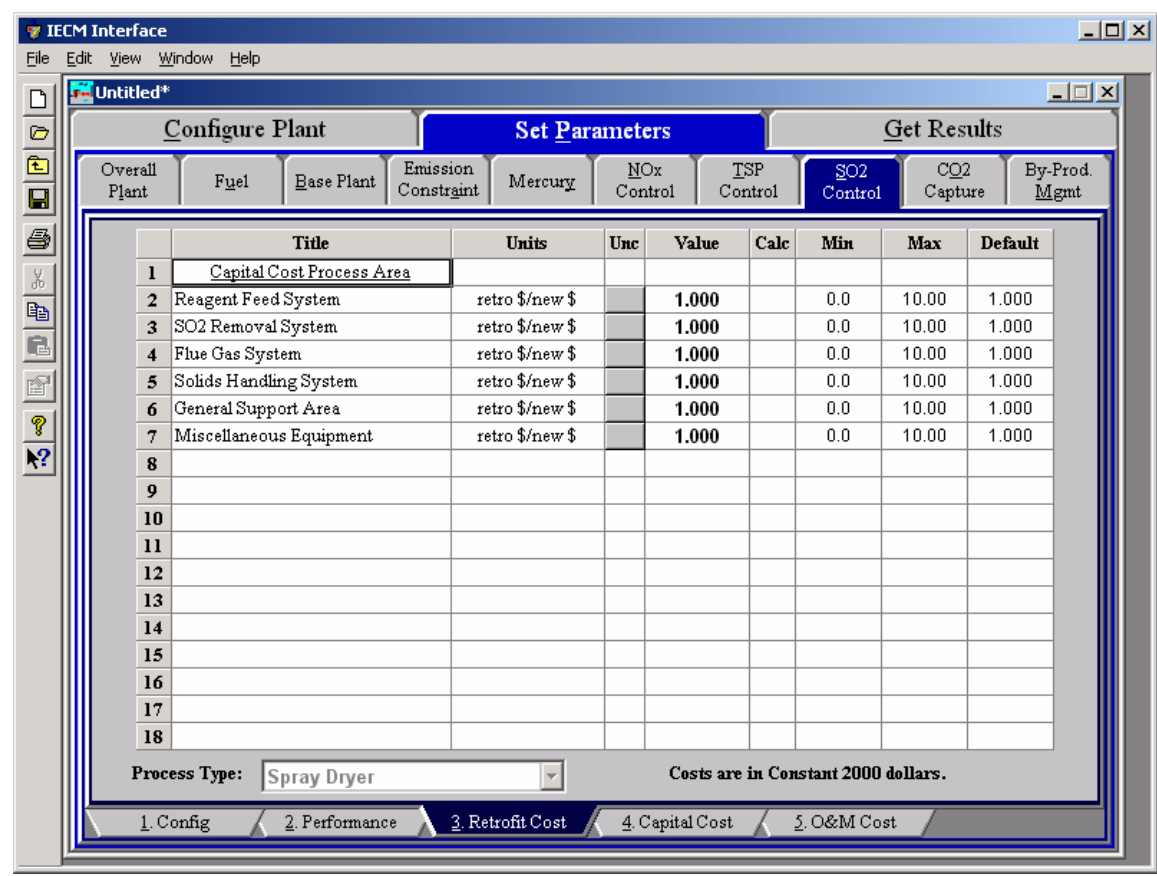

Spray Dryer - Retrofit Cost input screen.

Each parameter is described briefly below.

Reagent Feed System: This area includes all equipment for storage, handling and preparation of raw materials, reagents, and additives used.

$\mathrm{SO}_{2}$ Removal System: This area deals with the cost of equipment for $\mathrm{SO}_{2}$ scrubbing, such as absorption tower, recirculation pumps, and other equipment.

Flue Gas System: This area treats the cost of the duct work and fans required for flue gas distribution to $\mathrm{SO}_{2}$ system, plus gas reheat equipment.

Solids Handling System: This area includes the cost of the equipment for fixation, treatment, and transportation of all sludge/dry solids materials produced by scrubbing.

General Support Area: The cost associated with the equipment required to support FGD system operation such as makeup water and instrument air are treated here.

Miscellaneous Equipment: Any miscellaneous equipment is treated in this process area. 


\section{Spray Dryer Capital Cost Inputs}

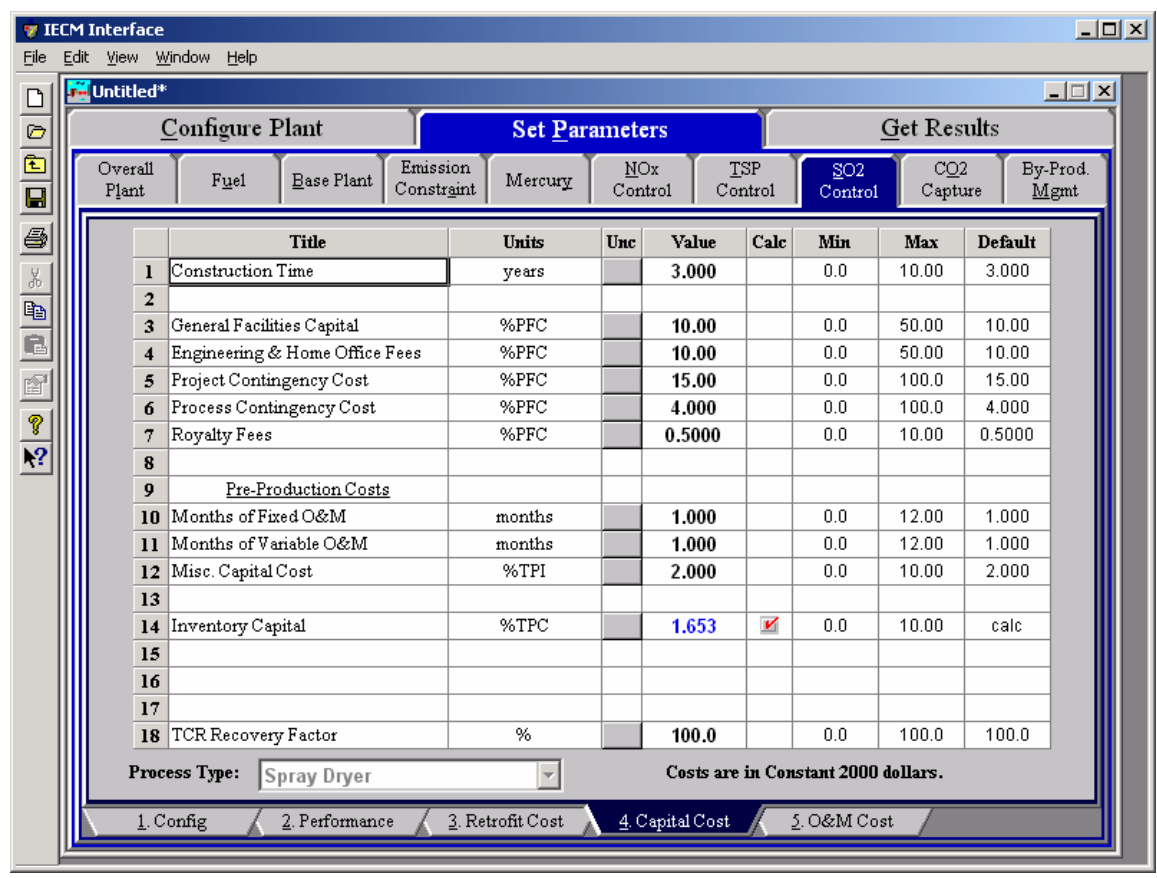

Spray Dryer - Capital Cost input screen.

Inputs for capital costs are entered on the Capital Cost input screen.

Construction Time: This is the idealized construction period in years. It is used to determine the allowance for funds used during construction (AFUDC).

General Facilities Capital (GFC): The general facilities include construction costs of roads, office buildings, shops, laboratories, etc. Sales taxes and freight costs are included implicitly. The cost typically ranges from $5-20 \%$.

Engineering \& Home Office Fees: The engineering \& home office fees are a percent of total direct capital cost. This is an overhead fee paid to the architect/engineering company. These fees typically range from 7$15 \%$.

Project Contingency Cost: This is factor covering the cost of additional equipment or other costs resulting from a more detailed design. Higher contingency factors will be applied to simplified or preliminary designs and lower factors to detailed or finalized designs.

Process Contingency Cost: This quantifies the design uncertainty and cost of a commercial-scale system. This is generally applied on an areaby-area basis. Higher contingency factors are applied to new regeneration systems tested at a pilot plant and lower factors to full-size or commercial systems.

Royalty Fees: Royalty charges may apply to some portions of generating units incorporating new proprietary technologies.

Pre-Production Costs: These costs consider the operator training, equipment checkout, major changes in unit equipment, extra maintenance, and inefficient use of fuel or other materials during start- 
up. These are typically applied to the O\&M costs over a specified period of time (months). The two time periods for fixed and variable O\&M costs are described below with the addition of a miscellaneous capital cost factor.

Months of Fixed O\&M: Time period of fixed operating costs used for preproduction to cover training, testing, major changes in equipment, and inefficiencies in start-up. This includes operating, maintenance, administrative and support labor. It also considers maintenance materials.

Months of Variable O\&M: Time period of variable operating costs used for preproduction to cover chemicals, water, consumables, and solid disposal charges in start-up, assuming 100\% load. This excludes any fuels.

Misc. Capital Cost: This is a percent of total plant investment (sum of TPC and AFUDC) to cover expected changes to equipment to bring the system up to full capacity.

Inventory Capital: Percent of the total direct capital for raw material supply based on $100 \%$ capacity during a 60 day period. These materials are considered storage. The inventory capital includes fuels, consumables, by-products, and spare parts. This is typically $0.5 \%$.

TCR Recovery Factor: The actual total capital required (TCR) as a percent of the TCR in a new power plant. This value is $100 \%$ for a new installation and may be set as low as $0 \%$ for a fabric filter that has been paid off.

\section{Spray O\&M Cost Inputs}

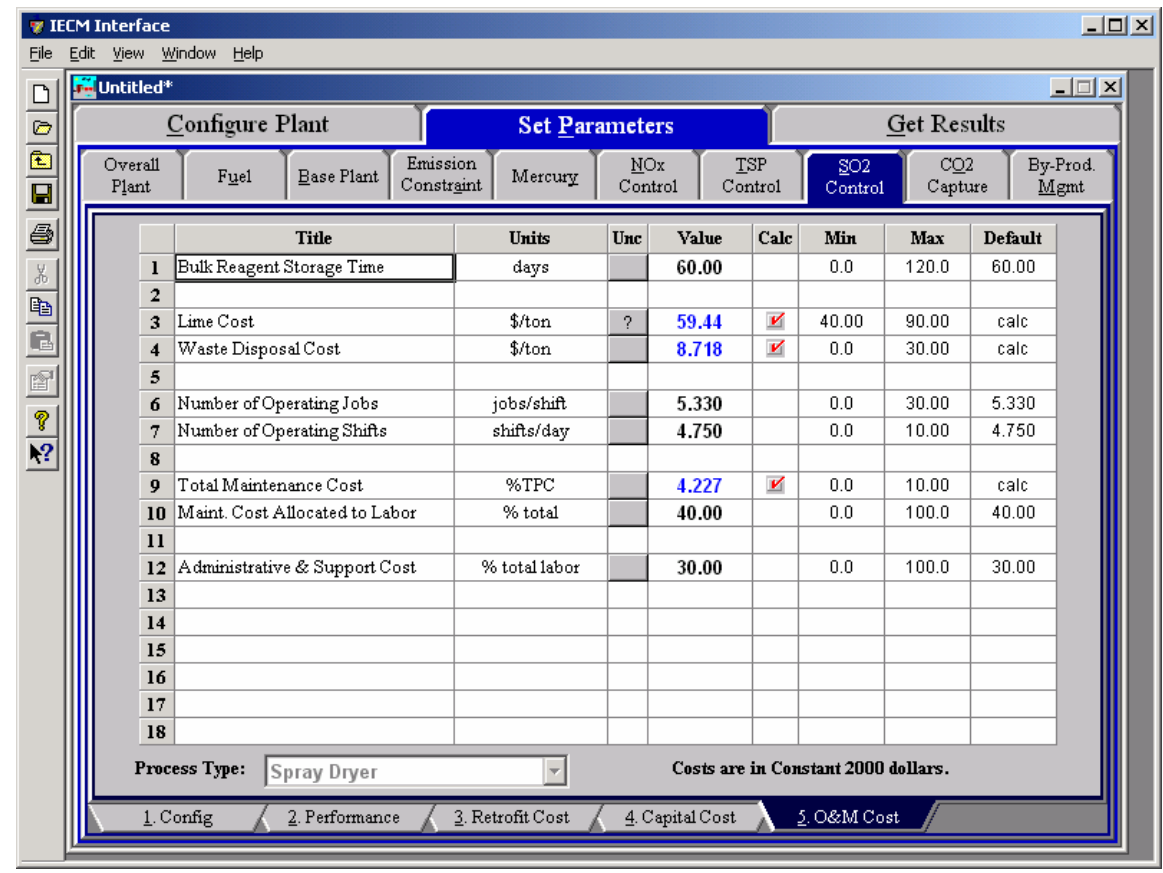

Spray Dryer-O\&M Cost input screen. 
Inputs for operation and maintenance are entered on the O\&M Cost input. Each parameter is described briefly below

Bulk Reagent Storage Time: This is the number of days of bulk storage of reagent. This factor is used to determine the inventory capital cost.

Lime Cost: This is the cost of Lime for the Wet FGD or Lime Spray Dryer system.

Waste Disposal Cost: This is the sludge disposal cost for the FGD system.

Number of Operating Jobs: This is the total number of operating jobs that are required to operate the plant per eight-hour shift.

Number of Operating Shifts: This is the total number of equivalent operating shifts in the plant per day. The number takes into consideration paid time off and weekend work ( 3 shifts/day * 7 days/5 day week * 52 weeks/(52 weeks -6 weeks PTO $)=4.75$ equiv. Shifts/day)

Total Maintenance Cost: This is the annual maintenance cost as a percentage of the total plant cost. Maintenance cost estimates can be developed separately for each process area.

Maint. Cost Allocated to Labor: Maintenance cost allocated to labor as a percentage of the total maintenance cost.

Administrative \& Support Cost: This is the percent of the total operating and maintenance labor associated with administrative and support labor.

\section{Spray Dryer Diagram}

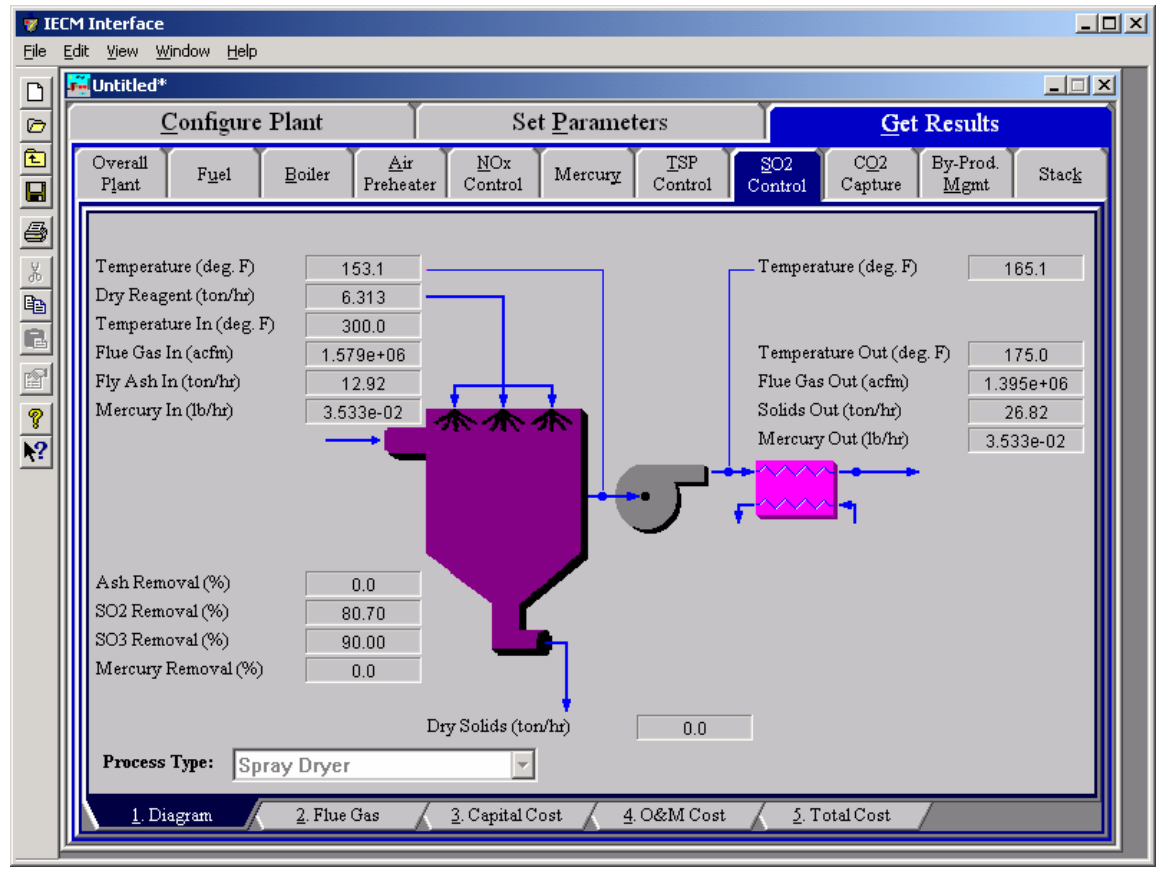

Spray Dryer - Diagram 
The Diagram result screen displays an icon for the Lime Spray Dryer $\mathrm{SO}_{2}$ control technology selected and values for major flows in and out of it. Each result is described briefly below:

\section{Reagent}

Dry Reagent: The total mass flow rate of lime, limestone or limestone with dibasic acid injected into the scrubber. This is a function of the $\mathrm{SO}_{2}$ removal efficiency, the reagent purity and the reagent stoichiometric (all performance input parameters). The reagent is assumed to be dry.

\section{Flue Gas Entering Dryer}

Temperature In: Temperature of the flue gas entering the scrubber. This is determined by the flue gas outlet temperature of the module upstream of the scrubber (e.g., a particulate removal technology).

Flue Gas In: Volumetric flow rate of flue gas entering the scrubber, based on the flue gas temperature entering the scrubber and atmospheric pressure.

Fly Ash In: Total solids mass flow rate in the flue gas entering the scrubber. This is determined by the solids exiting from the module upstream of the scrubber (e.g., a particulate removal technology).

Mercury In: Total mass of mercury entering the scrubber. The value is a sum of all the forms of mercury (elemental, oxidized, and particulate).

\section{Flue Gas Exiting Dryer}

Temperature: Temperature of the flue gas immediately after exiting the scrubber. This is a function of saturation temperature, and the flue gas component concentrations. This temperature is used to determine the flue gas bypass required.

Temperature: Temperature of the flue gas immediately after exiting the induced draft fan. This is a function of flue gas temperature exiting the scrubber, the flue gas bypass and the temperature rise across ID fan input parameter.

Temperature Out: Temperature of the flue gas immediately after exiting the reheater. This is determined by the gas temperature exiting reheater input parameter.

Flue Gas Out: Volumetric flow rate of the flue gas exiting the reheater, based on the flue gas temperature exiting the scrubber and atmospheric pressure.

Solids Out: Total solids mass flow rate in the flue gas exiting the reheater. This is a function of the ash removal parameter on the scrubber performance input screen.

Mercury Out: Total mass of mercury exiting the scrubber after the reheater. The value is a sum of all the forms of mercury (elemental, oxidized, and particulate).

\section{Spray Dryer Performance}

Ash Removal: Actual particulate removal efficiency in the scrubber. This is set by the scrubber performance input parameter.

$\mathrm{SO}_{2}$ Removal: Actual removal efficiency of $\mathrm{SO}_{2}$ in the scrubber. This is a function of the maximum removal efficiency (scrubber performance 
input parameter) and the emission constraint for $\mathrm{SO}_{2}$ (emission constraints input parameter). It is possible that the scrubber may over or under-comply with the emission constraint.

$\mathrm{SO}_{3}$ Removal: Percent of $\mathrm{SO}_{3}$ in the flue gas removed from the scrubber. The $\mathrm{SO}_{3}$ is assumed to combine with $\mathrm{H}_{2} \mathrm{O}$ and leave with the ash solids or sluice water as a sulfate (in the form of $\mathrm{H}_{2} \mathrm{SO}_{4}$ ).

Mercury Removal: Percent of the total mercury removed from the scrubber. The value reflects a weighted average based on the particular species of mercury present (elemental, oxidized, and particulate).

\section{Collected Solids}

Dry Solids: Total solids mass flow rate of solids removed from the scrubber. This is a function of the solids content in the flue gas and the particulate removal efficiency of the scrubber. The solids are assumed to be dry.

\section{Spray Dryer Flue Gas Results}

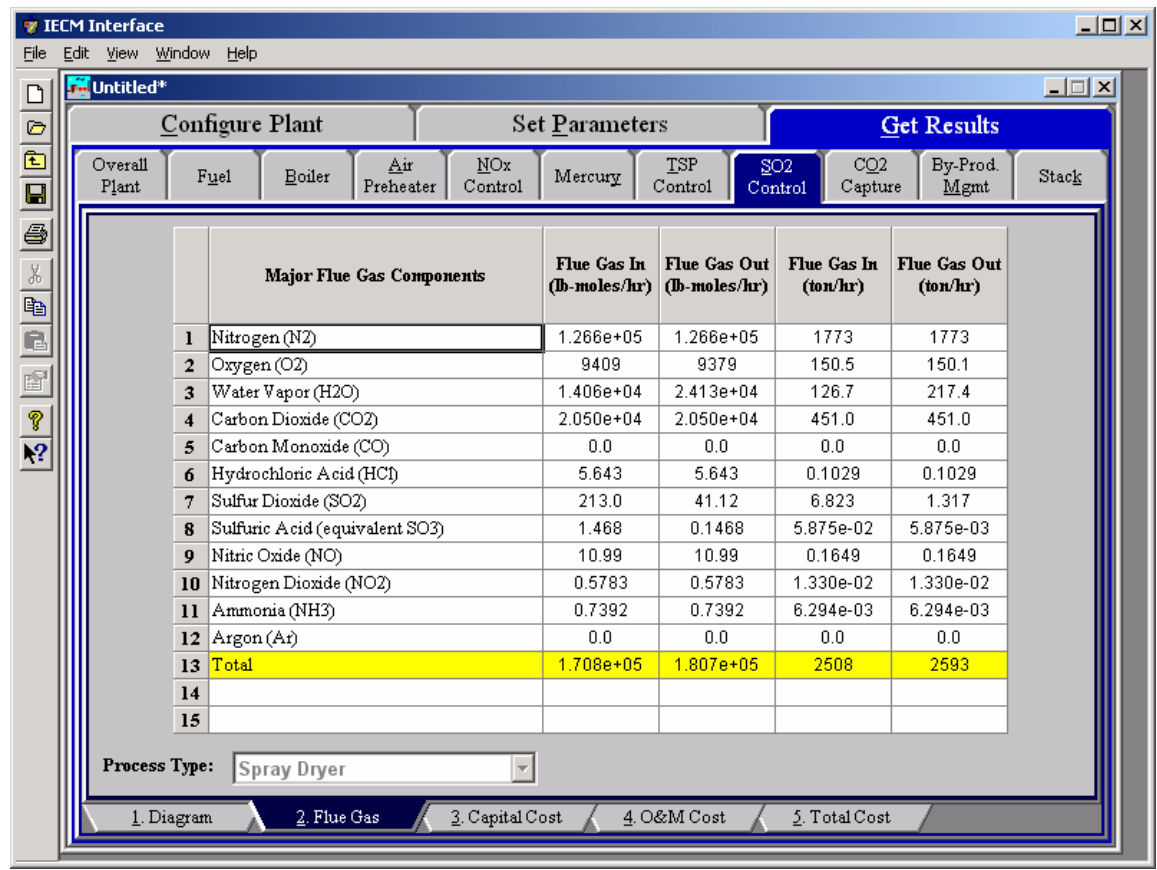

Spray Dryer - Flue Gas result screen.

\section{Major Flue Gas Components}

Each result is described briefly below:

Nitrogen $\left(\mathrm{N}_{2}\right)$ : Total mass of nitrogen.

Oxygen $\left(\mathrm{O}_{2}\right)$ : Total mass of oxygen.

Water Vapor $\left(\mathrm{H}_{2} \mathrm{O}\right)$ : Total mass of water vapor.

Carbon Dioxide $\left(\mathrm{CO}_{2}\right)$ : Total mass of carbon dioxide.

Carbon Monoxide (CO): Total mass of carbon monoxide.

Hydrochloric Acid $(\mathbf{H C l})$ : Total mass of hydrochloric acid. 
Sulfur Dioxide $\left(\mathrm{SO}_{2}\right)$ : Total mass of sulfur dioxide.

Sulfuric Acid (equivalent $\mathbf{S O}_{3}$ ): Total mass of sulfuric acid.

Nitric Oxide (NO): Total mass of nitric oxide.

Nitrogen Dioxide $\left(\mathrm{NO}_{2}\right)$ : Total mass of nitrogen dioxide.

Ammonia $\left(\mathrm{NH}_{3}\right)$ : Total mass of ammonia.

Argon (Ar): Total mass of argon.

Total: Total of the individual components listed above. This item is highlighted in yellow.

\section{Spray Dryer Capital Cost Results}

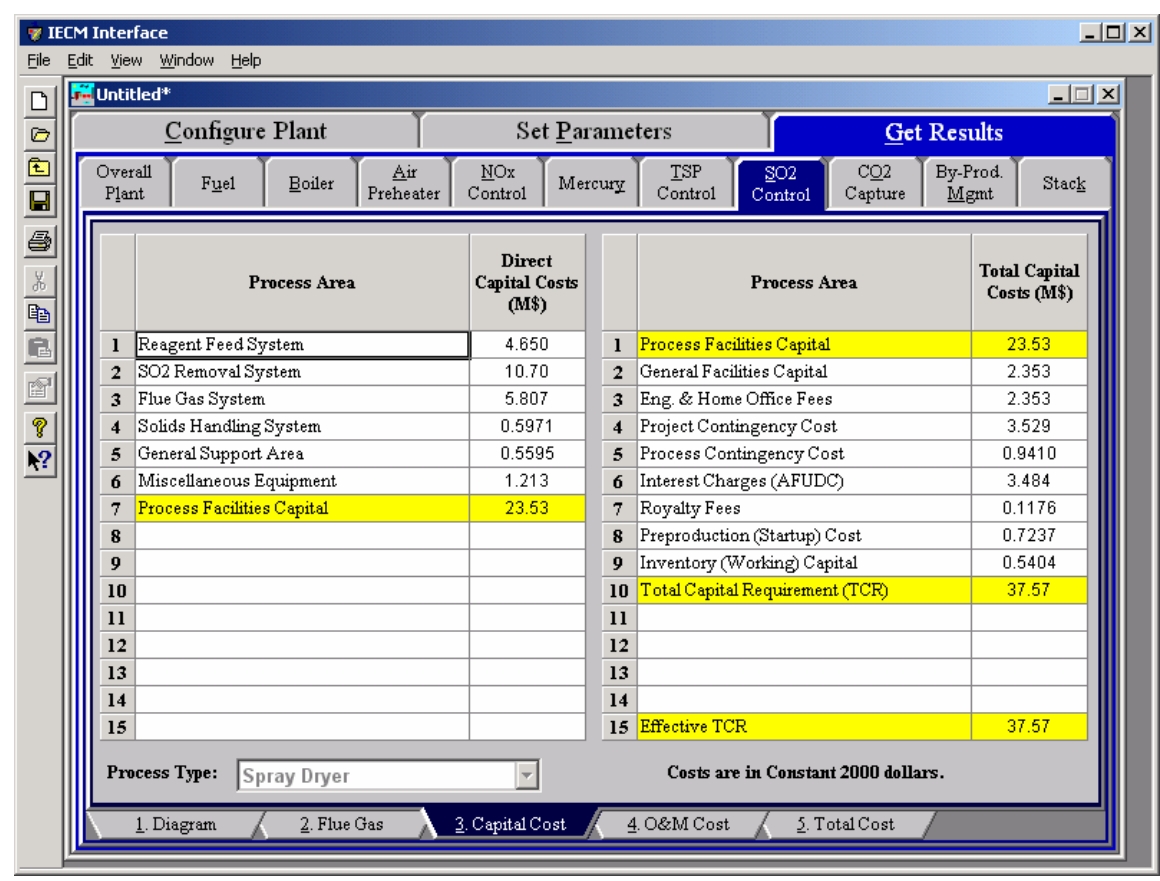

Spray Dryer - Capital Cost result screen.

The Capital Cost result screen displays tables for the direct and indirect capital costs. Each result is described briefly below:

Each process area direct capital cost is a reduced-form model based on regression analysis of data collected from several reports and analyses of particulate control technology units. They are described in general below. The primary factors in the model that effect the capital costs of the scrubbers are the flue gas flow rate through the scrubber, the composition of the flue gas, the reagent stoichiometry, and the reagent flow rate.

Reagent Feed System: This area includes all equipment for storage, handling and preparation of raw materials, reagents, and additives used.

$\mathrm{SO}_{2}$ Removal System: This area deals with the cost of equipment for $\mathrm{SO}_{2}$ scrubbing, such as absorption tower, recirculation pumps, and other equipment. 
Flue Gas System: This area treats the cost of the duct work and fans required for flue gas distribution to $\mathrm{SO}_{2}$ system, plus gas reheat equipment.

Solids Handling System: This area includes the cost of the equipment for fixation, treatment, and transportation of all sludge/dry solids materials produced by scrubbing.

General Support Area: The cost associated with the equipment required to support spray dryer system operation such as makeup water and instrument air are treated here.

Miscellaneous Equipment: Any miscellaneous equipment is treated in this process area.

Process Facilities Capital: The process facilities capital is the total constructed cost of all on-site processing and generating units listed above, including all direct and indirect construction costs. All sales taxes and freight costs are included where applicable implicitly. This result is highlighted in yellow.

General Facilities Capital: The general facilities include construction costs of roads, office buildings, shops, laboratories, etc. Sales taxes and freight costs are included implicitly.

Eng. \& Home Office Fees: The engineering \& home office fees are a percent of total direct capital cost. This is an overhead fee paid to the architect/engineering company.

Project Contingency Cost: Capital cost contingency factor covering the cost of additional equipment or other costs that would result from a more detailed design of a definitive project at the actual site.

Process Contingency Cost: Capital cost contingency factor applied to a new technology in an effort to quantify the uncertainty in the technical performance and cost of the commercial-scale equipment.

Interest Charges (AFUDC): Allowance for funds used during construction, also referred to as interest during construction, is the time value of the money used during construction and is based on an interest rate equal to the before-tax weighted cost of capital. This interest is compounded on an annual basis (end of year) during the construction period for all funds spent during the year or previous years.

Royalty Fees: Royalty charges may apply to some portions of generating units incorporating new proprietary technologies.

Preproduction (Startup) Cost: These costs consider the operator training, equipment checkout, major changes in unit equipment, extra maintenance, and inefficient use of fuel or other materials during startup.

Inventory (Working) Capital: The raw material supply based on $100 \%$ capacity during a 60 day period. These materials are considered storage. The inventory capital includes fuels, consumables, byproducts, and spare parts.

Total Capital Requirement (TCR): Money that is placed (capitalized) on the books of the utility on the service date. TCR includes all the items above. This result is highlighted in yellow. 
Effective TCR: The TCR of the spray dryer that is used in determining the total power plant cost. The effective TCR is determined by the "TCR Recovery Factor".

\section{Spray Dryer O\&M Results}

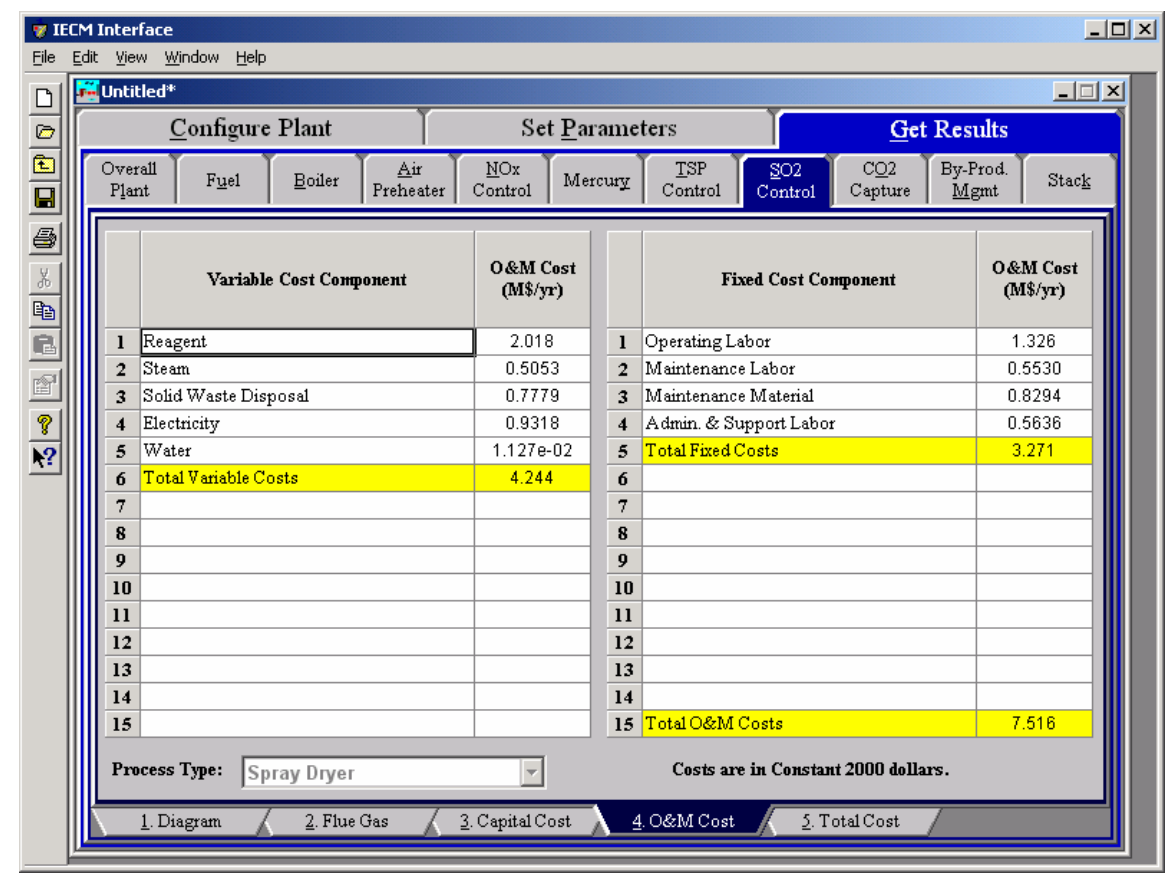

Spray Dryer - O\&M Cost result screen.

The O\&M Cost result screen displays tables for the variable and fixed operation and maintenance costs involved with the $\mathrm{SO}_{2}$ control technology. Each result is described briefly below:

\section{Variable Cost Components}

Variable operating costs and consumables are directly proportional to the amount of kilowatts produced and are referred to as incremental costs. All the costs are subject to inflation.

Reagent: Annual cost of lime or limestone injected into the scrubber on a wet basis. This is a function of the $\mathrm{SO}_{2}$ concentration in the flue gas and the reagent stoichiometric performance input value.

Steam: Annual cost of steam used for direct or reheat use in the scrubber. This is a function of the steam heat rate, reheat energy requirement and gross plant capacity.

Solid Waste Disposal: Total cost to dispose the collected flue gas waste solids. This does not consider by-product gypsum sold in commerce.

Power: Cost of power consumption of the scrubber. This is a function of the gross plant capacity and the scrubber energy penalty performance input parameter.

Water: Cost of water for reagent sluice in the scrubber. This is a function of the liquid to gas ratio performance input parameter for the wet FGD. 
The cost is a function of the flue gas flow rate and the slurry recycle ratio performance input parameter for the spray dryer.

Total Variable Costs: This is the sum of all the variable O\&M costs listed above. This result is highlighted in yellow.

\section{Fixed Cost Components}

Fixed operating costs are essentially independent of actual capacity factor, number of hours of operation, or amount of kilowatts produced. All the costs are subject to inflation.

Operating Labor: Operating labor cost is based on the operating labor rate, the number of personnel required to operate the plant per eighthour shift, and the average number of shifts per day over 40 hours per week and 52 weeks.

Maintenance Labor: The maintenance labor is determined as a fraction of the total maintenance cost.

Maintenance Material: The cost of maintenance material is the remainder of the total maintenance cost, considering the fraction associated with maintenance labor.

Admin. \& Support Labor: The administrative and support labor is the only overhead charge. It is taken as a fraction of the total operating and maintenance labor costs.

Total Fixed Costs: This is the sum of all the fixed O\&M costs listed above. This result is highlighted in yellow.

Total O\&M Costs: This is the sum of the total variable and total fixed O\&M costs. It is used to determine the base plant total revenue requirement. This result is highlighted in yellow.

\section{Spray Dryer Total Cost Results}

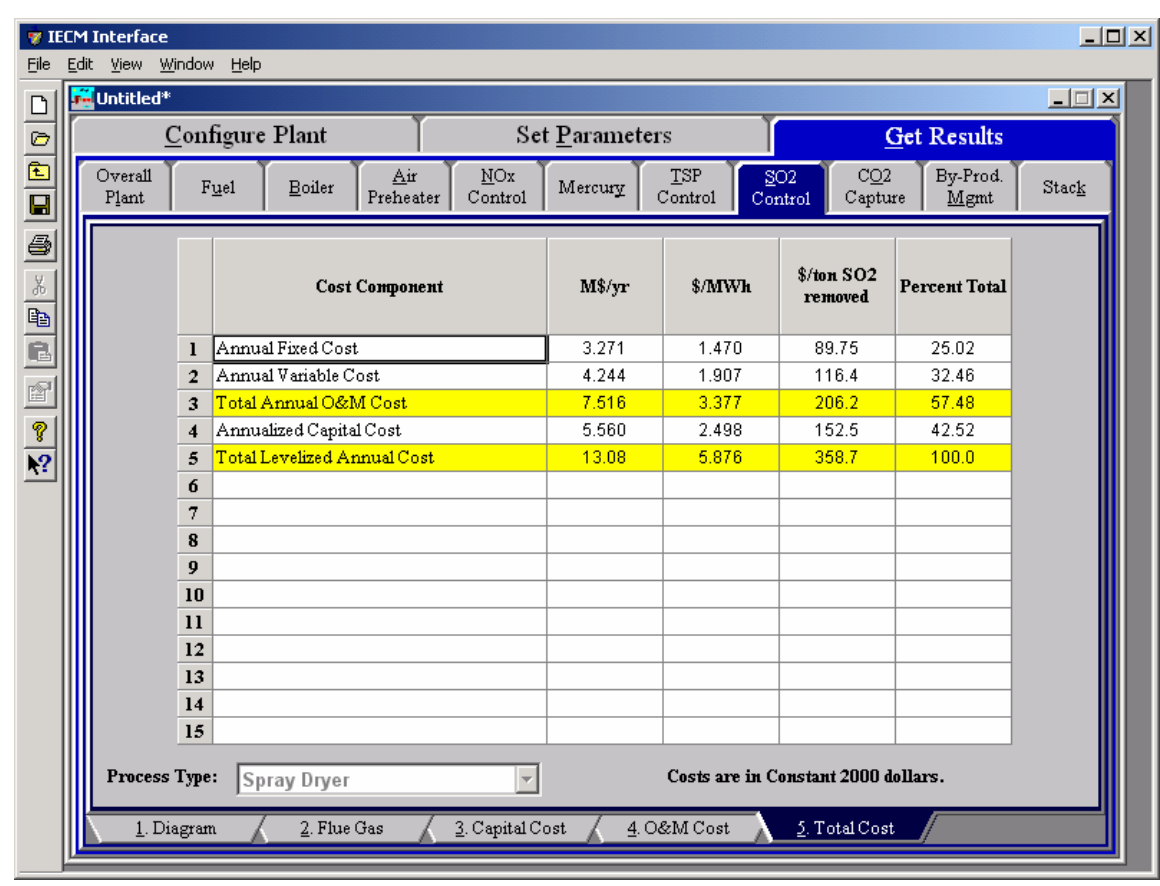


Spray Dryer - Total Cost result screen.

The Total Cost result screen displays a table which totals the annual fixed, variable, operations, maintenance, and capital costs. Each result is described briefly below.

Annual Fixed Cost: The operating and maintenance fixed costs are given as an annual total. This number includes all maintenance materials and all labor costs.

Annual Variable Cost: The operating and maintenance variables costs are given as an annual total. This includes all reagent, chemical, steam, and power costs.

Total Annual O\&M Cost: This is the sum of the annual fixed and variable operating and maintenance costs above. This result is highlighted in yellow.

Annualized Capital Cost: This is the total capital cost expressed on an annualized basis, taking into consideration the levelized carrying charge factor, or fixed charge factor, over the entire book life.

Total Levelized Annual Cost: The total annual cost is the sum of the total annual O\&M cost and annualized capital cost items above. This result is highlighted in yellow. 



\section{Power Block}

\section{Power Block Gas Turbine Inputs}

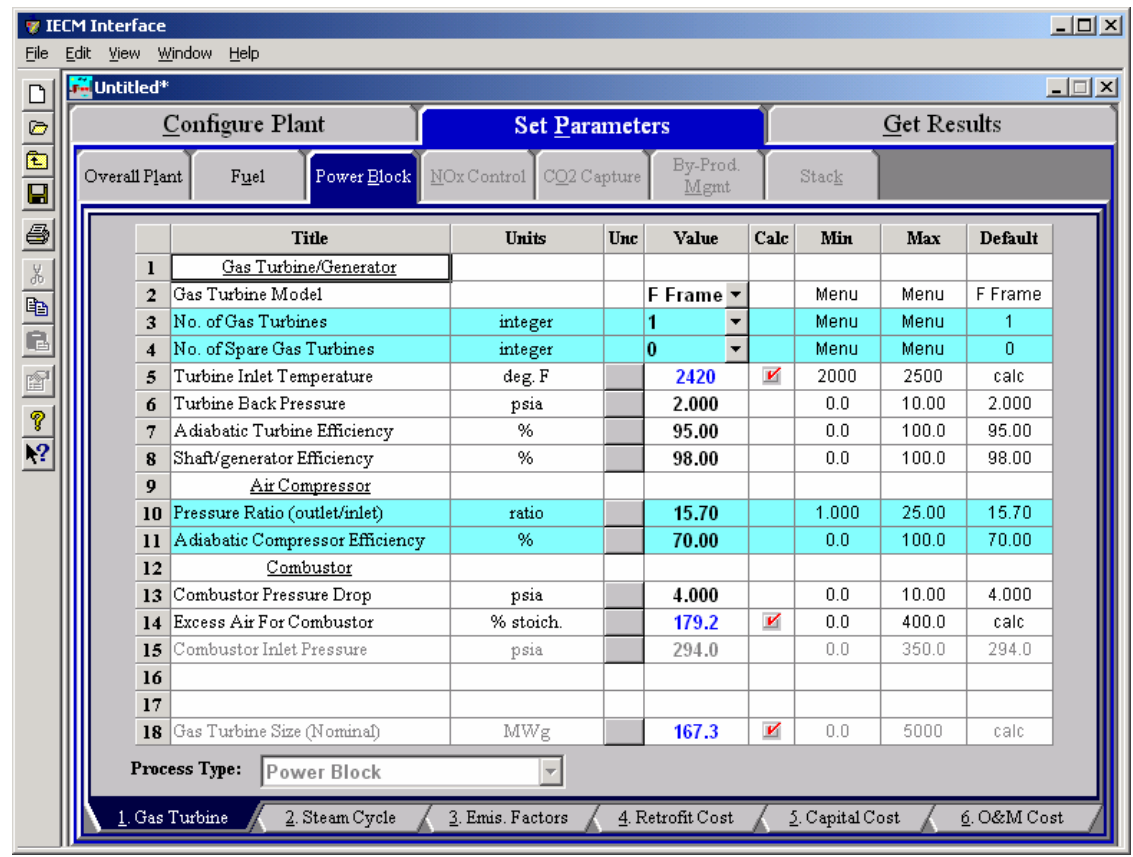

Power Block - Gas Turbine input screen.

\section{Gas Turbine/Generator}

Gas Turbine Model: This is a selection of the type of turbine model used (manufacturer types currently include "F" Frame and "H Frame). The type determines the inlet temperature, pressure ratio, and size parameters.

No. of Gas Turbines: This is the number of gas turbines. Since each turbine is able to produce a fixed output, the number of turbines will determine the plant size.

No. of Spare Gas Turbines: This is the number of spare gas turbines. The spare is primarily used for cost purposes.

Turbine Inlet Temperature: The turbine inlet temperature is carefully controlled to prevent damage or fatigue of the first stage stator and 
rotor blades. This temperature is one of the two most important parameters that impacts system efficiency.

Turbine Back Pressure: The turbine exit pressure must be higher than atmospheric pressure to provide a positive pressure on the flue gas exiting the turbine.

Adiabatic Turbine Efficiency: The adiabatic turbine efficiency adjusts for inefficiencies in real turbines. The ratio is an estimate of real to ideal performance.

Shaft/generator Efficiency: The combined shaft/generator efficiency adjusts for inefficiencies in generator and shaft between the compressor and the generator. The ratio is an estimate of real to ideal performance.

\section{Air Compressor}

Pressure Ratio (outlet/inlet): This is the ratio of the compressor exit pressure to the inlet ambient air pressure. Compression takes place approximately adiabatically.

Adiabatic Compressor Efficiency: The adiabatic compressor efficiency adjusts for inefficiencies in real compressors. The ratio is an estimate of real to ideal performance.

\section{Combustor}

Combustor Pressure Drop: Although the combustor operates at essentially constant pressure, a small pressure drop is typically observed in the combustor exit from the compressor exit.

Excess Air For Combustor: This is the excess theoretical air used for combustion. It is added to the stoichiometric air requirement calculated by the model. The value is calculated and based on the boiler type.

Combustor Inlet Pressure: The combustor inlet pressure is currently fixed at a single value.

Gas Turbine Size (Nominal): This is the electricity produced by one gas turbine. It eventually will include multiple turbine types. Multiple turbines must be specified for larger electricity generation. 


\section{Power Block Steam Cycle Inputs}

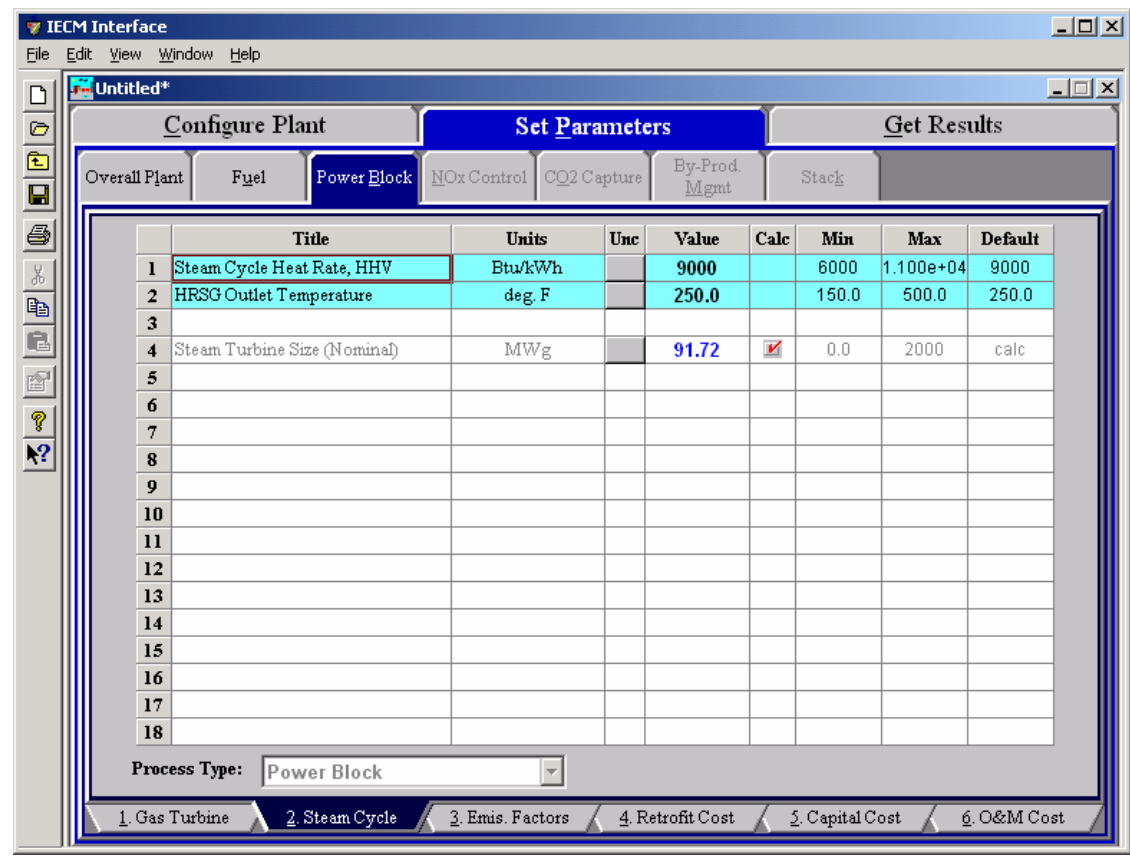

Power Block - Steam Cycle input screen

\section{Steam Cycle Input Parameters}

Steam Cycle Heat Rate, HHV: This is the steam cycle heat rate for the heat recovery steam generator.

HRSG Outlet Temperature: This is the desired output temperature from the heat recovery steam generator (HRSG).

Steam Turbine Size (Nominal): This is the net electricity produced by the steam turbine associated with the HRSG (steam cycle). 


\section{Power Block Emission Factors}

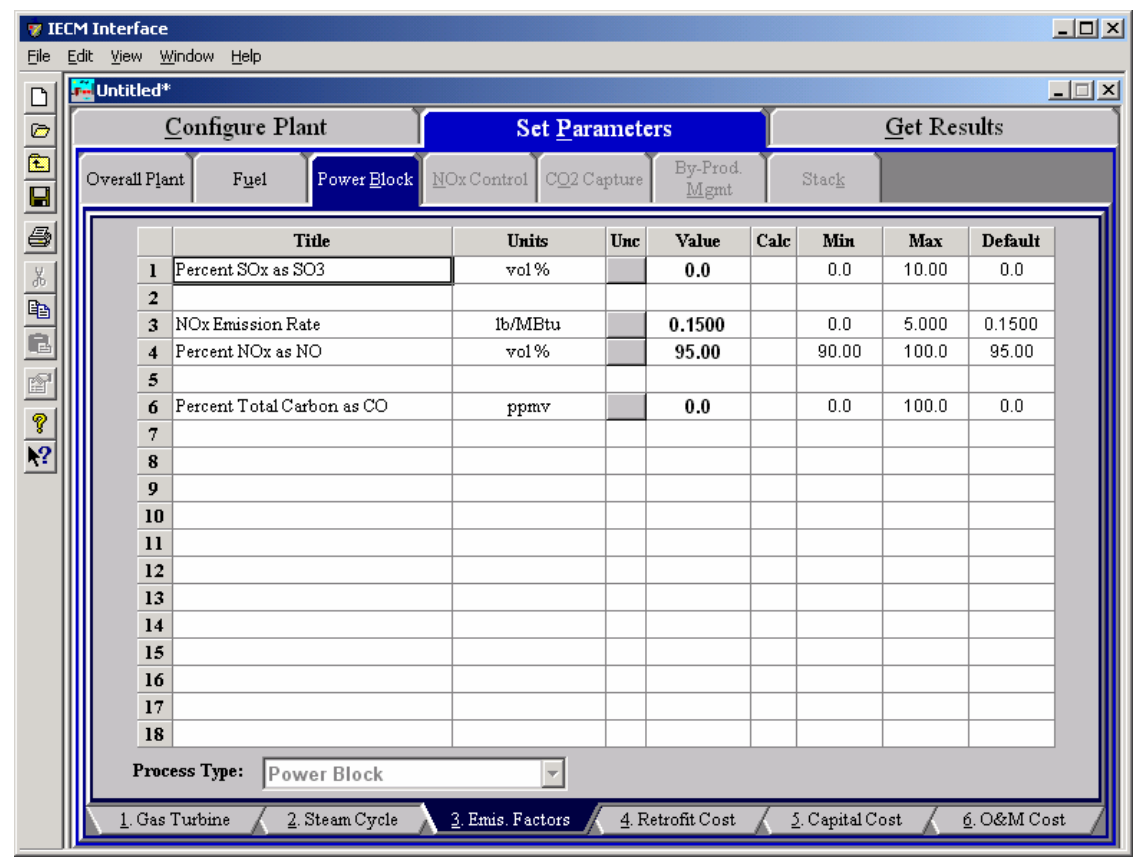

Power Block - Emission Factors input screen.

\section{Emission Factors Input Parameters}

Percent $\mathrm{SO}_{\mathrm{x}}$ as $\mathrm{SO}_{3}$ : This is the volume percent of $\mathrm{SO}_{3}$ in the $\mathrm{SO}_{\mathrm{x}}$.

$\mathrm{NO}_{\mathrm{x}}$ Emission Rate: This is the rate at which NOx is emitted from the plant based on the production of electricity.

Percent $\mathrm{NO}_{\mathrm{x}}$ as NO: This is the volume percent of $\mathrm{NO}_{\mathrm{x}}$ in the $\mathrm{NO}$

Percent Total Carbon as CO:This is the percent of the total carbon that is contained in the CO. 


\section{Power Block Retrofit Cost}

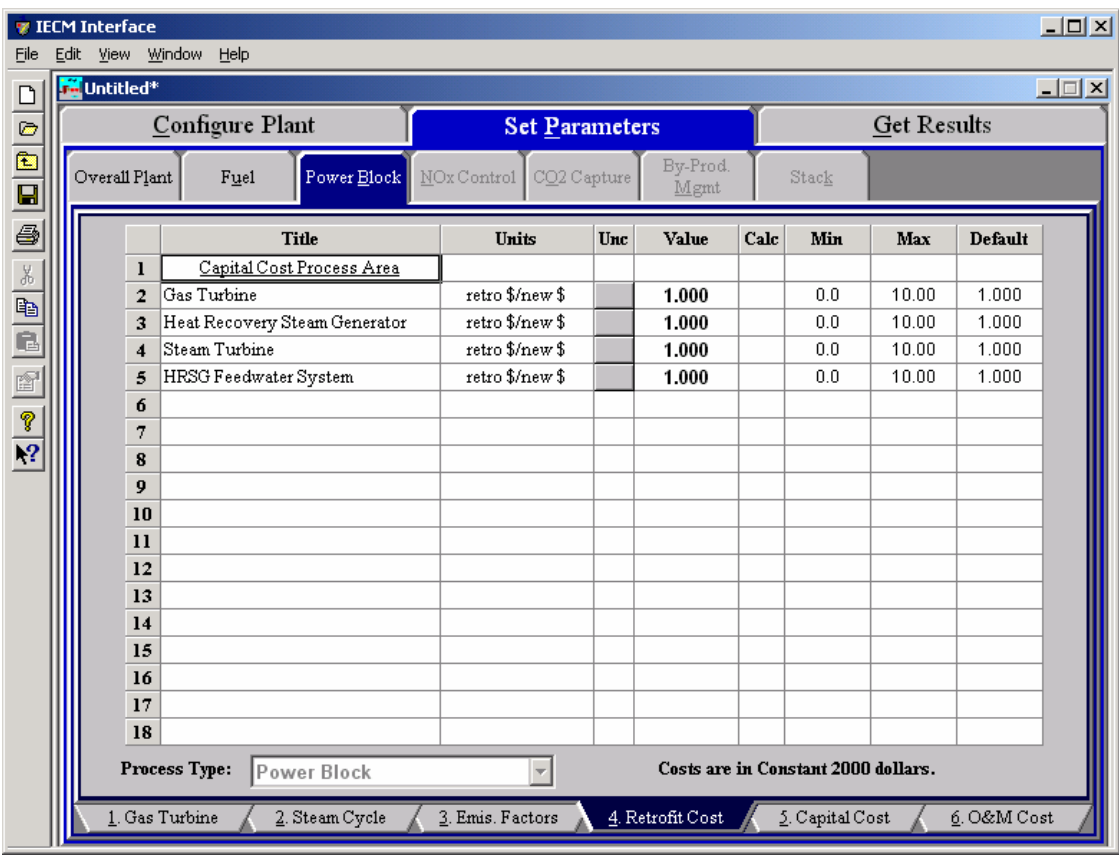

Power Block - Retrofit Cost input screen.

\section{Power Block Retrofit Cost Input Parameters}

Gas Turbine: The Gas Turbine retrofit factor is a ratio of the costs of retrofiting an existing facility versus a new facility, using the same equipment.

Heat Recovery Steam Generator: The Heat Recovery Steam Generator retrofit factor is a ratio of the costs of retrofiting an existing facility versus a new facility, using the same equipment.

Steam Turbine: The Steam Turbine retrofit factor is a ratio of the costs of retrofiting an existing facility versus a new facility, using the same equipment.

HRSG Feedwater System: The Boiler Feedwater retrofit factor is a ratio of the costs of retrofiting an existing facility versus a new facility, using the same equipment. 


\section{Power Block Capital Cost Inputs}

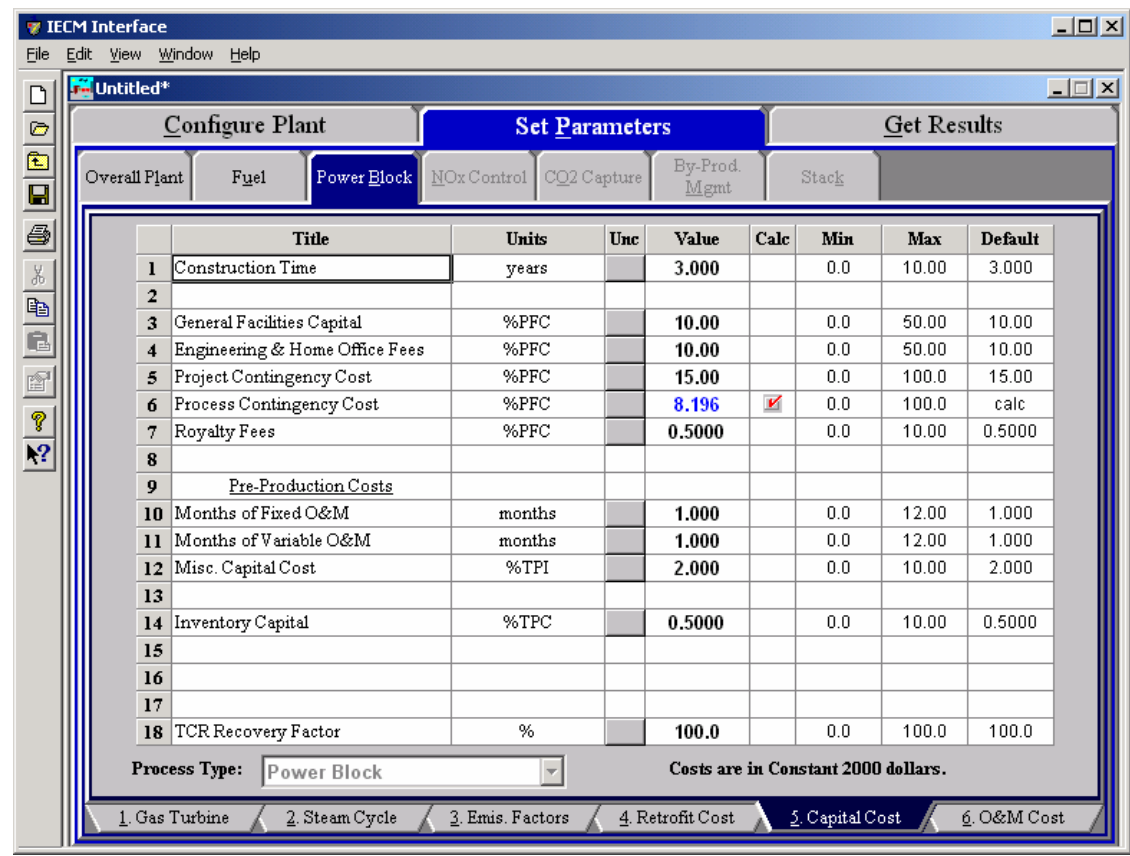

Power Block - Capital Cost input screen.

Inputs for capital costs are entered on the Capital Cost input screen.

Construction Time: This is the idealized construction period in years. It is used to determine the allowance for funds used during construction (AFUDC).

General Facilities Capital (GFC): The general facilities include construction costs of roads, office buildings, shops, laboratories, etc. Sales taxes and freight costs are included implicitly. The cost typically ranges from $5-20 \%$.

Engineering \& Home Office Fees: The engineering \& home office fees are a percent of total direct capital cost. This is an overhead fee paid to the architect/engineering company. These fees typically range from 7$15 \%$.

Project Contingency Cost: This is factor covering the cost of additional equipment or other costs resulting from a more detailed design. Higher contingency factors will be applied to simplified or preliminary designs and lower factors to detailed or finalized designs.

Process Contingency Cost: This quantifies the design uncertainty and cost of a commercial-scale system. This is generally applied on an areaby-area basis. Higher contingency factors are applied to new regeneration systems tested at a pilot plant and lower factors to full-size or commercial systems.

Royalty Fees: Royalty charges may apply to some portions of generating units incorporating new proprietary technologies.

Pre-Production Costs: These costs consider the operator training, equipment checkout, major changes in unit equipment, extra maintenance, and inefficient use of fuel or other materials during start- 
up. These are typically applied to the O\&M costs over a specified period of time (months). The two time periods for fixed and variable O\&M costs are described below with the addition of a miscellaneous capital cost factor.

Months of Fixed O\&M: Time period of fixed operating costs used for preproduction to cover training, testing, major changes in equipment, and inefficiencies in start-up. This includes operating, maintenance, administrative and support labor. It also considers maintenance materials.

Months of Variable O\&M: Time period of variable operating costs used for preproduction to cover chemicals, water, consumables, and solid disposal charges in start-up, assuming 100\% load. This excludes any fuels.

Misc. Capital Cost: This is a percent of total plant investment (sum of TPC and AFUDC) to cover expected changes to equipment to bring the system up to full capacity.

Inventory Capital: Percent of the total direct capital for raw material supply based on $100 \%$ capacity during a 60 day period. These materials are considered storage. The inventory capital includes fuels, consumables, by-products, and spare parts. This is typically $0.5 \%$.

TCR Recovery Factor: The actual total capital required (TCR) as a percent of the TCR in a new power plant. This value is $100 \%$ for a new installation and may be set as low as $0 \%$ for a fabric filter that has been paid off.

\section{Power Block O\&M Cost Inputs}

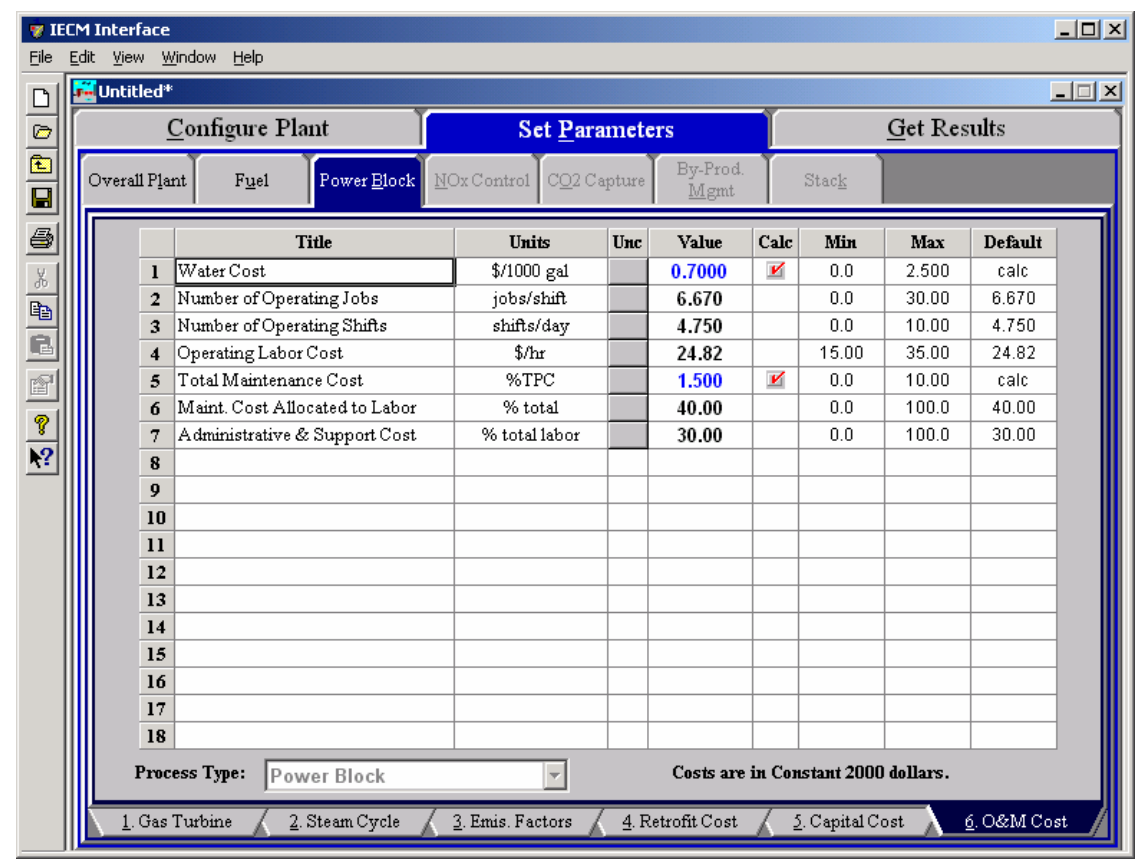

Power Block-O\&M Cost input screen.

Inputs for operating and maintenance costs are entered on the $\mathbf{O \& M}$ Cost input screen. 
Water Cost: Water is mainly required for cooling and also as process makeup. Tost of water may vary depending upon the location of the power plant.

Number of Operating Jobs: This is the total number of operating jobs that are required to operate the plant per eight-hour shift.

Number of Operating Shifts: This is the total number of equivalent operating shifts in the plant per day. The number takes into consideration paid time off and weekend work ( 3 shifts/day $* 7$ days/5 day week $* 52$ weeks $/(52$ weeks -6 weeks PTO $)=4.75$ equiv. Shifts/day)

Total Maintenance Cost: This is the annual maintenance cost as a percentage of the total plant cost. Maintenance cost estimates can be developed separately for each process area.

Maint. Cost Allocated to Labor: Maintenance cost allocated to labor as a percentage of the total maintenance cost.

Administrative \& Support Cost: This is the percent of the total operating and maintenance labor associated with administrative and support labor.

\section{Power Block HRSG/Steam Diagram}

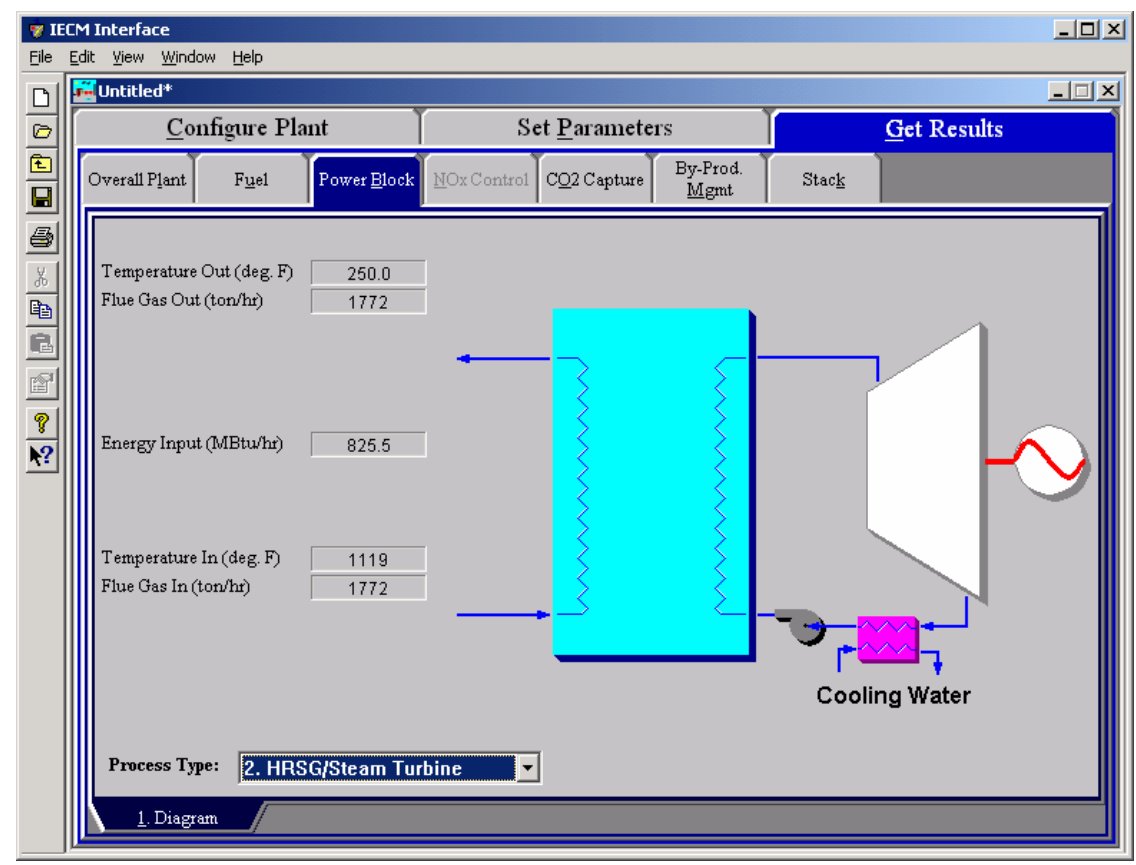

Power Block - HRSG/Steam Diagram results screen.

Flue Gas Exiting Heat Recovery Steam Generator

Temperature Out: Temperature of the flue gas exiting the amine scrubber system.

Flue Gas Out: Volumetric flow rate of the flue gas exiting the amine scrubber.

Flue Gas Entering Heat Recovery Steam Generator 
Energy Input: The energy required to power the heat recovery steam generator.

Temperature In: Temperature of the flue gas entering the amine system area, prior to any processing. This is determined by the flue gas outlet temperature of the process area upstream.

Flue Gas In: Volumetric flow rate of flue gas entering the amine system.

\section{Power Block Gas Turbine Diagram}

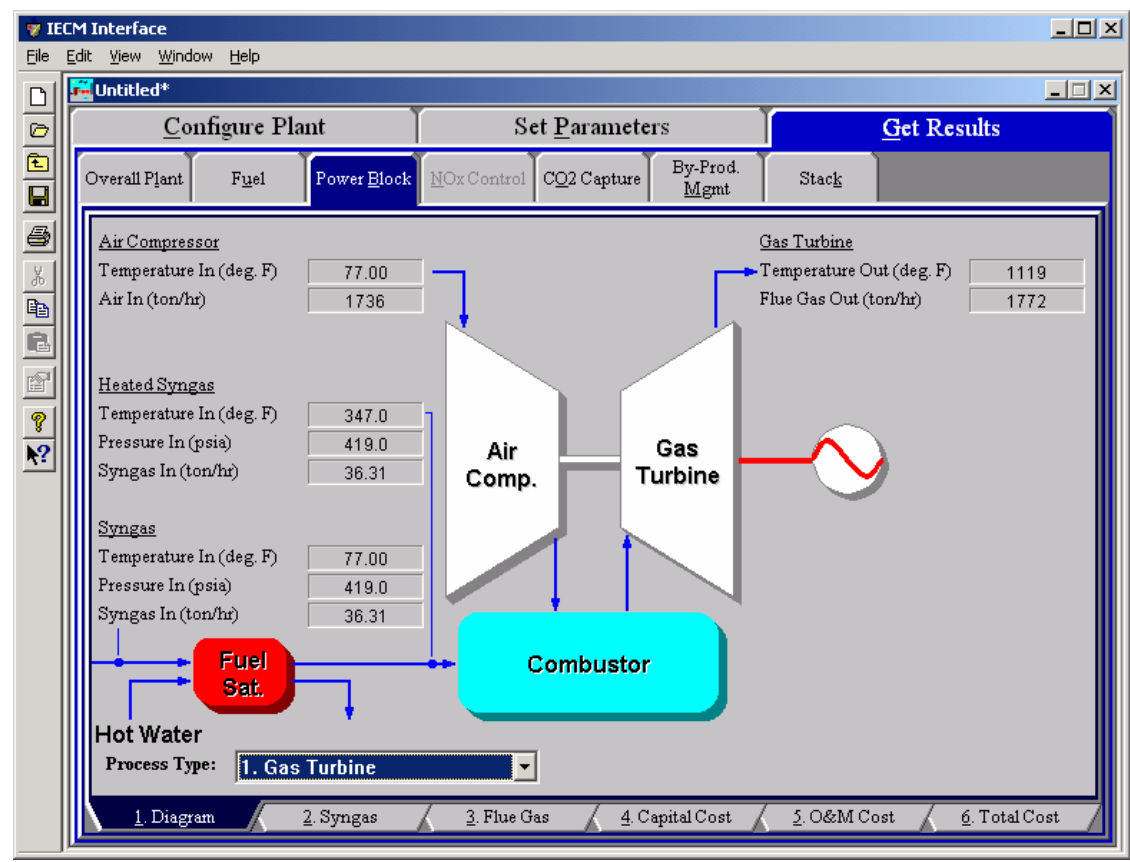

Power Block - Gas Turbine Diagram result screen.

\section{Air Entering Compressor}

Temperature In: Temperature of the flue gas entering the amine system area, prior to any processing. This is determined by the flue gas outlet temperature of the process area upstream.

Air In: Volumetric flow rate of the air at the burners, based on the air temperature at the burners and atmospheric pressure.

\section{Heated Syngas Entering Combustor}

Temperature In: Temperature of the flue gas entering the amine system area, prior to any processing. This is determined by the flue gas outlet temperature of the process area upstream.

Pressure In: This is the pressure of the heated syngas as it enters the combustor.

Syngas In: This is the mass flow rate of the heated syngas to the combustor.

\section{Syngas Entering Combustor}


Temperature In: Temperature of the flue gas entering the amine system area, prior to any processing. This is determined by the flue gas outlet temperature of the process area upstream.

Pressure In: This is the pressure of the synas as it enters the combustor.

Syngas In: This is the mass flow rate of the syngas to the combustor.

\section{Flue Gas Exiting Gas Turbine}

Temperature Out: Temperature of the flue gas exiting the amine scrubber system.

Flue Gas Out: Volumetric flow rate of the flue gas exiting the amine scrubber.

\section{Power Block Syngas Results}

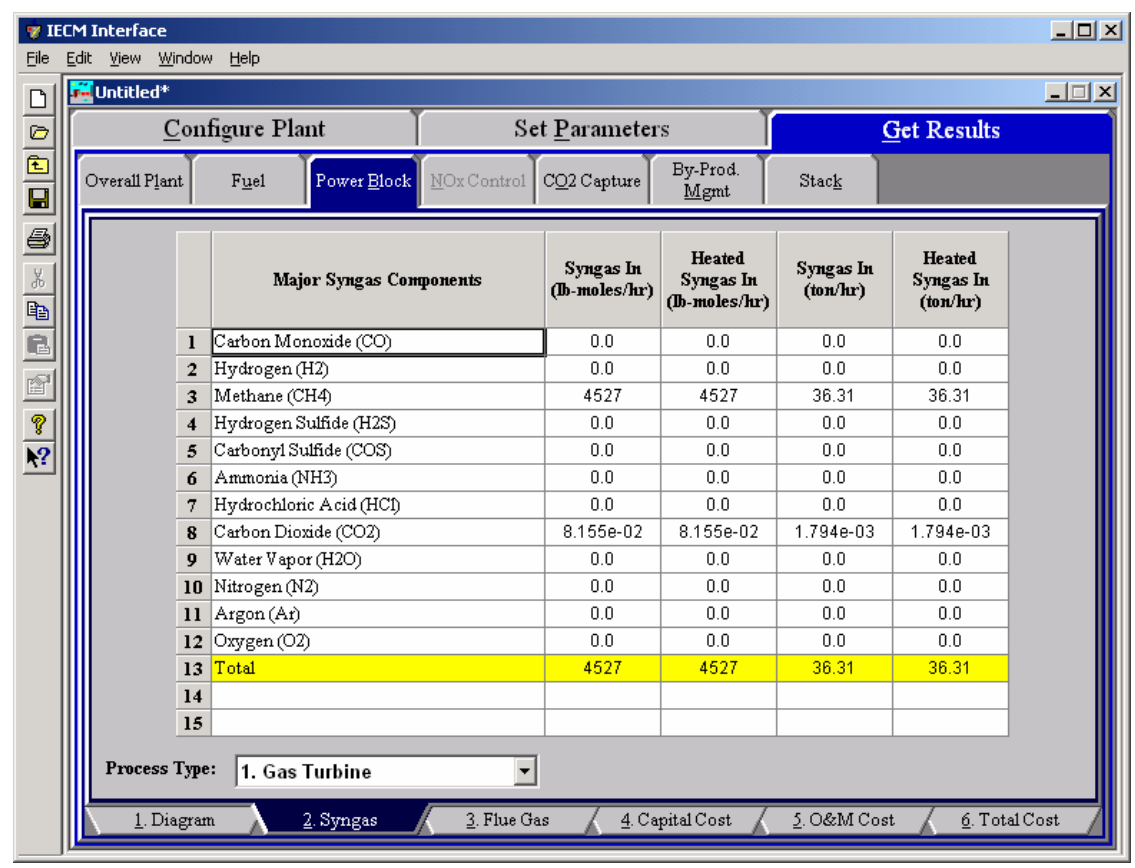

Power Block - Syngas result screen.

\section{Major Syngas Components}

Carbon Monoxide (CO): Flow rate of carbon monoxide in the syngas.

Hydrogen $\left(\mathbf{H}_{2}\right)$ : Flow rate of hydrogen in the syngas.

Methane $\left(\mathrm{CH}_{4}\right)$ : Flow rate of methane in the syngas.

Hydrogen Sulfide $\left(\mathrm{H}_{2} \mathrm{~S}\right)$ : Flow rate of hydrogen sulfide in the syngas.

Carbonyl Sulfide (COS): Flow rate of carbon sulfide in the syngas.

Ammonia $\left(\mathrm{NH}_{3}\right)$ : Flow rate of ammonia in the syngas.

Hydrochloric Acid $(\mathbf{H C l})$ : Flow rate of hydrochloric acid in the syngas.

Carbon Dioxide $\left(\mathrm{CO}_{2}\right)$ : Flow rate of carbon dioxide in the syngas.

Water Vapor $\left(\mathrm{H}_{2} \mathrm{O}\right)$ : Flow rate of water vapor in the syngas. 
Nitrogen $\left(\mathrm{N}_{2}\right)$ : Flow rate of nitrogen in the syngas.

Argon (Ar): Flow rate of argon in the syngas.

Oxygen $\left(\mathrm{O}_{2}\right)$ : Flow rate of oxygen in the syngas.

Total: Total flow rate of the syngas.

\section{Power Block Flue Gas Results}

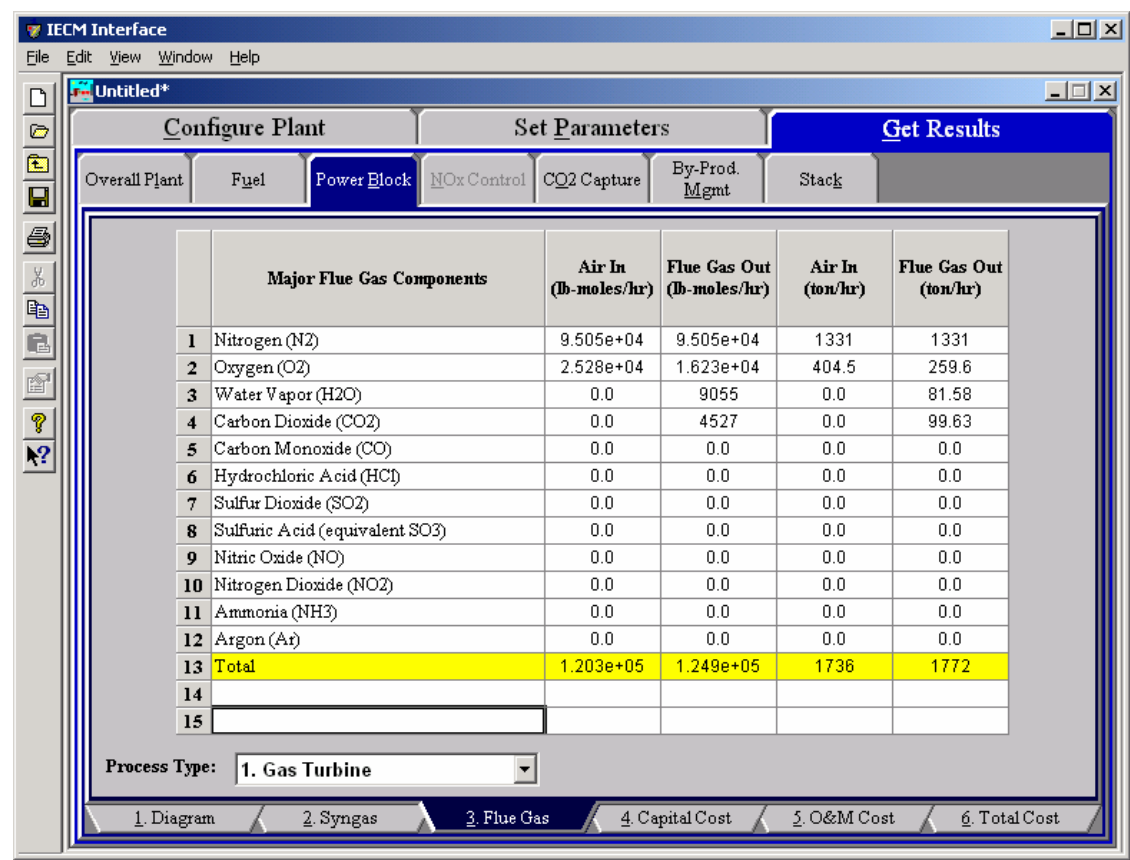

Power Block - Flue Gas results screen.

\section{Major Flue Gas Components}

Each result is described briefly below:

Nitrogen $\left(\mathbf{N}_{\mathbf{2}}\right)$ : Total mass of nitrogen.

Oxygen $\left(\mathrm{O}_{2}\right)$ : Total mass of oxygen.

Water Vapor $\left(\mathrm{H}_{2} \mathrm{O}\right)$ : Total mass of water vapor.

Carbon Dioxide $\left(\mathbf{C O}_{2}\right)$ : Total mass of carbon dioxide.

Carbon Monoxide (CO): Total mass of carbon monoxide.

Hydrochloric Acid (HCl): Total mass of hydrochloric acid.

Sulfur Dioxide $\left(\mathbf{S O}_{2}\right)$ : Total mass of sulfur dioxide.

Sulfuric Acid (equivalent $\mathbf{S O}_{3}$ ): Total mass of sulfuric acid.

Nitric Oxide (NO): Total mass of nitric oxide.

Nitrogen Dioxide $\left(\mathbf{N O}_{2}\right)$ : Total mass of nitrogen dioxide.

Ammonia $\left(\mathrm{NH}_{3}\right)$ : Total mass of ammonia.

Argon (Ar): Total mass of argon. 
Total: Total of the individual components listed above. This item is highlighted in yellow.

\section{Power Block Capital Cost Results}

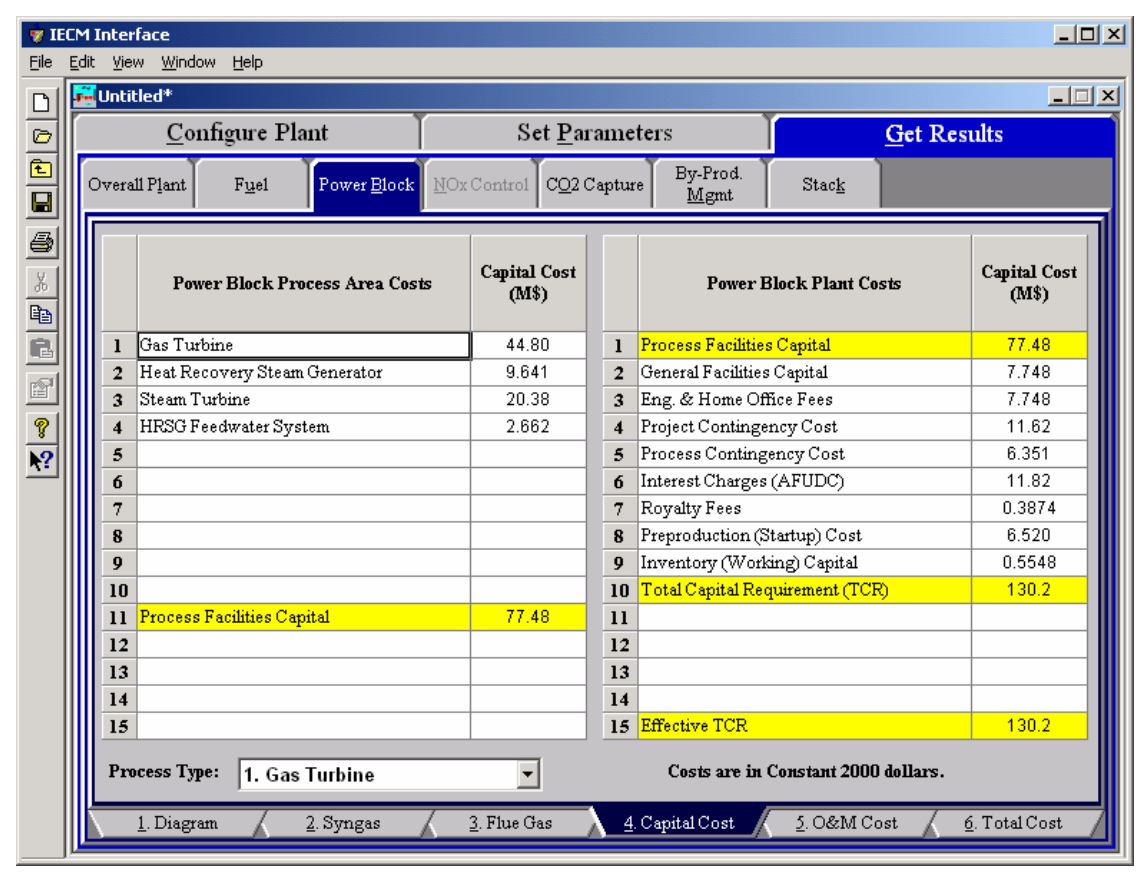

Power Block - Capital Cost results screen.

This result screen displays tables containing the Power Block Capital Costs. Each result is described briefly below:

\section{Power Block Process Area Costs}

Gas Turbine:The model bases the capital cost of the gas turbine on the turbine model used either manufacturer type "F" Frame or "H" Frame.

Heat Recovery Steam Generator:The heat recovery steam generator is a set of heat exchangers in which heat is removed from the gas turbine exhaust gas to generate steam for the steam turbine.

Steam Turbine: The cost of a stema turbine is expected to depend on the mass flow rate of steam through the system, the pressures in each stage, and the generator output

HRSG Feedwater System: The boiler feedwater system consists of equipment for handling raw water and polished water in the steam cycle, including a water mineralization unit for raw water, a dimineralized water storage tank, a condensate water, a condensate polishing unit, and a blowdown flash drum.

Process Facilities Capital: The process facilities capital is the total constructed cost of all on-site processing and generating units listed above, including all direct and indirect construction costs. All sales taxes and freight costs are included where applicable implicitly. This result is highlighted in yellow.

\section{Power Block Plant Costs}


Process Facilities Capital: (see definition above)

General Facilities Capital: The general facilities include construction costs of roads, office buildings, shops, laboratories, etc. Sales taxes and freight costs are included implicitly.

Eng. \& Home Office Fees: The engineering \& home office fees are a percent of total direct capital cost. This is an overhead fee paid to the architect/engineering company.

Project Contingency Cost: Capital cost contingency factor covering the cost of additional equipment or other costs that would result from a more detailed design of a definitive project at the actual site.

Process Contingency Cost: Capital cost contingency factor applied to a new technology in an effort to quantify the uncertainty in the technical performance and cost of the commercial-scale equipment.

Interest Charges (AFUDC): Allowance for funds used during construction, also referred to as interest during construction, is the time value of the money used during construction and is based on an interest rate equal to the before-tax weighted cost of capital. This interest is compounded on an annual basis (end of year) during the construction period for all funds spent during the year or previous years.

Royalty Fees: Royalty charges may apply to some portions of generating units incorporating new proprietary technologies.

Preproduction (Startup) Cost: These costs consider the operator training, equipment checkout, major changes in unit equipment, extra maintenance, and inefficient use of fuel or other materials during startup.

Inventory (Working) Capital: The raw material supply based on $100 \%$ capacity during a 60 day period. These materials are considered storage. The inventory capital includes fuels, consumables, byproducts, and spare parts.

Total Capital Requirement (TCR): Money that is placed (capitalized) on the books of the utility on the service date. TCR includes all the items above. This result is highlighted in yellow.

Effective TCR: The TCR of the spray dryer that is used in determining the total power plant cost. The effective TCR is determined by the "TCR Recovery Factor". 


\section{Power Block O\&M Cost Results}

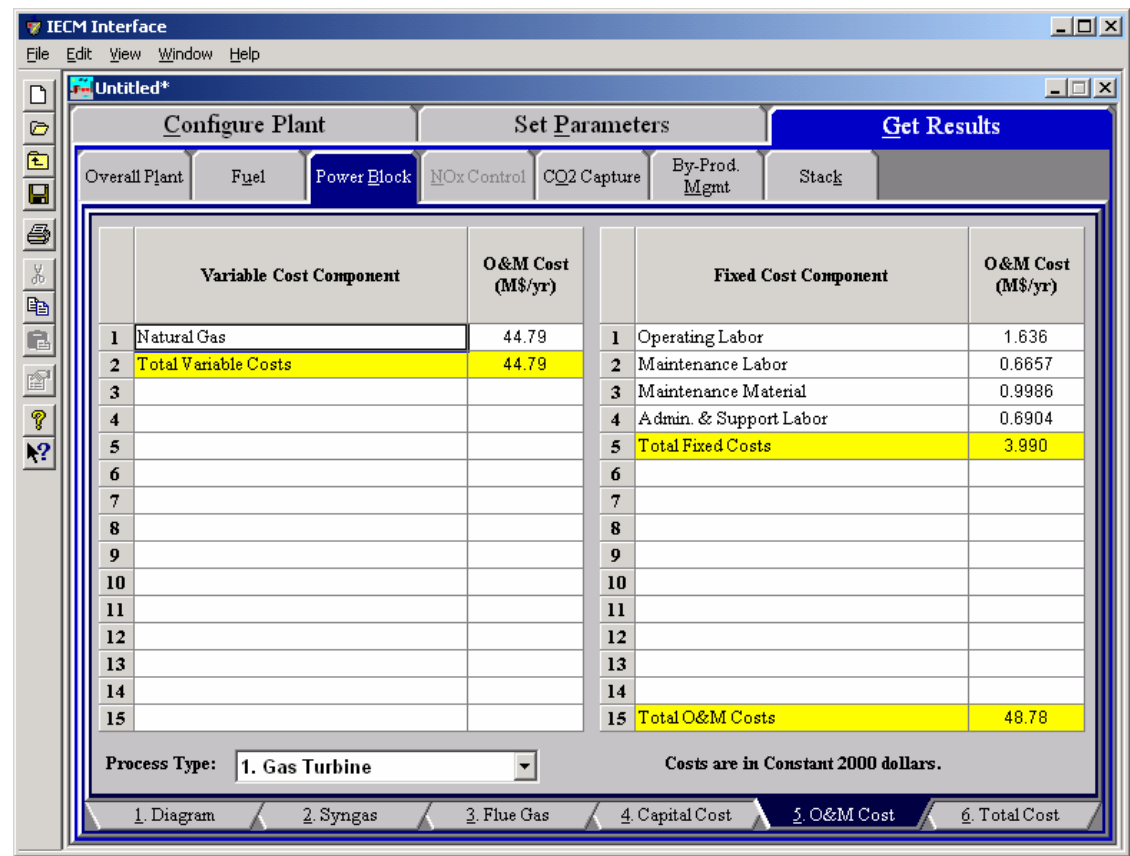

Power Block - O\&M Cost results screen.

\section{Variable Cost Component}

Natural Gas: Annual cost of natural gas supplied to the gas turbine.

Total Variable Costs: This is the sum of all the variable O\&M costs listed above. This result is highlighted in yellow.

\section{Fixed Cost Component}

Fixed operating costs are essentially independent of actual capacity factor, number of hours of operation, or amount of kilowatts produced. All the costs are subject to inflation.

Operating Labor: Operating labor cost is based on the operating labor rate, the number of personnel required to operate the plant per eighthour shift, and the average number of shifts per day over 40 hours per week and 52 weeks.

Maintenance Labor: The maintenance labor is determined as a fraction of the total maintenance cost.

Maintenance Material: The cost of maintenance material is the remainder of the total maintenance cost, considering the fraction associated with maintenance labor.

Admin. \& Support Labor: The administrative and support labor is the only overhead charge. It is taken as a fraction of the total operating and maintenance labor costs.

Total Fixed Costs: This is the sum of all the fixed O\&M costs listed above. This result is highlighted in yellow.

Total O\&M Costs: This is the sum of the total variable and total fixed O\&M costs. It is used to determine the base plant total revenue requirement. This result is highlighted in yellow. 


\section{Power Block Total Cost Results}

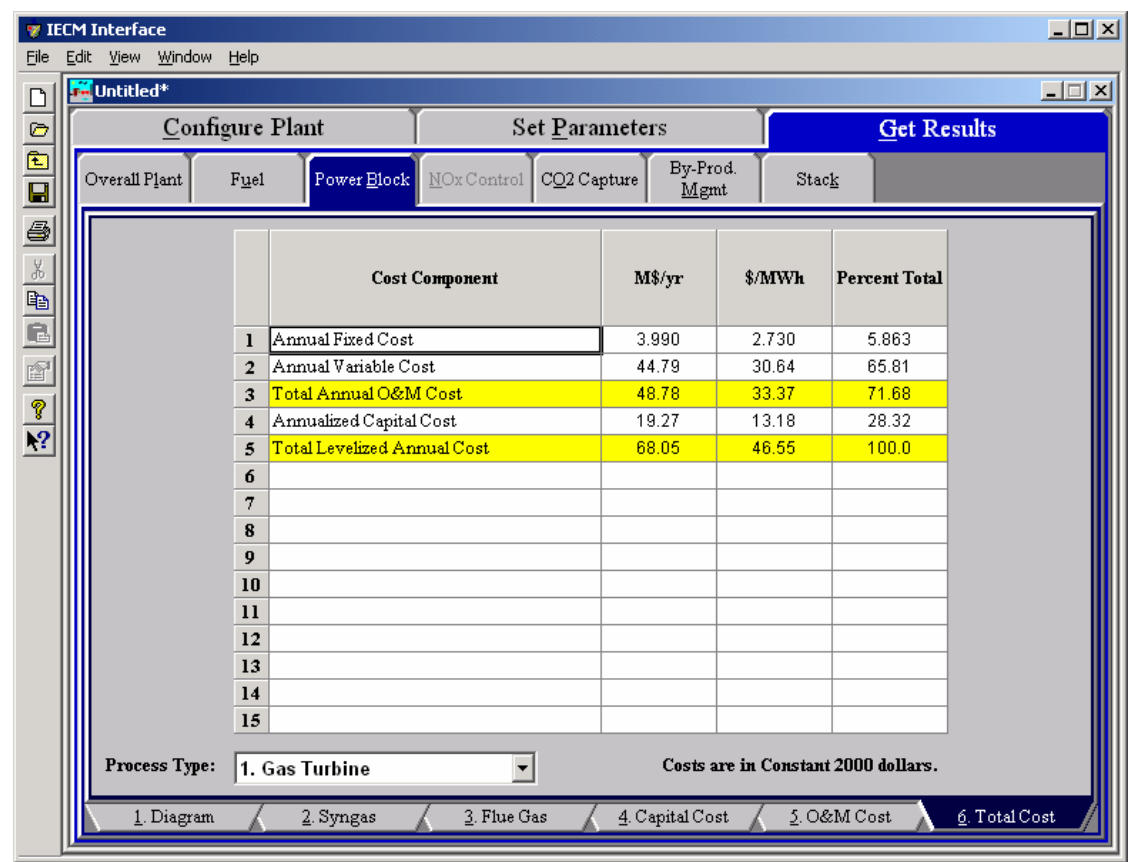

Power Block - Total Cost results screen.

The Total Cost result screen displays a table which totals the annual fixed, variable, operations, maintenance, and capital costs. Each result is described briefly below.

\section{Cost Component}

Annual Fixed Cost: The operating and maintenance fixed costs are given as an annual total. This number includes all maintenance materials and all labor costs.

Annual Variable Cost: The operating and maintenance variables costs are given as an annual total. This includes all reagent, chemical, steam, and power costs.

Total Annual O\&M Cost: This is the sum of the annual fixed and variable operating and maintenance costs above. This result is highlighted in yellow.

Annualized Capital Cost: This is the total capital cost expressed on an annualized basis, taking into consideration the levelized carrying charge factor, or fixed charge factor, over the entire book life.

Total Levelized Annual Cost: The total annual cost is the sum of the total annual O\&M cost and annualized capital cost items above. This result is highlighted in yellow. 



\section{Amine System}

\section{Amine System Configuration}

The screens under the Co2 Capture Technology Navigation Tab display and design flows and data related to the Amine System.

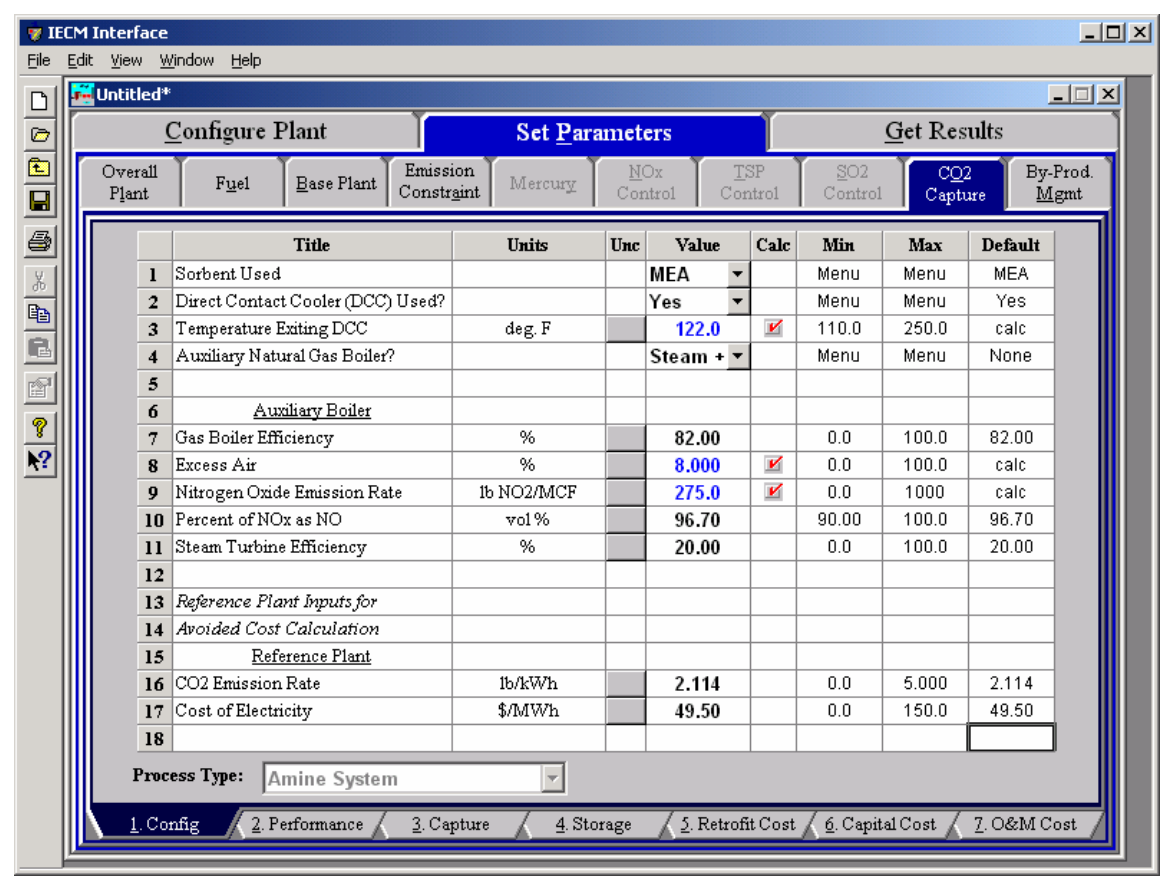

Amine System - Config. input screen.

Each configuration parameter is described briefly below.

Sorbent Used: MEA is the sorbent used in the system and the nominal values of various parameters are based on a process simulation model that uses MEA.

Direct Contact Cooler (DCC) Used: The default setting in the IECM chooses to include a DCC to cool the flue gas before it enters the amine system. The temperature of the flue gas affects the absorption reaction (absorption of $\mathrm{CO}_{2}$ in MEA sorbent is an exothermic process favored by lower temperatures). Also, the volumetric flow rate of the flue gas stream, which is a key determinant of the sizes of various equipments 
(direct contact cooler, flue gas blower, absorber), is directly related to the flue gas temperature. Hence lower flue gas temperature is desired. The typically acceptable range of flue gas temperature is about 50-60 ${ }^{\circ} \mathrm{C}$. If the flue gas is coming from wet sulfur scrubber, additional DCC may not be required. But in case of flue gas from NG-fired boiler, which often does not pass through a sulfur scrubber, DCC is a must.

Temperature Exiting DCC: The desirable temperature of the flue gas entering the $\mathrm{CO}_{2}$ capture system is about 45-50 deg C. If a direct contact cooler is installed upstream of $\mathrm{CO}_{2}$ capture system, then this temperature level may be achieved. Else, this is same as that obtained from the base plant. This variable is only displayed if a DCC is used.

Auxiliary Natural Gas Boiler?: A potential option to provide energy for the amine system is by adding an auxiliary Natural Gas (NG)-fired boiler. Often it would be combined with a steam turbine which could generate some additional power (mainly used to supply electrical energy demand of $\mathrm{CO}_{2}$ capture unit), and the LP steam would be then used for sorbent regeneration. Thus, the original steam cycle of the power plant remains unperturbed and the net power generation capacity of the power plant is not adversely affected. It comes at an additional cost of capital requirement for the boiler (and turbine) and the cost of supplemental NG fuel. Also, the combustion of NG leads to additional $\mathrm{CO}_{2}$ emissions (and $\mathrm{NO}_{\mathrm{x}}$ emissions). The Auxiliary Natural Gas options are:

- None

- Steam Only

- Steam + Power

\section{Auxiliary Boiler}

When an auxiliary natural gas boiler is selected the options for its use are either for Steam Only or for Steam + Power.

Steam Only: When the auxiliary natural gas boiler option has been selected and it is to be used for steam only then the following parameters are used in model calculations and are modifiable by the user.

- Gas Boiler Efficiency: This is the percentage of fuel input energy transferred to steam in the boiler. The model default is to calculate the boiler efficiency using standard algorithms described in the literature. The efficiency is a function of energy losses due to inefficient heat transfer across the preheater, latent heat of evaporation, incomplete combustion, radiation losses, and unaccounted losses.

- Excess Air:This is the excess theoretical air used for combustion. It is added to the stoichiometric air requirement calculated by the model. The value is calculated and based on the fuel type and boiler type.

- Nitrogen Oxide Emission Rate: This parameter establishes the level of $\mathrm{NO}_{\mathrm{x}}$ emissions from the boiler. The default values reflect the AP-42 EPA emission factors. It is a function of boiler firing method and the coal rank. The model calculates this value and expresses it in pounds of equivalent $\mathrm{NO}_{2}$ per ton of coal. 
- Percent of NOx as NO: This parameter establishes the level of nitric oxide (NO) in the flue gas stream. The remainder of the total $\mathrm{NO}_{\mathrm{x}}$ emissions is assumed to be nitrogen dioxide $\left(\mathrm{NO}_{2}\right)$. The default parameters reflect the AP-42 EPA emission factors and are dependent on the fuel type.

Steam + Power: When an auxiliary natural gas boiler is selected and is to be used for additional steam and power, then in addition to the above parameters the following parameter is displayed:

- Steam Turbine Efficiency: The steam turbine efficiency is the

\section{Reference Plant}

The following reference plant inputs are used in the Avoided Cost Calculation. Analysts commonly express the cost of an environmental control system in terms of either the cost per tonne of pollutant removed or the cost per tonne "avoided." For an energy-intensive system like amine scrubbers there is a big difference between the cost per tonne $\mathrm{CO}_{2}$ removed and the cost per tonne $\mathrm{CO}_{2}$ avoided based on net plant capacity. Since the purpose of adding a capture unit is to reduce the $\mathrm{CO}_{2}$ emissions per net kWh delivered, the cost of $\mathrm{CO}_{2}$ avoidance (relative to a reference plant with no $\mathrm{CO}_{2}$ control) is the economic indicator most widely used. It can be calculated as

$\mathbf{C O}_{2}$ Emission Rate: This is the emission rate for the reference power plant (without $\mathrm{CO}_{2}$ capture)

Cost of Electricity: This is the cost of electricity for the reference power plant (without $\mathrm{CO}_{2}$ capture)

\section{Amine System Performance Inputs}

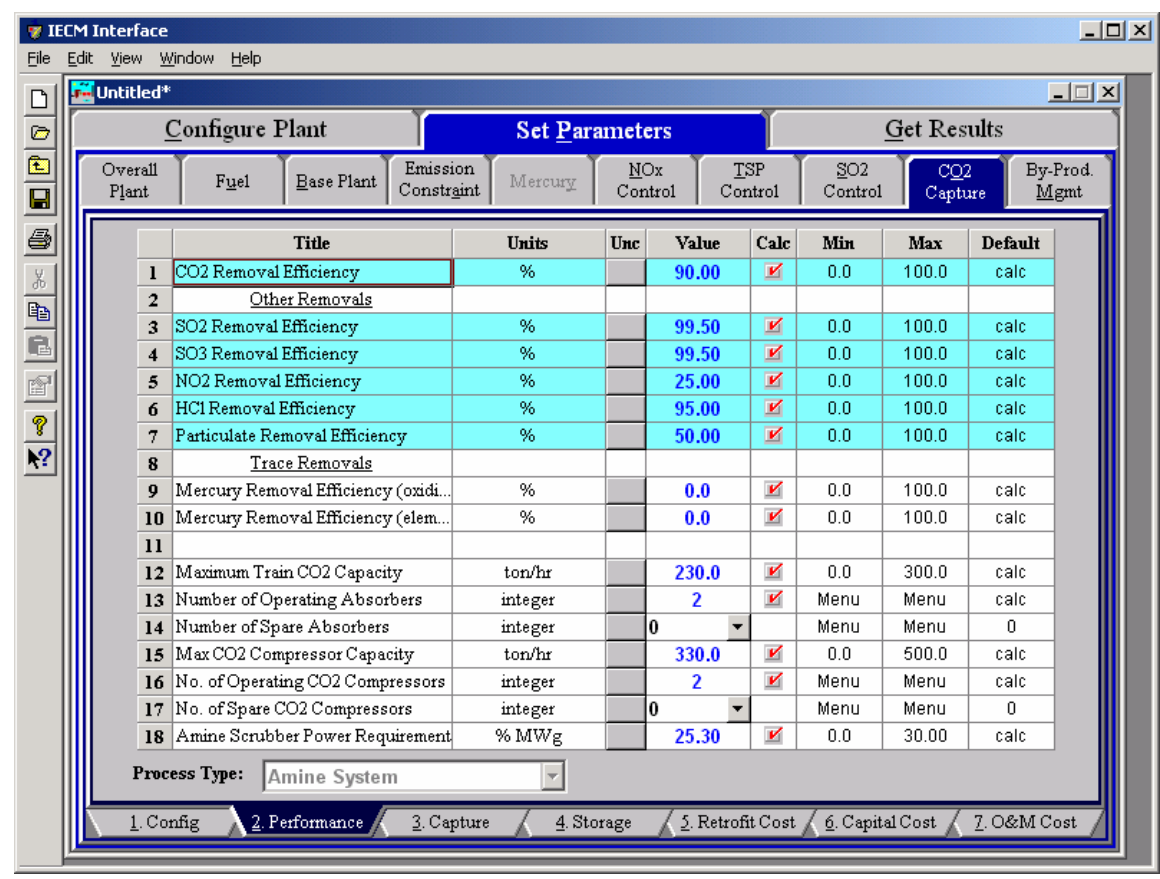

Amine System - Performance input screen.

\section{CO2 Removal}


$\mathrm{CO}_{2}$ Removal Efficiency: Most studies report the $\mathrm{CO}_{2}$ capture efficiency of the amine-based systems to be $90 \%$, with few others reporting as high as $96 \%$ capture efficiency. Here, it has been assumed to be $90 \%$ as nominal value, but the user can specify the desired level of $\mathrm{CO}_{2}$ capture efficiency.

\section{Other Removals}

The amine-based absorption system for $\mathrm{CO}_{2}$ removal is a wet scrubbing operation. This process also removes some other acid gases and particulate matter in addition to $\mathrm{CO}_{2}$ from the flue gas. These are listed below along with the annual average removal efficiencies:

- $\mathrm{SO}_{2}$ Removal Efficiency: 99.5\%

- $\mathrm{SO}_{3}$ Removal Efficiency: 99.5\%

- $\mathrm{NO}_{2}$ Removal Efficiency: 25\%

- HCl Removal Efficiency: 95\%

- Particulate Removal Efficiency: 50\%

\section{Trace Removals}

Removal of trace metals like mercury and other air toxics is not well-characterized for these systems but is anticipated to be similar to other wet scrubber systems. The model default is none, but the user may change these efficiencies.

- Mercury Removal Efficiency (oxidized):0\%

- Mercury Removal Efficiency (elemental): 0\%

\section{Amine System Performance Parameters}

Maximum Train $\mathrm{CO}_{2}$ Capacity: The default maximum train size has been assumed to be 5000 tonnes per day of $\mathrm{CO}_{2}$. Based on the actual $\mathrm{CO} 2$ capture rate the minimum number of trains required to be installed is determined. Different equipment has different maximum capacity limits.

Number of Operating Absorbers: This is the total number of operating absorber vessels. It is used primarily to calculate capital costs. The value must be an integer

Number of Spare Absorbers: This is the total number of spare absorber vessels. It is used primarily to calculate capital costs. The value must be an integer.

Max. $\mathrm{CO}_{2}$ Compressor Capacity: This the maximum amount of $\mathrm{CO}_{2}$ product that can be compressed per hour at the specified pressure.

No. of Operating $\mathrm{CO}_{2}$ Compressors: This is the total number of operating $\mathrm{CO}_{2}$ compressors. It is used primarily to calculate capital costs. The value must be an integer

No. of Spare $\mathbf{C O}_{2}$ Compressors: This is the total number of spare $\mathrm{CO}_{2}$ compressors. It is used primarily to calculate capital costs. The value must be an integer

Amine Scrubber Power Requirment:This is the equivalent electrical output of thermal (steam) energy used for reheat, plus the actual electrical output energy required for pumps and booster fans. 


\section{Amine System Capture Inputs}

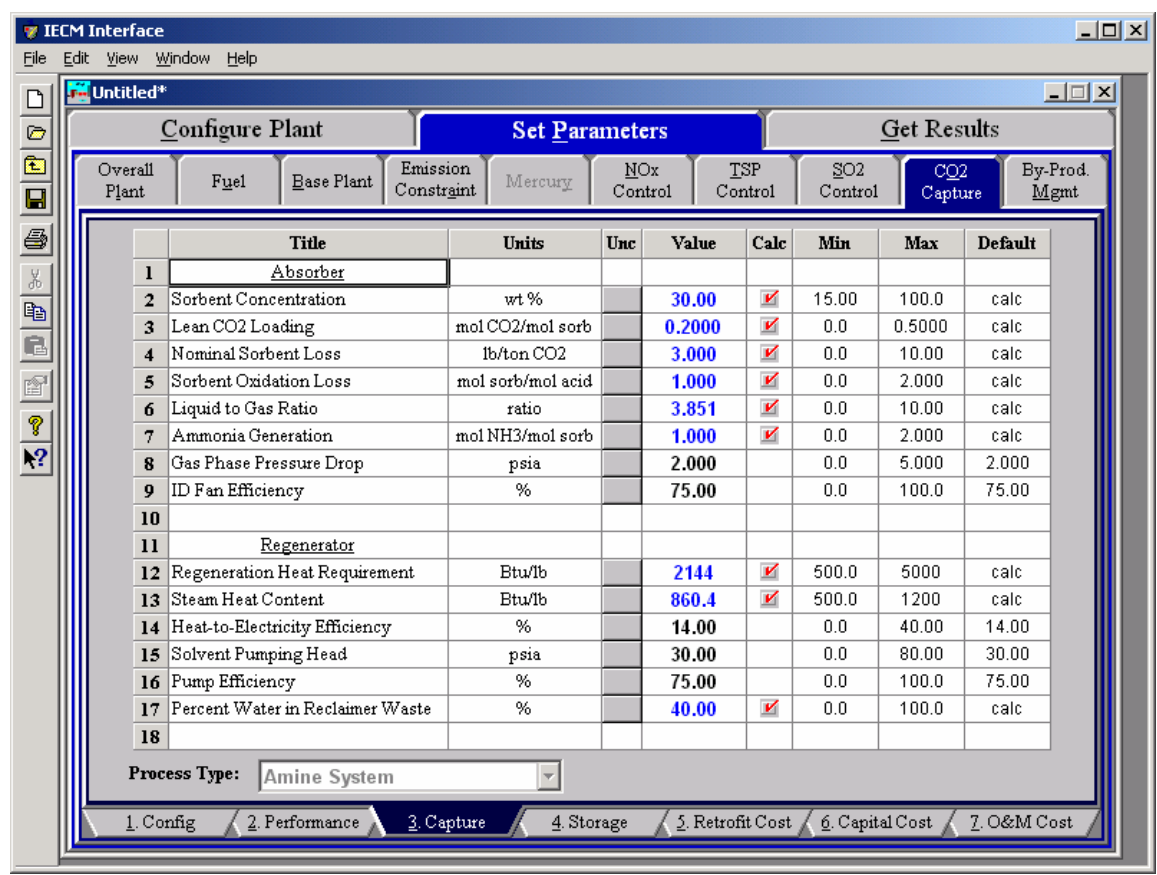

Amine System - Capture input screen.

\section{Absorber}

The absorber is the vessel where the flue gas makes contact with the MEA-based sorbent, and some of the $\mathrm{CO}_{2}$ from the flue gas is dissolved in the sorbent. The column may be plate-type or a packed one. Most of the $\mathrm{CO}_{2}$ absorbers are packed columns using some kind of polymer-based packing to provide large interfacial area.

Sorbent Concentration: The solvent used for $\mathrm{CO}_{2}$ absorption is a mixture of monoethanolamine (MEA) with water. MEA is a highly corrosive liquid, especially in the presence of oxygen and carbon dioxide, and hence needs to be diluted. Today the commercially available MEA-based technology supplied by Fluor Daniel uses 30\% w/w MEA solvent with the help of some corrosion inhibitors. Other suppliers, who do not use this inhibitor, prefer to use lower MEA concentrations in the range of $15 \%-20 \% \mathrm{w} / \mathrm{w}$. Here we use $30 \%$ as the nominal value for the solvent concentration and the user may choose any value between $15-40 \%$.

Lean CO2 Loading: Ideally, the solvent will be completely regenerated on application of heat in the regenerator section. Actually, even on applying heat, not all the MEA molecules are freed from $\mathrm{CO}_{2}$. So, the regenerated (or lean) solvent contains some "left-over" $\mathrm{CO}_{2}$. The level of lean solvent $\mathrm{CO}_{2}$ loading mainly depends upon the initial $\mathrm{CO}_{2}$ loading in the solvent and the amount of regeneration heat supplied, or alternatively, the regeneration heat requirement depends on the allowable level of lean sorbent loading. Here we use a nominal value of 0.2 based on the values reported in the literature, and the user may specify any desired value in the range (0.05-0.3).

Nominal Sorbent Loss: MEA is a reactive solvent. In spite of dilution with water and use of inhibitors, a small quantity of MEA is lost 
through various unwanted reactions, mainly the polymerization reaction (to form long-chained compounds) and the oxidation reaction forming organic acids and liberating ammonia. In general, this nominal loss of MEA is estimated as about $1.5 \mathrm{kgMEA} / \mathrm{mton} \mathrm{CO}_{2}$. It is also assumed that $50 \%$ of this MEA loss is due to polymerization and the remaining $50 \%$ of the MEA loss is due to oxidation to acids.

Sorbent Oxidation Loss: The sorbent oxidation loss variable is a ratio of the number moles of sorbent that are lost for every mole of acid formed due to oxidation of the sorbent.

Liquid to Gas Ratio: The liquid to gas ration is the ratio of total molar flow rate of the liquid (MEA sorbent plus water) to the total molar flow rate of flue gas being treated in the absorber. This is one of the parameters derived by the process simulation model.

Ammonia Generation:The oxidation of MEA to organic acids (oxalic, formic, etc.) also leads to formation of $\mathrm{NH}_{3}$. Each mole of MEA lost in oxidation, liberates a mole of ammonia $\left(\mathrm{NH}_{3}\right)$.

Gas Phase Pressure Drop: This is the pressure drop that the flue gas has to overcome as it passes through a very tall absorber column, countercurrent to the sorbent flow.

ID Fan Efficiency: The cooled flue gas is pressurized using a flue gas blower before it enters the absorber. This is the efficiency of the fan/blower to convert electrical energy input into mechanical work output. Typically, the value of blower efficiency is about $75 \%$.

\section{Regenerator}

The regenerator is the column where the weak intermediate compound (carbamate) formed between the MEA-based sorbent and dissolved $\mathrm{CO}_{2}$ is broken down with the application of heat and $\mathrm{CO}_{2}$ gets separated from the sorbent to leave reusable sorbent behind. In case of unhindered amines like MEA, the carbamate formed is stable and it takes large amount of energy to dissociate. It also consists of a flash separator where $\mathrm{CO}_{2}$ is separated from most of the moisture and evaporated sorbent, to give a fairly rich $\mathrm{CO}_{2}$ stream.

Regeneration Heat Requirement:This is the total amount of heat energy required in the reboiler for sorbent regeneration.

Steam Heat Content: The regeneration heat is provided in the form of LP steam extracted from the steam turbine (in case of coal-fired power plants and combined-cycle gas plants), through the reboiler (a heat exchanger). In case of simple cycle natural gas fired power plants, a heat recovery unit maybe required. This is the enthalpy or heat content of the steam used for solvent regeneration.

Heat-to-Electricity Efficiency: The energy penalty (extraction of LP steam) results in some loss of power generation capacity of the plant. The Heat-toElectricity Efficiency gives the equivalent loss of power generation capacity due to the heat requirement for solvent regeneration. From the data this efficiency has been found to lie in the range $(9,22)$ for a new plant and $(22,30)$ for retrofit cases. So, the nominal value (for this new plant application) has been taken as $14 \%$.

Solvent Pumping Head: The solvent has to flow through the absorber column (generally through packed media) countercurrent to the flue gas flowing upwards. So, some pressure loss is encountered in the absorber 
column and sufficient solvent head has to be provided to overcome these pressure losses. Solvent circulation pumps are used to provide the pressure head. A typical value is about $200 \mathrm{kPa}$ ( $30 \mathrm{psi})$.

Pump Efficiency: This is the efficiency of the solvent circulation pumps to convert electrical energy input into mechanical energy output.

Typically, the value is assumed to be $75 \%$.

Percent Water in Reclaimer Waste: Typically, the reclaimer waste contains about $40 \%$ water.

\section{Amine System Storage Inputs}

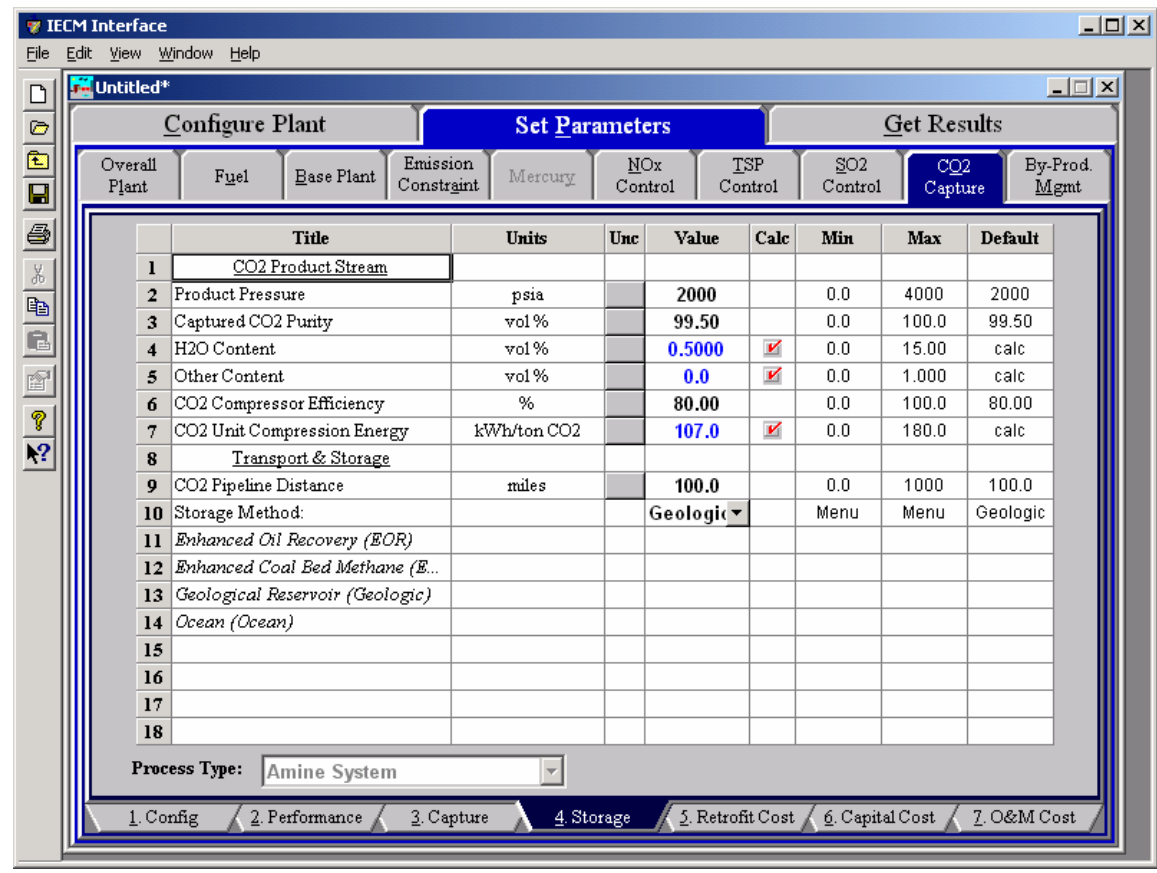

Amine System - Storage input screen

\section{$\mathrm{CO}_{2}$ Product Stream}

The concentrated $\mathrm{CO}_{2}$ product stream obtained from sorbent regeneration is compressed and dried using a multi-stage compressor with inter-stage cooling.

Product Pressure: The $\mathrm{CO}_{2}$ product may have to be carried over long distances. Hence it is necessary to compress (and liquefy) it to very high pressures, so that it maybe delivered to the required destination in liquid form and (as far as possible) without recompression facilities en route. The critical pressure for $\mathrm{CO}_{2}$ is about 1070 psig. The typically reported value of final pressure to which the product $\mathrm{CO}_{2}$ stream has to be pressurized using compressors, before it is transported is about 2000 psig.

Captured $\mathrm{CO}_{2}$ Purity: The final $\mathrm{CO}_{2}$ product has to meet certain specifications depending upon the mode of transport and final destination. Impurities such as nitrogen are undesirable as they may pose problems during compression and liquefaction of $\mathrm{CO}_{2}$. In order to avoid corrosion in the pipelines during transport, the moisture levels 
have to be controlled. The acceptable level of purity of $\mathrm{CO}_{2}$ product for most of the applications is about $99.8 \%$

$\mathrm{H}_{2} \mathrm{O}$ Content: This the volume percent of water contained in the final $\mathrm{CO}_{2}$ product.

Other Content:This the volume percent of any substances that are not water or $\mathrm{CO}_{2}$ contained in the final $\mathrm{CO}_{2}$ product.

$\mathbf{C O}_{2}$ Compressor Efficiency: This is the effective efficiency of the compressors used to compress $\mathrm{CO}_{2}$ to the desirable pressure.

Typically, the value of compressor efficiency ( $\eta_{\text {comp }}$ ) is about $80 \%$

$\mathrm{CO}_{2}$ Unit Compression Energy: This is the electrical energy required to compress a unit mass of $\mathrm{CO}_{2}$ product stream to the designated pressure. Compression of $\mathrm{CO}_{2}$ to high pressures takes lot of energy, and is a principle contributor to the overall energy penalty of a $\mathrm{CO}_{2}$ capture unit in a power plant.

\section{Transport \& Storage}

$\mathrm{CO}_{2}$ Pipeline Distance: The default mode of $\mathrm{CO}_{2}$ transportation is via pipelines. The user can specify the distance over which the $\mathrm{CO}_{2}$ needs to be carried.

Storage Method: The default option for $\mathrm{CO}_{2}$ disposal is underground geological storage.

- $\quad$ EOR - Enhanced Oil Recovery

- $\quad$ ECBM - Enhanced Coalbed Methane Recovery

- Geologic-Geological Reservoir

- Ocean

\section{Amine System Retrofit Cost Inputs}

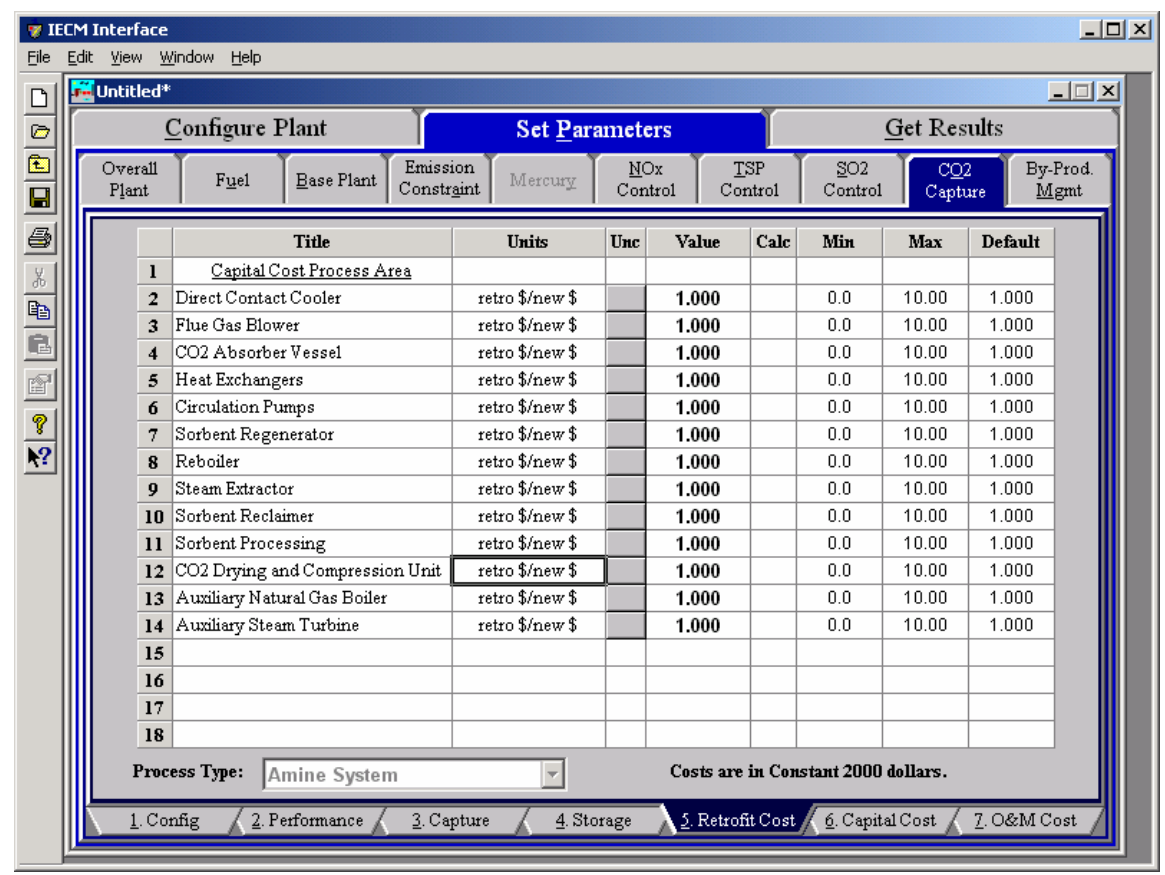




\section{Capital Cost Process Area}

The retrofit cost factor of each process is a multiplicative cost adjustment, which considers the cost of retrofitted capital equipment relative to similar equipment installed in a new plant. These factors affect the capital costs directly and the operating and maintenance costs indirectly.

Direct capital costs for each process area are calculated in the IECM. These calculations are reduced form equations derived from more sophisticated models and reports. The sum of the direct capital costs associated with each process area is defined as the process facilities capital (PFC). The retrofit cost factor provided for each of the process areas can be used as a tool for adjusting the anticipated costs and uncertainties across the process area separate from the other areas.

Uncertainty can be applied to the retrofit cost factor for each process area in each technology. Thus, uncertainty can be applied as a general factor across an entire process area, rather than as a specific uncertainty for the particular cost on the capital or O\&M input screens. Any uncertainty applied to a process area through the retrofit cost factor compounds any uncertainties specified later in the capital and O\&M cost input parameter screens.

The following are the Capital Cost Process Areas for the Amine System:

- Direct Contact Cooler

- $\quad$ Flue Gas Blower

- $\quad$ CO2 Absorber Vessel

- Heat Exchangers

- Circulation Pumps

- Sorbent Regenerator

- Reboiler

- Steam Extractor

- Sorbent Reclaimer

- Sorbent Processing

- CO2 Drying and Compression Unit

- Auxiliary Natural Gas Boiler

- Auxiliary Steam Turbine 


\section{Amine System Capital Cost Inputs}

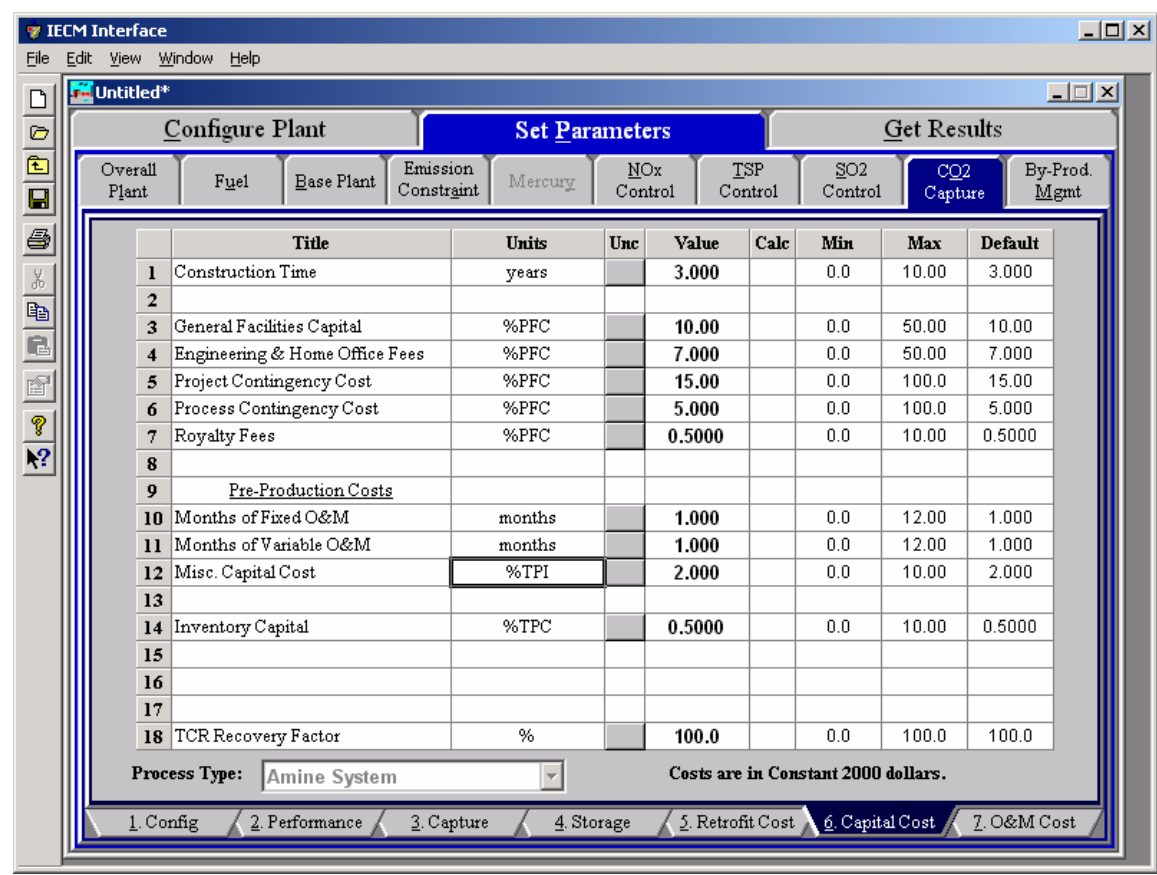

Amine System - Capital Cost input screen.

Inputs for capital costs are entered on the Capital Cost input screen.

Construction Time: This is the idealized construction period in years. It is used to determine the allowance for funds used during construction (AFUDC).

General Facilities Capital (GFC): The general facilities include construction costs of roads, office buildings, shops, laboratories, etc. Sales taxes and freight costs are included implicitly. The cost typically ranges from $5-20 \%$.

Engineering \& Home Office Fees: The engineering \& home office fees are a percent of total direct capital cost. This is an overhead fee paid to the architect/engineering company. These fees typically range from 7$15 \%$.

Project Contingency Cost: This is factor covering the cost of additional equipment or other costs resulting from a more detailed design. Higher contingency factors will be applied to simplified or preliminary designs and lower factors to detailed or finalized designs.

Process Contingency Cost: This quantifies the design uncertainty and cost of a commercial-scale system. This is generally applied on an areaby-area basis. Higher contingency factors are applied to new regeneration systems tested at a pilot plant and lower factors to full-size or commercial systems.

Royalty Fees: Royalty charges may apply to some portions of generating units incorporating new proprietary technologies.

Pre-Production Costs: These costs consider the operator training, equipment checkout, major changes in unit equipment, extra maintenance, and inefficient use of fuel or other materials during startup. These are typically applied to the O\&M costs over a specified 
period of time (months). The two time periods for fixed and variable O\&M costs are described below with the addition of a miscellaneous capital cost factor.

Months of Fixed O\&M: Time period of fixed operating costs used for preproduction to cover training, testing, major changes in equipment, and inefficiencies in start-up. This includes operating, maintenance, administrative and support labor. It also considers maintenance materials.

Months of Variable O\&M: Time period of variable operating costs used for preproduction to cover chemicals, water, consumables, and solid disposal charges in start-up, assuming 100\% load. This excludes any fuels.

Misc. Capital Cost: This is a percent of total plant investment (sum of TPC and AFUDC) to cover expected changes to equipment to bring the system up to full capacity.

Inventory Capital: Percent of the total direct capital for raw material supply based on $100 \%$ capacity during a 60 day period. These materials are considered storage. The inventory capital includes fuels, consumables, by-products, and spare parts. This is typically $0.5 \%$.

TCR Recovery Factor: The actual total capital required (TCR) as a percent of the TCR in a new power plant. This value is $100 \%$ for a new installation and may be set as low as $0 \%$ for a fabric filter that has been paid off.

\section{Amine System O\&M Cost Inputs}

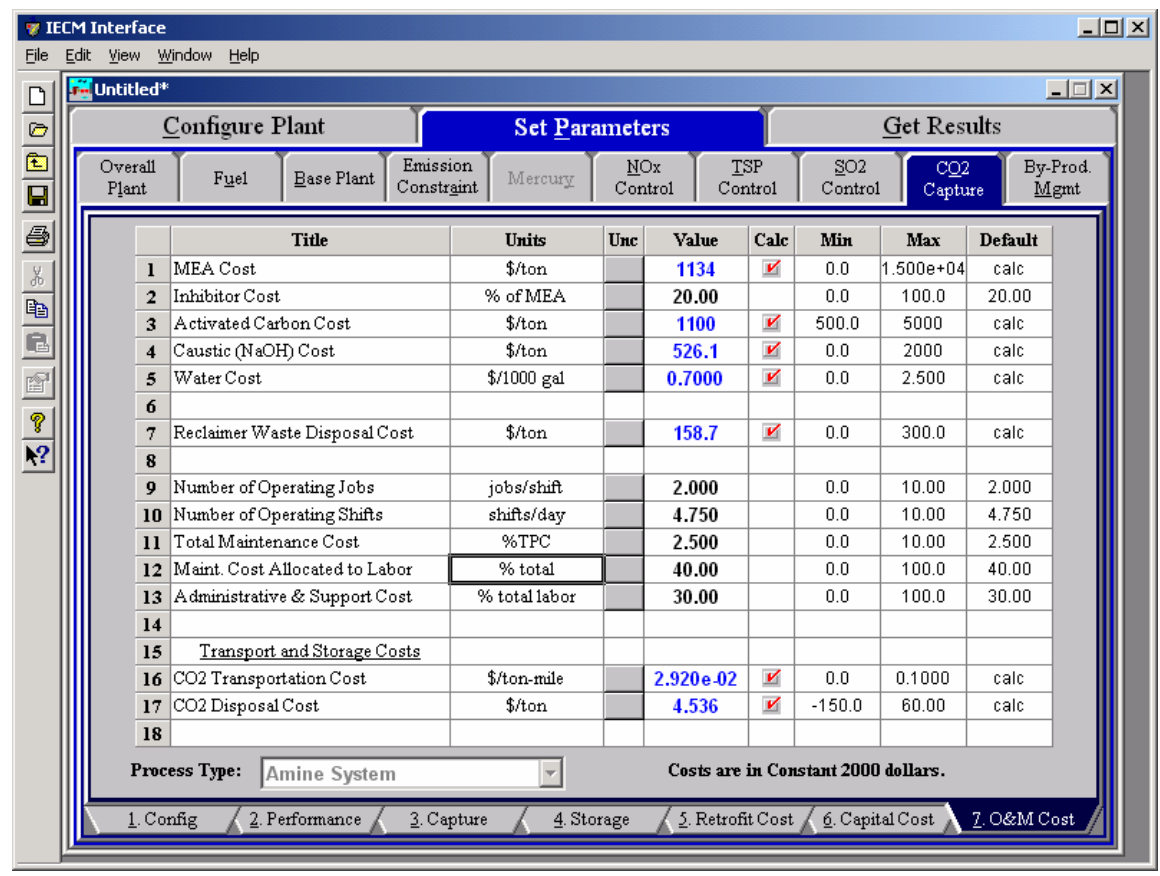

Amine System - O\&M Cost input screen.

Inputs for operation and maintenance are entered on the O\&M Cost input. Each parameter is described briefly below 
MEA Cost: This is the unit cost of the makeup MEA.

Inhibitor Cost: Addition of inhibitor makes it possible to use higher concentrations of MEA solvent in the system with minimal corrosion problems. Inhibitors are special compounds that come at a cost premium. The cost of inhibitor is estimated as a percent of the cost of MEA. The model default is $20 \%$.

Activated Carbon Cost: This is the cost of the activated carbon in \$ per ton.

Caustic (NaOH) Cost: This is the cost of the caustic in \$ per ton.

Water Cost: Water is mainly required for cooling and also as process makeup. Tost of water may vary depending upon the location of the power plant.

Reclaimer Waste Disposal Cost: The unit cost of waste disposal for the reclaimer waste.

Number of Operating Jobs: This is the total number of operating jobs that are required to operate the plant per eight-hour shift.

Number of Operating Shifts: This is the total number of equivalent operating shifts in the plant per day. The number takes into consideration paid time off and weekend work ( 3 shifts/day $* 7$ days/5 day week $* 52$ weeks/(52 weeks -6 weeks PTO $)=4.75$ equiv. Shifts/day)

Total Maintenance Cost: This is the annual maintenance cost as a percentage of the total plant cost. Maintenance cost estimates can be developed separately for each process area.

Maint. Cost Allocated to Labor: Maintenance cost allocated to labor as a percentage of the total maintenance cost.

Administrative \& Support Cost: This is the percent of the total operating and maintenance labor associated with administrative and support labor.

\section{Transport and Storage Costs}

CO2 Transportation Cost: Transportation of $\mathrm{CO}_{2}$ product is assumed to take place via pipelines. This is the unit cost of $\mathrm{CO}_{2}$ transport in \$/ton -mile.

CO2 Disposal Cost: This is the unit cost of $\mathrm{CO}_{2}$ disposal. Depending upon the method of $\mathrm{CO}_{2}$ disposal or storage, either there may be some revenue generated (Enhanced Oil Recovery, Coal Bed Methane) which may be treated as a "negative cost", or additional cost (all other disposal methods). 


\section{Amine System Diagram}

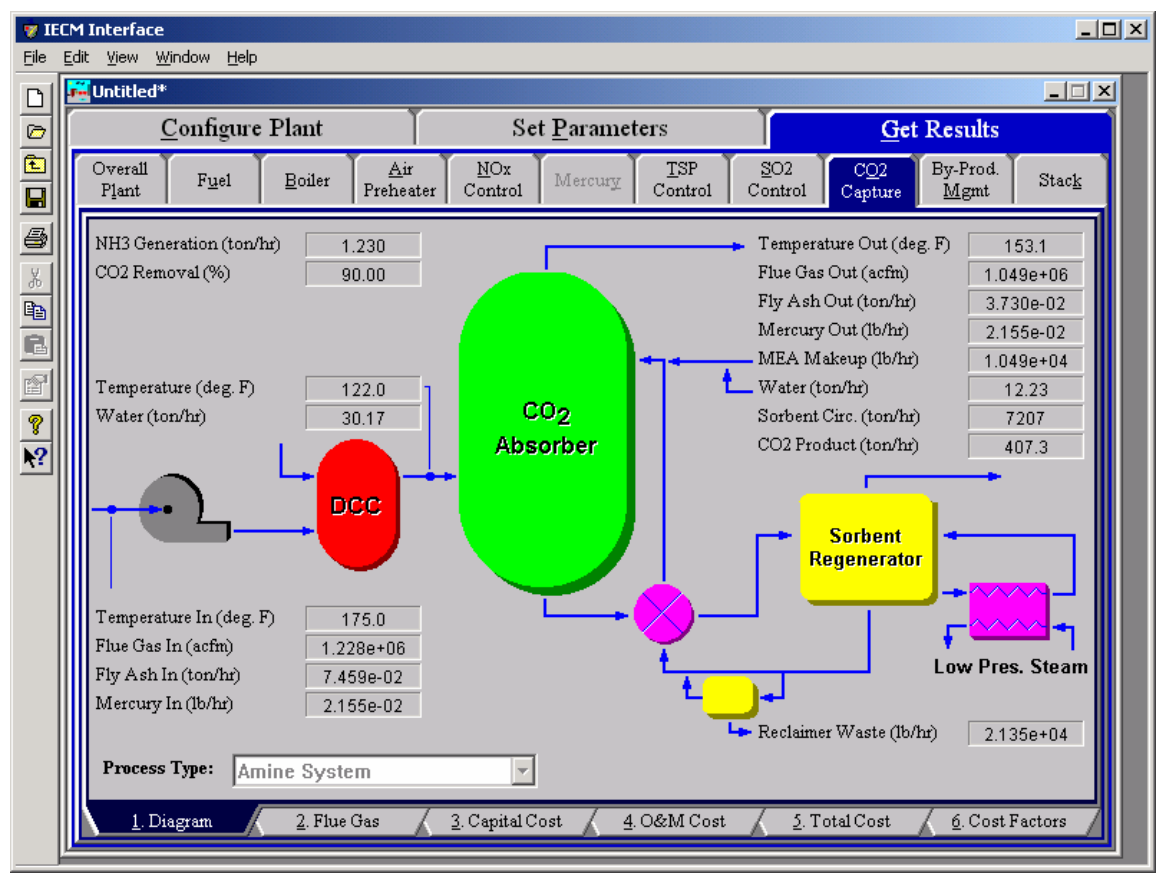

Amine System - Diagram.

\section{Reagent}

MEA Makeup: The mass flow rate of fresh MEA needed to replace the amount used in the process.

Water: This is the flow rate of water that is used to mix with the MEA Makeup.

\section{Flue Gas Entering Amine System}

Temperature In: Temperature of the flue gas entering the amine system area, prior to any processing. This is determined by the flue gas outlet temperature of the process area upstream.

Flue Gas In: Volumetric flow rate of flue gas entering the amine system.

Fly Ash In: Total solids mass flow rate in the flue gas entering the Amine System. This is determined by the solids exiting from the module upstream.

Mercury In: Total mass of mercury entering the Amine System. The value is a sum of all the forms of mercury (elemental, oxidized, and particulate).

Temperature: Temperature of the flue gas entering the amine scrubber system.

Water: This is the flow rate of water into the Direct Contact Cooler.

\section{Flue Gas Exiting Amine System}

Temperature Out: Temperature of the flue gas exiting the amine scrubber system. 
Flue Gas Out: Volumetric flow rate of the flue gas exiting the amine scrubber.

Fly Ash Out: Total solids mass flow rate in the flue gas exiting the amine scrubber.

Mercury Out: Total mass of mercury exiting the amine scrubber. The value is a sum of all the forms of mercury (elemental, oxidized, and particulate).

\section{Amine System Performance}

$\mathrm{CO}_{2}$ Product: Actual amount of $\mathrm{CO}_{2}$ produced as a result of the amine scrubbing.

$\mathbf{C O}_{2}$ Removal: Actual removal efficiency of $\mathrm{CO}_{2}$ in the amine scrubber.

$\mathrm{NH}_{3}$ Generation: The flow rate of ammonia by product produced in the amine scrubbing process.

Sorbent Circ.: The flow rate of the sorbent through the amine scrubber system.

Mercury Removal: Percent of the total mercury removed from the scrubber. The value reflects a weighted average based on the particular species of mercury present (elemental, oxidized, and particulate).

\section{Collected Solids}

Reclaimer Waste: Total solids mass flow rate of solids removed from the amine scrubber.

\section{Amine System Flue Gas Results}

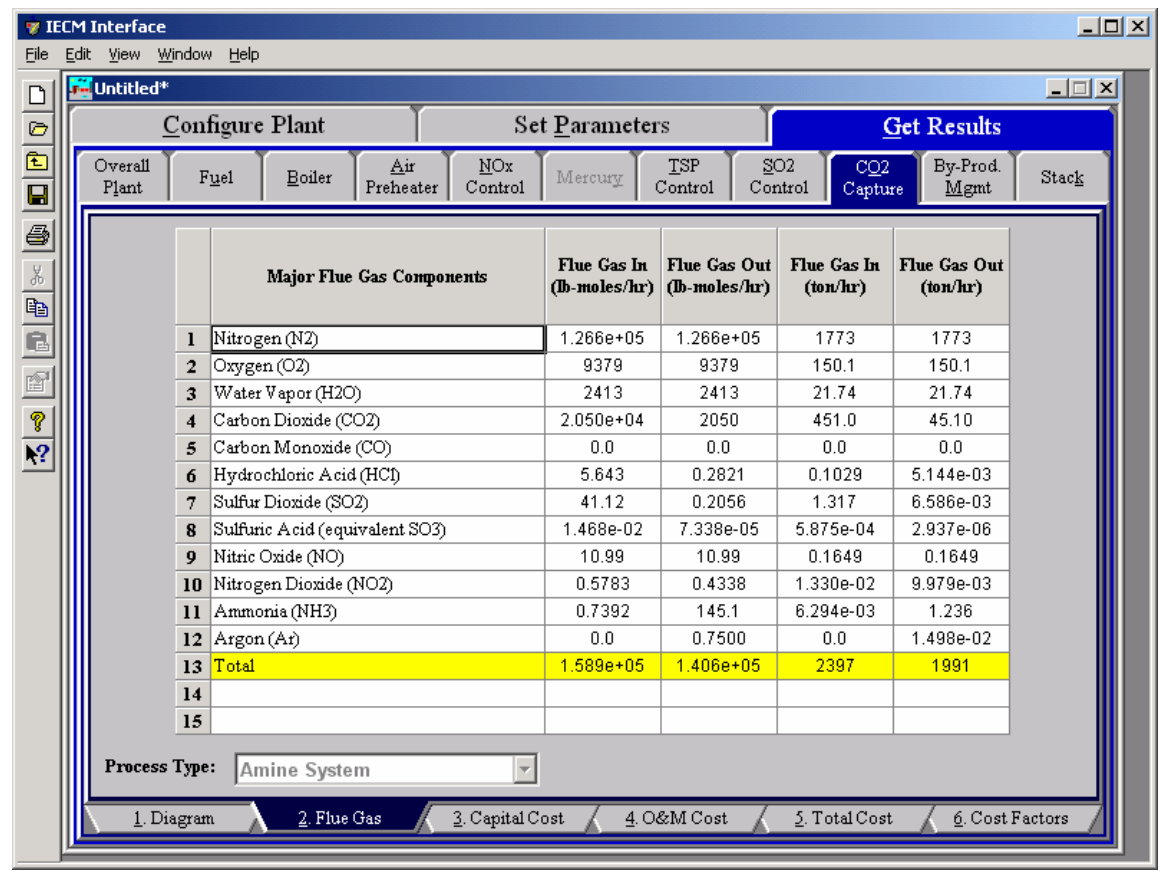

Amine System - Flue Gas result screen

Major Flue Gas Components 
Each result is described briefly below:

Nitrogen $\left(\mathrm{N}_{2}\right)$ : Total mass of nitrogen.

Oxygen $\left(\mathrm{O}_{2}\right)$ : Total mass of oxygen.

Water Vapor $\left(\mathrm{H}_{2} \mathrm{O}\right)$ : Total mass of water vapor.

Carbon Dioxide $\left(\mathrm{CO}_{2}\right)$ : Total mass of carbon dioxide.

Carbon Monoxide (CO): Total mass of carbon monoxide.

Hydrochloric Acid ( $\mathrm{HCl})$ : Total mass of hydrochloric acid.

Sulfur Dioxide $\left(\mathrm{SO}_{2}\right)$ : Total mass of sulfur dioxide.

Sulfuric Acid (equivalent $\mathbf{S O}_{3}$ ): Total mass of sulfuric acid.

Nitric Oxide (NO): Total mass of nitric oxide.

Nitrogen Dioxide $\left(\mathrm{NO}_{2}\right)$ : Total mass of nitrogen dioxide.

Ammonia $\left(\mathrm{NH}_{3}\right)$ : Total mass of ammonia.

Argon (Ar): Total mass of argon.

Total: Total of the individual components listed above. This item is highlighted in yellow.

\section{Amine System Capital Cost Results}

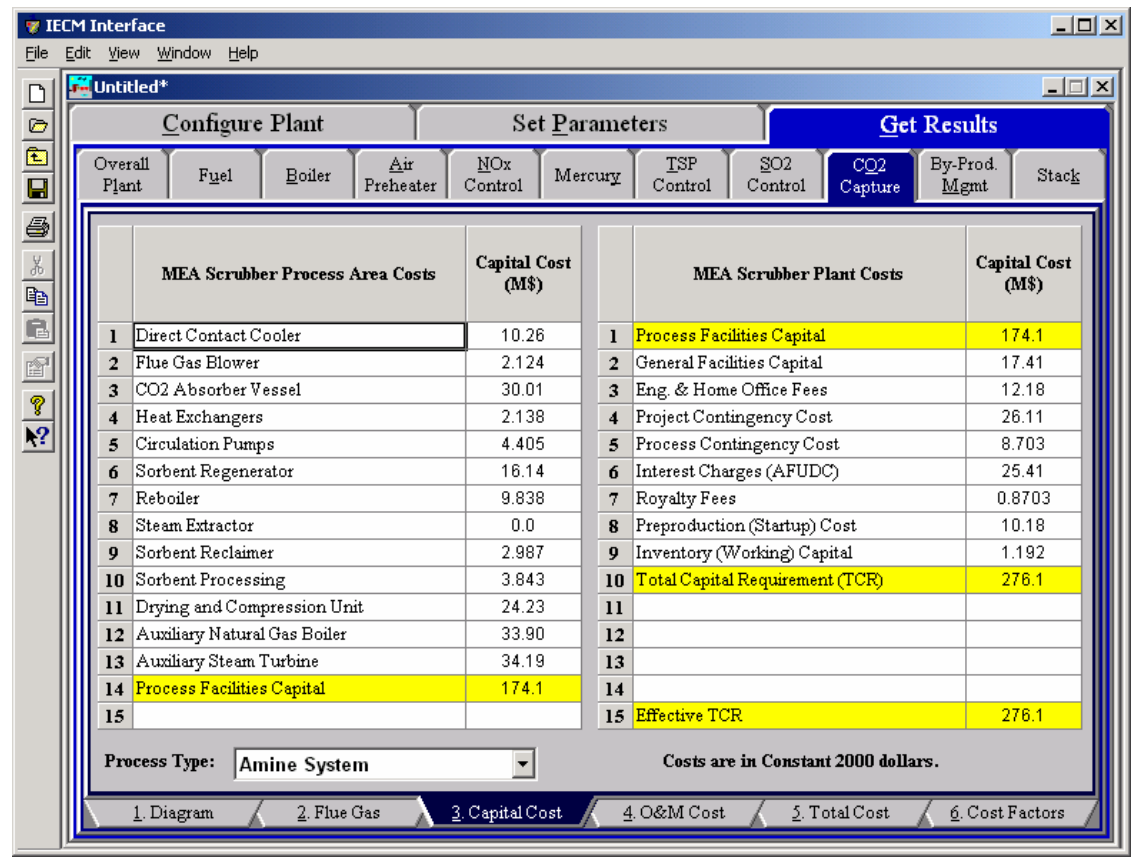

Amine System - Capital Cost result screen.

The Capital Cost result screen displays tables for the capital costs. Each result is described briefly below:

\section{MEA Scrubber Process Area Costs}

Direct Contact Cooler: This area includes the equipment required to cool the flue gas in order to improve absorption of $\mathrm{CO}_{2}$ into the amine 
sorbent. In case of coal-fired power plant applications that have a wet FGD (flue gas desulfurization) unit upstream of the amine system, the wet scrubber helps in substantial cooling of the flue gases, and additional cooler may not be required.

Flue Gas Blower: The flue gas has to overcome a substantial pressure drop as it passes through a very tall absorber column, countercurrent to the sorbent flow. Hence the cooled flue gas has to be pressurized using a blower before it enters the absorber.

$\mathrm{CO}_{2}$ Absorber Vessel: This is the vessel where the flue gas is made to contact with the MEA-based sorbent, and some of the $\mathrm{CO}_{2}$ from the flue gas gets dissolved in the sorbent. The column may be plate-type or a packed one. Most of the $\mathrm{CO}_{2}$ absorbers are packed columns using some kind of polymer-based packing to provide large interfacial area.

Heat Exchangers: The $\mathrm{CO}_{2}$-loaded sorbent needs to be heated in order to strip off $\mathrm{CO}_{2}$ and regenerate the sorbent. On the other hand, the regenerated (lean) sorbent coming out of the regenerator has to be cooled down before it could be circulated back to the absorber column. Hence these two sorbent streams are passed through a cross heat exchanger, where the rich $\left(\mathrm{CO}_{2}\right.$-loaded $)$ sorbent gets heated and the lean (regenerated) sorbent gets cooled.

Circulation Pumps: The cost associated with the equipment required to support FGD system operation such as makeup water and instrument air are treated here.

Sorbent Regenerator:This is the column where the weak intermediate compound (carbamate) formed between the MEA-based sorbent and dissolved $\mathrm{CO}_{2}$ is broken down with the application of heat and $\mathrm{CO}_{2}$ gets separated from the sorbent to leave reusable sorbent behind. In case of unhindered amines like MEA, the carbamate formed is stable and it takes large amount of energy to dissociate. It also consists of a flash separator where $\mathrm{CO}_{2}$ is separated from most of the moisture and evaporated sorbent, to give a fairly rich $\mathrm{CO}_{2}$ stream.

Reboiler: The regenerator is connected with a reboiler which is basically a heat exchanger where low-pressure steam extracted from the power plant is used to heat the loaded sorbent

Steam Extractor: In case of coal-fired power plants that generate electricity in a steam turbine, a part of the LP/IP steam has to be diverted to the reboiler for sorbent regeneration. Steam extractors are installed to take out steam from the steam turbines.

Sorbent Reclaimer: Presence of acid gas impurities $\left(\mathrm{SO}_{2}, \mathrm{SO}_{3}, \mathrm{NO}_{2}\right.$ and $\mathrm{HCl}$ ) in the flue gas leads to formation of heat stable salts in the sorbent stream, which can not be dissociated even on application of heat. In order to avoid accumulation of these salts in the sorbent stream and to recover some of this lost MEA sorbent, a part of the sorbent stream is periodically distilled in this vessel. Addition of caustic helps in freeing of some of the MEA. The recovered MEA is taken back to the sorbent stream while the bottom sludge (reclaimer waste) is sent for proper disposal.

Sorbent Processing: The regenerated sorbent has to be further cooled down even after passing through the rich/lean cross heat exchanger using a cooler, so that the sorbent temperature is brought back to acceptable level (about $40 \mathrm{deg} \mathrm{C}$ ). Also, in order to make up for the 
sorbent losses, a small quantity of fresh MEA sorbent has to be added to the sorbent stream. So, the sorbent processing area primarily consists of sorbent cooler, MEA storage tank, and a mixer. It also consists of an activated carbon bed filter that adsorbs impurities (degradation products of MEA) from the sorbent stream.

Drying and Compression Unit: The $\mathrm{CO}_{2}$ product may have to be carried to very long distances via pipelines. Hence it is desirable that it does not contain any moisture in order to avoid corrosion in the pipelines. Also, it has to be compressed to very high pressures so that it gets liquefied and can overcome the pressure losses during the pipeline transport. The multi-stage compression unit with inter-stage cooling and drying yields a final $\mathrm{CO}_{2}$ product at the specified pressure (about 2000 psig) that contains moisture and other impurities (e.g. $\mathrm{N}_{2}$ ) at acceptable levels.

Auxiliary Natural Gas Boiler: The cost of the Natural Gas boiler is estimated on the basis of the steam flow rate generated from the boiler.

Auxiliary Steam Turbine: The regeneration heat is provided in the form of LP steam extracted from the steam turbine (in case of coal-fired power plants and combined-cycle gas plants), through the reboiler (a heat exchanger). In case of simple cycle natural gas fired power plants, a heat recovery unit maybe required.

Process Facilities Capital: The process facilities capital is the total constructed cost of all on-site processing and generating units listed above, including all direct and indirect construction costs. All sales taxes and freight costs are included where applicable implicitly. This result is highlighted in yellow.

\section{MEA Scrubber Plant Costs}

Process Facilities Capital: (see definition above)

General Facilities Capital: The general facilities include construction costs of roads, office buildings, shops, laboratories, etc. Sales taxes and freight costs are included implicitly.

Eng. \& Home Office Fees: The engineering \& home office fees are a percent of total direct capital cost. This is an overhead fee paid to the architect/engineering company.

Project Contingency Cost: Capital cost contingency factor covering the cost of additional equipment or other costs that would result from a more detailed design of a definitive project at the actual site.

Process Contingency Cost: Capital cost contingency factor applied to a new technology in an effort to quantify the uncertainty in the technical performance and cost of the commercial-scale equipment.

Interest Charges (AFUDC): Allowance for funds used during construction, also referred to as interest during construction, is the time value of the money used during construction and is based on an interest rate equal to the before-tax weighted cost of capital. This interest is compounded on an annual basis (end of year) during the construction period for all funds spent during the year or previous years.

Royalty Fees: Royalty charges may apply to some portions of generating units incorporating new proprietary technologies. 
Preproduction (Startup) Cost: These costs consider the operator training, equipment checkout, major changes in unit equipment, extra maintenance, and inefficient use of fuel or other materials during startup.

Inventory (Working) Capital: The raw material supply based on 100\% capacity during a 60 day period. These materials are considered storage. The inventory capital includes fuels, consumables, byproducts, and spare parts.

Total Capital Requirement (TCR): Money that is placed (capitalized) on the books of the utility on the service date. TCR includes all the items above. This result is highlighted in yellow.

Effective TCR: The TCR of the spray dryer that is used in determining the total power plant cost. The effective TCR is determined by the "TCR Recovery Factor".

\section{Amine System O\&M Cost Results}

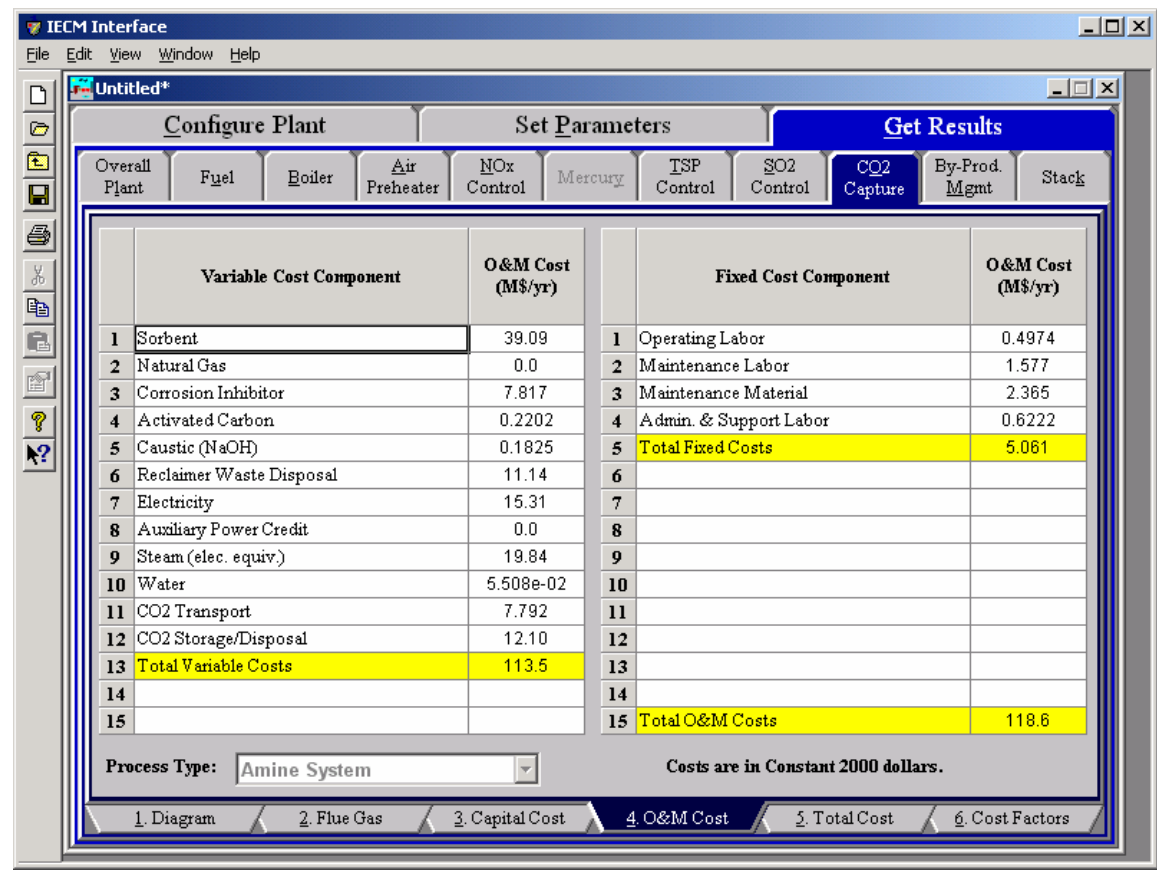

Amine System - O\&M Cost result screen.

The O\&M Cost result screen displays tables for the variable and fixed operation and maintenance costs involved with the $\mathbf{C O}_{\mathbf{2}}$ Capture technology. Each result is described briefly below:

\section{Variable Cost Components}

Variable operating costs and consumables are directly proportional to the amount of kilowatts produced and are referred to as incremental costs. All the costs are subject to inflation.

Sorbent: MEA is the default sorbent used in the system and this is the annual cost of the MEA. This is a function of the concentration of $\mathrm{CO} 2$ in the flue gas and the flue gas flow rate. 
Natural Gas: If the user has added an auxiliary natural gas boiler, the cost of the natural gas used to fuel the boiler is added here.

Corrosion Inhibitor: The inhibitor helps in two ways - reduced sorbent degradation and reduced equipment corrosion. This is the annual cost of the corrosion inhibitor.

Activated Carbon: This is the activated carbon cost for flue gas conditioning.

Caustic (NaOH): This is the annual cost of caustic. The presence of acid gas impurities $\left(\mathrm{SO}_{2}, \mathrm{SO}_{3}, \mathrm{NO}_{2}\right.$ and $\left.\mathrm{HCl}\right)$ in the flue gas leads to formation of heat stable salts in the sorbent stream, which can not be dissociated even on application of heat. In order to avoid accumulation of these salts in the sorbent stream and to recover some of this lost MEA sorbent, a part of the sorbent stream is periodically distilled in this vessel. Addition of caustic helps in freeing of some of the MEA. The recovered MEA is taken back to the sorbent stream while the bottom sludge (reclaimer waste) is sent for proper disposal.

Reclaimer Waste Disposal: This is the reclaimer waste disposal cost per year.

Electricity: The cost of electricity consumed by the Amine System.

Auxiliary Power Credit: An auxiliary natural gas boiler can be added by the user to provide steam and power for the Amine System. If it is added by the user then the additional power it provides is subtracted from the overall operating and maintenance cost, here.

Steam (elec. equiv.): Equivalent electrical penalty of the regeneration steam requirement

Water: This is the annual cost for water to the amine scrubber system, it is mainly required for cooling and also as process makeup.

$\mathrm{CO}_{2}$ Transport: The $\mathrm{CO}_{2}$ captured at the power plant site has to be carried to the appropriate storage/ disposal site. Transport of $\mathrm{CO}_{2}$ to a storage site is assumed to be via pipeline This is the annual cost of maintaining those pipelines.

$\mathrm{CO}_{2}$ Storage/Disposal: Once the $\mathrm{CO}_{2}$ is captured, it needs to be securely stored (sequestered). This cost is based upon the storage option chosen on the Amine System - Storage input screen.

Total Variable Costs: This is the sum of all the variable O\&M costs listed above. This result is highlighted in yellow.

\section{Fixed Cost Components}

Fixed operating costs are essentially independent of actual capacity factor, number of hours of operation, or amount of kilowatts produced. All the costs are subject to inflation.

Operating Labor: Operating labor cost is based on the operating labor rate, the number of personnel required to operate the plant per eighthour shift, and the average number of shifts per day over 40 hours per week and 52 weeks.

Maintenance Labor: The maintenance labor is determined as a fraction of the total maintenance cost. 
Maintenance Material: The cost of maintenance material is the remainder of the total maintenance cost, considering the fraction associated with maintenance labor.

Admin. \& Support Labor: The administrative and support labor is the only overhead charge. It is taken as a fraction of the total operating and maintenance labor costs.

Total Fixed Costs: This is the sum of all the fixed O\&M costs listed above. This result is highlighted in yellow.

Total O\&M Costs: This is the sum of the total variable and total fixed O\&M costs. It is used to determine the base plant total revenue requirement. This result is highlighted in yellow.

\section{Amine System Total Cost Results}

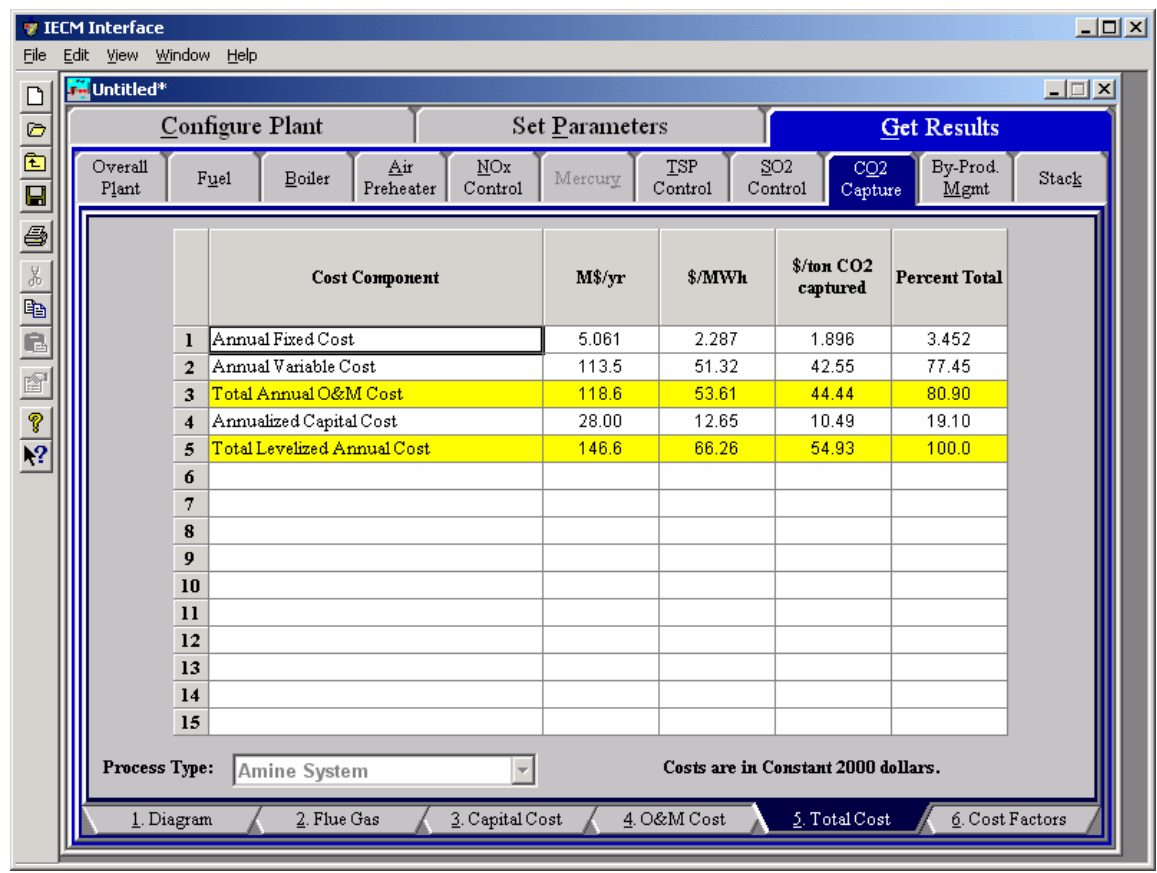

Amine System - Total Cost result screen.

The Total Cost result screen displays a table which totals the annual fixed, variable, operations and maintenance, and capital costs associated with the Amine System $\mathrm{CO}_{2}$ Control technology. Each result is described briefly below.

\section{Cost Component}

Annual Fixed Cost: The operating and maintenance fixed costs are given as an annual total. This number includes all maintenance materials and all labor costs.

Annual Variable Cost: The operating and maintenance variables costs are given as an annual total. This includes all reagent, chemical, steam, and power costs.

Total Annual O\&M Cost: This is the sum of the annual fixed and variable operating and maintenance costs above. This result is highlighted in yellow. 
Annualized Capital Cost: This is the total capital cost expressed on an annualized basis, taking into consideration the levelized carrying charge factor, or fixed charge factor, over the entire book life.

Total Levelized Annual Cost: The total annual cost is the sum of the total annual O\&M cost and annualized capital cost items above. This result is highlighted in yellow.

\section{Amine System Cost Factors Results}

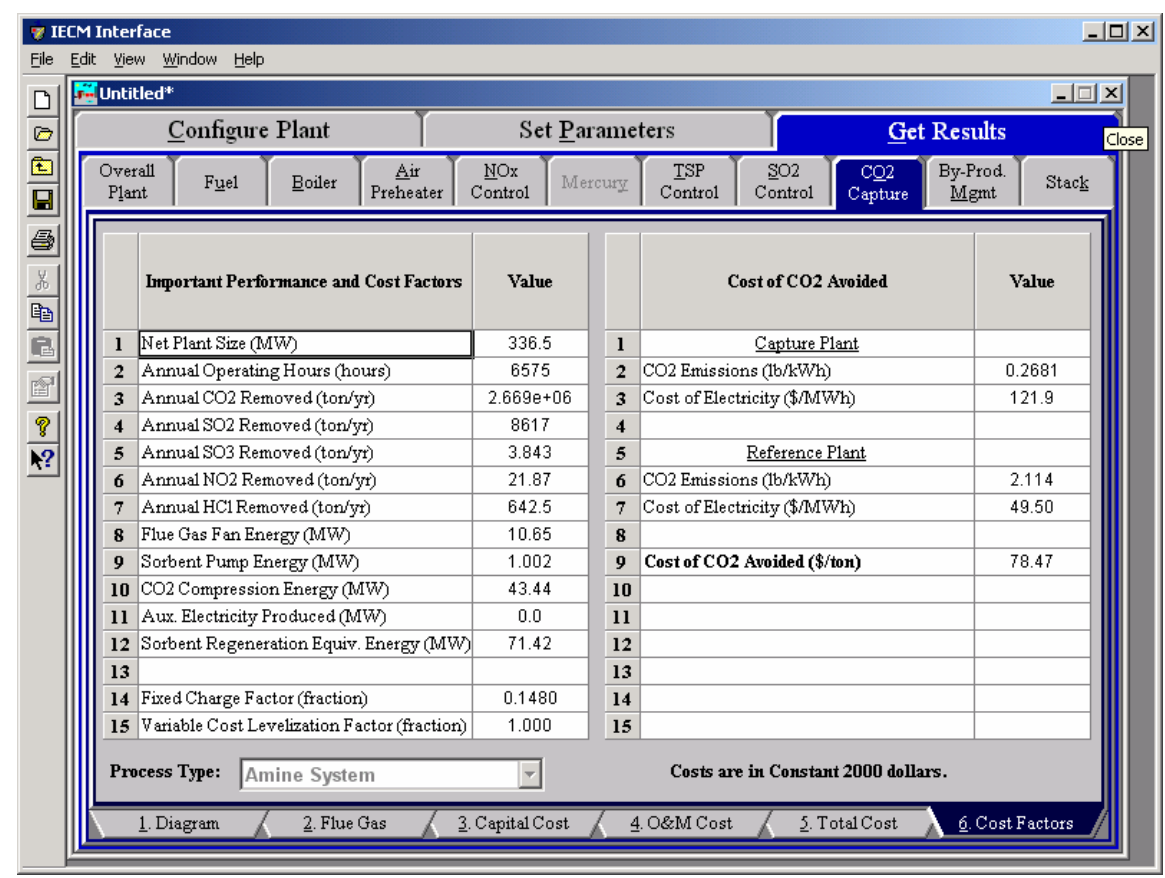

Amine System - Cost Factors result screen.

\section{Important Performance and Cost Factors}

This screen displays information that is key to the model calculations. The data is available else where in the model.

Net Plant Size (MW): This is the net plant capacity, which is the gross plant capacity minus the losses due to plant equipment and pollution equipment (energy penalties).

Annual Operating Hours (hours): This is the number of hours per year that the plant is in operation. If a plant runs 24 hours per day, seven days per week, with no outages, the calculation is 24 hours * 365 days. or 8,760 hours/year.

Annual $\mathrm{CO}_{2}$ Removed (ton/yr): This is thel amount of $\mathrm{CO}_{2}$ removed from the flue gas by the $\mathrm{CO}_{2}$ capture system per year.

Annual $\mathrm{SO}_{2}$ Removed (ton/yr): This is the amount of $\mathrm{SO}_{2}$ removed from the flue gas by the $\mathrm{CO}_{2}$ capture system per year.

Annual $\mathrm{SO}_{3}$ Removed (ton/yr): This is the amount of $\mathrm{SO}_{3}$ removed from the flue gas by the $\mathrm{CO}_{2}$ capture system per year.

Annual $\mathrm{NO}_{2}$ Removed (ton/yr): This is the amount of $\mathrm{NO}_{2}$ removed from the flue gas by the $\mathrm{CO}_{2}$ capture system per year. 
Annual $\mathrm{HCl}$ Removed (ton/yr): This is the amount of $\mathrm{HCl}$ removed from the flue gas by the $\mathrm{CO}_{2}$ capture system per year.

Flue Gas Fan Energy (MW): The flue gas has to be compressed in a flue gas blower so that it can overcome the pressure drop in the absorber tower. This is the electrical energy required by the blower.

Sorbent Pump Energy (MW):The solvent has to flow through the absorber column (generally through packed media) countercurrent to the flue gas flowing upwards. This is the energy required by the solvent circulation pumps to supply pressure to overcome the pressure losses encountered by the solvent in the absorber column.

$\mathrm{CO}_{2}$ Compression Energy (MW):This is the electrical energy required to compress the $\mathrm{CO}_{2}$ product stream to the designated pressure. Compression of $\mathrm{CO}_{2}$ to high pressures takes lot of energy, and is a principle contributor to the overall energy penalty of a $\mathrm{CO}_{2}$ capture unit in a power plant.

Aux. Electricity Produced (MW): If an auxiliary natural gas boiler is used to provide steam and power for the Amine System, this is the additional electricity that it produces.

Sorbent Regeneration Equiv. Energy (MW):This is the electrical equivalent of the energy required in the form of either steam or heat to regenerate the sorbent.

Fixed Charge Factor (fraction): The fixed charge factor is one of the most important parameters in the IECM. It determines the revenue required to finance the power plant based on the capital expenditures. Put another way, it is a levelized factor which accounts for the revenue per dollar of total plant cost that must be collected from customers in order to pay the carrying charges on that capital investment.

\section{Variable Cost Levelization Factor (fraction): \\ Cost of $\mathrm{CO}_{2}$ Avoided}

Many analysts like to express the cost of an environmental control system in terms of the cost per ton of pollutant removed or avoided. For energy-intensive $\mathrm{CO}_{2}$ controls there is a big difference between the cost per ton $\mathrm{CO}_{2}$ removed and the cost per ton "avoided" based on net plant capacity. Since the purpose of adding a $\mathrm{CO}_{2}$ unit is to reduce the $\mathrm{CO}_{2}$ emissions per net $\mathrm{kWh}$ delivered, the cost of $\mathrm{CO}_{2}$ avoidance is the economic indicator that is widely used in this field.

\section{Capture Plant}

$\mathbf{C O}_{2}$ Emissions (lb/kWh): This is the amount of $\mathrm{CO} 2$ vented to the air for every kilowatt hour of electricity produced in the power plant that is using $\mathrm{CO}_{2}$ Capture Technology.

Cost of Electricity (\$/MWh):The IECM framework calculates the cost of electricity (COE) for the overall Capture Plant by dividing the total annualized plant cost $(\$ / y r)$ by the net electricity generated $(\mathrm{kWh} / \mathrm{yr})$

\section{Reference Plant}

$\mathbf{C O}_{2}$ Emissions (lb/kWh): This is the amount of $\mathrm{CO} 2$ vented to the air for every kilowatt hour of electricity produced in the power plant with NO CO2 Capture. 
Cost of Electricity (\$/MWh):The IECM framework calculates the cost of electricity (COE) for the overall Reference Plant by dividing the total annualized plant cost $(\$ / y r)$ by the net electricity generated $(\mathrm{kWh} / \mathrm{yr})$

Cost of $\mathrm{CO}_{2}$ Avoided (\$/ton): This is the economic indicator widely used in the field, calculated as the difference between the cost of electricity in the capture plant and the reference plant divided by the difference between the $\mathrm{CO} 2$ emissions in the reference plant and the capture plant.

Cost of $\mathrm{CO}_{2}$ Avoided $=($ Cost of Electricity cap. - Cost of Electricity ref. $)$

$/\left(\mathrm{CO}_{2}\right.$ emissions ref. $_{-}-\mathrm{CO}_{2}$ emissions cap. $)$ 



\section{By Product Management}

The ByProduct Mg $\underline{\mathbf{g m t}}$ Technology Navigation Tab screens display and design the management of by products and waste disposal.

\section{By Product Management Performance Inputs}

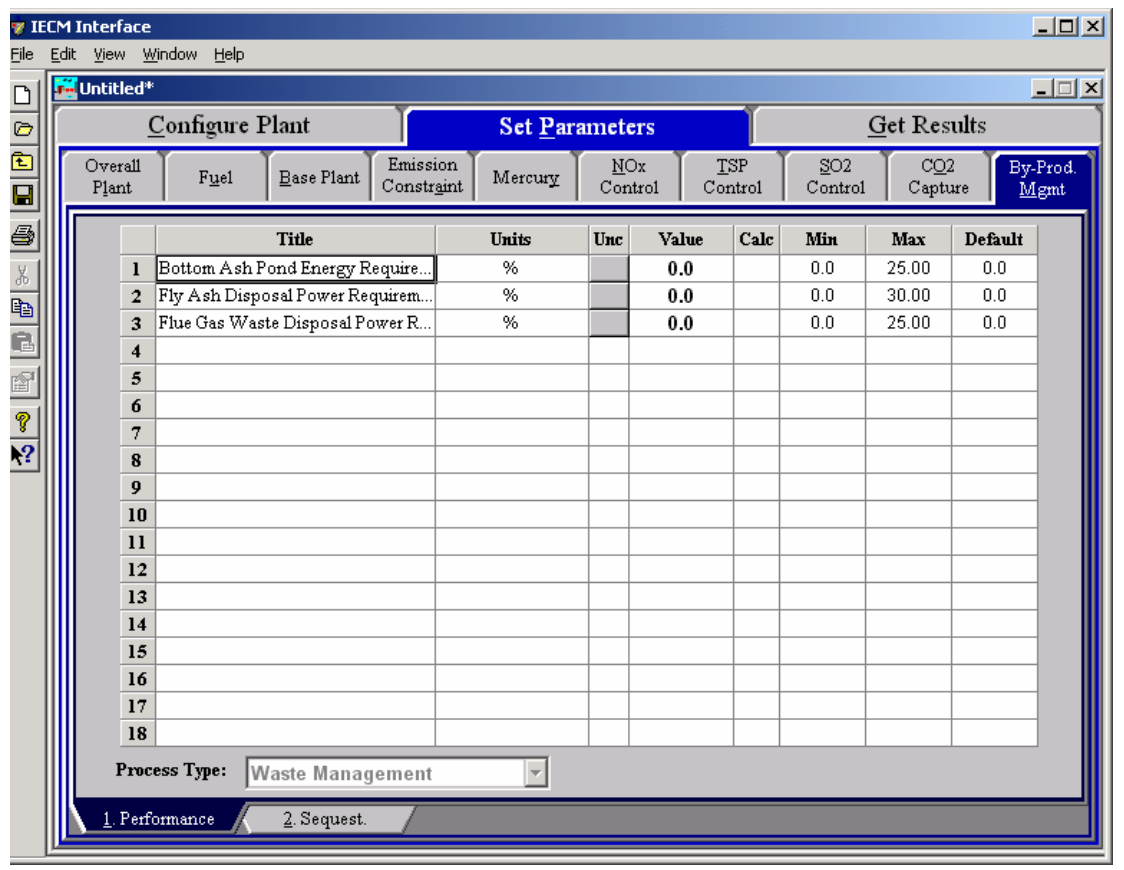

By Product Management - Performance input screen.

General inputs regarding solid waste management are entered on the Performance input screen. This screen is displayed for all plant configurations. One or more of the following By Product Management options will be shown on the input screen depending upon the options selected in the Configure Plant program area. Each of the possible parameters are described briefly below.

Bottom Ash PondEnergy Requirements: The energy requirement is zero by default. Any requirements are considered by the abatement technologies that dispose solids into the bottom ash pond. 
Fly Ash Disposal Power Requirements: The energy requirement is zero by default. Any requirements are considered by the abatement technologies that dispose of fly ash.

Flue Gas Waste Disposal Power Requirements: The energy requirement is zero by default. Any requirements are considered by the abatement technologies that dispose of flue gas waste.

\section{By Product Management Sequestration Input}

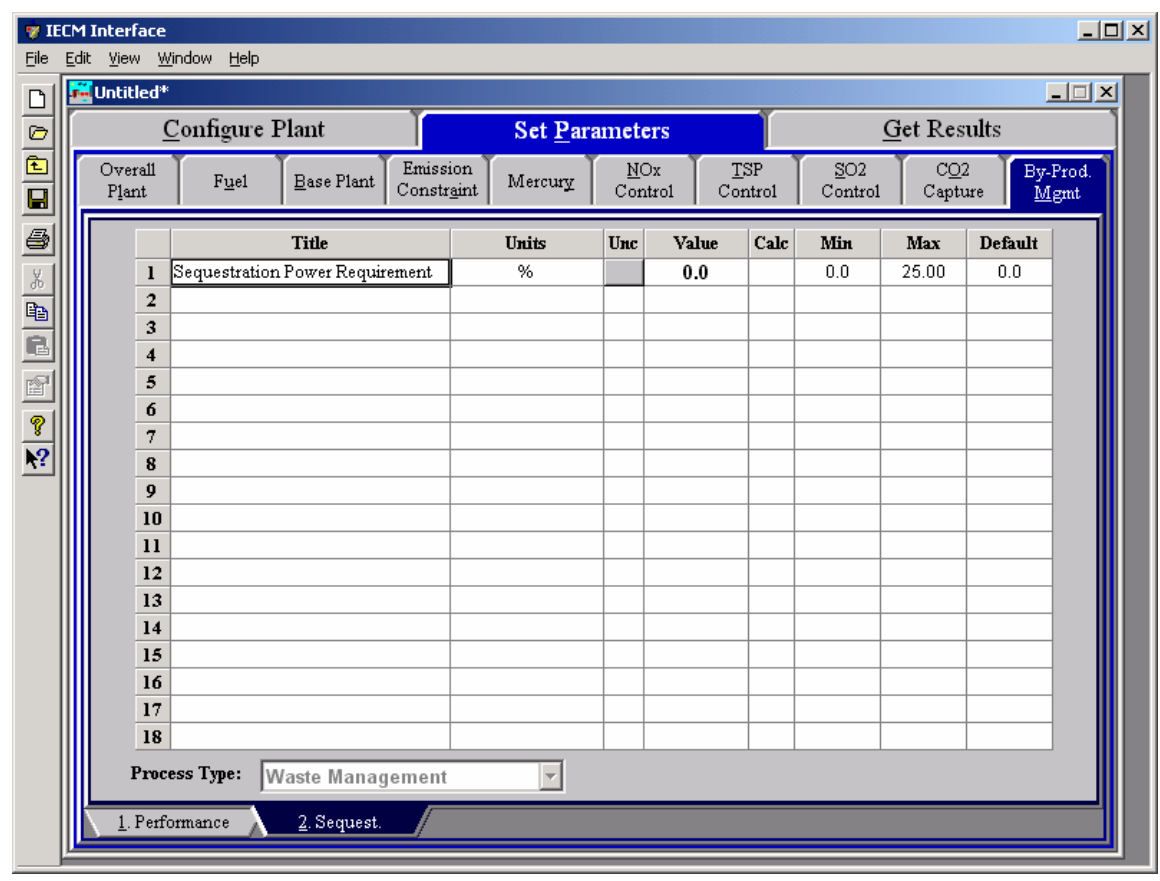

By Product Management - Sequestration input screen.

If the user has selected $\mathbf{C O}_{2}$ Capture in the Configure Plant program area this input screen will also be available. Its parameter is described briefly below.

Sequestration Power Requirement: The energy requirement is zero by default.

\section{By Products Management Bottom Ash Pond Diagram}

The By Product Management Technology Navigation Tab screens displays the flow rates of solid and liquid substances collected which require management (disposal or recovery). There are three By Product Management areas, Bottom Ash Pond, Flue Gas Treatment and Fly Ash Disposal. If $\mathbf{C O}_{2}$ Capture has been configured for the plant by the user then a Geological Reservoir is also available. These are accessed by the Process Type drop-down menu. Each 


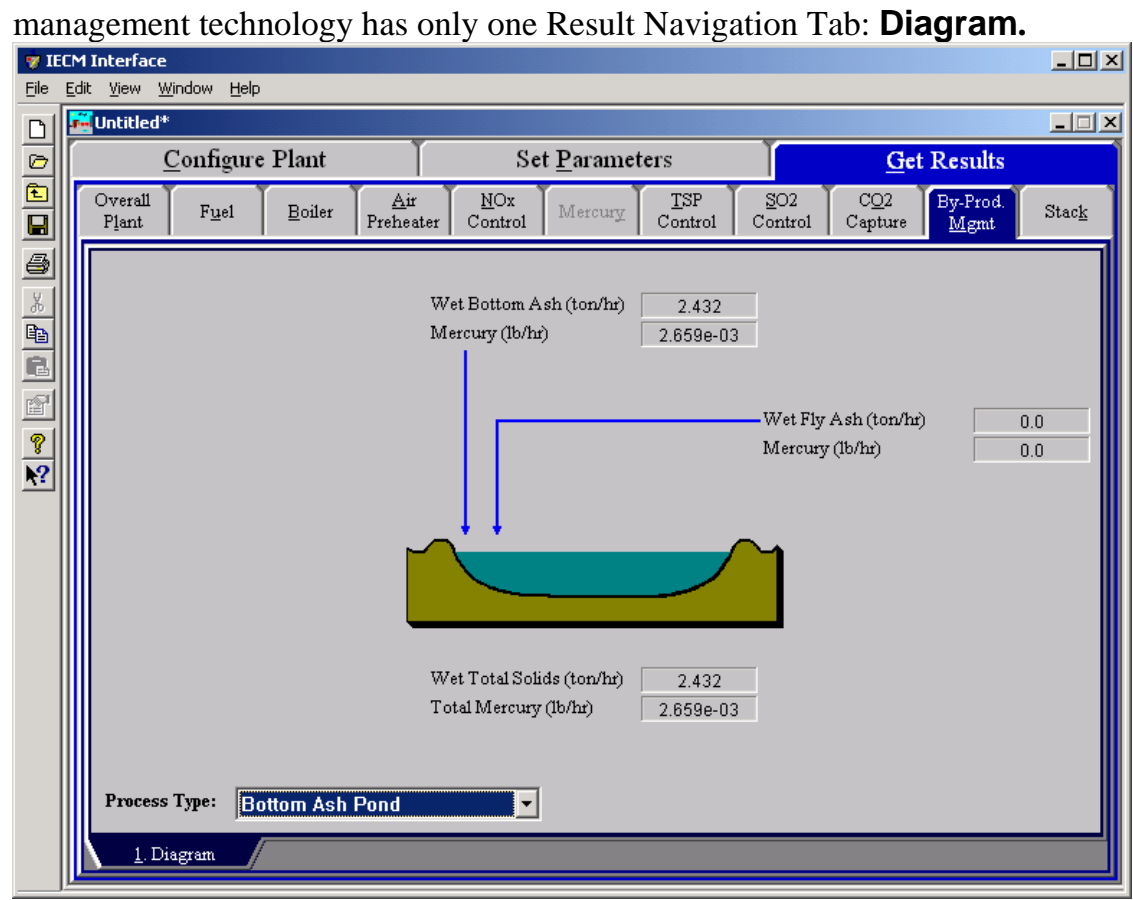

By Products Management Bottom Ash Pond-Diagram result screen

The Bottom Ash Pond Diagram result screen displays an icon for the Pond and values for major flows into it. Each result is described briefly below:

\section{Bottom Ash Pond Inputs}

Solids mixed with sluice water that are collected in the bottom of the boiler and by the particulate removal technologies are transported to the Pond for treatment. The IECM currently provides no additional treatment or consideration of these substances, and therefore simply reports the quantities entering the technology.

Wet Bottom Ash: Mass flow rate of bottom ash solids on a wet basis.

Mercury(contained in Bottom Ash): Mass flow rate of mercury present in the bottom ash solids on a wet basis.

Wet Fly Ash: Mass flow rate of total fly ash solids on a wet basis. This value is zero when the fly ash is disposed in a landfill.

Mercury(contained in Fly Ash): Mass flow rate of mercury present in the fly ash solids on a wet basis.

\section{Bottom Ash Pond - Totals}

Wet Total Solids: The sum of the fly ash and bottom ash solids on a wet basis.

Total Mercury: Mass flow rate of mercury present in the combined bottom ash and fly ash solids on a wet basis.

\section{By Products Management Flue Gas Treatment Diagram}

The By Product Management Technology Navigation Tab screens displays the flow rates of solid and liquid substances collected which require management (disposal or recovery). There are three By Product Management areas, Bottom 
Ash Pond, Flue Gas Treatment and Fly Ash Disposal. If $\mathbf{C O}_{2}$ Capture has been configured for the plant by the user then a Geological Reservoir is also available. These are accessed by the Process Type drop-down menu. Each management technology has only one Result Navigation Tab: Diagram.

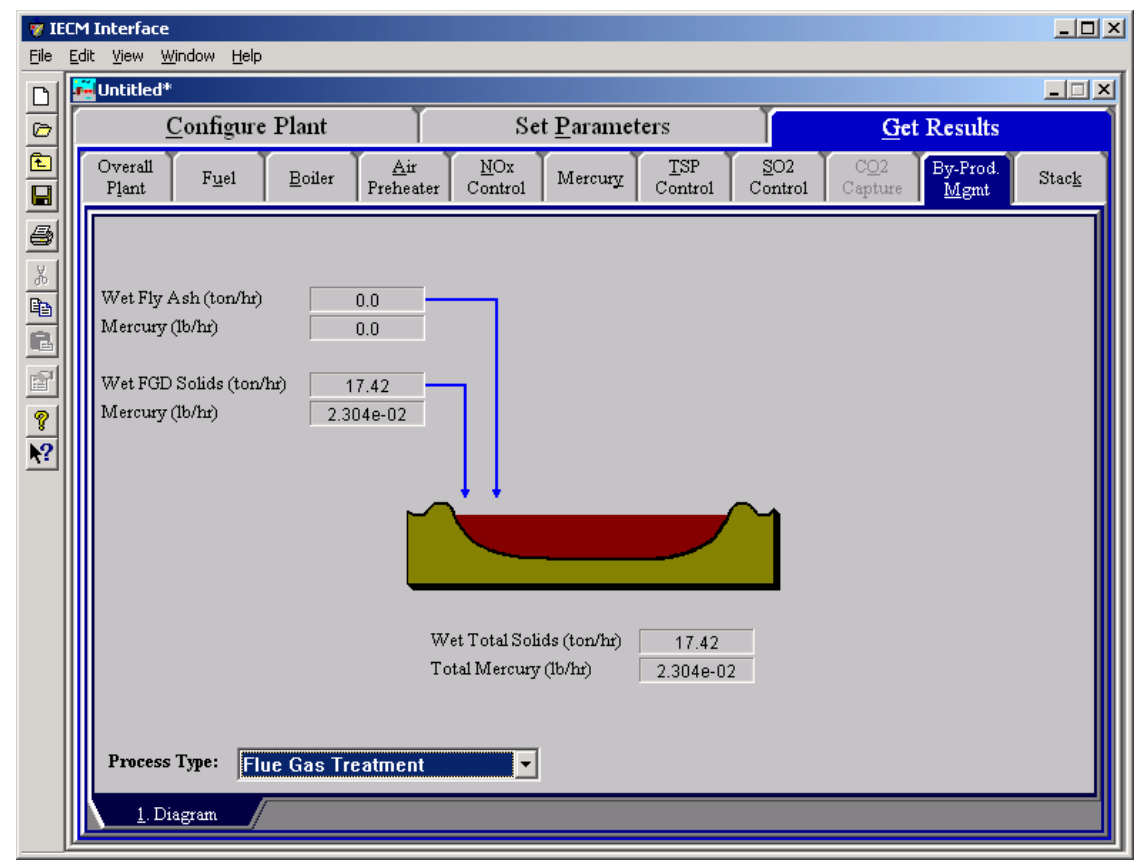

By Products Management Bottom Ash Pond-Diagram result screen

The Flue Gas Treatment Diagram result screen displays an icon for the Landfill and values for major flows into it. Each result is described briefly below:

\section{Flue Gas Treatment Inputs}

Solids mixed with sluice water that are collected in the bottom of the boiler and by the particulate removal technologies are transported to the Pond for treatment. The IECM currently provides no additional treatment or consideration of these substances, and therefore simply reports the quantities entering the technology.

Wet FGD Solids: Mass flow rate of wet FGD solids.

Mercury(contained in Wet FGD Solids): Mass flow rate of mercury present in the Wet FGD solids.

Wet Fly Ash: Mass flow rate of total fly ash solids on a wet basis. This value is zero when the fly ash is disposed in a landfill.

Mercury(contained in Fly Ash): Mass flow rate of mercury present in the fly ash solids on a wet basis.

\section{Flue Gas Treatment - Totals}

Wet Total Solids: The sum of the wet FGD solids and the fly ash on a wet basis.

Total Mercury: Mass flow rate of mercury present in the combined wet FGD solids and fly ash solids on a wet basis. 


\section{By Products Management Fly Ash Disposal Diagram}

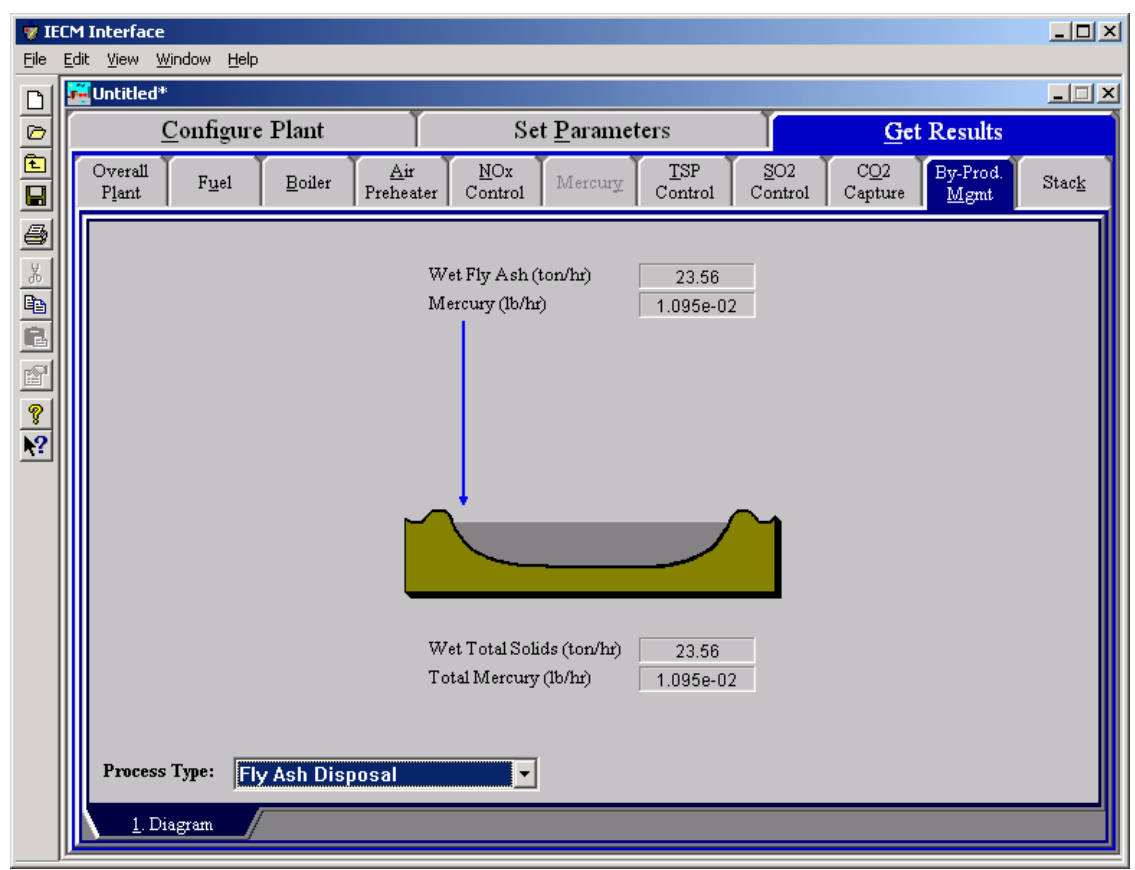

By Products Management Fly Ash Disposal_-Diagram result screen

The By Products Management Fly Ash Disposal Diagram result screen displays an icon for the Landfill and values for major flows into it. This screen is only an option if $\mathbf{C O}_{2}$ Capture has been configured for the plant by the user. Each result is described briefly below:

\section{Fly Ash Disposal Inputs}

Solids mixed with sluice water are collected in the particulate removal technologies and may be transported to the Landfill for treatment. The IECM currently provides no additional treatment or consideration of these substances, and therefore simply reports the quantities entering the technology.

Wet Fly Ash: Mass flow rate of total fly ash solids on a wet basis.

Mercury: Mass flow rate of mercury present in the fly ash solids on a wet basis.

\section{Fly Ash Disposal Totals}

Wet Total Solids: The sum of the fly ash and FGD solids on a wet basis.

Total Mercury: Mass flow rate of mercury present in the combined fly ash and FGD solids on a wet basis. 


\section{By Products Management Geological Resevoir Diagram}

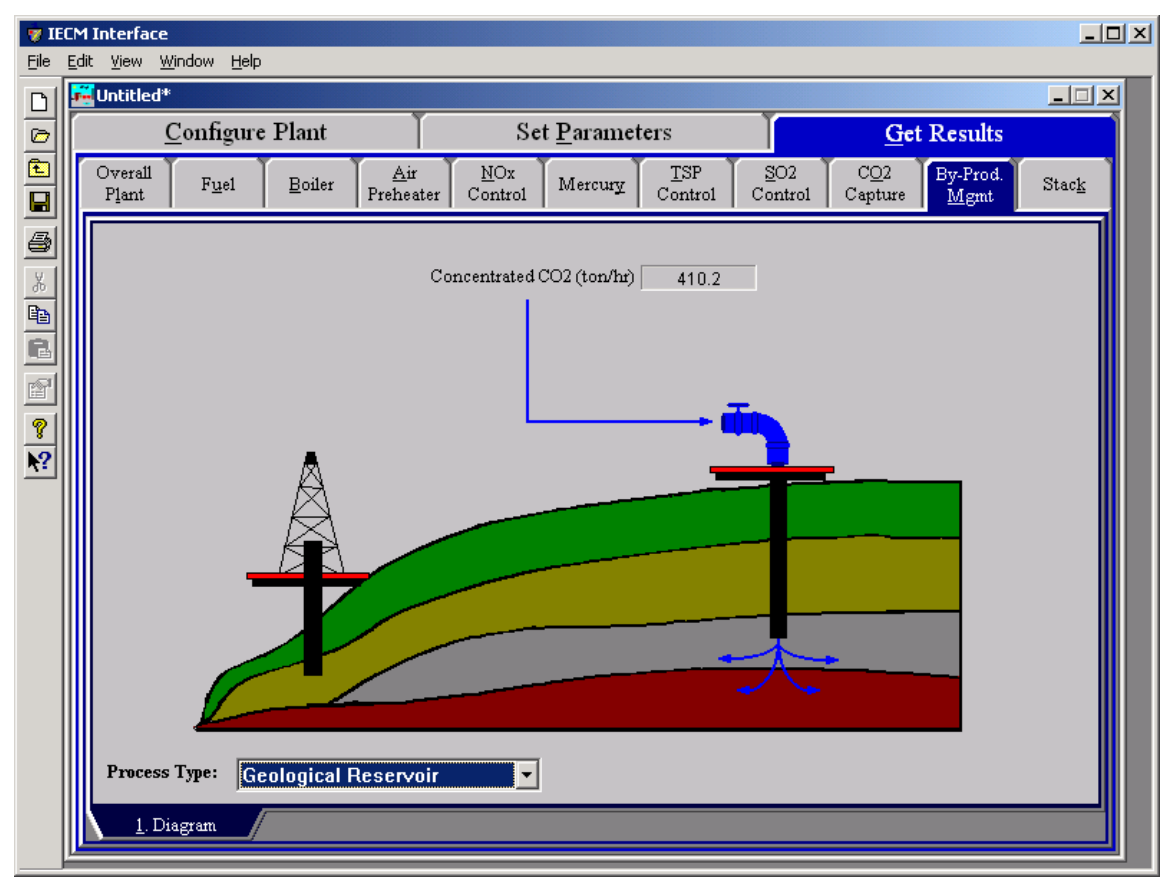

By Product Management - Geological Reservoir diagram.

The By Products Management Geological Resevoir Diagram result screen displays an icon for the Geological Reservoir and values for the concentrated $\mathrm{CO}_{2}$ that flows into it. The result is described briefly below:

Concentrated $\mathrm{CO}_{2}$ : Mass flow rate of $\mathrm{CO}_{2}$. 


\section{Stack}

\section{Stack Constraints Inputs}

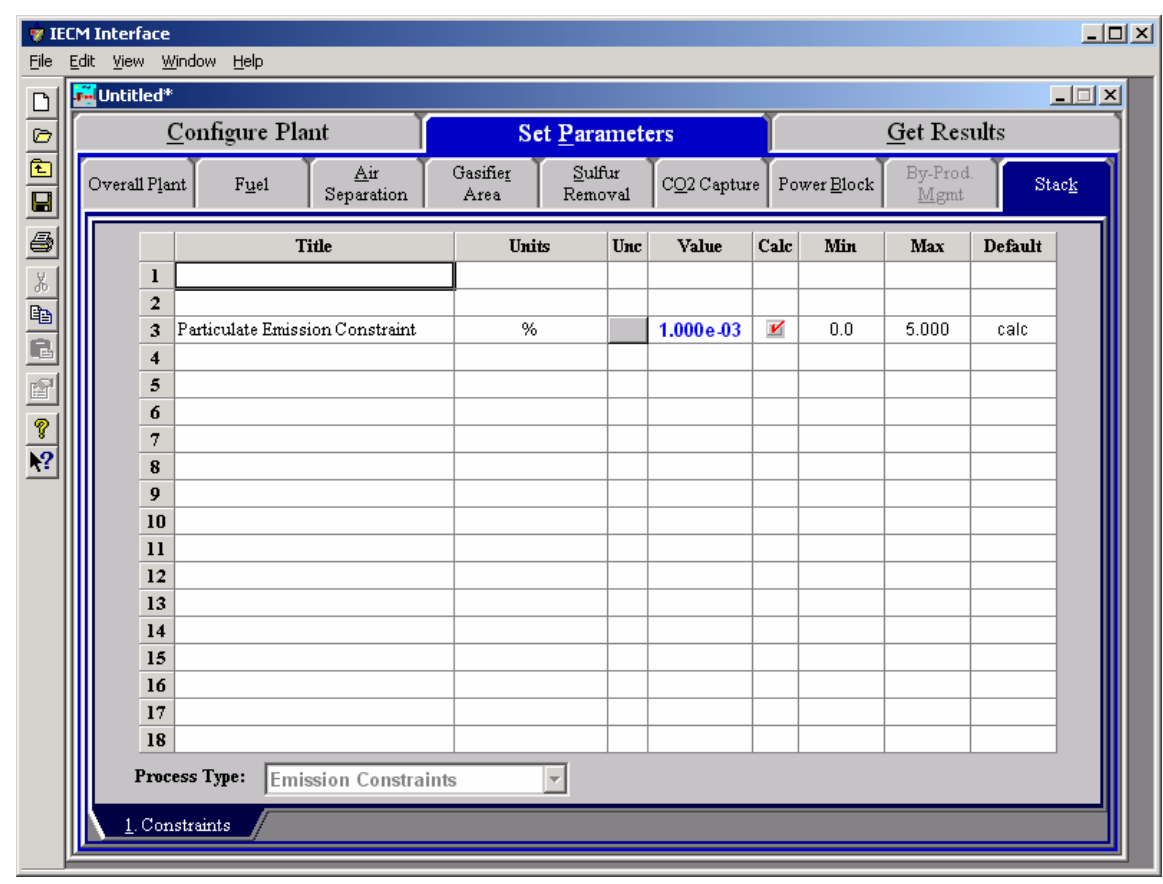

Stack-Contraints input screen.

The Stack Constraints input screen allows the user to enter the Particulate Emission Constriant as a percent. This screen is only available if the IGCC has been selected as the plant type. 


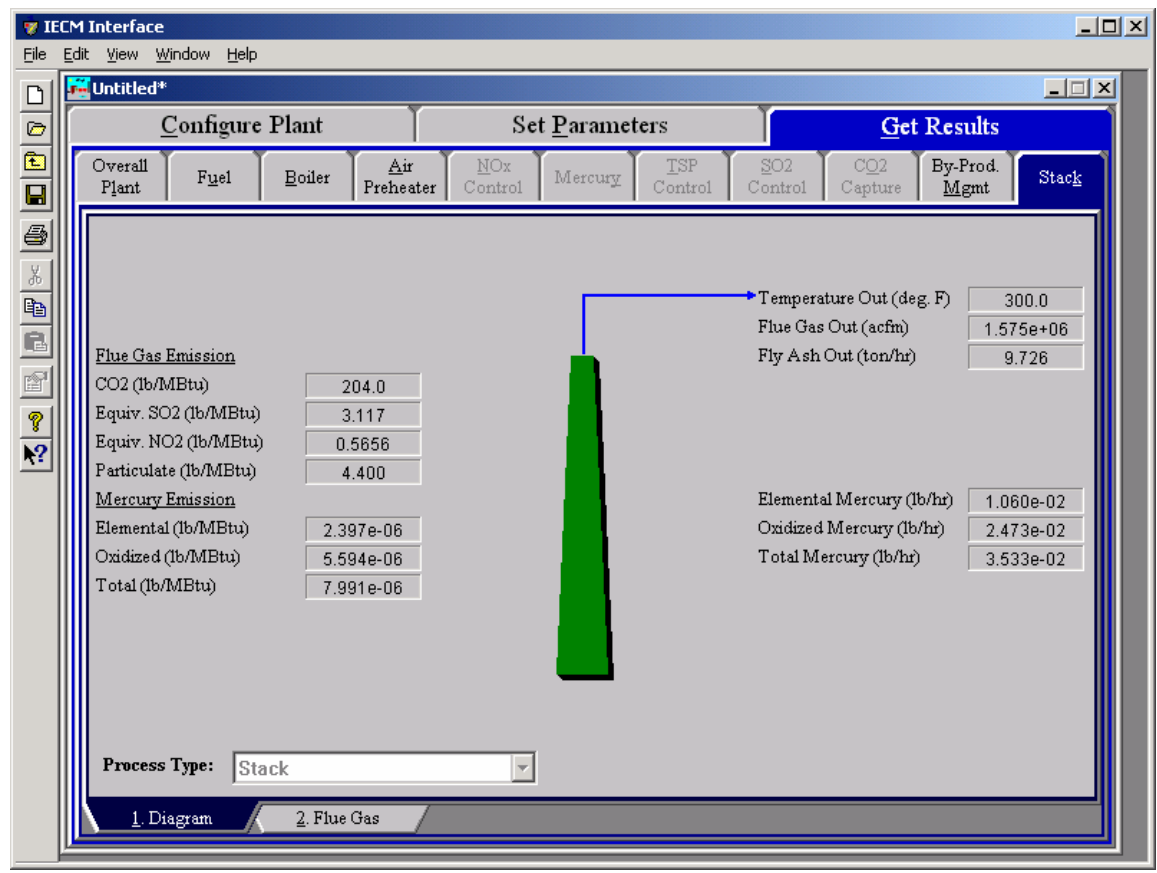

Stack-Diagram.

The Diagram result screen displays an icon for the stack and values for major flows out of it. Each result is described briefly below.

Temperature Out: Temperature of the flue gas exiting the stack.

Flue Gas Out: Volumetric flow rate of flue gas exiting the stack, based on the flue gas temperature exiting the stack and atmospheric pressure.

Fly Ash Out: Mass flow rate of solids in the flue gas exiting the stack.

\section{Flue Gas Emission}

$\mathrm{CO}_{2}$ : This is the number of pounds of $\mathbf{C O}_{2}$ vented to the air for every MBtu.

Equivalent $\mathrm{SO}_{2}$ : This is the number of pounds of Equivalent $\mathrm{SO}_{2}$ vented to the air for every MBtu.

Equivalent $\mathrm{NO}_{2}$ :This is the number of pounds of Equivalent $\mathrm{NO}_{2}$ vented to the air for every MBtu.

Particulate: This is the number of pounds of Particulate vented to the air for every MBtu.

\section{Mercury Emission}

Elemental: This is the number of pounds of Elemental Mercury vented to the air for every MBtu.

Oxidized: This is the number of pounds of Oxidized Mercury vented to the air for every MBtu.

Total: This is the number of pounds of Total Mercury vented to the air for every MBtu.

\section{Mercury Exiting Stack}


Elemental Mercury: Mass flow rate of elemental mercury $\left(\mathrm{Hg}^{0}\right)$ in the flue gas exiting the stack.

Oxidized Mercury: Mass flow rate of oxidized mercury $\left(\mathrm{Hg}^{+2}\right)$ in the flue gas exiting the stack.

Total Mercury: Mass flow rate of total mercury in the flue gas exiting the stack (elemental, oxidized, and particulate).

\section{Stack Flue Gas Results}

The FlueGas result screen displays a table of quantities of flue gas components exiting the stack. For each component, quantities are given in both moles and mass per hour.

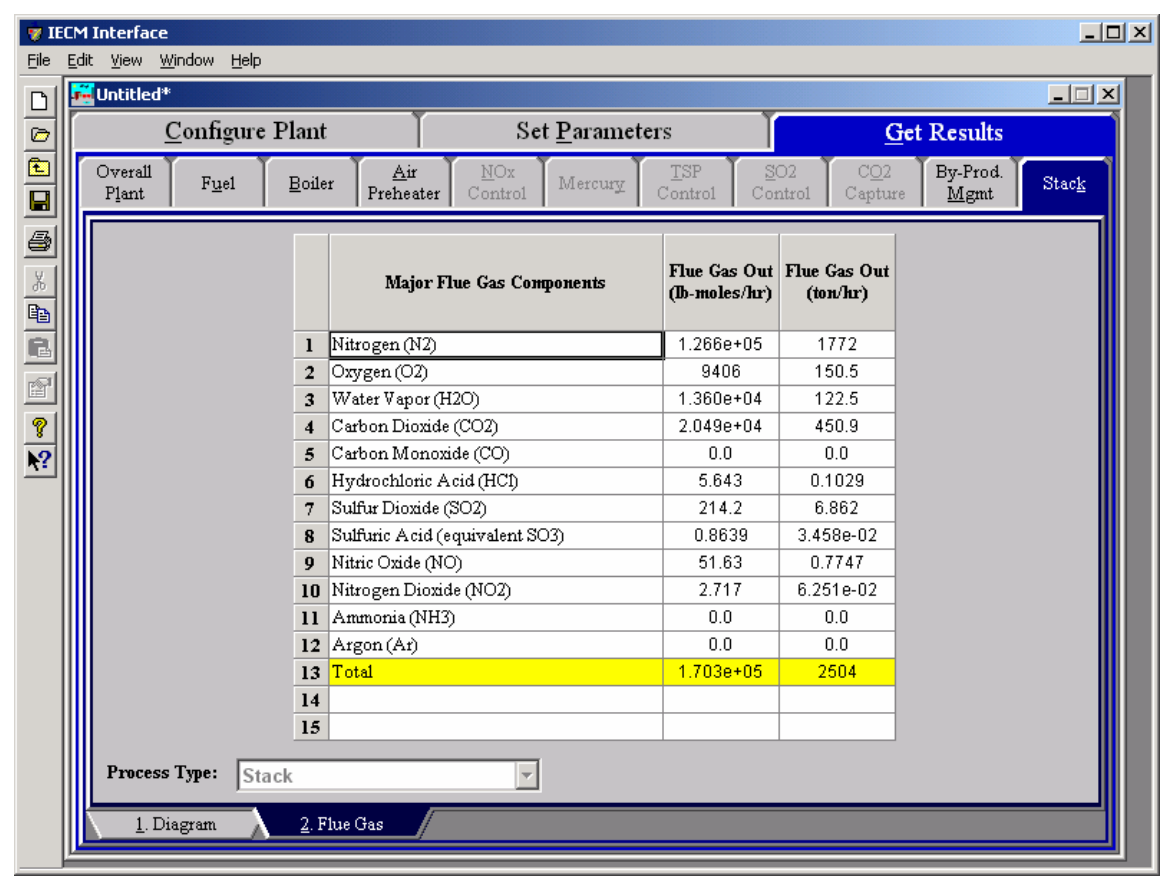

Stack Flue Gas result screen.

\section{Major Flue Gas Components}

Each result is described briefly below

Nitrogen $\left(\mathrm{N}_{2}\right)$ : Total mass of nitrogen.

Oxygen $\left(\mathrm{O}_{2}\right)$ : Total mass of oxygen.

Water Vapor $\left(\mathrm{H}_{2} \mathrm{O}\right)$ : Total mass of water vapor.

Carbon Dioxide $\left(\mathrm{CO}_{2}\right)$ : Total mass of carbon dioxide.

Carbon Monoxide (CO): Total mass of carbon monoxide.

Hydrochloric Acid ( $\mathbf{H C l})$ : Total mass of hydrochloric acid.

Sulfur Dioxide $\left(\mathrm{SO}_{2}\right)$ : Total mass of sulfur dioxide.

Sulfuric Acid (equivalent $\mathbf{S O}_{3}$ ): Total mass of sulfuric acid.

Nitric Oxide (NO): Total mass of nitric oxide.

Nitrogen Dioxide $\left(\mathrm{NO}_{2}\right)$ : Total mass of nitrogen dioxide. 
Ammonia $\left(\mathrm{NH}_{3}\right)$ : Total mass of ammonia.

Argon(Ar): Total mass ofargon.

Total: Total of the individual components listed above. This item is highlighted in yellow. 


\section{Units}

\section{Units Inputs}

Inputs may be entered using different units. Changing the units in which inputs are entered using the Input Tools floating palette is described in Getting Started. This section will describe the various unit settings in detail

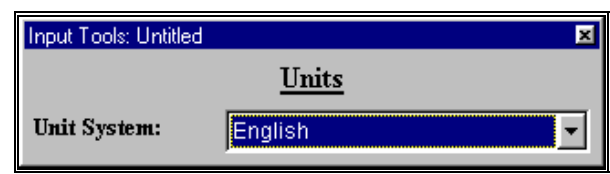

The Input Tools Floating Palette

\section{Unit System}

The Unit System option determines the unit system in which input values are entered. The choices are English and Metric. The default setting is English.

\section{Units Results}

Results may be displayed in different units. Changing the units in which results are displayed using the Result Tools floating palette is described in Getting Started. This section will describe the various unit settings in detail.

\begin{tabular}{|lll|}
\hline Result Tools: Untilled & \\
Result Type: & \multicolumn{1}{c|}{ Deterministic } \\
& \multicolumn{1}{|c|}{ Units } \\
Unit System: & English \\
Time Period: & Default \\
Perf. Table: & Default \\
Cost Table: & M\$(Cap), M\$Mr(0\&M) \\
& Revenue & - \\
Cost Year: & 1996 \\
Inflation Ctrl: & Constant & \\
\hline \hline
\end{tabular}

The Result Tools floating palette 


\section{Result Type}

The Result Type option determines the type of values displayed in the result tables. The choices available are Deterministic, Mean, Median (50th percentile), 2.5 percentile, 97.5 percentile, and Standard Deviation. The default setting is Deterministic.

\section{Unit System}

The Unit System option determines the unit system in which result values are displayed. The choices available are English and Metric. The default setting is English.

\section{Time Period}

The Time Period option determines the time period for which result values are displayed. The choices available are Default, Max Hourly and Annual Avg. The default setting is Default.

\section{Performance Table}

The Perf. Table option determines the units in which values are displayed on performance result screens. The choices available are Default, \% Total, mass $/ \mathbf{k W h}$, and mass/Btu in. The default setting is Default.

NOTE: The \% Total unit change can be used to determine the volume percent and weight percent of the components of the flue gas. This is possible when viewing the "Gas Summary" result table for any control technology.

\section{Cost Table}

The Cost Table option determines the units in which values are displayed on cost result screens. The choices available are $\mathbf{M} \$(C a p), \mathbf{M} \$ \mathbf{y r}(\mathbf{O} \& \mathbf{M})$ and $\$ / \mathbf{k W}(\mathrm{Cap})$, mills/kWh(O\&M). The default setting is $\mathbf{M} \$(\mathbf{C a p}), \mathbf{M} \$ \mathbf{y r}(\mathbf{O} \& \mathbf{M})$.

\section{Cost Year}

The Cost Year option determines the year for which values are displayed on cost result screens. You may choose any year between 1977 and 1998. The default setting is 1996.

\section{Inflation Control}

The Inflation Ctrl option determines the method by which inflation is calculated for cost result screens. The choices available are Constant and Current. The default setting is Constant. 


\section{Working with Graphs}

\section{Graphs and Values}

The table and diagram results are all deterministic values; that is, uncertainties are not taken into consideration. Graphs are probabilistic. This is different from previous versions of the IECM that used Analytica as a calculation engine. Analytica uses the expected value in table and diagram results, which takes into consideration the uncertainties defined.

The new IECM plots all samples on the CDF graph. The Analytica results in the Macintosh IECM version only displayed every fourth value (a value that could be adjusted within the model). By eliminating all but every fourth value, the Mac version of the IECM "smoothed" the graph.

\section{Copying Graphs}

If a graph window is active, you may use the Windows copy function to copy the graph to the clipboard. Do any one of the following:

- Pull down the Edit menu and select $\underline{\text { Copy. }}$

- $\quad$ Click the Copy button on the toolbar on the left side of the IECM window.

- Press Ctrl-C.

NOTE: When pasting the graph into other applications, it is not certain in which format Windows will paste the graph. Windows may paste a Bitmap image, a Windows Metafile, or a list of values taken from the graph. To be sure how the graph will be reproduced, use the Save $\underline{A} \mathbf{s}$ function to save the graph, then import it into the application.

\section{Saving Graphs}

To save a graph:

1. Make sure that the graph window is the active window.

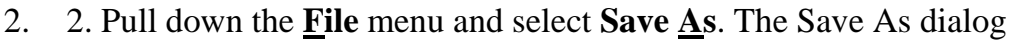
box will display 


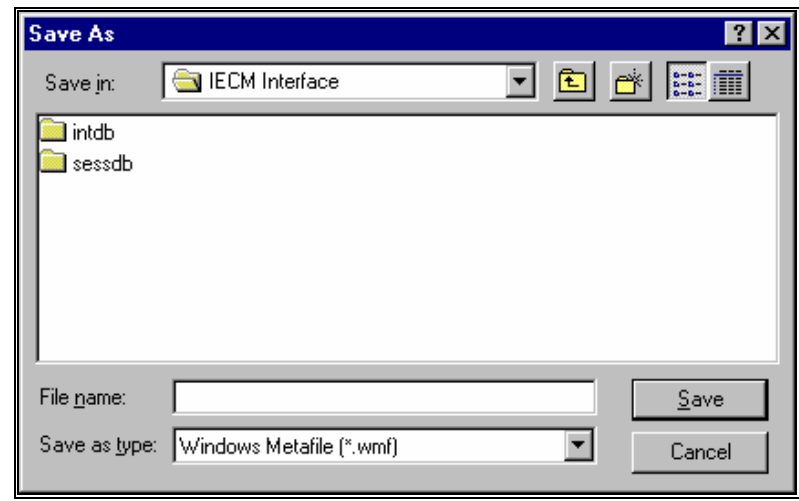

The Save As dialog box

3. Choose the folder to which you want to save from the pull-down Save in: box.

4. Type the filename in the File name: box.

5. Choose the file format you would like to use from the pull-down Save as type: box. Current options are:

- Windows Metafile (a drawing with separate objects)

- Windows Bitmap

- Text (the $\mathrm{x}$ and $\mathrm{y}$ coordinates)

6. Click the $\underline{\text { Save button. }}$ 


\section{Running a Probabilistic Analysis}

\section{Uncertainty Analysis}

As noted in the introduction, a unique feature of the IECM is its ability to analyze uncertainties probabilistically. You may assign probability distributions to any input parameter, including calculated parameters. The combined effect of all uncertain parameters is then calculated. This chapter describes again how to specify input probability distributions, and how to set several additional parameters needed to conduct a probabilistic analysis.

Even after probabilistic values have been set you do not have to use them. Probabilistic analysis can be turned on or off individually for technologies or input types or all at once. Turning the probabilistic calculations on and off for particular portions of the plant allows you to evaluate the major sources of uncertainty.

\section{Uncertainty Distributions}

The entry of uncertainty distributions is covered briefly in Getting Started This section gives a more detailed description of the process.

\section{Uncertainty Parameters}

Each uncertainty distribution requires one or more parameters. The table below lists the parameters and numerical value limits required for each distribution type.

\begin{tabular}{||l|c|c|c|c||}
\hline \hline Function & Operator & min or mean & mode & max or sdev \\
\hline Normal, Half- & $*$ & $\mathrm{x}>=0$ & $\mathrm{~N} / \mathrm{A}$ & $\mathrm{x}>0$ \\
normal(s) & + & $\mathrm{x}$ & $\mathrm{N} / \mathrm{A}$ & $\mathrm{x}>0$ \\
\hline LogNormal & $*$ & $\mathrm{x}>0$ & $\mathrm{~N} / \mathrm{A}$ & $\mathrm{x}>=1$ \\
& + & $\mathrm{x}>0$ & $\mathrm{~N} / \mathrm{A}$ & $\mathrm{x}>=1$ \\
\hline Uniform & $*$ & $\mathrm{x}>=0$ & $\mathrm{~N} / \mathrm{A}$ & $\mathrm{x}>=0$ \\
& + & $\mathrm{x}$ & $\mathrm{N} / \mathrm{A}$ & $\mathrm{x}$ \\
\hline Triangular & $*$ & $\mathrm{x}>=0$ & $\mathrm{x}>=0$ & $\mathrm{x}>=0$ \\
& + & $\mathrm{x}$ & $\mathrm{x}$ & $\mathrm{x}$ \\
\hline Fractiles & $*$ & $\mathrm{x}>=0$ & $\mathrm{~N} / \mathrm{A}$ & $\mathrm{N} / \mathrm{A}$
\end{tabular}




\begin{tabular}{||l|c|c|c|c||} 
& + & N/A & N/A & N/A \\
\hline Wedge & $*$ & $\mathrm{x}>=0$ & N/A & $\mathrm{x}>=0$ \\
& + & $\mathrm{x}$ & N/A & $\mathrm{x}$ \\
\hline
\end{tabular}

\section{Distribution Types}

Several types of probability distributions are provided with the IECM. Brief descriptions of each uncertainty distribution are included in the model when the uncertainty editor is selected; the information required, and additional notes, appear below. Distributions that are easiest to use are designated with a dagger $(\dagger)$. Consult a standard statistics reference for additional information.

\section{None}

None represents no uncertainty.

\section{Normal Distributions}

†Normal (mean, stddev) returns a continuous, normal Gaussian probability distribution with the specified mean and the standard deviation, stddev.

†Neghalf_Normal (mean, stddev) returns the lower half of a normal Gaussian probability distribution with the specified mean and the standard deviation, stddev.

†Half_Normal (mean, stddev) returns the upper half of a normal Gaussian probability distribution with the specified mean and the standard deviation, stddev.

This bell-shaped distribution is often assumed in statistical analysis as the basis for unbiased measurement errors. The normal distribution has infinite tails; however, over 99 percent of all values of the normal distribution lie within plus or minus three standard deviations of the mean. Thus, when used to represent uncertainty in physical quantities which much be greater than zero, the standard deviation should not be more than about 20 or 30 percent of the mean.

\section{Lognormal Distribution}

Lognormal (median, gsdev) returns a continuous lognormal probability distribution with the specified median and the geometric standard deviation, gsdev. The geometric standard deviation must be 1 or greater.

This distribution is usually used to represent uncertainty in physical quantities which must be positive values that are positively skewed, such as the ambient concentration of a pollutant. This distribution may be appropriate when uncertainties are expressed on a multiplicative order-of-magnitude basis (e.g., factor of 2) or when there is a probability of obtaining extreme large values.

\section{Uniform Distribution}

†Uniform (min, max) returns a continuous probability distribution in which every value between min and max has an equal chance of occurring.

Use this when you are able to specify a finite range of possible values, but are unable to decide which values in the range are more likely to occur than others. The use of the uniform distribution is also a signal that the details about uncertainty in the variable are not known. It is useful for screening studies. 


\section{Triangular Distribution}

†Triangular (min, mode, max) returns a continuous, triangular probability distribution bounded by min and max and with the specified mode.

Use this when you are able to specify both a finite range of possible values and a "most likely" (mode) value. The triangle distribution may be symmetric or skewed. Like the uniform distribution, this distribution indicates that additional details about uncertainty are not yet known. The triangle distribution is excellent for screening studies.

\section{Fractiles}

Fractiles. If $n$ is the number of elements in the list $L$, Fractiles $(\mathbf{L})$ returns a continuous probability distribution where the first element is the $0 \%$ fractile, the second is the $1 /(n-1)$ fractile, the third is the $2 /(n-1)$ fractile, and so on. (The values must be enclosed in square-brackets to register as a "list.")

This distribution looks like a histogram for large sample sizes and can be used to represent any arbitrary data or judgment about uncertainties in a parameter, when the parameter is continuous. It explicitly shows detail of the uncertainties. It is used in the IECM Model to represent all trace species data in the default databases. The finite range of possible values is divided into subintervals. Within each subinterval, the values are sampled uniformly according to a specified frequency for each subinterval.

\section{Wedge Distribution}

†Wedge (min, max) returns a continuous wedge-shaped probability distribution increasing linearly from $\mathbf{m i n}$ to max.

Use this when you are able to specify a finite range of possible values. The wedge distribution increases linearly from zero probability at the minimum value to the maximum probability at the maximum value. Like the uniform distribution, this distribution indicates that additional details about uncertainty are not yet known. This is a special case of the triangular distribution described below.

\section{Configuring Uncertainty in Results}

Some uncertainty parameters may be changed while results are displayed. These are modified using the Uncertainty Tools Floating Palette

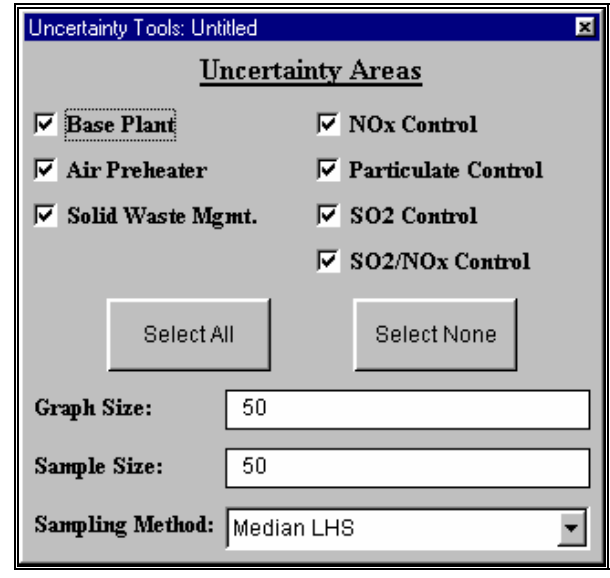

The Uncertainty Tools floating palette 


\section{Uncertainty Areas}

You may choose technology or technologies for which you would like results with uncertain values by clicking the box to the left of each technology. You may select all or none by clicking the buttons at the bottom of the palette.

\section{Graph Size}

The sample size determines the number of possible data points used to draw a graph. This parameter determines how many of the total samples to use for the graph. This value cannot exceed the sample size.

\section{Sample Size}

You can also specify the number of samples used with the sampling method. This is the number of iterations performed in a probabilistic analysis. The appropriate sample size depends on the number and types of uncertainty distributions that are specified, and on the accuracy with which the distribution is to be estimated (especially the tails of the distribution). A sample size of 100 is the default. The maximum is 200. The calculation time and memory requirements are proportional to this value.

\section{Sampling Methods}

Input and output variables are related to each other by model definitions defined for each variable. These relationships are generally referred to as the "decision tree." The model uses this decision tree to determine which input variables must be calculated to specify the output variable. Only those input variables necessary to specify the output variable value are calculated.

Since each input variable can be expressed as a non-singular distribution, a method of sampling the inputs must be determined. Several methods are available in the model, ranging from a deterministic or single "best guess" value to a completely random sampling of each input distribution. The sampling methods all produce sets of values for the inputs. These sets together form the "sampling space."

\section{Deterministic Evaluation}

Output values can be determined by using the most probable value for each input. This method is frequently referred to as the "best guess."

Input variables can be treated deterministically either by specifying only a single value, or by selecting the "Off" option for the "Uncertainty Distribution" pane. This option forces all uncertain parameters to be evaluated deterministically. Selecting the "Off" option forces each uncertainty function used in the decision tree to be evaluated using its expected value. This option overrides any particular uncertainty distribution types.

\section{Monte Carlo}

Monte Carlo is the simplest and best-known sampling method. It draws values at random from the uncertainty distribution of each input variable in the decision tree. For a particular sampling run, each input variable is randomly sampled once. The random samples from each input result in one final output value. This process is repeated $m$ times and results in a final solution set. This set can then be evaluated with standard statistical techniques to determine the mean, precision, and confidence. 
This method has the advantage of providing an easy method of determining the precision for a specific number of samples using standard statistical techniques. However, it suffers from requiring a large number of samples for a given precision. It also has the drawback of substantial noise in the resulting distribution. For these reasons, Latin Hypercube sampling is preferred as the model default.

\section{Latin Hypercube}

Latin Hypercube is a stratified sampling method that divides the sampling space into equally probable intervals, or strata. For each input variable, the method samples each interval in a random order. When the samples from each input variable are combined, one resultant output is determined. This process is repeated $m$ times, forming a final result of $m$ output values. These $m$ output values contain the uncertainty of the output variable, based on all the uncertainties of the entire set of input variables. The value $m$ is referred to as the sample size.

The model contains two variations of Latin Hypercube sampling: Random and Median. Random Latin Hypercube (RLH) samples each strata randomly, while the Median Latin Hypercube (MLH) samples each strata by its median value. (See: Diwekar, U.M. and J.R. Kalagnanam, (1997) "Efficient Sampling Technique for Optimization under Uncertainty,” AIChE Journal, Vol. 43, No. 2, pp. 440-7.) Median Latin Hypercube is the default sampling method.

Both forms of Latin Hypercube have the advantage of sampling more uniformly over the input distributions relative to Monte Carlo sampling, resulting in less noise in the final distribution. Another advantage is the reduced number of samples that must be taken to satisfy a given precision. Latin Hypercube has the drawback that the precision is more difficult to calculate using statistical methods. Finally, the output is random but not independent.

\section{Hammersley}

A new sampling technique has been added to the IECM which is more efficient than either the Monte Carlo or Latin-Hypercube sampling techniques. It is called the Hammersley sequence sampling technique. (See: Diwekar, U.M. and J.R.

Kalagnanam, (1997) "Efficient Sampling Technique for Optimization under Uncertainty,” AIChE Journal, Vol. 43, No. 2, pp. 440-7.) The sampling method is loosely based on the Monte Carlo method. However, instead of using a random number generator, it uses a quasi-random number generator based on Hammersley points to uniformly sample a unit hypercube. These points are an optimal design for placing $\mathrm{n}$ points on a k-dimensional hypercube. The sample points are then inverted over a cumulative probability distribution to define the sample set for any uncertainty variable.

Hammersley has the advantage of high precision and consistent behavior in addition to better computational efficiency. The method reduces the number of samples required relative to the other sampling methods for calculating uncertainty by a factor of 2 to 100. The actual sample reduction varies with the uncertainty function being sampled. 



\section{Appendix A - Introduction to Uncertainty Analysis}

\section{Uncertainty Analysis}

The following section is provided as a means of introducing uncertainty analysis as a tool for model design and operation. However, you should consult standard statistical and other texts (e.g., Morgan and Henrion, Uncertainty, Cambridge Press, 1990) to develop a more complete understanding of the subject.

\section{Introduction}




\section{Philosophy of Uncertainty Analysis}

The classical approach to probability theory requires that estimates for probability distributions be based on empirical data. However, in many practical cases, the available data may not be available or relevant to the problem at hand. Thus, statistical manipulation of data may be an insufficient basis for estimating uncertainty. Engineering analysis or judgments about the data may be required.

An alternative approach is the "Bayesian" view. It differs in how probability distributions are interpreted. The probability of an outcome is your "degree of belief" that the outcome will occur, based on all of the relevant information you currently have about the system. Thus, the probability distribution may be based on empirical data and/or other considerations, such as your own technically-informed judgments. The assessment of uncertainties requires thought about all possible outcomes and their likelihood, not just the "most likely" outcome. The advantage to thinking systematically and critically about uncertainties is the likelihood of anticipating otherwise overlooked problems, or identifying potential payoffs that might otherwise be overlooked.

\section{Types of Uncertain Quantities}

There are a number of types of uncertainty to consider when developing a probability distribution for a variable. Some of these are summarized briefly here.

Statistical error is associated with imperfections in measurement techniques. Statistical analysis of test data is thus one method for developing a representation of uncertainty in a variable.

Empirical measurements also involve systematic error. The mean value of a quantity may not converge to the "true" mean value because of biases in measurement and procedures. Such biases may arise from imprecise calibration, faulty reading of meters, and inaccuracies in the assumptions used to infer the actual quantity of interest from the observed readings of other quantities. Estimating the possible magnitude of systematic error may involve an element of engineering judgment.

Variability can be represented as a probability distribution. Some quantities are variable over time. For example, the composition of a coal (or perhaps a sorbent) may vary over time.

Uncertainty may also arise due to lack of actual experience with a process. This type of uncertainty often cannot be treated statistically, because it requires predictions about something that has yet to be built or tested. This type of uncertainty can be represented using technical estimates about the range and likelihood of possible outcomes. These judgments may be based on a theoretical foundation or experience with analogous systems.

\section{Encoding Uncertainties as Probability Distributions}

As indicated in the previous sections, there are two fundamental approaches for encoding uncertainty in terms of probability distributions. These include statistical estimation techniques and engineering judgments. A combination of both methods may be appropriate in many practical situations. For example, a statistical analysis of measured test data for a new emission control technology may be a starting point for thinking about uncertainties in a hypothetical commercial scale system. You must then consider the effect that systematic errors, variability, or uncertainties about 
scaling-up the process might have on interpreting test results for commercial-scale design applications.

\section{Statistical Techniques}

Statistical estimation techniques involve estimating probability distributions from available data. The fit of data to a particular probability distribution function can be evaluated using various statistical tests. For example, the cumulative probability distribution of a set of data may be plotted on "probability" paper. If the data plot as a straight line, then the distribution is normal. Procedures for fitting probability distribution functions are discussed in many standard texts on probability and are not reviewed here.

Such procedures can be utilized to obtain distribution functions for many of the power plant parameters in the IECM when data are available for operating plants. In other cases, especially where data are limited, expert technical judgments may be necessary to develop appropriate distribution functions for model parameters. The emphasis of the discussion below is on the situations where statistical analysis alone may be insufficient.

\section{Judgments about Uncertainties}

In making judgments about a probability distribution for a quantity, there are a number of approaches (heuristics) that people use which psychologists have observed. Some of these can lead to biases in the probability estimate. Three of the most common are briefly summarized.

1. Availability. The probability experts assign to a particular possible outcome may be linked to the ease (availability) with which they can recall past instances of the outcome. For example, if tests have yielded high sorbent utilization, it may be easier to imagine obtaining a high sorbent utilization in the future than obtaining lower utilization. Thus, one tends to expect experts to be biased toward outcomes they have recently observed or can easily imagine, as opposed to other possible outcomes that have not been observed in tests.

2. Representativeness has also been termed the "law of small numbers." People may tend to assume that the behavior they observe in a small set of data must be representative of the behavior of the system, which may not be completely characterized until substantially more data are collected. Thus, one should be cautious in inferring patterns from data with a small number of samples.

3. Anchoring and adjustment involves using a natural starting point as the basis for making adjustments. For example, an expert might choose to start with a "best guess" value, which represents perhaps an average or most likely (modal) value, and then make adjustments to the best guess to achieve "worst" and "best" outcomes as bounds. The "worst" and "best" outcomes may be intended to represent a 90 percent probability range for the variable. However, the adjustment from the central "best guess" value to the extreme values is often insufficient, with the result that the probability distribution is too tight and biased toward the central value. This phenomenon is overconfidence, because the expert's judgment reflects less uncertainty in the variable than it should. The "anchor" can be any value, not just a central value. For example, if an expert begins with a "worst" case value, the entire distribution may be biased toward that value. 
Judgments also may be biased for other reasons. One common concern is motivational bias. This bias may occur for reasons such as:
a. a person may want to influence a decision to go a certain way;
b. the person may perceive that they will be evaluated based on the outcome and might tend to be conservative in their estimates;
c. the person may want to suppress uncertainty that they actually believe is present in order to appear knowledgeable or authoritative; and
d. the expert has taken a strong stand in the past and does not want to appear to contradict himself by producing a distribution that lends credence to alternative views.

\section{Designing an Elicitation Protocol}

Studies of uncertainty judgment show that the most frequent problem encountered is overconfidence. Knowledge of how people make judgments about probability distributions can be used to design a procedure for eliciting these judgments. The appropriate procedure depends on the background of the expert and the quantity for which the judgment is being elicited. For example, if you have some prior knowledge about the shape of the distribution for the quantity, then it may be appropriate to ask you to think about extreme values of the distribution and then to draw the distribution yourself. On the other hand, if you have little statistical background, it may be more appropriate to ask you a series of questions. For example, you might be asked the probability of obtaining a value less than or equal to some value $\mathrm{x}$, and then the question is repeated for a few other values of $\mathrm{x}$. Your judgment can then be graphed by an elicitor, who would review the results of the elicitation with you to see if you are comfortable with your answers.

To overcome the typical problem of overconfidence, consider extreme high or low values before asking about central values of the distribution. In general, experts' judgments about uncertainties tend to improve when:

1. the expert is forced to consider how things could turn out differently than expected (e.g., high and low extremes); and

2. the expert is asked to list reasons for obtaining various outcomes.

While the development of expert judgments may be flawed in some respects, it does permit a more robust analysis of uncertainties in a process when limited data are available. Furthermore, in many ways, the assessment of probability distributions is qualitatively no different than selecting single "best guess" values for use in a deterministic estimate. For example, a "best guess" value often represents a judgment about the single most likely value that one expects to obtain. The "best guess" value may be selected after considering several possible values. The types of heuristics and biases discussed above may play a similar role in selecting the value. Thus, even when only a single "best guess" number is used in an analysis, a seasoned engineer usually has at least a "sense" for "how good that number really is." This may be why engineers are usually able to make judgments about uncertainties, because they implicitly make these types of judgments routinely.

\section{A Non-technical Example}

To illustrate the process of defining a subjective probability distribution, let's turn to a simple example of eating lunch in a cafeteria. How long does it take from the time 
you enter the cafeteria to the time you pay the cashier? Assume that you enter at 12:05 p.m. on a weekday and that you purchase your entire meal at the cafeteria. The answer you give may depend on your recent experiences in the cafeteria. Think about the shortest possible time that it could take (suppose nobody else is getting lunch) or the longest possible time (everyone shows up at the same time). What is the probability that it will take 2 minutes or less? 45 minutes or less? Is the probability that it takes 10 minutes or less greater than 50 percent? etc. After asking yourself a number of questions such as these, it should be possible to draw a distribution for your judgment regarding the time require to obtain and purchase lunch at the cafeteria. Such a distribution might take the form of a fractile distribution giving the probabilities of different waiting times to purchase lunch. For example, your evaluation may conclude that there is only a 1 percent (1 in 100) chance it will take one minute or less, a 60 percent chance of 1 to 10 minutes, a 25 percent likelihood of 10 to 15 minutes, and a 14 percent chance of up to 25 minutes. These probability intervals can be drawn as a histogram and translated into a fractile distribution for a probabilistic analysis.

\section{A Technical Example}

A second example focuses on a performance parameter for an advanced pollution control system. This parameter has an important effect on system performance and cost.

The example focuses on an assessment of uncertainty in the performance of an innovative emission control system for coal-fired power plants. In this system, a chemical sorbent circulates between a fluidized bed reactor, where $\mathrm{SO}_{2}$ in the flue gas is removed by chemical reaction with the sorbent, and a regenerator, in which $\mathrm{SO}_{2}$ is evolved in a reaction of the sulfated sorbent with methane. There is no commercial experience with this system; the largest test unit has been sized to handle $100 \mathrm{scfm}$ of flue gas. Furthermore, the test units have used batch, rather than continuous, regeneration.

One of the key parameters affecting the performance and cost of this system is the regeneration efficiency, which is defined as the fraction of the spent sorbent which is converted for reuse. In small-scale tests in which the regeneration efficiency has been estimated, the efficiency was found to be roughly 30 to 50 percent. In a more recent test, the regeneration efficiency was not measured due to instrumentation difficulties; however, it may have been lower than the previously obtained values. Regeneration residence times were typically greater than 30 minutes.

A detailed modeling study of the regenerator estimated that a properly sized and designed unit, coupled with heating of the sorbent to a sufficiently high reaction temperature, would result in a regeneration efficiency of just over 99 percent at a 30 minute residence time.

A potential problem that may be occurring in the test units is that regenerated sorbent in the regenerator may be reabsorbing some of the evolved $\mathrm{SO}_{2}$. However, this was not considered in the modeling study of the regenerator.

Based on this information, it appears that it may be possible to achieve the design target of over 99 percent regeneration efficiency. Clearly, however, it is possible that the actual efficiency may be substantially less than this target value. As a worst case, we might consider the known test results as a lower bound. Thus, there is a small chance the regeneration efficiency may be less than 50 percent. We expect the regeneration efficiency to tend toward the target value of 99.2 percent. Thus, to represent the expectation that the efficiency will be near the target value, but may be substantially less, we can use a negatively skewed distribution. In this case, we 
assume a triangle with a range from, say, 50 to 99.2 percent with a mode also at 99.2 percent. The triangle in this case gives us a distribution with a mean of about 83 percent and a median of about 85 percent. This type of triangular distribution, in which a minimum, maximum, and modal value are specified, is often a convenient way of expressing uncertainty distributions when a little information is available. 


\section{Appendix B - Technical Support}

\section{Reaching Technical Support}

Questions, issues or concers regarding the Integrated Environmental Control Model should be directed to:

\section{Carnegie Mellon University}

BERKENPAS, MICHAEL B.

Office: Baker Hall 128B

Location: Pittsburgh, PA 15213

Phone: (412) 268-1088

FAX: (412) 268-1089

Email: mikeb@cmu.edu 

